\title{
Morphological Awareness Test for Reading and Spelling (MATRS): Technical Report
}

\author{
Kenn Apel, PhD \\ University of South Carolina \\ Yaacov Petscher, PhD \\ Florida State University \\ Florida Center for Reading Research \\ Victoria S. Henbest, PhD \\ University of South Alabama
}

Funding Statement: The research reported here was supported by the Institute of Education Sciences, U.S. Department of Education, through Grant R305A170065 to the University of South Carolina. The opinions expressed are those of the authors and do not represent views of the Institute or the U.S. Department of Education.

Acknowledgements: We would like to acknowledge June Maranville and Alexis Mitchell for their significant contributions to the project. We also would like to thank the numerous research assistants, school districts, administrators, and teachers who participated in this project. 
Morphemes are the smallest units of meaning in a language. Morphemes often are classified as free morphemes or base words (i.e., simple, stand-alone words, such as cat, pack, or, sweet), or bound morphemes or affixes (i.e., prefixes and suffixes added onto base words, such as cats, repack, and sweetly). Children begin using free morphemes with their first spoken words and then begin using bound morphemes shortly thereafter, by one-and-a-half or two-years of age (Apel \& Masterson, 2012). The use of these morphemes are spontaneous; children are not actively thinking about the morphemes used in spoken conversations.

Morphological awareness is the ability to consciously think about and/or manipulate morphemes within a word. Children begin to demonstrate the rudiments of morphological awareness, albeit somewhat implicit awareness, as early as kindergarten (e.g., Bourassa \& Treiman, 2009; Treiman \& Cassar, 1996). Fully defined, morphological awareness is the awareness of spoken and printed forms ${ }^{1}$ of morphemes, the meaningful changes affixes bring to base words (e.g., how $\underline{-S}$ can causes a singular noun to become a plural noun or how un- means the opposite), the way printed affixes connect to base words (e.g., how some suffixes require a consonant to be doubled or dropped when attached to a written base word), and the relation between base words and their inflected or derived forms (e.g., knowing that a variety of words are related because they share the same base word, such as friend, friendly, friendship, and friending). This comprehensive definition best captures the multiple aspects of morphological awareness (Apel, 2014).

\section{Morphological Awareness and Its Relation to Reading and Spelling}

Students' performance on morphological awareness tasks significantly predicts their literacy abilities. Even when other known predictors of literacy abilities are considered, such as phonemic awareness and/or vocabulary, morphological awareness remains a unique predictor of literacy skills (e.g., Apel, Wilson-Fowler, Brimo, \& Perrin, 2012; Deacon \& Kirby, 2004; Deacon, Kirby, \& Casselman-Bell, 2009; Tighe \& Schatschneider, 2016). Because of the strong relation of morphological awareness to word-level reading, reading comprehension, and spelling, researchers and educators recognize the importance of targeting students' morphological awareness skills to help improve their literacy skills. This focus on morphological awareness to improve literacy abilities makes sense intuitively given written English is a morphophonemic system; that is, English spelling relies on morphemes as much as it does phonemes to produce written words and convey their meaning (e.g., Moats, 1995; Treiman, 1998). To provide specific or prescriptive morphological awareness instruction, a comprehensive morphological assessment tool is needed.

\section{Measures of Morphological Awareness}

In the past, a psychometrically-reliable and valid measure that represents conceptually a comprehensive definition of morphological awareness did not existed. Instead, researchers and practitioners used one or more tasks that had been suggested to assess morphological awareness. However, when tasks used to assess morphological awareness do not align with a comprehensive definition of morphological awareness and, thus, do not completely assess all aspects of this

\footnotetext{
${ }^{1}$ Our definition of morphological awareness includes the conscious awareness of printed morphological forms. By doing so, it may seem a confound exists between morphological awareness and orthographic knowledge. However, as we define morphological awareness, the awareness of affixes, and the means by which they connect to base words, is an aspect of written language specific to morphological forms versus more general orthographic knowledge.
} 
linguistic awareness skill, researchers cannot be assured that their findings represent those that may be found with a more comprehensive measure of the skill. Likewise, educators who wish to improve the literacy skills of their students risk the chance of under-identifying those who have deficits in areas of morphological awareness that may appreciably impact reading and writing skills. Further, optimal instructional practices for all types of learners may not be possible without a comprehensive morphological awareness assessment tool. With a well-designed, prescriptive assessment, educators are better informed of particular weaknesses in students' morphological awareness skills that then can help them select specific, targeted interventions for those students.

Given past limitations associated with assessing morphological awareness abilities, the goal of this project was to develop a comprehensive assessment of morphological awareness that represented an all-inclusive definition of this linguistic awareness skill. Specifically, through an iterative process across a three year time span, our two primary goals were to:

1) develop a reliable assessment tool that effectively represented the dimensionality of morphological awareness and contained test items that were most informative (i.e., most discriminative) for purposes of identifying students who struggle with their morphological awareness abilities, and

2) establish the convergent and predictive validity of the new assessment tool with measures of literacy and literacy-related skills.

As a result of those aims, we created a comprehensive, reliable and valid morphological awareness assessment tool for students in grades 1-6: the MATRS (Morphological Awareness Test for Reading and Spelling). We targeted these grades for our project because research has demonstrated significant and sustained growth in morphological awareness across these school years (e.g., Berninger, Abbott, Nagy, \& Carlisle 2010) and that morphological awareness interventions in these elementary school years lead to gains in morphological awareness and literacy skills in relatively short amounts of time (e.g., Apel, Brimo, Diehm, \& Apel, 2013; Bower, Kirby, \& Deacon, 2010; Goodwyn \& Ahn, 2013). The following sections detail the results of this project.

\section{Participants}

\section{Technical Documentation}

A total of 5,403 students across first through sixth grade served as participants. The students were recruited from both public and private schools in the southeastern region of the United States. Parents of all students provided their consent for their children to participate, using a consent form approved by the university institutional review board. Specific demographic information for all the students is contained in Table 1.

\section{Item-Calibration Full Sample}

Across the two years of the item calibration study, we worked in the Southeastern United States. A total of 4,060 students participated in the two-year item-validation study in grade 1 ( $N$ $=807)$, grade $2(N=726)$, grade $3(N=756)$, grade $4(N=644)$, grade $5(N=626)$, and grade 6 $(N=501)$. Full sample demographics were $53.97 \%$ female; race/ethnicity was $43.81 \%$ White, 37.70\% Black, 9\% Multi-racial, 5.24\% Latinx, 1.50\% Asian, <1\% Native American, and 1.75\% No response. Over $90 \%$ of the sample reported mother and father education having completed high school or a GED. Ninety-four percent of the sample spoke English as the primary language, $3 \%$ spoke Spanish as the primary language, and 3\% of the sample spoke another primary 
language in the home. The majority of students participating in the item calibration study did not receive services (70\%) with approximately $7 \%$ of the sample receiving speech-services only, $9 \%$ receiving reading/writing services only, 9\% receiving multiple services, and 5\% noting other services that were provided.

Year 1 Item-Calibration Sample. A total of 1,122 students participated in Year 1 of the item-validation study in grade $1(N=179)$, grade $2(N=224)$, grade $3(N=167)$, grade $4(N=$ 210), grade $5(N=180)$, and grade $6(N=162)$. The sample in Table 1 showed $54.37 \%$ female; race/ethnicity was $41.09 \%$ White, 38.32\% Black, 9.72\% Multi-racial, 6.42\% Latinx, 1.6\% Asian, $<1 \%$ Native American, and $2.5 \%$ No response. Over $90 \%$ of the sample reported mother and father education having completed high school or a GED. Approximately $94 \%$ percent of the sample spoke English as the primary language, 4.81\% spoke Spanish as the primary language, and $1 \%$ of the sample spoke another primary language in the home. The majority of students participating in the item calibration study did not receive services (66\%) with approximately 7\% of the sample receiving speech-services only, 8.65\% receiving reading/writing services only, $11.5 \%$ receiving multiple services, and 5\% noting other services that were provided.

Year 2 Item-Calibration Sample. A total of 2,939 students participated in Year 2 of the item-validation study in grade $1(N=628)$, grade $2(N=502)$, grade $3(N=589)$, grade $4(N=$ 434), grade $5(N=446)$, and grade $6(N=339)$. The sample in Table 1 showed $53.8 \%$ female; race/ethnicity was $44.85 \%$ White, $37.46 \%$ Black, 6.5\% Multi-racial, $4.8 \%$ Latinx, 1.5\% Asian, $<1 \%$ Native American, and $4.3 \%$ No response. Over $90 \%$ of the sample reported mother and father education having completed high school or a GED. Approximately $94 \%$ percent of the sample spoke English as the primary language, 2.96\% spoke Spanish as the primary language, and $3.5 \%$ of the sample spoke another primary language in the home. The majority of students participating in the item calibration study did not receive services (73\%) with approximately $7 \%$ of the sample receiving speech-services only, $8.54 \%$ receiving reading/writing services only, $8.4 \%$ receiving multiple services, and $2 \%$ noting other services that were provided.

\section{MATRS Task Validation Sample}

In Year 3 of the study, a total of 1,343 students participated in a validation study in grade $1(N=293)$, grade $2(N=262)$, grade $3(N=233)$, grade $4(N=197)$, grade $5(N=173)$, and grade $6(N=185)$. The sample in Table 1 showed $53.09 \%$ female; race/ethnicity was $50.04 \%$ White, 30.16\% Black, 7.97\% Multi-racial, 6.48\% Latinx, 1.71\% Asian, $<1 \%$ Native American, and $2.64 \%$ No response. Over $90 \%$ of the sample reported mother and father education having completed high school or a GED. Approximately 94\% percent of the sample spoke English as the primary language, $4.17 \%$ spoke Spanish as the primary language, and $1.64 \%$ of the sample spoke another primary language in the home. The majority of students participating in the item calibration study did not receive services (70\%) with approximately $10 \%$ of the sample receiving speech-services only, $8.19 \%$ receiving reading/writing services only, $10 \%$ receiving multiple services, and $1.87 \%$ noting other services that were provided. 
MATRS TECHNICAL MANUAL 5

\section{Measures}

The students were administered the Morphological Awareness Test for Reading and Spelling (MATRS) as well as tests measuring their word-level reading, reading comprehension skills, spelling, phonological awareness, and receptive vocabulary skills. These standardized, norm-referenced measures were administered following the instructions in the test manuals. The order of administration of the tests varied within and across grades and sessions.

\section{Morphological Awareness Tasks}

The students were administered the MATRS to assess all aspects of morphological awareness (e.g., Apel, 2014). Specifically, we assessed their awareness of a) morphemes in both spoken and written forms, b) affix meanings and how they can change the grammatical class of base words, c) how written prefixes attach to base words, and d) the relation between base words and their inflected and/or derived forms. MATRS involves eight subtests. Because two of the subtests required reading skills beyond an early developmental level, first and second grade students were administered only six subtests. For all subtests, at least 1 sample item was given before the subtest itself was administered. All subtest real-word items were grade-level appropriate (e.g., items on tasks administered to third grade students represented words considered to be third-grade level words; SPELL-Links Word List Maker, 2010; Zeno, Ivens, Millard, \& Duvvuri, 1995). No items represented low frequency words (Zeno et al., 1995). Pseudoword items were created from grade-level real words. Subtest items represented both inflected and derived forms of base words. The number of subtest items were the same for each grade.

The Segmenting subtest, similar to that used in Casalis, Cole, \& Sopo (2004), was administered to examine the students' ability to identify morphemes in spoken multi-morphemic words. The administrator verbally produced a word (e.g., unevenly) and the students were required to tap out how many "meaningful parts" (i.e., morphemes) they heard in the target word.

The Affix Identification subtest, used previously by Apel, Brimo, Diehm, \& Apel (2013), assessed the students' ability to identify real affixes in written pseudowords. The administrators presented the children with a number of printed pseudowords (e.g., lithful) and asked them to circle "add-ons" (i.e., true affixes) contained in those pseudowords.

The Affix Meaning subtest, which was similar to Mitchell and Brady’s (2014) task, students was presented in both spoken and written form. The students read and heard a verballypresented sentence containing a novel/pseudoword and then were asked to choose, from a group of four possibilities, the correct inflected or derived form of the pseudoword to match a provided definition. For example, the students heard the following sentence along with the four options that potentially fit the presented definition, "If edam means "sea," then which word means to go in the direction of the sea: edams, edamer, edamward, edamable.”).

The Suffix Choice subtest was a variation of a task used by Nagy, Berninger, \& Abbott (2006) and presented to students in written form. On the task, the students read sentences with a missing word and were asked to choose one of four possible inflected or derived forms to complete the sentence (e.g., Matthew was not known for being overly [friendly, friendship, friendliness, friends]). Because of the reading requirements for this task, only third through six grade students received the task.

Based on Apel et al. (2013), the students were asked to spell multi-morphemic words. On this Spelling Multi-Morphemic subtest, the students heard a word, heard it used in a sentence, 
and then heard the word again. They then were asked to spell the word on a provided sheet of paper.

On the Derivational Spelling subtest, taken from the work of Sangster and Deacon (2011), the students were provided a derived form of a base word both verbally and visually spelling (e.g., luck) in a sentence context and three phonologically-plausible written word endings (e.g., -y, -ie, -ey). The administrators then asked the students to circle the correct spelling of the word ending.

The final two subtests, Spoken Relatives and Written Relatives, were highly similar (e.g., both included inflectional and derivational items and both contained items that differed on how orthographically and phonologically transparent the base words were in their inflected and derived forms). The two tasks differed in the mode of administration and response (spoken vs. written) as well as the number of items contained on the task. That is, the Written Relatives task always contained more items than the Spoken Relatives task (e.g., 25 vs. 15). Used in other investigations (e.g., Apel et al. 2013; Casalis \& Cole, 2009), the students either heard or read a word, and then were asked to complete a spoken or written sentence with a related word. On both subtests, the provided word was either a base word that needed to be changed into an inflected or derived form or an inflected or derived form that needed to be modified to its base form.

\section{Literacy Tasks}

Reading. A number of measures were administered to assess the students' word-level reading and reading comprehension skills. For word-level reading, the students were administered subtests from either the Test of Word Reading Efficiency, Second Edition (TOWRE2; Torgesen, Wagner, \& Rashotte, 2012) or the Woodcock Reading Mastery Tests-Third Edition (WRMT-3; Woodcock, 2011). On the TOWRE-2, the Sight Word Efficiency subtest measured students real-word reading (i.e., stored knowledge of written words) skills. The Phonemic Decoding Efficiency subtest assessed the students' ability to decode pseudowords. The students were given 45 seconds to read as many real and pseudowords, respectively. The TOWRE-2 authors' manual reports an alternate form reliability of .93.

Word-level reading also was measured using the Word Identification and Word Attack subtests of the WRMT-3. The Word Identification subtest measured the students' abilities to read single real words in isolation. Split-half reliability for this subtest is .91. The Word Attack subtest measures the students' skills to decode single pseudowords until a ceiling is reached. Split-half reliability for this subtest is .89 .

We administered the Passage Comprehension subtest of the WRMT-3 to measure the students' reading comprehension skills. For this subtest, students read short passages and then stated the word(s) missing from those passage. Split-half reliability coefficients are between .84 and .96 for first through sixth grade students.

Spelling. The Test of Written Spelling-Fourth Edition (TWS-5; Larsen, Hammill, \& Moats, 2013) was used to assess students' spelling abilities. On this test, the examiner said a word, used it in a sentence, and then said the word again. The students then were required to spell the word. This task was conducted at the group level to decrease testing time; thus, all students were required to spell all 50 words on the test. However, each student's responses were scored according the measure's basal and ceiling guidelines. According to the authors' manual, test-retest reliability is .92 for grades one through five and .90 for grades six through eight.

Phonological Awareness. The Elision and Blending Words subtests from the Comprehensive Test of Phonological Processing-Second Edition (CTOPP-2; Wagner et al., 2013) were administered to measure the students’ phonological awareness skills. The Elision 
task measured the students' abilities to delete a whole word from a compound word, onsets from rime units, codas from words, and phonemes within rime units. The Blending Words task required that the students listen to sounds of words presented in segments and then blend the sounds together to create real words. According to the authors' manual, test-retest reliability for the Elision task ranges from.77 to .93 for the ages involved. For the Blending Words subtest, test-retest reliability ranged from .74 to .79 for the ages of our participants.

Receptive vocabulary. We administered the PPVT-4 (Dunn \& Dunn, 2007) to measure the students' receptive vocabulary skills. On this test, students point to one of four pictures that matches a verbally-presented word. The authors' manual states that split half reliability is .94.

\section{Procedures}

The students were administered all tasks within their school building. Typically, The Segmenting and Spoken Relatives tasks of the MATRS were administered one-to-one; the remaining MATRS tasks were administered in small groups of same-grade students. All normreferenced tasks were administered one-to-one except for the TWS-5, which was administered in small groups of same-grade students. All tasks were administered by individuals who were trained by the main investigators on all procedures used in the study. The students' parents were remunerated for the child's participation in the study. All tasks were scored by two different raters to ensure accuracy of scoring. Inter-scorer agreement was calculated for each task for $15 \%$ of the participants and average agreement ranged from .92-.99\% across all MATRS tasks and grades. A common-item nonequivalent research design was used to calibrate item and person properties on a common-scale across grade-levels for each proposed task.

\section{Data Analysis \\ Item Calibration}

Item analyses included data screening, multiple group item response modeling, reliability analysis, and differential item functioning.

Data Screening. Item-level p-values were calculated for each item by year and grade. Dichotomous items demonstrating floor effects (e.g., item means $>=95 \%$ incorrect) or ceiling effects (e.g., item means $>=95 \%$ correct) were flagged for potential removal prior to the item response theory modeling.

Multiple-Group Item Response Modeling (MG-IRM). A series of multiple-group unidimensional item response theory (IRT) models were fit to the item-level data corresponding to each of the individual tasks. Following the data screening processes, these analyses allowed for an evaluation of item properties, specifically the item difficulty. The multiple group approach to the item response modeling facilitated a vertical equating of the item parameters across groups (i.e., grade levels) as well as the vertical scaling of ability scores across groups. One aspect of IRT modeling is that the probability of item accuracy may be modeled according to constraints associated with particular item properties including the item difficulty, item discrimination, and pseudo-guessing parameter. For example, a one-parameter logistic response model (1PL) estimates each item's difficulty value but constrains the item discrimination value to equality across all items and fixes the pseudo-guessing parameter to 0 . The two-parameter logistic response model (2PL) estimates individual item difficulty and discrimination coefficients and fixes the pseudo-guessing parameter to 0 ; and the three-parameter logistic response model (3PL) 
estimates individual item difficult, discrimination, and pseudo-guessing coefficients. A variation of the 1PL models is the Rasch model whereby all item discrimination values are fixed to 1.0. A benefit of the Rasch model is that raw total scores from items can be more easily converted to a Rasch scale score compared to using other forms of IRT. This feature is particularly advantageous for paper-pencil delivered or hand-scored assessments.

Rasch models were fit using flexMIRT software (Cai, 2013) and were evaluated using local fit (i.e., performance of the individual items) and goodness-of-fit based on the $\mathrm{M}_{2}$ statistic (Maydeu-Olivares, 2013), the root mean square error of approximation based on $\mathrm{M}_{2}\left(\mathrm{RMSEA}_{2}\right)$, and the Tucker-Lewis Index (TLI). $\mathrm{M}_{2}$ is often sensitive to sample size in terms of rejecting the fitted model, thus, the RMSEA 2 is useful for determining adequate fit $(<.089)$, close fit $(<.05)$, or excellent fit [.05/(k-1), where $k=$ number of categories]. Marginal reliability was reported by grade for each of the administered tasks using Thissen and Orlando (2001) whereby the variance of theta minus the averaged error variances is divided by the variance of theta.

Differential Item Functioning (DIF). DIF was estimated using the difR package (Magis, Beland, \& Raiche, 2020) using the Mantel-Haenszel method (1959) for detecting uniform DIF. For each of the six MATRS tasks, DIF was tested for four primary contrasts: 1) Male vs. female, 2) White vs. Sample, 3) Black vs. Sample, and 4) Latinx vs. Sample. The Mantel-Haenszel chi-square statistic was reported for test by item and the chi-square was used to derive an effect size estimate (i.e., ETS delta scale; Holland \& Thayer, 1988). Effect size values $<=1.0$ are considered small, $1.0-1.5$ is moderate, and $>=1.5$ is considered large.

\section{Score Validity}

In Years 1 and 3 of the study, scores from the MATRS analyses were correlated with concurrently administered standardized assessments of reading and language. Year 1 validity analyses included grade based MATRS task correlations with the PPVT, TOWRE SWE, TOWRE PDE, and the TWS; multiple regression analyses tested the additive and interactive strength of MATRS scores explaining individual differences in the PPVT, TOWRE SWE, TOWRE PDE, and the TWS. Year 3 validity analyses included grade based, MATRS task concurrent correlations with the PPVT, TWS, Elision, Blending, and WRMT-3 LWID, WA, and PC subtests. Predictive relations were estimated between MATRS task scores and TWS along with the WRMT-3 LWID, WA, and PC tasks. For each set of concurrent and predictive correlations in Year 3, results are reported separately for Forms A and B of MATRS tasks that were administered in that portion of the study. Multiple regression analyses tested the additive strength of MATRS scores for each of Forms A and B explaining individual differences in the WRMT-3 LWID, WA, and PC tasks as well as the TWS.

\section{Data Screening}

\section{Results}

\section{Task 1}

The mean p-value (i.e., percent correct) for Task 1 items was $0.46(\mathrm{SD}=0.28)$ with a minimum of 0.00 and a maximum of 0.97 . Two items presented with floor effects (i.e., T1_12_6 and T1_13_23) and three items demonstrated ceiling effects (i.e., T1_14_4, T1_1_6, T1_6_3). Vertical linking items for Task 1 showed that 13 of the 20 items demonstrated reasonable increases in p-values by grade level. See Tables 2 and 10 for results. 


\section{Task 2}

Task 2 contained a mix of 2-category and 3-category items. The mean p-value (i.e., percent correct) for Task 2 dichotomous items was $0.34(\mathrm{SD}=0.22)$ with a minimum of 0.02 and a maximum of 0.95. Seven items presented with floor effects (i.e., T2_85_1, T2_76_2, T2_55_2, T2_72_1, T2_72_2, T2_75_12, T2_82_4) and one item demonstrated ceiling effects (i.e., T2_64_456). The mean response for Task 2 polytomous items was $1.23(\mathrm{SD}=0.14)$ with a minimum of 1.01 and a maximum of 1.62. Vertical linking items for Task 2 showed that 21 of the 25 items demonstrated reasonable increases in p-values by grade level. See Table 3 and 11 for results.

\section{Task 3}

The mean p-value (i.e., percent correct) for Task 3 items was $0.47(\mathrm{SD}=0.18$ ) with a minimum of 0.03 and a maximum of 0.88 . One item presented with floor effects (i.e., T3_40_1) and no items demonstrated ceiling effects. Vertical linking items for Task 3 showed that 40 of the 51 items demonstrated reasonable increases in p-values by grade level. See Table 4 and 12 for results.

\section{Task 4}

The mean p-value (i.e., percent correct) for Task 4 items was $0.65(\mathrm{SD}=0.17)$ with a minimum of 0.11 and a maximum of 0.94 . No items presented with floor or ceiling effects. Vertical linking items for Task 4 showed that 17 of the 23 items demonstrated reasonable increases in p-values by grade level. See Table 5 and 13 for results.

\section{Task 5}

The mean p-value (i.e., percent correct) for Task 5 items was $0.22(\mathrm{SD}=0.18)$ with a minimum of 0.00 and a maximum of 0.76 . A total of 49 items presented with floor effects and no items demonstrated ceiling effects. Vertical linking items for Task 5 showed that 27 of the 34 items demonstrated reasonable increases in p-values by grade level. See Table 6 and 14 for results.

\section{Task 6}

The mean p-value (i.e., percent correct) for Task 6 items was 0.60 (SD $=0.21$ ) with a minimum of 0.13 and a maximum of 0.99 . No items presented with floor effects and two items demonstrated ceiling effects (i.e., T6_31_6, T6_10_4). Vertical linking items for Task 6 showed that 30 of the 37 items demonstrated reasonable increases in p-values by grade level. See Table 7 and 15 for results.

\section{Task 7}

The mean p-value (i.e., percent correct) for Task 7 items was $0.51(\mathrm{SD}=0.31)$ with a minimum of 0.00 and a maximum of 0.97 . Seventeen items presented with floor effects and six items demonstrated ceiling effects (i.e., T7_17_23, T7_21_4, T7_17_4, T7_16_45, T7_22_2, T7_27_4). Vertical linking items for Task 7 showed that 19 of the 26 items demonstrated reasonable increases in p-values by grade level. See Table 8 and 16 for results.

\section{Task 8}

The mean p-value (i.e., percent correct) for Task 8 items was $0.34(\mathrm{SD}=0.24$ ) with a minimum of 0.00 and a maximum of 0.85 . Twenty-five items presented with floor effects and no items demonstrated ceiling effects. Vertical linking items for Task 8 showed that 19 of the 23 
items demonstrated reasonable increases in p-values by grade level. See Table 9 and 17 for results.

\section{Multiple-Group Item Response Modeling (MG-IRM)}

Test characteristic curves and test information functions are presented in Figures 1 and 2. Item characteristic curves are available upon request.

\section{Task 1}

Model fit for the Task 1 unidimensional MG-IRM resulted in a rejection of the null hypothesis of a correctly specified model $\left(\mathrm{M}_{2}=2003.46, p<.001\right)$ with poor fit also suggested by the TLI $=.82$. The RMSEA was estimated as 0.02 . The mean $b$ value was $0.25(\mathrm{SD}=1.50)$ with a minimum of -3.16 and a maximum of 3.08. Marginal reliability ranged from .67 in grade 6 to .78 in grade 1 . The vertical scale for Task 1 ability scores did not demonstrate consistent increases across the grade levels - decreasing from Grades 2 to 3 and remaining relatively constant from Grades 5 to 6 . See Table 18, 19, 27, and 28 for results.

\section{Task 2}

Based on the threshold sensitivity of the polytomous items, scores were recoded to be dichotomous. Model fit for the Task 2 unidimensional MG-IRM resulted in a rejection of the null hypothesis of a correctly specified model $\left(\mathrm{M}_{2}=16559.29, p<.001\right)$ with poor fit also suggested by the TLI $=.85$. The RMSEA was estimated as 0.03 . The mean $b$ value was $0.03(\mathrm{SD}=1.20)$ with a minimum of -1.50 and a maximum of 4.48 . Marginal reliability ranged from .80 in grade 1 to .92 in grades 5-6. The vertical scale for Task 2 ability scores demonstrated consistent increases from -1.96 in Grade 1 to 1.39 in Grade 6. See Table 18, 20, 27, and 28 for results.

\section{Task 3}

Model fit for the Task 3 unidimensional MG-IRM resulted in a fail-to-reject the null hypothesis of a correctly specified model $\left(\mathrm{M}_{2}=6365.34, p>.500\right)$ with acceptable fit also suggested by the TLI $=1.00$ and the RMSEA $=<.001$. The mean $b$ value was $0.03(\mathrm{SD}=0.74)$ with a minimum of -1.82 and a maximum of 2.29. Marginal reliability ranged from .82 in grade 1 to .94 in grade 4 . The vertical scale for Task 3 ability scores demonstrated consistent increases from -1.05 in Grade 1 to 0.76 in Grade 6 with similar ability in Grades 4 and 5. See Table 18, 21, 27, and 28 for results.

\section{Task 4}

Model fit for the Task 4 unidimensional MG-IRM resulted in a fail-to-reject the null hypothesis of a correctly specified model $\left(\mathrm{M}_{2}=1473.90, p>.500\right)$ with acceptable fit also suggested by the TLI $=1.00$ and the RMSEA $=<.001$. The mean $b$ value was $-0.94(\mathrm{SD}=1.09)$ with a minimum of -3.16 and a maximum of 3.05. Marginal reliability ranged from .89 in grade 6 to .91 in grades 3-4. The vertical scale for Task 4 ability scores demonstrated relatively consistent increases from -0.76 in Grade 3 to 0.76 in Grade 6 with similar ability in Grades 4 and 5. See Table 18, 22, 27, and 28 for results.

\section{Task 5}

Model fit for the Task 5 unidimensional MG-IRM resulted in a fail-to-reject the null hypothesis of a correctly specified model $\left(\mathrm{M}_{2}=1761.84, p>.500\right)$ with acceptable fit also suggested by the TLI $=1.00$ and the RMSEA $=<.001$. The mean $b$ value was $0.97(\mathrm{SD}=1.43)$ 
with a minimum of -3.22 and a maximum of 4.48. Marginal reliability ranged from .89 in grade 1 to .95 in grade 4 . The vertical scale for Task 5 ability scores demonstrated relatively consistent increases from -2.80 in Grade 3 to 0.00 in Grade 6. See Table 18, 23, 27, and 28 for results.

\section{Task 6}

Model fit for the Task 6 unidimensional MG-IRM resulted in a rejection of the null hypothesis of a correctly specified model $\left(\mathrm{M}_{2}=5508.05, p<.001\right)$ with acceptable fit suggested by the TLI $=.97$ and the RMSEA $=.01$. The mean $b$ value was $-0.21(\mathrm{SD}=0.99)$ with a minimum of -3.71 and a maximum of 2.84. Marginal reliability ranged from .84 in grade 1 to .90 in grades 3-4. The vertical scale for Task 6 ability scores demonstrated relatively consistent increases from -0.71 in Grade 1 to 1.26 in Grade 6 with similar ability in Grades 4 and 5. See Table 18, 24, 27, and 28 for results.

\section{Task 7}

Model fit for the Task 7 unidimensional MG-IRM resulted in a fail-to-reject the null hypothesis of a correctly specified model $\left(\mathrm{M}_{2}=1720.92, p>.500\right)$ with acceptable fit also suggested by the TLI $=1.00$ and the RMSEA $=<.001$. The mean $b$ value was $0.00(\mathrm{SD}=2.05)$ with a minimum of -4.09 and a maximum of 5.13. Marginal reliability ranged from .82 in grades 2 and 4 to .88 in grade 6 . The vertical scale for Task 7 ability scores did not demonstrate consistent increases across the grade levels - decreasing from Grades 4 to 5. See Table 18, 25, 27, and 28 for results.

\section{Task 8}

Model fit for the Task 8 unidimensional MG-IRM resulted in a fail-to-reject the null hypothesis of a correctly specified model $\left(\mathrm{M}_{2}=1983.86, p>.500\right)$ with acceptable fit also suggested by the TLI $=1.00$ and the RMSEA $=<.001$. The mean $b$ value was $0.69(\mathrm{SD}=1.76)$ with a minimum of -3.16 and a maximum of 6.03. Marginal reliability ranged from .93 in grades 5-6 to .95 in grade 3 . The vertical scale for Task 8 ability scores demonstrated relatively consistent increases from -1.23 in Grade 3 to 0.52 in Grade 6. See Table 18, 26, 27, and 28 for results.

\section{Differential Item Functioning (DIF)}

Results for the DIF analyses are reported in Tables 29-60. Across all tasks, five items demonstrated significant DIF (i.e., T3_1_1, T3_2_1, T4_20_3, T4_9_5, T6_14_456). Remaining items all presented with non-significant chi-square statistics and ETS delta values near 0.00 indicating no practically important DIF.

\section{Score Validity Year 1}

Grade 1. Correlations among MATRS ability scores ranged from .07 between Task 1 and Task 3 to .62 between Task 5 and Task 6. Correlations between MATRS ability scores with outcomes ranged from .00 between Task 1 and the PPVT to .48 between Task 5 and TOWRE PDE. Multiple regression $\mathrm{R}^{2}$ values for the additive MATRS model ranged from .08 for the PPVT to .27 for the TWS. Multiple regression $\mathrm{R}^{2}$ values for the MATRS 2-way interactions model ranged from .17 for the PPVT to .40 for the TWS. See Table 61 and 67 for results. 
Grade 2. Correlations among MATRS ability scores ranged from .21 between Task 1 and Task 5 to .72 between Task 5 and Task 6. Correlations between MATRS ability scores with outcomes ranged from .03 between Task 1 and the TWS to .53 between Task 5 and TWS. Multiple regression $\mathrm{R}^{2}$ values for the additive MATRS model ranged from .10 for the PDE to .30 for the TWS. Multiple regression $\mathrm{R}^{2}$ values for the MATRS 2-way interactions model ranged from .17 for the PDE to .42 for the TWS. See Table 62 and 67 for results.

Grade 3. Correlations among MATRS ability scores ranged from .08 between Task 1 and Task 4 to .74 between Task 5 and Task 8 . Correlations between MATRS ability scores with outcomes ranged from .00 between Task 1 and the TWS to .48 between Task 5 and TOWRE PDE. Multiple regression $\mathrm{R}^{2}$ values for the additive MATRS model ranged from .11 for the SWE to .27 for the TWS. Multiple regression $\mathrm{R}^{2}$ values for the MATRS 2-way interactions model ranged from .81 for the SWE, PDE, and TWS to .82 for the PPVT. See Table 63 and 67 for results.

Grade 4. Correlations among MATRS ability scores ranged from .06 between Task 1 and Task 6 to .77 between Task 4 and Task 8. Correlations between MATRS ability scores with outcomes ranged from .00 between Task 1 and the SWE to .49 between Task 5 and TWS. Multiple regression $\mathrm{R}^{2}$ values for the additive MATRS model ranged from .15 for the SWE to .26 for the TWS. Multiple regression $\mathrm{R}^{2}$ values for the MATRS 2-way interactions model ranged from .60 for the SWE to .67 for the TWS. See Table 64 and 67 for results.

Grade 5. Correlations among MATRS ability scores ranged from .11 between Task 1 and Task 7 to .77 between Task 5 and Task 8. Correlations between MATRS ability scores with outcomes ranged from .00 between Task 1 and the TWS to .51 between Task 5 and TWS. Multiple regression $\mathrm{R}^{2}$ values for the additive MATRS model ranged from .08 for the SWE to .27 for the TWS. Multiple regression $\mathrm{R}^{2}$ values for the MATRS 2-way interactions model ranged from .71 for the SWE to .76 for the TWS. See Table 65 and 67 for results.

Grade 6. Correlations among MATRS ability scores ranged from -.02 between Task 1 and Task 6 to .75 between Task 5 and Task 8. Correlations between MATRS ability scores with outcomes ranged from .00 between Task 1 and the PPVT to .48 between Task 6 and TWS. Multiple regression $\mathrm{R}^{2}$ values for the additive MATRS model ranged from .12 for the PDE to .35 for the TWS. Multiple regression $\mathrm{R}^{2}$ values for the MATRS 2-way interactions model ranged from .79 for the SWE to .86 for the PPVT. See Table 66 and 67 for results.

\section{Year 3}

Grade 1. Form A correlations among MATRS ability scores ranged from .04 between Task 1 and Task 6 to .36 between Task 3 and Task 7. Concurrent correlations between MATRS ability scores with outcomes ranged from .00 between Task 6 and LWID to .44 between Task 7 and PPVT. Predictive correlations between MATRS ability scores with outcomes ranged from .01 between Task 2 and WA to .56 between Task 5 and TWS. Multiple regression $\mathrm{R}^{2}$ values for the additive MATRS model ranged from .30 for LWID to .33 for the TWS. See Table 68 and 80 for results.

Form B correlations among MATRS ability scores ranged from .08 between Task 1 with Tasks 2 and Task 5 to .63 between Task 5 and Task 6. Concurrent correlations between MATRS ability scores with outcomes ranged from .05 between Task 1 and PC to .81 between Task 5 and 
TWS. Predictive correlations between MATRS ability scores with outcomes ranged from -.02 between Task 2 and TWS to .78 between Task 5 and TWS. Multiple regression $\mathrm{R}^{2}$ values for the additive MATRS model ranged from .44 for PC to .66 for the TWS. See Table 74 and 80 for results.

Grade 2. Form A correlations among MATRS ability scores ranged from .03 between Task 1 and Task 2 to .65 between Task 5 and Task 6. Concurrent correlations between MATRS ability scores with outcomes ranged from .05 between Task 1 and Elision to .75 between Task 5 and WA. Predictive correlations between MATRS ability scores with outcomes ranged from .24 between Task 2 and LWID and WA to .78 between Task 5 and TWS. Multiple regression R ${ }^{2}$ values for the additive MATRS model ranged from .47 for PC to .65 for the TWS. See Table 69 and 80 for results.

Form B correlations among MATRS ability scores ranged from .11 between Task 1 with Tasks 6 and Task 7 to .58 between Task 5 and Task 6. Concurrent correlations between MATRS ability scores with outcomes ranged from .09 between Task 1 and PPVT to .68 between Task 5 with PPVT and TWS. Predictive correlations between MATRS ability scores with outcomes ranged from .00 between Task 7 and PC to .79 between Task 5 and TWS. Multiple regression $\mathrm{R}^{2}$ values for the additive MATRS model ranged from .37 for PC to .65 for the TWS. See Table 75 and 80 for results.

Grade 3. Form A correlations among MATRS ability scores ranged from .00 between Task 1 with Tasks 5 and 8 to .65 between Task 5 and Task 8. Concurrent correlations between MATRS ability scores with outcomes ranged from .03 between Task 6 and Blending to .76 between Task 5 and WA. Predictive correlations between MATRS ability scores with outcomes ranged from .01 between Task 1 and WA to .76 between Task 5 and TWS. Multiple regression $\mathrm{R}^{2}$ values for the additive MATRS model ranged from .61 for WA to .71 for the LWID. See Table 70 and 80 for results.

Form B correlations among MATRS ability scores ranged from .01 between Task 1 and Task 2 to .73 between Task 5 and Task 7. Concurrent correlations between MATRS ability scores with outcomes ranged from .12 between Task 1 and Blending to .75 between Task 5 and LWID. Predictive correlations between MATRS ability scores with outcomes ranged from -.01 between Task 1 and WA to .78 between Task 5 and TWS. Multiple regression $\mathrm{R}^{2}$ values for the additive MATRS model ranged from .35 for PC to .69 for the TWS. See Table 76 and 80 for results.

Grade 4. Form A correlations among MATRS ability scores ranged from .02 between Task 1 with Task 7 to .73 between Task 5 and Task 8. Concurrent correlations between MATRS ability scores with outcomes ranged from .01 between Task 1 and TWS to .75 between Task 5 and WA. Predictive correlations between MATRS ability scores with outcomes ranged from .09 between Task 1 and TWS to .77 between Task 5 and LWID. Multiple regression $\mathrm{R}^{2}$ values for the additive MATRS model ranged from .46 for PC to .73 for the LWID. See Table 71 and 80 for results.

Form B correlations among MATRS ability scores ranged from .01 between Task 1 and Task 2 to .73 between Task 5 and Task 7. Concurrent correlations between MATRS ability scores with outcomes ranged from -.01 between Task 1 and PPVT to .77 between Task 8 and 
TWS. Predictive correlations between MATRS ability scores with outcomes ranged from .16 between Task 1 and LWID to .78 between Task 5 and TWS. Multiple regression $\mathrm{R}^{2}$ values for the additive MATRS model ranged from .42 for PC to .67 for the LWID. See Table 77 and 80 for results.

Grade 5. Form A correlations among MATRS ability scores ranged from .00 between Task 1 with Task 7 to .70 between Task 4 and Task 8. Concurrent correlations between MATRS ability scores with outcomes ranged from .14 between Task 1 and PC to .75 between Task 8 with and LWID and PC. Predictive correlations between MATRS ability scores with outcomes ranged from .04 between Task 1 and PC to .92 between Task 8 and TWS. Multiple regression $\mathrm{R}^{2}$ values for the additive MATRS model ranged from .76 for LWID to .92 for the TWS. See Table 72 and 80 for results.

Form B correlations among MATRS ability scores ranged from .09 between Task 1 and Task 3 to .65 between Task 4 and Task 8. Concurrent correlations between MATRS ability scores with outcomes ranged from .13 between Task 1 and Elision to .74 between Task 5 and LWID as well as between Task 8 and TWS. Predictive correlations between MATRS ability scores with outcomes ranged from .21 between Task 2 and WA as well as Task 7 and TWS to .75 between Task 4 and TWS. Multiple regression $\mathrm{R}^{2}$ values for the additive MATRS model ranged from .45 for PC to .70 for the TWS. See Table 78 and 80 for results.

Grade 6. Form A correlations among MATRS ability scores ranged from .05 between Task 1 with Task 4 to .70 between Task 4 and Task 8. Concurrent correlations between MATRS ability scores with outcomes ranged from -.01 between Task 1 and Elision to .84 between Task 8 and TWS. Predictive correlations between MATRS ability scores with outcomes ranged from .03 between Task 1 and PC to .82 between Task 5 and TWS. Multiple regression $\mathrm{R}^{2}$ values for the additive MATRS model ranged from .59 for WA to .86 for the TWS. See Table 73 and 80 for results.

Form B correlations among MATRS ability scores ranged from -.02 between Task 1 with Task 3 and Task 7 to .70 between Task 5 and Task 8. Concurrent correlations between MATRS ability scores with outcomes ranged from .00 between Task 1 with TWS, Elision, and Blending to .72 between Task 8 and TWS. Predictive correlations between MATRS ability scores with outcomes ranged from .22 between Task 4 and PC as well as Task 7 and TWS to .88 between Task 5 and LWID. Multiple regression $\mathrm{R}^{2}$ values for the additive MATRS model ranged from .59 for PC to .91 for the LWID. See Table 79 and 80 for results. 


\section{References}

Apel, K. (2014). A comprehensive definition of morphological awareness: Implications for assessment. Topics in Language Disorders, 34, 197-209. doi: 10.1097/ TLD.0000000000000019

Apel, K., Brimo, D., Diehm, E., \& Apel, L. (2013). Morphological awareness intervention with kindergarteners and first and second grade students from low SES homes: A feasibility study. Language, Speech, and Hearing Services in Schools, 44, 161-173. DOI: 10.1044/0161-1461(2012/12-0042

Apel, K., \& Masterson, J. J. (2012). Beyond baby talk: From speaking to spelling: A guide to language and literacy development for parents and caregivers. New York, NY: Three Rivers Press.

Apel, K., Wilson-Fowler, E.B., Brimo, D., \& Perrin, N.A. (2012). Metalinguistic contributions to reading and spelling in second and third grade students. Reading and Writing: An Interdisciplinary Journal, 25, 1283-1305. doi: 10.1007/s11145-011-9317-8

Berninger, V.W., Abbott, R.D., Nagy, W., \& Carlisle, J. (2010). Growth in phonological, orthographic, and morphological awareness in grades 1 to 6. Journal of Psycholinguistic Research, 39, 141-163. doi: 10.1007/s10936-009-9130-6

Bowers, P. N., Kirby, J. R., \& S. H. Deacon. (2010). The effects of morphological instruction on literacy skills: A systematic review of the literature. Review of Educational Research, 80, 144-179. doi:10.3102/0034654309359353

Bourassa, D., \& Treiman, R. (2009). Linguistic foundations of spelling development. In D. Wyse, R. Andrews, \& J. Hoffman (Eds.), Routledge international handbook of English, language and literacy teaching (pp. 182-192). London, UK: Routledge.

Cai L. (2013). flexMIRT® version 2: Flexible multilevel multidimensional item analysis and test scoring [Computer software]. Chapel Hill, NC: Vector Psychometric Group.

Casalis, S. \& Cole, P. (2009). On the relationship between morphological and phonological awareness: Effects of training in kindergarten and in first-grade reading. First Language, 29, 113-142. doi: 10.1177/0142723708097484

Casalis, S., Cole, P., \& Sopo, D. (2004). Morphological awareness in developmental dyslexia. Annals of Dyslexia, 54, 114-138.

Deacon, S.H. \& Kirby, J.R. (2004). Morphological awareness: Just "more phonological"? The roles of morphological and phonological awareness in reading development. Applied Psycholinguistics, 25, 223-238. doi: 10.1017.S0124716404001117

Deacon, S.H., Kirby, J.R., \& Casselman-Bell, M. (2009). How robust is the contribution of morphological awareness to general spelling outcomes? Reading Psychology, 30, 301318. doi: 10.1080/02702710802412057

Dunn, L. M., \& Dunn, D. M. (2007). Peabody Picture Vocabulary Test, Fourth Edition. Minneapolis, MN: Pearson Assessments.

Holland, P. W., \& Thayer, D. T. (1989). The kernel method of equating score distributions. ETS Research Report Series, 1989(1), i-45.

Larsen, S., Hammill, D. \& Moats, L. (2013). Test of Written Spelling, Fifth Edition. Austin, TX: Pro-Ed.

Magis, D., Beland, S., Raiche, G., \& Magis, M. D. (2020). Package 'difR'.

Mantel, N., \& Haenszel, W. (1959). Statistical aspects of the analysis of data from retrospective studies of disease. Journal of the national cancer institute, 22(4), 719-748. 
Maydeu-Olivares A. (2013). Goodness-of-fit assessment of item response theory models. Measurement: Interdisciplinary Research and Perspectives, 11, 71-101.

Mitchell, A. M., \& Brady, S. A. (2014). Assessing affix knowledge using both pseudoword and real-word measures. Topics in Language Disorders, 34, 210-227.

Moats, L. (1995). Spelling: Development, disability, and instruction. Baltimore: York Press.

Nagy, W., Berninger, V., \& Abbott, R. D. (2006). Contributions of morphology beyond phonology to literacy outcomes of upper elementary and middle-school students. Journal of Educational Psychology, 98, 134-147. doi: 10.1037/0022-0663.98.1.134

Sangster, L., \& Deacon, S. H. (2011). Development in children's sensitivity to the role of derivations in spelling. Canadian Journal of Experimental Psychology/Revue Canadienne de psychologie expérimentale, 65(2), 133.

SPELL-Links Word List Maker [Computer software]. Evanston, IL: Learning By Design, Inc., 2010.

Tighe, E. L., \& Schatschneider, C. (2016). Examining the relationships of component reading skills to reading comprehension in struggling adult readers: A meta-analysis. Journal of learning disabilities, 49(4), 395-409.

Torgesen, J. K., Wagner, R. K., Rashotte, C. A. (2012). Test of Word Reading Efficiency-Second Edition. Austin, TX: Pro-Ed.

Treiman, R. (1998). Beginning to spell in English. In C. Hulme \& R. M. Joshi (Eds.), Reading and spelling: Development and disorders (pp. 371-194). Mahwah, NJ: Erlbaum.

Treiman, R., \& Cassar, M. (1996). Effects of morphology on children's spelling of final consonant clusters. Journal of Experimental Child Psychology, 63, 141-170. doi:10.1006/jecp.1996.0045

Wagner, R. K., Torgesen, J. K., Rashotte, C. A., \& Pearson, N. A. (2013). Comprehensive Test of Phonological Processing-Second Edition (CTOPP-2). Austin, TX: PRO-ED.

Woodcock, R. (2011). The Woodcock Reading Mastery Test-Third Edition. Circle Pines, MN: American Guidance Service.

Zeno, S. M., Ivens, S. H., Millard, R. T., \& Duvvuri, R. (1995). The educator's word frequency guide. Brewster, NY: Touchstone Applied Science 
Table 1

Demographic characteristics by year

\begin{tabular}{|c|c|c|c|c|c|c|c|}
\hline \multirow[b]{2}{*}{ Demographic } & \multirow[b]{2}{*}{ Category } & \multicolumn{2}{|c|}{ Year 1} & \multicolumn{2}{|c|}{ Year 2} & \multicolumn{2}{|c|}{ Year 3} \\
\hline & & $\mathrm{N}$ & $\%$ & $\mathrm{~N}$ & $\%$ & $\mathrm{~N}$ & $\%$ \\
\hline \multirow[t]{2}{*}{ Sex } & Male & 512 & 45.63 & 1357 & 46.2 & 630 & 46.91 \\
\hline & Female & 610 & 54.37 & 1580 & 53.8 & 713 & 53.09 \\
\hline \multirow[t]{7}{*}{ Race/Ethnicity } & White & 461 & 41.09 & 1318 & 44.85 & 672 & 50.04 \\
\hline & Black & 430 & 38.32 & 1101 & 37.46 & 405 & 30.16 \\
\hline & Latinx & 72 & 6.42 & 141 & 4.8 & 87 & 6.48 \\
\hline & Asian & 18 & 1.6 & 44 & 1.5 & 23 & 1.71 \\
\hline & Native American & 4 & $<1 \%$ & 17 & $<1 \%$ & 3 & $<1 \%$ \\
\hline & Multi-racial & 109 & 9.72 & 192 & 6.5 & 107 & 7.97 \\
\hline & No Response & 28 & 2.5 & 126 & 4.3 & 46 & 2.64 \\
\hline \multicolumn{8}{|l|}{ Mother's } \\
\hline \multirow[t]{9}{*}{ Education } & Less than High School & 23 & 2.05 & 35 & 1.19 & 14 & 1.04 \\
\hline & Some High School & 63 & 5.62 & 158 & 5.38 & 57 & 4.24 \\
\hline & GED & 37 & 3.3 & 92 & 3.13 & 40 & 2.98 \\
\hline & High School Grad & 289 & 25.76 & 644 & 21.94 & 259 & 19.29 \\
\hline & Some College & 258 & 22.99 & 570 & 19.42 & 289 & 21.52 \\
\hline & Associate or Technical Degree & 138 & 12.3 & 327 & 11.14 & 144 & 10.72 \\
\hline & Bachelor's Degree & 151 & 13.46 & 537 & 18.3 & 233 & 17.35 \\
\hline & Graduate Degree & 102 & 9.09 & 382 & 13.02 & 228 & 16.98 \\
\hline & No Response & 61 & 5.43 & 94 & 6.48 & 79 & 5.88 \\
\hline \multicolumn{8}{|l|}{ Father's } \\
\hline \multirow[t]{9}{*}{ Education } & Less than High School & 25 & 2.23 & 46 & 1.57 & 21 & 1.56 \\
\hline & Some High School & 83 & 7.4 & 207 & 7.06 & 65 & 4.84 \\
\hline & GED & 50 & 4.46 & 123 & 4.19 & 60 & 4.47 \\
\hline & High School Grad & 373 & 33.24 & 791 & 26.96 & 369 & 27.48 \\
\hline & Some College & 193 & 17.2 & 411 & 14.01 & 243 & 18.09 \\
\hline & Associate or Technical Degree & 91 & 8.11 & 233 & 7.94 & 97 & 7.22 \\
\hline & Bachelor's Degree & 102 & 9.09 & 426 & 14.52 & 185 & 13.78 \\
\hline & Graduate Degree & 47 & 4.19 & 255 & 8.69 & 138 & 10.28 \\
\hline & No Response & 158 & 14.08 & 447 & 15.06 & 165 & 12.29 \\
\hline \multirow[t]{3}{*}{ Language } & English & 1053 & 93.85 & 2789 & 93.54 & 1265 & 94.19 \\
\hline & Spanish & 54 & 4.81 & 87 & 2.96 & 56 & 4.17 \\
\hline & Other & 15 & 1.34 & 63 & 3.5 & 22 & 1.64 \\
\hline \multicolumn{8}{|l|}{ Special } \\
\hline \multirow[t]{3}{*}{ Services } & No Services & 744 & 66.31 & 2137 & 72.71 & 942 & 70.14 \\
\hline & Speech Only & 82 & 7.31 & 215 & 7.32 & 131 & 9.75 \\
\hline & Language Only & 7 & 1 & 16 & 1 & 5 & $<1 \%$ \\
\hline
\end{tabular}


MATRS TECHNICAL MANUAL 18

\begin{tabular}{lcccccc} 
Reading/Writing Only & 97 & 8.65 & 251 & 8.54 & 110 & 8.19 \\
Multiple Services & 129 & 11.5 & 247 & 8.4 & 135 & 10.05 \\
Other & 63 & 5.23 & 73 & 2.03 & 20 & 1.87 \\
\hline
\end{tabular}


Table 2

Task 1 p-values and sample size

\begin{tabular}{|c|c|c|c|c|c|}
\hline Item & Year & Grade & Mean & $\mathrm{N}$ & Item Type \\
\hline T1_1_1 & 1 & 1 & 0.79 & 179 & Dichotomous \\
\hline T1_1_34 & 1 & 3 & 0.54 & 167 & Dichotomous \\
\hline T1_1_34 & 1 & 4 & 0.43 & 210 & Dichotomous \\
\hline T1_1_4 & 1 & 4 & 0.11 & 210 & Dichotomous \\
\hline T1_1_4 & 2 & 4 & 0.07 & 433 & Dichotomous \\
\hline T1_1_5 & 1 & 5 & 0.08 & 180 & Dichotomous \\
\hline T1_1_6 & 1 & 6 & 0.97 & 161 & Dichotomous \\
\hline T1_10_1 & 1 & 1 & 0.78 & 179 & Dichotomous \\
\hline T1_10_3 & 1 & 3 & 0.86 & 167 & Dichotomous \\
\hline T1_10_456 & 1 & 4 & 0.21 & 210 & Dichotomous \\
\hline T1_10_456 & 1 & 5 & 0.23 & 180 & Dichotomous \\
\hline T1_10_456 & 1 & 6 & 0.27 & 161 & Dichotomous \\
\hline T1_10_456 & 2 & 4 & 0.28 & 433 & Dichotomous \\
\hline T1_11_1 & 1 & 1 & 0.38 & 179 & Dichotomous \\
\hline T1_11_2 & 1 & 2 & 0.87 & 224 & Dichotomous \\
\hline T1_11_3 & 1 & 3 & 0.10 & 167 & Dichotomous \\
\hline T1_11_5 & 1 & 5 & 0.82 & 180 & Dichotomous \\
\hline T1_11_6 & 1 & 6 & 0.82 & 161 & Dichotomous \\
\hline T1_12_1 & 1 & 1 & 0.39 & 179 & Dichotomous \\
\hline T1_12_3 & 1 & 3 & 0.23 & 167 & Dichotomous \\
\hline T1_12_3 & 2 & 3 & 0.29 & 589 & Dichotomous \\
\hline T1_12_5 & 1 & 5 & 0.29 & 180 & Dichotomous \\
\hline T1_12_6 & 1 & 6 & 0.20 & 161 & Dichotomous \\
\hline T1_12_6 & 2 & 6 & 0.00 & 336 & Dichotomous \\
\hline T1_13_12 & 1 & 1 & 0.18 & 179 & Dichotomous \\
\hline T1_13_12 & 1 & 2 & 0.21 & 224 & Dichotomous \\
\hline T1_13_23 & 1 & 2 & 0.04 & 224 & Dichotomous \\
\hline T1_13_23 & 1 & 3 & 0.08 & 167 & Dichotomous \\
\hline T1_13_23 & 2 & 2 & 0.13 & 499 & Dichotomous \\
\hline T1_13_3 & 1 & 3 & 0.20 & 167 & Dichotomous \\
\hline T1_13_4 & 1 & 4 & 0.18 & 210 & Dichotomous \\
\hline T1_13_4 & 2 & 4 & 0.19 & 433 & Dichotomous \\
\hline T1_13_5 & 1 & 5 & 0.33 & 180 & Dichotomous \\
\hline T1_13_5 & 2 & 5 & 0.48 & 446 & Dichotomous \\
\hline T1_13_6 & 1 & 6 & 0.89 & 161 & Dichotomous \\
\hline T1_14_12 & 1 & 1 & 0.84 & 179 & Dichotomous \\
\hline T1_14_12 & 1 & 2 & 0.87 & 224 & Dichotomous \\
\hline
\end{tabular}




\begin{tabular}{|c|c|c|c|c|c|}
\hline T1_14_12 & 2 & 1 & 0.84 & 628 & Dichotomous \\
\hline T1_14_2 & 1 & 2 & 0.22 & 224 & Dichotomous \\
\hline T1_14_3 & 1 & 3 & 0.24 & 167 & Dichotomous \\
\hline T1_14_4 & 1 & 4 & 0.95 & 210 & Dichotomous \\
\hline T1_14_5 & 1 & 5 & 0.21 & 180 & Dichotomous \\
\hline T1_14_6 & 1 & 6 & 0.12 & 161 & Dichotomous \\
\hline T1_15_1 & 1 & 1 & 0.34 & 179 & Dichotomous \\
\hline T1_15_2 & 1 & 2 & 0.88 & 224 & Dichotomous \\
\hline T1_15_3 & 1 & 3 & 0.20 & 167 & Dichotomous \\
\hline T1_15_3 & 2 & 3 & 0.32 & 589 & Dichotomous \\
\hline T1_15_4 & 1 & 4 & 0.20 & 210 & Dichotomous \\
\hline T1_15_5 & 1 & 5 & 0.55 & 180 & Dichotomous \\
\hline T1_16_1 & 2 & 1 & 0.84 & 628 & Dichotomous \\
\hline T1_16_3 & 2 & 3 & 0.97 & 589 & Dichotomous \\
\hline T1_16_4 & 2 & 4 & 0.53 & 433 & Dichotomous \\
\hline T1_17_3 & 2 & 3 & 0.23 & 589 & Dichotomous \\
\hline T1_17_4 & 2 & 4 & 0.89 & 433 & Dichotomous \\
\hline T1_17_5 & 2 & 5 & 0.58 & 446 & Dichotomous \\
\hline T1_17_6 & 2 & 6 & 0.60 & 336 & Dichotomous \\
\hline T1_18_1 & 2 & 1 & 0.44 & 628 & Dichotomous \\
\hline T1_18_34 & 2 & 3 & 0.55 & 589 & Dichotomous \\
\hline T1_18_34 & 2 & 4 & 0.64 & 433 & Dichotomous \\
\hline T1_18_4 & 2 & 4 & 0.89 & 433 & Dichotomous \\
\hline T1_18_5 & 2 & 5 & 0.35 & 446 & Dichotomous \\
\hline T1_18_6 & 2 & 6 & 0.67 & 336 & Dichotomous \\
\hline T1_19_1 & 2 & 1 & 0.61 & 628 & Dichotomous \\
\hline T1_19_2 & 2 & 2 & 0.17 & 500 & Dichotomous \\
\hline T1_19_3 & 2 & 3 & 0.22 & 589 & Dichotomous \\
\hline T1_19_4 & 2 & 4 & 0.15 & 433 & Dichotomous \\
\hline T1_19_5 & 2 & 5 & 0.44 & 446 & Dichotomous \\
\hline T1_19_6 & 2 & 6 & 0.59 & 336 & Dichotomous \\
\hline T1_2_12 & 1 & 1 & 0.84 & 179 & Dichotomous \\
\hline T1_2_12 & 1 & 2 & 0.92 & 224 & Dichotomous \\
\hline T1_2_12 & 2 & 1 & 0.74 & 628 & Dichotomous \\
\hline T1_2_2 & 1 & 2 & 0.41 & 224 & Dichotomous \\
\hline T1_2_2 & 2 & 2 & 0.55 & 500 & Dichotomous \\
\hline T1_2_34 & 1 & 3 & 0.17 & 167 & Dichotomous \\
\hline T1_2_34 & 1 & 4 & 0.22 & 210 & Dichotomous \\
\hline T1_2_456 & 1 & 4 & 0.88 & 210 & Dichotomous \\
\hline T1_2_456 & 1 & 5 & 0.91 & 180 & Dichotomous \\
\hline T1_2_456 & 1 & 6 & 0.84 & 161 & Dichotomous \\
\hline
\end{tabular}




\begin{tabular}{|c|c|c|c|c|c|}
\hline T1_2_5 & 1 & 5 & 0.35 & 180 & Dichotomous \\
\hline T1_20_1 & 2 & 1 & 0.52 & 628 & Dichotomous \\
\hline T1_20_2 & 2 & 2 & 0.81 & 500 & Dichotomous \\
\hline T1_20_4 & 2 & 4 & 0.86 & 433 & Dichotomous \\
\hline T1_20_6 & 2 & 6 & 0.19 & 336 & Dichotomous \\
\hline T1_21_12 & 2 & 1 & 0.73 & 628 & Dichotomous \\
\hline T1_21_12 & 2 & 2 & 0.82 & 500 & Dichotomous \\
\hline T1_21_2 & 2 & 2 & 0.70 & 500 & Dichotomous \\
\hline T1_21_3 & 2 & 3 & 0.26 & 589 & Dichotomous \\
\hline T1_21_6 & 2 & 6 & 0.44 & 336 & Dichotomous \\
\hline T1_22_1 & 2 & 1 & 0.72 & 628 & Dichotomous \\
\hline T1_22_2 & 2 & 2 & 0.72 & 500 & Dichotomous \\
\hline T1_22_45 & 2 & 4 & 0.28 & 433 & Dichotomous \\
\hline T1_22_45 & 2 & 5 & 0.38 & 446 & Dichotomous \\
\hline T1_22_5 & 2 & 5 & 0.75 & 446 & Dichotomous \\
\hline T1_23_1 & 2 & 1 & 0.36 & 628 & Dichotomous \\
\hline T1_23_2 & 2 & 2 & 0.90 & 500 & Dichotomous \\
\hline T1_23_5 & 2 & 5 & 0.42 & 446 & Dichotomous \\
\hline T1_23_6 & 2 & 6 & 0.32 & 336 & Dichotomous \\
\hline T1_24_4 & 2 & 4 & 0.25 & 433 & Dichotomous \\
\hline T1_24_56 & 2 & 5 & 0.85 & 446 & Dichotomous \\
\hline T1_24_56 & 2 & 6 & 0.85 & 336 & Dichotomous \\
\hline T1_24_6 & 2 & 6 & 0.42 & 336 & Dichotomous \\
\hline T1_25_1 & 2 & 1 & 0.26 & 628 & Dichotomous \\
\hline T1_25_2 & 2 & 2 & 0.19 & 500 & Dichotomous \\
\hline T1_25_3 & 2 & 3 & 0.37 & 589 & Dichotomous \\
\hline T1_25_5 & 2 & 5 & 0.35 & 446 & Dichotomous \\
\hline T1_25_6 & 2 & 6 & 0.54 & 336 & Dichotomous \\
\hline T1_26_2 & 2 & 2 & 0.81 & 500 & Dichotomous \\
\hline T1_26_3 & 2 & 3 & 0.24 & 589 & Dichotomous \\
\hline T1_27_1 & 2 & 1 & 0.63 & 628 & Dichotomous \\
\hline T1_27_23 & 2 & 2 & 0.15 & 502 & Dichotomous \\
\hline T1_27_23 & 2 & 3 & 0.70 & 589 & Dichotomous \\
\hline T1_27_3 & 2 & 3 & 0.43 & 589 & Dichotomous \\
\hline T1_27_4 & 2 & 4 & 0.19 & 433 & Dichotomous \\
\hline T1_27_5 & 2 & 5 & 0.34 & 446 & Dichotomous \\
\hline T1_28_1 & 2 & 1 & 0.69 & 628 & Dichotomous \\
\hline T1_28_2 & 2 & 2 & 0.82 & 500 & Dichotomous \\
\hline T1_28_3 & 2 & 3 & 0.09 & 589 & Dichotomous \\
\hline T1_28_4 & 2 & 4 & 0.23 & 433 & Dichotomous \\
\hline T1_28_5 & 2 & 5 & 0.33 & 446 & Dichotomous \\
\hline
\end{tabular}




\begin{tabular}{|c|c|c|c|c|c|}
\hline T1_28_6 & 2 & 6 & 0.35 & 336 & Dichotomous \\
\hline T1_3_1 & 1 & 1 & 0.12 & 179 & Dichotomous \\
\hline T1_3_2 & 1 & 2 & 0.36 & 224 & Dichotomous \\
\hline T1_3_6 & 1 & 6 & 0.45 & 161 & Dichotomous \\
\hline T1_4_1 & 1 & 1 & 0.91 & 179 & Dichotomous \\
\hline T1_4_2 & 1 & 2 & 0.13 & 224 & Dichotomous \\
\hline T1_4_34 & 1 & 3 & 0.28 & 167 & Dichotomous \\
\hline T1_4_34 & 1 & 4 & 0.34 & 210 & Dichotomous \\
\hline T1_4_34 & 2 & 3 & 0.30 & 589 & Dichotomous \\
\hline T1_4_4 & 1 & 4 & 0.57 & 210 & Dichotomous \\
\hline T1_4_56 & 1 & 5 & 0.13 & 180 & Dichotomous \\
\hline T1_4_56 & 1 & 6 & 0.09 & 161 & Dichotomous \\
\hline T1_5_1 & 1 & 1 & 0.92 & 179 & Dichotomous \\
\hline T1_5_2 & 1 & 2 & 0.45 & 224 & Dichotomous \\
\hline T1_5_3 & 1 & 3 & 0.39 & 167 & Dichotomous \\
\hline T1_5_456 & 1 & 4 & 0.42 & 210 & Dichotomous \\
\hline T1_5_456 & 1 & 5 & 0.39 & 180 & Dichotomous \\
\hline T1_5_456 & 1 & 6 & 0.44 & 161 & Dichotomous \\
\hline T1_5_5 & 1 & 5 & 0.06 & 180 & Dichotomous \\
\hline T1_5_5 & 2 & 5 & 0.14 & 446 & Dichotomous \\
\hline T1_6_1 & 1 & 1 & 0.16 & 179 & Dichotomous \\
\hline T1_6_23 & 1 & 2 & 0.15 & 224 & Dichotomous \\
\hline T1_6_23 & 1 & 3 & 0.15 & 167 & Dichotomous \\
\hline T1_6_3 & 1 & 3 & 0.87 & 167 & Dichotomous \\
\hline T1_6_4 & 1 & 4 & 0.16 & 210 & Dichotomous \\
\hline T1_6_56 & 1 & 5 & 0.43 & 180 & Dichotomous \\
\hline T1_6_56 & 1 & 6 & 0.30 & 161 & Dichotomous \\
\hline T1_6_6 & 1 & 6 & 0.48 & 161 & Dichotomous \\
\hline T1_6_6 & 2 & 6 & 0.31 & 336 & Dichotomous \\
\hline T1_7_1 & 1 & 1 & 0.43 & 179 & Dichotomous \\
\hline T1_7_2 & 1 & 2 & 0.39 & 224 & Dichotomous \\
\hline T1_7_4 & 1 & 4 & 0.80 & 210 & Dichotomous \\
\hline T1_7_5 & 1 & 5 & 0.08 & 180 & Dichotomous \\
\hline T1_7_5 & 2 & 5 & 0.28 & 446 & Dichotomous \\
\hline T1_8_1 & 1 & 1 & 0.88 & 179 & Dichotomous \\
\hline T1_8_2 & 1 & 2 & 0.78 & 224 & Dichotomous \\
\hline T1_8_3 & 1 & 3 & 0.54 & 167 & Dichotomous \\
\hline T1_8_4 & 1 & 4 & 0.16 & 210 & Dichotomous \\
\hline T1_8_56 & 1 & 5 & 0.87 & 180 & Dichotomous \\
\hline T1_8_56 & 1 & 6 & 0.88 & 161 & Dichotomous \\
\hline T1_8_56 & 2 & 6 & 0.81 & 336 & Dichotomous \\
\hline
\end{tabular}


MATRS TECHNICAL MANUAL 23

\begin{tabular}{llllll} 
T1_8_6 & 1 & 6 & 0.88 & 161 & Dichotomous \\
T1_9_1 & 1 & 1 & 0.36 & 179 & Dichotomous \\
T1_9_1 & 2 & 1 & 0.36 & 628 & Dichotomous \\
T1_9_23 & 1 & 2 & 0.29 & 224 & Dichotomous \\
T1_9_23 & 1 & 3 & 0.37 & 167 & Dichotomous \\
T1_9_23 & 2 & 2 & 0.40 & 500 & Dichotomous \\
T1_9_4 & 1 & 4 & 0.27 & 210 & Dichotomous \\
T1_9_6 & 1 & 6 & 0.89 & 161 & Dichotomous \\
\hline
\end{tabular}


Table 3

Task 2 p-values and sample size

\begin{tabular}{lcccc}
\hline Item & Grade & Mean & N & Item Type \\
\hline T2_51_12 & 1 & 0.131 & 628 & Dichotomous \\
T2_51_12 & 2 & 0.322 & 500 & Dichotomous \\
T2_51_2 & 2 & 0.506 & 500 & Dichotomous \\
T2_51_3 & 3 & 0.341 & 589 & Dichotomous \\
T2_51_4 & 4 & 0.277 & 433 & Dichotomous \\
T2_51_5 & 5 & 0.713 & 446 & Dichotomous \\
T2_51_6 & 6 & 0.902 & 336 & Dichotomous \\
T2_52_1 & 1 & 0.143 & 628 & Dichotomous \\
T2_52_2 & 2 & 0.174 & 500 & Dichotomous \\
T2_52_3 & 3 & 0.314 & 589 & Dichotomous \\
T2_52_4 & 4 & 1.122 & 433 & Polytomous \\
T2_52_5 & 5 & 0.500 & 446 & Dichotomous \\
T2_52_6 & 6 & 0.530 & 336 & Dichotomous \\
T2_53_12 & 1 & 0.094 & 628 & Dichotomous \\
T2_53_12 & 2 & 0.224 & 500 & Dichotomous \\
T2_53_3 & 3 & 0.345 & 589 & Dichotomous \\
T2_53_4 & 4 & 0.536 & 433 & Dichotomous \\
T2_53_5 & 5 & 1.318 & 446 & Polytomous \\
T2_53_6 & 6 & 0.699 & 336 & Dichotomous \\
T2_54_1 & 1 & 0.377 & 628 & Dichotomous \\
T2_54_2 & 2 & 0.244 & 500 & Dichotomous \\
T2_54_3 & 3 & 0.375 & 589 & Dichotomous \\
T2_54_5 & 5 & 0.816 & 446 & Dichotomous \\
T2_54_6 & 6 & 0.551 & 336 & Dichotomous \\
T2_55_1 & 1 & 0.067 & 628 & Dichotomous \\
T2_55_2 & 2 & 0.026 & 500 & Dichotomous \\
T2_55_34 & 3 & 1.022 & 589 & Polytomous \\
T2_55_34 & 4 & 1.360 & 433 & Polytomous \\
T2_55_5 & 5 & 0.442 & 446 & Dichotomous \\
T2_56_12 & 1 & 0.084 & 628 & Dichotomous \\
T2_56_12 & 2 & 0.148 & 500 & Dichotomous \\
T2_56_34 & 3 & 0.671 & 589 & Dichotomous \\
T2_56_34 & 4 & 0.739 & 433 & Dichotomous \\
T2_56_6 & 6 & 1.015 & 336 & Polytomous \\
T2_57_1 & 1 & 0.311 & 628 & Dichotomous \\
T253 & 3 & 0.521 & 589 & Dichotomous \\
T25 & 5 & 0.742 & 446 & Dichotomous
\end{tabular}




\begin{tabular}{lllll} 
T2_57_6 & 6 & 1.345 & 336 & Polytomous \\
T2_58_12 & 1 & 0.078 & 628 & Dichotomous \\
T2_58_12 & 2 & 0.258 & 500 & Dichotomous \\
T2_58_2 & 2 & 0.192 & 500 & Dichotomous \\
T2_58_4 & 4 & 0.568 & 433 & Dichotomous \\
T2_58_5 & 5 & 1.309 & 446 & Polytomous \\
T2_58_6 & 6 & 0.714 & 336 & Dichotomous \\
T2_59_1 & 1 & 0.229 & 628 & Dichotomous \\
T2_59_34 & 3 & 0.569 & 589 & Dichotomous \\
T2_59_34 & 4 & 0.896 & 433 & Dichotomous \\
T2_59_5 & 5 & 0.814 & 446 & Dichotomous \\
T2_59_6 & 6 & 0.473 & 336 & Dichotomous \\
T2_60_1 & 1 & 0.175 & 628 & Dichotomous \\
T2_60_23 & 2 & 0.498 & 502 & Dichotomous \\
T2_60_23 & 3 & 0.603 & 589 & Dichotomous \\
T2_60_4 & 4 & 0.388 & 433 & Dichotomous \\
T2_60_6 & 6 & 0.762 & 336 & Dichotomous \\
T2_61_1 & 1 & 0.215 & 628 & Dichotomous \\
T2_61_2 & 2 & 0.456 & 500 & Dichotomous \\
T2_61_3 & 3 & 0.372 & 589 & Dichotomous \\
T2_61_45 & 4 & 0.335 & 433 & Dichotomous \\
T2_61_45 & 5 & 0.489 & 446 & Dichotomous \\
T2_61_56 & 5 & 1.184 & 446 & Polytomous \\
T2_61_56 & 6 & 1.330 & 336 & Polytomous \\
T2_61_6 & 6 & 1.280 & 336 & Polytomous \\
T2_62_1 & 1 & 0.170 & 628 & Dichotomous \\
T2_62_2 & 2 & 0.274 & 500 & Dichotomous \\
T2_62_3 & 3 & 0.397 & 589 & Dichotomous \\
T2_62_4 & 4 & 0.457 & 433 & Dichotomous \\
T2_62_5 & 5 & 0.531 & 446 & Dichotomous \\
T2_62_6 & 6 & 1.348 & 336 & Polytomous \\
T2_63_1 & 1 & 0.065 & 628 & Dichotomous \\
T2_63_2 & 2 & 0.480 & 500 & Dichotomous \\
T2_63_3 & 3 & 0.154 & 589 & Dichotomous \\
T2_63_4 & 4 & 0.501 & 433 & Dichotomous \\
T2_63_5 & 5 & 0.504 & 446 & Dichotomous \\
T2_63_6 & 6 & 0.565 & 336 & Dichotomous \\
T2_64_1 & 1 & 0.151 & 628 & Dichotomous \\
T2_64_2 & 2 & 0.260 & 500 & Dichotomous \\
T2_64_3 & 3 & 0.382 & 589 & Dichotomous \\
T2_64_456 & 4 & 0.947 & 433 & Dichotomous \\
& & & & \\
\hline
\end{tabular}




$\begin{array}{lllll}\text { T2_64_456 } & 5 & 1.085 & 446 & \text { Polytomous } \\ \text { T2_64_456 } & 6 & 1.208 & 336 & \text { Polytomous } \\ \text { T2_64_5 } & 5 & 0.565 & 446 & \text { Dichotomous } \\ \text { T2_65_1 } & 1 & 0.269 & 628 & \text { Dichotomous } \\ \text { T2_65_2 } & 2 & 0.524 & 500 & \text { Dichotomous } \\ \text { T2_65_4 } & 4 & 0.617 & 433 & \text { Dichotomous } \\ \text { T2_65_5 } & 5 & 0.164 & 446 & \text { Dichotomous } \\ \text { T2_65_6 } & 6 & 0.702 & 336 & \text { Dichotomous } \\ \text { T2_66_1 } & 1 & 0.153 & 628 & \text { Dichotomous } \\ \text { T2_66_22 } & 2 & 0.344 & 500 & \text { Dichotomous } \\ \text { T2_66_3 } & 3 & 0.508 & 589 & \text { Dichotomous } \\ \text { T2_66_4 } & 4 & 0.390 & 433 & \text { Dichotomous } \\ \text { T2_66_5 } & 5 & 0.388 & 446 & \text { Dichotomous } \\ \text { T2_66_6 } & 6 & 0.565 & 336 & \text { Dichotomous } \\ \text { T2_67_1 } & 1 & 0.182 & 628 & \text { Dichotomous } \\ \text { T2_67_2 } & 2 & 0.388 & 500 & \text { Dichotomous } \\ \text { T2_67_3 } & 3 & 0.435 & 589 & \text { Dichotomous } \\ \text { T2_67_4 } & 4 & 0.501 & 433 & \text { Dichotomous } \\ \text { T2_67_5 } & 5 & 0.484 & 446 & \text { Dichotomous } \\ \text { T2_67_6 } & 6 & 0.783 & 336 & \text { Dichotomous } \\ \text { T2_68_1 } & 1 & 0.166 & 628 & \text { Dichotomous } \\ \text { T2_68_2 } & 2 & 0.224 & 500 & \text { Dichotomous } \\ \text { T2_68_3 } & 3 & 0.363 & 589 & \text { Dichotomous } \\ \text { T2_68_4 } & 4 & 0.448 & 433 & \text { Dichotomous } \\ \text { T2_69_1 } & 1 & 0.209 & 628 & \text { Dichotomous } \\ \text { T2_69_2 } & 2 & 0.178 & 500 & \text { Dichotomous } \\ \text { T2_69_34 } & 3 & 0.587 & 589 & \text { Dichotomous } \\ \text { T2_69_34 } & 4 & 0.868 & 433 & \text { Dichotomous } \\ \text { T2_69_4 } & 4 & 0.386 & 433 & \text { Dichotomous } \\ \text { T2_69_5 } & 5 & 0.749 & 446 & \text { Dichotomous } \\ \text { T2_69_6 } & 6 & 1.625 & 336 & \text { Polytomous } \\ \text { T2_70_1 } & 1 & 0.137 & 628 & \text { Dichotomous } \\ \text { T2_70_23 } & 2 & 0.375 & 502 & \text { Dichotomous } \\ \text { T2_70_23 } & 3 & 0.416 & 589 & \text { Dichotomous } \\ \text { T2_70_3 } & 3 & 0.450 & 589 & \text { Dichotomous } \\ \text { T2_70_4 } & 4 & 1.021 & 433 & \text { Polytomous } \\ \text { T2_70_56 } & 5 & 1.206 & 446 & \text { Polytomous } \\ \text { T2_70_56 } & 6 & 1.182 & 336 & \text { Polytomous } \\ \text { T2_71_61 } & 6 & 1.244 & 336 & \text { Polytomous } \\ & 1 & 0.213 & 628 & \text { Dichotomous } \\ \text { T2 } & 3 & 0.241 & 589 & \text { Dichotomous }\end{array}$




\begin{tabular}{|c|c|c|c|c|}
\hline T2_71_456 & 4 & 0.185 & 433 & Dichotomous \\
\hline T2_71_456 & 5 & 0.426 & 446 & Dichotomous \\
\hline T2_71_456 & 6 & 0.423 & 336 & Dichotomous \\
\hline T2_71_56 & 5 & 0.646 & 446 & Dichotomous \\
\hline T2_71_56 & 6 & 0.664 & 336 & Dichotomous \\
\hline T2_72_1 & 1 & 0.027 & 628 & Dichotomous \\
\hline T2_72_2 & 2 & 0.028 & 500 & Dichotomous \\
\hline T2_72_4 & 4 & 0.263 & 433 & Dichotomous \\
\hline T2_72_5 & 5 & 1.170 & 446 & Polytomous \\
\hline T2_73_1 & 1 & 0.083 & 628 & Dichotomous \\
\hline T2_73_2 & 2 & 0.212 & 500 & Dichotomous \\
\hline T2_73_3 & 3 & 0.175 & 589 & Dichotomous \\
\hline T2_73_4 & 4 & 0.293 & 433 & Dichotomous \\
\hline T2_73_6 & 6 & 0.509 & 336 & Dichotomous \\
\hline T2_74_1 & 1 & 0.124 & 628 & Dichotomous \\
\hline T2_74_2 & 2 & 0.282 & 500 & Dichotomous \\
\hline T2_74_3 & 3 & 0.329 & 589 & Dichotomous \\
\hline T2_74_4 & 4 & 0.189 & 433 & Dichotomous \\
\hline T2_74_56 & 5 & 0.215 & 446 & Dichotomous \\
\hline T2_74_56 & 6 & 0.262 & 336 & Dichotomous \\
\hline T2_75_12 & 1 & 0.033 & 628 & Dichotomous \\
\hline T2_75_12 & 2 & 0.076 & 500 & Dichotomous \\
\hline T2_75_23 & 2 & 0.088 & 502 & Dichotomous \\
\hline T2_75_23 & 3 & 0.129 & 589 & Dichotomous \\
\hline T2_75_3 & 3 & 0.090 & 589 & Dichotomous \\
\hline T2_75_4 & 4 & 0.072 & 433 & Dichotomous \\
\hline T2_76_1 & 1 & 0.097 & 628 & Dichotomous \\
\hline T2_76_2 & 2 & 0.024 & 500 & Dichotomous \\
\hline T2_76_3 & 3 & 0.265 & 589 & Dichotomous \\
\hline T2_76_4 & 4 & 0.159 & 433 & Dichotomous \\
\hline T2_76_5 & 5 & 1.182 & 446 & Polytomous \\
\hline T2_77_1 & 1 & 0.100 & 628 & Dichotomous \\
\hline T2_77_2 & 2 & 0.290 & 500 & Dichotomous \\
\hline T2_77_34 & 3 & 0.430 & 589 & Dichotomous \\
\hline T2_77_34 & 4 & 0.630 & 433 & Dichotomous \\
\hline T2_77_456 & 4 & 0.182 & 433 & Dichotomous \\
\hline T2_77_456 & 5 & 0.233 & 446 & Dichotomous \\
\hline T2_77_456 & 6 & 0.265 & 336 & Dichotomous \\
\hline T2_77_5 & 5 & 1.209 & 446 & Polytomous \\
\hline T2_77_6 & 6 & 1.313 & 336 & Polytomous \\
\hline T2_78_1 & 1 & 0.108 & 628 & Dichotomous \\
\hline
\end{tabular}




$\begin{array}{lllll}\text { T2_78_23 } & 2 & 0.129 & 502 & \text { Dichotomous } \\ \text { T2_78_23 } & 3 & 0.238 & 589 & \text { Dichotomous } \\ \text { T2_78_3 } & 3 & 0.212 & 589 & \text { Dichotomous } \\ \text { T2_78_4 } & 4 & 0.383 & 433 & \text { Dichotomous } \\ \text { T2_78_5 } & 5 & 0.803 & 446 & \text { Dichotomous } \\ \text { T2_78_6 } & 6 & 0.839 & 336 & \text { Dichotomous } \\ \text { T2_79_1 } & 1 & 0.057 & 628 & \text { Dichotomous } \\ \text { T2_79_2 } & 2 & 0.390 & 500 & \text { Dichotomous } \\ \text { T2_79_3 } & 3 & 0.267 & 589 & \text { Dichotomous } \\ \text { T2_79_4 } & 4 & 0.330 & 433 & \text { Dichotomous } \\ \text { T2_79_56 } & 5 & 0.444 & 446 & \text { Dichotomous } \\ \text { T2_79_56 } & 6 & 0.521 & 336 & \text { Dichotomous } \\ \text { T2_79_6 } & 6 & 0.339 & 336 & \text { Dichotomous } \\ \text { T2_80_1 } & 1 & 0.212 & 628 & \text { Dichotomous } \\ \text { T2_80_2 } & 2 & 0.138 & 500 & \text { Dichotomous } \\ \text { T2_80_4 } & 4 & 0.423 & 433 & \text { Dichotomous } \\ \text { T2_80_5 } & 5 & 0.442 & 446 & \text { Dichotomous } \\ \text { T2_81_1 } & 1 & 0.099 & 628 & \text { Dichotomous } \\ \text { T2_81_2 } & 2 & 0.232 & 500 & \text { Dichotomous } \\ \text { T2_81_3 } & 3 & 0.090 & 589 & \text { Dichotomous } \\ \text { T2_81_456 } & 4 & 0.092 & 433 & \text { Dichotomous } \\ \text { T2_81_456 } & 5 & 0.083 & 446 & \text { Dichotomous } \\ \text { T2_81_456 } & 6 & 0.086 & 336 & \text { Dichotomous } \\ \text { T2_81_5 } & 5 & 0.545 & 446 & \text { Dichotomous } \\ \text { T2_81_6 } & 6 & 1.262 & 336 & \text { Polytomous } \\ \text { T2_82_1 } & 1 & 0.083 & 628 & \text { Dichotomous } \\ \text { T2_82_2 } & 2 & 0.146 & 500 & \text { Dichotomous } \\ \text { T2_82_3 } & 3 & 0.188 & 589 & \text { Dichotomous } \\ \text { T2_82_4 } & 4 & 0.046 & 433 & \text { Dichotomous } \\ \text { T2_82_5 } & 5 & 0.063 & 446 & \text { Dichotomous } \\ \text { T2_82_6 } & 6 & 0.137 & 336 & \text { Dichotomous } \\ \text { T2_83_1 } & 1 & 0.104 & 628 & \text { Dichotomous } \\ \text { T2_83_2 } & 2 & 0.328 & 500 & \text { Dichotomous } \\ \text { T2_83_3 } & 3 & 0.083 & 589 & \text { Dichotomous } \\ \text { T2_83_4 } & 4 & 0.125 & 433 & \text { Dichotomous } \\ \text { T2_83_5 } & 5 & 0.359 & 446 & \text { Dichotomous } \\ \text { T2_83_6 } & 6 & 0.560 & 336 & \text { Dichotomous } \\ \text { T2_84_1 } & 1 & 0.132 & 628 & \text { Dichotomous } \\ \text { T2_84_23 } & 2 & 0.185 & 502 & \text { Dichotomous } \\ \text { T2_84_23 } & 3 & 0.205 & 589 & \text { Dichotomous } \\ \text { T2_84_3 } & 3 & 0.258 & 589 & \text { Dichotomous }\end{array}$


MATRS TECHNICAL MANUAL 29

\begin{tabular}{lllll} 
T2_84_4 & 4 & 0.180 & 433 & Dichotomous \\
T2_84_5 & 5 & 0.074 & 446 & Dichotomous \\
T2_84_6 & 6 & 0.836 & 336 & Dichotomous \\
T2_85_1 & 1 & 0.022 & 628 & Dichotomous \\
T2_85_2 & 2 & 0.410 & 500 & Dichotomous \\
T2_85_3 & 3 & 0.107 & 589 & Dichotomous \\
T2_85_4 & 4 & 0.166 & 433 & Dichotomous \\
T2_85_5 & 5 & 0.296 & 446 & Dichotomous \\
T2_85_6 & 6 & 1.068 & 336 & Polytomous \\
\hline
\end{tabular}


Table 4

Task 3 p-values and sample size

\begin{tabular}{|c|c|c|c|c|c|}
\hline Item & Year & Grade & Mean & $\mathrm{N}$ & Item Type \\
\hline T3_1_2 & 1 & 2 & 0.63 & 224 & Dichotomous \\
\hline T3_1_3 & 1 & 3 & 0.43 & 167 & Dichotomous \\
\hline T3_1_5 & 1 & 5 & 0.70 & 180 & Dichotomous \\
\hline T3_1_6 & 1 & 6 & 0.73 & 161 & Dichotomous \\
\hline T3_10_12 & 1 & 1 & 0.24 & 179 & Dichotomous \\
\hline T3_10_12 & 1 & 2 & 0.36 & 224 & Dichotomous \\
\hline T3_10_2 & 1 & 2 & 0.60 & 224 & Dichotomous \\
\hline T3_10_3 & 1 & 3 & 0.38 & 167 & Dichotomous \\
\hline T3_10_4 & 1 & 4 & 0.52 & 210 & Dichotomous \\
\hline T3_10_5 & 1 & 5 & 0.51 & 180 & Dichotomous \\
\hline T3_10_6 & 1 & 6 & 0.48 & 161 & Dichotomous \\
\hline T3_11_12 & 1 & 1 & 0.27 & 179 & Dichotomous \\
\hline T3_11_12 & 1 & 2 & 0.53 & 224 & Dichotomous \\
\hline T3_11_2 & 1 & 2 & 0.58 & 224 & Dichotomous \\
\hline T3_11_34 & 1 & 3 & 0.25 & 167 & Dichotomous \\
\hline T3_11_34 & 1 & 4 & 0.24 & 210 & Dichotomous \\
\hline T3_11_4 & 1 & 4 & 0.70 & 210 & Dichotomous \\
\hline T3_11_5 & 1 & 5 & 0.79 & 180 & Dichotomous \\
\hline T3_11_5 & 2 & 5 & 0.82 & 446 & Dichotomous \\
\hline T3_12_12 & 1 & 1 & 0.44 & 179 & Dichotomous \\
\hline T3_12_12 & 1 & 2 & 0.26 & 224 & Dichotomous \\
\hline T3_12_2 & 1 & 2 & 0.34 & 224 & Dichotomous \\
\hline T3_12_3 & 1 & 3 & 0.61 & 167 & Dichotomous \\
\hline T3_12_3 & 2 & 3 & 0.75 & 589 & Dichotomous \\
\hline Т3_12_4 & 1 & 4 & 0.62 & 210 & Dichotomous \\
\hline T3_12_5 & 1 & 5 & 0.67 & 180 & Dichotomous \\
\hline T3_13_1 & 1 & 1 & 0.26 & 179 & Dichotomous \\
\hline T3_13_23 & 1 & 2 & 0.58 & 224 & Dichotomous \\
\hline T3_13_23 & 1 & 3 & 0.69 & 167 & Dichotomous \\
\hline T3_13_3 & 1 & 3 & 0.25 & 167 & Dichotomous \\
\hline T3_13_4 & 1 & 4 & 0.30 & 210 & Dichotomous \\
\hline T3_13_6 & 1 & 6 & 0.79 & 161 & Dichotomous \\
\hline T3_13_6 & 2 & 6 & 0.84 & 336 & Dichotomous \\
\hline T3_14_1 & 1 & 1 & 0.37 & 179 & Dichotomous \\
\hline T3_14_3 & 1 & 3 & 0.41 & 167 & Dichotomous \\
\hline T3_14_456 & 1 & 4 & 0.60 & 210 & Dichotomous \\
\hline T3_14_456 & 1 & 5 & 0.61 & 180 & Dichotomous \\
\hline
\end{tabular}




\begin{tabular}{|c|c|c|c|c|c|}
\hline T3_14_456 & 1 & 6 & 0.73 & 161 & Dichotomous \\
\hline T3_14_5 & 1 & 5 & 0.56 & 180 & Dichotomous \\
\hline T3_15_1 & 1 & 1 & 0.24 & 179 & Dichotomous \\
\hline T3_15_34 & 1 & 3 & 0.54 & 167 & Dichotomous \\
\hline T3_15_34 & 1 & 4 & 0.54 & 210 & Dichotomous \\
\hline T3_15_456 & 1 & 4 & 0.41 & 210 & Dichotomous \\
\hline T3_15_456 & 1 & 5 & 0.46 & 180 & Dichotomous \\
\hline T3_15_456 & 1 & 6 & 0.43 & 161 & Dichotomous \\
\hline T3_15_5 & 1 & 5 & 0.52 & 180 & Dichotomous \\
\hline T3_15_6 & 1 & 6 & 0.56 & 161 & Dichotomous \\
\hline T3_16_1 & 1 & 1 & 0.36 & 179 & Dichotomous \\
\hline T3_16_2 & 1 & 2 & 0.58 & 224 & Dichotomous \\
\hline T3_16_3 & 1 & 3 & 0.42 & 167 & Dichotomous \\
\hline T3_16_56 & 1 & 5 & 0.65 & 180 & Dichotomous \\
\hline T3_16_56 & 1 & 6 & 0.69 & 161 & Dichotomous \\
\hline T3_17_1 & 1 & 1 & 0.24 & 179 & Dichotomous \\
\hline T3_17_34 & 1 & 3 & 0.60 & 167 & Dichotomous \\
\hline T3_17_34 & 1 & 4 & 0.75 & 210 & Dichotomous \\
\hline T3_17_5 & 1 & 5 & 0.51 & 180 & Dichotomous \\
\hline T3_17_6 & 1 & 6 & 0.73 & 161 & Dichotomous \\
\hline T3_18_12 & 1 & 1 & 0.17 & 179 & Dichotomous \\
\hline T3_18_12 & 1 & 2 & 0.43 & 224 & Dichotomous \\
\hline T3_18_2 & 1 & 2 & 0.40 & 224 & Dichotomous \\
\hline T3_18_2 & 2 & 2 & 0.46 & 500 & Dichotomous \\
\hline T3_18_3 & 1 & 3 & 0.68 & 167 & Dichotomous \\
\hline T3_18_4 & 1 & 4 & 0.53 & 210 & Dichotomous \\
\hline T3_18_6 & 1 & 6 & 0.28 & 161 & Dichotomous \\
\hline T3_19_12 & 1 & 1 & 0.28 & 179 & Dichotomous \\
\hline T3_19_12 & 1 & 2 & 0.25 & 224 & Dichotomous \\
\hline T3_19_2 & 1 & 2 & 0.18 & 224 & Dichotomous \\
\hline T3_19_3 & 1 & 3 & 0.61 & 167 & Dichotomous \\
\hline T3_19_3 & 2 & 3 & 0.67 & 589 & Dichotomous \\
\hline T3_19_4 & 1 & 4 & 0.49 & 210 & Dichotomous \\
\hline T3_19_4 & 2 & 4 & 0.63 & 433 & Dichotomous \\
\hline T3_19_56 & 1 & 5 & 0.34 & 180 & Dichotomous \\
\hline T3_19_56 & 1 & 6 & 0.44 & 161 & Dichotomous \\
\hline T3_19_6 & 1 & 6 & 0.75 & 161 & Dichotomous \\
\hline T3_19_6 & 2 & 6 & 0.82 & 336 & Dichotomous \\
\hline T3_2_23 & 1 & 2 & 0.30 & 224 & Dichotomous \\
\hline T3_2_23 & 1 & 3 & 0.23 & 167 & Dichotomous \\
\hline T3_2_3 & 1 & 3 & 0.59 & 167 & Dichotomous \\
\hline
\end{tabular}




\begin{tabular}{|c|c|c|c|c|c|}
\hline T3_2_4 & 1 & 4 & 0.51 & 210 & Dichotomous \\
\hline T3_2_56 & 1 & 5 & 0.43 & 180 & Dichotomous \\
\hline T3_2_56 & 1 & 6 & 0.49 & 161 & Dichotomous \\
\hline T3_2_6 & 1 & 6 & 0.61 & 161 & Dichotomous \\
\hline T3_20_12 & 1 & 1 & 0.24 & 179 & Dichotomous \\
\hline T3_20_12 & 1 & 2 & 0.21 & 224 & Dichotomous \\
\hline T3_20_2 & 1 & 2 & 0.59 & 224 & Dichotomous \\
\hline T3_20_456 & 1 & 4 & 0.67 & 210 & Dichotomous \\
\hline T3_20_456 & 1 & 5 & 0.58 & 180 & Dichotomous \\
\hline T3_20_456 & 1 & 6 & 0.68 & 161 & Dichotomous \\
\hline T3_20_6 & 1 & 6 & 0.61 & 161 & Dichotomous \\
\hline T3_21_1 & 1 & 1 & 0.16 & 179 & Dichotomous \\
\hline T3_21_3 & 1 & 3 & 0.78 & 167 & Dichotomous \\
\hline T3_21_3 & 2 & 3 & 0.78 & 589 & Dichotomous \\
\hline T3_21_4 & 1 & 4 & 0.46 & 210 & Dichotomous \\
\hline T3_21_56 & 1 & 5 & 0.61 & 180 & Dichotomous \\
\hline T3_21_56 & 1 & 6 & 0.72 & 161 & Dichotomous \\
\hline T3_21_6 & 1 & 6 & 0.31 & 161 & Dichotomous \\
\hline T3_22_1 & 1 & 1 & 0.51 & 179 & Dichotomous \\
\hline T3_22_2 & 1 & 2 & 0.33 & 224 & Dichotomous \\
\hline T3_22_2 & 2 & 2 & 0.28 & 500 & Dichotomous \\
\hline T3_22_3 & 1 & 3 & 0.23 & 167 & Dichotomous \\
\hline T3_22_4 & 1 & 4 & 0.32 & 210 & Dichotomous \\
\hline T3_22_5 & 1 & 5 & 0.58 & 180 & Dichotomous \\
\hline T3_22_6 & 1 & 6 & 0.63 & 161 & Dichotomous \\
\hline T3_23_12 & 1 & 1 & 0.34 & 179 & Dichotomous \\
\hline T3_23_12 & 1 & 2 & 0.62 & 224 & Dichotomous \\
\hline T3_23_2 & 1 & 2 & 0.37 & 224 & Dichotomous \\
\hline T3_23_34 & 1 & 3 & 0.51 & 167 & Dichotomous \\
\hline T3_23_34 & 1 & 4 & 0.66 & 210 & Dichotomous \\
\hline T3_23_4 & 1 & 4 & 0.63 & 210 & Dichotomous \\
\hline T3_23_4 & 2 & 4 & 0.73 & 433 & Dichotomous \\
\hline T3_23_5 & 1 & 5 & 0.54 & 180 & Dichotomous \\
\hline T3_23_5 & 2 & 5 & 0.65 & 446 & Dichotomous \\
\hline T3_23_6 & 1 & 6 & 0.76 & 161 & Dichotomous \\
\hline T3_23_6 & 2 & 6 & 0.82 & 336 & Dichotomous \\
\hline T3_24_1 & 1 & 1 & 0.34 & 179 & Dichotomous \\
\hline T3_24_2 & 1 & 2 & 0.17 & 224 & Dichotomous \\
\hline T3_24_3 & 1 & 3 & 0.47 & 167 & Dichotomous \\
\hline T3_24_4 & 1 & 4 & 0.68 & 210 & Dichotomous \\
\hline T3_24_5 & 1 & 5 & 0.63 & 180 & Dichotomous \\
\hline
\end{tabular}




\begin{tabular}{|c|c|c|c|c|c|}
\hline T3_24_6 & 1 & 6 & 0.40 & 161 & Dichotomous \\
\hline T3_25_1 & 1 & 1 & 0.26 & 179 & Dichotomous \\
\hline T3_25_3 & 1 & 3 & 0.53 & 167 & Dichotomous \\
\hline T3_25_4 & 1 & 4 & 0.25 & 210 & Dichotomous \\
\hline T3_25_56 & 1 & 5 & 0.42 & 180 & Dichotomous \\
\hline T3_25_56 & 1 & 6 & 0.43 & 161 & Dichotomous \\
\hline T3_26_1 & 1 & 1 & 0.32 & 179 & Dichotomous \\
\hline T3_26_2 & 1 & 2 & 0.50 & 224 & Dichotomous \\
\hline T3_26_3 & 1 & 3 & 0.23 & 167 & Dichotomous \\
\hline T3_26_5 & 1 & 5 & 0.50 & 180 & Dichotomous \\
\hline T3_27_1 & 1 & 1 & 0.13 & 179 & Dichotomous \\
\hline T3_27_23 & 1 & 2 & 0.35 & 224 & Dichotomous \\
\hline T3_27_23 & 1 & 3 & 0.53 & 167 & Dichotomous \\
\hline T3_27_4 & 1 & 4 & 0.52 & 210 & Dichotomous \\
\hline T3_28_1 & 1 & 1 & 0.35 & 179 & Dichotomous \\
\hline T3_28_34 & 1 & 3 & 0.46 & 167 & Dichotomous \\
\hline T3_28_34 & 1 & 4 & 0.61 & 210 & Dichotomous \\
\hline T3_28_4 & 1 & 4 & 0.71 & 210 & Dichotomous \\
\hline T3_28_5 & 1 & 5 & 0.34 & 180 & Dichotomous \\
\hline T3_29_12 & 1 & 1 & 0.15 & 179 & Dichotomous \\
\hline T3_29_12 & 1 & 2 & 0.19 & 224 & Dichotomous \\
\hline T3_29_23 & 1 & 2 & 0.42 & 224 & Dichotomous \\
\hline T3_29_23 & 1 & 3 & 0.57 & 167 & Dichotomous \\
\hline T3_29_3 & 1 & 3 & 0.49 & 167 & Dichotomous \\
\hline T3_29_4 & 1 & 4 & 0.52 & 210 & Dichotomous \\
\hline T3_29_5 & 1 & 5 & 0.33 & 180 & Dichotomous \\
\hline T3_3_1 & 1 & 1 & 0.42 & 179 & Dichotomous \\
\hline T3_3_456 & 1 & 4 & 0.50 & 210 & Dichotomous \\
\hline T3_3_456 & 1 & 5 & 0.50 & 180 & Dichotomous \\
\hline T3_3_456 & 1 & 6 & 0.69 & 161 & Dichotomous \\
\hline T3_30_1 & 1 & 1 & 0.40 & 179 & Dichotomous \\
\hline T3_30_23 & 1 & 2 & 0.48 & 224 & Dichotomous \\
\hline T3_30_23 & 1 & 3 & 0.59 & 167 & Dichotomous \\
\hline T3_30_3 & 1 & 3 & 0.46 & 167 & Dichotomous \\
\hline T3_30_6 & 1 & 6 & 0.53 & 161 & Dichotomous \\
\hline T3_31_1 & 1 & 1 & 0.46 & 179 & Dichotomous \\
\hline T3_31_2 & 1 & 2 & 0.24 & 224 & Dichotomous \\
\hline T3_31_3 & 1 & 3 & 0.28 & 167 & Dichotomous \\
\hline T3_31_4 & 1 & 4 & 0.64 & 210 & Dichotomous \\
\hline T3_31_4 & 2 & 4 & 0.74 & 433 & Dichotomous \\
\hline T3_31_56 & 1 & 5 & 0.61 & 180 & Dichotomous \\
\hline
\end{tabular}




\begin{tabular}{|c|c|c|c|c|c|}
\hline T3_31_56 & 1 & 6 & 0.69 & 161 & Dichotomous \\
\hline T3_32_1 & 1 & 1 & 0.25 & 179 & Dichotomous \\
\hline T3_32_2 & 1 & 2 & 0.44 & 224 & Dichotomous \\
\hline T3_32_2 & 2 & 2 & 0.52 & 500 & Dichotomous \\
\hline T3_32_3 & 1 & 3 & 0.42 & 167 & Dichotomous \\
\hline T3_32_4 & 1 & 4 & 0.45 & 210 & Dichotomous \\
\hline T3_32_56 & 1 & 5 & 0.61 & 180 & Dichotomous \\
\hline T3_32_56 & 1 & 6 & 0.70 & 161 & Dichotomous \\
\hline T3_32_6 & 1 & 6 & 0.61 & 161 & Dichotomous \\
\hline T3_33_1 & 1 & 1 & 0.31 & 179 & Dichotomous \\
\hline T3_33_2 & 1 & 2 & 0.49 & 224 & Dichotomous \\
\hline T3_33_34 & 1 & 3 & 0.63 & 167 & Dichotomous \\
\hline T3_33_34 & 1 & 4 & 0.69 & 210 & Dichotomous \\
\hline T3_33_456 & 1 & 4 & 0.30 & 210 & Dichotomous \\
\hline T3_33_456 & 1 & 5 & 0.42 & 180 & Dichotomous \\
\hline T3_33_456 & 1 & 6 & 0.42 & 161 & Dichotomous \\
\hline T3_33_5 & 1 & 5 & 0.38 & 180 & Dichotomous \\
\hline T3_33_6 & 1 & 6 & 0.60 & 161 & Dichotomous \\
\hline T3_34_1 & 1 & 1 & 0.29 & 179 & Dichotomous \\
\hline T3_34_1 & 2 & 1 & 0.35 & 628 & Dichotomous \\
\hline T3_34_2 & 1 & 2 & 0.34 & 224 & Dichotomous \\
\hline T3_34_4 & 1 & 4 & 0.60 & 210 & Dichotomous \\
\hline T3_34_5 & 1 & 5 & 0.53 & 180 & Dichotomous \\
\hline T3_34_5 & 2 & 5 & 0.75 & 446 & Dichotomous \\
\hline T3_35_1 & 1 & 1 & 0.26 & 179 & Dichotomous \\
\hline T3_35_2 & 1 & 2 & 0.31 & 224 & Dichotomous \\
\hline T3_35_4 & 1 & 4 & 0.58 & 210 & Dichotomous \\
\hline T3_35_5 & 1 & 5 & 0.31 & 180 & Dichotomous \\
\hline T3_35_6 & 1 & 6 & 0.59 & 161 & Dichotomous \\
\hline T3_36_1 & 1 & 1 & 0.30 & 179 & Dichotomous \\
\hline T3_36_1 & 2 & 1 & 0.32 & 628 & Dichotomous \\
\hline T3_36_2 & 1 & 2 & 0.28 & 224 & Dichotomous \\
\hline T3_36_3 & 1 & 3 & 0.51 & 167 & Dichotomous \\
\hline T3_36_3 & 2 & 3 & 0.43 & 589 & Dichotomous \\
\hline T3_36_4 & 1 & 4 & 0.72 & 210 & Dichotomous \\
\hline T3_36_4 & 2 & 4 & 0.75 & 433 & Dichotomous \\
\hline T3_36_5 & 1 & 5 & 0.42 & 180 & Dichotomous \\
\hline T3_36_6 & 1 & 6 & 0.51 & 161 & Dichotomous \\
\hline T3_37_1 & 1 & 1 & 0.21 & 179 & Dichotomous \\
\hline T3_37_23 & 1 & 2 & 0.38 & 224 & Dichotomous \\
\hline T3_37_23 & 1 & 3 & 0.34 & 167 & Dichotomous \\
\hline
\end{tabular}




\begin{tabular}{|c|c|c|c|c|c|}
\hline T3_37_3 & 1 & 3 & 0.60 & 167 & Dichotomous \\
\hline T3_37_5 & 1 & 5 & 0.49 & 180 & Dichotomous \\
\hline T3_37_6 & 1 & 6 & 0.34 & 161 & Dichotomous \\
\hline T3_38_1 & 1 & 1 & 0.27 & 179 & Dichotomous \\
\hline T3_38_1 & 2 & 1 & 0.29 & 628 & Dichotomous \\
\hline T3_38_2 & 1 & 2 & 0.55 & 224 & Dichotomous \\
\hline T3_38_2 & 2 & 2 & 0.63 & 500 & Dichotomous \\
\hline T3_38_5 & 1 & 5 & 0.54 & 180 & Dichotomous \\
\hline T3_39_1 & 1 & 1 & 0.17 & 179 & Dichotomous \\
\hline T3_39_2 & 1 & 2 & 0.46 & 224 & Dichotomous \\
\hline T3_39_3 & 1 & 3 & 0.46 & 167 & Dichotomous \\
\hline T3_39_4 & 1 & 4 & 0.53 & 210 & Dichotomous \\
\hline T3_39_56 & 1 & 5 & 0.51 & 180 & Dichotomous \\
\hline T3_39_56 & 1 & 6 & 0.68 & 161 & Dichotomous \\
\hline T3_39_6 & 1 & 6 & 0.71 & 161 & Dichotomous \\
\hline T3_39_6 & 2 & 6 & 0.80 & 336 & Dichotomous \\
\hline T3_4_1 & 1 & 1 & 0.38 & 179 & Dichotomous \\
\hline T3_4_1 & 2 & 1 & 0.26 & 628 & Dichotomous \\
\hline T3_4_23 & 1 & 2 & 0.26 & 224 & Dichotomous \\
\hline T3_4_23 & 1 & 3 & 0.20 & 167 & Dichotomous \\
\hline T3_4_3 & 1 & 3 & 0.65 & 167 & Dichotomous \\
\hline T3_4_5 & 1 & 5 & 0.78 & 180 & Dichotomous \\
\hline T3_4_5 & 2 & 5 & 0.85 & 446 & Dichotomous \\
\hline T3_4_6 & 1 & 6 & 0.50 & 161 & Dichotomous \\
\hline T3_40_1 & 1 & 1 & 0.03 & 179 & Dichotomous \\
\hline T3_40_2 & 1 & 2 & 0.32 & 224 & Dichotomous \\
\hline T3_40_456 & 1 & 4 & 0.57 & 210 & Dichotomous \\
\hline T3_40_456 & 1 & 5 & 0.54 & 180 & Dichotomous \\
\hline T3_40_456 & 1 & 6 & 0.65 & 161 & Dichotomous \\
\hline T3_40_6 & 1 & 6 & 0.57 & 161 & Dichotomous \\
\hline T3_41_1 & 2 & 1 & 0.18 & 628 & Dichotomous \\
\hline T3_41_2 & 2 & 2 & 0.27 & 500 & Dichotomous \\
\hline T3_41_3 & 2 & 3 & 0.50 & 589 & Dichotomous \\
\hline T3_41_4 & 2 & 4 & 0.51 & 433 & Dichotomous \\
\hline T3_42_1 & 2 & 1 & 0.20 & 628 & Dichotomous \\
\hline T3_42_23 & 2 & 2 & 0.30 & 502 & Dichotomous \\
\hline T3_42_23 & 2 & 3 & 0.47 & 589 & Dichotomous \\
\hline T3_42_34 & 2 & 3 & 0.29 & 589 & Dichotomous \\
\hline T3_42_34 & 2 & 4 & 0.43 & 433 & Dichotomous \\
\hline T3_42_5 & 2 & 5 & 0.51 & 446 & Dichotomous \\
\hline T3_42_6 & 2 & 6 & 0.81 & 336 & Dichotomous \\
\hline
\end{tabular}




\begin{tabular}{|c|c|c|c|c|c|}
\hline T3_43_1 & 2 & 1 & 0.26 & 628 & Dichotomous \\
\hline T3_43_23 & 2 & 2 & 0.28 & 500 & Dichotomous \\
\hline T3_43_3 & 2 & 3 & 0.66 & 589 & Dichotomous \\
\hline T3_43_4 & 2 & 4 & 0.60 & 433 & Dichotomous \\
\hline T3_43_6 & 2 & 6 & 0.41 & 336 & Dichotomous \\
\hline T3_44_1 & 2 & 1 & 0.38 & 628 & Dichotomous \\
\hline T3_44_3 & 2 & 3 & 0.31 & 589 & Dichotomous \\
\hline T3_44_4 & 2 & 4 & 0.19 & 433 & Dichotomous \\
\hline T3_44_5 & 2 & 5 & 0.73 & 446 & Dichotomous \\
\hline T3_44_6 & 2 & 6 & 0.55 & 336 & Dichotomous \\
\hline T3_45_1 & 2 & 1 & 0.27 & 628 & Dichotomous \\
\hline T3_45_1.1 & 2 & 2 & 0.59 & 500 & Dichotomous \\
\hline T3_45_5 & 2 & 5 & 0.53 & 446 & Dichotomous \\
\hline T3_45_6 & 2 & 6 & 0.68 & 336 & Dichotomous \\
\hline T3_46_1 & 2 & 1 & 0.28 & 628 & Dichotomous \\
\hline T3_46_23 & 2 & 2 & 0.57 & 502 & Dichotomous \\
\hline T3_46_23 & 2 & 3 & 0.71 & 589 & Dichotomous \\
\hline T3_46_3 & 2 & 3 & 0.68 & 589 & Dichotomous \\
\hline T3_46_4 & 2 & 4 & 0.75 & 433 & Dichotomous \\
\hline T3_47_1 & 2 & 1 & 0.19 & 628 & Dichotomous \\
\hline T3_47_3 & 2 & 3 & 0.40 & 589 & Dichotomous \\
\hline T3_47_4 & 2 & 4 & 0.37 & 433 & Dichotomous \\
\hline T3_47_5 & 2 & 5 & 0.64 & 446 & Dichotomous \\
\hline T3_47_6 & 2 & 6 & 0.75 & 336 & Dichotomous \\
\hline T3_48_1 & 2 & 1 & 0.46 & 628 & Dichotomous \\
\hline T3_48_2 & 2 & 2 & 0.34 & 500 & Dichotomous \\
\hline T3_48_3 & 2 & 3 & 0.32 & 589 & Dichotomous \\
\hline T3_48_4 & 2 & 4 & 0.55 & 433 & Dichotomous \\
\hline T3_48_5 & 2 & 5 & 0.56 & 446 & Dichotomous \\
\hline T3_48_6 & 2 & 6 & 0.74 & 336 & Dichotomous \\
\hline T3_49_1 & 2 & 1 & 0.26 & 628 & Dichotomous \\
\hline T3_49_2 & 2 & 2 & 0.41 & 500 & Dichotomous \\
\hline T3_49_3 & 2 & 3 & 0.59 & 589 & Dichotomous \\
\hline T3_49_4 & 2 & 4 & 0.74 & 433 & Dichotomous \\
\hline T3_49_5 & 2 & 5 & 0.80 & 446 & Dichotomous \\
\hline T3_5_1 & 1 & 1 & 0.22 & 179 & Dichotomous \\
\hline T3_5_34 & 1 & 3 & 0.37 & 167 & Dichotomous \\
\hline T3_5_34 & 1 & 4 & 0.40 & 210 & Dichotomous \\
\hline T3_5_4 & 1 & 4 & 0.42 & 210 & Dichotomous \\
\hline T3_5_5 & 1 & 5 & 0.50 & 180 & Dichotomous \\
\hline T3_50_1 & 2 & 1 & 0.25 & 628 & Dichotomous \\
\hline
\end{tabular}




\begin{tabular}{|c|c|c|c|c|c|}
\hline T3_50_2 & 2 & 2 & 0.25 & 500 & Dichotomous \\
\hline T3_50_34 & 2 & 3 & 0.54 & 589 & Dichotomous \\
\hline T3_50_6 & 2 & 6 & 0.75 & 336 & Dichotomous \\
\hline T3_51_1 & 2 & 1 & 0.18 & 628 & Dichotomous \\
\hline T3_51_1.1 & 2 & 2 & 0.39 & 500 & Dichotomous \\
\hline T3_51_34 & 2 & 3 & 0.52 & 589 & Dichotomous \\
\hline T3_51_34 & 2 & 4 & 0.59 & 433 & Dichotomous \\
\hline T3_51_56 & 2 & 5 & 0.39 & 446 & Dichotomous \\
\hline T3_51_56 & 2 & 6 & 0.47 & 336 & Dichotomous \\
\hline T3_51_6 & 2 & 6 & 0.43 & 336 & Dichotomous \\
\hline T3_52_3 & 2 & 3 & 0.57 & 589 & Dichotomous \\
\hline T3_52_4 & 2 & 4 & 0.58 & 433 & Dichotomous \\
\hline T3_52_5 & 2 & 5 & 0.64 & 446 & Dichotomous \\
\hline T3_52_6 & 2 & 6 & 0.70 & 336 & Dichotomous \\
\hline T3_53_2 & 2 & 2 & 0.47 & 500 & Dichotomous \\
\hline T3_53_3 & 2 & 3 & 0.64 & 589 & Dichotomous \\
\hline T3_53_4 & 2 & 4 & 0.80 & 433 & Dichotomous \\
\hline T3_53_6 & 2 & 6 & 0.67 & 336 & Dichotomous \\
\hline T3_54_1 & 2 & 1 & 0.33 & 628 & Dichotomous \\
\hline T3_54_2 & 2 & 2 & 0.34 & 500 & Dichotomous \\
\hline T3_54_3 & 2 & 3 & 0.33 & 589 & Dichotomous \\
\hline T3_55_1 & 2 & 1 & 0.23 & 628 & Dichotomous \\
\hline T3_55_4 & 2 & 4 & 0.60 & 433 & Dichotomous \\
\hline T3_55_5 & 2 & 5 & 0.70 & 446 & Dichotomous \\
\hline T3_55_6 & 2 & 6 & 0.36 & 336 & Dichotomous \\
\hline T3_56_1 & 2 & 1 & 0.17 & 628 & Dichotomous \\
\hline T3_56_2 & 2 & 2 & 0.48 & 500 & Dichotomous \\
\hline T3_56_4 & 2 & 4 & 0.70 & 433 & Dichotomous \\
\hline T3_56_5 & 2 & 5 & 0.53 & 446 & Dichotomous \\
\hline T3_56_6 & 2 & 6 & 0.78 & 336 & Dichotomous \\
\hline T3_57_1 & 2 & 1 & 0.29 & 628 & Dichotomous \\
\hline T3_57_2 & 2 & 2 & 0.43 & 500 & Dichotomous \\
\hline T3_57_3 & 2 & 3 & 0.58 & 589 & Dichotomous \\
\hline T3_57_4 & 2 & 4 & 0.67 & 433 & Dichotomous \\
\hline T3_57_5 & 2 & 5 & 0.51 & 446 & Dichotomous \\
\hline T3_57_6 & 2 & 6 & 0.84 & 336 & Dichotomous \\
\hline T3_58_1 & 2 & 1 & 0.35 & 628 & Dichotomous \\
\hline T3_58_2 & 2 & 2 & 0.41 & 500 & Dichotomous \\
\hline T3_58_3 & 2 & 3 & 0.60 & 589 & Dichotomous \\
\hline T3_58_4 & 2 & 4 & 0.55 & 433 & Dichotomous \\
\hline T3_58_5 & 2 & 5 & 0.60 & 446 & Dichotomous \\
\hline
\end{tabular}




\begin{tabular}{|c|c|c|c|c|c|}
\hline T3_59_4 & 2 & 4 & 0.54 & 433 & Dichotomous \\
\hline T3_59_5 & 2 & 5 & 0.39 & 446 & Dichotomous \\
\hline T3_59_6 & 2 & 6 & 0.55 & 336 & Dichotomous \\
\hline T3_6_1 & 1 & 1 & 0.30 & 179 & Dichotomous \\
\hline T3_6_2 & 1 & 2 & 0.38 & 224 & Dichotomous \\
\hline T3_6_34 & 1 & 3 & 0.25 & 167 & Dichotomous \\
\hline T3_6_34 & 1 & 4 & 0.27 & 210 & Dichotomous \\
\hline T3_6_4 & 1 & 4 & 0.17 & 210 & Dichotomous \\
\hline T3_6_5 & 1 & 5 & 0.26 & 180 & Dichotomous \\
\hline T3_6_6 & 1 & 6 & 0.27 & 161 & Dichotomous \\
\hline T3_60_2 & 2 & 2 & 0.51 & 500 & Dichotomous \\
\hline T3_60_3 & 2 & 3 & 0.36 & 589 & Dichotomous \\
\hline T3_60_45 & 2 & 4 & 0.83 & 433 & Dichotomous \\
\hline T3_60_45 & 2 & 5 & 0.88 & 446 & Dichotomous \\
\hline T3_60_56 & 2 & 5 & 0.74 & 446 & Dichotomous \\
\hline T3_60_56 & 2 & 6 & 0.83 & 336 & Dichotomous \\
\hline T3_60_6 & 2 & 6 & 0.38 & 336 & Dichotomous \\
\hline T3_61_1 & 2 & 1 & 0.30 & 628 & Dichotomous \\
\hline T3_61_2 & 2 & 2 & 0.53 & 500 & Dichotomous \\
\hline T3_61_3 & 2 & 3 & 0.55 & 589 & Dichotomous \\
\hline T3_61_4 & 2 & 4 & 0.38 & 433 & Dichotomous \\
\hline T3_61_5 & 2 & 5 & 0.36 & 446 & Dichotomous \\
\hline T3_61_6 & 2 & 6 & 0.34 & 336 & Dichotomous \\
\hline T3_62_1 & 2 & 1 & 0.18 & 628 & Dichotomous \\
\hline T3_62_1 & 2 & 2 & 0.36 & 500 & Dichotomous \\
\hline T3_62_5 & 2 & 5 & 0.28 & 446 & Dichotomous \\
\hline T3_63_1 & 2 & 1 & 0.39 & 628 & Dichotomous \\
\hline T3_63_2 & 2 & 2 & 0.51 & 500 & Dichotomous \\
\hline T3_63_45 & 2 & 4 & 0.26 & 433 & Dichotomous \\
\hline T3_63_45 & 2 & 5 & 0.18 & 446 & Dichotomous \\
\hline T3_63_5 & 2 & 5 & 0.55 & 446 & Dichotomous \\
\hline T3_63_6 & 2 & 6 & 0.24 & 336 & Dichotomous \\
\hline T3_64_12 & 2 & 1 & 0.36 & 628 & Dichotomous \\
\hline T3_64_12 & 2 & 2 & 0.47 & 500 & Dichotomous \\
\hline T3_64_12.1 & 2 & 2 & 0.42 & 500 & Dichotomous \\
\hline T3_64_3 & 2 & 3 & 0.42 & 589 & Dichotomous \\
\hline T3_64_4 & 2 & 4 & 0.33 & 433 & Dichotomous \\
\hline T3_64_5 & 2 & 5 & 0.72 & 446 & Dichotomous \\
\hline T3_64_6 & 2 & 6 & 0.70 & 336 & Dichotomous \\
\hline T3_7_1 & 1 & 1 & 0.19 & 179 & Dichotomous \\
\hline T3_7_2 & 1 & 2 & 0.36 & 224 & Dichotomous \\
\hline
\end{tabular}




\begin{tabular}{llllll} 
T3_7_4 & 1 & 4 & 0.36 & 210 & Dichotomous \\
T3_7_6 & 1 & 6 & 0.39 & 161 & Dichotomous \\
T3_8_1 & 1 & 1 & 0.28 & 179 & Dichotomous \\
T3_8_23 & 1 & 2 & 0.20 & 224 & Dichotomous \\
T3_8_23 & 1 & 3 & 0.21 & 167 & Dichotomous \\
T3_8_3 & 1 & 3 & 0.31 & 167 & Dichotomous \\
T3_8_456 & 1 & 4 & 0.21 & 210 & Dichotomous \\
T3_8_456 & 1 & 5 & 0.31 & 180 & Dichotomous \\
T3_8_456 & 1 & 6 & 0.30 & 161 & Dichotomous \\
T3_8_5 & 1 & 5 & 0.58 & 180 & Dichotomous \\
T3_9_1 & 1 & 1 & 0.39 & 179 & Dichotomous \\
T3_9_2 & 1 & 2 & 0.47 & 224 & Dichotomous \\
T3_9_3 & 1 & 3 & 0.49 & 167 & Dichotomous \\
T3_9_456 & 1 & 4 & 0.24 & 210 & Dichotomous \\
T3_9_456 & 1 & 5 & 0.29 & 180 & Dichotomous \\
T3_9_456 & 1 & 6 & 0.38 & 161 & Dichotomous \\
T3_9_5 & 1 & 5 & 0.45 & 180 & Dichotomous \\
\hline
\end{tabular}


Table 5

Task 4 p-values and sample size

\begin{tabular}{lccccc}
\hline Item & Year & Grade & Mean & N & Item Type \\
\hline T4_1_3 & 1 & 3 & 0.43 & 167 & Dichotomous \\
T4_1_3 & 2 & 3 & 0.43 & 589 & Dichotomous \\
T4_1_456 & 1 & 4 & 0.35 & 210 & Dichotomous \\
T4_1_456 & 1 & 5 & 0.46 & 180 & Dichotomous \\
T4_1_456 & 1 & 6 & 0.39 & 161 & Dichotomous \\
T4_1_5 & 1 & 5 & 0.85 & 180 & Dichotomous \\
T4_10_3 & 1 & 3 & 0.63 & 167 & Dichotomous \\
T4_10_4 & 1 & 4 & 0.71 & 210 & Dichotomous \\
T4_11_3 & 1 & 3 & 0.78 & 167 & Dichotomous \\
T4_11_3 & 2 & 3 & 0.77 & 589 & Dichotomous \\
T4_11_56 & 1 & 5 & 0.45 & 180 & Dichotomous \\
T4_11_56 & 1 & 6 & 0.67 & 161 & Dichotomous \\
T4_11_6 & 1 & 6 & 0.58 & 161 & Dichotomous \\
T4_12_34 & 1 & 3 & 0.60 & 167 & Dichotomous \\
T4_12_34 & 1 & 4 & 0.74 & 210 & Dichotomous \\
T4_12_4 & 1 & 4 & 0.73 & 210 & Dichotomous \\
T4_12_4 & 2 & 4 & 0.75 & 433 & Dichotomous \\
T4_12_5 & 1 & 5 & 0.51 & 180 & Dichotomous \\
T4_12_6 & 1 & 6 & 0.50 & 161 & Dichotomous \\
T4_13_3 & 1 & 3 & 0.84 & 167 & Dichotomous \\
T4_13_4 & 1 & 4 & 0.44 & 210 & Dichotomous \\
T4_13_6 & 1 & 6 & 0.75 & 161 & Dichotomous \\
T4_14_3 & 1 & 3 & 0.77 & 167 & Dichotomous \\
T4_14_456 & 1 & 4 & 0.60 & 210 & Dichotomous \\
T4_14_456 & 1 & 5 & 0.44 & 180 & Dichotomous \\
T4_14_456 & 1 & 6 & 0.52 & 161 & Dichotomous \\
T4_14_56 & 1 & 5 & 0.38 & 180 & Dichotomous \\
T4_14_56 & 1 & 6 & 0.53 & 161 & Dichotomous \\
T4_14_6 & 1 & 6 & 0.15 & 161 & Dichotomous \\
T4_15_3 & 1 & 3 & 0.40 & 167 & Dichotomous \\
T4_15_456 & 1 & 4 & 0.58 & 210 & Dichotomous \\
T4_15_456 & 1 & 5 & 0.57 & 180 & Dichotomous \\
T4_15_456 & 1 & 6 & 0.67 & 161 & Dichotomous \\
T4_16_3 & 1 & 3 & 0.54 & 167 & Dichotomous \\
T4_16_4 & 1 & 4 & 0.73 & 210 & Dichotomous \\
T4_16_5 & 1 & 5 & 0.53 & 180 & Dichotomous \\
T4_16_6 & 1 & 6 & 0.76 & 161 & Dichotomous \\
& & & & & \\
\hline
\end{tabular}




\begin{tabular}{|c|c|c|c|c|c|}
\hline T4_17_3 & 1 & 3 & 0.70 & 167 & Dichotomous \\
\hline T4_17_4 & 1 & 4 & 0.78 & 210 & Dichotomous \\
\hline T4_17_5 & 1 & 5 & 0.71 & 180 & Dichotomous \\
\hline T4_17_6 & 1 & 6 & 0.70 & 161 & Dichotomous \\
\hline T4_18_3 & 1 & 3 & 0.58 & 167 & Dichotomous \\
\hline T4_18_5 & 1 & 5 & 0.49 & 180 & Dichotomous \\
\hline T4_18_6 & 1 & 6 & 0.72 & 161 & Dichotomous \\
\hline T4_19_3 & 1 & 3 & 0.37 & 167 & Dichotomous \\
\hline T4_19_5 & 1 & 5 & 0.55 & 180 & Dichotomous \\
\hline T4_19_5 & 2 & 5 & 0.62 & 446 & Dichotomous \\
\hline T4_2_3 & 1 & 3 & 0.57 & 167 & Dichotomous \\
\hline T4_2_4 & 1 & 4 & 0.85 & 210 & Dichotomous \\
\hline T4_2_5 & 1 & 5 & 0.71 & 180 & Dichotomous \\
\hline T4_20_4 & 1 & 4 & 0.81 & 210 & Dichotomous \\
\hline T4_20_4 & 2 & 4 & 0.80 & 433 & Dichotomous \\
\hline T4_20_5 & 1 & 5 & 0.66 & 180 & Dichotomous \\
\hline T4_20_6 & 1 & 6 & 0.55 & 161 & Dichotomous \\
\hline T4_21_3 & 1 & 3 & 0.42 & 167 & Dichotomous \\
\hline T4_21_4 & 1 & 4 & 0.50 & 210 & Dichotomous \\
\hline T4_21_5 & 1 & 5 & 0.12 & 180 & Dichotomous \\
\hline T4_22_3 & 1 & 3 & 0.75 & 167 & Dichotomous \\
\hline T4_22_456 & 1 & 4 & 0.77 & 210 & Dichotomous \\
\hline T4_22_456 & 1 & 5 & 0.85 & 180 & Dichotomous \\
\hline T4_22_456 & 1 & 6 & 0.81 & 161 & Dichotomous \\
\hline T4_22_56 & 1 & 5 & 0.63 & 180 & Dichotomous \\
\hline T4_22_56 & 1 & 6 & 0.57 & 161 & Dichotomous \\
\hline T4_22_6 & 1 & 6 & 0.50 & 161 & Dichotomous \\
\hline T4_23_34 & 1 & 3 & 0.62 & 167 & Dichotomous \\
\hline T4_23_34 & 1 & 4 & 0.72 & 210 & Dichotomous \\
\hline T4_23_56 & 1 & 5 & 0.74 & 180 & Dichotomous \\
\hline T4_23_56 & 1 & 6 & 0.74 & 161 & Dichotomous \\
\hline T4_23_56 & 2 & 5 & 0.83 & 446 & Dichotomous \\
\hline T4_24_3 & 1 & 3 & 0.66 & 167 & Dichotomous \\
\hline T4_24_4 & 1 & 4 & 0.84 & 210 & Dichotomous \\
\hline T4_24_6 & 1 & 6 & 0.61 & 161 & Dichotomous \\
\hline T4_25_34 & 1 & 3 & 0.40 & 167 & Dichotomous \\
\hline T4_25_34 & 1 & 4 & 0.63 & 210 & Dichotomous \\
\hline T4_25_4 & 1 & 4 & 0.52 & 210 & Dichotomous \\
\hline T4_25_5 & 1 & 5 & 0.78 & 180 & Dichotomous \\
\hline T4_26_3 & 2 & 3 & 0.69 & 589 & Dichotomous \\
\hline T4_26_5 & 2 & 5 & 0.91 & 446 & Dichotomous \\
\hline
\end{tabular}




\begin{tabular}{|c|c|c|c|c|c|}
\hline T4_27_23 & 2 & 3 & 0.32 & 589 & Dichotomous \\
\hline T4_27_23 & 2 & 4 & 0.43 & 433 & Dichotomous \\
\hline T4_27_4 & 2 & 4 & 0.60 & 433 & Dichotomous \\
\hline T4_27_6 & 2 & 6 & 0.49 & 336 & Dichotomous \\
\hline T4_28_23 & 2 & 3 & 0.69 & 589 & Dichotomous \\
\hline T4_28_23 & 2 & 4 & 0.75 & 433 & Dichotomous \\
\hline T4_28_4 & 2 & 4 & 0.68 & 433 & Dichotomous \\
\hline T4_29_3 & 2 & 3 & 0.76 & 589 & Dichotomous \\
\hline T4_29_34 & 2 & 4 & 0.76 & 433 & Dichotomous \\
\hline T4_29_5 & 2 & 5 & 0.69 & 446 & Dichotomous \\
\hline T4_29_6 & 2 & 6 & 0.87 & 336 & Dichotomous \\
\hline T4_3_3 & 1 & 3 & 0.82 & 167 & Dichotomous \\
\hline T4_3_3 & 2 & 3 & 0.80 & 589 & Dichotomous \\
\hline T4_3_4 & 1 & 4 & 0.62 & 210 & Dichotomous \\
\hline T4_3_5 & 1 & 5 & 0.66 & 180 & Dichotomous \\
\hline T4_30_3 & 2 & 3 & 0.67 & 589 & Dichotomous \\
\hline T4_30_4 & 2 & 4 & 0.74 & 433 & Dichotomous \\
\hline T4_30_5 & 2 & 5 & 0.58 & 446 & Dichotomous \\
\hline T4_30_6 & 2 & 6 & 0.29 & 336 & Dichotomous \\
\hline T4_31_4 & 2 & 4 & 0.77 & 433 & Dichotomous \\
\hline T4_31_56 & 2 & 5 & 0.82 & 446 & Dichotomous \\
\hline T4_31_56 & 2 & 6 & 0.88 & 336 & Dichotomous \\
\hline T4_31_6 & 2 & 6 & 0.53 & 336 & Dichotomous \\
\hline T4_32_3 & 2 & 3 & 0.84 & 589 & Dichotomous \\
\hline T4_32_3.1 & 2 & 3 & 0.72 & 589 & Dichotomous \\
\hline T4_32_4 & 2 & 4 & 0.44 & 433 & Dichotomous \\
\hline T4_32_6 & 2 & 6 & 0.48 & 336 & Dichotomous \\
\hline T4_33_4 & 2 & 4 & 0.83 & 433 & Dichotomous \\
\hline T4_33_6 & 2 & 6 & 0.94 & 336 & Dichotomous \\
\hline T4_34_3 & 2 & 3 & 0.71 & 589 & Dichotomous \\
\hline T4_34_4 & 2 & 4 & 0.78 & 433 & Dichotomous \\
\hline T4_34_6 & 2 & 6 & 0.87 & 336 & Dichotomous \\
\hline T4_35_3 & 2 & 3 & 0.72 & 589 & Dichotomous \\
\hline T4_35_6 & 2 & 6 & 0.75 & 336 & Dichotomous \\
\hline T4_36_34 & 2 & 4 & 0.72 & 433 & Dichotomous \\
\hline T4_36_6 & 2 & 6 & 0.65 & 336 & Dichotomous \\
\hline T4_37_3 & 2 & 3 & 0.39 & 589 & Dichotomous \\
\hline T4_37_4 & 2 & 4 & 0.60 & 433 & Dichotomous \\
\hline T4_38_3 & 2 & 3 & 0.75 & 589 & Dichotomous \\
\hline T4_38_45 & 2 & 4 & 0.59 & 433 & Dichotomous \\
\hline T4_38_45 & 2 & 5 & 0.70 & 446 & Dichotomous \\
\hline
\end{tabular}




\begin{tabular}{|c|c|c|c|c|c|}
\hline T4_38_6 & 2 & 6 & 0.77 & 336 & Dichotomous \\
\hline T4_39_3 & 2 & 3 & 0.29 & 589 & Dichotomous \\
\hline T4_39_6 & 2 & 6 & 0.58 & 336 & Dichotomous \\
\hline T4_4_3 & 1 & 3 & 0.80 & 167 & Dichotomous \\
\hline T4_4_5 & 1 & 5 & 0.58 & 180 & Dichotomous \\
\hline T4_4_5 & 2 & 5 & 0.71 & 446 & Dichotomous \\
\hline T4_4_6 & 1 & 6 & 0.11 & 161 & Dichotomous \\
\hline T4_4_6 & 2 & 6 & 0.14 & 336 & Dichotomous \\
\hline T4_40_3 & 2 & 3 & 0.59 & 589 & Dichotomous \\
\hline T4_41_3 & 2 & 3 & 0.69 & 589 & Dichotomous \\
\hline T4_41_45 & 2 & 4 & 0.82 & 433 & Dichotomous \\
\hline T4_41_45 & 2 & 5 & 0.87 & 446 & Dichotomous \\
\hline T4_42_3 & 2 & 3 & 0.50 & 589 & Dichotomous \\
\hline T4_43_34 & 2 & 4 & 0.46 & 433 & Dichotomous \\
\hline T4_43_5 & 2 & 5 & 0.74 & 446 & Dichotomous \\
\hline T4_43_6 & 2 & 6 & 0.84 & 336 & Dichotomous \\
\hline T4_45_56 & 2 & 5 & 0.77 & 446 & Dichotomous \\
\hline T4_45_56 & 2 & 6 & 0.82 & 336 & Dichotomous \\
\hline T4_46_56 & 2 & 5 & 0.83 & 446 & Dichotomous \\
\hline T4_46_56 & 2 & 6 & 0.88 & 336 & Dichotomous \\
\hline T4_47_5 & 2 & 5 & 0.76 & 446 & Dichotomous \\
\hline T4_48_5 & 2 & 5 & 0.46 & 446 & Dichotomous \\
\hline T4_49_5 & 2 & 5 & 0.61 & 446 & Dichotomous \\
\hline T4_5_3 & 1 & 3 & 0.67 & 167 & Dichotomous \\
\hline T4_5_4 & 1 & 4 & 0.71 & 210 & Dichotomous \\
\hline T4_5_56 & 1 & 5 & 0.82 & 180 & Dichotomous \\
\hline T4_5_56 & 1 & 6 & 0.80 & 161 & Dichotomous \\
\hline T4_5_6 & 1 & 6 & 0.87 & 161 & Dichotomous \\
\hline T4_5_6 & 2 & 6 & 0.92 & 336 & Dichotomous \\
\hline T4_50_5 & 2 & 5 & 0.73 & 446 & Dichotomous \\
\hline T4_51_5 & 2 & 5 & 0.71 & 446 & Dichotomous \\
\hline T4_52_5 & 2 & 5 & 0.88 & 446 & Dichotomous \\
\hline T4_6_3 & 1 & 3 & 0.66 & 167 & Dichotomous \\
\hline T4_6_456 & 1 & 4 & 0.53 & 210 & Dichotomous \\
\hline T4_6_456 & 1 & 5 & 0.56 & 180 & Dichotomous \\
\hline T4_6_456 & 1 & 6 & 0.66 & 161 & Dichotomous \\
\hline T4_7_34 & 1 & 3 & 0.60 & 167 & Dichotomous \\
\hline T4_7_34 & 1 & 4 & 0.79 & 210 & Dichotomous \\
\hline T4_7_34 & 2 & 4 & 0.74 & 433 & Dichotomous \\
\hline T4_7_4 & 1 & 4 & 0.74 & 210 & Dichotomous \\
\hline T4_7_5 & 1 & 5 & 0.57 & 180 & Dichotomous \\
\hline
\end{tabular}




$\begin{array}{llllll}\text { T4_7_6 } & 1 & 6 & 0.77 & 161 & \text { Dichotomous } \\ \text { T4_7_6 } & 2 & 6 & 0.75 & 336 & \text { Dichotomous } \\ \text { T4_8_34 } & 1 & 3 & 0.75 & 167 & \text { Dichotomous } \\ \text { T4_8_34 } & 1 & 4 & 0.92 & 210 & \text { Dichotomous } \\ \text { T4_8_4 } & 1 & 4 & 0.55 & 210 & \text { Dichotomous } \\ \text { T4_8_5 } & 1 & 5 & 0.15 & 180 & \text { Dichotomous } \\ \text { T4_8_6 } & 1 & 6 & 0.63 & 161 & \text { Dichotomous } \\ \text { T4_9_3 } & 1 & 3 & 0.85 & 167 & \text { Dichotomous } \\ \text { T4_9_4 } & 1 & 4 & 0.83 & 210 & \text { Dichotomous } \\ \text { T4_9_6 } & 1 & 6 & 0.60 & 161 & \text { Dichotomous }\end{array}$


Table 6

Task 5 p-values and sample size

\begin{tabular}{lccccc}
\hline Item & Year & Grade & Mean & N & Item Type \\
\hline T5_1_1 & 1 & 1 & 0.27 & 179 & Dichotomous \\
T5_1_4 & 1 & 4 & 0.18 & 210 & Dichotomous \\
T5_1_4 & 2 & 5 & 0.16 & 446 & Dichotomous \\
T5_1_5 & 1 & 5 & 0.17 & 180 & Dichotomous \\
T5_1_6 & 1 & 6 & 0.16 & 161 & Dichotomous \\
T5_1_6 & 2 & 6 & 0.23 & 336 & Dichotomous \\
T5_10_12 & 1 & 1 & 0.01 & 179 & Dichotomous \\
T5_10_12 & 1 & 2 & 0.04 & 224 & Dichotomous \\
T5_10_12 & 2 & 2 & 0.07 & 500 & Dichotomous \\
T5_10_23 & 1 & 2 & 0.07 & 224 & Dichotomous \\
T5_10_23 & 1 & 3 & 0.19 & 167 & Dichotomous \\
T5_10_34 & 1 & 3 & 0.22 & 167 & Dichotomous \\
T5_10_34 & 1 & 4 & 0.32 & 210 & Dichotomous \\
T5_10_5 & 1 & 5 & 0.27 & 180 & Dichotomous \\
T5_10_6 & 1 & 6 & 0.04 & 161 & Dichotomous \\
T5_11_1 & 1 & 1 & 0.21 & 179 & Dichotomous \\
T5_11_1 & 2 & 1 & 0.16 & 628 & Dichotomous \\
T5_11_23 & 1 & 2 & 0.39 & 224 & Dichotomous \\
T5_11_23 & 1 & 3 & 0.63 & 167 & Dichotomous \\
T5_11_3 & 1 & 3 & 0.01 & 167 & Dichotomous \\
T5_12_1 & 1 & 1 & 0.04 & 179 & Dichotomous \\
T5_12_1 & 2 & 1 & 0.04 & 628 & Dichotomous \\
T5_12_2 & 1 & 2 & 0.38 & 224 & Dichotomous \\
T5_12_34 & 1 & 3 & 0.01 & 167 & Dichotomous \\
T5_12_34 & 1 & 4 & 0.03 & 210 & Dichotomous \\
T5_12_4 & 1 & 4 & 0.29 & 210 & Dichotomous \\
T5_12_56 & 1 & 5 & 0.13 & 180 & Dichotomous \\
T5_12_56 & 1 & 6 & 0.20 & 161 & Dichotomous \\
T5_13_1 & 1 & 1 & 0.00 & 179 & Dichotomous \\
T5_13_2 & 1 & 2 & 0.12 & 224 & Dichotomous \\
T5_13_4 & 1 & 4 & 0.25 & 210 & Dichotomous \\
T5_13_5 & 1 & 5 & 0.43 & 180 & Dichotomous \\
T5_13_6 & 1 & 6 & 0.48 & 161 & Dichotomous \\
T5_14_1 & 1 & 1 & 0.01 & 179 & Dichotomous \\
T5_14_2 & 1 & 2 & 0.05 & 224 & Dichotomous \\
T5_14_34 & 1 & 3 & 0.03 & 167 & Dichotomous \\
T5_14_34 & 1 & 4 & 0.08 & 210 & Dichotomous
\end{tabular}




\begin{tabular}{|c|c|c|c|c|c|}
\hline T5_14_4 & 1 & 4 & 0.54 & 210 & Dichotomous \\
\hline T5_15_1 & 1 & 1 & 0.01 & 179 & Dichotomous \\
\hline T5_15_2 & 1 & 2 & 0.08 & 224 & Dichotomous \\
\hline T5_15_2 & 2 & 2 & 0.09 & 500 & Dichotomous \\
\hline T5_15_456 & 1 & 4 & 0.14 & 210 & Dichotomous \\
\hline T5_15_456 & 1 & 5 & 0.38 & 180 & Dichotomous \\
\hline T5_15_456 & 1 & 6 & 0.47 & 161 & Dichotomous \\
\hline T5_15_5 & 1 & 5 & 0.13 & 180 & Dichotomous \\
\hline T5_15_6 & 1 & 6 & 0.16 & 161 & Dichotomous \\
\hline T5_16_1 & 1 & 1 & 0.02 & 179 & Dichotomous \\
\hline T5_16_3 & 1 & 3 & 0.10 & 167 & Dichotomous \\
\hline T5_16_4 & 1 & 4 & 0.36 & 210 & Dichotomous \\
\hline T5_16_5 & 1 & 5 & 0.09 & 180 & Dichotomous \\
\hline T5_17_1 & 1 & 1 & 0.06 & 179 & Dichotomous \\
\hline T5_17_2 & 1 & 2 & 0.00 & 224 & Dichotomous \\
\hline T5_17_34 & 1 & 3 & 0.18 & 167 & Dichotomous \\
\hline T5_17_34 & 1 & 4 & 0.35 & 210 & Dichotomous \\
\hline T5_17_4 & 1 & 4 & 0.09 & 210 & Dichotomous \\
\hline T5_17_5 & 1 & 5 & 0.13 & 180 & Dichotomous \\
\hline T5_18_12 & 1 & 1 & 0.07 & 179 & Dichotomous \\
\hline T5_18_12 & 1 & 2 & 0.35 & 224 & Dichotomous \\
\hline T5_18_2 & 1 & 2 & 0.47 & 224 & Dichotomous \\
\hline T5_18_3 & 1 & 3 & 0.05 & 167 & Dichotomous \\
\hline T5_18_5 & 1 & 5 & 0.08 & 180 & Dichotomous \\
\hline T5_19_1 & 1 & 1 & 0.04 & 179 & Dichotomous \\
\hline T5_19_2 & 1 & 2 & 0.41 & 224 & Dichotomous \\
\hline T5_19_3 & 1 & 3 & 0.03 & 167 & Dichotomous \\
\hline T5_19_4 & 1 & 4 & 0.00 & 210 & Dichotomous \\
\hline T5_19_5 & 1 & 5 & 0.26 & 180 & Dichotomous \\
\hline T5_2_1 & 1 & 1 & 0.08 & 179 & Dichotomous \\
\hline T5_2_2 & 1 & 2 & 0.50 & 224 & Dichotomous \\
\hline T5_2_2.1 & 2 & 2 & 0.50 & 500 & Dichotomous \\
\hline T5_2_4 & 1 & 4 & 0.48 & 210 & Dichotomous \\
\hline T5_2_6 & 1 & 6 & 0.11 & 161 & Dichotomous \\
\hline T5_2_6 & 2 & 6 & 0.22 & 336 & Dichotomous \\
\hline T5_20_1 & 1 & 1 & 0.01 & 179 & Dichotomous \\
\hline T5_20_2 & 1 & 2 & 0.30 & 224 & Dichotomous \\
\hline T5_20_3 & 1 & 3 & 0.00 & 167 & Dichotomous \\
\hline T5_20_4 & 1 & 4 & 0.32 & 210 & Dichotomous \\
\hline T5_20_4 & 2 & 4 & 0.23 & 433 & Dichotomous \\
\hline T5_20_56 & 1 & 5 & 0.01 & 180 & Dichotomous \\
\hline
\end{tabular}




\begin{tabular}{|c|c|c|c|c|c|}
\hline T5_20_56 & 1 & 6 & 0.07 & 161 & Dichotomous \\
\hline T5_20_6 & 1 & 6 & 0.34 & 161 & Dichotomous \\
\hline T5_21_1 & 1 & 1 & 0.15 & 179 & Dichotomous \\
\hline T5_21_2 & 1 & 2 & 0.29 & 224 & Dichotomous \\
\hline T5_21_3 & 1 & 3 & 0.47 & 167 & Dichotomous \\
\hline T5_21_3 & 2 & 3 & 0.42 & 589 & Dichotomous \\
\hline T5_21_456 & 1 & 4 & 0.08 & 210 & Dichotomous \\
\hline T5_21_456 & 1 & 5 & 0.12 & 180 & Dichotomous \\
\hline T5_21_456 & 1 & 6 & 0.18 & 161 & Dichotomous \\
\hline T5_21_5 & 1 & 5 & 0.26 & 180 & Dichotomous \\
\hline T5_22_1 & 1 & 1 & 0.16 & 179 & Dichotomous \\
\hline T5_22_2 & 1 & 2 & 0.09 & 224 & Dichotomous \\
\hline T5_22_3 & 1 & 3 & 0.28 & 167 & Dichotomous \\
\hline T5_22_4 & 1 & 4 & 0.47 & 210 & Dichotomous \\
\hline T5_22_5 & 1 & 5 & 0.04 & 180 & Dichotomous \\
\hline T5_22_6 & 1 & 6 & 0.45 & 161 & Dichotomous \\
\hline T5_23_1 & 1 & 1 & 0.00 & 179 & Dichotomous \\
\hline T5_23_23 & 1 & 2 & 0.01 & 224 & Dichotomous \\
\hline T5_23_23 & 1 & 3 & 0.07 & 167 & Dichotomous \\
\hline T5_23_3 & 1 & 3 & 0.10 & 167 & Dichotomous \\
\hline T5_23_4 & 1 & 4 & 0.01 & 210 & Dichotomous \\
\hline T5_23_5 & 1 & 5 & 0.33 & 180 & Dichotomous \\
\hline T5_23_6 & 1 & 6 & 0.55 & 161 & Dichotomous \\
\hline T5_24_12 & 1 & 1 & 0.03 & 179 & Dichotomous \\
\hline T5_24_12 & 1 & 2 & 0.27 & 224 & Dichotomous \\
\hline T5_24_2 & 1 & 2 & 0.33 & 224 & Dichotomous \\
\hline T5_24_3 & 1 & 3 & 0.32 & 167 & Dichotomous \\
\hline T5_24_4 & 1 & 4 & 0.37 & 210 & Dichotomous \\
\hline T5_24_6 & 1 & 6 & 0.37 & 161 & Dichotomous \\
\hline T5_25_1 & 1 & 1 & 0.04 & 179 & Dichotomous \\
\hline T5_25_2 & 1 & 2 & 0.19 & 224 & Dichotomous \\
\hline T5_25_3 & 1 & 3 & 0.08 & 167 & Dichotomous \\
\hline T5_25_456 & 1 & 4 & 0.14 & 210 & Dichotomous \\
\hline T5_25_456 & 1 & 5 & 0.13 & 180 & Dichotomous \\
\hline T5_25_456 & 1 & 6 & 0.23 & 161 & Dichotomous \\
\hline T5_25_5 & 1 & 5 & 0.14 & 180 & Dichotomous \\
\hline T5_25_6 & 1 & 6 & 0.11 & 161 & Dichotomous \\
\hline T5_26_1 & 2 & 1 & 0.07 & 628 & Dichotomous \\
\hline T5_26_23 & 2 & 2 & 0.57 & 502 & Dichotomous \\
\hline T5_26_23 & 2 & 3 & 0.64 & 589 & Dichotomous \\
\hline T5_26_45 & 2 & 4 & 0.55 & 433 & Dichotomous \\
\hline
\end{tabular}




\begin{tabular}{|c|c|c|c|c|c|}
\hline T5_26_45 & 2 & 5 & 0.59 & 446 & Dichotomous \\
\hline T5_26_56 & 2 & 5 & 0.56 & 446 & Dichotomous \\
\hline T5_26_56 & 2 & 6 & 0.63 & 336 & Dichotomous \\
\hline T5_26_6 & 2 & 6 & 0.09 & 336 & Dichotomous \\
\hline T5_27_1 & 2 & 1 & 0.10 & 628 & Dichotomous \\
\hline T5_27_3 & 2 & 3 & 0.43 & 589 & Dichotomous \\
\hline T5_27_4 & 2 & 4 & 0.12 & 433 & Dichotomous \\
\hline T5_27_5 & 2 & 5 & 0.50 & 446 & Dichotomous \\
\hline T5_27_6 & 2 & 6 & 0.33 & 336 & Dichotomous \\
\hline T5_28_1 & 2 & 1 & 0.10 & 628 & Dichotomous \\
\hline T5_28_2 & 2 & 2 & 0.24 & 500 & Dichotomous \\
\hline T5_28_3 & 2 & 3 & 0.13 & 589 & Dichotomous \\
\hline T5_28_4 & 2 & 4 & 0.46 & 433 & Dichotomous \\
\hline T5_28_5 & 2 & 5 & 0.31 & 446 & Dichotomous \\
\hline T5_29_1 & 2 & 1 & 0.02 & 628 & Dichotomous \\
\hline T5_29_3 & 2 & 3 & 0.15 & 589 & Dichotomous \\
\hline T5_3_1 & 1 & 1 & 0.20 & 179 & Dichotomous \\
\hline T5_3_2 & 1 & 2 & 0.00 & 224 & Dichotomous \\
\hline T5_3_34 & 1 & 3 & 0.07 & 167 & Dichotomous \\
\hline T5_3_34 & 1 & 4 & 0.16 & 210 & Dichotomous \\
\hline T5_3_56 & 1 & 5 & 0.38 & 180 & Dichotomous \\
\hline T5_3_56 & 1 & 6 & 0.45 & 161 & Dichotomous \\
\hline T5_30_12 & 2 & 1 & 0.08 & 628 & Dichotomous \\
\hline T5_30_12 & 2 & 2 & 0.28 & 500 & Dichotomous \\
\hline T5_30_3 & 2 & 3 & 0.42 & 589 & Dichotomous \\
\hline T5_30_4 & 2 & 4 & 0.08 & 433 & Dichotomous \\
\hline T5_30_56 & 2 & 5 & 0.43 & 446 & Dichotomous \\
\hline T5_30_56 & 2 & 6 & 0.52 & 336 & Dichotomous \\
\hline T5_31_34 & 2 & 3 & 0.19 & 589 & Dichotomous \\
\hline T5_31_34 & 2 & 4 & 0.31 & 433 & Dichotomous \\
\hline T5_31_4 & 2 & 4 & 0.21 & 433 & Dichotomous \\
\hline T5_31_5 & 2 & 5 & 0.36 & 446 & Dichotomous \\
\hline T5_31_6 & 2 & 6 & 0.27 & 336 & Dichotomous \\
\hline T5_32_12 & 2 & 1 & 0.06 & 628 & Dichotomous \\
\hline T5_32_12 & 2 & 2 & 0.38 & 500 & Dichotomous \\
\hline T5_32_2 & 2 & 2 & 0.04 & 500 & Dichotomous \\
\hline T5_32_3 & 2 & 3 & 0.44 & 589 & Dichotomous \\
\hline T5_32_4 & 2 & 4 & 0.10 & 433 & Dichotomous \\
\hline T5_32_5 & 2 & 5 & 0.33 & 446 & Dichotomous \\
\hline T5_32_6 & 2 & 6 & 0.08 & 336 & Dichotomous \\
\hline T5_33_1 & 2 & 1 & 0.37 & 628 & Dichotomous \\
\hline
\end{tabular}




\begin{tabular}{|c|c|c|c|c|c|}
\hline T5_33_2 & 2 & 2 & 0.40 & 500 & Dichotomous \\
\hline T5_33_4 & 2 & 4 & 0.52 & 433 & Dichotomous \\
\hline T5_33_6 & 2 & 6 & 0.21 & 336 & Dichotomous \\
\hline T5_34_1 & 2 & 1 & 0.03 & 628 & Dichotomous \\
\hline T5_34_2 & 2 & 2 & 0.13 & 500 & Dichotomous \\
\hline T5_34_34 & 2 & 3 & 0.53 & 589 & Dichotomous \\
\hline T5_34_34 & 2 & 4 & 0.69 & 433 & Dichotomous \\
\hline T5_34_4 & 2 & 4 & 0.39 & 433 & Dichotomous \\
\hline T5_34_5 & 2 & 5 & 0.37 & 446 & Dichotomous \\
\hline T5_34_6 & 2 & 6 & 0.76 & 336 & Dichotomous \\
\hline T5_35_2 & 2 & 2 & 0.33 & 500 & Dichotomous \\
\hline T5_35_5 & 2 & 5 & 0.07 & 446 & Dichotomous \\
\hline T5_36_1 & 2 & 1 & 0.06 & 628 & Dichotomous \\
\hline T5_36_3 & 2 & 3 & 0.11 & 589 & Dichotomous \\
\hline T5_36_45 & 2 & 4 & 0.39 & 433 & Dichotomous \\
\hline T5_36_45 & 2 & 5 & 0.54 & 446 & Dichotomous \\
\hline T5_36_6 & 2 & 6 & 0.17 & 336 & Dichotomous \\
\hline T5_37_1 & 2 & 1 & 0.56 & 628 & Dichotomous \\
\hline T5_37_3 & 2 & 3 & 0.30 & 589 & Dichotomous \\
\hline T5_37_4 & 2 & 4 & 0.39 & 433 & Dichotomous \\
\hline T5_37_56 & 2 & 5 & 0.42 & 446 & Dichotomous \\
\hline T5_37_56 & 2 & 6 & 0.43 & 336 & Dichotomous \\
\hline T5_38_1 & 2 & 1 & 0.19 & 628 & Dichotomous \\
\hline T5_38_2 & 2 & 2 & 0.06 & 500 & Dichotomous \\
\hline T5_38_4 & 2 & 4 & 0.30 & 433 & Dichotomous \\
\hline T5_38_6 & 2 & 6 & 0.30 & 336 & Dichotomous \\
\hline T5_39_1 & 2 & 1 & 0.16 & 628 & Dichotomous \\
\hline T5_39_23 & 2 & 2 & 0.12 & 502 & Dichotomous \\
\hline T5_39_23 & 2 & 3 & 0.28 & 589 & Dichotomous \\
\hline T5_39_3 & 2 & 3 & 0.27 & 589 & Dichotomous \\
\hline T5_39_4 & 2 & 4 & 0.11 & 433 & Dichotomous \\
\hline T5_39_5 & 2 & 5 & 0.26 & 446 & Dichotomous \\
\hline T5_4_1 & 1 & 1 & 0.01 & 179 & Dichotomous \\
\hline T5_4_2 & 1 & 2 & 0.03 & 224 & Dichotomous \\
\hline T5_4_3 & 1 & 3 & 0.11 & 167 & Dichotomous \\
\hline T5_4_4 & 1 & 4 & 0.25 & 210 & Dichotomous \\
\hline T5_4_5 & 1 & 5 & 0.11 & 180 & Dichotomous \\
\hline T5_40_1 & 2 & 1 & 0.06 & 628 & Dichotomous \\
\hline T5_40_2 & 2 & 2 & 0.38 & 500 & Dichotomous \\
\hline T5_40_3 & 2 & 3 & 0.52 & 589 & Dichotomous \\
\hline T5_40_5 & 2 & 5 & 0.21 & 446 & Dichotomous \\
\hline
\end{tabular}




\begin{tabular}{|c|c|c|c|c|c|}
\hline T5_40_6 & 2 & 6 & 0.28 & 336 & Dichotomous \\
\hline T5_41_1 & 2 & 1 & 0.04 & 628 & Dichotomous \\
\hline T5_41_2 & 2 & 2 & 0.61 & 500 & Dichotomous \\
\hline T5_41_3 & 2 & 3 & 0.39 & 589 & Dichotomous \\
\hline T5_41_5 & 2 & 5 & 0.35 & 446 & Dichotomous \\
\hline T5_41_6 & 2 & 6 & 0.04 & 336 & Dichotomous \\
\hline T5_42_1 & 2 & 1 & 0.29 & 628 & Dichotomous \\
\hline T5_42_2 & 2 & 2 & 0.19 & 500 & Dichotomous \\
\hline T5_42_3 & 2 & 3 & 0.15 & 589 & Dichotomous \\
\hline T5_42_6 & 2 & 6 & 0.41 & 336 & Dichotomous \\
\hline T5_43_2 & 2 & 2 & 0.17 & 500 & Dichotomous \\
\hline T5_43_4 & 2 & 4 & 0.44 & 433 & Dichotomous \\
\hline T5_43_5 & 2 & 5 & 0.32 & 446 & Dichotomous \\
\hline T5_43_6 & 2 & 6 & 0.19 & 336 & Dichotomous \\
\hline T5_5_12 & 1 & 1 & 0.00 & 179 & Dichotomous \\
\hline T5_5_12 & 1 & 2 & 0.01 & 224 & Dichotomous \\
\hline T5_5_3 & 1 & 3 & 0.05 & 167 & Dichotomous \\
\hline T5_5_6 & 1 & 6 & 0.14 & 161 & Dichotomous \\
\hline T5_5_6 & 2 & 6 & 0.14 & 336 & Dichotomous \\
\hline T5_6_1 & 1 & 1 & 0.02 & 179 & Dichotomous \\
\hline T5_6_2 & 1 & 2 & 0.09 & 224 & Dichotomous \\
\hline T5_6_3 & 1 & 3 & 0.01 & 167 & Dichotomous \\
\hline T5_6_456 & 1 & 4 & 0.21 & 210 & Dichotomous \\
\hline T5_6_456 & 1 & 5 & 0.22 & 180 & Dichotomous \\
\hline T5_6_456 & 1 & 6 & 0.31 & 161 & Dichotomous \\
\hline T5_6_456 & 2 & 5 & 0.26 & 446 & Dichotomous \\
\hline T5_6_56 & 1 & 5 & 0.09 & 180 & Dichotomous \\
\hline T5_6_56 & 1 & 6 & 0.14 & 161 & Dichotomous \\
\hline T5_6_6 & 1 & 6 & 0.67 & 161 & Dichotomous \\
\hline T5_7_1 & 1 & 1 & 0.00 & 179 & Dichotomous \\
\hline T5_7_2 & 1 & 2 & 0.42 & 224 & Dichotomous \\
\hline T5_7_2 & 2 & 2 & 0.40 & 500 & Dichotomous \\
\hline T5_7_3 & 1 & 3 & 0.28 & 167 & Dichotomous \\
\hline T5_7_4 & 1 & 4 & 0.59 & 210 & Dichotomous \\
\hline T5_7_4 & 2 & 4 & 0.51 & 433 & Dichotomous \\
\hline T5_7_56 & 1 & 5 & 0.05 & 180 & Dichotomous \\
\hline T5_7_56 & 1 & 6 & 0.03 & 161 & Dichotomous \\
\hline T5_7_6 & 1 & 6 & 0.25 & 161 & Dichotomous \\
\hline T5_8_1 & 1 & 1 & 0.00 & 179 & Dichotomous \\
\hline T5_8_23 & 1 & 2 & 0.00 & 224 & Dichotomous \\
\hline T5_8_23 & 1 & 3 & 0.03 & 167 & Dichotomous \\
\hline
\end{tabular}




\begin{tabular}{llllll} 
T5_8_3 & 1 & 3 & 0.24 & 167 & Dichotomous \\
T5_8_3 & 2 & 3 & 0.24 & 589 & Dichotomous \\
T5_8_456 & 1 & 4 & 0.01 & 210 & Dichotomous \\
T5_8_456 & 1 & 5 & 0.01 & 180 & Dichotomous \\
T5_8_456 & 1 & 6 & 0.01 & 161 & Dichotomous \\
T5_8_5 & 1 & 5 & 0.16 & 180 & Dichotomous \\
T5_8_5 & 2 & 5 & 0.23 & 446 & Dichotomous \\
T5_8_6 & 1 & 6 & 0.01 & 161 & Dichotomous \\
T5_9_1 & 1 & 1 & 0.11 & 179 & Dichotomous \\
T5_9_1 & 2 & 1 & 0.08 & 628 & Dichotomous \\
T5_9_3 & 1 & 3 & 0.17 & 167 & Dichotomous \\
T5_9_3 & 2 & 3 & 0.27 & 589 & Dichotomous \\
T5_9_4 & 1 & 4 & 0.20 & 210 & Dichotomous \\
T5_9_4 & 2 & 4 & 0.18 & 433 & Dichotomous \\
T5_9_5 & 1 & 5 & 0.12 & 180 & Dichotomous \\
T5_9_6 & 1 & 6 & 0.27 & 161 & Dichotomous \\
\hline
\end{tabular}


Table 7

Task 6 p-values and sample size

\begin{tabular}{lccccc}
\hline Item & Year & Grade & Mean & N & Item Type \\
\hline T6_1_1 & 1 & 1 & 0.58 & 179 & Dichotomous \\
T6_1_1 & 2 & 1 & 0.51 & 628 & Dichotomous \\
T6_1_2 & 1 & 2 & 0.45 & 224 & Dichotomous \\
T6_1_2 & 2 & 2 & 0.51 & 500 & Dichotomous \\
T6_1_34 & 1 & 3 & 0.54 & 167 & Dichotomous \\
T6_1_34 & 1 & 4 & 0.65 & 210 & Dichotomous \\
T6_1_34 & 2 & 3 & 0.61 & 589 & Dichotomous \\
T6_1_456 & 1 & 4 & 0.68 & 210 & Dichotomous \\
T6_1_456 & 1 & 5 & 0.77 & 180 & Dichotomous \\
T6_1_456 & 1 & 6 & 0.78 & 161 & Dichotomous \\
T6_1_56 & 1 & 5 & 0.72 & 180 & Dichotomous \\
T6_1_56 & 1 & 6 & 0.80 & 161 & Dichotomous \\
T6_1_6 & 1 & 6 & 0.83 & 161 & Dichotomous \\
T6_1_6 & 2 & 6 & 0.81 & 336 & Dichotomous \\
T6_10_12 & 1 & 1 & 0.46 & 179 & Dichotomous \\
T6_10_12 & 1 & 2 & 0.41 & 224 & Dichotomous \\
T6_10_2 & 1 & 2 & 0.53 & 224 & Dichotomous \\
T6_10_34 & 1 & 3 & 0.62 & 167 & Dichotomous \\
T6_10_34 & 1 & 4 & 0.76 & 210 & Dichotomous \\
T6_10_4 & 1 & 4 & 0.98 & 210 & Dichotomous \\
T6_10_5 & 1 & 5 & 0.64 & 180 & Dichotomous \\
T6_10_6 & 1 & 6 & 0.90 & 161 & Dichotomous \\
T6_11_1 & 1 & 1 & 0.23 & 179 & Dichotomous \\
T6_11_23 & 1 & 2 & 0.55 & 224 & Dichotomous \\
T6_11_23 & 1 & 3 & 0.56 & 167 & Dichotomous \\
T6_11_3 & 1 & 3 & 0.73 & 167 & Dichotomous \\
T6_11_4 & 1 & 4 & 0.84 & 210 & Dichotomous \\
T6_11_5 & 1 & 5 & 0.71 & 180 & Dichotomous \\
T6_11_6 & 1 & 6 & 0.80 & 161 & Dichotomous \\
T6_12_1 & 1 & 1 & 0.58 & 179 & Dichotomous \\
T6_12_2 & 1 & 2 & 0.64 & 224 & Dichotomous \\
T6_12_3 & 1 & 3 & 0.14 & 167 & Dichotomous \\
T6_12_3.1 & 2 & 3 & 0.20 & 589 & Dichotomous \\
T6_12_4 & 1 & 4 & 0.79 & 210 & Dichotomous \\
T6_12_5 & 1 & 5 & 0.81 & 180 & Dichotomous \\
T6_6 & 1 & 6 & 0.89 & 161 & Dichotomous \\
& 1 & 1 & 0.45 & 179 & Dichotomous
\end{tabular}




\begin{tabular}{|c|c|c|c|c|c|}
\hline T6_13_23 & 1 & 2 & 0.63 & 224 & Dichotomous \\
\hline T6_13_23 & 1 & 3 & 0.60 & 167 & Dichotomous \\
\hline T6_13_3 & 1 & 3 & 0.87 & 167 & Dichotomous \\
\hline T6_13_5 & 1 & 5 & 0.91 & 180 & Dichotomous \\
\hline T6_14_1 & 1 & 1 & 0.32 & 179 & Dichotomous \\
\hline T6_14_2 & 1 & 2 & 0.38 & 224 & Dichotomous \\
\hline T6_15_12 & 1 & 1 & 0.56 & 179 & Dichotomous \\
\hline T6_15_12 & 1 & 2 & 0.44 & 224 & Dichotomous \\
\hline T6_15_2 & 1 & 2 & 0.78 & 224 & Dichotomous \\
\hline T6_15_2 & 2 & 2 & 0.84 & 500 & Dichotomous \\
\hline T6_15_3 & 1 & 3 & 0.41 & 167 & Dichotomous \\
\hline T6_15_5 & 1 & 5 & 0.69 & 180 & Dichotomous \\
\hline T6_16_12 & 1 & 1 & 0.42 & 179 & Dichotomous \\
\hline T6_16_12 & 1 & 2 & 0.57 & 224 & Dichotomous \\
\hline T6_16_2 & 1 & 2 & 0.55 & 224 & Dichotomous \\
\hline T6_16_3 & 1 & 3 & 0.57 & 167 & Dichotomous \\
\hline T6_16_3 & 2 & 3 & 0.54 & 589 & Dichotomous \\
\hline T6_16_4 & 1 & 4 & 0.93 & 210 & Dichotomous \\
\hline T6_16_4 & 2 & 4 & 0.90 & 433 & Dichotomous \\
\hline T6_16_5 & 1 & 5 & 0.86 & 180 & Dichotomous \\
\hline T6_16_5 & 2 & 5 & 0.89 & 446 & Dichotomous \\
\hline T6_16_6 & 1 & 6 & 0.81 & 161 & Dichotomous \\
\hline T6_17_1 & 1 & 1 & 0.48 & 179 & Dichotomous \\
\hline T6_17_23 & 1 & 2 & 0.42 & 224 & Dichotomous \\
\hline T6_17_23 & 1 & 3 & 0.81 & 167 & Dichotomous \\
\hline T6_17_3 & 1 & 3 & 0.37 & 167 & Dichotomous \\
\hline T6_17_4 & 1 & 4 & 0.82 & 210 & Dichotomous \\
\hline T6_17_5 & 1 & 5 & 0.90 & 180 & Dichotomous \\
\hline T6_18_1 & 1 & 1 & 0.22 & 179 & Dichotomous \\
\hline T6_18_2 & 1 & 2 & 0.63 & 224 & Dichotomous \\
\hline T6_18_3 & 1 & 3 & 0.72 & 167 & Dichotomous \\
\hline T6_18_4 & 1 & 4 & 0.86 & 210 & Dichotomous \\
\hline T6_18_4 & 2 & 4 & 0.82 & 433 & Dichotomous \\
\hline T6_18_56 & 1 & 5 & 0.37 & 180 & Dichotomous \\
\hline T6_18_56 & 1 & 6 & 0.43 & 161 & Dichotomous \\
\hline T6_19_12 & 1 & 1 & 0.43 & 179 & Dichotomous \\
\hline T6_19_12 & 1 & 2 & 0.74 & 224 & Dichotomous \\
\hline T6_19_2 & 1 & 2 & 0.54 & 224 & Dichotomous \\
\hline T6_19_3 & 1 & 3 & 0.93 & 167 & Dichotomous \\
\hline T6_19_4 & 1 & 4 & 0.66 & 210 & Dichotomous \\
\hline T6_19_5 & 1 & 5 & 0.63 & 180 & Dichotomol \\
\hline
\end{tabular}




\begin{tabular}{|c|c|c|c|c|c|}
\hline T6_2_1 & 1 & 1 & 0.45 & 179 & Dichotomous \\
\hline T6_2_1 & 2 & 1 & 0.37 & 628 & Dichotomous \\
\hline T6_2_3 & 1 & 3 & 0.78 & 167 & Dichotomous \\
\hline T6_2_3 & 2 & 3 & 0.76 & 589 & Dichotomous \\
\hline T6_2_4 & 1 & 4 & 0.76 & 210 & Dichotomous \\
\hline T6_2_4 & 2 & 4 & 0.70 & 433 & Dichotomous \\
\hline T6_2_56 & 1 & 5 & 0.70 & 180 & Dichotomous \\
\hline T6_2_56 & 1 & 6 & 0.81 & 161 & Dichotomous \\
\hline T6_2_56 & 2 & 5 & 0.73 & 446 & Dichotomous \\
\hline T6_20_1 & 1 & 1 & 0.21 & 179 & Dichotomous \\
\hline T6_20_23 & 1 & 2 & 0.31 & 224 & Dichotomous \\
\hline T6_20_23 & 1 & 3 & 0.44 & 167 & Dichotomous \\
\hline T6_20_4 & 1 & 4 & 0.79 & 210 & Dichotomous \\
\hline T6_20_4 & 2 & 4 & 0.84 & 433 & Dichotomous \\
\hline T6_20_5 & 1 & 5 & 0.43 & 180 & Dichotomous \\
\hline T6_20_5 & 2 & 5 & 0.48 & 446 & Dichotomous \\
\hline T6_20_6 & 1 & 6 & 0.81 & 161 & Dichotomous \\
\hline T6_21_12 & 1 & 1 & 0.30 & 179 & Dichotomous \\
\hline T6_21_12 & 1 & 2 & 0.42 & 224 & Dichotomous \\
\hline T6_21_34 & 1 & 3 & 0.24 & 167 & Dichotomous \\
\hline T6_21_34 & 1 & 4 & 0.42 & 210 & Dichotomous \\
\hline T6_21_4 & 1 & 4 & 0.63 & 210 & Dichotomous \\
\hline T6_22_1 & 1 & 1 & 0.23 & 179 & Dichotomous \\
\hline T6_22_2 & 1 & 2 & 0.41 & 224 & Dichotomous \\
\hline T6_22_3 & 1 & 3 & 0.57 & 167 & Dichotomous \\
\hline T6_22_456 & 1 & 4 & 0.79 & 210 & Dichotomous \\
\hline T6_22_456 & 1 & 5 & 0.89 & 180 & Dichotomous \\
\hline T6_22_456 & 1 & 6 & 0.91 & 161 & Dichotomous \\
\hline T6_22_5 & 1 & 5 & 0.76 & 180 & Dichotomous \\
\hline T6_22_6 & 1 & 6 & 0.45 & 161 & Dichotomous \\
\hline T6_23_1 & 1 & 1 & 0.49 & 179 & Dichotomous \\
\hline T6_23_2 & 1 & 2 & 0.29 & 224 & Dichotomous \\
\hline T6_23_3 & 1 & 3 & 0.80 & 167 & Dichotomous \\
\hline T6_23_4 & 1 & 4 & 0.61 & 210 & Dichotomous \\
\hline T6_23_6 & 1 & 6 & 0.80 & 161 & Dichotomous \\
\hline T6_24_1 & 1 & 1 & 0.61 & 179 & Dichotomous \\
\hline T6_24_34 & 1 & 3 & 0.62 & 167 & Dichotomous \\
\hline T6_24_34 & 1 & 4 & 0.80 & 210 & Dichotomous \\
\hline T6_24_4 & 1 & 4 & 0.60 & 210 & Dichotomous \\
\hline T6_24_5 & 1 & 5 & 0.63 & 180 & Dichotomous \\
\hline T6_24_6 & 1 & 6 & 0.31 & 161 & Dichotomous \\
\hline
\end{tabular}




\begin{tabular}{|c|c|c|c|c|c|}
\hline T6_25_1 & 1 & 1 & 0.35 & 179 & Dichotomous \\
\hline T6_25_4 & 1 & 4 & 0.88 & 210 & Dichotomous \\
\hline T6_25_6 & 1 & 6 & 0.86 & 161 & Dichotomous \\
\hline T6_26_12 & 2 & 1 & 0.23 & 628 & Dichotomous \\
\hline T6_26_12 & 2 & 2 & 0.48 & 500 & Dichotomous \\
\hline T6_26_2 & 2 & 2 & 0.85 & 500 & Dichotomous \\
\hline T6_26_3 & 2 & 3 & 0.68 & 589 & Dichotomous \\
\hline T6_26_4 & 2 & 4 & 0.37 & 433 & Dichotomous \\
\hline T6_26_5 & 2 & 5 & 0.78 & 446 & Dichotomous \\
\hline T6_27_1 & 2 & 1 & 0.18 & 628 & Dichotomous \\
\hline T6_27_3 & 2 & 3 & 0.70 & 589 & Dichotomous \\
\hline T6_27_45 & 2 & 4 & 0.47 & 433 & Dichotomous \\
\hline T6_27_45 & 2 & 5 & 0.62 & 446 & Dichotomous \\
\hline T6_27_5 & 2 & 5 & 0.68 & 446 & Dichotomous \\
\hline T6_27_6 & 2 & 6 & 0.91 & 336 & Dichotomous \\
\hline T6_28_1 & 2 & 1 & 0.30 & 628 & Dichotomous \\
\hline T6_28_2 & 2 & 2 & 0.55 & 500 & Dichotomous \\
\hline T6_28_3 & 2 & 3 & 0.51 & 589 & Dichotomous \\
\hline T6_28_45 & 2 & 4 & 0.46 & 433 & Dichotomous \\
\hline T6_28_45 & 2 & 5 & 0.53 & 446 & Dichotomous \\
\hline T6_28_56 & 2 & 5 & 0.90 & 446 & Dichotomous \\
\hline T6_28_56 & 2 & 6 & 0.93 & 336 & Dichotomous \\
\hline T6_28_6 & 2 & 6 & 0.79 & 336 & Dichotomous \\
\hline T6_29_1 & 2 & 1 & 0.41 & 628 & Dichotomous \\
\hline T6_29_2 & 2 & 2 & 0.26 & 500 & Dichotomous \\
\hline T6_29_3 & 2 & 3 & 0.44 & 589 & Dichotomous \\
\hline T6_29_4 & 2 & 4 & 0.48 & 433 & Dichotomous \\
\hline T6_29_5 & 2 & 5 & 0.30 & 446 & Dichotomous \\
\hline T6_3_1 & 1 & 1 & 0.19 & 179 & Dichotomous \\
\hline T6_3_1 & 2 & 1 & 0.14 & 628 & Dichotomous \\
\hline T6_3_2 & 1 & 2 & 0.21 & 224 & Dichotomous \\
\hline T6_3_2 & 2 & 2 & 0.21 & 500 & Dichotomous \\
\hline T6_3_3 & 1 & 3 & 0.49 & 167 & Dichotomous \\
\hline T6_3_4 & 1 & 4 & 0.77 & 210 & Dichotomous \\
\hline T6_3_5 & 1 & 5 & 0.80 & 180 & Dichotomous \\
\hline T6_30_1 & 2 & 1 & 0.44 & 628 & Dichotomous \\
\hline T6_30_3 & 2 & 3 & 0.45 & 589 & Dichotomous \\
\hline T6_30_5 & 2 & 5 & 0.77 & 446 & Dichotomous \\
\hline T6_30_6 & 2 & 6 & 0.88 & 336 & Dichotomous \\
\hline T6_31_1 & 2 & 1 & 0.38 & 628 & Dichotomous \\
\hline T6_31_2 & 2 & 2 & 0.42 & 500 & Dichotomous \\
\hline
\end{tabular}




\begin{tabular}{|c|c|c|c|c|c|}
\hline T6_31_4 & 2 & 4 & 0.56 & 433 & Dichotomous \\
\hline T6_31_5 & 2 & 5 & 0.84 & 446 & Dichotomous \\
\hline T6_31_6 & 2 & 6 & 0.99 & 336 & Dichotomous \\
\hline T6_32_1 & 2 & 1 & 0.13 & 628 & Dichotomous \\
\hline T6_32_2 & 2 & 2 & 0.14 & 500 & Dichotomous \\
\hline T6_32_3 & 2 & 3 & 0.70 & 589 & Dichotomous \\
\hline T6_32_56 & 2 & 5 & 0.61 & 446 & Dichotomous \\
\hline T6_32_56 & 2 & 6 & 0.69 & 336 & Dichotomous \\
\hline T6_33_3 & 2 & 3 & 0.75 & 589 & Dichotomous \\
\hline T6_33_4 & 2 & 4 & 0.54 & 433 & Dichotomous \\
\hline T6_33_5 & 2 & 5 & 0.84 & 446 & Dichotomous \\
\hline T6_34_1 & 2 & 1 & 0.50 & 628 & Dichotomous \\
\hline T6_34_3 & 2 & 3 & 0.74 & 589 & Dichotomous \\
\hline T6_34_4 & 2 & 4 & 0.59 & 433 & Dichotomous \\
\hline T6_34_5 & 2 & 5 & 0.65 & 446 & Dichotomous \\
\hline T6_34_6 & 2 & 6 & 0.74 & 336 & Dichotomous \\
\hline T6_35_1 & 2 & 1 & 0.58 & 628 & Dichotomous \\
\hline T6_35_2 & 2 & 2 & 0.50 & 500 & Dichotomous \\
\hline T6_35_4 & 2 & 4 & 0.54 & 433 & Dichotomous \\
\hline T6_35_5 & 2 & 5 & 0.57 & 446 & Dichotomous \\
\hline T6_35_6 & 2 & 6 & 0.79 & 336 & Dichotomous \\
\hline T6_36_1 & 2 & 1 & 0.38 & 628 & Dichotomous \\
\hline T6_36_23 & 2 & 2 & 0.34 & 502 & Dichotomous \\
\hline T6_36_23 & 2 & 3 & 0.55 & 589 & Dichotomous \\
\hline T6_36_3 & 2 & 3 & 0.67 & 589 & Dichotomous \\
\hline T6_36_4 & 2 & 4 & 0.58 & 433 & Dichotomous \\
\hline T6_36_5 & 2 & 5 & 0.87 & 446 & Dichotomous \\
\hline T6_36_6 & 2 & 6 & 0.62 & 336 & Dichotomous \\
\hline T6_37_1 & 2 & 1 & 0.31 & 628 & Dichotomous \\
\hline T6_37_3 & 2 & 3 & 0.48 & 589 & Dichotomous \\
\hline T6_37_5 & 2 & 5 & 0.43 & 446 & Dichotomous \\
\hline T6_38_2 & 2 & 2 & 0.36 & 500 & Dichotomous \\
\hline T6_38_3 & 2 & 3 & 0.23 & 589 & Dichotomous \\
\hline T6_38_4 & 2 & 4 & 0.39 & 433 & Dichotomous \\
\hline T6_38_5 & 2 & 5 & 0.67 & 446 & Dichotomous \\
\hline T6_38_6 & 2 & 6 & 0.85 & 336 & Dichotomous \\
\hline T6_39_1 & 2 & 1 & 0.47 & 628 & Dichotomous \\
\hline T6_39_2 & 2 & 2 & 0.78 & 500 & Dichotomous \\
\hline T6_39_3 & 2 & 3 & 0.39 & 589 & Dichotomous \\
\hline T6_4_1 & 1 & 1 & 0.28 & 179 & Dichotomous \\
\hline T6_4_2 & 1 & 2 & 0.82 & 224 & Dichotomous \\
\hline
\end{tabular}




\begin{tabular}{|c|c|c|c|c|c|}
\hline T6_4_2 & 2 & 2 & 0.81 & 500 & Dichotomous \\
\hline T6_4_3 & 1 & 3 & 0.84 & 167 & Dichotomous \\
\hline T6_4_456 & 1 & 4 & 0.44 & 210 & Dichotomous \\
\hline T6_4_456 & 1 & 5 & 0.47 & 180 & Dichotomous \\
\hline T6_4_456 & 1 & 6 & 0.78 & 161 & Dichotomous \\
\hline T6_40_1 & 2 & 1 & 0.23 & 628 & Dichotomous \\
\hline T6_40_2 & 2 & 2 & 0.28 & 500 & Dichotomous \\
\hline T6_40_34 & 2 & 3 & 0.69 & 589 & Dichotomous \\
\hline T6_40_34 & 2 & 4 & 0.82 & 433 & Dichotomous \\
\hline T6_40_4 & 2 & 4 & 0.82 & 433 & Dichotomous \\
\hline T6_40_6 & 2 & 6 & 0.91 & 336 & Dichotomous \\
\hline T6_41_12 & 2 & 1 & 0.28 & 628 & Dichotomous \\
\hline T6_41_12 & 2 & 2 & 0.56 & 500 & Dichotomous \\
\hline T6_41_2 & 2 & 2 & 0.75 & 500 & Dichotomous \\
\hline T6_41_6 & 2 & 6 & 0.47 & 336 & Dichotomous \\
\hline T6_42_1 & 2 & 1 & 0.20 & 628 & Dichotomous \\
\hline T6_42_23 & 2 & 2 & 0.46 & 502 & Dichotomous \\
\hline T6_42_23 & 2 & 3 & 0.54 & 589 & Dichotomous \\
\hline T6_42_4 & 2 & 4 & 0.93 & 433 & Dichotomous \\
\hline T6_42_6 & 2 & 6 & 0.67 & 336 & Dichotomous \\
\hline T6_43_1 & 2 & 1 & 0.47 & 628 & Dichotomous \\
\hline T6_43_2 & 2 & 2 & 0.68 & 500 & Dichotomous \\
\hline T6_43_34 & 2 & 3 & 0.51 & 589 & Dichotomous \\
\hline T6_43_34 & 2 & 4 & 0.66 & 433 & Dichotomous \\
\hline T6_43_4 & 2 & 4 & 0.76 & 433 & Dichotomous \\
\hline T6_43_5 & 2 & 5 & 0.93 & 446 & Dichotomous \\
\hline T6_43_6 & 2 & 6 & 0.71 & 336 & Dichotomous \\
\hline T6_44_2 & 2 & 2 & 0.48 & 500 & Dichotomous \\
\hline T6_44_3 & 2 & 3 & 0.72 & 589 & Dichotomous \\
\hline T6_44_4 & 2 & 4 & 0.30 & 433 & Dichotomous \\
\hline T6_44_6 & 2 & 6 & 0.20 & 336 & Dichotomous \\
\hline T6_45_1 & 2 & 1 & 0.25 & 628 & Dichotomous \\
\hline T6_45_2 & 2 & 2 & 0.66 & 500 & Dichotomous \\
\hline T6_45_3 & 2 & 3 & 0.31 & 589 & Dichotomous \\
\hline T6_45_4 & 2 & 4 & 0.78 & 433 & Dichotomous \\
\hline T6_45_56 & 2 & 5 & 0.62 & 446 & Dichotomous \\
\hline T6_45_56 & 2 & 6 & 0.76 & 336 & Dichotomous \\
\hline T6_45_6 & 2 & 6 & 0.85 & 336 & Dichotomous \\
\hline T6_46_1 & 2 & 1 & 0.31 & 628 & Dichotomous \\
\hline T6_46_2 & 2 & 2 & 0.66 & 500 & Dichotomous \\
\hline T6_46_4 & 2 & 4 & 0.82 & 433 & Dichotomous \\
\hline
\end{tabular}




\begin{tabular}{|c|c|c|c|c|c|}
\hline T6_46_5 & 2 & 5 & 0.69 & 446 & Dichotomous \\
\hline T6_46_6 & 2 & 6 & 0.89 & 336 & Dichotomous \\
\hline T6_47_1 & 2 & 1 & 0.49 & 628 & Dichotomous \\
\hline T6_47_2 & 2 & 2 & 0.55 & 499 & Dichotomous \\
\hline T6_47_3 & 2 & 3 & 0.57 & 589 & Dichotomous \\
\hline T6_47_5 & 2 & 5 & 0.64 & 446 & Dichotomous \\
\hline T6_47_6 & 2 & 6 & 0.57 & 336 & Dichotomous \\
\hline T6_48_1 & 2 & 1 & 0.24 & 628 & Dichotomous \\
\hline T6_48_4 & 2 & 4 & 0.61 & 433 & Dichotomous \\
\hline T6_48_6 & 2 & 6 & 0.91 & 336 & Dichotomous \\
\hline T6_49_2 & 2 & 2 & 0.59 & 500 & Dichotomous \\
\hline T6_49_4 & 2 & 4 & 0.83 & 433 & Dichotomous \\
\hline T6_49_5 & 2 & 5 & 0.65 & 446 & Dichotomous \\
\hline T6_5_1 & 1 & 1 & 0.48 & 179 & Dichotomous \\
\hline T6_5_2 & 1 & 2 & 0.41 & 224 & Dichotomous \\
\hline T6_5_34 & 1 & 3 & 0.37 & 167 & Dichotomous \\
\hline T6_5_34 & 1 & 4 & 0.47 & 210 & Dichotomous \\
\hline T6_5_4 & 1 & 4 & 0.82 & 210 & Dichotomous \\
\hline T6_5_56 & 1 & 5 & 0.78 & 180 & Dichotomous \\
\hline T6_5_56 & 1 & 6 & 0.65 & 161 & Dichotomous \\
\hline T6_5_6 & 1 & 6 & 0.86 & 161 & Dichotomous \\
\hline T6_5_6 & 2 & 6 & 0.79 & 336 & Dichotomous \\
\hline T6_6_1 & 1 & 1 & 0.45 & 179 & Dichotomous \\
\hline T6_6_23 & 1 & 2 & 0.46 & 224 & Dichotomous \\
\hline T6_6_23 & 1 & 3 & 0.41 & 167 & Dichotomous \\
\hline T6_6_56 & 1 & 5 & 0.56 & 180 & Dichotomous \\
\hline T6_6_56 & 1 & 6 & 0.84 & 161 & Dichotomous \\
\hline T6_6_6 & 1 & 6 & 0.81 & 161 & Dichotomous \\
\hline T6_6_6 & 2 & 6 & 0.78 & 336 & Dichotomous \\
\hline T6_7_1 & 1 & 1 & 0.26 & 179 & Dichotomous \\
\hline T6_7_1 & 2 & 1 & 0.18 & 628 & Dichotomous \\
\hline T6_7_2 & 1 & 2 & 0.56 & 224 & Dichotomous \\
\hline T6_7_5 & 1 & 5 & 0.81 & 180 & Dichotomous \\
\hline T6_7_6 & 1 & 6 & 0.39 & 161 & Dichotomous \\
\hline T6_7_6 & 2 & 6 & 0.45 & 336 & Dichotomous \\
\hline T6_8_1 & 1 & 1 & 0.26 & 179 & Dichotomous \\
\hline T6_8_2 & 1 & 2 & 0.77 & 224 & Dichotomous \\
\hline T6_8_3 & 1 & 3 & 0.80 & 167 & Dichotomous \\
\hline T6_8_456 & 1 & 4 & 0.92 & 210 & Dichotomous \\
\hline T6_8_456 & 1 & 5 & 0.94 & 180 & Dichotomous \\
\hline T6_8_456 & 1 & 6 & 0.90 & 161 & Dichotomous \\
\hline
\end{tabular}


MATRS TECHNICAL MANUAL 59

\begin{tabular}{llllll} 
T6_8_5 & 1 & 5 & 0.37 & 180 & Dichotomous \\
T6_8_6 & 1 & 6 & 0.80 & 161 & Dichotomous \\
T6_9_1 & 1 & 1 & 0.37 & 179 & Dichotomous \\
T6_9_3 & 1 & 3 & 0.73 & 167 & Dichotomous \\
T6_9_3 & 2 & 3 & 0.65 & 589 & Dichotomous \\
T6_9_5 & 1 & 5 & 0.77 & 180 & Dichotomous \\
T6_9_5 & 2 & 5 & 0.78 & 446 & Dichotomous \\
T6_9_6 & 1 & 6 & 0.45 & 161 & Dichotomous \\
\hline
\end{tabular}


Table 8

Task 7 p-values and sample size

\begin{tabular}{lccccc}
\hline Item & Year & Grade & Mean & N & Item Type \\
\hline T7_1_1 & 1 & 1 & 0.31 & 179 & Dichotomous \\
T7_1_2 & 1 & 2 & 0.00 & 224 & Dichotomous \\
T7_1_3 & 1 & 3 & 0.01 & 167 & Dichotomous \\
T7_1_56 & 1 & 5 & 0.14 & 180 & Dichotomous \\
T7_1_56 & 1 & 6 & 0.29 & 161 & Dichotomous \\
T7_10_1 & 1 & 1 & 0.12 & 179 & Dichotomous \\
T7_10_3 & 1 & 3 & 0.92 & 167 & Dichotomous \\
T7_10_4 & 1 & 4 & 0.52 & 210 & Dichotomous \\
T7_10_5 & 1 & 5 & 0.68 & 180 & Dichotomous \\
T7_10_6 & 1 & 6 & 0.60 & 161 & Dichotomous \\
T7_10_6 & 2 & 6 & 0.64 & 336 & Dichotomous \\
T7_11_12 & 1 & 1 & 0.00 & 179 & Dichotomous \\
T7_11_12 & 1 & 2 & 0.00 & 224 & Dichotomous \\
T7_11_2 & 1 & 2 & 0.84 & 224 & Dichotomous \\
T7_11_3 & 1 & 3 & 0.83 & 167 & Dichotomous \\
T7_11_3 & 2 & 3 & 0.81 & 589 & Dichotomous \\
T7_11_4 & 1 & 4 & 0.20 & 210 & Dichotomous \\
T7_11_4 & 2 & 4 & 0.19 & 433 & Dichotomous \\
T7_11_5 & 1 & 5 & 0.50 & 180 & Dichotomous \\
T7_11_5 & 2 & 5 & 0.56 & 446 & Dichotomous \\
T7_12_1 & 1 & 1 & 0.66 & 179 & Dichotomous \\
T7_12_23 & 1 & 2 & 0.25 & 224 & Dichotomous \\
T7_12_23 & 1 & 3 & 0.27 & 167 & Dichotomous \\
T7_12_5 & 1 & 5 & 0.08 & 180 & Dichotomous \\
T7_12_5 & 2 & 5 & 0.13 & 446 & Dichotomous \\
T7_13_1 & 1 & 1 & 0.94 & 179 & Dichotomous \\
T7_13_1 & 2 & 1 & 0.87 & 628 & Dichotomous \\
T7_13_2 & 1 & 2 & 0.79 & 224 & Dichotomous \\
T7_13_4 & 1 & 4 & 0.94 & 210 & Dichotomous \\
T7_13_5 & 1 & 5 & 0.77 & 180 & Dichotomous \\
T7_14_1 & 1 & 1 & 0.74 & 179 & Dichotomous \\
T7_14_1 & 2 & 1 & 0.70 & 628 & Dichotomous \\
T7_14_3 & 1 & 3 & 0.53 & 167 & Dichotomous \\
T7_14_3 & 2 & 3 & 0.70 & 589 & Dichotomous \\
T7_14_4 & 1 & 4 & 0.83 & 210 & Dichotomous \\
T7_14_6 & 1 & 6 & 0.76 & 161 & Dichotomous \\
T7_14_6 & 2 & 6 & 0.87 & 336 & Dichotomous
\end{tabular}




\begin{tabular}{|c|c|c|c|c|c|}
\hline T7_15_1 & 1 & 1 & 0.76 & 179 & Dichotomous \\
\hline T7_15_23 & 1 & 2 & 0.29 & 224 & Dichotomous \\
\hline T7_15_23 & 1 & 3 & 0.46 & 167 & Dichotomous \\
\hline T7_15_34 & 1 & 3 & 0.15 & 167 & Dichotomous \\
\hline T7_15_34 & 1 & 4 & 0.26 & 210 & Dichotomous \\
\hline T7_15_456 & 1 & 4 & 0.30 & 210 & Dichotomous \\
\hline T7_15_456 & 1 & 5 & 0.32 & 180 & Dichotomous \\
\hline T7_15_456 & 1 & 6 & 0.28 & 161 & Dichotomous \\
\hline T7_15_56 & 1 & 5 & 0.88 & 180 & Dichotomous \\
\hline T7_15_56 & 1 & 6 & 0.91 & 161 & Dichotomous \\
\hline T7_15_6 & 1 & 6 & 0.70 & 161 & Dichotomous \\
\hline T7_15_6 & 2 & 6 & 0.49 & 336 & Dichotomous \\
\hline T7_16_1 & 2 & 1 & 0.35 & 628 & Dichotomous \\
\hline T7_16_2 & 2 & 2 & 0.01 & 500 & Dichotomous \\
\hline T7_16_3 & 2 & 3 & 0.92 & 589 & Dichotomous \\
\hline T7_16_45 & 2 & 4 & 0.94 & 433 & Dichotomous \\
\hline T7_16_45 & 2 & 5 & 0.95 & 446 & Dichotomous \\
\hline T7_16_56 & 2 & 5 & 0.88 & 446 & Dichotomous \\
\hline T7_16_56 & 2 & 6 & 0.86 & 336 & Dichotomous \\
\hline T7_16_6 & 2 & 6 & 0.85 & 336 & Dichotomous \\
\hline T7_17_1 & 2 & 1 & 0.04 & 628 & Dichotomous \\
\hline T7_17_23 & 2 & 2 & 0.89 & 502 & Dichotomous \\
\hline T7_17_23 & 2 & 3 & 0.97 & 589 & Dichotomous \\
\hline T7_17_3 & 2 & 3 & 0.92 & 589 & Dichotomous \\
\hline T7_17_4 & 2 & 4 & 0.96 & 433 & Dichotomous \\
\hline T7_17_56 & 2 & 5 & 0.03 & 446 & Dichotomous \\
\hline T7_17_56 & 2 & 6 & 0.04 & 336 & Dichotomous \\
\hline T7_17_6 & 2 & 6 & 0.75 & 336 & Dichotomous \\
\hline T7_18_1 & 2 & 1 & 0.16 & 628 & Dichotomous \\
\hline T7_18_4 & 2 & 4 & 0.51 & 433 & Dichotomous \\
\hline T7_18_5 & 2 & 5 & 0.51 & 446 & Dichotomous \\
\hline T7_19_1 & 2 & 1 & 0.56 & 628 & Dichotomous \\
\hline T7_19_2 & 2 & 2 & 0.85 & 500 & Dichotomous \\
\hline T7_19_3 & 2 & 3 & 0.01 & 589 & Dichotomous \\
\hline T7_19_4 & 2 & 4 & 0.57 & 433 & Dichotomous \\
\hline T7_19_5 & 2 & 5 & 0.60 & 446 & Dichotomous \\
\hline T7_2_1 & 1 & 1 & 0.58 & 179 & Dichotomous \\
\hline T7_2_2 & 1 & 2 & 0.37 & 224 & Dichotomous \\
\hline T7_2_2 & 2 & 2 & 0.36 & 500 & Dichotomous \\
\hline T7_2_34 & 1 & 3 & 0.07 & 167 & Dichotomous \\
\hline T7_2_34 & 1 & 4 & 0.11 & 210 & Dichotomous \\
\hline
\end{tabular}




\begin{tabular}{|c|c|c|c|c|c|}
\hline T7_2_4 & 1 & 4 & 0.50 & 210 & Dichotomous \\
\hline T7_2_5 & 1 & 5 & 0.19 & 180 & Dichotomous \\
\hline T7_2_6 & 1 & 6 & 0.57 & 161 & Dichotomous \\
\hline T7_20_1 & 2 & 1 & 0.54 & 628 & Dichotomous \\
\hline T7_20_2 & 2 & 2 & 0.90 & 500 & Dichotomous \\
\hline T7_20_3 & 2 & 3 & 0.88 & 589 & Dichotomous \\
\hline T7_20_4 & 2 & 4 & 0.08 & 433 & Dichotomous \\
\hline T7_20_5 & 2 & 5 & 0.80 & 446 & Dichotomous \\
\hline T7_21_1 & 2 & 1 & 0.44 & 628 & Dichotomous \\
\hline T7_21_2 & 2 & 2 & 0.46 & 500 & Dichotomous \\
\hline T7_21_4 & 2 & 4 & 0.97 & 433 & Dichotomous \\
\hline T7_21_6 & 2 & 6 & 0.79 & 336 & Dichotomous \\
\hline T7_22_12 & 2 & 1 & 0.66 & 628 & Dichotomous \\
\hline T7_22_12 & 2 & 2 & 0.81 & 500 & Dichotomous \\
\hline T7_22_2 & 2 & 2 & 0.95 & 500 & Dichotomous \\
\hline T7_22_3 & 2 & 3 & 0.66 & 589 & Dichotomous \\
\hline T7_22_6 & 2 & 6 & 0.58 & 336 & Dichotomous \\
\hline T7_23_5 & 2 & 5 & 0.57 & 446 & Dichotomous \\
\hline T7_24_2 & 2 & 2 & 0.14 & 500 & Dichotomous \\
\hline T7_24_3 & 2 & 3 & 0.29 & 589 & Dichotomous \\
\hline T7_24_4 & 2 & 4 & 0.18 & 433 & Dichotomous \\
\hline T7_24_5 & 2 & 5 & 0.39 & 446 & Dichotomous \\
\hline T7_24_6 & 2 & 6 & 0.51 & 336 & Dichotomous \\
\hline T7_25_1 & 2 & 1 & 0.69 & 628 & Dichotomous \\
\hline T7_25_5 & 2 & 5 & 0.81 & 446 & Dichotomous \\
\hline T7_25_6 & 2 & 6 & 0.47 & 336 & Dichotomous \\
\hline T7_26_1 & 2 & 1 & 0.50 & 628 & Dichotomous \\
\hline T7_26_23 & 2 & 2 & 0.90 & 502 & Dichotomous \\
\hline T7_26_23 & 2 & 3 & 0.91 & 589 & Dichotomous \\
\hline T7_26_4 & 2 & 4 & 0.74 & 433 & Dichotomous \\
\hline T7_26_5 & 2 & 5 & 0.23 & 446 & Dichotomous \\
\hline T7_27_1 & 2 & 1 & 0.78 & 628 & Dichotomous \\
\hline T7_27_2 & 2 & 2 & 0.66 & 500 & Dichotomous \\
\hline T7_27_3 & 2 & 3 & 0.72 & 589 & Dichotomous \\
\hline T7_27_4 & 2 & 4 & 0.95 & 433 & Dichotomous \\
\hline T7_27_6 & 2 & 6 & 0.70 & 336 & Dichotomous \\
\hline T7_28_1 & 2 & 1 & 0.82 & 628 & Dichotomous \\
\hline T7_28_2 & 2 & 2 & 0.39 & 500 & Dichotomous \\
\hline T7_28_3 & 2 & 3 & 0.83 & 589 & Dichotomous \\
\hline T7_28_6 & 2 & 6 & 0.39 & 336 & Dichotomous \\
\hline T7_29_12 & 2 & 1 & 0.19 & 628 & Dichotomous \\
\hline
\end{tabular}




\begin{tabular}{|c|c|c|c|c|c|}
\hline T7_29_12 & 2 & 2 & 0.37 & 500 & Dichotomous \\
\hline T7_29_12.1 & 2 & 2 & 0.41 & 500 & Dichotomous \\
\hline T7_29_34 & 2 & 3 & 0.84 & 589 & Dichotomous \\
\hline T7_29_34 & 2 & 4 & 0.89 & 433 & Dichotomous \\
\hline T7_29_4 & 2 & 4 & 0.00 & 433 & Dichotomous \\
\hline T7_29_56 & 2 & 5 & 0.40 & 446 & Dichotomous \\
\hline T7_29_56 & 2 & 6 & 0.38 & 336 & Dichotomous \\
\hline T7_29_6 & 2 & 6 & 0.90 & 336 & Dichotomous \\
\hline T7_3_1 & 1 & 1 & 0.12 & 179 & Dichotomous \\
\hline T7_3_1 & 2 & 1 & 0.06 & 628 & Dichotomous \\
\hline T7_3_3 & 1 & 3 & 0.03 & 167 & Dichotomous \\
\hline T7_3_456 & 1 & 4 & 0.72 & 210 & Dichotomous \\
\hline T7_3_456 & 1 & 5 & 0.72 & 180 & Dichotomous \\
\hline T7_3_456 & 1 & 6 & 0.70 & 161 & Dichotomous \\
\hline T7_3_5 & 1 & 5 & 0.03 & 180 & Dichotomous \\
\hline T7_3_6 & 1 & 6 & 0.14 & 161 & Dichotomous \\
\hline T7_30_1 & 2 & 1 & 0.55 & 628 & Dichotomous \\
\hline T7_30_3 & 2 & 3 & 0.31 & 589 & Dichotomous \\
\hline T7_30_45 & 2 & 4 & 0.92 & 433 & Dichotomous \\
\hline T7_30_45 & 2 & 5 & 0.88 & 446 & Dichotomous \\
\hline T7_30_5 & 2 & 5 & 0.46 & 446 & Dichotomous \\
\hline T7_30_6 & 2 & 6 & 0.72 & 336 & Dichotomous \\
\hline T7_31_1 & 2 & 1 & 0.85 & 628 & Dichotomous \\
\hline T7_31_34 & 2 & 3 & 0.60 & 589 & Dichotomous \\
\hline T7_31_34 & 2 & 4 & 0.68 & 433 & Dichotomous \\
\hline T7_31_5 & 2 & 5 & 0.60 & 446 & Dichotomous \\
\hline T7_31_6 & 2 & 6 & 0.64 & 336 & Dichotomous \\
\hline T7_32_2 & 2 & 2 & 0.80 & 500 & Dichotomous \\
\hline T7_32_3 & 2 & 3 & 0.07 & 589 & Dichotomous \\
\hline T7_32_4 & 2 & 4 & 0.06 & 433 & Dichotomous \\
\hline T7_32_5 & 2 & 5 & 0.52 & 446 & Dichotomous \\
\hline T7_32_6 & 2 & 6 & 0.82 & 336 & Dichotomous \\
\hline T7_33_1 & 2 & 1 & 0.83 & 628 & Dichotomous \\
\hline T7_33_2 & 2 & 2 & 0.75 & 500 & Dichotomous \\
\hline T7_33_3 & 2 & 3 & 0.88 & 589 & Dichotomous \\
\hline T7_33_4 & 2 & 4 & 0.05 & 433 & Dichotomous \\
\hline T7_4_1 & 1 & 1 & 0.01 & 179 & Dichotomous \\
\hline T7_4_23 & 1 & 2 & 0.13 & 224 & Dichotomous \\
\hline T7_4_23 & 1 & 3 & 0.20 & 167 & Dichotomous \\
\hline T7_4_23 & 2 & 2 & 0.15 & 500 & Dichotomous \\
\hline T7_4_3 & 1 & 3 & 0.78 & 167 & Dichotomous \\
\hline
\end{tabular}




\begin{tabular}{|c|c|c|c|c|c|}
\hline T7_4_4 & 1 & 4 & 0.09 & 210 & Dichotomous \\
\hline T7_4_5 & 1 & 5 & 0.35 & 180 & Dichotomous \\
\hline T7_4_5 & 2 & 5 & 0.53 & 446 & Dichotomous \\
\hline T7_4_6 & 1 & 6 & 0.61 & 161 & Dichotomous \\
\hline T7_5_1 & 1 & 1 & 0.79 & 179 & Dichotomous \\
\hline T7_5_2 & 1 & 2 & 0.46 & 224 & Dichotomous \\
\hline T7_5_2 & 2 & 2 & 0.39 & 500 & Dichotomous \\
\hline T7_5_3 & 1 & 3 & 0.31 & 167 & Dichotomous \\
\hline T7_5_4 & 1 & 4 & 0.82 & 210 & Dichotomous \\
\hline T7_5_56 & 1 & 5 & 0.01 & 180 & Dichotomous \\
\hline T7_5_56 & 1 & 6 & 0.01 & 161 & Dichotomous \\
\hline T7_6_12 & 1 & 1 & 0.03 & 179 & Dichotomous \\
\hline T7_6_12 & 1 & 2 & 0.09 & 224 & Dichotomous \\
\hline T7_6_2 & 1 & 2 & 0.92 & 224 & Dichotomous \\
\hline T7_6_3 & 1 & 3 & 0.17 & 167 & Dichotomous \\
\hline T7_6_3 & 2 & 3 & 0.16 & 589 & Dichotomous \\
\hline T7_6_4 & 1 & 4 & 0.46 & 210 & Dichotomous \\
\hline T7_7_1 & 1 & 1 & 0.41 & 179 & Dichotomous \\
\hline T7_7_2 & 1 & 2 & 0.35 & 224 & Dichotomous \\
\hline T7_7_4 & 1 & 4 & 0.81 & 210 & Dichotomous \\
\hline T7_7_4 & 2 & 4 & 0.69 & 433 & Dichotomous \\
\hline T7_7_5 & 1 & 5 & 0.02 & 180 & Dichotomous \\
\hline T7_7_6 & 1 & 6 & 0.09 & 161 & Dichotomous \\
\hline T7_8_12 & 1 & 1 & 0.15 & 179 & Dichotomous \\
\hline T7_8_12 & 1 & 2 & 0.29 & 224 & Dichotomous \\
\hline T7_8_2 & 1 & 2 & 0.88 & 224 & Dichotomous \\
\hline T7_8_3 & 1 & 3 & 0.43 & 167 & Dichotomous \\
\hline T7_8_6 & 1 & 6 & 0.28 & 161 & Dichotomous \\
\hline T7_9_1 & 1 & 1 & 0.13 & 179 & Dichotomous \\
\hline T7_9_2 & 1 & 2 & 0.91 & 224 & Dichotomous \\
\hline T7_9_34 & 1 & 3 & 0.13 & 167 & Dichotomous \\
\hline T7_9_34 & 1 & 4 & 0.27 & 210 & Dichotomous \\
\hline T7_9_34 & 2 & 4 & 0.37 & 433 & Dichotomous \\
\hline T7_9_456 & 1 & 4 & 0.70 & 210 & Dichotomous \\
\hline T7_9_456 & 1 & 5 & 0.78 & 180 & Dichotomous \\
\hline T7_9_456 & 1 & 6 & 0.82 & 161 & Dichotomous \\
\hline T7_9_5 & 1 & 5 & 0.69 & 180 & Dichotomous \\
\hline T7_9_6 & 1 & 6 & 0.35 & 161 & Dichotomous \\
\hline
\end{tabular}


Table 9

Task 8 p-values and sample size

\begin{tabular}{lccccc}
\hline Item & Year & Grade & Mean & N & Item Type \\
\hline T8_1_3 & 1 & 3 & 0.00 & 167 & Dichotomous \\
T8_1_4 & 1 & 4 & 0.33 & 210 & Dichotomous \\
T8_1_4 & 2 & 4 & 0.37 & 433 & Dichotomous \\
T8_1_5 & 1 & 5 & 0.21 & 180 & Dichotomous \\
T8_1_5 & 2 & 5 & 0.13 & 446 & Dichotomous \\
T8_1_6 & 1 & 6 & 0.01 & 161 & Dichotomous \\
T8_1_6 & 2 & 6 & 0.01 & 336 & Dichotomous \\
T8_10_3 & 1 & 3 & 0.00 & 167 & Dichotomous \\
T8_10_3 & 2 & 3 & 0.00 & 589 & Dichotomous \\
T8_10_4 & 1 & 4 & 0.14 & 210 & Dichotomous \\
T8_10_5 & 1 & 5 & 0.31 & 180 & Dichotomous \\
T8_10_6 & 1 & 6 & 0.73 & 161 & Dichotomous \\
T8_11_3 & 1 & 3 & 0.09 & 167 & Dichotomous \\
T8_11_3 & 2 & 3 & 0.15 & 589 & Dichotomous \\
T8_11_5 & 1 & 5 & 0.14 & 180 & Dichotomous \\
T8_11_5 & 2 & 5 & 0.16 & 446 & Dichotomous \\
T8_12_3 & 1 & 3 & 0.03 & 167 & Dichotomous \\
T8_12_4 & 1 & 4 & 0.04 & 210 & Dichotomous \\
T8_12_56 & 1 & 5 & 0.00 & 180 & Dichotomous \\
T8_12_56 & 1 & 6 & 0.00 & 161 & Dichotomous \\
T8_13_3 & 1 & 3 & 0.10 & 167 & Dichotomous \\
T8_13_456 & 1 & 4 & 0.70 & 210 & Dichotomous \\
T8_13_456 & 1 & 5 & 0.69 & 180 & Dichotomous \\
T8_13_456 & 1 & 6 & 0.83 & 161 & Dichotomous \\
T8_13_456 & 2 & 5 & 0.69 & 446 & Dichotomous \\
T8_13_5 & 1 & 5 & 0.12 & 180 & Dichotomous \\
T8_14_3 & 1 & 3 & 0.47 & 167 & Dichotomous \\
T8_14_5 & 1 & 5 & 0.61 & 180 & Dichotomous \\
T8_15_3 & 1 & 3 & 0.23 & 167 & Dichotomous \\
T8_15_4 & 1 & 4 & 0.71 & 210 & Dichotomous \\
T8_15_4 & 2 & 4 & 0.35 & 433 & Dichotomous \\
T8_15_5 & 1 & 5 & 0.12 & 180 & Dichotomous \\
T8_15_6 & 1 & 6 & 0.67 & 161 & Dichotomous \\
T8_16_34 & 1 & 3 & 0.07 & 167 & Dichotomous \\
T8_16_34 & 1 & 4 & 0.28 & 210 & Dichotomous \\
T8_16_4 & 1 & 4 & 0.23 & 210 & Dichotomous \\
T8_17_3 & 1 & 3 & 0.38 & 167 & Dichotomous
\end{tabular}




\begin{tabular}{|c|c|c|c|c|c|}
\hline T8_17_4 & 1 & 4 & 0.83 & 210 & Dichotomous \\
\hline T8_17_6 & 1 & 6 & 0.50 & 161 & Dichotomous \\
\hline T8_18_34 & 1 & 3 & 0.21 & 167 & Dichotomous \\
\hline T8_18_34 & 1 & 4 & 0.44 & 210 & Dichotomous \\
\hline T8_18_5 & 1 & 5 & 0.14 & 180 & Dichotomous \\
\hline T8_19_3 & 1 & 3 & 0.25 & 167 & Dichotomous \\
\hline T8_19_5 & 1 & 5 & 0.36 & 180 & Dichotomous \\
\hline T8_19_6 & 1 & 6 & 0.39 & 161 & Dichotomous \\
\hline T8_2_3 & 1 & 3 & 0.01 & 167 & Dichotomous \\
\hline T8_2_4 & 1 & 4 & 0.35 & 210 & Dichotomous \\
\hline T8_2_4 & 2 & 4 & 0.36 & 433 & Dichotomous \\
\hline T8_2_5 & 1 & 5 & 0.08 & 180 & Dichotomous \\
\hline T8_2_5 & 2 & 5 & 0.08 & 446 & Dichotomous \\
\hline T8_2_6 & 1 & 6 & 0.10 & 161 & Dichotomous \\
\hline T8_2_6 & 2 & 6 & 0.07 & 336 & Dichotomous \\
\hline T8_20_34 & 1 & 3 & 0.28 & 167 & Dichotomous \\
\hline T8_20_34 & 1 & 4 & 0.56 & 210 & Dichotomous \\
\hline T8_20_456 & 1 & 4 & 0.53 & 210 & Dichotomous \\
\hline T8_20_456 & 1 & 5 & 0.47 & 179 & Dichotomous \\
\hline T8_20_456 & 1 & 6 & 0.56 & 161 & Dichotomous \\
\hline T8_20_5 & 1 & 5 & 0.38 & 180 & Dichotomous \\
\hline T8_20_6 & 1 & 6 & 0.85 & 161 & Dichotomous \\
\hline T8_21_34 & 1 & 3 & 0.44 & 167 & Dichotomous \\
\hline T8_21_34 & 1 & 4 & 0.73 & 210 & Dichotomous \\
\hline T8_21_4 & 1 & 4 & 0.60 & 210 & Dichotomous \\
\hline T8_21_6 & 1 & 6 & 0.32 & 161 & Dichotomous \\
\hline T8_22_3 & 1 & 3 & 0.13 & 167 & Dichotomous \\
\hline T8_22_5 & 1 & 5 & 0.29 & 180 & Dichotomous \\
\hline T8_22_6 & 1 & 6 & 0.06 & 161 & Dichotomous \\
\hline T8_23_3 & 1 & 3 & 0.28 & 167 & Dichotomous \\
\hline T8_23_4 & 1 & 4 & 0.28 & 210 & Dichotomous \\
\hline T8_23_5 & 1 & 5 & 0.53 & 180 & Dichotomous \\
\hline T8_23_6 & 1 & 6 & 0.73 & 161 & Dichotomous \\
\hline T8_24_3 & 1 & 3 & 0.19 & 167 & Dichotomous \\
\hline T8_24_456 & 1 & 4 & 0.41 & 210 & Dichotomous \\
\hline T8_24_456 & 1 & 5 & 0.54 & 180 & Dichotomous \\
\hline T8_24_456 & 1 & 6 & 0.63 & 161 & Dichotomous \\
\hline T8_24_6 & 1 & 6 & 0.50 & 161 & Dichotomous \\
\hline T8_25_3 & 1 & 3 & 0.38 & 167 & Dichotomous \\
\hline T8_25_456 & 1 & 4 & 0.02 & 210 & Dichotomous \\
\hline T8_25_456 & 1 & 5 & 0.03 & 180 & Dichotomous \\
\hline
\end{tabular}




\begin{tabular}{|c|c|c|c|c|c|}
\hline T8_25_456 & 1 & 6 & 0.11 & 161 & Dichotomous \\
\hline T8_25_5 & 1 & 5 & 0.66 & 180 & Dichotomous \\
\hline T8_25_6 & 1 & 6 & 0.85 & 161 & Dichotomous \\
\hline T8_26_3 & 2 & 3 & 0.79 & 589 & Dichotomous \\
\hline T8_26_4 & 2 & 4 & 0.80 & 433 & Dichotomous \\
\hline T8_26_5 & 2 & 5 & 0.04 & 446 & Dichotomous \\
\hline T8_27_3 & 2 & 3 & 0.14 & 589 & Dichotomous \\
\hline T8_27_4 & 2 & 4 & 0.85 & 433 & Dichotomous \\
\hline T8_27_56 & 2 & 5 & 0.21 & 446 & Dichotomous \\
\hline T8_27_56 & 2 & 6 & 0.30 & 336 & Dichotomous \\
\hline T8_28_3 & 2 & 3 & 0.47 & 589 & Dichotomous \\
\hline T8_28_6 & 2 & 6 & 0.27 & 336 & Dichotomous \\
\hline T8_29_3 & 2 & 3 & 0.75 & 589 & Dichotomous \\
\hline T8_29_4 & 2 & 4 & 0.22 & 433 & Dichotomous \\
\hline T8_29_5 & 2 & 5 & 0.48 & 446 & Dichotomous \\
\hline T8_29_6 & 2 & 6 & 0.04 & 336 & Dichotomous \\
\hline T8_3_3 & 1 & 3 & 0.00 & 167 & Dichotomous \\
\hline T8_3_4 & 1 & 4 & 0.23 & 210 & Dichotomous \\
\hline T8_3_4 & 2 & 4 & 0.27 & 433 & Dichotomous \\
\hline T8_3_56 & 1 & 5 & 0.04 & 180 & Dichotomous \\
\hline T8_3_56 & 1 & 6 & 0.01 & 161 & Dichotomous \\
\hline T8_3_56 & 2 & 6 & 0.03 & 336 & Dichotomous \\
\hline T8_30_3 & 2 & 3 & 0.32 & 589 & Dichotomous \\
\hline T8_30_6 & 2 & 6 & 0.64 & 336 & Dichotomous \\
\hline T8_31_45 & 2 & 4 & 0.30 & 433 & Dichotomous \\
\hline T8_31_45 & 2 & 5 & 0.40 & 446 & Dichotomous \\
\hline T8_31_5 & 2 & 5 & 0.52 & 446 & Dichotomous \\
\hline T8_31_6 & 2 & 6 & 0.17 & 336 & Dichotomous \\
\hline T8_32_3 & 2 & 3 & 0.54 & 589 & Dichotomous \\
\hline T8_32_4 & 2 & 4 & 0.33 & 433 & Dichotomous \\
\hline T8_32_6 & 2 & 6 & 0.70 & 336 & Dichotomous \\
\hline T8_33_3 & 2 & 3 & 0.16 & 589 & Dichotomous \\
\hline T8_33_4 & 2 & 4 & 0.11 & 433 & Dichotomous \\
\hline T8_33_5 & 2 & 5 & 0.42 & 446 & Dichotomous \\
\hline T8_34_3 & 2 & 3 & 0.48 & 589 & Dichotomous \\
\hline T8_34_4 & 2 & 4 & 0.43 & 433 & Dichotomous \\
\hline T8_34_6 & 2 & 6 & 0.02 & 336 & Dichotomous \\
\hline T8_35_4 & 2 & 4 & 0.25 & 433 & Dichotomous \\
\hline T8_35_5 & 2 & 5 & 0.66 & 446 & Dichotomous \\
\hline T8_35_6 & 2 & 6 & 0.25 & 336 & Dichotomous \\
\hline T8_36_3 & 2 & 3 & 0.64 & 589 & Dichotomous \\
\hline
\end{tabular}




\begin{tabular}{|c|c|c|c|c|c|}
\hline T8_36_34 & 2 & 4 & 0.22 & 433 & Dichotomous \\
\hline T8_36_5 & 2 & 5 & 0.35 & 446 & Dichotomous \\
\hline T8_36_6 & 2 & 6 & 0.59 & 336 & Dichotomous \\
\hline T8_37_34 & 2 & 3 & 0.43 & 589 & Dichotomous \\
\hline T8_37_34 & 2 & 4 & 0.50 & 433 & Dichotomous \\
\hline T8_37_6 & 2 & 6 & 0.38 & 336 & Dichotomous \\
\hline T8_38_4 & 2 & 4 & 0.61 & 433 & Dichotomous \\
\hline T8_38_5 & 2 & 5 & 0.21 & 446 & Dichotomous \\
\hline T8_38_6 & 2 & 6 & 0.32 & 336 & Dichotomous \\
\hline T8_39_56 & 2 & 5 & 0.48 & 446 & Dichotomous \\
\hline T8_39_56 & 2 & 6 & 0.69 & 336 & Dichotomous \\
\hline T8_39_6 & 2 & 6 & 0.83 & 336 & Dichotomous \\
\hline T8_4_3 & 1 & 3 & 0.13 & 167 & Dichotomous \\
\hline T8_4_4 & 1 & 4 & 0.00 & 210 & Dichotomous \\
\hline T8_4_56 & 1 & 5 & 0.08 & 180 & Dichotomous \\
\hline T8_4_56 & 1 & 6 & 0.16 & 161 & Dichotomous \\
\hline T8_40_3 & 2 & 3 & 0.48 & 589 & Dichotomous \\
\hline T8_40_5 & 2 & 5 & 0.44 & 446 & Dichotomous \\
\hline T8_40_6 & 2 & 6 & 0.33 & 336 & Dichotomous \\
\hline T8_41_3 & 2 & 3 & 0.00 & 589 & Dichotomous \\
\hline T8_41_4 & 2 & 4 & 0.36 & 433 & Dichotomous \\
\hline T8_41_5 & 2 & 5 & 0.66 & 446 & Dichotomous \\
\hline T8_42_3 & 2 & 3 & 0.49 & 589 & Dichotomous \\
\hline T8_42_5 & 2 & 5 & 0.64 & 446 & Dichotomous \\
\hline T8_42_6 & 2 & 6 & 0.42 & 336 & Dichotomous \\
\hline T8_43_3 & 2 & 3 & 0.49 & 589 & Dichotomous \\
\hline T8_43_45 & 2 & 4 & 0.38 & 433 & Dichotomous \\
\hline T8_43_45 & 2 & 5 & 0.54 & 446 & Dichotomous \\
\hline T8_43_56 & 2 & 5 & 0.24 & 446 & Dichotomous \\
\hline T8_43_56 & 2 & 6 & 0.34 & 336 & Dichotomous \\
\hline T8_43_6 & 2 & 6 & 0.13 & 336 & Dichotomous \\
\hline T8_44_3 & 2 & 3 & 0.35 & 589 & Dichotomous \\
\hline T8_44_4 & 2 & 4 & 0.00 & 433 & Dichotomous \\
\hline T8_44_5 & 2 & 5 & 0.04 & 446 & Dichotomous \\
\hline T8_44_6 & 2 & 6 & 0.71 & 336 & Dichotomous \\
\hline T8_45_3 & 2 & 3 & 0.48 & 589 & Dichotomous \\
\hline T8_45_4 & 2 & 4 & 0.48 & 433 & Dichotomous \\
\hline T8_45_5 & 2 & 5 & 0.47 & 446 & Dichotomous \\
\hline T8_46_34 & 2 & 3 & 0.54 & 589 & Dichotomous \\
\hline T8_46_34 & 2 & 4 & 0.75 & 433 & Dichotomous \\
\hline T8_46_4 & 2 & 4 & 0.60 & 433 & Dichotomous \\
\hline
\end{tabular}




\begin{tabular}{llllll} 
T8_46_5 & 2 & 5 & 0.55 & 446 & Dichotomous \\
T8_46_6 & 2 & 6 & 0.48 & 336 & Dichotomous \\
T8_47_3 & 2 & 3 & 0.50 & 589 & Dichotomous \\
T8_47_4 & 2 & 4 & 0.43 & 433 & Dichotomous \\
T8_48_3 & 2 & 3 & 0.16 & 589 & Dichotomous \\
T8_48_4 & 2 & 4 & 0.11 & 433 & Dichotomous \\
T8_48_5 & 2 & 5 & 0.44 & 446 & Dichotomous \\
T8_49_3 & 2 & 3 & 0.54 & 589 & Dichotomous \\
T8_49_4 & 2 & 4 & 0.13 & 433 & Dichotomous \\
T8_49_5 & 2 & 5 & 0.22 & 446 & Dichotomous \\
T8_49_6 & 2 & 6 & 0.21 & 336 & Dichotomous \\
T8_5_3 & 1 & 3 & 0.20 & 167 & Dichotomous \\
T8_5_4 & 1 & 4 & 0.07 & 210 & Dichotomous \\
T8_5_56 & 1 & 5 & 0.23 & 180 & Dichotomous \\
T8_5_56 & 1 & 6 & 0.37 & 161 & Dichotomous \\
T8_5_6 & 1 & 6 & 0.62 & 161 & Dichotomous \\
T8_6_3 & 1 & 3 & 0.47 & 167 & Dichotomous \\
T8_6_4 & 1 & 4 & 0.61 & 210 & Dichotomous \\
T8_6_5 & 1 & 5 & 0.83 & 180 & Dichotomous \\
T8_7_34 & 1 & 3 & 0.00 & 167 & Dichotomous \\
T8_7_34 & 1 & 4 & 0.00 & 210 & Dichotomous \\
T8_7_4 & 1 & 4 & 0.13 & 210 & Dichotomous \\
T8_7_5 & 1 & 5 & 0.19 & 180 & Dichotomous \\
T8_7_6 & 1 & 6 & 0.07 & 161 & Dichotomous \\
T8_7_6 & 2 & 6 & 0.10 & 336 & Dichotomous \\
T8_8_3 & 1 & 3 & 0.05 & 167 & Dichotomous \\
T8_8_3 & 2 & 3 & 0.06 & 589 & Dichotomous \\
T8_8_4 & 1 & 4 & 0.70 & 210 & Dichotomous \\
T8_8_56 & 1 & 5 & 0.59 & 180 & Dichotomous \\
T8_8_56 & 1 & 6 & 0.66 & 161 & Dichotomous \\
T8_9_3 & 1 & 3 & 0.45 & 167 & Dichotomous \\
T8_9_3 & 2 & 3 & 0.54 & 589 & Dichotomous \\
T8_9_456 & 1 & 4 & 0.06 & 210 & Dichotomous \\
T8_9_456 & 1 & 5 & 0.10 & 180 & Dichotomous \\
T8_9_456 & 1 & 6 & 0.16 & 161 & Dichotomous \\
T8_9_6 & 1 & 6 & 0.08 & 161 & Dichotomous \\
\hline & & & & & \\
\hline
\end{tabular}


Table 10

Task 1 vertical linking item p-values by grade level

\begin{tabular}{lcccccc}
\hline Item & Grade 1 & Grade 2 & Grade 3 & Grade 4 & Grade 5 & Grade 6 \\
\hline T1_2_12 & 76.2 & 91.5 & & & & \\
T1_13_12 & 17.9 & 20.5 & & & & \\
T1_14_12 & 83.9 & 87.1 & & & & \\
T1_21_12 & 72.9 & 81.6 & & & & \\
T1_6_23 & & 15.2 & 13.2 & & & \\
T1_9_23 & & 36.5 & 35.3 & & & \\
T1_13_23 & & 10.4 & 7.8 & & & \\
T1_27_23 & & 15.1 & 70.5 & & & \\
T1_1_34 & & 54.5 & 42.9 & & \\
T1_2_34 & & 15.6 & 21.9 & & \\
T1_4_34 & & 29.1 & 33.8 & & \\
T1_18_34 & & & 55.3 & 63.5 & & \\
T1_22_45 & & & & 28.2 & 38.3 & \\
T1_4_56 & & & & & 13.3 & 9.3 \\
T1_6_56 & & & & & 42.8 & 29.8 \\
T1_8_56 & & & & & 84.4 & 83.3 \\
T1_24_56 & & & & 86.7 & 90.6 & 84.4 \\
T1_2_456 & & & & 41.9 & 39.4 & 44.1 \\
T1_5_456 & & & & 26 & 22.8 & 26.7 \\
T1_10_456 & & & & & \\
\hline
\end{tabular}

Note. Items highlighted in green were used for grade-based vertical links. 
Table 11

Task 2 vertical linking item p-values by grade level

\begin{tabular}{|c|c|c|c|c|c|c|}
\hline Item & $\begin{array}{l}\text { Grade } \\
1\end{array}$ & $\begin{array}{l}\text { Grade } \\
2\end{array}$ & $\begin{array}{l}\text { Grade } \\
3\end{array}$ & $\begin{array}{l}\text { Grade } \\
4\end{array}$ & $\begin{array}{l}\text { Grade } \\
5\end{array}$ & $\begin{array}{l}\text { Grade } \\
6\end{array}$ \\
\hline T2_51_12 & 13 & 32 & & & & \\
\hline T2_53_12 & 9 & 22 & & & & \\
\hline T2_56_12 & 8 & 15 & & & & \\
\hline T2_58_12 & 8 & 26 & & & & \\
\hline T2_75_12 & 3 & 8 & & & & \\
\hline T2_60_23 & & 50 & 60 & & & \\
\hline T2_70_23 & & 38 & 42 & & & \\
\hline T2_75_23 & & 9 & 13 & & & \\
\hline T2_78_23 & & 13 & 24 & & & \\
\hline T2_84_23 & & 19 & 21 & & & \\
\hline T2_55_34 & & & 76 & 88 & & \\
\hline T2_56_34 & & & 67 & 74 & & \\
\hline T2_59_34 & & & 48 & 75 & & \\
\hline T2_69_34 & & & 56 & 77 & & \\
\hline T2_77_34 & & & 43 & 63 & & \\
\hline T2_61_45 & & & & 34 & 49 & \\
\hline T2_61_56 & & & & & 83 & 87 \\
\hline T2_70_56 & & & & & 84 & 85 \\
\hline T2_71_56 & & & & & 65 & 66 \\
\hline T2_74_56 & & & & & 22 & 26 \\
\hline T2_79_56 & & & & & 44 & 52 \\
\hline T2_64_456 & & & & 70 & 78 & 81 \\
\hline T2_71_456 & & & & 19 & 43 & 42 \\
\hline T2_77_456 & & & & 18 & 23 & 27 \\
\hline T2_81_456 & & & & 9 & 8 & 9 \\
\hline
\end{tabular}


Table 12

Task 3 vertical linking item p-values by grade level

\begin{tabular}{|c|c|c|c|c|c|c|}
\hline Item & $\begin{array}{c}\text { Grade } \\
1\end{array}$ & $\begin{array}{c}\text { Grade } \\
2\end{array}$ & $\begin{array}{c}\text { Grade } \\
3\end{array}$ & $\begin{array}{c}\text { Grade } \\
4\end{array}$ & $\begin{array}{c}\text { Grade } \\
5\end{array}$ & $\begin{array}{c}\text { Grade } \\
6\end{array}$ \\
\hline T3_10_12 & 24 & 35.7 & & & & \\
\hline T3_11_12 & 27.4 & 52.7 & & & & \\
\hline T3_12_12 & 43.6 & 26.3 & & & & \\
\hline T3_18_12 & 17.3 & 42.9 & & & & \\
\hline T3_19_12 & 27.9 & 24.6 & & & & \\
\hline T3_20_12 & 24 & 21.4 & & & & \\
\hline T3_23_12 & 34.1 & 61.6 & & & & \\
\hline T3_29_12 & 15.1 & 18.8 & & & & \\
\hline T3_64_12 & 36.5 & 47 & & & & \\
\hline T3_42_23 & & 29.9 & 46.5 & & & \\
\hline T3_43_23 & & 28 & & & & \\
\hline T3_46_23 & & 57 & 70.6 & & & \\
\hline T3_2_23 & & 30.4 & 23.4 & & & \\
\hline T3_4_23 & & 25.9 & 19.8 & & & \\
\hline T3_8_23 & & 19.6 & 21 & & & \\
\hline T3_13_23 & & 58 & 68.9 & & & \\
\hline T3_27_23 & & 35.3 & 52.7 & & & \\
\hline T3_29_23 & & 41.5 & 56.9 & & & \\
\hline T3_30_23 & & 47.8 & 58.7 & & & \\
\hline T3_37_23 & & 37.9 & 34.1 & & & \\
\hline T3_5_34 & & & 37.1 & 40.5 & & \\
\hline T3_42_34 & & & 28.5 & 43 & & \\
\hline T3_6_34 & & & 24.6 & 27.1 & & \\
\hline T3_11_34 & & & 24.6 & 23.8 & & \\
\hline T3_15_34 & & & 53.9 & 54.3 & & \\
\hline T3_17_34 & & & 59.9 & 75.2 & & \\
\hline T3_23_34 & & & 50.9 & 65.7 & & \\
\hline T3_28_34 & & & 45.5 & 61 & & \\
\hline T3_33_34 & & & 62.9 & 68.6 & & \\
\hline T3_50_34 & & & 54 & & & \\
\hline T3_51_34 & & & 52.3 & 59.4 & & \\
\hline T3_60_45 & & & & 83.1 & 88.3 & \\
\hline T3_63_45 & & & & 26.1 & 18.4 & \\
\hline T3_2_56 & & & & & 42.8 & 49.1 \\
\hline T3_16_56 & & & & & 65 & 68.9 \\
\hline T3_19_56 & & & & & 34.4 & 44.1 \\
\hline
\end{tabular}


MATRS TECHNICAL MANUAL 73

T3_21_56

T3_25_56

T3_31_56

T3_32_56

T3_39_56

T3_51_56

T3_60_56

T3_3_456

T3_8_456

T3_9_456

T3_14_456

T3_15_456

T3_20_456

T3_33_456

T3_40_456

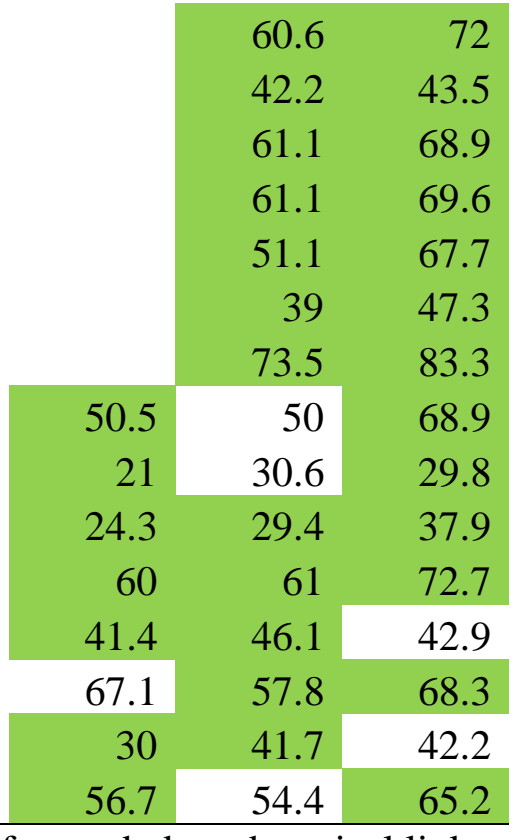

Note. Items highlighted in green were used for grade-based vertical links. 
Table 13

Task 4 vertical linking item p-values by grade level

\begin{tabular}{|c|c|c|c|c|}
\hline Item & $\begin{array}{c}\text { Grade } \\
3 \\
\end{array}$ & $\begin{array}{c}\text { Grade } \\
4\end{array}$ & $\begin{array}{c}\text { Grade } \\
5 \\
\end{array}$ & $\begin{array}{c}\text { Grade } \\
6 \\
\end{array}$ \\
\hline T4_7_34 & 60.5 & 75.6 & & \\
\hline T4_8_34 & 75.4 & 91.9 & & \\
\hline T4_12_34 & 60.5 & 73.8 & & \\
\hline T4_23_34 & 62.3 & 71.9 & & \\
\hline T4_25_34 & 39.5 & 63.3 & & \\
\hline T4_29_34 & & 76.2 & & \\
\hline T4_36_34 & & 72.3 & & \\
\hline T4_43_34 & & 45.7 & & \\
\hline T4_38_45 & & 58.7 & 70.2 & \\
\hline T4_41_45 & & 81.5 & 86.8 & \\
\hline T4_5_56 & & & 81.7 & 80.1 \\
\hline T4_11_56 & & & 45 & 67.7 \\
\hline T4_14_56 & & & 37.8 & 53.4 \\
\hline T4_22_56 & & & 62.8 & 56.5 \\
\hline T4_23_56 & & & 80.7 & 73.9 \\
\hline T4_31_56 & & & 81.8 & 87.8 \\
\hline T4_45_56 & & & 76.7 & 82.4 \\
\hline T4_46_56 & & & 83.2 & 87.5 \\
\hline T4_1_456 & & 34.8 & 46.1 & 38.5 \\
\hline T4_6_456 & & 53.3 & 55.6 & 66.5 \\
\hline T4_14_456 & & 60 & 43.9 & 51.6 \\
\hline T4_15_456 & & 58.1 & 57.2 & 67.1 \\
\hline T4_22_456 & & 76.7 & 85 & 80.7 \\
\hline
\end{tabular}

Note. Items highlighted in green were used for grade-based vertical links. 
Table 14

Task 5 vertical linking item p-values by grade level

\begin{tabular}{|c|c|c|c|c|c|c|}
\hline Item & $\begin{array}{c}\text { Grade } \\
1\end{array}$ & $\begin{array}{c}\text { Grade } \\
2\end{array}$ & $\begin{array}{c}\text { Grade } \\
3 \\
\end{array}$ & $\begin{array}{c}\text { Grade } \\
4\end{array}$ & $\begin{array}{c}\text { Grade } \\
5\end{array}$ & $\begin{array}{c}\text { Grade } \\
6\end{array}$ \\
\hline T5_5_12 & 0 & 1.3 & & & & \\
\hline T5_10_12 & 1.1 & 6.4 & & & & \\
\hline T5_18_12 & 6.7 & 34.8 & & & & \\
\hline T5_24_12 & 2.8 & 27.2 & & & & \\
\hline T5_30_12 & 7.6 & 27.6 & & & & \\
\hline T5_32_12 & 5.9 & 37.6 & & & & \\
\hline T5_26_23 & & 57 & 63.7 & & & \\
\hline T5_39_23 & & 11.8 & 27.7 & & & \\
\hline T5_8_23 & & 0 & 3 & & & \\
\hline T5_10_23 & & 7.1 & 18.6 & & & \\
\hline T5_11_23 & & 39.3 & 63.5 & & & \\
\hline T5_23_23 & & 1.3 & 6.6 & & & \\
\hline T5_3_34 & & & 7.2 & 16.2 & & \\
\hline T5_10_34 & & & 22.2 & 31.9 & & \\
\hline T5_12_34 & & & 1.2 & 2.9 & & \\
\hline T5_14_34 & & & 3 & 7.6 & & \\
\hline T5_17_34 & & & 18 & 34.8 & & \\
\hline T5_31_34 & & & 19 & 30.9 & & \\
\hline T5_34_34 & & & 53.5 & 68.6 & & \\
\hline T5_26_45 & & & & 55.5 & 58.7 & \\
\hline T5_36_45 & & & & 39.5 & 54.3 & \\
\hline T5_26_56 & & & & & 55.8 & 63.1 \\
\hline T5_30_56 & & & & & 42.8 & 52.1 \\
\hline T5_37_56 & & & & & 41.9 & 42.6 \\
\hline T5_3_56 & & & & & 38.3 & 45.3 \\
\hline T5_6_56 & & & & & 9.4 & 13.7 \\
\hline T5_7_56 & & & & & 5 & 3.1 \\
\hline T5_12_56 & & & & & 13.3 & 20.5 \\
\hline T5_20_56 & & & & & 1 & 7.5 \\
\hline T5_6_456 & & & & 21.4 & 24.8 & 31.1 \\
\hline T5_8_456 & & & & 1.4 & 1.1 & 1.2 \\
\hline T5_15_456 & & & & 13.8 & 38.3 & 47.2 \\
\hline T5_21_456 & & & & 7.6 & 12.2 & 18 \\
\hline T5_25_456 & & & & 14.3 & 13.3 & 23 \\
\hline
\end{tabular}

Note. Items highlighted in green were used for grade-based vertical links. 
Table 15

Task 6 vertical linking item p-values by grade level

\begin{tabular}{|c|c|c|c|c|c|c|}
\hline Item & Grade 1 & Grade 2 & Grade 3 & Grade 4 & Grade 5 & Grade 6 \\
\hline T6_10_12 & 45.8 & 40.6 & & & & \\
\hline T6_15_12 & 55.9 & 44.2 & & & & \\
\hline T6_16_12 & 42.5 & 56.7 & & & & \\
\hline T6_19_12 & 43 & 74.1 & & & & \\
\hline T6_21_12 & 29.6 & 42 & & & & \\
\hline T6_26_12 & 22.9 & 48.2 & & & & \\
\hline T6_41_12 & 28.3 & 55.6 & & & & \\
\hline T6_6_23 & & 46.4 & 40.7 & & & \\
\hline T6_11_23 & & 54.9 & 56.3 & & & \\
\hline T6_13_23 & & 62.5 & 59.9 & & & \\
\hline T6_17_23 & & 41.5 & 81.4 & & & \\
\hline T6_20_23 & & 30.8 & 44.3 & & & \\
\hline T6_36_23 & & 33.9 & 55 & & & \\
\hline T6_42_23 & & 45.8 & 54.5 & & & \\
\hline T6_1_34 & & & 59.9 & 65.2 & & \\
\hline T6_5_34 & & & 37.1 & 46.7 & & \\
\hline T6_10_34 & & & 62.3 & 76.2 & & \\
\hline T6_21_34 & & & 24 & 42.4 & & \\
\hline T6_24_34 & & & 61.7 & 80.5 & & \\
\hline T6_12_3.1 & & & 20 & & & \\
\hline T6_40_34 & & & 69.3 & 81.8 & & \\
\hline T6_43_34 & & & 51.4 & 66.3 & & \\
\hline T6_27_45 & & & & 46.7 & 62.1 & \\
\hline T6_28_45 & & & & 46 & 52.9 & \\
\hline T6_1_56 & & & & & 71.7 & 79.5 \\
\hline T6_2_56 & & & & & 72.2 & 80.7 \\
\hline T6_5_56 & & & & & 77.8 & 65.2 \\
\hline T6_6_56 & & & & & 56.1 & 84.5 \\
\hline T6_18_56 & & & & & 37.2 & 42.9 \\
\hline T6_28_56 & & & & & 89.7 & 92.6 \\
\hline T6_32_56 & & & & & 61.4 & 69 \\
\hline T6_45_56 & & & & & 61.7 & 75.9 \\
\hline T6_1_456 & & & & 68.1 & 77.2 & 77.6 \\
\hline T6_4_456 & & & & 43.8 & 46.7 & 77.6 \\
\hline T6_8_456 & & & & 92.4 & 93.9 & 90.1 \\
\hline T6_14_456 & & & & 74.3 & 87.2 & 82.6 \\
\hline T6_22_456 & & & & 79 & 88.9 & 90.7 \\
\hline
\end{tabular}


Note. Items highlighted in green were used for grade-based vertical links.

Table 16

Task 7 vertical linking item p-values by grade level

\begin{tabular}{|c|c|c|c|c|c|c|}
\hline Item & $\begin{array}{c}\text { Grade } \\
1\end{array}$ & $\begin{array}{c}\text { Grade } \\
2\end{array}$ & $\begin{array}{c}\text { Grade } \\
3\end{array}$ & $\begin{array}{c}\text { Grade } \\
4\end{array}$ & $\begin{array}{c}\text { Grade } \\
5\end{array}$ & $\begin{array}{c}\text { Grade } \\
6\end{array}$ \\
\hline T7_6_12 & 3.4 & 9.4 & & & & \\
\hline T7_8_12 & 14.5 & 29 & & & & \\
\hline T7_11_12 & 0 & 0.4 & & & & \\
\hline T7_22_12 & 66.4 & 81.4 & & & & \\
\hline T7_29_12 & 18.9 & 37 & & & & \\
\hline T7_4_23 & & 14.5 & 20.4 & & & \\
\hline T7_12_23 & & 24.6 & 26.9 & & & \\
\hline T7_15_23 & & 28.6 & 45.5 & & & \\
\hline T7_17_23 & & 89 & 96.8 & & & \\
\hline T7_26_23 & & 89.6 & 91.3 & & & \\
\hline T7_2_34 & & & 7.1 & 11.4 & & \\
\hline T7_9_34 & & & 12.6 & 33.4 & & \\
\hline T7_15_34 & & & 15 & 26.2 & & \\
\hline T7_29_34 & & & 83.7 & 89.1 & & \\
\hline T7_31_34 & & & & 59.9 & 68.1 & \\
\hline T7_16_45 & & & & 94.2 & 95.1 & \\
\hline T7_30_45 & & & & 91.7 & 87.9 & \\
\hline T7_1_56 & & & & & 14.4 & 29.2 \\
\hline T7_5_56 & & & & & 0.6 & 1.2 \\
\hline T7_15_56 & & & & & 87.8 & 91.3 \\
\hline T7_16_56 & & & & & 87.7 & 86.3 \\
\hline T7_17_56 & & & & & 2.9 & 3.6 \\
\hline T7_29_56 & & & & & 40.1 & 38.4 \\
\hline T7_3_456 & & & & 72.4 & 71.7 & 70.2 \\
\hline T7_9_456 & & & & 70 & 77.8 & 82 \\
\hline T7_15_456 & & & & 30.5 & 32.2 & 28 \\
\hline
\end{tabular}

Note. Items highlighted in green were used for grade-based vertical links. 
Table 17

Task 8 vertical linking item p-values by grade level

\begin{tabular}{|c|c|c|c|c|}
\hline Item & $\begin{array}{c}\text { Grade } \\
3 \\
\end{array}$ & $\begin{array}{c}\text { Grade } \\
4 \\
\end{array}$ & $\begin{array}{c}\text { Grade } \\
5 \\
\end{array}$ & $\begin{array}{c}\text { Grade } \\
6 \\
\end{array}$ \\
\hline T8_7_34 & 0 & 0.5 & & \\
\hline T8_16_34 & 6.6 & 27.6 & & \\
\hline T8_18_34 & 21 & 43.8 & & \\
\hline T8_20_34 & 27.5 & 56.2 & & \\
\hline T8_21_34 & 44.3 & 72.9 & & \\
\hline T8_3_56 & & & 3.9 & 2.2 \\
\hline T8_37_34 & 43 & 49.7 & & \\
\hline T8_46_34 & 54.3 & 75.3 & & \\
\hline T8_36_34 & & 21.9 & & \\
\hline T8_31_45 & & 30.5 & 39.7 & \\
\hline T8_43_45 & & 37.9 & 54 & \\
\hline T8_4_56 & & & 8.3 & 16.1 \\
\hline T8_5_56 & & & 23.3 & 36.6 \\
\hline T8_8_56 & & & 58.9 & 66.5 \\
\hline T8_12_56 & & & 0 & 0 \\
\hline T8_27_56 & & & 20.9 & 30.1 \\
\hline T8_39_56 & & & 48 & 68.8 \\
\hline T8_43_56 & & & 23.5 & 33.6 \\
\hline T8_9_456 & & 5.7 & 10 & 15.5 \\
\hline T8_13_456 & & 70.5 & 68.7 & 83.2 \\
\hline T8_20_456 & & 53.3 & 46.9 & 55.9 \\
\hline T8_24_456 & & 41.4 & 53.9 & 63.4 \\
\hline T8_25_456 & & 2.4 & 3.3 & 10.6 \\
\hline
\end{tabular}

Note. Items highlighted in green were used for grade-based vertical links. 
Table 18

Task-based Rasch model fit

\begin{tabular}{lcccccc}
\hline Tasks & M2 & df & p-value & f0hat & RMSEA & TLI \\
\hline Task 1 & 2003.46 & 867 & $<.001$ & 0.49 & 0.02 & 0.82 \\
Task 2 & 16559.29 & 4604 & $<.001$ & 4.08 & 0.03 & 0.85 \\
Task 3 & 6365.34 & 8085 & 1.00 & 1.57 & $<.001$ & 1.00 \\
Task 4 & 1473.90 & 2426 & 1.00 & 0.58 & $<.001$ & 1.00 \\
Task 5 & 1761.84 & 3210 & 1.00 & 0.43 & $<.001$ & 1.00 \\
Task 6 & 5508.05 & 4802 & $<.001$ & 1.36 & 0.01 & 0.97 \\
Task 7 & 1720.92 & 2010 & 1.00 & 0.42 & $<.001$ & 1.00 \\
Task 8 & 1983.96 & 3059 & 1.00 & 0.79 & $<.001$ & 1.00 \\
\hline
\end{tabular}

Note. $\mathrm{M} 2=\mathrm{M} 2$ statistic, $\mathrm{df}=$ degrees of freedom, RMSEA $=$ root mean square error of approximation, TLI $=$ Tucker-Lewis Index 
Table 19

Task 1 Rasch model item parameters

\begin{tabular}{|c|c|c|}
\hline Item & $\mathrm{a}$ & $\mathrm{b}$ \\
\hline T1_1_1 & 1 & -2.038 \\
\hline T1_1_5 & 1 & 2.725 \\
\hline T1_10_456 & 1 & 1.537 \\
\hline T1_11_1 & 1 & 0.093 \\
\hline T1_11_6 & 1 & -1.616 \\
\hline T1_12_1 & 1 & 0.040 \\
\hline T1_12_3 & 1 & 0.674 \\
\hline T1_12_5 & 1 & 0.973 \\
\hline T1_12_6 & 1 & 3.081 \\
\hline T1_13_12 & 1 & 1.423 \\
\hline T1_13_3 & 1 & 1.245 \\
\hline T1_13_4 & 1 & 1.519 \\
\hline T1_13_5 & 1 & 0.330 \\
\hline T1_14_12 & 1 & -2.477 \\
\hline T1_14_2 & 1 & 1.406 \\
\hline T1_14_3 & 1 & 0.941 \\
\hline T1_14_5 & 1 & 1.528 \\
\hline T1_14_6 & 1 & 2.563 \\
\hline T1_15_1 & 1 & 0.283 \\
\hline T1_15_2 & 1 & -2.495 \\
\hline T1_15_3 & 1 & 0.592 \\
\hline T1_15_4 & 1 & 1.473 \\
\hline T1_16_1 & 1 & -2.515 \\
\hline T1_17_3 & 1 & 0.960 \\
\hline T1_17_5 & 1 & -0.303 \\
\hline T1_17_6 & 1 & -0.553 \\
\hline T1_18_1 & 1 & -0.261 \\
\hline T1_18_34 & 1 & -0.918 \\
\hline T1_18_5 & 1 & 0.861 \\
\hline T1_19_3 & 1 & 1.047 \\
\hline T1_19_5 & 1 & 0.380 \\
\hline T1_2_12 & 1 & -2.072 \\
\hline T1_2_2 & 1 & -0.079 \\
\hline T1_2_34 & 1 & 1.447 \\
\hline T1_2_5 & 1 & 0.623 \\
\hline T1_20_1 & 1 & -0.654 \\
\hline T1_20_2 & 1 & -1.799 \\
\hline
\end{tabular}




$\begin{array}{lcc}\text { T1_20_6 } & 1 & 1.660 \\ \text { T1_21_12 } & 1 & -1.803 \\ \text { T1_21_2 } & 1 & -1.074 \\ \text { T1_21_3 } & 1 & 0.803 \\ \text { T1_21_6 } & 1 & 0.238 \\ \text { T1_22_1 } & 1 & -1.678 \\ \text { T1_22_2 } & 1 & -1.212 \\ \text { T1_22_45 } & 1 & 0.731 \\ \text { T1_23_1 } & 1 & 0.142 \\ \text { T1_23_2 } & 1 & -2.755 \\ \text { T1_23_6 } & 1 & 0.858 \\ \text { T1_24_4 } & 1 & 0.979 \\ \text { T1_25_1 } & 1 & 0.691 \\ \text { T1_25_3 } & 1 & 0.149 \\ \text { T1_25_5 } & 1 & 0.861 \\ \text { T1_25_6 } & 1 & -0.211 \\ \text { T1_26_2 } & 1 & -1.850 \\ \text { T1_26_3 } & 1 & 0.915 \\ \text { T1_27_1 } & 1 & -1.190 \\ \text { T1_27_3 } & 1 & -0.148 \\ \text { T1_27_4 } & 1 & 1.481 \\ \text { T1_27_5 } & 1 & 0.938 \\ \text { T1_28_1 } & 1 & -1.516 \\ \text { T1_28_2 } & 1 & -1.886 \\ \text { T1_28_4 } & 1 & 1.167 \\ \text { T1_28_5 } & 1 & 0.962 \\ \text { T1_28_6 } & 1 & 0.693 \\ \text { T1_3_1 } & 1 & 1.824 \\ \text { T1_3_2 } & 1 & 0.608 \\ \text { T1_3_6 } & 1 & 0.445 \\ \text { T1_4_1 } & 1 & -3.031 \\ \text { T1_4_2 } & 1 & 2.126 \\ \text { T1_4_34 } & 1 & 0.591 \\ \text { T1_4_4 } & 1 & -0.639 \\ \text { T1_4_56 } & 1 & 2.984 \\ \text { T1_5_1 } & 1 & -3.158 \\ \text { T1_5_2 } & 1 & 0.151 \\ \text { T1_5_456 } & 1 & 0.548 \\ \text { T1_5_5 } & 1 & 2.516 \\ \text { T1_6_1 } & 1 & 1.451 \\ \text { T1_6_23 } & 1 & 2.346\end{array}$




\begin{tabular}{llc} 
T1_6_4 & 1 & 1.832 \\
T1_7_1 & 1 & -0.159 \\
T1_7_5 & 1 & 1.566 \\
T1_8_1 & 1 & -2.779 \\
T1_8_2 & 1 & -1.622 \\
T1_8_3 & 1 & -0.608 \\
T1_8_4 & 1 & 1.877 \\
T1_9_1 & 1 & 0.121 \\
T1_9_23 & 1 & 0.833 \\
T1_9_4 & 1 & 1.106 \\
\hline
\end{tabular}

Note. $\mathrm{a}=$ item discrimination, $\mathrm{b}=$ item difficulty 
Table 20

Task 2 Rasch model item parameters

\begin{tabular}{llc}
\hline Item & $\mathrm{a}$ & $\mathrm{b}$ \\
\hline T2_51_12 & 1 & 0.26141 \\
T2_51_2 & 1 & -0.8091 \\
T2_51_3 & 1 & 0.61501 \\
T2_51_4 & 1 & 1.86916 \\
T2_51_5 & 1 & -0.113 \\
T2_51_6 & 1 & -1.5054 \\
T2_52_1 & 1 & 0.19671 \\
T2_52_2 & 1 & 1.21216 \\
T2_52_3 & 1 & 0.77653 \\
T2_52_5 & 1 & 1.17356 \\
T2_52_6 & 1 & 1.31385 \\
T2_53_12 & 1 & 0.80187 \\
T2_53_3 & 1 & 0.59607 \\
T2_53_4 & 1 & 0.43278 \\
T2_53_6 & 1 & 0.31218 \\
T2_54_1 & 1 & -1.3237 \\
T2_54_2 & 1 & 0.68961 \\
T2_54_3 & 1 & 0.43129 \\
T2_54_6 & 1 & 1.18807 \\
T2_55_1 & 1 & 1.14671 \\
T2_55_2 & 1 & 3.51855 \\
T2_55_5 & 1 & 1.49468 \\
T2_56_12 & 1 & 1.20506 \\
T2_56_34 & 1 & -0.9704 \\
T2_57_1 & 1 & -0.9548 \\
T2_57_5 & 1 & -0.3245 \\
T2_58_12 & 1 & 0.74235 \\
T2_58_2 & 1 & 1.06346 \\
T2_58_4 & 1 & 0.2737 \\
T2_58_6 & 1 & 0.22084 \\
T2_59_1 & 1 & -0.4625 \\
T2_59_5 & 1 & -0.9291 \\
T2_59_6 & 1 & 1.60127 \\
T2_60_1 & 1 & -0.0657 \\
T2_60_23 & 1 & -0.7536 \\
& 1 & 1.22344 \\
T2_6 & 1 & -0.1221 \\
\hline
\end{tabular}




$\begin{array}{lll}\text { T2_61_1 } & 1 & -0.3539 \\ \text { T2_61_2 } & 1 & -0.5403 \\ \text { T2_61_3 } & 1 & 0.44963 \\ \text { T2_61_45 } & 1 & 1.37523 \\ \text { T2_62_1 } & 1 & -0.0287 \\ \text { T2_62_2 } & 1 & 0.49593 \\ \text { T2_62_3 } & 1 & 0.31173 \\ \text { T2_62_4 } & 1 & 0.85571 \\ \text { T2_62_5 } & 1 & 1.00092 \\ \text { T2_63_1 } & 1 & 1.17358 \\ \text { T2_63_2 } & 1 & -0.6742 \\ \text { T2_63_3 } & 1 & 1.88129 \\ \text { T2_63_4 } & 1 & 0.62643 \\ \text { T2_63_5 } & 1 & 1.1487 \\ \text { T2_63_6 } & 1 & 1.1073 \\ \text { T2_64_1 } & 1 & 0.1278 \\ \text { T2_64_2 } & 1 & 0.58949 \\ \text { T2_64_3 } & 1 & 0.39479 \\ \text { T2_64_5 } & 1 & 0.81432 \\ \text { T2_65_1 } & 1 & -0.7058 \\ \text { T2_65_2 } & 1 & -0.9023 \\ \text { T2_65_5 } & 1 & 3.24614 \\ \text { T2_65_6 } & 1 & 0.29279 \\ \text { T2_66_1 } & 1 & 0.11372 \\ \text { T2_66_22 } & 1 & 0.07026 \\ \text { T2_66_3 } & 1 & -0.2582 \\ \text { T2_66_4 } & 1 & 1.21095 \\ \text { T2_66_5 } & 1 & 1.78242 \\ \text { T2_66_6 } & 1 & 1.09078 \\ \text { T2_67_1 } & 1 & -0.1159 \\ \text { T2_67_2 } & 1 & -0.1734 \\ \text { T2_67_3 } & 1 & 0.11629 \\ \text { T2_67_4 } & 1 & 0.61436 \\ \text { T2_67_5 } & 1 & 1.24793 \\ \text { T2_68_1 } & 1 & 0.01056 \\ \text { T2_68_2 } & 1 & 0.82597 \\ \text { T2_68_3 } & 1 & 0.49908 \\ \text { T2_68_4 } & 1 & 0.90415 \\ \text { T2_69_1 } & 1 & -0.3203 \\ \text { T2_69_2 } & 1 & 1.17797 \\ \text { T2_69_4 } & 1 & 1.23594\end{array}$




\begin{tabular}{|c|c|c|}
\hline T2_69_5 & 1 & -0.3796 \\
\hline T2_70_1 & 1 & 0.26566 \\
\hline T2_70_23 & 1 & 0.0771 \\
\hline T2_70_3 & 1 & 0.03738 \\
\hline T2_71_1 & 1 & -0.3539 \\
\hline T2_71_3 & 1 & 1.2193 \\
\hline T2_71_456 & 1 & 1.98121 \\
\hline T2_72_1 & 1 & 2.14178 \\
\hline T2_72_2 & 1 & 3.4322 \\
\hline T2_72_4 & 1 & 1.94609 \\
\hline T2_73_1 & 1 & 0.89267 \\
\hline T2_73_2 & 1 & 0.91737 \\
\hline T2_73_3 & 1 & 1.70782 \\
\hline T2_73_4 & 1 & 1.7599 \\
\hline T2_73_6 & 1 & 1.40854 \\
\hline T2_74_1 & 1 & 0.39018 \\
\hline T2_74_2 & 1 & 0.44429 \\
\hline T2_74_3 & 1 & 0.68606 \\
\hline T2_74_4 & 1 & 2.45259 \\
\hline T2_74_56 & 1 & 2.82531 \\
\hline T2_75_23 & 1 & 2.13903 \\
\hline T2_75_3 & 1 & 2.59537 \\
\hline T2_75_4 & 1 & 3.7023 \\
\hline T2_76_1 & 1 & 0.69787 \\
\hline T2_76_2 & 1 & 3.60888 \\
\hline T2_76_3 & 1 & 1.06729 \\
\hline T2_76_4 & 1 & 2.6905 \\
\hline T2_77_1 & 1 & 0.66195 \\
\hline T2_77_2 & 1 & 0.39437 \\
\hline T2_77_34 & 1 & 0.06246 \\
\hline T2_77_456 & 1 & 2.67508 \\
\hline T2_78_1 & 1 & 0.55995 \\
\hline T2_78_23 & 1 & 1.39152 \\
\hline T2_78_3 & 1 & 1.42903 \\
\hline T2_78_4 & 1 & 1.24847 \\
\hline T2_78_5 & 1 & -0.8183 \\
\hline T2_79_1 & 1 & 1.32483 \\
\hline T2_79_2 & 1 & -0.1912 \\
\hline T2_79_3 & 1 & 1.05564 \\
\hline T2_79_4 & 1 & 1.52975 \\
\hline T2_79_56 & 1 & 1.4272 \\
\hline
\end{tabular}




\begin{tabular}{llc} 
T2_79_6 & 1 & 2.33034 \\
T2_80_1 & 1 & -0.3427 \\
T2_80_2 & 1 & 1.5374 \\
T2_80_4 & 1 & 1.0381 \\
T2_80_5 & 1 & 1.49468 \\
T2_81_1 & 1 & 0.6816 \\
T2_81_2 & 1 & 0.76937 \\
T2_81_3 & 1 & 2.59537 \\
T2_81_456 & 1 & 4.11886 \\
T2_81_5 & 1 & 0.92507 \\
T2_82_1 & 1 & 0.89267 \\
T2_82_2 & 1 & 1.46462 \\
T2_82_3 & 1 & 1.60373 \\
T2_82_4 & 1 & 4.2642 \\
T2_82_5 & 1 & 4.48596 \\
T2_82_6 & 1 & 3.77744 \\
T2_83_1 & 1 & 0.62339 \\
T2_83_2 & 1 & 0.16305 \\
T2_83_3 & 1 & 2.6945 \\
T2_83_4 & 1 & 3.03105 \\
T2_83_6 & 1 & 1.14025 \\
T2_84_1 & 1 & 0.32775 \\
T2_84_23 & 1 & 1.32685 \\
T2_84_3 & 1 & 1.11149 \\
T2_84_4 & 1 & 2.52784 \\
T2_84_5 & 1 & 4.25762 \\
T2_85_1 & 1 & 2.35009 \\
T2_85_2 & 1 & -0.3006 \\
T2_85_3 & 1 & 2.36935 \\
T2_85_4 & 1 & 2.64276 \\
T2_85_5 & 1 & 2.32505 \\
\hline Tot_
\end{tabular}

Note. $\mathrm{a}=$ item discrimination, $\mathrm{b}=$ item difficulty 
Table 21

Task 3 Rasch model item parameters

\begin{tabular}{lcc}
\hline Item & $\mathrm{a}$ & $\mathrm{b}$ \\
\hline T3_1_2 & 1 & -1.0435 \\
T3_1_3 & 1 & 0.31377 \\
T3_1_5 & 1 & -0.9483 \\
T3_1_6 & 1 & -0.672 \\
T3_10_12 & 1 & 0.23693 \\
T3_10_2 & 1 & -0.9348 \\
T3_10_3 & 1 & 0.5502 \\
T3_10_4 & 1 & 0.02133 \\
T3_10_5 & 1 & 0.05449 \\
T3_10_6 & 1 & 0.83032 \\
T3_11_12 & 1 & -0.325 \\
T3_11_2 & 1 & -0.8228 \\
T3_11_34 & 1 & 1.27905 \\
T3_11_4 & 1 & -0.9938 \\
T3_11_5 & 1 & -1.4987 \\
T3_12_12 & 1 & 0.77096 \\
T3_12_2 & 1 & 0.3417 \\
T3_12_3 & 1 & -1.0903 \\
T3_12_4 & 1 & -0.4989 \\
T3_12_5 & 1 & -0.7496 \\
T3_13_23 & 1 & -0.8852 \\
T3_13_6 & 1 & -1.1724 \\
T3_14_1 & 1 & -0.467 \\
T3_14_3 & 1 & 0.40142 \\
T3_14_456 & 1 & -0.4668 \\
T3_14_5 & 1 & -0.1637 \\
T3_15_1 & 1 & 0.2266 \\
T3_15_34 & 1 & -0.1118 \\
T3_15_456 & 1 & 0.45244 \\
T3_15_5 & 1 & 0.02607 \\
T3_15_6 & 1 & 0.39387 \\
T3_16_1 & 1 & -0.4398 \\
T3_16_2 & 1 & -0.7992 \\
T3_16_3 & 1 & 0.34287 \\
T3_16_56 & 1 & -0.5454 \\
T3_17_1 & 1 & 0.2266 \\
T3_17_34 & 1 & -1.2826 \\
\end{tabular}




$\begin{array}{llc}\text { T3_17_5 } & 1 & 0.09571 \\ \text { T3_17_6 } & 1 & -0.672 \\ \text { T3_18_12 } & 1 & 0.18231 \\ \text { T3_18_2 } & 1 & -0.1477 \\ \text { T3_18_3 } & 1 & -0.9575 \\ \text { T3_18_4 } & 1 & -0.0253 \\ \text { T3_19_3 } & 1 & -0.7745 \\ \text { T3_19_4 } & 1 & -0.0877 \\ \text { T3_19_56 } & 1 & 0.98191 \\ \text { T3_19_6 } & 1 & -0.9486 \\ \text { T3_2_3 } & 1 & -0.4646 \\ \text { T3_2_4 } & 1 & 0.06794 \\ \text { T3_2_56 } & 1 & 0.4889 \\ \text { T3_2_56 } & 1 & 0.76332 \\ \text { T3_2_6 } & 1 & 0.07004 \\ \text { T3_20_12 } & 1 & 1.09473 \\ \text { T3_20_2 } & 1 & -0.8655 \\ \text { T3_20_456 } & 1 & -0.3024 \\ \text { T3_20_6 } & 1 & 0.07004 \\ \text { T3_21_1 } & 1 & 0.81117 \\ \text { T3_21_3 } & 1 & -1.5166 \\ \text { T3_21_4 } & 1 & 0.30123 \\ \text { T3_21_56 } & 1 & -0.4936 \\ \text { T3_21_6 } & 1 & 1.76067 \\ \text { T3_22_1 } & 1 & -1.1158 \\ \text { T3_22_2 } & 1 & 0.57168 \\ \text { T3_22_3 } & 1 & 1.35516 \\ \text { T3_22_4 } & 1 & 1.0536 \\ \text { T3_22_5 } & 1 & -0.3032 \\ \text { T3_22_6 } & 1 & -0.0021 \\ \text { T3_23_12 } & 1 & -0.7021 \\ \text { T3_23_2 } & 1 & 0.20864 \\ \text { T3_23_34 } & 1 & -0.4185 \\ \text { T3_23_4 } & 1 & -0.7338 \\ \text { T3_23_5 } & 1 & -0.2587 \\ \text { T3_23_6 } & 1 & -0.9799 \\ \text { T3_24_1 } & 1 & -0.3062 \\ \text { T3_24_3 } & 1 & 0.09075 \\ \text { T3_24_4 } & 1 & -0.8278 \\ \text { T3_24_5 } & 1 & -0.5451 \\ \text { T3_24_6 } & 1 & 1.23729 \\ \end{array}$




\begin{tabular}{|c|c|c|}
\hline T3_25_1 & 1 & 0.11704 \\
\hline T3_25_3 & 1 & -0.2113 \\
\hline T3_25_56 & 1 & 1.06767 \\
\hline T3_26_1 & 1 & -0.2267 \\
\hline T3_26_2 & 1 & -0.4205 \\
\hline T3_26_3 & 1 & 1.39415 \\
\hline T3_26_5 & 1 & 0.12414 \\
\hline T3_27_1 & 1 & 1.01619 \\
\hline T3_27_23 & 1 & 0.07127 \\
\hline T3_27_4 & 1 & 0.02133 \\
\hline T3_28_1 & 1 & -0.385 \\
\hline T3_28_34 & 1 & -0.1612 \\
\hline T3_28_4 & 1 & -1.0508 \\
\hline T3_28_5 & 1 & 0.96557 \\
\hline T3_29_12 & 1 & 1.11674 \\
\hline T3_29_23 & 1 & -0.182 \\
\hline T3_29_3 & 1 & 0 \\
\hline T3_29_4 & 1 & -0.0159 \\
\hline T3_29_5 & 1 & 0.99717 \\
\hline T3_3_1 & 1 & -0.7127 \\
\hline T3_3_456 & 1 & -0.0866 \\
\hline T3_30_1 & 1 & -0.6083 \\
\hline T3_30_23 & 1 & -0.3872 \\
\hline T3_30_3 & 1 & 0.14101 \\
\hline T3_30_6 & 1 & 0.5301 \\
\hline T3_31_1 & 1 & -0.8889 \\
\hline T3_31_3 & 1 & 1.0981 \\
\hline T3_31_4 & 1 & -0.7806 \\
\hline T3_31_56 & 1 & -0.4254 \\
\hline T3_32_1 & 1 & 0.14698 \\
\hline T3_32_2 & 1 & -0.418 \\
\hline T3_32_3 & 1 & 0.34287 \\
\hline T3_32_4 & 1 & 0.3876 \\
\hline T3_32_56 & 1 & -0.4424 \\
\hline T3_32_6 & 1 & 0.10575 \\
\hline T3_33_1 & 1 & -0.1541 \\
\hline T3_33_2 & 1 & -0.3784 \\
\hline T3_33_34 & 1 & -0.7822 \\
\hline T3_33_456 & 1 & 0.88605 \\
\hline T3_33_5 & 1 & 0.75003 \\
\hline T3_33_6 & 1 & 0.14124 \\
\hline
\end{tabular}




$\begin{array}{llc}\text { T3_34_1 } & 1 & -0.2526 \\ \text { T3_34_2 } & 1 & 0.3417 \\ \text { T3_34_4 } & 1 & -0.399 \\ \text { T3_34_5 } & 1 & -0.6672 \\ \text { T3_35_1 } & 1 & 0.11704 \\ \text { T3_35_4 } & 1 & -0.3003 \\ \text { T3_35_5 } & 1 & 1.162 \\ \text { T3_35_6 } & 1 & 0.22075 \\ \text { T3_36_1 } & 1 & -0.1446 \\ \text { T3_36_2 } & 1 & 0.66756 \\ \text { T3_36_3 } & 1 & 0.28228 \\ \text { T3_36_4 } & 1 & -1.0253 \\ \text { T3_36_5 } & 1 & 0.51802 \\ \text { T3_36_6 } & 1 & 0.66266 \\ \text { T3_37_1 } & 1 & 0.43762 \\ \text { T3_37_23 } & 1 & 0.73466 \\ \text { T3_37_3 } & 1 & -0.5505 \\ \text { T3_37_5 } & 1 & 0.17797 \\ \text { T3_37_6 } & 1 & 1.6228 \\ \text { T3_38_1 } & 1 & -0.0238 \\ \text { T3_38_2 } & 1 & -0.9417 \\ \text { T3_38_5 } & 1 & -0.1114 \\ \text { T3_39_1 } & 1 & 0.72071 \\ \text { T3_39_2 } & 1 & -0.2541 \\ \text { T3_39_3 } & 1 & 0.14101 \\ \text { T3_39_4 } & 1 & -0.0253 \\ \text { T3_39_56 } & 1 & -0.0901 \\ \text { T3_39_6 } & 1 & -0.7833 \\ \text { T3_4_1 } & 1 & -0.0035 \\ \text { T3_4_23 } & 1 & 0.79815 \\ \text { T3_4_3 } & 1 & -0.786 \\ \text { T3_4_5 } & 1 & -1.6686 \\ \text { T3_4_6 } & 1 & 0.69625 \\ \text { T3_40_456 } & 1 & -0.1957 \\ \text { T3_40_6 } & 1 & 0.35953 \\ \text { T3_41_2 } & 1 & 0.75152 \\ \text { T3_41_3 } & 1 & 0.09876 \\ \text { T3_41_4 } & 1 & 0.39009 \\ \text { T3_42_23 } & 1 & 0.39037 \\ \text { T3_42_34 } & 1 & 1.04393 \\ \text { T3_42_5 } & 1 & 0.38654 \\ \text { T2 } & & \end{array}$




$\begin{array}{lll}\text { T3_42_6 } & 1 & -0.9785 \\ \text { T3_43_23 } & 1 & 0.65907 \\ \text { T3_43_3 } & 1 & -0.7582 \\ \text { T3_43_4 } & 1 & -0.0576 \\ \text { T3_43_6 } & 1 & 1.27633 \\ \text { T3_44_1 } & 1 & -0.469 \\ \text { T3_44_3 } & 1 & 1.08561 \\ \text { T3_44_5 } & 1 & -0.8278 \\ \text { T3_44_6 } & 1 & 0.57437 \\ \text { T3_45_1 } & 1 & 0.12003 \\ \text { T3_45_5 } & 1 & 0.28615 \\ \text { T3_45_6 } & 1 & -0.1085 \\ \text { T3_46_1 } & 1 & 0.0546 \\ \text { T3_46_23 } & 1 & -0.8873 \\ \text { T3_46_3 } & 1 & -0.8635 \\ \text { T3_46_4 } & 1 & -0.9349 \\ \text { T3_47_1 } & 1 & 0.61594 \\ \text { T3_47_3 } & 1 & 0.61111 \\ \text { T3_47_4 } & 1 & 1.12844 \\ \text { T3_47_5 } & 1 & -0.3128 \\ \text { T3_47_6 } & 1 & -0.5557 \\ \text { T3_48_1 } & 1 & -0.8289 \\ \text { T3_48_2 } & 1 & 0.3254 \\ \text { T3_48_3 } & 1 & 1.02937 \\ \text { T3_48_4 } & 1 & 0.19663 \\ \text { T3_48_5 } & 1 & 0.12808 \\ \text { T3_48_6 } & 1 & -0.4783 \\ \text { T3_49_1 } & 1 & 0.14735 \\ \text { T3_49_2 } & 1 & 0.00696 \\ \text { T3_49_3 } & 1 & -0.3625 \\ \text { T3_49_4 } & 1 & -0.8742 \\ \text { T3_49_5 } & 1 & -1.3232 \\ \text { T3_5153_34 } & 1 & 0.59011 \\ \text { T3_5_4 } & 1 & 0.50613 \\ \text { T3_5_5 } & 1 & 0.12414 \\ \text { T3_50_1 } & 1 & 0.20951 \\ \text { T3_50_2 } & 1 & 0.88017 \\ \text { T35534 } & 1 & -0.1316 \\ & 1 & -0.5557 \\ \text { T3 } 31 & 1 & 0.65056 \\ \text { T3 } 34.037\end{array}$




\begin{tabular}{|c|c|c|}
\hline T3_51_56 & 1 & 1.0055 \\
\hline T3_51_6 & 1 & 1.19893 \\
\hline T3_52_3 & 1 & -0.2926 \\
\hline T3_52_4 & 1 & 0.02408 \\
\hline T3_52_5 & 1 & -0.3128 \\
\hline T3_52_6 & 1 & -0.2128 \\
\hline T3_53_2 & 1 & -0.2695 \\
\hline T3_53_3 & 1 & -0.6567 \\
\hline T3_53_4 & 1 & -1.2925 \\
\hline T3_53_6 & 1 & -0.0405 \\
\hline T3_54_1 & 1 & -0.2267 \\
\hline T3_54_2 & 1 & 0.3254 \\
\hline T3_54_3 & 1 & 0.9734 \\
\hline T3_55_1 & 1 & 0.31442 \\
\hline T3_55_4 & 1 & -0.0576 \\
\hline T3_55_5 & 1 & -0.6615 \\
\hline T3_55_6 & 1 & 1.56466 \\
\hline T3_56_1 & 1 & 0.76104 \\
\hline T3_56_2 & 1 & -0.3422 \\
\hline T3_56_4 & 1 & -0.6191 \\
\hline T3_56_5 & 1 & 0.28615 \\
\hline T3_56_6 & 1 & -0.7805 \\
\hline T3_57_1 & 1 & -0.0056 \\
\hline T3_57_2 & 1 & -0.1065 \\
\hline T3_57_3 & 1 & -0.3188 \\
\hline T3_57_4 & 1 & -0.4552 \\
\hline T3_57_5 & 1 & 0.38654 \\
\hline T3_57_6 & 1 & -1.2551 \\
\hline T3_58_1 & 1 & -0.3301 \\
\hline T3_58_2 & 1 & 0.01678 \\
\hline T3_58_3 & 1 & -0.4232 \\
\hline T3_58_4 & 1 & 0.21943 \\
\hline T3_58_5 & 1 & -0.0627 \\
\hline T3_59_4 & 1 & 0.2422 \\
\hline T3_59_5 & 1 & 1.02855 \\
\hline T3_59_6 & 1 & 0.5437 \\
\hline T3_6_2 & 1 & 0.16397 \\
\hline T3_6_34 & 1 & 1.33174 \\
\hline T3_6_5 & 1 & 1.44545 \\
\hline T3_60_2 & 1 & -0.4845 \\
\hline T3_60_3 & 1 & 0.80164 \\
\hline
\end{tabular}




\begin{tabular}{lll} 
T3_60_45 & 1 & -1.8169 \\
T3_60_56 & 1 & -0.9788 \\
T3_60_6 & 1 & 1.46801 \\
T3_61_1 & 1 & -0.0739 \\
T3_61_2 & 1 & -0.589 \\
T3_61_3 & 1 & -0.1574 \\
T3_61_4 & 1 & 1.11571 \\
T3_61_5 & 1 & 1.18538 \\
T3_61_6 & 1 & 1.6466 \\
T3_62_1 & 1 & 0.68267 \\
T3_62_5 & 1 & 1.69519 \\
T3_63_2 & 1 & -0.5035 \\
T3_63_5 & 1 & 0.15171 \\
T3_63_6 & 1 & 2.29092 \\
T3_64_12 & 1 & -0.3509 \\
T3_64_3 & 1 & 0.48234 \\
T3_64_4 & 1 & 1.33505 \\
T3_64_5 & 1 & -0.7715 \\
T3_64_6 & 1 & -0.2128 \\
T3_7_2 & 1 & 0.23148 \\
T3_7_4 & 1 & 0.86789 \\
T3_7_6 & 1 & 1.34038 \\
T3_8_1 & 1 & -0.0348 \\
T3_8_456 & 1 & 1.81893 \\
T3_8_5 & 1 & -0.2768 \\
T3_9_1 & 1 & -0.5554 \\
T3_9_2 & 1 & -0.2941 \\
T3_9_3 & 1 & 0 \\
T3_9_456 & 1 & 1.39649 \\
T3_9_5 & 1 & 0.37324 \\
\hline Tate.
\end{tabular}

Note. $\mathrm{a}=$ item discrimination, $\mathrm{b}=$ item difficulty 
Table 22

Task 4 Rasch model item parameters

\begin{tabular}{llc}
\hline Item & $\mathrm{a}$ & $\mathrm{b}$ \\
\hline T4_1_3 & 1 & -0.2883 \\
T4_1_456 & 1 & 0.58736 \\
T4_1_5 & 1 & -2.1536 \\
T4_10_3 & 1 & -1.5619 \\
T4_10_4 & 1 & -1.2242 \\
T4_11_3 & 1 & -2.4473 \\
T4_11_56 & 1 & 0.12851 \\
T4_11_6 & 1 & 0.41862 \\
T4_12_34 & 1 & -1.3755 \\
T4_12_4 & 1 & -1.5098 \\
T4_12_5 & 1 & 0.0076 \\
T4_12_6 & 1 & 0.80894 \\
T4_13_3 & 1 & -3.0524 \\
T4_13_4 & 1 & 0.33718 \\
T4_13_6 & 1 & -0.6401 \\
T4_14_3 & 1 & -2.4539 \\
T4_14_456 & 1 & 0.55863 \\
T4_14_56 & 1 & 0.69144 \\
T4_14_6 & 1 & 3.04586 \\
T4_15_3 & 1 & -0.176 \\
T4_15_456 & 1 & -0.2303 \\
T4_16_3 & 1 & -1.02 \\
T4_16_4 & 1 & -1.3126 \\
T4_16_5 & 1 & -0.1103 \\
T4_16_6 & 1 & -0.734 \\
T4_17_3 & 1 & -1.9811 \\
T4_17_4 & 1 & -1.6965 \\
T4_17_5 & 1 & -1.1064 \\
T4_17_6 & 1 & -0.2683 \\
T4_18_3 & 1 & -1.22 \\
T4_18_5 & 1 & 0.09585 \\
T4_18_6 & 1 & -0.4218 \\
T4_19_3 & 1 & 0.00352 \\
T4_19_5 & 1 & -0.3463 \\
T4_2_3 & 1 & -1.1844 \\
& 1 & -2.2749 \\
T4_5 & 1 & -1.0717 \\
\hline
\end{tabular}




$\begin{array}{llc}\text { T4_20_4 } & 1 & -1.9933 \\ \text { T4_20_5 } & 1 & -0.81 \\ \text { T4_20_6 } & 1 & 0.55326 \\ \text { T4_21_3 } & 1 & -0.2757 \\ \text { T4_21_4 } & 1 & 0.04778 \\ \text { T4_22_3 } & 1 & -2.3559 \\ \text { T4_22_456 } & 1 & -1.8421 \\ \text { T4_22_56 } & 1 & -0.617 \\ \text { T4_22_6 } & 1 & 0.80894 \\ \text { T4_23_34 } & 1 & -1.3671 \\ \text { T4_23_56 } & 1 & -0.5492 \\ \text { T4_24_3 } & 1 & -1.7333 \\ \text { T4_24_4 } & 1 & -2.2212 \\ \text { T4_24_6 } & 1 & 0.24041 \\ \text { T4_25_34 } & 1 & -0.4651 \\ \text { T4_25_4 } & 1 & -0.1081 \\ \text { T4_25_5 } & 1 & -1.5989 \\ \text { T4_26_3 } & 1 & -1.7968 \\ \text { T4_26_5 } & 1 & -2.8012 \\ \text { T4_27_4 } & 1 & -0.618 \\ \text { T4_27_6 } & 1 & 0.82401 \\ \text { T4_28_4 } & 1 & -1.119 \\ \text { T4_29_3 } & 1 & -2.3154 \\ \text { T4_29_34 } & 1 & -1.694 \\ \text { T4_29_5 } & 1 & -0.8138 \\ \text { T4_29_6 } & 1 & -1.6169 \\ \text { T4_3_3 } & 1 & -2.6619 \\ \text { T4_3_4 } & 1 & -0.6641 \\ \text { T4_3_5 } & 1 & -0.81 \\ \text { T4_30_3 } & 1 & -1.6856 \\ \text { T4_30_4 } & 1 & -1.5286 \\ \text { T4_30_5 } & 1 & -0.1756 \\ \text { T4_30_6 } & 1 & 1.91016 \\ \text { T4_31_4 } & 1 & -1.7533 \\ \text { T4_31_56 } & 1 & -1.7311 \\ \text { T4_31_6 } & 1 & 0.64176 \\ \text { T4_32_3 } & 1 & -3.0368 \\ \text { T4_32_4 } & 1 & 0.30704 \\ \end{array}$




$\begin{array}{lll}\text { T4_34_4 } & 1 & -1.8696 \\ \text { T4_34_6 } & 1 & -1.5826 \\ \text { T4_35_3 } & 1 & -2.0282 \\ \text { T4_35_6 } & 1 & -0.6097 \\ \text { T4_36_34 } & 1 & -1.4226 \\ \text { T4_36_6 } & 1 & 0.02213 \\ \text { T4_37_3 } & 1 & -0.0332 \\ \text { T4_37_4 } & 1 & -0.5901 \\ \text { T4_38_3 } & 1 & -2.2502 \\ \text { T4_38_45 } & 1 & -0.711 \\ \text { T4_38_6 } & 1 & -0.7848 \\ \text { T4_39_3 } & 1 & 0.5501 \\ \text { T4_39_6 } & 1 & 0.37238 \\ \text { T4_4_3 } & 1 & -2.7274 \\ \text { T4_4_5 } & 1 & -0.7657 \\ \text { T4_40_3 } & 1 & -1.1881 \\ \text { T4_41_3 } & 1 & -1.7856 \\ \text { T4_41_45 } & 1 & -2.1855 \\ \text { T4_42_3 } & 1 & -0.6423 \\ \text { T4_43_34 } & 1 & 0.22692 \\ \text { T4_43_5 } & 1 & -1.1163 \\ \text { T4_43_6 } & 1 & -1.2969 \\ \text { T4_45_56 } & 1 & -1.2823 \\ \text { T4_46_56 } & 1 & -1.7953 \\ \text { T4_47_5 } & 1 & -1.2623 \\ \text { T4_48_5 } & 1 & 0.45047 \\ \text { T4_49_5 } & 1 & -0.318 \\ \text { T4_5_3 } & 1 & -1.7779 \\ \text { T4_5_4 } & 1 & -1.1928 \\ \text { T4_7_4 } & 1 & -1.4114 \\ \text { T4_756 } & 1 & -0.2935 \\ \text { T4_5_6 } & 1 & -0.6984 \\ \text { T4_50_5 } & 1 & -2.0305 \\ \text { T4_51_5 } & 1 & -1.0858 \\ \text { T4_52_5 } & 1 & -2.378 \\ \text { T4_6_3 } & 1 & -1.7333 \\ \text { T4_6_456 } & 1 & -0.1292 \\ \text { T4_34 } & 1 & -1.5547 \\ \end{array}$




\begin{tabular}{lll} 
T4_8_34 & 1 & -2.6896 \\
T4_8_4 & 1 & -0.2651 \\
T4_8_6 & 1 & 0.13521 \\
T4_9_3 & 1 & -3.1598 \\
T4_9_4 & 1 & -2.1414 \\
T4_9_6 & 1 & 0.27507 \\
\hline
\end{tabular}

Note. $\mathrm{a}=$ item discrimination, $\mathrm{b}=$ item difficulty 
Table 23

Task 5 Rasch model item parameters

\begin{tabular}{lcc}
\hline Item & a & b \\
\hline T5_1_1 & 1 & -1.2578 \\
T5_1_4 & 1 & 1.68805 \\
T5_1_5 & 1 & 1.793 \\
T5_1_6 & 1 & 2.01513 \\
T5_10_12 & 1 & 2.99988 \\
T5_10_23 & 1 & 1.92229 \\
T5_10_34 & 1 & 0.70336 \\
T5_10_5 & 1 & 0.98158 \\
T5_10_6 & 1 & 4.05378 \\
T5_11_1 & 1 & -0.4613 \\
T5_11_23 & 1 & -1.0609 \\
T5_12_1 & 1 & 1.73034 \\
T5_12_2 & 1 & -0.3603 \\
T5_12_4 & 1 & 0.67497 \\
T5_12_56 & 1 & 2.10083 \\
T5_13_2 & 1 & 1.95805 \\
T5_13_4 & 1 & 1.01835 \\
T5_13_5 & 1 & -0.0317 \\
T5_13_6 & 1 & 0.11551 \\
T5_14_1 & 1 & 3.2515 \\
T5_14_2 & 1 & 3.10209 \\
T5_14_4 & 1 & -1.1296 \\
T5_15_2 & 1 & 2.54775 \\
T5_15_456 & 1 & 0.78137 \\
T5_15_5 & 1 & 2.2838 \\
T5_15_6 & 1 & 2.35398 \\
T5_16_1 & 1 & 2.89226 \\
T5_16_3 & 1 & 2.31043 \\
T5_16_4 & 1 & 0.13086 \\
T5_16_5 & 1 & 2.75841 \\
T5_17_34 & 1 & 0.68814 \\
T5_17_4 & 1 & 2.77091 \\
T5_17_5 & 1 & 2.21989 \\
T5_18_12 & 1 & 0.17159 \\
T5_18_2 & 1 & -1.0222 \\
T5_18_3 & 1 & 3.16489 \\
T5_18_5 & 1 & 2.84577
\end{tabular}




\begin{tabular}{|c|c|c|}
\hline T5_19_2 & 1 & -0.5939 \\
\hline T5_19_5 & 1 & 1.10562 \\
\hline T5_2_1 & 1 & 0.8945 \\
\hline T5_2_2 & 1 & -1.2173 \\
\hline T5_2_4 & 1 & -0.7065 \\
\hline T5_2_6 & 1 & 2.25438 \\
\hline T5_20_2 & 1 & 0.20945 \\
\hline T5_20_4 & 1 & 0.94419 \\
\hline T5_20_6 & 1 & 0.98079 \\
\hline T5_21_1 & 1 & -0.0773 \\
\hline T5_21_2 & 1 & 0.25064 \\
\hline T5_21_3 & 1 & -0.5295 \\
\hline T5_21_456 & 1 & 2.49516 \\
\hline T5_21_5 & 1 & 1.10562 \\
\hline T5_22_1 & 1 & -0.2777 \\
\hline T5_22_3 & 1 & 0.5558 \\
\hline T5_22_4 & 1 & -0.6843 \\
\hline T5_22_6 & 1 & 0.30806 \\
\hline T5_23_23 & 1 & 3.56187 \\
\hline T5_23_3 & 1 & 2.22093 \\
\hline T5_23_5 & 1 & 0.54875 \\
\hline T5_23_6 & 1 & -0.3052 \\
\hline T5_24_12 & 1 & 0.79286 \\
\hline T5_24_2 & 1 & -0.0056 \\
\hline T5_24_3 & 1 & 0.21678 \\
\hline T5_24_4 & 1 & 0.04361 \\
\hline T5_24_6 & 1 & 0.81496 \\
\hline T5_25_3 & 1 & 2.51415 \\
\hline T5_25_456 & 1 & 1.90342 \\
\hline T5_25_5 & 1 & 2.14157 \\
\hline T5_25_6 & 1 & 2.90791 \\
\hline T5_26_1 & 1 & 1.04584 \\
\hline T5_26_23 & 1 & -1.7826 \\
\hline T5_26_45 & 1 & -1.0944 \\
\hline T5_26_56 & 1 & -0.7738 \\
\hline T5_26_6 & 1 & 3.27201 \\
\hline T5_27_1 & 1 & 0.38137 \\
\hline T5_27_3 & 1 & -0.5304 \\
\hline T5_27_4 & 1 & 2.40179 \\
\hline T5_27_5 & 1 & -0.3809 \\
\hline T5_27_6 & 1 & 1.10901 \\
\hline
\end{tabular}




$\begin{array}{lcc}\text { T5_28_1 } & 1 & 0.40539 \\ \text { T5_28_2 } & 1 & 0.8431 \\ \text { T5_28_3 } & 1 & 1.94891 \\ \text { T5_28_4 } & 1 & -0.5042 \\ \text { T5_28_5 } & 1 & 0.90731 \\ \text { T5_29_1 } & 1 & 2.37879 \\ \text { T5_29_3 } & 1 & 1.77019 \\ \text { T5_3_1 } & 1 & -0.6256 \\ \text { T5_3_56 } & 1 & 0.2823 \\ \text { T5_30_12 } & 1 & 0.62719 \\ \text { T5_30_3 } & 1 & -0.4304 \\ \text { T5_30_4 } & 1 & 3.06635 \\ \text { T5_30_56 } & 1 & -0.0026 \\ \text { T5_31_34 } & 1 & 0.98787 \\ \text { T5_31_4 } & 1 & 1.41396 \\ \text { T5_31_5 } & 1 & 0.53611 \\ \text { T5_31_6 } & 1 & 1.51952 \\ \text { T5_32_12 } & 1 & 0.25696 \\ \text { T5_32_2 } & 1 & 3.75743 \\ \text { T5_32_3 } & 1 & -0.5763 \\ \text { T5_32_4 } & 1 & 2.7121 \\ \text { T5_32_5 } & 1 & 0.73544 \\ \text { T5_32_6 } & 1 & 3.42081 \\ \text { T5_33_1 } & 1 & -2.037 \\ \text { T5_33_2 } & 1 & -0.4737 \\ \text { T5_33_4 } & 1 & -0.969 \\ \text { T5_33_6 } & 1 & 1.99359 \\ \text { T5_34_1 } & 1 & 2.04684 \\ \text { T5_34_2 } & 1 & 1.99821 \\ \text { T5_34_34 } & 1 & -1.6126 \\ \text { T5_34_4 } & 1 & -0.0489 \\ \text { T5_34_5 } & 1 & 0.44174 \\ \text { T5_34_6 } & 1 & -1.6984 \\ \text { T5_35_2 } & 1 & 0.1061 \\ \text { T5_35_5 } & 1 & 3.43429 \\ \text { T5_36_1 } & 1 & 1.09331 \\ \text { T5_36_3 } & 1 & 2.30585 \\ \text { T5_36_45 } & 1 & -0.3815 \\ \text { T5_36_6 } & 1 & 2.31315 \\ \text { T5_37_1 } & 1 & -3.2195 \\ \text { T5_37_3 } & 1 & 0.41973\end{array}$




\begin{tabular}{|c|c|c|}
\hline T5_37_4 & 1 & 0.01931 \\
\hline T5_38_1 & 1 & -0.593 \\
\hline T5_38_2 & 1 & 3.14984 \\
\hline T5_38_4 & 1 & 0.67189 \\
\hline T5_38_6 & 1 & 1.28929 \\
\hline T5_39_1 & 1 & -0.364 \\
\hline T5_39_23 & 1 & 1.19727 \\
\hline T5_39_3 & 1 & 0.65232 \\
\hline T5_39_4 & 1 & 2.52952 \\
\hline T5_39_5 & 1 & 1.25564 \\
\hline T5_4_3 & 1 & 2.13557 \\
\hline T5_4_4 & 1 & 0.97817 \\
\hline T5_4_5 & 1 & 2.44628 \\
\hline T5_40_1 & 1 & 1.19521 \\
\hline T5_40_2 & 1 & -0.2743 \\
\hline T5_40_3 & 1 & -1.1518 \\
\hline T5_40_5 & 1 & 1.68861 \\
\hline T5_40_6 & 1 & 1.47672 \\
\hline T5_41_1 & 1 & 1.84451 \\
\hline T5_41_2 & 1 & -1.9508 \\
\hline T5_41_3 & 1 & -0.2088 \\
\hline T5_41_5 & 1 & 0.59962 \\
\hline T5_41_6 & 1 & 4.25696 \\
\hline T5_42_1 & 1 & -1.5064 \\
\hline T5_42_2 & 1 & 1.29141 \\
\hline T5_42_3 & 1 & 1.71335 \\
\hline T5_42_6 & 1 & 0.61519 \\
\hline T5_43_2 & 1 & 1.53127 \\
\hline T5_43_4 & 1 & -0.3801 \\
\hline T5_43_5 & 1 & 0.85747 \\
\hline T5_43_6 & 1 & 2.1243 \\
\hline T5_5_6 & 1 & 2.67443 \\
\hline T5_6_2 & 1 & 2.3439 \\
\hline T5_6_456 & 1 & 1.29628 \\
\hline T5_6_56 & 1 & 2.67977 \\
\hline T5_6_6 & 1 & -1.1245 \\
\hline T5_7_2 & 1 & -0.5339 \\
\hline T5_7_3 & 1 & 0.51197 \\
\hline T5_7_4 & 1 & -1.0451 \\
\hline T5_7_56 & 1 & 4.47808 \\
\hline T5_7_6 & 1 & 1.62736 \\
\hline
\end{tabular}




\begin{tabular}{lll} 
T5_8_3 & 1 & 0.8841 \\
T5_8_5 & 1 & 1.6457 \\
T5_9_1 & 1 & 0.73405 \\
T5_9_3 & 1 & 0.78661 \\
T5_9_4 & 1 & 1.62064 \\
T5_9_5 & 1 & 2.34974 \\
T5_9_6 & 1 & 1.49068 \\
\hline
\end{tabular}

Note. $\mathrm{a}=$ item discrimination, $\mathrm{b}=$ item difficulty 
Table 24

Task 6 Rasch model item parameters

\begin{tabular}{|c|c|c|}
\hline Item & $\mathrm{a}$ & $\mathrm{b}$ \\
\hline T6_1_1 & 1 & -0.826 \\
\hline T6_1_2 & 1 & 0.10417 \\
\hline T6_1_34 & 1 & -0.0461 \\
\hline T6_1_456 & 1 & -0.378 \\
\hline T6_1_56 & 1 & -0.3131 \\
\hline T6_1_6 & 1 & -0.5597 \\
\hline T6_10_2 & 1 & -0.1404 \\
\hline T6_10_34 & 1 & -0.3744 \\
\hline T6_10_4 & 1 & -3.7048 \\
\hline T6_10_5 & 1 & 0.08835 \\
\hline T6_10_6 & 1 & -1.4306 \\
\hline T6_11_1 & 1 & 0.72991 \\
\hline T6_11_3 & 1 & -0.6861 \\
\hline T6_11_4 & 1 & -1.2553 \\
\hline T6_11_5 & 1 & -0.2591 \\
\hline T6_11_6 & 1 & -0.32 \\
\hline T6_12_1 & 1 & -0.9947 \\
\hline T6_12_2 & 1 & -0.6569 \\
\hline T6_12_4 & 1 & -0.7959 \\
\hline T6_12_5 & 1 & -0.9295 \\
\hline T6_12_6 & 1 & -1.3609 \\
\hline T6_13_1 & 1 & -0.3803 \\
\hline T6_13_3 & 1 & -1.7014 \\
\hline T6_13_5 & 1 & -1.8379 \\
\hline T6_14_2 & 1 & 0.56041 \\
\hline T6_15_2 & 1 & -1.7811 \\
\hline T6_15_3 & 1 & 0.98014 \\
\hline T6_15_5 & 1 & -0.1676 \\
\hline T6_16_12 & 1 & -0.3049 \\
\hline T6_16_2 & 1 & -0.2245 \\
\hline T6_16_3 & 1 & 0.21501 \\
\hline T6_16_4 & 1 & -2.1211 \\
\hline T6_16_5 & 1 & -1.489 \\
\hline T6_16_6 & 1 & -0.4229 \\
\hline T6_17_1 & 1 & -0.5341 \\
\hline T6_17_23 & 1 & -0.215 \\
\hline T6_17_4 & 1 & -1.0908 \\
\hline
\end{tabular}




$\begin{array}{lll}\text { T6_17_5 } & 1 & -1.7682 \\ \text { T6_18_1 } & 1 & 0.83715 \\ \text { T6_18_2 } & 1 & -0.5901 \\ \text { T6_18_3 } & 1 & -0.6258 \\ \text { T6_18_4 } & 1 & -1.2241 \\ \text { T6_18_56 } & 1 & 1.56703 \\ \text { T6_19_12 } & 1 & -0.7769 \\ \text { T6_19_2 } & 1 & -0.2034 \\ \text { T6_19_3 } & 1 & -2.5385 \\ \text { T6_19_4 } & 1 & -0.0236 \\ \text { T6_19_5 } & 1 & 0.14412 \\ \text { T6_2_3 } & 1 & -1.049 \\ \text { T6_2_4 } & 1 & -0.4117 \\ \text { T6_2_56 } & 1 & -0.3417 \\ \text { T6_20_1 } & 1 & 0.91177 \\ \text { T6_20_23 } & 1 & 0.87406 \\ \text { T6_20_4 } & 1 & -1.1585 \\ \text { T6_20_5 } & 1 & 0.96984 \\ \text { T6_20_6 } & 1 & -0.4764 \\ \text { T6_21_12 } & 1 & 0.3752 \\ \text { T6_21_34 } & 1 & 1.49531 \\ \text { T6_21_4 } & 1 & 0.1607 \\ \text { T6_22_1 } & 1 & 0.76508 \\ \text { T6_22_2 } & 1 & 0.40773 \\ \text { T6_22_3 } & 1 & 0.17839 \\ \text { T6_22_456 } & 1 & -1.2372 \\ \text { T6_22_5 } & 1 & -0.5529 \\ \text { T6_22_6 } & 1 & 1.71247 \\ \text { T6_23_1 } & 1 & -0.5851 \\ \text { T6_23_2 } & 1 & 1.01075 \\ \text { T6_23_3 } & 1 & -1.1119 \\ \text { T6_23_4 } & 1 & 0.26316 \\ \text { T6_23_6 } & 1 & -0.3708 \\ \text { T6_24_1 } & 1 & -1.1257 \\ \text { T6_24_34 } & 1 & -0.5005 \\ \text { T6_24_4 } & 1 & 0.27904 \\ \text { T6_24_5 } & 1 & 0.11632 \\ \text { T6_24_6 } & 1 & 2.42356 \\ \text { T6_25_1 } & 1 & 0.11607 \\ \text { T6_25_4 } & 1 & -1.5825 \\ \text { T6_25_6 } & 1 & -0.9127 \\ \end{array}$




$\begin{array}{lll}\text { T6_26_12 } & 1 & 0.45373 \\ \text { T6_26_2 } & 1 & -2.0445 \\ \text { T6_26_3 } & 1 & -0.5301 \\ \text { T6_26_4 } & 1 & 1.32826 \\ \text { T6_26_5 } & 1 & -0.7039 \\ \text { T6_27_1 } & 1 & 1.02354 \\ \text { T6_27_3 } & 1 & -0.6486 \\ \text { T6_27_45 } & 1 & 0.52765 \\ \text { T6_27_5 } & 1 & -0.0777 \\ \text { T6_27_6 } & 1 & -1.5097 \\ \text { T6_28_1 } & 1 & 0.26847 \\ \text { T6_28_2 } & 1 & -0.1116 \\ \text { T6_28_3 } & 1 & 0.37638 \\ \text { T6_28_45 } & 1 & 0.77817 \\ \text { T6_28_56 } & 1 & -1.7137 \\ \text { T6_28_6 } & 1 & -0.3627 \\ \text { T6_29_1 } & 1 & -0.2848 \\ \text { T6_29_3 } & 1 & 0.73635 \\ \text { T6_29_4 } & 1 & 0.81869 \\ \text { T6_29_5 } & 1 & 1.82179 \\ \text { T6_3_1 } & 1 & 1.25036 \\ \text { T6_3_2 } & 1 & 1.69622 \\ \text { T6_3_3 } & 1 & 0.55144 \\ \text { T6_3_4 } & 1 & -0.6944 \\ \text { T6_3_5 } & 1 & -0.8486 \\ \text { T6_30_1 } & 1 & -0.4726 \\ \text { T6_30_3 } & 1 & 0.68319 \\ \text { T6_30_5 } & 1 & -0.6035 \\ \text { T6_30_6 } & 1 & -1.0573 \\ \text { T6_31_1 } & 1 & -0.1706 \\ \text { T6_31_2 } & 1 & 0.55433 \\ \text { T6_31_4 } & 1 & 0.38047 \\ \text { T6_31_5 } & 1 & -1.1093 \\ \text { T6_31_6 } & 1 & -3.5715 \\ \text { T6_32_1 } & 1 & 1.43431 \\ \text { T6_32_3 } & 1 & -0.6877 \\ \text { T6_32_56 } & 1 & 0.24907 \\ \text { T6_33_3 } & 1 & -0.9863 \\ \text { T6_33_4 } & 1 & 0.49563 \\ \text { T6_33_5 } & 1 & -1.1093 \\ \text { T6_34_1 } & 1 & -0.7239\end{array}$




$\begin{array}{llc}\text { T6_34_3 } & 1 & -0.9115 \\ \text { T6_34_4 } & 1 & 0.26335 \\ \text { T6_34_5 } & 1 & 0.02533 \\ \text { T6_34_6 } & 1 & -0.0266 \\ \text { T6_35_1 } & 1 & -1.118 \\ \text { T6_35_2 } & 1 & 0.11673 \\ \text { T6_35_4 } & 1 & 0.48416 \\ \text { T6_35_5 } & 1 & 0.47428 \\ \text { T6_35_6 } & 1 & -0.404 \\ \text { T6_36_1 } & 1 & -0.1551 \\ \text { T6_36_23 } & 1 & 0.54537 \\ \text { T6_36_3 } & 1 & -0.4547 \\ \text { T6_36_4 } & 1 & 0.29878 \\ \text { T6_36_5 } & 1 & -1.4057 \\ \text { T6_36_6 } & 1 & 0.62182 \\ \text { T6_37_1 } & 1 & 0.18314 \\ \text { T6_37_3 } & 1 & 0.53523 \\ \text { T6_37_5 } & 1 & 1.11281 \\ \text { T6_38_2 } & 1 & 0.8782 \\ \text { T6_38_4 } & 1 & 1.26039 \\ \text { T6_38_5 } & 1 & -0.0456 \\ \text { T6_38_6 } & 1 & -0.7641 \\ \text { T6_39_1 } & 1 & -0.569 \\ \text { T6_39_2 } & 1 & -1.493 \\ \text { T6_39_3 } & 1 & 1.00155 \\ \text { T6_4_2 } & 1 & -1.7151 \\ \text { T6_4_3 } & 1 & -1.4329 \\ \text { T6_4_456 } & 1 & 0.72642 \\ \text { T6_40_1 } & 1 & 0.68037 \\ \text { T6_40_34 } & 1 & -0.8256 \\ \text { T6_40_4 } & 1 & -1.1651 \\ \text { T6_40_6 } & 1 & -1.5097 \\ \text { T6_41_12 } & 1 & 0.12259 \\ \text { T6_41_2 } & 1 & -1.2833 \\ \text { T6_41_6 } & 1 & 1.37532 \\ \text { T6_42_1 } & 1 & 0.82813 \\ \text { T6_42_23 } & 1 & 0.26822 \\ \text { T6_42_4 } & 1 & -2.4886 \\ \text { T6_42_6 } & 1 & 0.36032 \\ \text { T6_43_1 } & 1 & -0.5986 \\ \text { T6_43_2 } & 1 & -0.8105\end{array}$




$\begin{array}{llc}\text { T6_43_34 } & 1 & 0.13624 \\ \text { T6_43_4 } & 1 & -0.7767 \\ \text { T6_43_5 } & 1 & -2.162 \\ \text { T6_43_6 } & 1 & 0.14784 \\ \text { T6_44_2 } & 1 & 0.22023 \\ \text { T6_44_3 } & 1 & -0.7673 \\ \text { T6_44_4 } & 1 & 1.73352 \\ \text { T6_44_6 } & 1 & 2.83826 \\ \text { T6_45_1 } & 1 & 0.55031 \\ \text { T6_45_2 } & 1 & -0.7298 \\ \text { T6_45_3 } & 1 & 1.45073 \\ \text { T6_45_4 } & 1 & -0.8521 \\ \text { T6_45_56 } & 1 & 0.09281 \\ \text { T6_45_6 } & 1 & -0.8424 \\ \text { T6_46_1 } & 1 & 0.23406 \\ \text { T6_46_2 } & 1 & -0.707 \\ \text { T6_46_4 } & 1 & -1.2011 \\ \text { T6_46_5 } & 1 & -0.1632 \\ \text { T6_46_6 } & 1 & -1.1826 \\ \text { T6_47_1 } & 1 & -0.6944 \\ \text { T6_47_2 } & 1 & -0.1056 \\ \text { T6_47_3 } & 1 & 0.03145 \\ \text { T6_47_6 } & 1 & 0.8283 \\ \text { T6_48_1 } & 1 & 0.60864 \\ \text { T6_48_4 } & 1 & 0.13185 \\ \text { T6_48_6 } & 1 & -1.4304 \\ \text { T6_49_2 } & 1 & -0.3127 \\ \text { T6_49_4 } & 1 & -1.2751 \\ \text { T6_49_5 } & 1 & 0.06927 \\ \text { T6_5_1 } & 1 & -0.5341 \\ \text { T6_5_2 } & 1 & 0.40773 \\ \text { T6_5_34 } & 1 & 1.04932 \\ \text { T6_5_4 } & 1 & -1.0517 \\ \text { T6_5_6 } & 1 & -0.5276 \\ \text { T6_6_1 } & 1 & -0.3803 \\ \text { T6_6_56 } & 1 & 0.03556 \\ \text { T6_6_6 } & 1 & -0.3327 \\ \text { T6_7_1 } & 1 & 0.89611 \\ \text { T6_7_5 } & 1 & -0.9295 \\ \text { T6_7_6 } & 1 & 1.62963 \\ \text { T6_8_1 } & 1 & 0.56668\end{array}$




\begin{tabular}{lll} 
T6_8_2 & 1 & -1.4271 \\
T6_8_3 & 1 & -1.1547 \\
T6_8_456 & 1 & -2.2734 \\
T6_8_6 & 1 & -0.32 \\
T6_9_1 & 1 & -0.01 \\
T6_9_3 & 1 & -0.4294 \\
T6_9_5 & 1 & -0.6942 \\
T6_9_6 & 1 & 1.71247 \\
\hline
\end{tabular}

Note. $\mathrm{a}=\mathrm{item}$ discrimination, $\mathrm{b}=$ item difficulty 
Table 25

Task 7 Rasch model item parameters

\begin{tabular}{|c|c|c|}
\hline Item & $\mathrm{a}$ & $\mathrm{b}$ \\
\hline T7_1_1 & 1 & 0.87475 \\
\hline T7_1_56 & 1 & 1.84209 \\
\hline T7_10_3 & 1 & -2.7437 \\
\hline T7_10_4 & 1 & 0.00561 \\
\hline T7_10_5 & 1 & -1.1081 \\
\hline T7_10_6 & 1 & -0.2969 \\
\hline T7_11_2 & 1 & -1.7978 \\
\hline T7_11_3 & 1 & -1.5275 \\
\hline T7_11_4 & 1 & 1.88106 \\
\hline T7_11_5 & 1 & -0.2544 \\
\hline T7_12_1 & 1 & -0.9509 \\
\hline T7_12_23 & 1 & 1.54757 \\
\hline T7_12_5 & 1 & 2.49427 \\
\hline T7_13_1 & 1 & -2.8602 \\
\hline T7_13_2 & 1 & -1.4418 \\
\hline T7_13_4 & 1 & -3.2539 \\
\hline T7_13_5 & 1 & -1.69 \\
\hline T7_14_1 & 1 & -1.3804 \\
\hline T7_14_3 & 1 & -0.4921 \\
\hline T7_14_4 & 1 & -1.8537 \\
\hline T7_14_6 & 1 & -1.7668 \\
\hline T7_15_1 & 1 & -1.5187 \\
\hline T7_15_23 & 1 & 0.95548 \\
\hline T7_15_23 & 1 & 0.95548 \\
\hline T7_15_34 & 1 & 1.80041 \\
\hline T7_15_34 & 1 & 1.80041 \\
\hline T7_15_456 & 1 & 0.97534 \\
\hline T7_15_56 & 1 & -2.809 \\
\hline T7_15_6 & 1 & 0.08484 \\
\hline T7_16_1 & 1 & 0.58341 \\
\hline T7_16_2 & 1 & 5.1372 \\
\hline T7_16_45 & 1 & -3.4193 \\
\hline T7_17_1 & 1 & 3.66475 \\
\hline T7_17_23 & 1 & -2.8475 \\
\hline T7_17_3 & 1 & -2.5641 \\
\hline T7_17_4 & 1 & -3.5697 \\
\hline T7_17_56 & 1 & 4.22391 \\
\hline
\end{tabular}




\begin{tabular}{|c|c|c|}
\hline T7_17_6 & 1 & -1.0953 \\
\hline T7_18_1 & 1 & 1.81321 \\
\hline T7_18_4 & 1 & 0.09202 \\
\hline T7_18_5 & 1 & 0.02691 \\
\hline T7_19_1 & 1 & -0.553 \\
\hline T7_19_2 & 1 & -1.8506 \\
\hline T7_19_4 & 1 & -0.2506 \\
\hline T7_19_5 & 1 & -0.4759 \\
\hline T7_2_1 & 1 & -0.5425 \\
\hline T7_2_2 & 1 & 1.00586 \\
\hline T7_2_34 & 1 & 2.91215 \\
\hline T7_2_4 & 1 & 0.07792 \\
\hline T7_2_5 & 1 & 1.56373 \\
\hline T7_2_6 & 1 & 0.0772 \\
\hline T7_20_1 & 1 & -0.5014 \\
\hline T7_20_2 & 1 & -2.3411 \\
\hline T7_20_3 & 1 & -2.0534 \\
\hline T7_20_4 & 1 & 3.02256 \\
\hline T7_20_5 & 1 & -1.751 \\
\hline T7_21_1 & 1 & 0.07078 \\
\hline T7_21_2 & 1 & 0.53169 \\
\hline T7_21_4 & 1 & -4.0916 \\
\hline T7_21_6 & 1 & -1.4019 \\
\hline T7_22_12 & 1 & -1.3009 \\
\hline T7_22_2 & 1 & -3.2898 \\
\hline T7_22_3 & 1 & -0.3898 \\
\hline T7_22_6 & 1 & -0.0222 \\
\hline T7_23_5 & 1 & -0.2833 \\
\hline T7_24_2 & 1 & 2.49469 \\
\hline T7_24_3 & 1 & 1.59997 \\
\hline T7_24_4 & 1 & 1.99654 \\
\hline T7_24_5 & 1 & 0.66986 \\
\hline T7_24_6 & 1 & 0.32782 \\
\hline T7_25_1 & 1 & -1.3456 \\
\hline T7_25_5 & 1 & -1.8413 \\
\hline T7_25_6 & 1 & 0.56811 \\
\hline T7_26_1 & 1 & -0.2791 \\
\hline T7_26_23 & 1 & -2.4432 \\
\hline T7_26_4 & 1 & -1.1946 \\
\hline T7_26_5 & 1 & 1.60826 \\
\hline T7_27_1 & 1 & -1.9454 \\
\hline
\end{tabular}




\begin{tabular}{|c|c|c|}
\hline T7_27_2 & 1 & -0.5276 \\
\hline T7_27_3 & 1 & -0.7684 \\
\hline T7_27_4 & 1 & -3.4532 \\
\hline T7_27_6 & 1 & -0.7359 \\
\hline T7_28_1 & 1 & -2.2611 \\
\hline T7_28_2 & 1 & 0.89116 \\
\hline T7_28_3 & 1 & -1.6242 \\
\hline T7_28_6 & 1 & 0.97178 \\
\hline T7_29_12 & 1 & 1.30084 \\
\hline T7_29_34 & 1 & -1.9953 \\
\hline T7_29_6 & 1 & -2.472 \\
\hline T7_3_1 & 1 & 2.90477 \\
\hline T7_3_3 & 1 & 4.28917 \\
\hline T7_3_5 & 1 & 3.79137 \\
\hline T7_3_6 & 1 & 2.82243 \\
\hline T7_30_1 & 1 & -0.5014 \\
\hline T7_30_3 & 1 & 1.45501 \\
\hline T7_30_5 & 1 & 0.257 \\
\hline T7_30_6 & 1 & -0.8754 \\
\hline T7_31_1 & 1 & -2.5496 \\
\hline T7_31_34 & 1 & -0.8222 \\
\hline T7_31_5 & 1 & -0.453 \\
\hline T7_31_6 & 1 & -0.3647 \\
\hline T7_32_2 & 1 & -1.3865 \\
\hline T7_32_3 & 1 & 3.58366 \\
\hline T7_32_4 & 1 & 3.33922 \\
\hline T7_32_5 & 1 & -0.044 \\
\hline T7_32_6 & 1 & -1.6849 \\
\hline T7_33_1 & 1 & -2.3158 \\
\hline T7_33_2 & 1 & -1.0256 \\
\hline T7_33_3 & 1 & -2.1321 \\
\hline T7_4_1 & 1 & 5.00093 \\
\hline T7_4_23 & 1 & 2.33941 \\
\hline T7_4_3 & 1 & -1.469 \\
\hline T7_4_4 & 1 & 2.91766 \\
\hline T7_4_5 & 1 & 0.10684 \\
\hline T7_4_6 & 1 & -0.1971 \\
\hline T7_5_1 & 1 & -1.7687 \\
\hline T7_5_2 & 1 & 0.73032 \\
\hline T7_5_3 & 1 & 1.21994 \\
\hline T7_5_4 & 1 & -1.82 \\
\hline
\end{tabular}




\begin{tabular}{lll} 
T7_6_12 & 1 & 3.23101 \\
T7_6_2 & 1 & -2.7067 \\
T7_6_3 & 1 & 2.42831 \\
T7_6_4 & 1 & 0.31916 \\
T7_7_1 & 1 & 0.31601 \\
T7_72 & 1 & 1.02804 \\
T7_7_4 & 1 & -1.1192 \\
T7_75 & 1 & 4.12467 \\
T7_7_6 & 1 & 3.39666 \\
T7_8_12 & 1 & 1.63188 \\
T7_8_2 & 1 & -2.1778 \\
T7_8_3 & 1 & 0.4903 \\
T7_8_6 & 1 & 1.77678 \\
T7_9_1 & 1 & 2.22174 \\
T7_9_2 & 1 & -2.4708 \\
T7_9_34 & 1 & 1.25276 \\
T7_9_34 & 1 & 1.25276 \\
T7_9_456 & 1 & -1.7333 \\
T7_9_5 & 1 & -1.1943 \\
T7_9_6 & 1 & 1.32752 \\
\hline
\end{tabular}

Note. $\mathrm{a}=$ item discrimination, $\mathrm{b}=$ item difficulty 
Table 26

Task 8 Rasch model item parameters

\begin{tabular}{llc}
\hline Item & a & b \\
\hline T8_1_4 & 1 & 0.4904902 \\
T8_1_5 & 1 & 2.2597594 \\
T8_1_6 & 1 & 6.029227 \\
T8_10_4 & 1 & 2.2928346 \\
T8_10_5 & 1 & 1.1309922 \\
T8_10_6 & 1 & -1.0310562 \\
T8_11_3 & 1 & 1.5954088 \\
T8_11_5 & 1 & 2.2597594 \\
T8_13_3 & 1 & 1.704317 \\
T8_13_456 & 1 & -1.7091784 \\
T8_13_5 & 1 & 2.7080697 \\
T8_14_3 & 1 & -1.3445502 \\
T8_14_5 & 1 & -0.6853117 \\
T8_15_3 & 1 & 0.3676555 \\
T8_15_4 & 1 & -0.203495 \\
T8_15_5 & 1 & 2.7762404 \\
T8_15_6 & 1 & -0.5704266 \\
T8_16_34 & 1 & 1.4877083 \\
T8_16_4 & 1 & 1.5067139 \\
T8_17_3 & 1 & -0.737473 \\
T8_17_6 & 1 & 0.5765528 \\
T8_18_34 & 1 & 0.3093492 \\
T8_18_5 & 1 & 2.5129547 \\
T8_19_3 & 1 & 0.1538021 \\
T8_19_5 & 1 & 0.7988559 \\
T8_19_6 & 1 & 1.3315698 \\
T8_2_4 & 1 & 0.5197879 \\
T8_2_5 & 1 & 3.2834606 \\
T8_2_6 & 1 & 3.8858431 \\
T8_20_34 & 1 & -0.340116 \\
T8_20_456 & 1 & -0.1427499 \\
T8_20_5 & 1 & 0.6849993 \\
T8_21_34 & 1 & -1.4662177 \\
T8_21_4 & 1 & -0.7918268 \\
T8_223 & 1 & 1.4070831 \\
T8_6 & 1 & 1.281905 \\
T8 & 1 & 4.3471762 \\
\hline
\end{tabular}




$\begin{array}{llc}\text { T8_23_3 } & 1 & -0.0765295 \\ \text { T8_23_4 } & 1 & 1.1712234 \\ \text { T8_23_5 } & 1 & -0.2561326 \\ \text { T8_23_6 } & 1 & -1.0310562 \\ \text { T8_24_3 } & 1 & 0.7573266 \\ \text { T8_24_456 } & 1 & -0.0189005 \\ \text { T8_24_6 } & 1 & 0.6158123 \\ \text { T8_25_3 } & 1 & -0.737473 \\ \text { T8_25_456 } & 1 & 4.0485955 \\ \text { T8_25_5 } & 1 & -1.0476005 \\ \text { T8_26_3 } & 1 & -3.1559243 \\ \text { T8_26_4 } & 1 & -2.586426 \\ \text { T8_26_5 } & 1 & 3.9311429 \\ \text { T8_27_3 } & 1 & 1.6261383 \\ \text { T8_27_4 } & 1 & -3.1164032 \\ \text { T8_27_56 } & 1 & 1.7968769 \\ \text { T8_28_3 } & 1 & -0.7708413 \\ \text { T8_28_6 } & 1 & 1.9514409 \\ \text { T8_29_3 } & 1 & -2.7663578 \\ \text { T8_29_4 } & 1 & 1.3349741 \\ \text { T8_29_5 } & 1 & 0.074408 \\ \text { T8_29_6 } & 1 & 4.6315134 \\ \text { T8_3_4 } & 1 & 1.1434102 \\ \text { T8_30_3 } & 1 & 0.1823708 \\ \text { T8_30_6 } & 1 & -0.2711124 \\ \text { T8_31_45 } & 1 & 0.6277822 \\ \text { T8_31_5 } & 1 & -0.1852504 \\ \text { T8_31_6 } & 1 & 2.7265738 \\ \text { T8_32_3 } & 1 & -1.2281413 \\ \text { T8_32_4 } & 1 & 0.5229873 \\ \text { T8_32_6 } & 1 & -0.6391653 \\ \text { T8_33_3 } & 1 & 1.4726143 \\ \text { T8_33_4 } & 1 & 2.4343965 \\ \text { T8_33_5 } & 1 & 0.3980395 \\ \text { T8_34_3 } & 1 & -0.8459867 \\ \text { T8_34_4 } & 1 & -0.026547 \\ \text { T8_34_6 } & 1 & 5.1385733 \\ \text { T8_35_4 } & 1 & 1.0772144 \\ \text { T8_3 } & 1 & -1.0140907 \\ \text { T86 } & 1 & 2.120651 \\ \text { T8 } & 1 & -1.9389986\end{array}$




$\begin{array}{llc}\text { T8_36_5 } & 1 & 0.8435032 \\ \text { T8_36_6 } & 1 & 0.069691 \\ \text { T8_37_34 } & 1 & -0.4921632 \\ \text { T8_37_6 } & 1 & 1.2754213 \\ \text { T8_38_4 } & 1 & -1.1559426 \\ \text { T8_38_5 } & 1 & 1.746311 \\ \text { T8_38_6 } & 1 & 1.6539919 \\ \text { T8_39_56 } & 1 & -0.173702 \\ \text { T8_39_6 } & 1 & -1.6379441 \\ \text { T8_4_3 } & 1 & 1.4070831 \\ \text { T8_4_56 } & 1 & 3.1091566 \\ \text { T8_40_3 } & 1 & -0.8459867 \\ \text { T8_40_5 } & 1 & 0.3201082 \\ \text { T8_40_6 } & 1 & 1.5616442 \\ \text { T8_41_4 } & 1 & 0.3527305 \\ \text { T8_41_5 } & 1 & -1.0140907 \\ \text { T8_42_3 } & 1 & -0.8890642 \\ \text { T8_42_5 } & 1 & -0.8992375 \\ \text { T8_42_6 } & 1 & 1.0500342 \\ \text { T8_43_3 } & 1 & -0.9214495 \\ \text { T8_43_45 } & 1 & -0.0224037 \\ \text { T8_43_56 } & 1 & 1.5512495 \\ \text { T8_43_6 } & 1 & 3.1640352 \\ \text { T8_44_3 } & 1 & -0.0292716 \\ \text { T8_44_5 } & 1 & 4.0944967 \\ \text { T8_44_6 } & 1 & -0.7012798 \\ \text { T8_45_3 } & 1 & -0.8137475 \\ \text { T8_45_4 } & 1 & -0.3476586 \\ \text { T8_45_5 } & 1 & 0.1260995 \\ \text { T8_46_34 } & 1 & -1.6082697 \\ \text { T8_46_4 } & 1 & -1.0966882 \\ \text { T8_46_5 } & 1 & -0.3431061 \\ \text { T8_46_6 } & 1 & 0.7090312 \\ \text { T8_47_3 } & 1 & -0.9430801 \\ \text { T8_47_4 } & 1 & -0.0276708 \\ \text { T8_48_3 } & 1 & 1.455891 \\ \text { T8_48_4 } & 1 & 2.4647076 \\ \text { T8_48_5 } & 1 & 0.3201082 \\ \text { T8_3 } & 1 & -1.2504366 \\ \text { T8 } & 1 & 2.1599743 \\ \text { T8 } & & 1.6436804\end{array}$




\begin{tabular}{llc} 
T8_49_6 & 1 & 2.4144632 \\
T8_5_3 & 1 & 0.6625793 \\
T8_5_4 & 1 & 3.2074819 \\
T8_5_56 & 1 & 1.5531612 \\
T8_5_6 & 1 & -0.2155743 \\
T8_6_3 & 1 & -1.3445502 \\
T8_6_4 & 1 & -0.8622252 \\
T8_6_5 & 1 & -2.3942523 \\
T8_7_4 & 1 & 2.4445985 \\
T8_7_5 & 1 & 2.0358949 \\
T8_7_6 & 1 & 3.6645676 \\
T8_8_3 & 1 & 2.6859605 \\
T8_8_4 & 1 & -1.5057799 \\
T8_8_56 & 1 & -0.556179 \\
T8_9_3 & 1 & -1.2437147 \\
T8_9_456 & 1 & 3.157444 \\
T8_9_6 & 1 & 4.0179161 \\
\hline
\end{tabular}

Note. $\mathrm{a}=$ item discrimination, $\mathrm{b}=$ item difficulty 
Table 27

Task-based marginal reliability by grade

\begin{tabular}{ccccccccc}
\hline Grade & Task 1 & Task 2 & Task 3 & Task 4 & Task 5 & Task 6 & Task 7 & Task 8 \\
\hline 1 & 0.78 & 0.80 & 0.82 & - & 0.89 & 0.84 & 0.86 & - \\
2 & 0.76 & 0.88 & 0.89 & - & 0.94 & 0.89 & 0.82 & - \\
3 & 0.72 & 0.89 & 0.92 & 0.91 & 0.93 & 0.90 & 0.84 & 0.95 \\
4 & 0.77 & 0.90 & 0.93 & 0.91 & 0.95 & 0.90 & 0.82 & 0.94 \\
5 & 0.76 & 0.92 & 0.93 & 0.90 & 0.93 & 0.85 & 0.85 & 0.93 \\
6 & 0.67 & 0.92 & 0.94 & 0.89 & 0.92 & 0.87 & 0.88 & 0.93 \\
\hline
\end{tabular}


Table 28

Task-based vertical scale by task and grade

\begin{tabular}{ccccccccc}
\hline Grade & Task 1 & Task 2 & Task 3 & Task 4 & Task 5 & Task 6 & Task 7 & Task 8 \\
\hline 1 & -0.57 & $\mathbf{- 1 . 9 6}$ & $\mathbf{- 1 . 0 5}$ & & $\mathbf{- 2 . 8 0}$ & $\mathbf{- 0 . 7 1}$ & $\mathbf{- 0 . 4 7}$ & \\
2 & -0.08 & $\mathbf{- 0 . 8 0}$ & $\mathbf{- 0 . 4 3}$ & & $\mathbf{- 1 . 1 7}$ & $\mathbf{0 . 0 0}$ & $\mathbf{0 . 0 0}$ & \\
3 & -0.48 & $\mathbf{0 . 0 0}$ & $\mathbf{0 . 0 0}$ & $\mathbf{- 0 . 7 6}$ & $\mathbf{- 0 . 9 8}$ & $\mathbf{0 . 4 4}$ & $\mathbf{0 . 3 5}$ & $\mathbf{- 1 . 2 3}$ \\
4 & -0.40 & $\mathbf{0 . 5 9}$ & $\mathbf{0 . 3 3}$ & $\mathbf{- 0 . 0 6}$ & $\mathbf{- 0 . 8 7}$ & $\mathbf{0 . 7 2}$ & $\mathbf{0 . 4 3}$ & $\mathbf{- 0 . 4 7}$ \\
5 & 0.00 & $\mathbf{1 . 0 7}$ & $\mathbf{0 . 3 5}$ & $\mathbf{0 . 0 0}$ & $\mathbf{- 0 . 4 6}$ & $\mathbf{0 . 7 7}$ & -0.02 & $\mathbf{0 . 0 0}$ \\
6 & 0.03 & $\mathbf{1 . 3 9}$ & $\mathbf{0 . 7 6}$ & $\mathbf{0 . 7 6}$ & $\mathbf{0 . 0 0}$ & $\mathbf{1 . 2 6}$ & 0.38 & $\mathbf{0 . 5 2}$ \\
\hline
\end{tabular}

Note. $0.00=$ location point for each task; values are scaled theta scores. Bold values reflect final selected grades for tasks. 
Table 29

Task 1 DIF: Male vs. female

\begin{tabular}{|c|c|c|c|}
\hline Item & ChiSq & pvalue & EffectSize \\
\hline T1_1_1 & 0.000 & 0.991 & -0.005 \\
\hline T1_2_12 & 0.000 & 0.996 & 0.004 \\
\hline T1_3_1 & 0.003 & 0.954 & 0.003 \\
\hline T1_4_1 & 0.000 & 0.997 & -0.006 \\
\hline T1_5_1 & 0.000 & 0.989 & -0.005 \\
\hline T1_6_1 & 0.027 & 0.870 & 0.018 \\
\hline T1_7_1 & 0.003 & 0.959 & 0.002 \\
\hline T1_8_1 & 0.001 & 0.979 & -0.015 \\
\hline T1_9_1 & 0.034 & 0.854 & 0.035 \\
\hline T1_10_1 & 0.004 & 0.953 & 0.002 \\
\hline T1_11_1 & 0.050 & 0.822 & 0.027 \\
\hline T1_12_1 & 0.019 & 0.890 & 0.014 \\
\hline T1_13_12 & 0.000 & 0.991 & -0.010 \\
\hline T1_14_12 & 0.024 & 0.878 & -0.023 \\
\hline T1_15_1 & 0.012 & 0.913 & 0.010 \\
\hline T1_2_2 & 0.032 & 0.858 & 0.013 \\
\hline T1_3_2 & 0.020 & 0.888 & 0.022 \\
\hline T1_4_2 & 0.000 & 0.996 & 0.000 \\
\hline T1_5_2 & 0.006 & 0.937 & 0.013 \\
\hline T1_6_23 & 0.202 & 0.654 & 0.047 \\
\hline T1_7_2 & 0.024 & 0.878 & 0.024 \\
\hline T1_8_2 & 0.002 & 0.967 & -0.010 \\
\hline T1_9_23 & 0.290 & 0.590 & 0.042 \\
\hline T1_11_2 & 0.000 & 0.992 & -0.006 \\
\hline T1_13_23 & 0.258 & 0.612 & 0.043 \\
\hline T1_14_2 & 0.002 & 0.970 & 0.007 \\
\hline T1_15_2 & 0.003 & 0.958 & 0.009 \\
\hline T1_1_34 & 0.000 & 0.993 & -0.002 \\
\hline T1_2_34 & 0.013 & 0.908 & 0.019 \\
\hline T1_4_34 & 0.038 & 0.846 & -0.014 \\
\hline T1_5_3 & 0.009 & 0.925 & -0.005 \\
\hline T1_6_3 & 0.000 & 0.989 & -0.021 \\
\hline T1_8_3 & 0.002 & 0.967 & -0.030 \\
\hline T1_10_3 & 0.001 & 0.970 & -0.014 \\
\hline T1_11_3 & 0.090 & 0.764 & 0.025 \\
\hline T1_12_3 & 0.011 & 0.917 & -0.018 \\
\hline T1_13_3 & 0.073 & 0.788 & 0.021 \\
\hline
\end{tabular}




$\begin{array}{llll} & & \\ \text { T1_14_3 } & 0.040 & 0.841 & 0.011 \\ \text { T1_15_3 } & 0.008 & 0.930 & -0.019 \\ \text { T1_1_4 } & 0.698 & 0.403 & -0.080 \\ \text { T1_2_456 } & 0.000 & 0.988 & 0.012 \\ \text { T1_4_4 } & 0.058 & 0.810 & -0.017 \\ \text { T1_5_456 } & 0.278 & 0.598 & -0.066 \\ \text { T1_6_4 } & 0.067 & 0.796 & -0.020 \\ \text { T1_7_4 } & 0.000 & 0.986 & 0.016 \\ \text { T1_8_4 } & 0.137 & 0.711 & -0.037 \\ \text { T1_9_4 } & 0.069 & 0.793 & -0.021 \\ \text { T1_10_456 } & 0.037 & 0.847 & 0.014 \\ \text { T1_13_4 } & 0.148 & 0.700 & -0.013 \\ \text { T1_14_4 } & 0.000 & 0.987 & 0.022 \\ \text { T1_15_4 } & 0.033 & 0.856 & -0.009 \\ \text { T1_1_5 } & 0.012 & 0.912 & 0.014 \\ \text { T1_2_5 } & 0.049 & 0.825 & 0.030 \\ \text { T1_4_56 } & 0.014 & 0.906 & 0.014 \\ \text { T1_5_5 } & 0.071 & 0.789 & 0.030 \\ \text { T1_6_56 } & 0.004 & 0.949 & 0.005 \\ \text { T1_7_5 } & 0.088 & 0.767 & 0.035 \\ \text { T1_8_56 } & 0.032 & 0.858 & 0.001 \\ \text { T1_11_5 } & 0.001 & 0.970 & 0.002 \\ \text { T1_12_5 } & 0.000 & 0.998 & -0.003 \\ \text { T1_13_5 } & 0.015 & 0.901 & -0.035 \\ \text { T1_14_5 } & 0.007 & 0.936 & 0.009 \\ \text { T1_15_5 } & 0.001 & 0.979 & 0.001 \\ \text { T1_1_6 } & 0.002 & 0.964 & 0.008 \\ \text { T1_3_6 } & 0.012 & 0.915 & 0.018 \\ \text { T1_6_6 } & 0.028 & 0.867 & 0.008 \\ \text { T1_8_6 } & 0.002 & 0.964 & 0.008 \\ \text { T1_9_6 } & 0.000 & 0.997 & 0.002 \\ \text { T1_11_6 } & 0.002 & 0.961 & -0.011 \\ \text { T1_12_6 } & 0.139 & 0.709 & 0.039 \\ \text { T1_13_6 } & 0.002 & 0.964 & -0.010 \\ \text { T1_14_6 } & 0.000 & 0.998 & 0.002 \\ \text { T1_16_1 } & 0.074 & 0.785 & -0.029 \\ \text { T1_18_1 } & 0.085 & 0.771 & -0.032 \\ \text { T1_19_1 } & 0.001 & 0.974 & 0.019 \\ \text { T1_20_1 } & 0.136 & 0.712 & -0.044 \\ \text { T1_22_1 } & 0.022 & 0.881 & -0.012\end{array}$




$\begin{array}{llll} & & & \\ \text { T1_23_1 } & 0.021 & 0.884 & -0.011 \\ \text { T1_25_1 } & 0.071 & 0.791 & -0.031 \\ \text { T1_27_1 } & 0.001 & 0.980 & 0.009 \\ \text { T1_28_1 } & 0.001 & 0.983 & 0.023 \\ \text { T1_19_2 } & 0.043 & 0.835 & 0.019 \\ \text { T1_20_2 } & 0.002 & 0.966 & -0.023 \\ \text { T1_21_2 } & 0.000 & 0.984 & -0.019 \\ \text { T1_22_2 } & 0.010 & 0.920 & -0.032 \\ \text { T1_23_2 } & 0.000 & 0.988 & -0.016 \\ \text { T1_25_2 } & 0.059 & 0.808 & 0.024 \\ \text { T1_26_2 } & 0.043 & 0.837 & -0.047 \\ \text { T1_27_23 } & 0.057 & 0.812 & 0.047 \\ \text { T1_28_2 } & 0.002 & 0.967 & -0.006 \\ \text { T1_16_3 } & 0.005 & 0.946 & 0.034 \\ \text { T1_17_3 } & 0.079 & 0.779 & -0.025 \\ \text { T1_18_34 } & 0.087 & 0.768 & 0.001 \\ \text { T1_19_3 } & 0.006 & 0.941 & 0.005 \\ \text { T1_21_3 } & 0.029 & 0.864 & -0.009 \\ \text { T1_25_3 } & 0.001 & 0.971 & 0.012 \\ \text { T1_26_3 } & 0.093 & 0.760 & -0.028 \\ \text { T1_27_3 } & 0.013 & 0.911 & 0.000 \\ \text { T1_28_3 } & 0.148 & 0.701 & -0.041 \\ \text { T1_16_4 } & 0.005 & 0.943 & 0.041 \\ \text { T1_17_4 } & 0.001 & 0.979 & 0.035 \\ \text { T1_18_4 } & 0.001 & 0.978 & 0.021 \\ \text { T1_19_4 } & 0.140 & 0.708 & -0.033 \\ \text { T1_20_4 } & 0.004 & 0.951 & 0.040 \\ \text { T1_22_45 } & 0.115 & 0.734 & -0.032 \\ \text { T1_24_4 } & 0.031 & 0.861 & -0.002 \\ \text { T1_27_4 } & 0.054 & 0.817 & -0.011 \\ \text { T1_28_4 } & 0.094 & 0.760 & -0.022 \\ \text { T1_17_5 } & 0.153 & 0.695 & 0.052 \\ \text { T1_18_5 } & 0.005 & 0.944 & -0.022 \\ \text { T1_19_5 } & 0.003 & 0.958 & 0.001 \\ \text { T1_22_5 } & 0.033 & 0.856 & 0.021 \\ \text { T1_23_5 } & 0.086 & 0.770 & 0.037 \\ \text { T1_24_56 } & 0.227 & 0.634 & 0.041 \\ \text { T1_25_5 } & 0.008 & 0.929 & -0.024 \\ \text { T1_17_6 } & 0.014 & 0.905 & 0.000\end{array}$




\begin{tabular}{llll} 
T1_18_6 & 0.000 & 0.995 & -0.020 \\
T1_19_6 & 0.033 & 0.856 & 0.011 \\
T1_20_6 & 0.023 & 0.880 & 0.007 \\
T1_21_6 & 0.013 & 0.910 & 0.000 \\
T1_23_6 & 0.003 & 0.956 & -0.009 \\
T1_24_6 & 0.012 & 0.915 & 0.000 \\
T1_25_6 & 0.079 & 0.779 & -0.062 \\
T1_28_6 & 0.001 & 0.978 & -0.012 \\
\hline
\end{tabular}


Table 30

Task 1 DIF: White vs. Sample

\begin{tabular}{lccc}
\hline Item & ChiSq & pvalue & EffectSize \\
\hline T1_1_1 & 0.003 & 0.960 & 0.014 \\
T1_2_12 & 0.049 & 0.825 & -0.080 \\
T1_3_1 & 0.002 & 0.963 & -0.006 \\
T1_4_1 & 0.001 & 0.980 & 0.011 \\
T1_5_1 & 0.000 & 0.999 & 0.006 \\
T1_6_1 & 0.000 & 0.983 & -0.002 \\
T1_7_1 & 0.004 & 0.950 & -0.008 \\
T1_8_1 & 0.003 & 0.959 & 0.014 \\
T1_9_1 & 0.483 & 0.487 & 0.072 \\
T1_10_1 & 0.006 & 0.937 & -0.010 \\
T1_11_1 & 0.001 & 0.978 & 0.010 \\
T1_12_1 & 0.010 & 0.919 & 0.021 \\
T1_13_12 & 0.228 & 0.633 & 0.019 \\
T1_14_12 & 0.228 & 0.633 & -0.033 \\
T1_15_1 & 0.037 & 0.848 & -0.027 \\
T1_2_2 & 0.489 & 0.484 & 0.044 \\
T1_3_2 & 0.234 & 0.629 & 0.015 \\
T1_4_2 & 0.473 & 0.492 & 0.046 \\
T1_5_2 & 0.341 & 0.560 & 0.029 \\
T1_6_23 & 0.446 & 0.504 & 0.056 \\
T1_7_2 & 0.059 & 0.808 & -0.021 \\
T1_8_2 & 0.057 & 0.812 & -0.023 \\
T1_9_23 & 0.001 & 0.981 & -0.036 \\
T1_11_2 & 0.016 & 0.899 & -0.040 \\
T1_13_23 & 0.386 & 0.534 & 0.054 \\
T1_14_2 & 0.197 & 0.657 & 0.009 \\
T1_15_2 & 0.005 & 0.944 & -0.049 \\
T1_1_34 & 0.001 & 0.973 & -0.005 \\
T1_2_34 & 0.021 & 0.884 & 0.027 \\
T1_4_34 & 0.011 & 0.918 & 0.022 \\
T1_5_3 & 0.012 & 0.913 & -0.004 \\
T1_6_3 & 0.000 & 0.985 & 0.009 \\
T1_8_3 & 0.001 & 0.972 & 0.007 \\
T1_10_3 & 0.007 & 0.933 & -0.001 \\
T1_11_3 & 0.017 & 0.897 & -0.008 \\
T1_12_3 & 0.119 & 0.730 & 0.000 \\
T1_3 & 0.031 & 0.861 & -0.014 \\
& & &
\end{tabular}




$\begin{array}{llll}\text { T1_14_3 } & 0.000 & 0.989 & 0.014 \\ \text { T1_15_3 } & 0.108 & 0.743 & 0.001 \\ \text { T1_1_4 } & 0.089 & 0.766 & -0.010 \\ \text { T1_2_456 } & 0.005 & 0.942 & -0.054 \\ \text { T1_4_4 } & 0.025 & 0.873 & 0.014 \\ \text { T1_5_456 } & 0.048 & 0.826 & -0.073 \\ \text { T1_6_4 } & 0.075 & 0.784 & 0.032 \\ \text { T1_7_4 } & 0.025 & 0.875 & -0.038 \\ \text { T1_8_4 } & 0.017 & 0.896 & 0.010 \\ \text { T1_9_4 } & 0.023 & 0.879 & 0.014 \\ \text { T1_10_456 } & 0.016 & 0.901 & -0.010 \\ \text { T1_13_4 } & 0.108 & 0.742 & -0.013 \\ \text { T1_14_4 } & 0.001 & 0.970 & -0.004 \\ \text { T1_15_4 } & 0.045 & 0.833 & 0.022 \\ \text { T1_1_5 } & 0.000 & 0.986 & -0.001 \\ \text { T1_2_5 } & 0.004 & 0.951 & -0.008 \\ \text { T1_4_56 } & 0.151 & 0.698 & 0.035 \\ \text { T1_5_5 } & 0.364 & 0.546 & 0.075 \\ \text { T1_6_56 } & 0.024 & 0.876 & 0.001 \\ \text { T1_7_5 } & 0.274 & 0.601 & 0.062 \\ \text { T1_8_56 } & 0.015 & 0.904 & 0.046 \\ \text { T1_11_5 } & 0.000 & 0.993 & 0.007 \\ \text { T1_12_5 } & 0.004 & 0.952 & -0.008 \\ \text { T1_13_5 } & 0.000 & 0.997 & -0.019 \\ \text { T1_14_5 } & 0.040 & 0.842 & -0.029 \\ \text { T1_15_5 } & 0.000 & 0.994 & 0.002 \\ \text { T1_1_6 } & 0.000 & 0.994 & -0.030 \\ \text { T1_3_6 } & 0.050 & 0.824 & 0.007 \\ \text { T1_6_6 } & 0.240 & 0.624 & -0.056 \\ \text { T1_8_6 } & 0.004 & 0.952 & -0.040 \\ \text { T1_9_6 } & 0.004 & 0.952 & -0.017 \\ \text { T1_11_6 } & 0.001 & 0.973 & -0.021 \\ \text { T1_12_6 } & 0.065 & 0.799 & -0.023 \\ \text { T1_13_6 } & 0.000 & 0.995 & -0.030 \\ \text { T1_14_6 } & 0.177 & 0.674 & 0.037 \\ \text { T1_16_1 } & 0.108 & 0.742 & 0.013 \\ \text { T1_18_1 } & 0.311 & 0.577 & 0.048 \\ \text { T1_19_1 } & 0.010 & 0.920 & -0.018 \\ \text { T1_20_1 } & 0.556 & 0.456 & 0.075 \\ \text { T1_21_12 } & 0.125 & 0.724 & 0.023 \\ \text { T1_22_1 } & 0.004 & 0.950 & -0.024 \\ & & & \end{array}$




\begin{tabular}{|c|c|c|c|}
\hline T1_23_1 & 0.287 & 0.593 & 0.047 \\
\hline T1_25_1 & 0.117 & 0.733 & 0.020 \\
\hline T1_27_1 & 0.189 & 0.664 & 0.031 \\
\hline T1_28_1 & 0.600 & 0.439 & 0.083 \\
\hline T1_19_2 & 0.008 & 0.929 & -0.011 \\
\hline T1_20_2 & 0.024 & 0.876 & -0.020 \\
\hline T1_21_2 & 0.068 & 0.794 & 0.046 \\
\hline T1_22_2 & 0.107 & 0.743 & -0.047 \\
\hline T1_23_2 & 0.016 & 0.900 & -0.014 \\
\hline T1_25_2 & 0.083 & 0.774 & -0.039 \\
\hline T1_26_2 & 0.073 & 0.787 & -0.038 \\
\hline T1_27_23 & 0.396 & 0.529 & -0.051 \\
\hline T1_28_2 & 0.162 & 0.688 & -0.056 \\
\hline T1_16_3 & 0.000 & 0.998 & 0.042 \\
\hline T1_17_3 & 0.276 & 0.599 & -0.044 \\
\hline T1_18_34 & 0.246 & 0.620 & 0.023 \\
\hline T1_19_3 & 0.074 & 0.786 & -0.006 \\
\hline T1_21_3 & 0.065 & 0.799 & -0.004 \\
\hline T1_25_3 & 0.011 & 0.917 & 0.020 \\
\hline T1_26_3 & 0.084 & 0.773 & -0.008 \\
\hline T1_27_3 & 0.061 & 0.804 & 0.075 \\
\hline T1_28_3 & 0.377 & 0.539 & -0.059 \\
\hline T1_16_4 & 0.024 & 0.878 & 0.026 \\
\hline T1_17_4 & 0.018 & 0.894 & 0.033 \\
\hline T1_18_4 & 0.005 & 0.946 & 0.042 \\
\hline T1_19_4 & 0.316 & 0.574 & -0.037 \\
\hline T1_20_4 & 0.036 & 0.850 & 0.024 \\
\hline T1_22_45 & 0.025 & 0.876 & 0.057 \\
\hline T1_24_4 & 0.333 & 0.564 & -0.038 \\
\hline T1_27_4 & 0.280 & 0.597 & -0.032 \\
\hline T1_28_4 & 0.789 & 0.374 & -0.085 \\
\hline T1_17_5 & 0.285 & 0.594 & 0.058 \\
\hline T1_18_5 & 0.000 & 0.993 & -0.027 \\
\hline T1_19_5 & 0.251 & 0.617 & 0.054 \\
\hline T1_22_5 & 0.000 & 0.989 & -0.024 \\
\hline T1_23_5 & 0.012 & 0.912 & -0.043 \\
\hline T1_24_56 & 0.004 & 0.950 & 0.039 \\
\hline T1_25_5 & 0.015 & 0.902 & -0.002 \\
\hline T1_27_5 & 0.077 & 0.782 & 0.021 \\
\hline T1_28_5 & 0.008 & 0.927 & -0.006 \\
\hline T1_17_6 & 0.008 & 0.930 & 0.031 \\
\hline
\end{tabular}




\begin{tabular}{llll} 
T1_18_6 & 0.145 & 0.704 & -0.009 \\
T1_19_6 & 0.041 & 0.840 & 0.016 \\
T1_20_6 & 0.439 & 0.508 & -0.054 \\
T1_21_6 & 0.012 & 0.912 & 0.065 \\
T1_23_6 & 0.204 & 0.652 & -0.023 \\
T1_24_6 & 0.314 & 0.575 & -0.039 \\
T1_25_6 & 0.046 & 0.830 & 0.013 \\
T1_28_6 & 0.061 & 0.805 & 0.007 \\
\hline
\end{tabular}


Table 31

Task 1 DIF: Black vs. Sample

\begin{tabular}{|c|c|c|c|}
\hline Item & ChiSq & pvalue & EffectSize \\
\hline T1_1_1 & 0.000 & 0.986 & 0.010 \\
\hline T1_2_12 & 0.169 & 0.681 & 0.080 \\
\hline T1_3_1 & 0.000 & 0.996 & 0.002 \\
\hline T1_4_1 & 0.004 & 0.950 & -0.007 \\
\hline T1_5_1 & 0.001 & 0.980 & -0.001 \\
\hline T1_6_1 & 0.001 & 0.975 & -0.002 \\
\hline T1_7_1 & 0.000 & 0.987 & 0.010 \\
\hline T1_8_1 & 0.000 & 0.987 & 0.000 \\
\hline T1_9_1 & 0.991 & 0.320 & -0.085 \\
\hline T1_10_1 & 0.000 & 0.998 & 0.002 \\
\hline T1_11_1 & 0.000 & 0.995 & 0.004 \\
\hline T1_12_1 & 0.025 & 0.874 & -0.021 \\
\hline T1_13_12 & 0.187 & 0.665 & -0.016 \\
\hline T1_14_12 & 0.609 & 0.435 & 0.014 \\
\hline T1_15_1 & 0.007 & 0.934 & -0.010 \\
\hline T1_2_2 & 0.730 & 0.393 & -0.084 \\
\hline T1_3_2 & 0.104 & 0.748 & -0.004 \\
\hline T1_4_2 & 0.348 & 0.555 & -0.045 \\
\hline T1_5_2 & 0.309 & 0.578 & -0.039 \\
\hline T1_6_23 & 0.148 & 0.700 & -0.028 \\
\hline T1_7_2 & 0.048 & 0.827 & 0.013 \\
\hline T1_8_2 & 0.009 & 0.924 & 0.033 \\
\hline T1_9_23 & 0.027 & 0.871 & 0.001 \\
\hline T1_11_2 & 0.001 & 0.972 & 0.042 \\
\hline T1_13_23 & 0.231 & 0.631 & -0.049 \\
\hline T1_14_2 & 0.205 & 0.651 & -0.024 \\
\hline T1_15_2 & 0.001 & 0.979 & 0.044 \\
\hline T1_1_34 & 0.299 & 0.584 & 0.054 \\
\hline T1_2_34 & 0.056 & 0.812 & 0.007 \\
\hline T1_4_34 & 0.046 & 0.830 & -0.005 \\
\hline T1_5_3 & 0.006 & 0.938 & -0.001 \\
\hline T1_6_3 & 0.001 & 0.977 & -0.022 \\
\hline T1_8_3 & 0.000 & 0.998 & -0.012 \\
\hline T1_10_3 & 0.005 & 0.943 & -0.002 \\
\hline T1_11_3 & 0.029 & 0.864 & 0.014 \\
\hline T1_12_3 & 0.066 & 0.797 & 0.016 \\
\hline T1_13_3 & 0.010 & 0.921 & 0.003 \\
\hline
\end{tabular}




$\begin{array}{llll}\text { T1_14_3 } & 0.002 & 0.962 & -0.005 \\ \text { T1_15_3 } & 0.000 & 0.998 & -0.021 \\ \text { T1_1_4 } & 0.687 & 0.407 & 0.060 \\ \text { T1_2_456 } & 0.007 & 0.931 & -0.001 \\ \text { T1_4_4 } & 0.000 & 0.989 & -0.016 \\ \text { T1_5_456 } & 0.147 & 0.702 & 0.046 \\ \text { T1_6_4 } & 0.013 & 0.908 & 0.004 \\ \text { T1_7_4 } & 0.060 & 0.807 & 0.023 \\ \text { T1_8_4 } & 0.048 & 0.826 & 0.020 \\ \text { T1_9_4 } & 0.010 & 0.921 & 0.001 \\ \text { T1_10_456 } & 1.059 & 0.303 & 0.095 \\ \text { T1_13_4 } & 0.631 & 0.427 & 0.053 \\ \text { T1_14_4 } & 0.002 & 0.969 & -0.026 \\ \text { T1_15_4 } & 0.005 & 0.946 & -0.004 \\ \text { T1_1_5 } & 0.198 & 0.657 & 0.037 \\ \text { T1_2_5 } & 0.183 & 0.669 & 0.034 \\ \text { T1_4_56 } & 0.003 & 0.954 & -0.010 \\ \text { T1_5_5 } & 0.128 & 0.721 & -0.048 \\ \text { T1_6_56 } & 0.015 & 0.904 & -0.020 \\ \text { T1_7_5 } & 0.073 & 0.787 & -0.035 \\ \text { T1_8_56 } & 0.014 & 0.907 & -0.092 \\ \text { T1_11_5 } & 0.019 & 0.892 & -0.012 \\ \text { T1_12_5 } & 0.127 & 0.721 & 0.023 \\ \text { T1_13_5 } & 0.000 & 0.988 & 0.016 \\ \text { T1_14_5 } & 0.190 & 0.663 & 0.035 \\ \text { T1_15_5 } & 0.002 & 0.970 & -0.026 \\ \text { T1_1_6 } & 0.000 & 0.998 & 0.034 \\ \text { T1_3_6 } & 0.080 & 0.777 & -0.010 \\ \text { T1_6_6 } & 0.062 & 0.803 & 0.011 \\ \text { T1_8_6 } & 0.000 & 0.998 & 0.039 \\ \text { T1_9_6 } & 0.006 & 0.941 & 0.023 \\ \text { T1_11_6 } & 0.001 & 0.982 & 0.030 \\ \text { T1_12_6 } & 0.250 & 0.617 & 0.052 \\ \text { T1_13_6 } & 0.000 & 0.992 & 0.035 \\ \text { T1_14_6 } & 0.209 & 0.648 & -0.037 \\ \text { T1_16_1 } & 0.300 & 0.584 & -0.017 \\ \text { T1_18_1 } & 0.460 & 0.498 & -0.042 \\ \text { T1_19_1 } & 0.136 & 0.712 & 0.008 \\ \text { T1_22_1 } & 0.874 & 0.350 & -0.079 \\ \text { T1_1_12 } & 0.316 & 0.574 & -0.026 \\ \text { T1 } & 0.079 & 0.779 & 0.019\end{array}$




\begin{tabular}{|c|c|c|c|}
\hline T1_23_1 & 0.716 & 0.398 & -0.070 \\
\hline T1_25_1 & 0.524 & 0.469 & -0.053 \\
\hline T1_27_1 & 0.821 & 0.365 & -0.078 \\
\hline T1_28_1 & 0.843 & 0.359 & -0.080 \\
\hline T1_19_2 & 0.000 & 0.987 & -0.006 \\
\hline T1_20_2 & 0.018 & 0.893 & 0.015 \\
\hline T1_21_2 & 0.039 & 0.844 & -0.040 \\
\hline T1_22_2 & 0.114 & 0.736 & 0.047 \\
\hline T1_23_2 & 0.030 & 0.863 & 0.021 \\
\hline T1_25_2 & 0.062 & 0.804 & 0.033 \\
\hline T1_26_2 & 0.075 & 0.784 & 0.039 \\
\hline T1_27_23 & 0.006 & 0.940 & 0.000 \\
\hline T1_28_2 & 0.123 & 0.726 & 0.049 \\
\hline T1_16_3 & 0.000 & 0.997 & -0.011 \\
\hline T1_17_3 & 0.007 & 0.933 & 0.008 \\
\hline T1_18_34 & 0.128 & 0.720 & -0.010 \\
\hline T1_19_3 & 0.017 & 0.896 & 0.015 \\
\hline T1_21_3 & 0.017 & 0.898 & -0.030 \\
\hline T1_25_3 & 0.015 & 0.903 & -0.031 \\
\hline T1_26_3 & 0.012 & 0.915 & 0.009 \\
\hline T1_27_3 & 0.219 & 0.640 & -0.080 \\
\hline T1_28_3 & 0.069 & 0.793 & 0.035 \\
\hline T1_16_4 & 0.165 & 0.685 & 0.010 \\
\hline T1_17_4 & 0.030 & 0.863 & -0.029 \\
\hline T1_18_4 & 0.010 & 0.920 & -0.040 \\
\hline T1_19_4 & 0.449 & 0.503 & 0.052 \\
\hline T1_20_4 & 0.094 & 0.760 & -0.008 \\
\hline T1_22_45 & 0.001 & 0.974 & -0.003 \\
\hline T1_24_4 & 0.406 & 0.524 & 0.046 \\
\hline T1_27_4 & 0.286 & 0.593 & 0.031 \\
\hline T1_28_4 & 0.855 & 0.355 & 0.091 \\
\hline T1_17_5 & 0.170 & 0.680 & -0.019 \\
\hline T1_18_5 & 0.026 & 0.873 & 0.018 \\
\hline T1_19_5 & 0.404 & 0.525 & -0.054 \\
\hline T1_22_5 & 0.000 & 0.989 & 0.043 \\
\hline T1_23_5 & 0.017 & 0.896 & 0.024 \\
\hline T1_24_56 & 0.002 & 0.963 & -0.031 \\
\hline T1_25_5 & 0.365 & 0.546 & -0.051 \\
\hline T1_27_5 & 0.158 & 0.691 & -0.018 \\
\hline T1_28_5 & 0.014 & 0.905 & 0.024 \\
\hline T1_17_6 & 0.021 & 0.884 & -0.041 \\
\hline
\end{tabular}


MATRS TECHNICAL MANUAL 130

\begin{tabular}{llll} 
T1_18_6 & 0.104 & 0.747 & -0.015 \\
T1_19_6 & 0.060 & 0.807 & -0.027 \\
T1_20_6 & 0.667 & 0.414 & 0.062 \\
T1_21_6 & 0.011 & 0.918 & -0.047 \\
T1_23_6 & 0.557 & 0.456 & 0.051 \\
T1_24_6 & 0.274 & 0.601 & 0.018 \\
T1_25_6 & 0.188 & 0.665 & 0.002 \\
T1_28_6 & 0.125 & 0.724 & -0.008 \\
\hline
\end{tabular}


Table 32

Task 1 DIF: Latinx vs. Sample

\begin{tabular}{|c|c|c|c|}
\hline Item & ChiSq & pvalue & EffectSize \\
\hline T1_1_1 & 0.001 & 0.973 & -0.020 \\
\hline T1_2_12 & 0.166 & 0.683 & 0.010 \\
\hline T1_3_1 & 0.046 & 0.831 & 0.042 \\
\hline T1_4_1 & 0.001 & 0.978 & -0.022 \\
\hline T1_5_1 & 0.000 & 0.983 & -0.025 \\
\hline T1_6_1 & 0.036 & 0.849 & 0.031 \\
\hline T1_7_1 & 0.006 & 0.939 & -0.005 \\
\hline T1_8_1 & 0.001 & 0.977 & -0.041 \\
\hline T1_9_1 & 0.094 & 0.759 & 0.007 \\
\hline T1_10_1 & 0.001 & 0.977 & -0.039 \\
\hline T1_11_1 & 0.031 & 0.860 & 0.028 \\
\hline T1_12_1 & 0.011 & 0.916 & 0.004 \\
\hline T1_13_12 & 0.055 & 0.814 & 0.036 \\
\hline T1_14_12 & 0.065 & 0.799 & -0.042 \\
\hline T1_15_1 & 0.019 & 0.890 & 0.015 \\
\hline T1_2_2 & 0.379 & 0.538 & 0.159 \\
\hline T1_3_2 & 0.000 & 0.991 & -0.015 \\
\hline T1_4_2 & 0.003 & 0.958 & 0.021 \\
\hline T1_5_2 & 0.005 & 0.943 & 0.028 \\
\hline T1_6_23 & 0.001 & 0.982 & -0.007 \\
\hline T1_7_2 & 0.000 & 0.988 & -0.001 \\
\hline T1_8_2 & 0.000 & 0.985 & -0.027 \\
\hline T1_9_23 & 0.063 & 0.802 & 0.035 \\
\hline T1_11_2 & 0.000 & 0.988 & -0.026 \\
\hline T1_13_23 & 0.091 & 0.763 & 0.055 \\
\hline T1_14_2 & 0.017 & 0.897 & 0.047 \\
\hline T1_15_2 & 0.001 & 0.983 & -0.028 \\
\hline T1_1_34 & 0.794 & 0.373 & -0.208 \\
\hline T1_2_34 & 0.197 & 0.657 & -0.058 \\
\hline T1_4_34 & 0.059 & 0.809 & -0.056 \\
\hline T1_5_3 & 0.027 & 0.870 & 0.061 \\
\hline T1_6_3 & 0.000 & 0.985 & 0.002 \\
\hline T1_8_3 & 0.000 & 0.992 & -0.012 \\
\hline T1_10_3 & 0.002 & 0.964 & 0.023 \\
\hline T1_11_3 & 0.000 & 0.990 & -0.019 \\
\hline T1_12_3 & 0.000 & 0.996 & -0.082 \\
\hline T1_13_3 & 0.000 & 0.998 & -0.014 \\
\hline
\end{tabular}




$\begin{array}{llll} & & \\ \text { T1_14_3 } & 0.001 & 0.981 & -0.023 \\ \text { T1_15_3 } & 0.131 & 0.718 & 0.058 \\ \text { T1_1_4 } & 0.000 & 0.989 & 0.025 \\ \text { T1_2_456 } & 0.046 & 0.831 & 0.155 \\ \text { T1_4_4 } & 0.080 & 0.778 & 0.001 \\ \text { T1_5_456 } & 0.035 & 0.852 & 0.149 \\ \text { T1_6_4 } & 0.347 & 0.556 & -0.106 \\ \text { T1_7_4 } & 0.011 & 0.919 & 0.064 \\ \text { T1_8_4 } & 0.259 & 0.611 & -0.078 \\ \text { T1_9_4 } & 0.140 & 0.708 & -0.032 \\ \text { T1_10_456 } & 0.408 & 0.523 & -0.117 \\ \text { T1_13_4 } & 0.000 & 0.990 & -0.014 \\ \text { T1_14_4 } & 0.000 & 0.992 & 0.102 \\ \text { T1_15_4 } & 0.235 & 0.628 & -0.070 \\ \text { T1_1_5 } & 0.105 & 0.746 & -0.060 \\ \text { T1_2_5 } & 0.026 & 0.871 & -0.005 \\ \text { T1_4_56 } & 0.281 & 0.596 & -0.088 \\ \text { T1_5_5 } & 0.104 & 0.747 & -0.091 \\ \text { T1_6_56 } & 0.020 & 0.888 & 0.046 \\ \text { T1_7_5 } & 0.051 & 0.822 & -0.054 \\ \text { T1_8_56 } & 0.028 & 0.866 & 0.236 \\ \text { T1_11_5 } & 0.003 & 0.954 & 0.032 \\ \text { T1_12_5 } & 0.013 & 0.910 & 0.013 \\ \text { T1_13_5 } & 0.012 & 0.914 & 0.087 \\ \text { T1_14_5 } & 0.038 & 0.846 & -0.016 \\ \text { T1_15_5 } & 0.027 & 0.869 & -0.005 \\ \text { T1_1_6 } & 0.000 & 0.999 & 0.031 \\ \text { T1_3_6 } & 0.036 & 0.849 & -0.033 \\ \text { T1_6_6 } & 0.002 & 0.965 & 0.172 \\ \text { T1_8_6 } & 0.001 & 0.980 & 0.022 \\ \text { T1_9_6 } & 0.001 & 0.973 & 0.019 \\ \text { T1_11_6 } & 0.004 & 0.951 & 0.009 \\ \text { T1_12_6 } & 1.319 & 0.251 & -0.215 \\ \text { T1_13_6 } & 0.012 & 0.915 & -0.005 \\ \text { T1_14_6 } & 0.020 & 0.887 & -0.019 \\ \text { T1_16_1 } & 0.013 & 0.908 & -0.021 \\ \text { T1_18_1 } & 0.002 & 0.961 & -0.030 \\ \text { T1_19_1 } & 0.000 & 0.988 & -0.077 \\ \text { T1_20_1 } & 0.054 & 0.816 & 0.024 \\ \text { T1_22_1 } & 0.002 & 0.964 & -0.044\end{array}$




$\begin{array}{llll} & & & \\ \text { T1_23_1 } & 0.076 & 0.784 & 0.037 \\ \text { T1_25_1 } & 0.098 & 0.755 & 0.055 \\ \text { T1_27_1 } & 0.192 & 0.662 & 0.086 \\ \text { T1_28_1 } & 0.003 & 0.959 & -0.041 \\ \text { T1_19_2 } & 0.041 & 0.839 & 0.035 \\ \text { T1_20_2 } & 0.001 & 0.983 & -0.052 \\ \text { T1_21_2 } & 0.015 & 0.904 & 0.005 \\ \text { T1_22_2 } & 0.002 & 0.961 & -0.017 \\ \text { T1_23_2 } & 0.001 & 0.981 & -0.045 \\ \text { T1_25_2 } & 0.103 & 0.748 & 0.073 \\ \text { T1_26_2 } & 0.000 & 0.998 & -0.060 \\ \text { T1_27_23 } & 0.732 & 0.392 & 0.164 \\ \text { T1_28_2 } & 0.000 & 0.998 & -0.036 \\ \text { T1_16_3 } & 0.002 & 0.961 & -0.101 \\ \text { T1_17_3 } & 0.060 & 0.807 & 0.028 \\ \text { T1_18_34 } & 0.002 & 0.968 & -0.167 \\ \text { T1_19_3 } & 0.124 & 0.725 & 0.066 \\ \text { T1_21_3 } & 0.008 & 0.929 & -0.022 \\ \text { T1_25_3 } & 0.008 & 0.930 & -0.019 \\ \text { T1_26_3 } & 0.006 & 0.938 & -0.102 \\ \text { T1_27_3 } & 0.036 & 0.849 & 0.009 \\ \text { T1_28_3 } & 0.131 & 0.717 & 0.069 \\ \text { T1_16_4 } & 0.005 & 0.942 & -0.069 \\ \text { T1_17_4 } & 0.001 & 0.970 & -0.134 \\ \text { T1_18_4 } & 0.011 & 0.918 & -0.151 \\ \text { T1_19_4 } & 0.180 & 0.672 & 0.052 \\ \text { T1_20_4 } & 0.001 & 0.973 & -0.105 \\ \text { T1_22_45 } & 0.003 & 0.960 & -0.170 \\ \text { T1_24_4 } & 0.153 & 0.696 & 0.039 \\ \text { T1_27_4 } & 0.131 & 0.717 & 0.031 \\ \text { T1_28_4 } & 0.187 & 0.665 & 0.052 \\ \text { T1_17_5 } & 0.009 & 0.927 & -0.074 \\ \text { T1_18_5 } & 0.000 & 0.989 & -0.039 \\ \text { T1_19_5 } & 0.005 & 0.945 & 0.005 \\ \text { T1_22_5 } & 0.043 & 0.835 & 0.048 \\ \text { T1_23_5 } & 0.176 & 0.675 & 0.119 \\ \text { T1_24_56 } & 0.025 & 0.875 & 0.210 \\ \text { T1_25_5 } & 0.157 & 0.692 & 0.112 \\ \text { T1_17_6 } & 0.073 & 0.787 & 0.058\end{array}$




\begin{tabular}{llll} 
T1_18_6 & 0.000 & 0.997 & 0.146 \\
T1_19_6 & 0.041 & 0.840 & 0.077 \\
T1_20_6 & 0.506 & 0.477 & -0.101 \\
T1_21_6 & 0.408 & 0.523 & -0.072 \\
T1_23_6 & 0.816 & 0.367 & -0.166 \\
T1_24_6 & 0.011 & 0.918 & 0.108 \\
T1_25_6 & 0.388 & 0.533 & -0.063 \\
T1_28_6 & 0.344 & 0.558 & -0.053 \\
\hline
\end{tabular}


Table 33

Task 2 DIF: Male vs. female

\begin{tabular}{|c|c|c|c|}
\hline ItemID & ChiSq & pvalue & EffectSize \\
\hline T2_51_12 & 0.644 & 0.422 & 0.074 \\
\hline T2_53_12 & 0.347 & 0.556 & 0.032 \\
\hline T2_56_12 & 0.468 & 0.494 & 0.057 \\
\hline T2_58_12 & 0.085 & 0.771 & -0.004 \\
\hline T2_75_12 & 0.441 & 0.506 & 0.053 \\
\hline T2_60_23 & 0.002 & 0.961 & -0.027 \\
\hline T2_70_23 & 0.054 & 0.816 & -0.050 \\
\hline T2_75_23 & 0.183 & 0.669 & 0.047 \\
\hline T2_78_23 & 0.031 & 0.860 & 0.008 \\
\hline T2_84_23 & 0.062 & 0.803 & -0.048 \\
\hline T2_55_34 & 0.154 & 0.695 & 0.003 \\
\hline T2_56_34 & 0.127 & 0.722 & 0.003 \\
\hline T2_59_34 & 0.159 & 0.690 & -0.013 \\
\hline T2_69_34 & 0.098 & 0.755 & -0.006 \\
\hline T2_77_34 & 0.263 & 0.608 & -0.029 \\
\hline T2_61_45 & 0.836 & 0.361 & -0.086 \\
\hline T2_61_56 & 0.079 & 0.778 & 0.028 \\
\hline T2_70_56 & 0.089 & 0.765 & 0.005 \\
\hline T2_71_56 & 0.009 & 0.924 & 0.003 \\
\hline T2_74_56 & 0.003 & 0.956 & 0.002 \\
\hline T2_79_56 & 0.044 & 0.833 & -0.027 \\
\hline T2_64_456 & 0.240 & 0.624 & -0.001 \\
\hline T2_71_456 & 0.507 & 0.476 & -0.055 \\
\hline T2_77_456 & 0.768 & 0.381 & -0.080 \\
\hline T2_81_456 & 0.636 & 0.425 & -0.066 \\
\hline T2_52_1 & 0.291 & 0.590 & 0.055 \\
\hline T2_54_1 & 0.001 & 0.981 & -0.031 \\
\hline T2_55_1 & 0.145 & 0.704 & 0.037 \\
\hline T2_57_1 & 0.008 & 0.928 & -0.007 \\
\hline T2_59_1 & 0.003 & 0.954 & -0.011 \\
\hline T2_60_1 & 0.008 & 0.928 & -0.009 \\
\hline T2_61_1 & 0.063 & 0.803 & 0.021 \\
\hline T2_62_1 & 0.024 & 0.876 & -0.042 \\
\hline T2_63_1 & 0.027 & 0.869 & 0.006 \\
\hline T2_64_1 & 0.000 & 0.996 & -0.014 \\
\hline T2_65_1 & 0.000 & 0.985 & -0.017 \\
\hline T2_66_1 & 0.000 & 0.995 & -0.014 \\
\hline
\end{tabular}




$\begin{array}{llll}\text { T2_67_1 } & 0.128 & 0.721 & 0.033 \\ \text { T2_68_1 } & 0.058 & 0.810 & 0.016 \\ \text { T2_69_1 } & 0.085 & 0.770 & 0.024 \\ \text { T2_70_1 } & 0.032 & 0.859 & 0.009 \\ \text { T2_71_1 } & 0.006 & 0.938 & -0.009 \\ \text { T2_72_1 } & 0.041 & 0.839 & 0.013 \\ \text { T2_73_1 } & 0.112 & 0.738 & 0.031 \\ \text { T2_74_1 } & 0.031 & 0.860 & 0.006 \\ \text { T2_76_1 } & 0.027 & 0.869 & 0.004 \\ \text { T2_77_1 } & 0.000 & 0.988 & -0.015 \\ \text { T2_78_1 } & 0.035 & 0.852 & 0.009 \\ \text { T2_79_1 } & 0.075 & 0.784 & 0.022 \\ \text { T2_80_1 } & 0.063 & 0.802 & 0.017 \\ \text { T2_81_1 } & 0.033 & 0.857 & 0.005 \\ \text { T2_82_1 } & 0.003 & 0.956 & -0.008 \\ \text { T2_83_1 } & 0.000 & 0.987 & -0.016 \\ \text { T2_84_1 } & 0.016 & 0.899 & 0.001 \\ \text { T2_85_1 } & 0.025 & 0.875 & 0.005 \\ \text { T2_51_2 } & 0.318 & 0.573 & 0.053 \\ \text { T2_52_2 } & 0.186 & 0.666 & 0.035 \\ \text { T2_54_2 } & 0.247 & 0.619 & 0.044 \\ \text { T2_55_2 } & 0.122 & 0.727 & 0.025 \\ \text { T2_58_2 } & 0.403 & 0.526 & 0.065 \\ \text { T2_61_2 } & 0.241 & 0.624 & 0.044 \\ \text { T2_62_2 } & 0.374 & 0.541 & 0.061 \\ \text { T2_64_2 } & 0.210 & 0.647 & 0.040 \\ \text { T2_65_2 } & 0.185 & 0.668 & 0.035 \\ \text { T2_66_22 } & 0.353 & 0.553 & 0.059 \\ \text { T2_67_2 } & 0.062 & 0.803 & 0.010 \\ \text { T2_68_2 } & 0.137 & 0.712 & 0.027 \\ \text { T2_69_2 } & 0.230 & 0.632 & 0.045 \\ \text { T2_73_2 } & 0.212 & 0.645 & 0.041 \\ \text { T2_74_2 } & 0.061 & 0.805 & 0.011 \\ \text { T2_83_2 } & 0.209 & 0.647 & 0.040 \\ \text { T2_77_2 } & 0.076 & 0.782 & 0.015 \\ \text { T2_79_2 } & 0.303 & 0.582 & 0.054 \\ \text { T2_2 } & 0.390 & 0.532 & 0.062 \\ \text { T2 } & 0.002 & 0.961 & -0.019\end{array}$




$\begin{array}{llll}\text { T2_72_2 } & 0.140 & 0.709 & 0.028 \\ \text { T2_82_2 } & 0.093 & 0.760 & 0.017 \\ \text { T2_51_3 } & 0.000 & 0.987 & 0.002 \\ \text { T2_52_3 } & 0.228 & 0.633 & -0.059 \\ \text { T2_53_3 } & 0.067 & 0.795 & -0.031 \\ \text { T2_54_3 } & 0.046 & 0.830 & -0.024 \\ \text { T2_61_3 } & 0.350 & 0.554 & -0.077 \\ \text { T2_63_3 } & 0.029 & 0.865 & -0.014 \\ \text { T2_64_3 } & 0.136 & 0.712 & -0.046 \\ \text { T2_66_3 } & 0.185 & 0.668 & -0.051 \\ \text { T2_67_3 } & 0.098 & 0.754 & -0.038 \\ \text { T2_68_3 } & 0.059 & 0.809 & -0.025 \\ \text { T2_70_3 } & 0.104 & 0.747 & -0.040 \\ \text { T2_71_3 } & 0.021 & 0.885 & -0.017 \\ \text { T2_74_3 } & 0.015 & 0.904 & -0.009 \\ \text { T2_76_3 } & 0.002 & 0.961 & 0.020 \\ \text { T2_78_3 } & 0.000 & 0.999 & 0.013 \\ \text { T2_79_3 } & 0.036 & 0.850 & -0.017 \\ \text { T2_81_3 } & 0.072 & 0.788 & -0.031 \\ \text { T2_82_3 } & 0.020 & 0.889 & -0.012 \\ \text { T2_84_3 } & 0.124 & 0.725 & -0.043 \\ \text { T2_57_3 } & 0.087 & 0.769 & -0.041 \\ \text { T2_62_3 } & 0.000 & 0.989 & 0.009 \\ \text { T2_73_3 } & 0.039 & 0.844 & -0.019 \\ \text { T2_75_3 } & 0.017 & 0.896 & -0.010 \\ \text { T2_83_3 } & 0.121 & 0.728 & -0.042 \\ \text { T2_85_3 } & 0.045 & 0.832 & -0.023 \\ \text { T2_51_4 } & 0.019 & 0.892 & 0.011 \\ \text { T2_53_4 } & 0.081 & 0.776 & -0.008 \\ \text { T2_60_4 } & 0.112 & 0.738 & -0.016 \\ \text { T2_62_4 } & 0.034 & 0.854 & 0.006 \\ \text { T2_63_4 } & 0.227 & 0.634 & -0.035 \\ \text { T2_66_4 } & 0.010 & 0.921 & 0.016 \\ \text { T2_67_4 } & 0.142 & 0.706 & -0.020 \\ \text { T2_68_4 } & 0.283 & 0.595 & -0.044 \\ \text { T2_69_4 } & 0.089 & 0.766 & -0.010 \\ \text { T2772_4 } & 0.044 & 0.834 & 0.003 \\ \text { T279_4 } & 0.108 & 0.743 & -0.015 \\ \text { T244 } & 0.155 & 0.694 & -0.027 \\ \text { T2 } & 0.049 & 0.826 & 0.000 \\ \text { T2 } & 0.130 & 0.719 & -0.022\end{array}$




\begin{tabular}{|c|c|c|c|}
\hline T2_80_4 & 0.144 & 0.705 & -0.022 \\
\hline T2_82_4 & 0.110 & 0.740 & -0.016 \\
\hline T2_83_4 & 0.180 & 0.671 & -0.031 \\
\hline T2_84_4 & 0.205 & 0.651 & -0.034 \\
\hline T2_52_4 & 0.000 & 0.988 & 0.022 \\
\hline T2_58_4 & 0.074 & 0.786 & -0.006 \\
\hline T2_65_4 & 0.479 & 0.489 & -0.052 \\
\hline T2_70_4 & 0.070 & 0.792 & -0.024 \\
\hline T2_74_4 & 0.216 & 0.642 & -0.035 \\
\hline T2_76_4 & 0.281 & 0.596 & -0.046 \\
\hline T2_85_4 & 0.119 & 0.730 & -0.020 \\
\hline T2_52_5 & 0.025 & 0.875 & -0.011 \\
\hline T2_55_5 & 0.082 & 0.774 & -0.030 \\
\hline T2_57_5 & 0.008 & 0.931 & -0.001 \\
\hline T2_59_5 & 0.001 & 0.976 & 0.007 \\
\hline T2_62_5 & 0.050 & 0.824 & -0.016 \\
\hline T2_63_5 & 0.037 & 0.848 & -0.017 \\
\hline T2_64_5 & 0.116 & 0.734 & -0.040 \\
\hline T2_65_5 & 0.000 & 0.988 & 0.012 \\
\hline T2_66_5 & 0.002 & 0.968 & 0.001 \\
\hline T2_67_5 & 0.079 & 0.779 & -0.029 \\
\hline T2_69_5 & 0.031 & 0.860 & -0.016 \\
\hline T2_80_5 & 0.051 & 0.821 & -0.029 \\
\hline T2_81_5 & 0.189 & 0.663 & -0.054 \\
\hline T2_84_5 & 0.144 & 0.704 & -0.042 \\
\hline T2_85_5 & 0.044 & 0.835 & -0.021 \\
\hline T2_51_5 & 0.009 & 0.924 & 0.028 \\
\hline T2_53_5 & 0.020 & 0.887 & -0.003 \\
\hline T2_54_5 & 0.043 & 0.835 & -0.015 \\
\hline T2_58_5 & 0.009 & 0.926 & -0.024 \\
\hline T2_72_5 & 0.001 & 0.979 & -0.002 \\
\hline T2_76_5 & 0.046 & 0.830 & -0.028 \\
\hline T2_77_5 & 0.000 & 0.995 & -0.013 \\
\hline T2_78_5 & 0.136 & 0.712 & -0.045 \\
\hline T2_82_5 & 0.067 & 0.796 & -0.027 \\
\hline T2_83_5 & 0.038 & 0.845 & -0.024 \\
\hline T2_52_6 & 0.000 & 0.992 & 0.008 \\
\hline T2_53_6 & 0.022 & 0.882 & 0.028 \\
\hline T2_54_6 & 0.015 & 0.901 & 0.025 \\
\hline T2_59_6 & 0.082 & 0.775 & 0.044 \\
\hline T2_60_6 & 0.000 & 0.990 & -0.002 \\
\hline
\end{tabular}




\begin{tabular}{lllc} 
T2_63_6 & 0.035 & 0.851 & 0.032 \\
T2_65_6 & 0.012 & 0.914 & -0.011 \\
T2_66_6 & 0.034 & 0.854 & 0.030 \\
T2_73_6 & 0.000 & 1.000 & 0.003 \\
T2_79_6 & 0.035 & 0.851 & 0.029 \\
T2_82_6 & 0.007 & 0.932 & -0.010 \\
T2_83_6 & 0.007 & 0.934 & 0.022 \\
T2_51_6 & 0.001 & 0.978 & -0.003 \\
T2_56_6 & 0.000 & 0.995 & 0.002 \\
T2_57_6 & 0.017 & 0.895 & 0.013 \\
T2_58_6 & 0.023 & 0.879 & -0.018 \\
T2_61_6 & 0.000 & 0.993 & 0.013 \\
T2_62_6 & 0.003 & 0.956 & -0.003 \\
T2_67_6 & 0.012 & 0.911 & 0.007 \\
T2_69_6 & 0.000 & 0.995 & 0.010 \\
T2_70_6 & 0.006 & 0.938 & 0.020 \\
T2_77_6 & 0.055 & 0.815 & 0.027 \\
T2_78_6 & 0.008 & 0.929 & 0.002 \\
T2_81_6 & 0.007 & 0.934 & -0.003 \\
T2_84_6 & 0.069 & 0.793 & -0.017 \\
T2_85_6 & 0.003 & 0.954 & 0.019 \\
\hline
\end{tabular}


Table 34

Task 2 DIF: White vs. Sample

\begin{tabular}{lccc}
\hline ItemID & ChiSq & pvalue & EffectSize \\
\hline T2_51_12 & 0.056 & 0.813 & 0.042 \\
T2_53_12 & 0.048 & 0.828 & 0.034 \\
T2_56_12 & 0.016 & 0.900 & 0.027 \\
T2_58_12 & 0.027 & 0.870 & -0.021 \\
T2_75_12 & 0.005 & 0.943 & 0.019 \\
T2_60_23 & 0.201 & 0.654 & 0.094 \\
T2_70_23 & 0.000 & 0.993 & 0.018 \\
T2_75_23 & 0.023 & 0.881 & -0.002 \\
T2_78_23 & 0.007 & 0.933 & 0.006 \\
T2_84_23 & 0.624 & 0.430 & -0.087 \\
T2_55_34 & 0.265 & 0.607 & -0.013 \\
T2_56_34 & 0.029 & 0.866 & 0.021 \\
T2_59_34 & 0.514 & 0.473 & -0.057 \\
T2_69_34 & 0.056 & 0.814 & 0.021 \\
T2_77_34 & 0.157 & 0.692 & -0.006 \\
T2_61_45 & 0.115 & 0.734 & 0.015 \\
T2_61_56 & 0.362 & 0.547 & 0.002 \\
T2_70_56 & 0.032 & 0.859 & -0.045 \\
T2_71_56 & 0.041 & 0.839 & -0.027 \\
T2_74_56 & 0.933 & 0.334 & 0.080 \\
T2_79_56 & 0.149 & 0.699 & -0.006 \\
T2_64_456 & 0.112 & 0.738 & 0.009 \\
T2_71_456 & 0.069 & 0.792 & 0.019 \\
T2_77_456 & 0.730 & 0.393 & 0.079 \\
T2_81_456 & 0.194 & 0.660 & 0.037 \\
T2_52_1 & 0.029 & 0.866 & 0.024 \\
T2_54_1 & 0.068 & 0.794 & 0.036 \\
T2_55_1 & 0.003 & 0.955 & 0.009 \\
T2_57_1 & 0.160 & 0.689 & 0.060 \\
T2_59_1 & 0.043 & 0.836 & 0.031 \\
T2_60_1 & 0.144 & 0.705 & 0.059 \\
T2_61_1 & 0.060 & 0.807 & 0.035 \\
T2_62_1 & 0.008 & 0.929 & 0.014 \\
T2_63_1 & 0.014 & 0.907 & 0.020 \\
T2_64_1 & 0.003 & 0.960 & 0.009 \\
T2_65_1 & 0.025 & 0.876 & 0.029 \\
T2_66_1 & 0.006 & 0.940 & -0.014 \\
\end{tabular}




\begin{tabular}{|c|c|c|c|}
\hline T2_67_1 & 0.003 & 0.958 & 0.008 \\
\hline T2_68_1 & 0.029 & 0.866 & 0.025 \\
\hline T2_69_1 & 0.006 & 0.941 & 0.012 \\
\hline T2_70_1 & 0.004 & 0.948 & -0.010 \\
\hline T2_71_1 & 0.064 & 0.800 & -0.035 \\
\hline T2_72_1 & 0.000 & 0.994 & 0.003 \\
\hline T2_73_1 & 0.010 & 0.922 & 0.013 \\
\hline T2_74_1 & 0.011 & 0.915 & 0.021 \\
\hline T2_76_1 & 0.081 & 0.776 & 0.045 \\
\hline T2_77_1 & 0.016 & 0.900 & 0.022 \\
\hline T2_78_1 & 0.012 & 0.913 & 0.012 \\
\hline T2_79_1 & 0.001 & 0.979 & 0.005 \\
\hline T2_80_1 & 0.122 & 0.727 & 0.045 \\
\hline T2_81_1 & 0.001 & 0.973 & 0.009 \\
\hline T2_82_1 & 0.143 & 0.706 & 0.057 \\
\hline T2_83_1 & 0.071 & 0.790 & 0.044 \\
\hline T2_84_1 & 0.016 & 0.901 & 0.023 \\
\hline T2_85_1 & 0.007 & 0.932 & 0.013 \\
\hline T2_51_2 & 0.001 & 0.978 & 0.009 \\
\hline T2_52_2 & 0.072 & 0.789 & -0.038 \\
\hline T2_54_2 & 0.000 & 0.985 & 0.009 \\
\hline T2_55_2 & 0.000 & 0.996 & 0.000 \\
\hline T2_58_2 & 0.142 & 0.707 & 0.059 \\
\hline T2_61_2 & 0.003 & 0.955 & 0.013 \\
\hline T2_62_2 & 0.072 & 0.788 & -0.039 \\
\hline T2_64_2 & 0.082 & 0.775 & 0.045 \\
\hline T2_65_2 & 0.106 & 0.745 & 0.053 \\
\hline T2_66_22 & 0.107 & 0.743 & 0.053 \\
\hline T2_67_2 & 0.058 & 0.809 & 0.041 \\
\hline T2_68_2 & 0.023 & 0.880 & -0.022 \\
\hline T2_69_2 & 0.111 & 0.739 & -0.049 \\
\hline T2_73_2 & 0.120 & 0.729 & 0.055 \\
\hline T2_74_2 & 0.079 & 0.778 & 0.046 \\
\hline T2_76_2 & 0.000 & 0.993 & 0.006 \\
\hline T2_77_2 & 0.002 & 0.967 & -0.005 \\
\hline T2_79_2 & 0.014 & 0.907 & 0.023 \\
\hline T2_80_2 & 0.010 & 0.920 & -0.016 \\
\hline T2_81_2 & 0.002 & 0.967 & 0.012 \\
\hline T2_83_2 & 0.083 & 0.773 & 0.046 \\
\hline T2_85_2 & 0.002 & 0.967 & 0.011 \\
\hline T2_63_2 & 0.089 & 0.766 & -0.041 \\
\hline
\end{tabular}




\begin{tabular}{|c|c|c|c|}
\hline T2_72_2 & 0.000 & 0.988 & 0.001 \\
\hline T2_82_2 & 0.010 & 0.922 & 0.018 \\
\hline T2_51_3 & 0.040 & 0.841 & -0.013 \\
\hline T2_52_3 & 0.000 & 0.991 & 0.018 \\
\hline T2_53_3 & 0.021 & 0.884 & 0.041 \\
\hline T2_54_3 & 0.000 & 0.988 & 0.014 \\
\hline T2_61_3 & 0.070 & 0.792 & -0.019 \\
\hline T2_63_3 & 0.169 & 0.681 & -0.046 \\
\hline T2_64_3 & 0.047 & 0.828 & 0.050 \\
\hline T2_66_3 & 0.000 & 0.985 & 0.026 \\
\hline T2_67_3 & 0.007 & 0.932 & 0.034 \\
\hline T2_68_3 & 0.018 & 0.893 & -0.002 \\
\hline T2_70_3 & 0.058 & 0.809 & 0.058 \\
\hline T2_71_3 & 0.000 & 0.984 & 0.014 \\
\hline T2_74_3 & 0.003 & 0.959 & 0.029 \\
\hline T2_76_3 & 0.050 & 0.824 & -0.016 \\
\hline T2_78_3 & 0.000 & 0.985 & 0.012 \\
\hline T2_79_3 & 0.148 & 0.701 & -0.039 \\
\hline T2_81_3 & 0.142 & 0.707 & -0.041 \\
\hline T2_82_3 & 0.005 & 0.943 & 0.005 \\
\hline T2_84_3 & 0.082 & 0.774 & -0.023 \\
\hline T2_57_3 & 0.077 & 0.782 & -0.013 \\
\hline T2_62_3 & 0.013 & 0.909 & 0.002 \\
\hline T2_73_3 & 0.094 & 0.760 & -0.029 \\
\hline T2_75_3 & 0.035 & 0.852 & -0.014 \\
\hline T2_83_3 & 0.050 & 0.823 & -0.018 \\
\hline T2_85_3 & 0.039 & 0.843 & -0.013 \\
\hline T2_51_4 & 0.160 & 0.689 & -0.037 \\
\hline T2_53_4 & 0.002 & 0.969 & 0.014 \\
\hline T2_60_4 & 0.000 & 0.995 & 0.017 \\
\hline T2_62_4 & 0.001 & 0.977 & 0.014 \\
\hline T2_63_4 & 0.001 & 0.972 & 0.030 \\
\hline T2_66_4 & 0.116 & 0.734 & -0.030 \\
\hline T2_67_4 & 0.093 & 0.761 & -0.025 \\
\hline T2_68_4 & 0.050 & 0.824 & -0.011 \\
\hline T2_69_4 & 0.032 & 0.859 & -0.006 \\
\hline T2_72_4 & 0.171 & 0.679 & -0.040 \\
\hline T2_73_4 & 0.034 & 0.855 & -0.006 \\
\hline T2_75_4 & 0.042 & 0.838 & -0.011 \\
\hline T2_78_4 & 0.248 & 0.619 & -0.051 \\
\hline T2_79_4 & 0.000 & 0.988 & 0.022 \\
\hline
\end{tabular}




$\begin{array}{llll}\text { T2_80_4 } & 0.008 & 0.930 & 0.007 \\ \text { T2_82_4 } & 0.068 & 0.794 & -0.019 \\ \text { T2_83_4 } & 0.031 & 0.861 & -0.006 \\ \text { T2_84_4 } & 0.000 & 0.997 & 0.019 \\ \text { T2_52_4 } & 0.010 & 0.919 & 0.002 \\ \text { T2_58_4 } & 0.028 & 0.866 & -0.004 \\ \text { T2_65_4 } & 0.392 & 0.531 & -0.070 \\ \text { T2_70_4 } & 0.077 & 0.781 & -0.005 \\ \text { T2_74_4 } & 0.029 & 0.865 & -0.006 \\ \text { T2_76_4 } & 0.041 & 0.840 & -0.009 \\ \text { T2_85_4 } & 0.036 & 0.850 & -0.008 \\ \text { T2_52_5 } & 0.595 & 0.441 & 0.052 \\ \text { T2_55_5 } & 0.367 & 0.545 & 0.032 \\ \text { T2_57_5 } & 0.135 & 0.714 & -0.008 \\ \text { T2_59_5 } & 0.117 & 0.732 & -0.013 \\ \text { T2_62_5 } & 0.233 & 0.630 & 0.017 \\ \text { T2_63_5 } & 0.219 & 0.640 & 0.010 \\ \text { T2_64_5 } & 0.359 & 0.549 & 0.026 \\ \text { T2_65_5 } & 0.809 & 0.368 & 0.073 \\ \text { T2_66_5 } & 0.231 & 0.631 & 0.016 \\ \text { T2_67_5 } & 0.507 & 0.477 & 0.045 \\ \text { T2_69_5 } & 0.119 & 0.730 & -0.011 \\ \text { T2_80_5 } & 0.360 & 0.548 & 0.026 \\ \text { T2_81_5 } & 0.110 & 0.740 & -0.007 \\ \text { T2_84_5 } & 0.477 & 0.490 & 0.048 \\ \text { T2_85_5 } & 0.645 & 0.422 & 0.061 \\ \text { T2_51_5 } & 0.005 & 0.942 & -0.051 \\ \text { T2_53_5 } & 0.514 & 0.474 & -0.011 \\ \text { T2_54_5 } & 0.007 & 0.934 & -0.038 \\ \text { T2_58_5 } & 0.664 & 0.415 & 0.015 \\ \text { T2_72_5 } & 0.317 & 0.574 & -0.003 \\ \text { T2_76_5 } & 0.196 & 0.658 & 0.013 \\ \text { T2_77_5 } & 0.000 & 0.988 & -0.037 \\ \text { T2_78_5 } & 0.105 & 0.746 & -0.019 \\ \text { T2_82_5 } & 0.675 & 0.411 & 0.067 \\ \text { T2_83_5 } & 0.755 & 0.385 & 0.068 \\ \text { T2_52_6 } & 0.041 & 0.839 & -0.030 \\ \text { T2_53_6 } & 0.000 & 0.997 & -0.006 \\ \text { T2_54_6 } & 0.043 & 0.836 & 0.026 \\ \text { T2_59_6 } & 0.001 & 0.977 & 0.007 \\ \text { T2_60_6 } & 0.003 & 0.959 & 0.005\end{array}$




\begin{tabular}{llll} 
T2_63_6 & 0.019 & 0.892 & 0.019 \\
T2_65_6 & 0.015 & 0.904 & -0.021 \\
T2_66_6 & 0.005 & 0.946 & -0.015 \\
T2_73_6 & 0.009 & 0.925 & 0.015 \\
T2_79_6 & 0.020 & 0.887 & -0.021 \\
T2_82_6 & 0.000 & 0.998 & -0.001 \\
T2_83_6 & 0.000 & 0.991 & -0.006 \\
T2_51_6 & 0.000 & 0.989 & 0.000 \\
T2_56_6 & 0.024 & 0.876 & -0.007 \\
T2_57_6 & 0.009 & 0.923 & 0.008 \\
T2_58_6 & 0.000 & 0.994 & 0.000 \\
T2_61_6 & 0.002 & 0.963 & -0.010 \\
T2_62_6 & 0.001 & 0.977 & -0.004 \\
T2_67_6 & 0.016 & 0.901 & -0.012 \\
T2_69_6 & 0.004 & 0.950 & -0.002 \\
T2_70_6 & 0.056 & 0.814 & 0.024 \\
T2_77_6 & 0.034 & 0.854 & 0.010 \\
T2_78_6 & 0.036 & 0.850 & -0.023 \\
T2_81_6 & 0.000 & 0.998 & -0.001 \\
T2_84_6 & 0.071 & 0.790 & 0.041 \\
T2_85_6 & 0.000 & 0.993 & 0.007 \\
\hline
\end{tabular}


Table 35

Task 2 DIF: Black vs. Sample

\begin{tabular}{|c|c|c|c|}
\hline ItemID & ChiSq & pvalue & EffectSize \\
\hline T2_51_12 & 0.000 & 0.999 & -0.027 \\
\hline T2_53_12 & 0.000 & 0.999 & -0.016 \\
\hline T2_56_12 & 0.001 & 0.980 & -0.016 \\
\hline T2_58_12 & 0.091 & 0.762 & 0.020 \\
\hline T2_75_12 & 0.005 & 0.942 & -0.006 \\
\hline T2_60_23 & 0.146 & 0.703 & -0.101 \\
\hline T2_70_23 & 0.271 & 0.603 & 0.037 \\
\hline T2_75_23 & 0.031 & 0.861 & -0.009 \\
\hline T2_78_23 & 0.136 & 0.713 & 0.015 \\
\hline T2_84_23 & 1.037 & 0.309 & 0.108 \\
\hline T2_55_34 & 0.747 & 0.387 & 0.022 \\
\hline T2_56_34 & 0.029 & 0.864 & -0.019 \\
\hline T2_59_34 & 0.239 & 0.625 & 0.027 \\
\hline T2_69_34 & 0.027 & 0.870 & -0.030 \\
\hline T2_77_34 & 0.008 & 0.929 & -0.033 \\
\hline T2_61_45 & 0.196 & 0.658 & -0.009 \\
\hline T2_61_56 & 0.640 & 0.424 & 0.023 \\
\hline T2_70_56 & 0.002 & 0.966 & 0.070 \\
\hline T2_71_56 & 0.007 & 0.933 & 0.044 \\
\hline T2_74_56 & 0.722 & 0.396 & -0.068 \\
\hline T2_79_56 & 0.208 & 0.648 & -0.001 \\
\hline T2_64_456 & 0.000 & 0.995 & 0.050 \\
\hline T2_71_456 & 0.021 & 0.885 & -0.002 \\
\hline T2_77_456 & 0.180 & 0.671 & -0.036 \\
\hline T2_81_456 & 0.186 & 0.666 & -0.037 \\
\hline T2_52_1 & 0.006 & 0.938 & -0.004 \\
\hline T2_54_1 & 0.161 & 0.688 & -0.047 \\
\hline T2_55_1 & 0.018 & 0.893 & -0.011 \\
\hline T2_57_1 & 0.497 & 0.481 & -0.094 \\
\hline T2_59_1 & 0.035 & 0.851 & -0.024 \\
\hline T2_60_1 & 0.304 & 0.582 & -0.075 \\
\hline T2_61_1 & 0.042 & 0.838 & -0.021 \\
\hline T2_62_1 & 0.200 & 0.655 & -0.056 \\
\hline T2_63_1 & 0.121 & 0.728 & -0.045 \\
\hline T2_64_1 & 0.090 & 0.764 & -0.036 \\
\hline T2_65_1 & 0.197 & 0.657 & -0.061 \\
\hline T2_66_1 & 0.024 & 0.876 & -0.012 \\
\hline
\end{tabular}




\begin{tabular}{|c|c|c|c|}
\hline T2_67_1 & 0.117 & 0.733 & -0.038 \\
\hline T2_68_1 & 0.188 & 0.665 & -0.055 \\
\hline T2_69_1 & 0.007 & 0.936 & -0.001 \\
\hline T2_70_1 & 0.004 & 0.948 & -0.002 \\
\hline T2_71_1 & 0.000 & 0.997 & 0.011 \\
\hline T2_72_1 & 0.091 & 0.762 & -0.037 \\
\hline T2_73_1 & 0.007 & 0.931 & -0.003 \\
\hline T2_74_1 & 0.066 & 0.797 & -0.033 \\
\hline T2_76_1 & 0.108 & 0.743 & -0.041 \\
\hline T2_77_1 & 0.127 & 0.721 & -0.047 \\
\hline T2_78_1 & 0.027 & 0.870 & -0.011 \\
\hline T2_79_1 & 0.022 & 0.882 & -0.013 \\
\hline T2_80_1 & 0.124 & 0.725 & -0.041 \\
\hline T2_81_1 & 0.062 & 0.804 & -0.031 \\
\hline T2_82_1 & 0.221 & 0.638 & -0.064 \\
\hline T2_83_1 & 0.183 & 0.669 & -0.058 \\
\hline T2_84_1 & 0.145 & 0.703 & -0.050 \\
\hline T2_85_1 & 0.036 & 0.849 & -0.019 \\
\hline T2_51_2 & 0.000 & 0.998 & -0.028 \\
\hline T2_52_2 & 0.238 & 0.626 & 0.046 \\
\hline T2_54_2 & 0.057 & 0.811 & 0.009 \\
\hline T2_55_2 & 0.077 & 0.782 & 0.018 \\
\hline T2_58_2 & 0.004 & 0.953 & -0.017 \\
\hline T2_61_2 & 0.000 & 0.999 & -0.032 \\
\hline T2_62_2 & 0.190 & 0.663 & 0.038 \\
\hline T2_64_2 & 0.005 & 0.945 & -0.015 \\
\hline T2_65_2 & 0.006 & 0.941 & -0.044 \\
\hline T2_66_22 & 0.060 & 0.806 & -0.068 \\
\hline T2_67_2 & 0.000 & 1.000 & -0.027 \\
\hline T2_68_2 & 0.138 & 0.710 & 0.029 \\
\hline T2_69_2 & 0.278 & 0.598 & 0.053 \\
\hline T2_73_2 & 0.000 & 0.990 & -0.033 \\
\hline T2_74_2 & 0.002 & 0.964 & -0.020 \\
\hline T2_76_2 & 0.088 & 0.767 & 0.020 \\
\hline T2_77_2 & 0.107 & 0.744 & 0.022 \\
\hline T2_79_2 & 0.073 & 0.787 & 0.013 \\
\hline T2_80_2 & 0.110 & 0.740 & 0.025 \\
\hline T2_81_2 & 0.065 & 0.799 & 0.009 \\
\hline T2_83_2 & 0.008 & 0.928 & -0.014 \\
\hline T2_85_2 & 0.098 & 0.754 & 0.019 \\
\hline T2_63_2 & 0.130 & 0.719 & 0.024 \\
\hline
\end{tabular}




\begin{tabular}{|c|c|c|c|}
\hline T2_72_2 & 0.106 & 0.745 & 0.025 \\
\hline T2_82_2 & 0.037 & 0.848 & 0.004 \\
\hline T2_51_3 & 0.000 & 0.998 & -0.009 \\
\hline T2_52_3 & 0.003 & 0.955 & 0.002 \\
\hline T2_53_3 & 0.141 & 0.708 & -0.066 \\
\hline T2_54_3 & 0.001 & 0.983 & -0.004 \\
\hline T2_61_3 & 0.005 & 0.946 & 0.001 \\
\hline T2_63_3 & 0.055 & 0.815 & 0.030 \\
\hline T2_64_3 & 0.168 & 0.682 & -0.070 \\
\hline T2_66_3 & 0.026 & 0.872 & -0.036 \\
\hline T2_67_3 & 0.142 & 0.706 & -0.067 \\
\hline T2_68_3 & 0.016 & 0.901 & -0.030 \\
\hline T2_70_3 & 0.163 & 0.686 & -0.072 \\
\hline T2_71_3 & 0.005 & 0.942 & -0.022 \\
\hline T2_74_3 & 0.082 & 0.775 & -0.055 \\
\hline T2_76_3 & 0.019 & 0.890 & -0.031 \\
\hline T2_78_3 & 0.000 & 0.990 & -0.007 \\
\hline T2_79_3 & 0.004 & 0.949 & 0.005 \\
\hline T2_81_3 & 0.008 & 0.928 & 0.010 \\
\hline T2_82_3 & 0.011 & 0.916 & -0.025 \\
\hline T2_84_3 & 0.006 & 0.937 & 0.004 \\
\hline T2_57_3 & 0.020 & 0.889 & -0.006 \\
\hline T2_62_3 & 0.003 & 0.954 & -0.021 \\
\hline T2_73_3 & 0.029 & 0.866 & 0.021 \\
\hline T2_75_3 & 0.001 & 0.979 & 0.001 \\
\hline T2_83_3 & 0.004 & 0.949 & 0.006 \\
\hline T2_85_3 & 0.000 & 1.000 & -0.004 \\
\hline T2_51_4 & 0.407 & 0.524 & 0.063 \\
\hline T2_53_4 & 0.047 & 0.829 & 0.000 \\
\hline T2_60_4 & 0.007 & 0.931 & -0.016 \\
\hline T2_62_4 & 0.004 & 0.950 & -0.018 \\
\hline T2_63_4 & 0.045 & 0.832 & 0.000 \\
\hline T2_66_4 & 0.151 & 0.698 & 0.027 \\
\hline T2_67_4 & 0.109 & 0.741 & 0.019 \\
\hline T2_68_4 & 0.067 & 0.796 & 0.007 \\
\hline T2_69_4 & 0.078 & 0.780 & 0.010 \\
\hline T2_72_4 & 0.108 & 0.743 & 0.019 \\
\hline T2_73_4 & 0.104 & 0.747 & 0.017 \\
\hline T2_75_4 & 0.156 & 0.693 & 0.029 \\
\hline T2_78_4 & 0.394 & 0.530 & 0.061 \\
\hline T2_79_4 & 0.017 & 0.898 & -0.011 \\
\hline
\end{tabular}




\begin{tabular}{|c|c|c|c|}
\hline T2_80_4 & 0.003 & 0.960 & -0.025 \\
\hline T2_82_4 & 0.131 & 0.718 & 0.024 \\
\hline T2_83_4 & 0.209 & 0.648 & 0.038 \\
\hline T2_84_4 & 0.053 & 0.818 & 0.004 \\
\hline T2_52_4 & 0.226 & 0.634 & 0.032 \\
\hline T2_58_4 & 0.038 & 0.845 & -0.002 \\
\hline T2_65_4 & 0.364 & 0.546 & 0.064 \\
\hline T2_70_4 & 0.155 & 0.694 & 0.027 \\
\hline T2_74_4 & 0.150 & 0.699 & 0.027 \\
\hline T2_76_4 & 0.201 & 0.654 & 0.036 \\
\hline T2_85_4 & 0.135 & 0.713 & 0.024 \\
\hline T2_52_5 & 0.802 & 0.371 & -0.049 \\
\hline T2_55_5 & 0.646 & 0.422 & -0.040 \\
\hline T2_57_5 & 0.201 & 0.654 & 0.022 \\
\hline T2_59_5 & 0.073 & 0.788 & 0.048 \\
\hline T2_62_5 & 0.285 & 0.593 & 0.001 \\
\hline T2_63_5 & 0.194 & 0.660 & 0.015 \\
\hline T2_64_5 & 0.500 & 0.480 & -0.017 \\
\hline T2_65_5 & 1.302 & 0.254 & -0.089 \\
\hline T2_66_5 & 0.207 & 0.649 & 0.006 \\
\hline T2_67_5 & 0.784 & 0.376 & -0.051 \\
\hline T2_69_5 & 0.141 & 0.707 & 0.032 \\
\hline T2_80_5 & 0.561 & 0.454 & -0.028 \\
\hline T2_81_5 & 0.180 & 0.672 & 0.017 \\
\hline T2_84_5 & 0.947 & 0.331 & -0.070 \\
\hline T2_85_5 & 1.210 & 0.271 & -0.083 \\
\hline T2_51_5 & 0.032 & 0.859 & 0.062 \\
\hline T2_53_5 & 0.560 & 0.454 & 0.026 \\
\hline T2_54_5 & 0.000 & 0.999 & 0.086 \\
\hline T2_58_5 & 0.983 & 0.321 & 0.007 \\
\hline T2_72_5 & 0.274 & 0.601 & 0.032 \\
\hline T2_76_5 & 0.368 & 0.544 & 0.012 \\
\hline T2_77_5 & 0.050 & 0.822 & 0.047 \\
\hline T2_78_5 & 0.191 & 0.663 & 0.027 \\
\hline T2_82_5 & 1.208 & 0.272 & -0.089 \\
\hline T2_83_5 & 0.671 & 0.413 & -0.043 \\
\hline T2_52_6 & 0.115 & 0.735 & 0.033 \\
\hline T2_53_6 & 0.053 & 0.818 & 0.022 \\
\hline T2_54_6 & 0.026 & 0.871 & 0.006 \\
\hline T2_59_6 & 0.025 & 0.873 & 0.008 \\
\hline T2_60_6 & 0.008 & 0.929 & -0.001 \\
\hline
\end{tabular}




\begin{tabular}{llll} 
T2_63_6 & 0.057 & 0.811 & 0.018 \\
T2_65_6 & 0.067 & 0.796 & 0.027 \\
T2_66_6 & 0.007 & 0.934 & 0.000 \\
T2_73_6 & 0.041 & 0.841 & 0.011 \\
T2_79_6 & 0.018 & 0.895 & 0.007 \\
T2_82_6 & 0.034 & 0.854 & 0.014 \\
T2_83_6 & 0.007 & 0.935 & -0.001 \\
T2_51_6 & 0.014 & 0.906 & 0.003 \\
T2_56_6 & 0.090 & 0.764 & 0.008 \\
T2_57_6 & 0.022 & 0.883 & -0.002 \\
T2_58_6 & 0.026 & 0.872 & 0.006 \\
T2_61_6 & 0.094 & 0.760 & 0.026 \\
T2_62_6 & 0.021 & 0.884 & 0.001 \\
T2_67_6 & 0.062 & 0.804 & 0.007 \\
T2_69_6 & 0.008 & 0.930 & -0.001 \\
T2_70_6 & 0.029 & 0.864 & -0.001 \\
T2_77_6 & 0.003 & 0.957 & -0.014 \\
T2_78_6 & 0.137 & 0.711 & 0.023 \\
T2_81_6 & 0.004 & 0.948 & 0.000 \\
T2_84_6 & 0.027 & 0.868 & -0.044 \\
T2_85_6 & 0.006 & 0.940 & -0.005 \\
\hline
\end{tabular}


Table 36

Task 2 DIF: Latinx vs. Sample

\begin{tabular}{|c|c|c|c|}
\hline ItemID & ChiSq & pvalue & EffectSize \\
\hline T2_51_12 & 0.117 & 0.733 & 0.090 \\
\hline T2_53_12 & 0.017 & 0.895 & 0.020 \\
\hline T2_56_12 & 0.086 & 0.769 & 0.065 \\
\hline T2_58_12 & 0.328 & 0.567 & 0.157 \\
\hline T2_75_12 & 0.134 & 0.715 & 0.088 \\
\hline T2_60_23 & 0.394 & 0.530 & 0.187 \\
\hline T2_70_23 & 0.086 & 0.769 & -0.107 \\
\hline T2_75_23 & 0.141 & 0.707 & 0.123 \\
\hline T2_78_23 & 0.028 & 0.867 & 0.060 \\
\hline T2_84_23 & 0.048 & 0.827 & 0.071 \\
\hline T2_55_34 & 0.190 & 0.663 & -0.032 \\
\hline T2_56_34 & 0.107 & 0.744 & -0.224 \\
\hline T2_59_34 & 0.055 & 0.815 & -0.014 \\
\hline T2_69_34 & 0.000 & 0.986 & -0.119 \\
\hline T2_77_34 & 0.008 & 0.929 & -0.071 \\
\hline T2_61_45 & 0.161 & 0.689 & 0.015 \\
\hline T2_61_56 & 0.001 & 0.979 & -0.020 \\
\hline T2_70_56 & 0.162 & 0.688 & 0.009 \\
\hline T2_71_56 & 0.115 & 0.735 & 0.011 \\
\hline T2_74_56 & 0.336 & 0.562 & -0.110 \\
\hline T2_79_56 & 0.086 & 0.770 & 0.007 \\
\hline T2_64_456 & 0.003 & 0.956 & -0.125 \\
\hline T2_71_456 & 0.455 & 0.500 & -0.163 \\
\hline T2_77_456 & 0.131 & 0.717 & -0.089 \\
\hline T2_81_456 & 0.037 & 0.848 & -0.040 \\
\hline T2_52_1 & 0.029 & 0.864 & 0.022 \\
\hline T2_54_1 & 0.020 & 0.889 & 0.004 \\
\hline T2_55_1 & 0.098 & 0.754 & 0.062 \\
\hline T2_57_1 & 0.142 & 0.706 & 0.068 \\
\hline T2_59_1 & 0.013 & 0.910 & 0.003 \\
\hline T2_60_1 & 0.085 & 0.770 & 0.054 \\
\hline T2_61_1 & 0.003 & 0.955 & -0.011 \\
\hline T2_62_1 & 0.216 & 0.642 & 0.096 \\
\hline T2_63_1 & 0.111 & 0.739 & 0.065 \\
\hline T2_64_1 & 0.081 & 0.776 & 0.050 \\
\hline T2_65_1 & 0.050 & 0.824 & 0.028 \\
\hline T2_66_1 & 0.111 & 0.739 & 0.060 \\
\hline
\end{tabular}




\begin{tabular}{|c|c|c|c|}
\hline T2_67_1 & 0.088 & 0.767 & 0.046 \\
\hline T2_68_1 & 0.057 & 0.812 & 0.033 \\
\hline T2_69_1 & 0.000 & 0.995 & -0.055 \\
\hline T2_70_1 & 0.029 & 0.864 & 0.016 \\
\hline T2_71_1 & 0.281 & 0.596 & 0.125 \\
\hline T2_72_1 & 0.111 & 0.740 & 0.070 \\
\hline T2_73_1 & 0.081 & 0.776 & 0.045 \\
\hline T2_74_1 & 0.112 & 0.738 & 0.065 \\
\hline T2_76_1 & 0.175 & 0.676 & 0.093 \\
\hline T2_77_1 & 0.163 & 0.687 & 0.089 \\
\hline T2_78_1 & 0.198 & 0.657 & 0.101 \\
\hline T2_79_1 & 0.112 & 0.738 & 0.066 \\
\hline T2_80_1 & 0.004 & 0.952 & -0.012 \\
\hline T2_81_1 & 0.151 & 0.698 & 0.084 \\
\hline T2_82_1 & 0.075 & 0.785 & 0.052 \\
\hline T2_83_1 & 0.106 & 0.745 & 0.067 \\
\hline T2_84_1 & 0.065 & 0.799 & 0.036 \\
\hline T2_85_1 & 0.071 & 0.790 & 0.046 \\
\hline T2_51_2 & 0.062 & 0.803 & 0.096 \\
\hline T2_52_2 & 0.039 & 0.843 & 0.079 \\
\hline T2_54_2 & 0.007 & 0.932 & 0.040 \\
\hline T2_55_2 & 0.004 & 0.947 & 0.031 \\
\hline T2_58_2 & 0.017 & 0.895 & -0.051 \\
\hline T2_61_2 & 0.077 & 0.782 & 0.107 \\
\hline T2_62_2 & 0.105 & 0.746 & 0.118 \\
\hline T2_64_2 & 0.016 & 0.900 & 0.058 \\
\hline T2_65_2 & 0.094 & 0.760 & 0.113 \\
\hline T2_66_22 & 0.053 & 0.818 & 0.091 \\
\hline T2_67_2 & 0.023 & 0.879 & 0.066 \\
\hline T2_68_2 & 0.001 & 0.979 & 0.022 \\
\hline T2_69_2 & 0.037 & 0.848 & 0.076 \\
\hline T2_73_2 & 0.001 & 0.979 & -0.016 \\
\hline T2_74_2 & 0.001 & 0.978 & 0.026 \\
\hline T2_76_2 & 0.006 & 0.941 & 0.037 \\
\hline T2_77_2 & 0.001 & 0.976 & 0.028 \\
\hline T2_79_2 & 0.000 & 0.995 & 0.020 \\
\hline T2_80_2 & 0.010 & 0.921 & 0.044 \\
\hline T2_81_2 & 0.002 & 0.965 & 0.033 \\
\hline T2_83_2 & 0.038 & 0.845 & -0.067 \\
\hline T2_85_2 & 0.000 & 0.991 & -0.010 \\
\hline T2_63_2 & 0.000 & 0.992 & 0.017 \\
\hline
\end{tabular}




\begin{tabular}{|c|c|c|c|}
\hline T2_72_2 & 0.000 & 0.998 & -0.012 \\
\hline T2_82_2 & 0.001 & 0.981 & 0.000 \\
\hline T2_51_3 & 0.054 & 0.816 & 0.068 \\
\hline T2_52_3 & 0.000 & 0.985 & -0.025 \\
\hline T2_53_3 & 0.013 & 0.908 & 0.030 \\
\hline T2_54_3 & 0.002 & 0.964 & -0.042 \\
\hline T2_61_3 & 0.036 & 0.849 & 0.058 \\
\hline T2_63_3 & 0.013 & 0.909 & 0.030 \\
\hline T2_64_3 & 0.014 & 0.906 & 0.034 \\
\hline T2_66_3 & 0.000 & 0.995 & -0.001 \\
\hline T2_67_3 & 0.001 & 0.970 & 0.007 \\
\hline T2_68_3 & 0.020 & 0.887 & 0.036 \\
\hline T2_70_3 & 0.000 & 0.989 & -0.005 \\
\hline T2_71_3 & 0.009 & 0.926 & 0.023 \\
\hline T2_74_3 & 0.000 & 0.996 & -0.002 \\
\hline T2_76_3 & 0.036 & 0.850 & 0.056 \\
\hline T2_78_3 & 0.003 & 0.954 & 0.015 \\
\hline T2_79_3 & 0.093 & 0.760 & 0.092 \\
\hline T2_81_3 & 0.020 & 0.888 & 0.042 \\
\hline T2_82_3 & 0.066 & 0.798 & 0.071 \\
\hline T2_84_3 & 0.044 & 0.833 & 0.058 \\
\hline T2_57_3 & 0.017 & 0.896 & 0.046 \\
\hline T2_62_3 & 0.021 & 0.884 & 0.040 \\
\hline T2_73_3 & 0.000 & 0.985 & -0.024 \\
\hline T2_75_3 & 0.001 & 0.981 & -0.010 \\
\hline T2_83_3 & 0.000 & 0.995 & -0.007 \\
\hline T2_85_3 & 0.028 & 0.867 & 0.048 \\
\hline T2_51_4 & 0.076 & 0.784 & 0.020 \\
\hline T2_53_4 & 0.000 & 0.995 & -0.065 \\
\hline T2_60_4 & 0.064 & 0.801 & 0.014 \\
\hline T2_62_4 & 0.026 & 0.871 & -0.017 \\
\hline T2_63_4 & 0.001 & 0.972 & -0.075 \\
\hline T2_66_4 & 0.016 & 0.899 & -0.022 \\
\hline T2_67_4 & 0.041 & 0.840 & -0.001 \\
\hline T2_68_4 & 0.070 & 0.792 & 0.016 \\
\hline T2_69_4 & 0.074 & 0.786 & 0.022 \\
\hline T2_72_4 & 0.074 & 0.785 & 0.027 \\
\hline T2_73_4 & 0.071 & 0.790 & 0.019 \\
\hline T2_75_4 & 0.100 & 0.752 & 0.042 \\
\hline T2_78_4 & 0.050 & 0.823 & 0.008 \\
\hline T2_79_4 & 0.043 & 0.837 & 0.005 \\
\hline
\end{tabular}




\begin{tabular}{|c|c|c|c|}
\hline T2_80_4 & 0.000 & 0.986 & -0.070 \\
\hline T2_82_4 & 0.130 & 0.718 & 0.056 \\
\hline T2_83_4 & 0.096 & 0.756 & 0.039 \\
\hline T2_84_4 & 0.043 & 0.836 & 0.007 \\
\hline T2_52_4 & 0.004 & 0.953 & -0.072 \\
\hline T2_58_4 & 0.008 & 0.930 & -0.041 \\
\hline T2_65_4 & 0.026 & 0.872 & -0.020 \\
\hline T2_70_4 & 0.008 & 0.931 & -0.078 \\
\hline T2_74_4 & 0.096 & 0.757 & 0.039 \\
\hline T2_76_4 & 0.073 & 0.788 & 0.028 \\
\hline T2_85_4 & 0.072 & 0.789 & 0.027 \\
\hline T2_52_5 & 0.006 & 0.936 & -0.001 \\
\hline T2_55_5 & 0.001 & 0.973 & -0.027 \\
\hline T2_57_5 & 0.000 & 0.989 & -0.054 \\
\hline T2_59_5 & 0.003 & 0.958 & -0.062 \\
\hline T2_62_5 & 0.000 & 0.984 & -0.036 \\
\hline T2_63_5 & 0.021 & 0.884 & -0.087 \\
\hline T2_64_5 & 0.004 & 0.948 & 0.004 \\
\hline T2_65_5 & 0.049 & 0.825 & 0.055 \\
\hline T2_66_5 & 0.019 & 0.891 & -0.078 \\
\hline T2_67_5 & 0.004 & 0.952 & -0.003 \\
\hline T2_69_5 & 0.000 & 0.990 & -0.026 \\
\hline T2_80_5 & 0.006 & 0.941 & 0.005 \\
\hline T2_81_5 & 0.010 & 0.919 & 0.007 \\
\hline T2_84_5 & 0.062 & 0.804 & 0.058 \\
\hline T2_85_5 & 0.073 & 0.787 & 0.057 \\
\hline T2_51_5 & 0.001 & 0.979 & -0.053 \\
\hline T2_53_5 & 0.006 & 0.939 & -0.013 \\
\hline T2_54_5 & 0.000 & 0.993 & -0.055 \\
\hline T2_58_5 & 0.000 & 0.996 & -0.048 \\
\hline T2_72_5 & 0.050 & 0.823 & 0.002 \\
\hline T2_76_5 & 0.106 & 0.745 & -0.011 \\
\hline T2_77_5 & 0.078 & 0.780 & 0.005 \\
\hline T2_78_5 & 0.000 & 0.995 & -0.020 \\
\hline T2_82_5 & 0.102 & 0.750 & 0.079 \\
\hline T2_83_5 & 0.001 & 0.978 & -0.039 \\
\hline T2_52_6 & 0.359 & 0.549 & -0.073 \\
\hline T2_53_6 & 0.402 & 0.526 & -0.075 \\
\hline T2_54_6 & 0.781 & 0.377 & -0.139 \\
\hline T2_59_6 & 0.813 & 0.367 & -0.164 \\
\hline T2_60_6 & 0.105 & 0.746 & 0.025 \\
\hline
\end{tabular}




\begin{tabular}{llll} 
T2_63_6 & 0.601 & 0.438 & -0.120 \\
T2_65_6 & 0.166 & 0.684 & -0.005 \\
T2_66_6 & 0.199 & 0.655 & -0.016 \\
T2_73_6 & 0.343 & 0.558 & -0.066 \\
T2_79_6 & 0.324 & 0.569 & -0.059 \\
T2_82_6 & 0.703 & 0.402 & -0.157 \\
T2_83_6 & 0.328 & 0.567 & -0.057 \\
T2_51_6 & 0.082 & 0.775 & 0.048 \\
T2_56_6 & 0.070 & 0.791 & 0.031 \\
T2_57_6 & 0.603 & 0.437 & -0.025 \\
T2_58_6 & 0.085 & 0.771 & 0.049 \\
T2_61_6 & 0.840 & 0.359 & -0.106 \\
T2_62_6 & 0.192 & 0.661 & 0.069 \\
T2_67_6 & 0.101 & 0.751 & 0.028 \\
T2_69_6 & 0.189 & 0.664 & 0.042 \\
T2_70_6 & 0.424 & 0.515 & -0.072 \\
T2_77_6 & 0.735 & 0.391 & -0.038 \\
T2_78_6 & 0.001 & 0.975 & 0.105 \\
T2_81_6 & 0.010 & 0.922 & 0.074 \\
T2_84_6 & 0.293 & 0.588 & 0.008 \\
T2_85_6 & 0.227 & 0.634 & 0.010 \\
\hline
\end{tabular}


Table 37

Task 3 DIF: Male vs. female

\begin{tabular}{|c|c|c|c|}
\hline ItemID & ChiSq & pvalue & EffectSize \\
\hline T3_10_12 & 0.000 & 0.999 & -0.005 \\
\hline T3_11_12 & 0.026 & 0.872 & -0.033 \\
\hline T3_12_12 & 0.018 & 0.892 & 0.016 \\
\hline T3_18_12 & 0.019 & 0.892 & -0.030 \\
\hline T3_19_12 & 0.022 & 0.883 & 0.019 \\
\hline T3_20_12 & 0.081 & 0.776 & 0.037 \\
\hline T3_23_12 & 0.003 & 0.955 & -0.018 \\
\hline T3_29_12 & 0.002 & 0.967 & -0.015 \\
\hline T3_64_12 & 0.002 & 0.963 & -0.017 \\
\hline T3_42_23 & 0.001 & 0.975 & 0.017 \\
\hline T3_43_23 & 0.027 & 0.870 & 0.004 \\
\hline T3_46_23 & 0.002 & 0.969 & 0.003 \\
\hline T3_2_23 & 0.033 & 0.857 & 0.012 \\
\hline T3_4_23 & 0.190 & 0.663 & 0.050 \\
\hline T3_8_23 & 0.014 & 0.905 & 0.003 \\
\hline T3_13_23 & 0.018 & 0.894 & 0.003 \\
\hline T3_27_23 & 0.006 & 0.939 & -0.032 \\
\hline T3_29_23 & 0.045 & 0.832 & 0.015 \\
\hline T3_30_23 & 0.000 & 0.992 & -0.018 \\
\hline T3_37_23 & 0.010 & 0.921 & -0.035 \\
\hline T3_5_34 & 0.017 & 0.897 & -0.015 \\
\hline T3_42_34 & 0.257 & 0.612 & -0.006 \\
\hline T3_6_34 & 0.029 & 0.865 & -0.023 \\
\hline T3_11_34 & 0.042 & 0.838 & -0.028 \\
\hline T3_15_34 & 0.024 & 0.877 & -0.018 \\
\hline T3_17_34 & 0.013 & 0.909 & 0.027 \\
\hline T3_23_34 & 0.001 & 0.979 & 0.013 \\
\hline T3_28_34 & 0.000 & 0.993 & 0.001 \\
\hline T3_33_34 & 0.005 & 0.945 & 0.019 \\
\hline T3_50_34 & 0.000 & 0.991 & 0.034 \\
\hline T3_51_34 & 0.145 & 0.703 & 0.015 \\
\hline T3_60_45 & 0.307 & 0.580 & -0.058 \\
\hline T3_63_45 & 0.046 & 0.830 & 0.058 \\
\hline T3_2_56 & 0.000 & 0.988 & -0.003 \\
\hline T3_16_56 & 0.010 & 0.919 & -0.027 \\
\hline T3_19_56 & 0.007 & 0.936 & 0.005 \\
\hline T3_21_56 & 0.000 & 0.995 & -0.011 \\
\hline
\end{tabular}




\begin{tabular}{|c|c|c|c|}
\hline T3_25_56 & 0.000 & 0.998 & -0.001 \\
\hline T3_31_56 & 0.019 & 0.891 & 0.015 \\
\hline T3_32_56 & 0.014 & 0.908 & 0.010 \\
\hline T3_39_56 & 0.003 & 0.953 & 0.001 \\
\hline T3_51_56 & 0.001 & 0.977 & -0.032 \\
\hline T3_60_56 & 0.006 & 0.939 & -0.053 \\
\hline T3_3_456 & 0.000 & 0.992 & 0.022 \\
\hline T3_8_456 & 0.122 & 0.727 & -0.037 \\
\hline T3_9_456 & 0.122 & 0.727 & -0.034 \\
\hline T3_14_456 & 0.005 & 0.945 & 0.029 \\
\hline T3_15_456 & 0.126 & 0.723 & -0.034 \\
\hline T3_20_456 & 0.211 & 0.646 & -0.051 \\
\hline T3_33_456 & 0.017 & 0.898 & -0.001 \\
\hline T3_40_456 & 0.131 & 0.717 & -0.043 \\
\hline T3_1_1 & Inf & 0.000 & $\mathrm{NaN}$ \\
\hline T3_2_1 & Inf & 0.000 & $\mathrm{NaN}$ \\
\hline T3_3_1 & 0.013 & 0.908 & 0.014 \\
\hline T3_4_1 & 0.271 & 0.603 & 0.072 \\
\hline T3_5_1 & 0.059 & 0.809 & 0.033 \\
\hline T3_6_1 & 0.011 & 0.915 & 0.012 \\
\hline T3_7_1 & 0.000 & 0.997 & -0.007 \\
\hline T3_8_1 & 0.020 & 0.887 & 0.018 \\
\hline T3_9_1 & 0.038 & 0.846 & 0.026 \\
\hline T3_13_1 & 0.003 & 0.960 & -0.015 \\
\hline T3_14_1 & 0.000 & 0.990 & -0.010 \\
\hline T3_15_1 & 0.000 & 0.996 & -0.003 \\
\hline T3_16_1 & 0.001 & 0.971 & 0.002 \\
\hline T3_17_1 & 0.000 & 0.998 & -0.007 \\
\hline T3_21_1 & 0.000 & 0.997 & -0.007 \\
\hline T3_22_1 & 0.017 & 0.896 & 0.016 \\
\hline T3_24_1 & 0.001 & 0.979 & -0.012 \\
\hline T3_25_1 & 0.008 & 0.928 & 0.010 \\
\hline T3_26_1 & 0.000 & 0.991 & -0.005 \\
\hline T3_27_1 & 0.001 & 0.974 & -0.012 \\
\hline T3_28_1 & 0.038 & 0.846 & 0.026 \\
\hline T3_30_1 & 0.002 & 0.967 & 0.003 \\
\hline T3_31_1 & 0.038 & 0.845 & 0.026 \\
\hline T3_32_1 & 0.017 & 0.898 & 0.016 \\
\hline T3_33_1 & 0.000 & 0.989 & -0.001 \\
\hline T3_34_1 & 0.000 & 0.994 & -0.012 \\
\hline T3_35_1 & 0.004 & 0.952 & -0.017 \\
\hline
\end{tabular}




\begin{tabular}{|c|c|c|c|}
\hline T3_36_1 & 0.033 & 0.855 & -0.034 \\
\hline T3_37_1 & 0.001 & 0.971 & -0.013 \\
\hline T3_38_1 & 0.039 & 0.844 & 0.023 \\
\hline T3_39_1 & 0.001 & 0.972 & 0.002 \\
\hline T3_40_1 & 0.001 & 0.973 & 0.002 \\
\hline T3_41_1 & 0.031 & 0.860 & 0.024 \\
\hline T3_42_1 & 0.044 & 0.834 & 0.028 \\
\hline T3_43_1 & 0.001 & 0.979 & -0.007 \\
\hline T3_44_1 & 0.014 & 0.905 & -0.021 \\
\hline T3_45_1 & 0.043 & 0.835 & 0.033 \\
\hline T3_46_1 & 0.159 & 0.690 & 0.057 \\
\hline T3_47_1 & 0.001 & 0.975 & 0.007 \\
\hline T3_48_1 & 0.016 & 0.898 & 0.021 \\
\hline T3_49_1 & 0.125 & 0.723 & -0.050 \\
\hline T3_50_1 & 0.065 & 0.798 & -0.035 \\
\hline T3_51_1 & 0.000 & 0.998 & -0.003 \\
\hline T3_54_1 & 0.192 & 0.661 & 0.061 \\
\hline T3_55_1 & 0.001 & 0.978 & 0.007 \\
\hline T3_56_1 & 0.000 & 0.987 & -0.003 \\
\hline T3_57_1 & 0.002 & 0.968 & 0.015 \\
\hline T3_58_1 & 0.034 & 0.855 & -0.022 \\
\hline T3_61_1 & 0.000 & 0.983 & 0.007 \\
\hline T3_62_1 & 0.000 & 0.996 & -0.023 \\
\hline T3_63_1 & 0.010 & 0.919 & 0.015 \\
\hline T3_1_2 & 0.004 & 0.950 & -0.013 \\
\hline T3_6_2 & 0.000 & 0.984 & 0.005 \\
\hline T3_7_2 & 0.021 & 0.885 & -0.025 \\
\hline T3_9_2 & 0.006 & 0.937 & -0.015 \\
\hline T3_10_2 & 0.016 & 0.899 & -0.023 \\
\hline T3_11_2 & 0.042 & 0.838 & 0.032 \\
\hline T3_12_2 & 0.002 & 0.961 & -0.011 \\
\hline T3_16_2 & 0.005 & 0.944 & -0.014 \\
\hline T3_18_2 & 0.012 & 0.914 & -0.005 \\
\hline T3_19_2 & 0.009 & 0.926 & 0.015 \\
\hline T3_20_2 & 0.000 & 0.996 & 0.002 \\
\hline T3_22_2 & 0.036 & 0.849 & 0.009 \\
\hline T3_23_2 & 0.021 & 0.886 & -0.024 \\
\hline T3_24_2 & 0.000 & 0.991 & 0.003 \\
\hline T3_26_2 & 0.000 & 0.984 & -0.007 \\
\hline T3_31_2 & 0.020 & 0.887 & 0.023 \\
\hline T3_32_2 & 0.031 & 0.860 & 0.006 \\
\hline
\end{tabular}




\begin{tabular}{|c|c|c|c|}
\hline T3_33_2 & 0.001 & 0.980 & -0.008 \\
\hline T3_34_2 & 0.010 & 0.921 & -0.018 \\
\hline T3_35_2 & 0.011 & 0.916 & -0.019 \\
\hline T3_36_2 & 0.005 & 0.945 & -0.014 \\
\hline T3_38_2 & 0.030 & 0.863 & -0.053 \\
\hline T3_39_2 & 0.016 & 0.899 & -0.022 \\
\hline T3_40_2 & 0.003 & 0.958 & -0.011 \\
\hline T3_41_2 & 0.196 & 0.658 & 0.048 \\
\hline T3_48_2 & 0.135 & 0.713 & 0.038 \\
\hline T3_49_2 & 0.052 & 0.820 & 0.014 \\
\hline T3_50_2 & 0.168 & 0.682 & 0.043 \\
\hline T3_53_2 & 0.015 & 0.901 & -0.002 \\
\hline T3_54_2 & 0.152 & 0.696 & 0.040 \\
\hline T3_56_2 & 0.013 & 0.910 & -0.001 \\
\hline T3_57_2 & 0.054 & 0.816 & 0.016 \\
\hline T3_58_2 & 0.175 & 0.676 & 0.043 \\
\hline T3_60_2 & 0.063 & 0.801 & 0.018 \\
\hline T3_61_2 & 0.101 & 0.751 & 0.027 \\
\hline T3_63_2 & 0.002 & 0.962 & -0.012 \\
\hline T3_1_3 & 0.011 & 0.916 & 0.002 \\
\hline T3_2_3 & 0.052 & 0.819 & 0.019 \\
\hline T3_4_3 & 0.014 & 0.907 & 0.003 \\
\hline T3_8_3 & 0.003 & 0.955 & -0.006 \\
\hline T3_9_3 & 0.014 & 0.906 & 0.003 \\
\hline T3_10_3 & 0.058 & 0.810 & 0.022 \\
\hline T3_12_3 & 0.003 & 0.957 & 0.022 \\
\hline T3_13_3 & 0.019 & 0.890 & 0.006 \\
\hline T3_14_3 & 0.002 & 0.966 & -0.007 \\
\hline T3_16_3 & 0.065 & 0.799 & 0.024 \\
\hline T3_18_3 & 0.021 & 0.885 & 0.007 \\
\hline T3_19_3 & 0.074 & 0.785 & -0.032 \\
\hline T3_21_3 & 0.071 & 0.791 & -0.032 \\
\hline T3_22_3 & 0.006 & 0.939 & -0.003 \\
\hline T3_24_3 & 0.002 & 0.962 & -0.007 \\
\hline T3_25_3 & 0.046 & 0.830 & 0.018 \\
\hline T3_26_3 & 0.007 & 0.932 & -0.001 \\
\hline T3_29_3 & 0.051 & 0.822 & 0.019 \\
\hline T3_30_3 & 0.004 & 0.951 & -0.005 \\
\hline T3_31_3 & 0.029 & 0.864 & 0.011 \\
\hline T3_32_3 & 0.085 & 0.770 & 0.029 \\
\hline T3_36_3 & 0.000 & 0.994 & 0.015 \\
\hline
\end{tabular}




\begin{tabular}{|c|c|c|c|}
\hline T3_37_3 & 0.000 & 0.992 & -0.021 \\
\hline T3_39_3 & 0.022 & 0.883 & 0.008 \\
\hline T3_41_3 & 0.019 & 0.891 & 0.007 \\
\hline T3_43_3 & 0.044 & 0.835 & -0.002 \\
\hline T3_44_3 & 0.036 & 0.849 & -0.002 \\
\hline T3_46_3 & 0.153 & 0.696 & -0.030 \\
\hline T3_47_3 & 0.037 & 0.848 & -0.001 \\
\hline T3_48_3 & 0.018 & 0.892 & 0.007 \\
\hline T3_49_3 & 0.109 & 0.742 & -0.022 \\
\hline T3_52_3 & 0.004 & 0.949 & 0.020 \\
\hline T3_53_3 & 0.011 & 0.918 & 0.049 \\
\hline T3_54_3 & 0.014 & 0.907 & 0.049 \\
\hline T3_57_3 & 0.045 & 0.833 & 0.065 \\
\hline T3_58_3 & 0.164 & 0.686 & -0.032 \\
\hline T3_60_3 & 0.182 & 0.670 & -0.035 \\
\hline T3_61_3 & 0.003 & 0.956 & 0.021 \\
\hline T3_64_3 & 0.010 & 0.921 & 0.048 \\
\hline T3_2_4 & 0.000 & 0.988 & 0.022 \\
\hline T3_5_4 & 0.086 & 0.770 & -0.024 \\
\hline T3_6_4 & 0.049 & 0.825 & -0.012 \\
\hline T3_7_4 & 0.041 & 0.840 & -0.009 \\
\hline T3_10_4 & 0.017 & 0.898 & 0.003 \\
\hline T3_11_4 & 0.001 & 0.973 & 0.017 \\
\hline T3_12_4 & 0.013 & 0.911 & 0.005 \\
\hline T3_13_4 & 0.124 & 0.725 & -0.029 \\
\hline T3_18_4 & 0.036 & 0.850 & -0.009 \\
\hline T3_19_4 & 0.159 & 0.690 & 0.003 \\
\hline T3_21_4 & 0.004 & 0.952 & 0.012 \\
\hline T3_22_4 & 0.149 & 0.700 & -0.033 \\
\hline T3_23_4 & 0.050 & 0.823 & 0.028 \\
\hline T3_24_4 & 0.006 & 0.938 & 0.010 \\
\hline T3_25_4 & 0.019 & 0.891 & 0.000 \\
\hline T3_27_4 & 0.001 & 0.978 & 0.016 \\
\hline T3_28_4 & 0.010 & 0.920 & 0.006 \\
\hline T3_29_4 & 0.002 & 0.963 & 0.015 \\
\hline T3_31_4 & 0.008 & 0.930 & 0.047 \\
\hline T3_32_4 & 0.094 & 0.759 & -0.024 \\
\hline T3_34_4 & 0.000 & 0.987 & 0.018 \\
\hline T3_35_4 & 0.001 & 0.975 & 0.015 \\
\hline T3_36_4 & 0.053 & 0.818 & 0.029 \\
\hline T3_39_4 & 0.000 & 0.984 & 0.028 \\
\hline
\end{tabular}




\begin{tabular}{|c|c|c|c|}
\hline T3_41_4 & 0.028 & 0.867 & 0.011 \\
\hline T3_43_4 & 0.000 & 0.987 & 0.042 \\
\hline T3_44_4 & 0.042 & 0.838 & 0.002 \\
\hline T3_46_4 & 0.026 & 0.873 & 0.012 \\
\hline T3_47_4 & 0.019 & 0.892 & 0.058 \\
\hline T3_48_4 & 0.028 & 0.868 & 0.010 \\
\hline T3_49_4 & 0.008 & 0.929 & 0.022 \\
\hline T3_52_4 & 0.002 & 0.965 & 0.028 \\
\hline T3_53_4 & 0.023 & 0.878 & 0.013 \\
\hline T3_55_4 & 0.037 & 0.848 & 0.007 \\
\hline T3_56_4 & 0.007 & 0.933 & 0.023 \\
\hline T3_57_4 & 0.017 & 0.895 & 0.016 \\
\hline T3_58_4 & 0.054 & 0.817 & 0.001 \\
\hline T3_59_4 & 0.009 & 0.926 & 0.021 \\
\hline T3_61_4 & 0.005 & 0.943 & 0.024 \\
\hline T3_64_4 & 0.002 & 0.963 & 0.028 \\
\hline T3_1_5 & 0.001 & 0.971 & 0.001 \\
\hline T3_4_5 & 0.133 & 0.716 & -0.077 \\
\hline T3_5_5 & 0.003 & 0.957 & 0.003 \\
\hline T3_6_5 & 0.003 & 0.960 & 0.004 \\
\hline T3_8_5 & 0.000 & 0.989 & -0.012 \\
\hline T3_9_5 & 0.000 & 0.987 & -0.011 \\
\hline T3_10_5 & 0.002 & 0.967 & -0.016 \\
\hline T3_11_5 & 0.058 & 0.810 & -0.060 \\
\hline T3_12_5 & 0.003 & 0.958 & -0.018 \\
\hline T3_14_5 & 0.005 & 0.944 & -0.020 \\
\hline T3_15_5 & 0.000 & 0.998 & -0.004 \\
\hline T3_17_5 & 0.008 & 0.930 & 0.008 \\
\hline T3_22_5 & 0.001 & 0.976 & 0.001 \\
\hline T3_23_5 & 0.000 & 0.988 & -0.020 \\
\hline T3_24_5 & 0.005 & 0.945 & -0.020 \\
\hline T3_26_5 & 0.016 & 0.899 & -0.030 \\
\hline T3_28_5 & 0.026 & 0.872 & 0.018 \\
\hline T3_29_5 & 0.006 & 0.937 & 0.008 \\
\hline T3_33_5 & 0.024 & 0.878 & 0.018 \\
\hline T3_34_5 & 0.016 & 0.899 & -0.042 \\
\hline T3_35_5 & 0.000 & 0.987 & -0.012 \\
\hline T3_36_5 & 0.001 & 0.978 & -0.001 \\
\hline T3_37_5 & 0.000 & 0.994 & -0.004 \\
\hline T3_38_5 & 0.013 & 0.911 & -0.026 \\
\hline T3_42_5 & 0.045 & 0.832 & 0.023 \\
\hline
\end{tabular}


MATRS TECHNICAL MANUAL 161

\begin{tabular}{llll} 
T3_44_5 & 0.051 & 0.822 & -0.047 \\
T3_45_5 & 0.044 & 0.834 & 0.021 \\
T3_47_5 & 0.042 & 0.839 & -0.044 \\
T3_48_5 & 0.069 & 0.792 & -0.052 \\
T3_49_5 & 0.004 & 0.951 & -0.022 \\
T3_52_5 & 0.037 & 0.849 & -0.041 \\
T3_55_5 & 0.494 & 0.482 & -0.117 \\
T3_56_5 & 0.005 & 0.946 & -0.024 \\
\hline
\end{tabular}


Table 38

Task 3 DIF: White vs. Sample

\begin{tabular}{|c|c|c|c|}
\hline ItemID & ChiSq & pvalue & EffectSize \\
\hline T3_10_12 & 0.079 & 0.778 & -0.007 \\
\hline T3_11_12 & 0.150 & 0.698 & 0.008 \\
\hline T3_12_12 & 0.476 & 0.490 & 0.055 \\
\hline T3_18_12 & 0.026 & 0.873 & -0.024 \\
\hline T3_19_12 & 0.434 & 0.510 & 0.051 \\
\hline T3_20_12 & 0.018 & 0.894 & -0.028 \\
\hline T3_23_12 & 0.083 & 0.774 & -0.007 \\
\hline T3_29_12 & 0.345 & 0.557 & 0.041 \\
\hline T3_64_12 & 0.969 & 0.325 & -0.032 \\
\hline T3_42_23 & 1.372 & 0.241 & -0.079 \\
\hline T3_43_23 & 0.253 & 0.615 & -0.026 \\
\hline T3_46_23 & 0.084 & 0.773 & 0.154 \\
\hline T3_2_23 & 0.327 & 0.568 & 0.018 \\
\hline T3_4_23 & 0.399 & 0.528 & 0.029 \\
\hline T3_8_23 & 0.199 & 0.656 & 0.002 \\
\hline T3_13_23 & 0.121 & 0.728 & -0.017 \\
\hline T3_27_23 & 0.549 & 0.459 & 0.044 \\
\hline T3_29_23 & 0.446 & 0.504 & 0.033 \\
\hline T3_30_23 & 0.144 & 0.705 & -0.012 \\
\hline T3_37_23 & 0.632 & 0.427 & 0.053 \\
\hline T3_5_34 & 0.023 & 0.880 & -0.006 \\
\hline T3_42_34 & 0.967 & 0.325 & -0.077 \\
\hline T3_6_34 & 0.071 & 0.790 & 0.013 \\
\hline T3_11_34 & 0.070 & 0.791 & 0.011 \\
\hline T3_15_34 & 0.044 & 0.834 & 0.002 \\
\hline T3_17_34 & 0.051 & 0.822 & 0.006 \\
\hline T3_23_34 & 0.009 & 0.926 & -0.014 \\
\hline T3_28_34 & 0.073 & 0.787 & 0.009 \\
\hline T3_33_34 & 0.009 & 0.923 & -0.014 \\
\hline T3_50_34 & 0.000 & 0.987 & 0.031 \\
\hline T3_51_34 & 0.002 & 0.968 & 0.072 \\
\hline T3_60_45 & 0.011 & 0.918 & -0.031 \\
\hline T3_63_45 & 0.004 & 0.953 & 0.005 \\
\hline T3_2_56 & 0.282 & 0.596 & 0.018 \\
\hline T3_16_56 & 0.101 & 0.751 & -0.017 \\
\hline T3_19_56 & 0.173 & 0.677 & 0.002 \\
\hline T3_21_56 & 0.315 & 0.574 & 0.019 \\
\hline
\end{tabular}




\begin{tabular}{lccc} 
T3_25_56 & 0.733 & 0.392 & 0.061 \\
T3_31_56 & 0.130 & 0.719 & -0.009 \\
T3_32_56 & 0.256 & 0.613 & 0.013 \\
T3_39_56 & 0.182 & 0.669 & -0.003 \\
T3_51_56 & 0.011 & 0.915 & 0.001 \\
T3_60_56 & 0.057 & 0.812 & 0.015 \\
T3_3_456 & 0.355 & 0.551 & -0.004 \\
T3_8_456 & 1.129 & 0.288 & 0.060 \\
T3_9_456 & 0.515 & 0.473 & 0.019 \\
T3_14_456 & 0.443 & 0.506 & -0.001 \\
T3_15_456 & 1.070 & 0.301 & 0.052 \\
T3_20_456 & 0.174 & 0.676 & -0.038 \\
T3_33_456 & 0.954 & 0.329 & 0.050 \\
T3_40_456 & 0.383 & 0.536 & -0.008 \\
T3_1_1 & Inf & 0.000 & NaN \\
T3_2_1 & Inf & 0.000 & NaN \\
T3_3_1 & 0.053 & 0.818 & -0.016 \\
T3_4_1 & 0.482 & 0.488 & -0.024 \\
T3_5_1 & 0.048 & 0.826 & -0.016 \\
T3_6_1 & 0.011 & 0.915 & 0.002 \\
T3_7_1 & 0.067 & 0.795 & -0.021 \\
T3_8_1 & 0.017 & 0.896 & -0.002 \\
T3_9_1 & 0.010 & 0.922 & 0.003 \\
T3_13_1 & 0.063 & 0.802 & -0.020 \\
T3_14_1 & 0.006 & 0.938 & 0.006 \\
T3_15_1 & 0.002 & 0.962 & 0.011 \\
T3_16_1 & 0.042 & 0.837 & -0.013 \\
T3_17_1 & 0.116 & 0.733 & -0.034 \\
T3_21_1 & 0.107 & 0.744 & -0.031 \\
T3_22_1 & 0.009 & 0.924 & 0.003 \\
T3_24_1 & 0.091 & 0.762 & -0.028 \\
T3_25_1 & 0.005 & 0.942 & 0.007 \\
T3_26_1 & 0.107 & 0.743 & -0.032 \\
T3_27_1 & 0.019 & 0.892 & -0.003 \\
T3_28_1 & 0.012 & 0.915 & 0.001 \\
T3_30_1 & 0.064 & 0.800 & -0.020 \\
T3_31_1 & 0.010 & 0.919 & 0.002 \\
T3_32_1 & 0.007 & 0.933 & 0.005 \\
T3_33_1 & 0.147 & 0.701 & -0.041 \\
T3_1 & 0.800 & 0.371 & -0.045 \\
T3 & 0.067 & 0.796 & -0.022 \\
\hline
\end{tabular}




$\begin{array}{llll}\text { T3_36_1 } & 0.883 & 0.347 & -0.062 \\ \text { T3_37_1 } & 0.032 & 0.857 & -0.010 \\ \text { T3_38_1 } & 0.096 & 0.756 & 0.030 \\ \text { T3_39_1 } & 0.076 & 0.783 & -0.023 \\ \text { T3_40_1 } & 0.030 & 0.863 & -0.009 \\ \text { T3_41_1 } & 0.275 & 0.600 & -0.027 \\ \text { T3_42_1 } & 0.577 & 0.447 & -0.063 \\ \text { T3_43_1 } & 0.252 & 0.616 & -0.020 \\ \text { T3_44_1 } & 0.208 & 0.649 & -0.010 \\ \text { T3_45_1 } & 0.978 & 0.323 & -0.087 \\ \text { T3_46_1 } & 0.013 & 0.909 & 0.037 \\ \text { T3_47_1 } & 0.247 & 0.619 & -0.021 \\ \text { T3_48_1 } & 0.256 & 0.613 & -0.016 \\ \text { T3_49_1 } & 0.368 & 0.544 & -0.037 \\ \text { T3_50_1 } & 0.158 & 0.691 & -0.004 \\ \text { T3_51_1 } & 0.342 & 0.559 & -0.035 \\ \text { T3_54_1 } & 0.015 & 0.903 & 0.032 \\ \text { T3_55_1 } & 0.099 & 0.753 & 0.007 \\ \text { T3_56_1 } & 0.363 & 0.547 & -0.039 \\ \text { T3_57_1 } & 0.171 & 0.679 & -0.004 \\ \text { T3_58_1 } & 0.006 & 0.937 & 0.041 \\ \text { T3_61_1 } & 0.000 & 0.987 & 0.056 \\ \text { T3_62_1 } & 1.497 & 0.221 & -0.056 \\ \text { T3_63_1 } & 0.082 & 0.774 & 0.011 \\ \text { T3_1_2 } & 0.307 & 0.580 & 0.014 \\ \text { T3_6_2 } & 0.426 & 0.514 & 0.030 \\ \text { T3_7_2 } & 0.419 & 0.517 & 0.030 \\ \text { T3_9_2 } & 0.402 & 0.526 & 0.026 \\ \text { T3_10_2 } & 0.040 & 0.841 & -0.039 \\ \text { T3_11_2 } & 0.536 & 0.464 & 0.041 \\ \text { T3_12_2 } & 0.338 & 0.561 & 0.020 \\ \text { T3_16_2 } & 0.193 & 0.660 & -0.003 \\ \text { T3_18_2 } & 0.116 & 0.734 & 0.036 \\ \text { T3_19_2 } & 0.746 & 0.388 & 0.063 \\ \text { T3_20_2 } & 0.177 & 0.674 & -0.006 \\ \text { T3_22_2 } & 0.082 & 0.775 & 0.025 \\ \text { T3_23_2 } & 0.592 & 0.442 & 0.048 \\ \text { T3_24_2 } & 0.794 & 0.373 & 0.067 \\ \text { T3_32_2 } & 0.458 & 0.499 & 0.033 \\ \text { T3_2 } & 0.780 & 0.377 & 0.065 \\ \text { T3 } & 0.327 & 0.568 & 0.066\end{array}$




$\begin{array}{llll}\text { T3_33_2 } & 0.108 & 0.742 & -0.019 \\ \text { T3_34_2 } & 0.342 & 0.559 & 0.020 \\ \text { T3_35_2 } & 0.449 & 0.503 & 0.033 \\ \text { T3_36_2 } & 0.475 & 0.491 & 0.036 \\ \text { T3_38_2 } & 0.071 & 0.790 & 0.020 \\ \text { T3_39_2 } & 0.284 & 0.594 & 0.012 \\ \text { T3_40_2 } & 0.537 & 0.464 & 0.042 \\ \text { T3_41_2 } & 0.079 & 0.779 & 0.007 \\ \text { T3_48_2 } & 0.096 & 0.757 & 0.005 \\ \text { T3_49_2 } & 0.119 & 0.730 & 0.001 \\ \text { T3_50_2 } & 0.445 & 0.505 & -0.049 \\ \text { T3_53_2 } & 0.240 & 0.624 & -0.023 \\ \text { T3_54_2 } & 0.482 & 0.487 & -0.052 \\ \text { T3_56_2 } & 0.068 & 0.795 & 0.014 \\ \text { T3_57_2 } & 0.057 & 0.811 & 0.015 \\ \text { T3_58_2 } & 0.020 & 0.888 & 0.030 \\ \text { T3_60_2 } & 0.000 & 0.989 & 0.054 \\ \text { T3_61_2 } & 0.001 & 0.978 & 0.048 \\ \text { T3_63_2 } & 0.042 & 0.837 & 0.086 \\ \text { T3_1_3 } & 0.014 & 0.906 & -0.019 \\ \text { T3_2_3 } & 0.000 & 0.992 & 0.005 \\ \text { T3_4_3 } & 0.006 & 0.941 & -0.012 \\ \text { T3_8_3 } & 0.002 & 0.970 & -0.007 \\ \text { T3_9_3 } & 0.010 & 0.921 & -0.016 \\ \text { T3_10_3 } & 0.000 & 0.998 & 0.005 \\ \text { T3_12_3 } & 0.158 & 0.691 & 0.102 \\ \text { T3_13_3 } & 0.005 & 0.943 & 0.016 \\ \text { T3_14_3 } & 0.007 & 0.932 & 0.017 \\ \text { T3_16_3 } & 0.000 & 0.994 & -0.001 \\ \text { T3_18_3 } & 0.008 & 0.931 & -0.014 \\ \text { T3_19_3 } & 0.082 & 0.775 & 0.085 \\ \text { T3_21_3 } & 0.042 & 0.837 & 0.074 \\ \text { T3_22_3 } & 0.035 & 0.851 & 0.032 \\ \text { T3_24_3 } & 0.015 & 0.903 & 0.022 \\ \text { T3_25_3 } & 0.007 & 0.934 & 0.017 \\ \text { T3_26_3 } & 0.017 & 0.897 & -0.020 \\ \text { T3_29_3 } & 0.024 & 0.877 & -0.024 \\ \text { T3_30_3 } & 0.027 & 0.871 & -0.025 \\ \text { T3_36_33 } & 0.004 & 0.952 & 0.013 \\ \text { T3 } & 0.016 & 0.899 & -0.020 \\ \text { T3 } & 0.040 & 0.841 & 0.005\end{array}$




$\begin{array}{llll}\text { T3_37_3 } & 0.000 & 0.983 & -0.004 \\ \text { T3_39_3 } & 0.000 & 0.994 & 0.001 \\ \text { T3_41_3 } & 0.122 & 0.726 & 0.091 \\ \text { T3_43_3 } & 0.006 & 0.939 & 0.050 \\ \text { T3_44_3 } & 0.001 & 0.972 & 0.024 \\ \text { T3_46_3 } & 0.001 & 0.971 & 0.044 \\ \text { T3_47_3 } & 0.000 & 0.990 & 0.030 \\ \text { T3_48_3 } & 0.038 & 0.845 & 0.064 \\ \text { T3_49_3 } & 0.007 & 0.934 & 0.051 \\ \text { T3_52_3 } & 0.238 & 0.626 & 0.113 \\ \text { T3_53_3 } & 0.052 & 0.820 & 0.073 \\ \text { T3_54_3 } & 0.096 & 0.756 & -0.017 \\ \text { T3_57_3 } & 0.026 & 0.871 & 0.062 \\ \text { T3_58_3 } & 0.143 & 0.705 & 0.096 \\ \text { T3_60_3 } & 0.010 & 0.921 & 0.049 \\ \text { T3_61_3 } & 0.027 & 0.870 & 0.064 \\ \text { T3_64_3 } & 0.151 & 0.698 & 0.094 \\ \text { T3_2_4 } & 0.022 & 0.883 & -0.006 \\ \text { T3_5_4 } & 0.019 & 0.889 & -0.008 \\ \text { T3_6_4 } & 0.121 & 0.728 & 0.025 \\ \text { T3_7_4 } & 0.069 & 0.793 & 0.012 \\ \text { T3_10_4 } & 0.000 & 0.985 & -0.037 \\ \text { T3_11_4 } & 0.019 & 0.891 & -0.010 \\ \text { T3_12_4 } & 0.041 & 0.840 & 0.002 \\ \text { T3_13_4 } & 0.218 & 0.641 & 0.042 \\ \text { T3_18_4 } & 0.076 & 0.784 & 0.012 \\ \text { T3_19_4 } & 0.000 & 0.996 & 0.003 \\ \text { T3_21_4 } & 0.024 & 0.878 & -0.005 \\ \text { T3_22_4 } & 0.011 & 0.916 & -0.011 \\ \text { T3_23_4 } & 0.069 & 0.793 & -0.041 \\ \text { T3_24_4 } & 0.007 & 0.932 & -0.016 \\ \text { T3_25_4 } & 0.052 & 0.820 & 0.007 \\ \text { T3_27_4 } & 0.005 & 0.944 & -0.019 \\ \text { T3_28_4 } & 0.050 & 0.822 & 0.005 \\ \text { T3_29_4 } & 0.029 & 0.865 & -0.005 \\ \text { T3_31_4 } & 0.015 & 0.903 & -0.021 \\ \text { T3_32_4 } & 0.064 & 0.800 & 0.010 \\ \text { T3_34_4 } & 0.057 & 0.811 & 0.006 \\ \text { T3_35_4 } & 0.006 & 0.941 & -0.017 \\ \text { T3_39_4 } & 0.011 & 0.917 & -0.014\end{array}$




$\begin{array}{llcc}\text { T3_41_4 } & 0.000 & 0.986 & 0.030 \\ \text { T3_43_4 } & 0.011 & 0.916 & 0.016 \\ \text { T3_44_4 } & 0.327 & 0.567 & -0.057 \\ \text { T3_46_4 } & 0.000 & 0.998 & 0.032 \\ \text { T3_47_4 } & 0.006 & 0.941 & 0.019 \\ \text { T3_48_4 } & 0.162 & 0.688 & -0.028 \\ \text { T3_49_4 } & 0.013 & 0.911 & 0.016 \\ \text { T3_52_4 } & 0.037 & 0.847 & 0.004 \\ \text { T3_53_4 } & 0.000 & 0.998 & 0.033 \\ \text { T3_55_4 } & 0.136 & 0.712 & -0.023 \\ \text { T3_56_4 } & 0.000 & 0.994 & 0.031 \\ \text { T3_57_4 } & 0.012 & 0.913 & 0.015 \\ \text { T3_58_4 } & 0.097 & 0.755 & -0.014 \\ \text { T3_59_4 } & 0.153 & 0.696 & -0.026 \\ \text { T3_61_4 } & 0.050 & 0.822 & 0.069 \\ \text { T3_64_4 } & 0.001 & 0.979 & 0.026 \\ \text { T3_1_5 } & 0.034 & 0.854 & 0.018 \\ \text { T3_4_5 } & 0.000 & 0.990 & -0.058 \\ \text { T3_5_5 } & 0.093 & 0.761 & 0.035 \\ \text { T3_6_5 } & 0.002 & 0.967 & -0.003 \\ \text { T3_8_5 } & 0.007 & 0.933 & 0.003 \\ \text { T3_9_5 } & 0.021 & 0.884 & 0.012 \\ \text { T3_10_5 } & 0.030 & 0.863 & 0.016 \\ \text { T3_11_5 } & 0.125 & 0.724 & -0.005 \\ \text { T3_12_5 } & 0.023 & 0.878 & 0.014 \\ \text { T3_14_5 } & 0.052 & 0.820 & 0.025 \\ \text { T3_15_5 } & 0.027 & 0.871 & 0.015 \\ \text { T3_17_5 } & 0.020 & 0.888 & 0.013 \\ \text { T3_22_5 } & 0.006 & 0.941 & -0.026 \\ \text { T3_23_5 } & 0.162 & 0.688 & 0.006 \\ \text { T3_24_5 } & 0.000 & 0.984 & -0.008 \\ \text { T3_26_5 } & 0.052 & 0.820 & 0.024 \\ \text { T3_28_5 } & 0.050 & 0.823 & 0.022 \\ \text { T3_29_5 } & 0.018 & 0.894 & 0.010 \\ \text { T3_33_5 } & 0.078 & 0.780 & 0.030 \\ \text { T3_34_5 } & 0.106 & 0.744 & -0.007 \\ \text { T3_35_5 } & 0.005 & 0.941 & 0.002 \\ \text { T3_36_5 } & 0.024 & 0.876 & 0.012 \\ \text { T3_37_5 } & 0.093 & 0.761 & 0.035 \\ \text { T3_5 } & 0.024 & 0.877 & 0.012 \\ \text { T2_5 } & 0.156 & 0.693 & 0.019\end{array}$


MATRS TECHNICAL MANUAL 168

\begin{tabular}{llll} 
T3_44_5 & 0.051 & 0.822 & -0.007 \\
T3_45_5 & 0.190 & 0.663 & 0.025 \\
T3_47_5 & 0.012 & 0.914 & -0.025 \\
T3_48_5 & 0.292 & 0.589 & 0.041 \\
T3_49_5 & 0.024 & 0.876 & -0.019 \\
T3_52_5 & 0.088 & 0.767 & 0.005 \\
T3_55_5 & 0.000 & 0.993 & -0.041 \\
T3_56_5 & 0.151 & 0.698 & 0.018 \\
\hline
\end{tabular}


Table 39

Task 3 DIF: Black vs. Sample

\begin{tabular}{|c|c|c|c|}
\hline ItemID & ChiSq & pvalue & EffectSize \\
\hline T3_10_12 & 0.057 & 0.811 & 0.003 \\
\hline T3_11_12 & 0.208 & 0.648 & -0.029 \\
\hline T3_12_12 & 0.232 & 0.630 & -0.035 \\
\hline T3_18_12 & 0.061 & 0.805 & 0.001 \\
\hline T3_19_12 & 0.382 & 0.536 & -0.056 \\
\hline T3_20_12 & 0.002 & 0.967 & 0.051 \\
\hline T3_23_12 & 0.105 & 0.746 & -0.009 \\
\hline T3_29_12 & 0.143 & 0.705 & -0.019 \\
\hline T3_64_12 & 0.936 & 0.333 & 0.029 \\
\hline T3_42_23 & 1.052 & 0.305 & 0.075 \\
\hline T3_43_23 & 0.470 & 0.493 & 0.039 \\
\hline T3_46_23 & 0.085 & 0.771 & -0.139 \\
\hline T3_2_23 & 0.347 & 0.556 & -0.027 \\
\hline T3_4_23 & 0.370 & 0.543 & -0.032 \\
\hline T3_8_23 & 0.576 & 0.448 & -0.054 \\
\hline T3_13_23 & 0.206 & 0.650 & -0.003 \\
\hline T3_27_23 & 0.327 & 0.567 & -0.023 \\
\hline T3_29_23 & 0.290 & 0.590 & -0.019 \\
\hline T3_30_23 & 0.056 & 0.814 & 0.029 \\
\hline T3_37_23 & 0.541 & 0.462 & -0.050 \\
\hline T3_5_34 & 0.008 & 0.928 & 0.003 \\
\hline T3_42_34 & 0.296 & 0.586 & 0.041 \\
\hline T3_6_34 & 0.000 & 0.996 & 0.018 \\
\hline T3_11_34 & 0.065 & 0.799 & -0.023 \\
\hline T3_15_34 & 0.036 & 0.850 & -0.012 \\
\hline T3_17_34 & 0.013 & 0.909 & -0.002 \\
\hline T3_23_34 & 0.018 & 0.895 & -0.005 \\
\hline T3_28_34 & 0.065 & 0.800 & -0.018 \\
\hline T3_33_34 & 0.017 & 0.896 & -0.005 \\
\hline T3_50_34 & 0.198 & 0.656 & -0.077 \\
\hline T3_51_34 & 0.002 & 0.964 & -0.062 \\
\hline T3_60_45 & 0.006 & 0.937 & 0.025 \\
\hline T3_63_45 & 0.160 & 0.689 & -0.034 \\
\hline T3_2_56 & 0.206 & 0.650 & -0.013 \\
\hline T3_16_56 & 0.199 & 0.655 & -0.008 \\
\hline T3_19_56 & 0.128 & 0.720 & 0.003 \\
\hline T3_21_56 & 0.152 & 0.697 & 0.001 \\
\hline
\end{tabular}




$\begin{array}{lccc}\text { T3_25_56 } & 0.575 & 0.449 & -0.054 \\ \text { T3_31_56 } & 0.142 & 0.707 & 0.001 \\ \text { T3_32_56 } & 0.130 & 0.719 & 0.004 \\ \text { T3_39_56 } & 0.212 & 0.645 & -0.010 \\ \text { T3_51_56 } & 0.163 & 0.686 & -0.039 \\ \text { T3_60_56 } & 0.060 & 0.807 & -0.013 \\ \text { T3_3_456 } & 0.060 & 0.807 & 0.032 \\ \text { T3_8456 } & 0.830 & 0.362 & -0.066 \\ \text { T3_9_456 } & 0.510 & 0.475 & -0.043 \\ \text { T3_14_456 } & 0.249 & 0.618 & -0.005 \\ \text { T3_15_456 } & 0.712 & 0.399 & -0.055 \\ \text { T3_20456 } & 0.119 & 0.730 & 0.018 \\ \text { T3_33_456 } & 0.308 & 0.579 & -0.022 \\ \text { T3_40_456 } & 0.355 & 0.551 & -0.014 \\ \text { T3_1_1 } & \text { Inf } & 0.000 & \text { NaN } \\ \text { T3_2_1 } & \text { Inf } & 0.000 & \text { NaN } \\ \text { T3_3_1 } & 0.072 & 0.789 & 0.027 \\ \text { T3_4_1 } & 0.536 & 0.464 & 0.045 \\ \text { T3_5_1 } & 0.019 & 0.891 & 0.007 \\ \text { T3_6_1 } & 0.004 & 0.948 & -0.005 \\ \text { T3_7_1 } & 0.045 & 0.832 & 0.018 \\ \text { T3_8_1 } & 0.016 & 0.901 & 0.005 \\ \text { T3_9_1 } & 0.011 & 0.918 & 0.002 \\ \text { T3_13_1 } & 0.057 & 0.811 & 0.023 \\ \text { T3_14_1 } & 0.010 & 0.921 & 0.001 \\ \text { T3_15_1 } & 0.017 & 0.896 & 0.006 \\ \text { T3_16_1 } & 0.008 & 0.929 & 0.000 \\ \text { T3_17_1 } & 0.126 & 0.723 & 0.040 \\ \text { T3_21_1 } & 0.186 & 0.666 & 0.052 \\ \text { T3_22_1 } & 0.025 & 0.876 & 0.010 \\ \text { T3_24_1 } & 0.104 & 0.747 & 0.036 \\ \text { T3_25_1 } & 0.000 & 0.998 & -0.014 \\ \text { T3_6611 } & 0.156 & 0.693 & 0.047 \\ \text { T3_27_1 } & 0.029 & 0.866 & 0.011 \\ \text { T3_28_1 } & 0.000 & 0.990 & -0.017 \\ \text { T3_30_1 } & 0.138 & 0.710 & 0.043 \\ \text { T3_31_1 } & 0.017 & 0.896 & 0.006 \\ \text { T3_32_1 } & 0.012 & 0.912 & 0.003 \\ \text { T3_33_1 } & 0.167 & 0.683 & 0.049 \\ \text { T3_34_1 } & 1.298 & 0.255 & 0.098 \\ \text { T3_35_1 } & 0.064 & 0.800 & 0.025\end{array}$




$\begin{array}{llll} & & & \\ \text { T3_36_1 } & 0.444 & 0.505 & 0.043 \\ \text { T3_37_1 } & 0.023 & 0.881 & 0.009 \\ \text { T3_38_1 } & 0.109 & 0.742 & -0.014 \\ \text { T3_39_1 } & 0.057 & 0.811 & 0.022 \\ \text { T3_40_1 } & 0.016 & 0.899 & 0.005 \\ \text { T3_41_1 } & 0.254 & 0.614 & 0.033 \\ \text { T3_42_1 } & 0.477 & 0.490 & 0.063 \\ \text { T3_43_1 } & 0.046 & 0.831 & -0.012 \\ \text { T3_44_1 } & 0.016 & 0.900 & -0.023 \\ \text { T3_45_1 } & 0.773 & 0.379 & 0.083 \\ \text { T3_46_1 } & 0.053 & 0.817 & -0.011 \\ \text { T3_47_1 } & 0.182 & 0.670 & 0.019 \\ \text { T3_48_1 } & 0.206 & 0.650 & 0.019 \\ \text { T3_49_1 } & 0.151 & 0.698 & 0.017 \\ \text { T3_50_1 } & 0.098 & 0.755 & 0.003 \\ \text { T3_51_1 } & 0.370 & 0.543 & 0.049 \\ \text { T3_54_1 } & 0.039 & 0.845 & -0.014 \\ \text { T3_55_1 } & 0.200 & 0.655 & 0.021 \\ \text { T3_56_1 } & 0.122 & 0.727 & 0.012 \\ \text { T3_57_1 } & 0.042 & 0.838 & -0.014 \\ \text { T3_58_1 } & 0.000 & 0.991 & -0.050 \\ \text { T3_61_1 } & 0.009 & 0.925 & -0.060 \\ \text { T3_62_1 } & 1.420 & 0.234 & 0.053 \\ \text { T3_63_1 } & 0.046 & 0.830 & -0.013 \\ \text { T3_1_2 } & 0.161 & 0.689 & -0.005 \\ \text { T3_6_2 } & 0.141 & 0.707 & -0.002 \\ \text { T3_7_2 } & 0.224 & 0.636 & -0.017 \\ \text { T3_9_2 } & 0.354 & 0.552 & -0.035 \\ \text { T3_10_2 } & 0.041 & 0.840 & 0.026 \\ \text { T3_11_2 } & 0.319 & 0.572 & -0.031 \\ \text { T3_12_2 } & 0.227 & 0.634 & -0.017 \\ \text { T3_16_2 } & 0.241 & 0.624 & -0.019 \\ \text { T3_18_2 } & 0.007 & 0.936 & -0.026 \\ \text { T3_19_2 } & 0.507 & 0.477 & -0.055 \\ \text { T3_20_2 } & 0.178 & 0.673 & -0.008 \\ \text { T3_22_2 } & 0.000 & 0.999 & -0.006 \\ \text { T3_23_2 } & 0.377 & 0.540 & -0.039 \\ \text { T3_32_2 } & 0.491 & 0.483 & -0.053 \\ \text { T3_2 } & 0.189 & 0.664 & -0.011 \\ \text { T3 } & 0.519 & 0.471 & -0.056 \\ \text { T3 } & 0.001 & 0.977 & -0.014\end{array}$




\begin{tabular}{|c|c|c|c|}
\hline T3_33_2 & 0.111 & 0.739 & 0.005 \\
\hline T3_34_2 & 0.150 & 0.699 & -0.004 \\
\hline T3_35_2 & 0.429 & 0.513 & -0.045 \\
\hline T3_36_2 & 0.238 & 0.625 & -0.020 \\
\hline T3_38_2 & 0.047 & 0.828 & -0.043 \\
\hline T3_39_2 & 0.188 & 0.664 & -0.011 \\
\hline T3_40_2 & 0.417 & 0.519 & -0.043 \\
\hline T3_41_2 & 0.370 & 0.543 & 0.028 \\
\hline T3_48_2 & 0.312 & 0.577 & 0.019 \\
\hline T3_49_2 & 0.490 & 0.484 & 0.039 \\
\hline T3_50_2 & 1.034 & 0.309 & 0.088 \\
\hline T3_53_2 & 0.356 & 0.551 & 0.025 \\
\hline T3_54_2 & 0.604 & 0.437 & 0.050 \\
\hline T3_56_2 & 0.060 & 0.807 & -0.031 \\
\hline T3_57_2 & 0.313 & 0.576 & 0.018 \\
\hline T3_58_2 & 0.147 & 0.701 & -0.010 \\
\hline T3_60_2 & 0.207 & 0.649 & 0.002 \\
\hline T3_61_2 & 0.015 & 0.902 & -0.050 \\
\hline T3_63_2 & 0.005 & 0.946 & -0.058 \\
\hline T3_1_3 & 0.001 & 0.975 & 0.000 \\
\hline T3_2_3 & 0.001 & 0.976 & 0.000 \\
\hline T3_4_3 & 0.012 & 0.913 & 0.028 \\
\hline T3_8_3 & 0.000 & 0.994 & 0.009 \\
\hline T3_9_3 & 0.002 & 0.961 & 0.017 \\
\hline Т3_10_3 & 0.008 & 0.927 & -0.010 \\
\hline T3_12_3 & 0.319 & 0.572 & -0.086 \\
\hline T3_13_3 & 0.001 & 0.981 & 0.001 \\
\hline T3_14_3 & 0.002 & 0.963 & 0.017 \\
\hline T3_16_3 & 0.001 & 0.981 & 0.001 \\
\hline T3_18_3 & 0.003 & 0.955 & 0.019 \\
\hline T3_19_3 & 0.314 & 0.575 & -0.086 \\
\hline T3_21_3 & 0.147 & 0.702 & -0.059 \\
\hline T3_22_3 & 0.030 & 0.862 & -0.022 \\
\hline T3_24_3 & 0.024 & 0.877 & -0.019 \\
\hline T3_25_3 & 0.014 & 0.907 & -0.013 \\
\hline T3_26_3 & 0.000 & 0.993 & 0.012 \\
\hline T3_29_3 & 0.014 & 0.906 & 0.029 \\
\hline T3_30_3 & 0.033 & 0.856 & 0.038 \\
\hline T3_31_3 & 0.046 & 0.831 & -0.028 \\
\hline T3_32_3 & 0.000 & 0.997 & 0.005 \\
\hline T3_36_3 & 0.000 & 0.985 & -0.004 \\
\hline
\end{tabular}




$\begin{array}{llll}\text { T3_37_3 } & 0.002 & 0.964 & 0.018 \\ \text { T3_39_3 } & 0.005 & 0.942 & -0.006 \\ \text { T3_41_3 } & 0.194 & 0.660 & -0.077 \\ \text { T3_43_3 } & 0.139 & 0.709 & -0.065 \\ \text { T3_44_3 } & 0.065 & 0.799 & -0.044 \\ \text { T3_46_3 } & 0.078 & 0.781 & -0.051 \\ \text { T3_47_3 } & 0.228 & 0.633 & -0.080 \\ \text { T3_48_3 } & 0.061 & 0.805 & -0.043 \\ \text { T3_49_3 } & 0.185 & 0.667 & -0.075 \\ \text { T3_52_3 } & 0.495 & 0.482 & -0.118 \\ \text { T3_53_3 } & 0.144 & 0.704 & -0.066 \\ \text { T3_54_3 } & 0.125 & 0.723 & -0.060 \\ \text { T3_57_3 } & 0.228 & 0.633 & -0.080 \\ \text { T3_58_3 } & 0.779 & 0.378 & -0.145 \\ \text { T3_60_3 } & 0.188 & 0.664 & -0.070 \\ \text { T3_61_3 } & 0.541 & 0.462 & -0.122 \\ \text { T3_64_3 } & 0.354 & 0.552 & -0.097 \\ \text { T3_244 } & 0.011 & 0.919 & -0.007 \\ \text { T3_5_4 } & 0.001 & 0.973 & 0.002 \\ \text { T3_6_4 } & 0.007 & 0.936 & -0.007 \\ \text { T3_7_4 } & 0.014 & 0.905 & -0.012 \\ \text { T3_10_4 } & 0.026 & 0.872 & 0.037 \\ \text { T3_11_4 } & 0.006 & 0.941 & -0.003 \\ \text { T3_12_4 } & 0.004 & 0.948 & -0.004 \\ \text { T3_13_4 } & 0.038 & 0.845 & -0.024 \\ \text { T3_18_4 } & 0.008 & 0.928 & -0.008 \\ \text { T3_19_4 } & 0.042 & 0.837 & 0.007 \\ \text { T3_21_4 } & 0.019 & 0.891 & -0.014 \\ \text { T3_22_4 } & 0.002 & 0.969 & 0.016 \\ \text { T3_23_4 } & 0.193 & 0.661 & 0.046 \\ \text { T3_24_4 } & 0.000 & 0.993 & 0.010 \\ \text { T3_25_4 } & 0.034 & 0.854 & -0.022 \\ \text { T3_27_4 } & 0.006 & 0.940 & -0.005 \\ \text { T3_28_4 } & 0.004 & 0.953 & -0.002 \\ \text { T3_29_4 } & 0.000 & 0.987 & 0.011 \\ \text { T3_31_4 } & 0.005 & 0.945 & -0.011 \\ \text { T3_32_4 } & 0.011 & 0.918 & -0.009 \\ \text { T3_34_4 } & 0.000 & 0.997 & 0.013 \\ \text { T3_35_4 } & 0.009 & 0.923 & 0.027 \\ \text { T3_36_4 } & 0.137 & 0.711 & 0.032 \\ \text { T3_39_4 } & 0.018 & 0.895 & -0.012 \\ & & & \end{array}$




$\begin{array}{llll}\text { T3_41_4 } & 0.001 & 0.983 & -0.030 \\ \text { T3_43_4 } & 0.000 & 0.997 & -0.039 \\ \text { T3_44_4 } & 0.121 & 0.729 & 0.023 \\ \text { T3_46_4 } & 0.000 & 0.995 & -0.032 \\ \text { T3_47_4 } & 0.013 & 0.908 & -0.055 \\ \text { T3_48_4 } & 0.081 & 0.776 & 0.011 \\ \text { T3_49_4 } & 0.132 & 0.717 & 0.022 \\ \text { T3_52_4 } & 0.000 & 0.998 & -0.032 \\ \text { T3_53_4 } & 0.001 & 0.973 & -0.028 \\ \text { T3_55_4 } & 0.363 & 0.547 & 0.059 \\ \text { T3_56_4 } & 0.002 & 0.969 & -0.027 \\ \text { T3_57_4 } & 0.035 & 0.853 & -0.004 \\ \text { T3_58_4 } & 0.117 & 0.732 & 0.019 \\ \text { T3_59_4 } & 0.160 & 0.689 & 0.028 \\ \text { T3_61_4 } & 0.048 & 0.828 & -0.070 \\ \text { T3_64_4 } & 0.000 & 0.991 & -0.038 \\ \text { T3_1_5 } & 0.000 & 0.992 & -0.009 \\ \text { T3_4_5 } & 0.111 & 0.739 & 0.013 \\ \text { T3_5_5 } & 0.024 & 0.878 & -0.034 \\ \text { T3_6_5 } & 0.001 & 0.971 & -0.016 \\ \text { T3_8_5 } & 0.003 & 0.954 & -0.020 \\ \text { T3_9_5 } & 0.000 & 0.992 & -0.009 \\ \text { T3_10_5 } & 0.000 & 0.994 & -0.005 \\ \text { T3_11_5 } & 0.349 & 0.555 & -0.029 \\ \text { T3_12_5 } & 0.041 & 0.841 & -0.042 \\ \text { T3_14_5 } & 0.024 & 0.876 & -0.035 \\ \text { T3_15_5 } & 0.010 & 0.922 & -0.026 \\ \text { T3_17_5 } & 0.020 & 0.888 & -0.032 \\ \text { T3_22_5 } & 0.000 & 0.990 & -0.003 \\ \text { T3_23_5 } & 0.244 & 0.621 & -0.019 \\ \text { T3_24_5 } & 0.019 & 0.890 & -0.032 \\ \text { T3_26_5 } & 0.000 & 0.989 & -0.014 \\ \text { T3_28_5 } & 0.004 & 0.951 & 0.004 \\ \text { T3_29_5 } & 0.030 & 0.864 & 0.020 \\ \text { T3_33_5 } & 0.000 & 0.996 & -0.004 \\ \text { T3_34_5 } & 0.041 & 0.841 & 0.028 \\ \text { T3_35_5 } & 0.010 & 0.920 & -0.025 \\ \text { T3_36_5 } & 0.000 & 0.997 & -0.006 \\ \text { T3_37_5 } & 0.015 & 0.902 & -0.030 \\ \text { T3_38_5 } & 0.001 & 0.977 & -0.015 \\ \text { T3_42_5 } & 0.299 & 0.585 & -0.016\end{array}$




\begin{tabular}{lllc} 
T3_44_5 & 0.068 & 0.794 & 0.029 \\
T3_45_5 & 0.217 & 0.641 & -0.004 \\
T3_47_5 & 0.049 & 0.824 & 0.034 \\
T3_48_5 & 0.627 & 0.429 & -0.054 \\
T3_49_5 & 0.065 & 0.799 & 0.031 \\
T3_52_5 & 0.105 & 0.747 & 0.017 \\
T3_55_5 & 0.000 & 0.991 & 0.068 \\
T3_56_5 & 0.179 & 0.672 & 0.001 \\
\hline
\end{tabular}


Table 40

Task 3 DIF: Latinx vs. Sample

\begin{tabular}{|c|c|c|c|}
\hline ItemID & ChiSq & pvalue & EffectSize \\
\hline T3_10_12 & 0.044 & 0.834 & 0.025 \\
\hline T3_11_12 & 0.126 & 0.723 & 0.073 \\
\hline T3_12_12 & 0.090 & 0.764 & 0.053 \\
\hline T3_18_12 & 0.260 & 0.610 & 0.123 \\
\hline T3_19_12 & 0.080 & 0.777 & 0.050 \\
\hline T3_20_12 & 0.036 & 0.850 & 0.015 \\
\hline T3_23_12 & 0.268 & 0.605 & 0.125 \\
\hline T3_29_12 & 0.086 & 0.769 & 0.050 \\
\hline T3_64_12 & 0.218 & 0.641 & 0.020 \\
\hline T3_42_23 & 0.000 & 0.993 & -0.104 \\
\hline T3_43_23 & 0.032 & 0.859 & 0.026 \\
\hline T3_46_23 & 0.000 & 0.991 & -0.129 \\
\hline T3_2_23 & 0.000 & 0.992 & -0.026 \\
\hline T3_4_23 & 0.001 & 0.976 & 0.005 \\
\hline T3_8_23 & 0.126 & 0.723 & 0.112 \\
\hline T3_13_23 & 0.014 & 0.906 & 0.033 \\
\hline T3_27_23 & 0.011 & 0.917 & -0.064 \\
\hline T3_29_23 & 0.002 & 0.967 & 0.009 \\
\hline T3_30_23 & 0.001 & 0.982 & -0.038 \\
\hline T3_37_23 & 0.000 & 0.989 & -0.035 \\
\hline T3_5_34 & 0.180 & 0.671 & -0.036 \\
\hline T3_42_34 & 0.931 & 0.335 & 0.137 \\
\hline T3_6_34 & 0.435 & 0.510 & -0.119 \\
\hline T3_11_34 & 0.252 & 0.616 & -0.069 \\
\hline T3_15_34 & 0.230 & 0.631 & -0.057 \\
\hline T3_17_34 & 0.388 & 0.534 & -0.107 \\
\hline T3_23_34 & 0.167 & 0.683 & -0.032 \\
\hline T3_28_34 & 0.465 & 0.495 & -0.129 \\
\hline T3_33_34 & 0.078 & 0.779 & 0.012 \\
\hline T3_50_34 & 0.198 & 0.657 & 0.086 \\
\hline T3_51_34 & 0.272 & 0.602 & -0.016 \\
\hline T3_60_45 & 0.003 & 0.957 & -0.202 \\
\hline T3_63_45 & 0.905 & 0.341 & 0.172 \\
\hline T3_2_56 & 0.125 & 0.723 & -0.019 \\
\hline T3_16_56 & 0.008 & 0.928 & 0.071 \\
\hline T3_19_56 & 0.318 & 0.573 & -0.085 \\
\hline T3_21_56 & 0.339 & 0.561 & -0.091 \\
\hline
\end{tabular}




\begin{tabular}{|c|c|c|c|}
\hline T3_25_56 & 0.040 & 0.841 & 0.032 \\
\hline T3_31_56 & 0.080 & 0.778 & 0.003 \\
\hline T3_32_56 & 0.139 & 0.710 & -0.028 \\
\hline T3_39_56 & 0.093 & 0.760 & -0.002 \\
\hline T3_51_56 & 0.012 & 0.913 & 0.080 \\
\hline T3_60_56 & 0.086 & 0.769 & 0.039 \\
\hline T3_3_456 & 0.821 & 0.365 & -0.084 \\
\hline T3_8_456 & 1.237 & 0.266 & -0.159 \\
\hline T3_9_456 & 0.798 & 0.372 & -0.082 \\
\hline T3_14_456 & 0.921 & 0.337 & -0.103 \\
\hline T3_15_456 & 1.161 & 0.281 & -0.134 \\
\hline T3_20_456 & 0.441 & 0.507 & 0.000 \\
\hline T3_33_456 & 0.724 & 0.395 & -0.064 \\
\hline T3_40_456 & 0.185 & 0.667 & 0.071 \\
\hline T3_1_1 & Inf & 0.000 & $\mathrm{NaN}$ \\
\hline T3_2_1 & Inf & 0.000 & $\mathrm{NaN}$ \\
\hline T3_3_1 & 0.029 & 0.864 & 0.022 \\
\hline T3_4_1 & 0.291 & 0.590 & 0.057 \\
\hline T3_5_1 & 0.027 & 0.871 & 0.020 \\
\hline T3_6_1 & 0.034 & 0.853 & 0.028 \\
\hline T3_7_1 & 0.009 & 0.926 & -0.002 \\
\hline T3_8_1 & 0.034 & 0.854 & 0.027 \\
\hline T3_9_1 & 0.036 & 0.850 & 0.028 \\
\hline T3_13_1 & 0.075 & 0.785 & 0.057 \\
\hline Т3_14_1 & 0.038 & 0.846 & 0.031 \\
\hline T3_15_1 & 0.045 & 0.833 & 0.036 \\
\hline T3_16_1 & 0.004 & 0.951 & -0.013 \\
\hline T3_17_1 & 0.008 & 0.931 & -0.004 \\
\hline T3_21_1 & 0.037 & 0.848 & 0.030 \\
\hline T3_22_1 & 0.032 & 0.859 & 0.025 \\
\hline T3_24_1 & 0.000 & 0.995 & -0.034 \\
\hline T3_25_1 & 0.070 & 0.792 & 0.053 \\
\hline T3_26_1 & 0.000 & 0.984 & -0.026 \\
\hline T3_27_1 & 0.084 & 0.772 & 0.063 \\
\hline T3_28_1 & 0.046 & 0.830 & 0.038 \\
\hline T3_30_1 & 0.001 & 0.972 & -0.045 \\
\hline T3_31_1 & 0.005 & 0.946 & -0.011 \\
\hline T3_32_1 & 0.042 & 0.839 & 0.035 \\
\hline T3_33_1 & 0.010 & 0.920 & 0.001 \\
\hline T3_34_1 & 0.049 & 0.825 & -0.041 \\
\hline T3_35_1 & 0.080 & 0.777 & 0.060 \\
\hline
\end{tabular}




$\begin{array}{llll}\text { T3_36_1 } & 0.621 & 0.431 & 0.130 \\ \text { T3_37_1 } & 0.055 & 0.815 & 0.044 \\ \text { T3_38_1 } & 0.252 & 0.616 & 0.050 \\ \text { T3_39_1 } & 0.071 & 0.789 & 0.055 \\ \text { T3_40_1 } & 0.047 & 0.829 & 0.039 \\ \text { T3_41_1 } & 0.000 & 0.999 & -0.054 \\ \text { T3_42_1 } & 0.035 & 0.852 & -0.001 \\ \text { T3_43_1 } & 0.127 & 0.721 & 0.049 \\ \text { T3_44_1 } & 0.118 & 0.731 & 0.042 \\ \text { T3_45_1 } & 0.106 & 0.744 & 0.037 \\ \text { T3_46_1 } & 0.083 & 0.774 & 0.019 \\ \text { T3_47_1 } & 0.041 & 0.839 & 0.007 \\ \text { T3_48_1 } & 0.002 & 0.969 & -0.102 \\ \text { T3_49_1 } & 0.277 & 0.599 & 0.096 \\ \text { T3_50_1 } & 0.033 & 0.856 & -0.005 \\ \text { T3_51_1 } & 0.014 & 0.908 & -0.023 \\ \text { T3_54_1 } & 0.007 & 0.936 & -0.026 \\ \text { T3_55_1 } & 0.110 & 0.740 & 0.040 \\ \text { T3_56_1 } & 0.118 & 0.732 & 0.044 \\ \text { T3_57_1 } & 0.024 & 0.878 & -0.012 \\ \text { T3_58_1 } & 0.067 & 0.796 & 0.015 \\ \text { T3_61_1 } & 0.204 & 0.652 & 0.074 \\ \text { T3_62_1 } & 0.311 & 0.577 & 0.065 \\ \text { T3_63_1 } & 0.026 & 0.873 & -0.123 \\ \text { T3_1_2 } & 0.003 & 0.955 & -0.042 \\ \text { T3_6_2 } & 0.002 & 0.967 & -0.039 \\ \text { T3_7_2 } & 0.062 & 0.804 & 0.082 \\ \text { T3_9_2 } & 0.023 & 0.879 & 0.051 \\ \text { T3_10_2 } & 0.013 & 0.911 & 0.037 \\ \text { T3_11_2 } & 0.027 & 0.869 & -0.079 \\ \text { T3_12_2 } & 0.001 & 0.976 & -0.035 \\ \text { T3_31_2 } & 0.003 & 0.958 & 0.016 \\ \text { T3_16_2 } & 0.017 & 0.896 & 0.043 \\ \text { T3_18_2 } & 0.022 & 0.881 & 0.006 \\ \text { T3_19_2 } & 0.001 & 0.976 & -0.015 \\ \text { T3_20_2 } & 0.014 & 0.905 & 0.039 \\ \text { T3_22_2 } & 0.062 & 0.803 & 0.038 \\ \text { T3_23_2 } & 0.001 & 0.978 & -0.016 \\ \text { T3242 } & 0.001 & 0.979 & -0.009 \\ \text { T32 } & 0.000 & 0.994 & -0.002 \\ \text { T3 } & 0.038 & 0.846 & -0.136\end{array}$




$\begin{array}{llll}\text { T3_33_2 } & 0.006 & 0.936 & 0.027 \\ \text { T3_34_2 } & 0.001 & 0.981 & -0.008 \\ \text { T3_35_2 } & 0.020 & 0.888 & 0.046 \\ \text { T3_36_2 } & 0.002 & 0.963 & -0.039 \\ \text { T3_38_2 } & 0.053 & 0.817 & 0.025 \\ \text { T3_39_2 } & 0.011 & 0.918 & 0.034 \\ \text { T3_40_2 } & 0.004 & 0.949 & 0.021 \\ \text { T3_41_2 } & 0.024 & 0.876 & 0.017 \\ \text { T3_48_2 } & 0.144 & 0.705 & 0.088 \\ \text { T3_49_2 } & 0.000 & 0.999 & -0.060 \\ \text { T3_50_2 } & 0.257 & 0.612 & 0.135 \\ \text { T3_53_2 } & 0.009 & 0.925 & -0.004 \\ \text { T3_54_2 } & 0.005 & 0.942 & -0.012 \\ \text { T3_56_2 } & 0.030 & 0.862 & 0.023 \\ \text { T3_57_2 } & 0.074 & 0.786 & 0.053 \\ \text { T3_58_2 } & 0.030 & 0.863 & 0.025 \\ \text { T3_60_2 } & 0.001 & 0.977 & -0.049 \\ \text { T3_61_2 } & 0.020 & 0.889 & 0.007 \\ \text { T3_63_2 } & 0.000 & 0.989 & -0.034 \\ \text { T3_1_3 } & 0.003 & 0.959 & -0.034 \\ \text { T3_2_3 } & 0.000 & 0.993 & -0.015 \\ \text { T3_4_3 } & 0.030 & 0.862 & -0.075 \\ \text { T3_8_3 } & 0.009 & 0.924 & 0.039 \\ \text { T3_9_3 } & 0.001 & 0.976 & -0.007 \\ \text { T3_10_3 } & 0.001 & 0.975 & -0.004 \\ \text { T3_12_3 } & 0.009 & 0.927 & -0.129 \\ \text { T3_13_3 } & 0.000 & 0.995 & -0.019 \\ \text { T3_14_3 } & 0.027 & 0.870 & -0.072 \\ \text { T3_16_3 } & 0.001 & 0.974 & -0.029 \\ \text { T3_32_3 } & 0.001 & 0.976 & -0.007 \\ \text { T3_18_3 } & 0.014 & 0.908 & -0.056 \\ \text { T3_19_3 } & 0.019 & 0.891 & -0.145 \\ \text { T3_21_3 } & 0.000 & 0.990 & -0.096 \\ \text { T3_22_3 } & 0.000 & 1.000 & -0.017 \\ \text { T3_24_3 } & 0.000 & 0.999 & -0.016 \\ \text { T3_25_3 } & 0.001 & 0.972 & -0.005 \\ \text { T3_26_3 } & 0.005 & 0.943 & -0.041 \\ \text { T323 } & 0.000 & 1.000 & 0.007 \\ \text { T333 } & 0.000 & 0.983 & 0.014 \\ \text { T3 } & 0.012 & 0.912 & 0.044 \\ \text { T3 } & & 0.912 & -0.028\end{array}$




$\begin{array}{llll}\text { T3_37_3 } & 0.000 & 1.000 & -0.017 \\ \text { T3_39_3 } & 0.004 & 0.950 & -0.038 \\ \text { T3_41_3 } & 0.012 & 0.913 & -0.025 \\ \text { T3_43_3 } & 0.025 & 0.875 & -0.010 \\ \text { T3_44_3 } & 0.087 & 0.769 & 0.038 \\ \text { T3_46_3 } & 0.003 & 0.957 & -0.047 \\ \text { T3_47_3 } & 0.101 & 0.751 & 0.044 \\ \text { T3_48_3 } & 0.037 & 0.847 & 0.005 \\ \text { T3_49_3 } & 0.038 & 0.846 & 0.003 \\ \text { T3_52_3 } & 0.000 & 0.994 & -0.064 \\ \text { T3_53_3 } & 0.002 & 0.967 & -0.049 \\ \text { T3_54_3 } & 0.110 & 0.740 & 0.049 \\ \text { T3_57_3 } & 0.138 & 0.711 & 0.061 \\ \text { T3_58_3 } & 0.102 & 0.750 & 0.043 \\ \text { T3_60_3 } & 0.044 & 0.834 & 0.011 \\ \text { T3_61_3 } & 0.124 & 0.725 & 0.055 \\ \text { T3_64_3 } & 0.138 & 0.710 & 0.064 \\ \text { T3_2_4 } & 0.378 & 0.539 & -0.102 \\ \text { T3_5_4 } & 0.387 & 0.534 & -0.106 \\ \text { T3_6_4 } & 0.290 & 0.590 & -0.076 \\ \text { T3_7_4 } & 0.191 & 0.663 & -0.044 \\ \text { T3_10_4 } & 0.116 & 0.734 & -0.010 \\ \text { T3_11_4 } & 0.069 & 0.793 & 0.021 \\ \text { T3_12_4 } & 0.369 & 0.544 & -0.100 \\ \text { T3_13_4 } & 0.384 & 0.536 & -0.099 \\ \text { T3_18_4 } & 0.144 & 0.705 & -0.019 \\ \text { T3_19_4 } & 0.006 & 0.940 & -0.026 \\ \text { T3_21_4 } & 0.133 & 0.716 & -0.020 \\ \text { T3_22_4 } & 0.392 & 0.531 & -0.101 \\ \text { T3_23_4 } & 0.309 & 0.578 & -0.184 \\ \text { T3_24_4 } & 0.077 & 0.781 & 0.013 \\ \text { T3_25_4 } & 0.069 & 0.793 & 0.016 \\ \text { T3_27_4 } & 0.029 & 0.864 & 0.049 \\ \text { T3_28_4 } & 0.060 & 0.807 & 0.026 \\ \text { T3_29_4 } & 0.235 & 0.628 & -0.059 \\ \text { T3_31_4 } & 0.124 & 0.724 & -0.119 \\ \text { T3_32_4 } & 0.494 & 0.482 & -0.127 \\ \text { T3_39_4 } & 0.030 & 0.863 & 0.048\end{array}$




\begin{tabular}{|c|c|c|c|}
\hline T3_41_4 & 0.065 & 0.799 & -0.013 \\
\hline T3_43_4 & 0.115 & 0.735 & 0.013 \\
\hline T3_44_4 & 0.340 & 0.560 & 0.101 \\
\hline T3_46_4 & 0.005 & 0.942 & -0.075 \\
\hline T3_47_4 & 0.172 & 0.679 & 0.042 \\
\hline T3_48_4 & 0.182 & 0.670 & 0.042 \\
\hline T3_49_4 & 0.002 & 0.967 & -0.136 \\
\hline T3_52_4 & 0.133 & 0.715 & 0.022 \\
\hline T3_53_4 & 0.000 & 0.993 & -0.122 \\
\hline T3_55_4 & 0.013 & 0.910 & -0.061 \\
\hline T3_56_4 & 0.010 & 0.920 & -0.066 \\
\hline T3_57_4 & 0.000 & 0.992 & -0.101 \\
\hline T3_58_4 & 0.000 & 0.987 & -0.101 \\
\hline T3_59_4 & 0.052 & 0.820 & -0.023 \\
\hline T3_61_4 & 0.222 & 0.638 & 0.061 \\
\hline T3_64_4 & 0.177 & 0.674 & 0.043 \\
\hline T3_1_5 & 0.167 & 0.683 & -0.078 \\
\hline T3_4_5 & 0.027 & 0.870 & 0.103 \\
\hline T3_5_5 & 0.076 & 0.783 & -0.034 \\
\hline T3_6_5 & 0.031 & 0.859 & -0.006 \\
\hline T3_8_5 & 0.033 & 0.855 & 0.000 \\
\hline T3_9_5 & 0.034 & 0.854 & -0.005 \\
\hline T3_10_5 & 0.045 & 0.832 & -0.013 \\
\hline T3_11_5 & 0.009 & 0.925 & 0.077 \\
\hline T3_12_5 & 0.000 & 0.989 & 0.077 \\
\hline T3_14_5 & 0.008 & 0.927 & 0.027 \\
\hline T3_15_5 & 0.005 & 0.946 & 0.036 \\
\hline T3_17_5 & 0.043 & 0.835 & -0.015 \\
\hline T3_22_5 & 0.011 & 0.917 & 0.022 \\
\hline T3_23_5 & 0.065 & 0.799 & -0.068 \\
\hline T3_24_5 & 0.000 & 0.995 & 0.081 \\
\hline T3_26_5 & 0.106 & 0.745 & -0.048 \\
\hline T3_28_5 & 0.064 & 0.801 & -0.029 \\
\hline T3_29_5 & 0.149 & 0.700 & -0.074 \\
\hline T3_33_5 & 0.046 & 0.830 & -0.015 \\
\hline T3_34_5 & 0.205 & 0.651 & -0.131 \\
\hline T3_35_5 & 0.026 & 0.873 & -0.001 \\
\hline T3_36_5 & 0.009 & 0.924 & 0.025 \\
\hline T3_37_5 & 0.002 & 0.965 & 0.044 \\
\hline T3_38_5 & 0.006 & 0.938 & 0.031 \\
\hline T3_42_5 & 0.001 & 0.971 & -0.068 \\
\hline
\end{tabular}




\begin{tabular}{llll} 
T3_44_5 & 0.016 & 0.900 & -0.098 \\
T3_45_5 & 0.004 & 0.951 & -0.012 \\
T3_47_5 & 0.002 & 0.969 & -0.070 \\
T3_48_5 & 0.001 & 0.980 & -0.046 \\
T3_49_5 & 0.001 & 0.979 & -0.067 \\
T3_52_5 & 0.001 & 0.980 & -0.040 \\
T3_55_5 & 0.004 & 0.951 & -0.011 \\
T3_56_5 & 0.005 & 0.943 & -0.078 \\
\hline
\end{tabular}


Table 41

Task 4 DIF: Male vs. female

\begin{tabular}{lccc}
\hline ItemID & ChiSq & pvalue & EffectSize \\
\hline T4_7_34 & 0.007 & 0.934 & 0.025 \\
T4_8_34 & 0.030 & 0.863 & -0.019 \\
T4_12_34 & 0.000 & 0.989 & 0.010 \\
T4_23_34 & 0.004 & 0.951 & 0.019 \\
T4_25_34 & 0.000 & 0.991 & 0.012 \\
T4_29_34 & 0.020 & 0.886 & 0.011 \\
T4_36_34 & 0.025 & 0.875 & 0.056 \\
T4_43_34 & 0.011 & 0.918 & 0.013 \\
T4_38_45 & 0.055 & 0.814 & 0.068 \\
T4_41_45 & 0.130 & 0.719 & 0.086 \\
T4_5_56 & 0.000 & 0.992 & -0.011 \\
T4_11_56 & 0.020 & 0.887 & -0.029 \\
T4_14_56 & 0.004 & 0.952 & 0.005 \\
T4_22_56 & 0.073 & 0.787 & -0.043 \\
T4_23_56 & 0.000 & 0.993 & -0.015 \\
T4_31_56 & 0.007 & 0.936 & -0.047 \\
T4_45_56 & 0.008 & 0.930 & -0.045 \\
T4_46_56 & 0.001 & 0.973 & -0.039 \\
T4_1_456 & 0.000 & 0.997 & 0.021 \\
T4_6_456 & 0.038 & 0.845 & -0.006 \\
T4_14_456 & 0.217 & 0.641 & -0.048 \\
T4_15_456 & 0.000 & 0.985 & 0.010 \\
T4_22_456 & 0.000 & 0.995 & 0.021 \\
T4_1_3 & 0.000 & 0.989 & -0.022 \\
T4_2_3 & 0.018 & 0.893 & 0.004 \\
T4_3_3 & 0.086 & 0.770 & -0.058 \\
T4_4_3 & 0.000 & 0.985 & -0.013 \\
T4_5_3 & 0.000 & 0.995 & -0.019 \\
T4_6_3 & 0.000 & 0.988 & -0.017 \\
T4_9_3 & 0.008 & 0.929 & -0.034 \\
T4_10_3 & 0.000 & 0.986 & -0.013 \\
T4_11_3 & 0.000 & 0.995 & -0.015 \\
T4_13_3 & 0.020 & 0.888 & -0.040 \\
T4_14_3 & 0.000 & 0.992 & -0.017 \\
T4_15_3 & 0.000 & 0.990 & -0.020 \\
T4_3 & 0.058 & 0.810 & 0.019 \\
T4_3 & 0.001 & 0.975 & -0.011 \\
\hline
\end{tabular}




\begin{tabular}{|c|c|c|c|}
\hline T4_18_3 & 0.022 & 0.881 & 0.006 \\
\hline T4_19_3 & 0.007 & 0.936 & -0.003 \\
\hline T4_20_3 & Inf & 0.000 & $\mathrm{NaN}$ \\
\hline T4_21_3 & 0.005 & 0.944 & -0.004 \\
\hline T4_22_3 & 0.002 & 0.966 & -0.027 \\
\hline T4_24_3 & 0.019 & 0.890 & 0.005 \\
\hline T4_26_3 & 0.000 & 0.989 & 0.006 \\
\hline T4_29_3 & 0.003 & 0.959 & -0.008 \\
\hline T4_30_3 & 0.068 & 0.794 & -0.035 \\
\hline T4_32_3 & 0.023 & 0.880 & -0.019 \\
\hline T4_34_3 & 0.000 & 1.000 & -0.002 \\
\hline T4_35_3 & 0.000 & 0.984 & -0.003 \\
\hline T4_37_3 & 0.013 & 0.908 & 0.019 \\
\hline T4_38_3 & 0.081 & 0.776 & -0.039 \\
\hline T4_39_3 & 0.028 & 0.868 & -0.020 \\
\hline T4_40_3 & 0.012 & 0.915 & 0.016 \\
\hline T4_41_3 & 0.028 & 0.867 & -0.023 \\
\hline T4_42_3 & 0.005 & 0.944 & -0.010 \\
\hline T4_2_4 & 0.000 & 0.997 & 0.023 \\
\hline T4_3_4 & 0.148 & 0.700 & -0.031 \\
\hline T4_5_4 & 0.032 & 0.859 & -0.003 \\
\hline T4_7_4 & 0.046 & 0.831 & -0.008 \\
\hline T4_8_4 & 0.001 & 0.979 & 0.017 \\
\hline T4_9_4 & 0.013 & 0.909 & 0.007 \\
\hline T4_10_4 & 0.000 & 0.989 & 0.025 \\
\hline T4_12_4 & 0.005 & 0.944 & 0.074 \\
\hline T4_13_4 & 0.058 & 0.810 & -0.012 \\
\hline T4_16_4 & 0.014 & 0.906 & 0.006 \\
\hline T4_17_4 & 0.029 & 0.866 & -0.002 \\
\hline T4_20_4 & 0.109 & 0.741 & 0.013 \\
\hline T4_21_4 & 0.005 & 0.943 & 0.012 \\
\hline T4_24_4 & 0.000 & 0.997 & 0.023 \\
\hline T4_25_4 & 0.026 & 0.872 & 0.000 \\
\hline T4_27_4 & 0.000 & 0.998 & 0.033 \\
\hline T4_28_4 & 0.061 & 0.804 & 0.068 \\
\hline T4_30_4 & 0.008 & 0.930 & 0.018 \\
\hline T4_31_4 & 0.000 & 0.994 & 0.031 \\
\hline T4_32_4 & 0.026 & 0.871 & 0.005 \\
\hline T4_33_4 & 0.000 & 0.993 & 0.035 \\
\hline T4_34_4 & 0.078 & 0.780 & 0.074 \\
\hline T4_37_4 & 0.000 & 0.987 & 0.036 \\
\hline
\end{tabular}




$\begin{array}{lccc}\text { T4_1_5 } & 0.003 & 0.959 & -0.017 \\ \text { T4_2_5 } & 0.006 & 0.939 & 0.007 \\ \text { T4_3_5 } & 0.002 & 0.969 & 0.002 \\ \text { T4_4_5 } & 0.000 & 0.984 & -0.018 \\ \text { T4_7_5 } & 0.002 & 0.966 & 0.002 \\ \text { T4_8_5 } & 0.006 & 0.937 & 0.008 \\ \text { T4_9_5 } & \text { Inf } & 0.000 & \text { NaN } \\ \text { T4_12_5 } & 0.001 & 0.974 & 0.001 \\ \text { T4_16_5 } & 0.001 & 0.973 & 0.001 \\ \text { T4_17_5 } & 0.020 & 0.887 & 0.017 \\ \text { T4_18_5 } & 0.005 & 0.945 & 0.006 \\ \text { T4_19_5 } & 0.004 & 0.950 & -0.020 \\ \text { T4_20_5 } & 0.000 & 0.985 & -0.012 \\ \text { T4_21_5 } & 0.030 & 0.863 & 0.022 \\ \text { T4_26_5 } & 0.000 & 0.989 & 0.001 \\ \text { T4_29_5 } & 0.023 & 0.879 & 0.020 \\ \text { T4_30_5 } & 0.016 & 0.899 & -0.023 \\ \text { T4_43_5 } & 0.001 & 0.973 & -0.011 \\ \text { T4_47_5 } & 0.005 & 0.945 & -0.015 \\ \text { T4_48_5 } & 0.007 & 0.933 & -0.017 \\ \text { T4_49_5 } & 0.018 & 0.894 & -0.022 \\ \text { T4_50_5 } & 0.012 & 0.913 & 0.015 \\ \text { T4_51_5 } & 0.009 & 0.925 & -0.020 \\ \text { T4_52_5 } & 0.001 & 0.977 & 0.003 \\ \text { T4_25_5 } & 0.013 & 0.911 & -0.026 \\ \text { T4_4_6 } & 0.223 & 0.637 & 0.047 \\ \text { T4_5_6 } & 0.000 & 0.993 & -0.029 \\ \text { T4_7_6 } & 0.002 & 0.968 & -0.032 \\ \text { T4_8_6 } & 0.025 & 0.873 & 0.025 \\ \text { T4_9_6 } & 0.000 & 0.998 & -0.004 \\ \text { T4_11_6 } & 0.000 & 0.999 & -0.002 \\ \text { T4_12_6 } & 0.011 & 0.917 & 0.017 \\ \text { T4_13_6 } & 0.015 & 0.902 & 0.021 \\ \text { T4_14_6 } & 0.003 & 0.958 & -0.009 \\ \text { T4_16_6 } & 0.013 & 0.910 & 0.020 \\ \text { T4_17_6 } & 0.006 & 0.941 & 0.014 \\ \text { T4_18_6 } & 0.015 & 0.903 & 0.021 \\ \text { T466 } & 0.010 & 0.920 & 0.017 \\ \text { T46 } & 0.007 & 0.932 & -0.015 \\ \text { T46 } & 0.001 & 0.980 & 0.006 \\ \text { T4 } & 0.008 & 0.930 & -0.009\end{array}$




\begin{tabular}{llll} 
T4_29_6 & 0.000 & 0.998 & -0.023 \\
T4_30_6 & 0.001 & 0.979 & -0.016 \\
T4_31_6 & 0.000 & 0.993 & -0.019 \\
T4_32_6 & 0.001 & 0.982 & -0.017 \\
T4_33_6 & 0.018 & 0.893 & -0.047 \\
T4_34_6 & 0.000 & 0.993 & -0.023 \\
T4_35_6 & 0.000 & 0.991 & -0.025 \\
T4_36_6 & 0.005 & 0.943 & -0.012 \\
T4_38_6 & 0.036 & 0.849 & 0.005 \\
T4_39_6 & 0.001 & 0.974 & -0.017 \\
T4_43_6 & 0.008 & 0.928 & -0.009 \\
\hline
\end{tabular}


Table 42

Task 4 DIF: White vs. Sample

\begin{tabular}{lccc}
\hline ItemID & ChiSq & pvalue & EffectSize \\
\hline T4_7_34 & 0.133 & 0.715 & -0.046 \\
T4_8_34 & 0.009 & 0.927 & -0.048 \\
T4_12_34 & 0.007 & 0.933 & -0.045 \\
T4_23_34 & 0.033 & 0.855 & 0.000 \\
T4_25_34 & 0.052 & 0.820 & 0.008 \\
T4_29_34 & 0.055 & 0.814 & 0.008 \\
T4_36_34 & 0.068 & 0.795 & 0.004 \\
T4_43_34 & 0.195 & 0.659 & -0.024 \\
T4_38_45 & 0.002 & 0.963 & -0.022 \\
T4_41_45 & 0.011 & 0.917 & -0.051 \\
T4_5_56 & 0.033 & 0.856 & -0.022 \\
T4_11_56 & 0.025 & 0.875 & -0.020 \\
T4_14_56 & 0.322 & 0.570 & 0.039 \\
T4_22_56 & 0.115 & 0.734 & 0.005 \\
T4_23_56 & 0.493 & 0.483 & -0.026 \\
T4_31_56 & 0.078 & 0.780 & -0.004 \\
T4_45_56 & 0.180 & 0.671 & 0.019 \\
T4_46_56 & 0.274 & 0.601 & 0.035 \\
T4_1_456 & 0.713 & 0.399 & 0.050 \\
T4_6_456 & 0.277 & 0.599 & -0.007 \\
T4_14_456 & 0.229 & 0.632 & -0.010 \\
T4_15_456 & 0.352 & 0.553 & 0.008 \\
T4_22_456 & 0.219 & 0.640 & -0.018 \\
T4_1_3 & 0.031 & 0.861 & 0.060 \\
T4_2_3 & 0.003 & 0.954 & 0.018 \\
T4_3_3 & 0.008 & 0.931 & 0.110 \\
T4_4_3 & 0.001 & 0.981 & 0.001 \\
T4_5_3 & 0.038 & 0.845 & -0.024 \\
T4_6_3 & 0.017 & 0.895 & -0.016 \\
T4_9_3 & 0.015 & 0.904 & -0.013 \\
T4_10_3 & 0.011 & 0.917 & -0.011 \\
T4_11_3 & 0.050 & 0.823 & 0.065 \\
T4_13_3 & 0.027 & 0.869 & -0.021 \\
T4_14_3 & 0.012 & 0.914 & -0.011 \\
T4_15_3 & 0.011 & 0.915 & -0.012 \\
T4_16_3 & 0.024 & 0.876 & -0.017 \\
T4_17_3 & 0.000 & 0.991 & 0.003 \\
\end{tabular}




$\begin{array}{lccc}\text { T4_18_3 } & 0.026 & 0.872 & -0.020 \\ \text { T4_19_3 } & 0.000 & 0.986 & 0.001 \\ \text { T4_20_3 } & \text { Inf } & 0.000 & \text { NaN } \\ \text { T4_21_3 } & 0.005 & 0.945 & -0.007 \\ \text { T4_22_3 } & 0.002 & 0.969 & -0.001 \\ \text { T4_24_3 } & 0.000 & 0.991 & 0.011 \\ \text { T4_26_3 } & 0.000 & 0.990 & 0.087 \\ \text { T4_29_3 } & 0.006 & 0.937 & 0.099 \\ \text { T4_30_3 } & 0.217 & 0.642 & 0.020 \\ \text { T4_32_3 } & 0.022 & 0.881 & 0.111 \\ \text { T4_34_3 } & 0.005 & 0.941 & 0.071 \\ \text { T4_35_3 } & 0.017 & 0.897 & 0.105 \\ \text { T4_37_3 } & 0.450 & 0.502 & -0.020 \\ \text { T4_38_3 } & 0.003 & 0.957 & 0.079 \\ \text { T4_39_3 } & 0.832 & 0.362 & -0.054 \\ \text { T4_40_3 } & 0.004 & 0.949 & 0.074 \\ \text { T4_41_3 } & 0.023 & 0.880 & 0.111 \\ \text { T4_42_3 } & 0.001 & 0.970 & 0.073 \\ \text { T4_2_4 } & 0.000 & 0.992 & -0.036 \\ \text { T4_3_4 } & 0.037 & 0.847 & -0.008 \\ \text { T4_5_4 } & 0.009 & 0.926 & -0.019 \\ \text { T4_7_4 } & 0.071 & 0.790 & 0.004 \\ \text { T4_8_4 } & 0.043 & 0.835 & 0.000 \\ \text { T4_9_4 } & 0.004 & 0.951 & -0.026 \\ \text { T4_10_4 } & 0.000 & 1.000 & -0.032 \\ \text { T4_12_4 } & 0.166 & 0.684 & -0.057 \\ \text { T4_13_4 } & 0.024 & 0.878 & -0.006 \\ \text { T4_16_4 } & 0.074 & 0.785 & 0.008 \\ \text { T4_17_4 } & 0.047 & 0.828 & -0.001 \\ \text { T4_20_4 } & 0.010 & 0.919 & -0.010 \\ \text { T4_21_4 } & 0.186 & 0.667 & 0.032 \\ \text { T4_24_4 } & 0.002 & 0.967 & -0.027 \\ \text { T4_25_4 } & 0.174 & 0.676 & 0.028 \\ \text { T4_27_4 } & 0.132 & 0.716 & -0.012 \\ \text { T4_28_4 } & 0.135 & 0.714 & -0.012 \\ \text { T4_30_4 } & 0.091 & 0.763 & -0.002 \\ \text { T4_31_4 } & 0.009 & 0.924 & 0.028 \\ \text { T4_32_4 } & 0.339 & 0.560 & -0.044 \\ \text { T4344 } & 0.128 & 0.721 & -0.008 \\ \text { T4_4 } & 0.141 & 0.708 & -0.012 \\ \text { T4 } & 0.099 & 0.754 & -0.005\end{array}$




$\begin{array}{lccc}\text { T4_1_5 } & 0.006 & 0.939 & 0.010 \\ \text { T4_2_5 } & 0.007 & 0.931 & -0.020 \\ \text { T4_3_5 } & 0.026 & 0.873 & 0.022 \\ \text { T4_4_5 } & 0.838 & 0.360 & 0.063 \\ \text { T4_7_5 } & 0.002 & 0.965 & 0.005 \\ \text { T4_8_5 } & 0.001 & 0.975 & 0.003 \\ \text { T4_9_5 } & \text { Inf } & 0.000 & \text { NaN } \\ \text { T4_12_5 } & 0.008 & 0.928 & 0.013 \\ \text { T4_16_5 } & 0.000 & 0.996 & -0.001 \\ \text { T4_17_5 } & 0.026 & 0.871 & 0.023 \\ \text { T4_18_5 } & 0.013 & 0.910 & 0.015 \\ \text { T4_19_5 } & 0.895 & 0.344 & 0.072 \\ \text { T4_20_5 } & 0.036 & 0.850 & 0.026 \\ \text { T4_21_5 } & 0.005 & 0.945 & 0.008 \\ \text { T4_26_5 } & 0.001 & 0.978 & -0.079 \\ \text { T4_29_5 } & 0.000 & 0.987 & -0.073 \\ \text { T4_30_5 } & 0.003 & 0.954 & -0.076 \\ \text { T4_43_5 } & 0.003 & 0.954 & -0.060 \\ \text { T4_47_5 } & 0.128 & 0.721 & -0.017 \\ \text { T4_48_5 } & 0.056 & 0.813 & -0.027 \\ \text { T4_49_5 } & 0.316 & 0.574 & 0.017 \\ \text { T4_50_5 } & 0.203 & 0.652 & -0.001 \\ \text { T4_51_5 } & 0.220 & 0.639 & 0.001 \\ \text { T4_52_5 } & 0.021 & 0.885 & -0.049 \\ \text { T4_25_5 } & 0.012 & 0.911 & 0.015 \\ \text { T4_4_6 } & 0.063 & 0.802 & 0.024 \\ \text { T4_5_6 } & 0.052 & 0.819 & 0.019 \\ \text { T4_7_6 } & 0.213 & 0.644 & 0.054 \\ \text { T4_8_6 } & 0.067 & 0.795 & -0.002 \\ \text { T4_9_6 } & 0.093 & 0.760 & 0.004 \\ \text { T4_11_6 } & 0.065 & 0.799 & -0.003 \\ \text { T4_12_6 } & 0.363 & 0.547 & 0.047 \\ \text { T4_13_6 } & 0.011 & 0.916 & -0.026 \\ \text { T4_14_6 } & 0.356 & 0.551 & 0.049 \\ \text { T4_16_6 } & 0.054 & 0.816 & -0.007 \\ \text { T4_17_6 } & 0.013 & 0.908 & -0.024 \\ \text { T4_18_6 } & 0.047 & 0.828 & -0.009 \\ \text { T4_20_6 } & 0.140 & 0.709 & 0.014 \\ \text { T427_6 } & 0.325 & 0.569 & 0.043 \\ \text { T466 } & 0.056 & 0.814 & -0.006 \\ \text { T4 } & 0.001 & 0.977 & 0.036\end{array}$




\begin{tabular}{llll} 
T4_29_6 & 0.000 & 0.989 & 0.025 \\
T4_30_6 & 0.008 & 0.930 & 0.013 \\
T4_31_6 & 0.130 & 0.718 & -0.026 \\
T4_32_6 & 0.007 & 0.932 & 0.013 \\
T4_33_6 & 0.004 & 0.947 & 0.042 \\
T4_34_6 & 0.009 & 0.926 & 0.014 \\
T4_35_6 & 0.025 & 0.875 & 0.056 \\
T4_36_6 & 0.006 & 0.940 & 0.042 \\
T4_38_6 & 0.003 & 0.960 & 0.039 \\
T4_39_6 & 0.027 & 0.870 & 0.003 \\
T4_43_6 & 0.026 & 0.872 & 0.055 \\
\hline
\end{tabular}


Table 43

Task 4 DIF: Black vs. Sample

\begin{tabular}{|c|c|c|c|}
\hline ItemID & ChiSq & pvalue & EffectSize \\
\hline T4_7_34 & 0.275 & 0.600 & 0.051 \\
\hline T4_8_34 & 0.009 & 0.926 & 0.029 \\
\hline T4_12_34 & 0.006 & 0.939 & 0.025 \\
\hline T4_23_34 & 0.003 & 0.957 & 0.019 \\
\hline T4_25_34 & 0.000 & 0.994 & 0.010 \\
\hline T4_29_34 & 0.091 & 0.763 & 0.006 \\
\hline T4_36_34 & 0.178 & 0.673 & 0.025 \\
\hline T4_43_34 & 0.224 & 0.636 & 0.031 \\
\hline T4_38_45 & 0.017 & 0.895 & 0.026 \\
\hline T4_41_45 & 0.020 & 0.887 & 0.077 \\
\hline T4_5_56 & 0.042 & 0.838 & -0.006 \\
\hline T4_11_56 & 0.000 & 0.994 & 0.027 \\
\hline T4_14_56 & 0.030 & 0.863 & -0.006 \\
\hline T4_22_56 & 0.036 & 0.849 & -0.004 \\
\hline T4_23_56 & 0.447 & 0.504 & 0.020 \\
\hline T4_31_56 & 0.061 & 0.805 & 0.000 \\
\hline T4_45_56 & 0.043 & 0.836 & 0.006 \\
\hline T4_46_56 & 0.393 & 0.531 & -0.058 \\
\hline T4_1_456 & 0.018 & 0.892 & 0.009 \\
\hline T4_6_456 & 0.000 & 0.995 & 0.032 \\
\hline T4_14_456 & 0.000 & 0.985 & 0.034 \\
\hline T4_15_456 & 0.098 & 0.755 & -0.010 \\
\hline T4_22_456 & 0.001 & 0.978 & 0.033 \\
\hline T4_1_3 & 0.018 & 0.893 & -0.064 \\
\hline T4_2_3 & 0.005 & 0.943 & -0.016 \\
\hline T4_3_3 & 0.084 & 0.772 & -0.087 \\
\hline T4_4_3 & 0.000 & 0.983 & 0.004 \\
\hline T4_5_3 & 0.014 & 0.906 & 0.019 \\
\hline T4_6_3 & 0.007 & 0.932 & 0.015 \\
\hline T4_9_3 & 0.012 & 0.914 & 0.017 \\
\hline T4_10_3 & 0.013 & 0.909 & 0.019 \\
\hline T4_11_3 & 0.000 & 0.996 & -0.043 \\
\hline T4_13_3 & 0.005 & 0.946 & 0.012 \\
\hline T4_14_3 & 0.001 & 0.973 & -0.010 \\
\hline T4_15_3 & 0.023 & 0.879 & 0.024 \\
\hline T4_16_3 & 0.009 & 0.926 & 0.013 \\
\hline T4_17_3 & 0.000 & 0.999 & 0.001 \\
\hline
\end{tabular}




\begin{tabular}{|c|c|c|c|}
\hline T4_18_3 & 0.016 & 0.900 & 0.021 \\
\hline T4_19_3 & 0.006 & 0.940 & -0.016 \\
\hline T4_20_3 & Inf & 0.000 & $\mathrm{NaN}$ \\
\hline T4_21_3 & 0.001 & 0.975 & -0.008 \\
\hline T4_22_3 & 0.002 & 0.969 & 0.007 \\
\hline T4_24_3 & 0.000 & 0.995 & -0.003 \\
\hline T4_26_3 & 0.053 & 0.818 & -0.078 \\
\hline T4_29_3 & 0.081 & 0.776 & -0.084 \\
\hline T4_30_3 & 0.001 & 0.983 & -0.038 \\
\hline T4_32_3 & 0.087 & 0.768 & -0.086 \\
\hline T4_34_3 & 0.000 & 0.997 & -0.044 \\
\hline T4_35_3 & 0.035 & 0.852 & -0.071 \\
\hline T4_37_3 & 0.011 & 0.916 & -0.019 \\
\hline T4_38_3 & 0.028 & 0.867 & -0.070 \\
\hline T4_39_3 & 0.105 & 0.746 & 0.012 \\
\hline T4_40_3 & 0.018 & 0.894 & -0.063 \\
\hline T4_41_3 & 0.041 & 0.839 & -0.076 \\
\hline T4_42_3 & 0.011 & 0.916 & -0.059 \\
\hline T4_2_4 & 0.000 & 0.996 & 0.014 \\
\hline T4_3_4 & 0.007 & 0.935 & -0.003 \\
\hline T4_5_4 & 0.010 & 0.922 & 0.029 \\
\hline T4_7_4 & 0.000 & 0.986 & 0.008 \\
\hline T4_8_4 & 0.001 & 0.978 & 0.003 \\
\hline T4_9_4 & 0.000 & 0.995 & 0.016 \\
\hline T4_10_4 & 0.005 & 0.944 & 0.022 \\
\hline T4_12_4 & 0.528 & 0.468 & 0.082 \\
\hline T4_13_4 & 0.005 & 0.945 & -0.007 \\
\hline T4_16_4 & 0.025 & 0.875 & -0.016 \\
\hline T4_17_4 & 0.013 & 0.911 & -0.009 \\
\hline T4_20_4 & 0.076 & 0.783 & 0.014 \\
\hline T4_21_4 & 0.017 & 0.898 & -0.011 \\
\hline T4_24_4 & 0.015 & 0.902 & 0.032 \\
\hline T4_25_4 & 0.008 & 0.929 & -0.004 \\
\hline T4_27_4 & 0.141 & 0.707 & 0.017 \\
\hline T4_28_4 & 0.129 & 0.719 & 0.014 \\
\hline T4_30_4 & 0.133 & 0.715 & 0.016 \\
\hline T4_31_4 & 0.038 & 0.846 & -0.010 \\
\hline T4_32_4 & 0.235 & 0.628 & 0.033 \\
\hline T4_33_4 & 0.158 & 0.691 & 0.019 \\
\hline T4_34_4 & 0.159 & 0.690 & 0.020 \\
\hline T4_37_4 & 0.045 & 0.832 & -0.006 \\
\hline
\end{tabular}




$\begin{array}{lccc}\text { T4_1_5 } & 0.001 & 0.970 & -0.036 \\ \text { T4_2_5 } & 0.014 & 0.907 & -0.007 \\ \text { T4_3_5 } & 0.000 & 1.000 & -0.030 \\ \text { T4_4_5 } & 0.563 & 0.453 & -0.060 \\ \text { T4_7_5 } & 0.010 & 0.919 & -0.046 \\ \text { T4_8_5 } & 0.052 & 0.819 & 0.011 \\ \text { T4_9_5 } & \text { Inf } & 0.000 & \text { NaN } \\ \text { T4_12_5 } & 0.006 & 0.940 & -0.014 \\ \text { T4_16_5 } & 0.002 & 0.963 & -0.018 \\ \text { T4_17_5 } & 0.000 & 0.989 & -0.028 \\ \text { T4_18_5 } & 0.001 & 0.980 & -0.033 \\ \text { T4_19_5 } & 0.579 & 0.447 & -0.067 \\ \text { T4_20_5 } & 0.001 & 0.977 & -0.035 \\ \text { T4_21_5 } & 0.059 & 0.807 & 0.014 \\ \text { T4_26_5 } & 0.004 & 0.952 & 0.101 \\ \text { T4_29_5 } & 0.064 & 0.801 & 0.043 \\ \text { T4_30_5 } & 0.000 & 0.998 & 0.084 \\ \text { T4_43_5 } & 0.002 & 0.963 & 0.095 \\ \text { T4_47_5 } & 0.061 & 0.806 & 0.046 \\ \text { T4_48_5 } & 0.215 & 0.643 & 0.006 \\ \text { T4_49_5 } & 0.507 & 0.477 & -0.026 \\ \text { T4_50_5 } & 0.462 & 0.497 & -0.020 \\ \text { T4_51_5 } & 0.341 & 0.559 & -0.006 \\ \text { T4_52_5 } & 0.035 & 0.851 & 0.058 \\ \text { T4_25_5 } & 0.012 & 0.912 & -0.048 \\ \text { T4_4_6 } & 0.009 & 0.925 & -0.011 \\ \text { T4_5_6 } & 0.000 & 0.996 & 0.002 \\ \text { T4_22_6 } & 0.225 & 0.635 & -0.022 \\ \text { T4_7_6 } & 0.072 & 0.789 & -0.036 \\ \text { T4_8_6 } & 0.033 & 0.855 & 0.022 \\ \text { T4_9_6 } & 0.196 & 0.658 & -0.017 \\ \text { T4_11_6 } & 0.036 & 0.850 & 0.022 \\ \text { T4_12_6 } & 0.336 & 0.562 & -0.037 \\ \text { T4_13_6 } & 0.016 & 0.899 & 0.031 \\ \text { T4_14_6 } & 0.295 & 0.587 & -0.035 \\ \text { T416_6 } & 0.024 & 0.878 & 0.028 \\ \text { T4_6 } & 0.004 & 0.949 & 0.041 \\ \text { T46 } & 0.002 & 0.961 & 0.044 \\ \text { T46 } & 0.117 & 0.733 & -0.002 \\ \text { T4 } & 0.048 & 0.827 & -0.012\end{array}$




$\begin{array}{llll}\text { T4_29_6 } & 0.009 & 0.925 & -0.033 \\ \text { T4_30_6 } & 0.113 & 0.737 & 0.007 \\ \text { T4_31_6 } & 0.110 & 0.740 & 0.005 \\ \text { T4_32_6 } & 0.071 & 0.790 & -0.004 \\ \text { T4_33_6 } & 0.002 & 0.969 & -0.059 \\ \text { T4_34_6 } & 0.039 & 0.844 & -0.018 \\ \text { T4_35_6 } & 0.003 & 0.953 & -0.038 \\ \text { T4_36_6 } & 0.000 & 0.991 & -0.044 \\ \text { T4_38_6 } & 0.006 & 0.937 & -0.035 \\ \text { T4_39_6 } & 0.123 & 0.725 & 0.006 \\ \text { T4_43_6 } & 0.003 & 0.960 & -0.060\end{array}$


Table 44

Task 4 DIF: Latinx vs. Sample

\begin{tabular}{lccc}
\hline ItemID & ChiSq & pvalue & EffectSize \\
\hline T4_7_34 & 0.01 & 0.94 & -0.07 \\
T4_8_34 & 0.04 & 0.84 & 0.04 \\
T4_12_34 & 0.01 & 0.90 & 0.07 \\
T4_23_34 & 0.42 & 0.52 & -0.09 \\
T4_25_34 & 0.19 & 0.67 & -0.04 \\
T4_29_34 & 0.00 & 1.00 & -0.15 \\
T4_36_34 & 0.01 & 0.92 & -0.12 \\
T4_43_34 & 0.15 & 0.70 & -0.01 \\
T4_38_45 & 0.27 & 0.61 & -0.04 \\
T4_41_45 & 0.02 & 0.89 & -0.28 \\
T4_5_56 & 0.00 & 0.98 & 0.10 \\
T4_11_56 & 0.04 & 0.84 & 0.02 \\
T4_14_56 & 0.47 & 0.49 & -0.13 \\
T4_22_56 & 0.06 & 0.80 & 0.01 \\
T4_23_56 & 0.01 & 0.90 & 0.01 \\
T4_31_56 & 0.01 & 0.91 & 0.06 \\
T4_45_56 & 0.09 & 0.76 & -0.01 \\
T4_46_56 & 0.00 & 0.95 & 0.08 \\
T4_1_456 & 1.25 & 0.26 & -0.17 \\
T4_6_456 & 0.97 & 0.32 & -0.08 \\
T4_14_456 & 0.54 & 0.46 & -0.04 \\
T4_15_456 & 0.27 & 0.60 & 0.04 \\
T4_22_456 & 0.55 & 0.46 & -0.01 \\
T4_1_3 & 0.30 & 0.59 & 0.01 \\
T4_2_3 & 0.01 & 0.94 & -0.05 \\
T4_3_3 & 0.05 & 0.83 & -0.12 \\
T4_4_3 & 0.00 & 0.97 & -0.01 \\
T4_5_3 & 0.00 & 0.99 & 0.00 \\
T4_6_3 & 0.00 & 0.99 & 0.00 \\
T4_9_3 & 0.00 & 1.00 & -0.02 \\
T4_10_3 & 0.01 & 0.92 & -0.05 \\
T4_11_3 & 0.02 & 0.89 & -0.14 \\
T4_13_3 & 0.01 & 0.94 & 0.03 \\
T4_14_3 & 0.00 & 0.99 & 0.01 \\
T4_15_3 & 0.00 & 0.99 & 0.01 \\
T4_16_3 & 0.00 & 0.95 & -0.04 \\
T4_17_3 & 0.00 & 0.98 & -0.03 \\
\end{tabular}




\begin{tabular}{|c|c|c|c|}
\hline T4_18_3 & 0.00 & 0.99 & -0.02 \\
\hline T4_19_3 & 0.00 & 0.99 & 0.00 \\
\hline T4_20_3 & Inf & 0.00 & $\mathrm{NaN}$ \\
\hline T4_21_3 & 0.00 & 0.97 & -0.01 \\
\hline T4_22_3 & 0.00 & 0.99 & -0.01 \\
\hline T4_24_3 & 0.00 & 0.99 & -0.02 \\
\hline T4_26_3 & 0.19 & 0.67 & -0.03 \\
\hline T4_29_3 & 0.03 & 0.85 & -0.11 \\
\hline T4_30_3 & 0.11 & 0.74 & -0.06 \\
\hline T4_32_3 & 0.07 & 0.79 & -0.09 \\
\hline T4_34_3 & 0.02 & 0.89 & -0.12 \\
\hline T4_35_3 & 0.16 & 0.69 & -0.05 \\
\hline T4_37_3 & 0.51 & 0.47 & 0.07 \\
\hline T4_38_3 & 0.17 & 0.68 & -0.05 \\
\hline T4_39_3 & 0.44 & 0.51 & 0.06 \\
\hline T4_40_3 & 0.13 & 0.72 & -0.05 \\
\hline T4_41_3 & 0.04 & 0.84 & -0.10 \\
\hline T4_42_3 & 0.11 & 0.74 & -0.05 \\
\hline T4_2_4 & 0.00 & 0.97 & 0.11 \\
\hline T4_3_4 & 0.11 & 0.74 & 0.01 \\
\hline T4_5_4 & 0.11 & 0.74 & 0.00 \\
\hline T4_7_4 & 0.26 & 0.61 & -0.05 \\
\hline T4_8_4 & 0.20 & 0.66 & -0.03 \\
\hline T4_9_4 & 0.09 & 0.76 & 0.01 \\
\hline T4_10_4 & 0.06 & 0.81 & 0.04 \\
\hline T4_12_4 & 0.06 & 0.81 & -0.12 \\
\hline T4_13_4 & 0.00 & 0.96 & 0.10 \\
\hline T4_16_4 & 0.09 & 0.77 & 0.02 \\
\hline T4_17_4 & 0.03 & 0.85 & 0.06 \\
\hline T4_20_4 & 0.03 & 0.87 & -0.10 \\
\hline T4_21_4 & 0.29 & 0.59 & -0.07 \\
\hline T4_24_4 & 0.06 & 0.81 & 0.04 \\
\hline T4_25_4 & 0.48 & 0.49 & -0.11 \\
\hline T4_27_4 & 0.15 & 0.70 & -0.02 \\
\hline T4_28_4 & 0.05 & 0.83 & -0.08 \\
\hline T4_30_4 & 0.02 & 0.88 & -0.10 \\
\hline T4_31_4 & 0.01 & 0.92 & -0.12 \\
\hline T4_32_4 & 0.41 & 0.52 & 0.07 \\
\hline T4_33_4 & 0.00 & 0.98 & -0.14 \\
\hline T4_34_4 & 0.00 & 0.96 & -0.14 \\
\hline T4_37_4 & 0.10 & 0.76 & -0.05 \\
\hline
\end{tabular}




\begin{tabular}{|c|c|c|c|}
\hline T4_1_5 & 0.02 & 0.88 & 0.00 \\
\hline T4_2_5 & 0.06 & 0.81 & -0.04 \\
\hline T4_3_5 & 0.04 & 0.83 & -0.02 \\
\hline T4_4_5 & 0.14 & 0.71 & -0.12 \\
\hline T4_7_5 & 0.00 & 0.99 & 0.07 \\
\hline T4_8_5 & 0.11 & 0.74 & -0.06 \\
\hline T4_9_5 & Inf & 0.00 & $\mathrm{NaN}$ \\
\hline T4_12_5 & 0.03 & 0.87 & -0.01 \\
\hline T4_16_5 & 0.00 & 0.98 & 0.04 \\
\hline T4_17_5 & 0.03 & 0.86 & -0.01 \\
\hline T4_18_5 & 0.00 & 1.00 & 0.07 \\
\hline T4_19_5 & 0.23 & 0.63 & -0.15 \\
\hline T4_20_5 & 0.02 & 0.89 & 0.00 \\
\hline T4_21_5 & 0.06 & 0.80 & -0.04 \\
\hline T4_26_5 & 0.00 & 1.00 & -0.06 \\
\hline T4_29_5 & 0.02 & 0.88 & 0.02 \\
\hline T4_30_5 & 0.05 & 0.82 & 0.04 \\
\hline T4_43_5 & 0.00 & 0.98 & -0.02 \\
\hline T4_47_5 & 0.00 & 0.97 & -0.02 \\
\hline T4_48_5 & 0.11 & 0.75 & 0.07 \\
\hline T4_49_5 & 0.00 & 1.00 & -0.03 \\
\hline T4_50_5 & 0.03 & 0.86 & 0.03 \\
\hline T4_51_5 & 0.00 & 0.97 & -0.07 \\
\hline T4_52_5 & 0.01 & 0.91 & 0.00 \\
\hline T4_25_5 & 0.00 & 0.99 & 0.07 \\
\hline T4_4_6 & 0.66 & 0.42 & -0.11 \\
\hline T4_5_6 & 0.06 & 0.80 & 0.11 \\
\hline T4_7_6 & 0.33 & 0.57 & -0.01 \\
\hline T4_8_6 & 0.02 & 0.89 & -0.01 \\
\hline T4_9_6 & 0.01 & 0.94 & 0.00 \\
\hline T4_11_6 & 0.01 & 0.92 & 0.00 \\
\hline T4_12_6 & 0.04 & 0.84 & -0.04 \\
\hline T4_13_6 & 0.00 & 0.99 & 0.03 \\
\hline T4_14_6 & 0.08 & 0.78 & -0.06 \\
\hline T4_16_6 & 0.01 & 0.93 & 0.00 \\
\hline T4_17_6 & 0.00 & 0.99 & 0.04 \\
\hline T4_18_6 & 0.07 & 0.80 & -0.06 \\
\hline T4_20_6 & 0.01 & 0.92 & 0.00 \\
\hline T4_22_6 & 0.04 & 0.84 & -0.03 \\
\hline T4_24_6 & 0.04 & 0.85 & -0.03 \\
\hline T4_27_6 & 0.34 & 0.56 & -0.07 \\
\hline
\end{tabular}




\begin{tabular}{llll} 
T4_29_6 & 0.00 & 0.97 & 0.17 \\
T4_30_6 & 0.51 & 0.48 & -0.12 \\
T4_31_6 & 0.02 & 0.88 & 0.07 \\
T4_32_6 & 0.33 & 0.56 & -0.07 \\
T4_33_6 & 0.00 & 0.99 & 0.14 \\
T4_34_6 & 0.11 & 0.74 & 0.03 \\
T4_35_6 & 0.24 & 0.63 & -0.03 \\
T4_36_6 & 0.08 & 0.78 & 0.04 \\
T4_38_6 & 0.01 & 0.92 & 0.10 \\
T4_39_6 & 0.16 & 0.69 & -0.01 \\
T4_43_6 & 0.00 & 0.95 & 0.12 \\
\hline
\end{tabular}


Table 45

Task 5 DIF: Male vs. female

\begin{tabular}{lccc}
\hline ItemID & ChiSq & pvalue & EffectSize \\
\hline T5_10_12 & 0.036 & 0.849 & 0.006 \\
T5_18_12 & 0.042 & 0.837 & 0.020 \\
T5_24_12 & 0.018 & 0.894 & 0.009 \\
T5_30_12 & 0.057 & 0.812 & 0.042 \\
T5_32_12 & 0.012 & 0.912 & 0.025 \\
T5_26_23 & 0.038 & 0.846 & 0.040 \\
T5_39_23 & 0.056 & 0.813 & -0.029 \\
T5_10_23 & 0.030 & 0.864 & 0.010 \\
T5_11_23 & 0.097 & 0.756 & -0.069 \\
T5_23_23 & 0.030 & 0.862 & 0.011 \\
T5_3_34 & 0.000 & 0.987 & 0.001 \\
T5_10_34 & 0.000 & 1.000 & -0.001 \\
T5_14_34 & 0.000 & 0.984 & -0.003 \\
T5_17_34 & 0.010 & 0.920 & -0.018 \\
T5_31_34 & 0.171 & 0.679 & -0.021 \\
T5_34_34 & 0.230 & 0.632 & -0.029 \\
T5_26_45 & 0.271 & 0.603 & -0.059 \\
T5_36_45 & 0.054 & 0.816 & -0.016 \\
T5_26_56 & 0.041 & 0.840 & 0.000 \\
T5_30_56 & 0.024 & 0.878 & -0.006 \\
T5_37_56 & 0.001 & 0.978 & -0.025 \\
T5_3_56 & 0.003 & 0.959 & 0.006 \\
T5_6_56 & 0.017 & 0.896 & 0.018 \\
T5_7_56 & 0.001 & 0.974 & 0.003 \\
T5_12_56 & 0.055 & 0.815 & 0.034 \\
T5_20_56 & 0.006 & 0.938 & 0.009 \\
T5_6_456 & 0.037 & 0.848 & 0.036 \\
T5_8_456 & 0.012 & 0.911 & -0.008 \\
T5_15_456 & 0.000 & 0.996 & 0.011 \\
T5_21_456 & 0.004 & 0.947 & 0.000 \\
T5_25_456 & 0.003 & 0.959 & 0.001 \\
T5_1_1 & 0.009 & 0.923 & 0.006 \\
T5_2_1 & 0.048 & 0.826 & 0.025 \\
T5_3_1 & 0.000 & 0.990 & -0.007 \\
T5_4_1 & 0.023 & 0.880 & 0.014 \\
T5_6_1 & 0.015 & 0.902 & 0.010 \\
T5_1 & 0.018 & 0.894 & 0.011 \\
\end{tabular}




$\begin{array}{llll}\text { T5_8_1 } & 0.018 & 0.894 & 0.011 \\ \text { T5_9_1 } & 0.047 & 0.828 & -0.034 \\ \text { T5_11_1 } & 0.000 & 0.995 & 0.003 \\ \text { T5_12_1 } & 0.005 & 0.941 & -0.010 \\ \text { T5_13_1 } & 0.018 & 0.894 & 0.011 \\ \text { T5_14_1 } & 0.019 & 0.891 & 0.012 \\ \text { T5_15_1 } & 0.014 & 0.905 & 0.009 \\ \text { T5_16_1 } & 0.024 & 0.876 & 0.015 \\ \text { T5_17_1 } & 0.028 & 0.868 & 0.016 \\ \text { T5_19_1 } & 0.043 & 0.835 & 0.023 \\ \text { T5_20_1 } & 0.014 & 0.905 & 0.009 \\ \text { T5_21_1 } & 0.008 & 0.930 & 0.004 \\ \text { T5_22_1 } & 0.012 & 0.915 & 0.007 \\ \text { T5_23_1 } & 0.018 & 0.894 & 0.011 \\ \text { T5_25_1 } & 0.014 & 0.907 & 0.009 \\ \text { T5_26_1 } & 0.013 & 0.911 & -0.005 \\ \text { T5_27_1 } & 0.016 & 0.901 & -0.008 \\ \text { T5_28_1 } & 0.050 & 0.823 & -0.023 \\ \text { T5_29_1 } & 0.066 & 0.797 & -0.027 \\ \text { T5_33_1 } & 0.000 & 0.984 & 0.018 \\ \text { T5_34_1 } & 0.025 & 0.873 & -0.012 \\ \text { T5_36_1 } & 0.069 & 0.793 & -0.029 \\ \text { T5_37_1 } & 0.010 & 0.922 & -0.002 \\ \text { T5_38_1 } & 0.238 & 0.626 & -0.062 \\ \text { T5_39_1 } & 0.284 & 0.594 & -0.068 \\ \text { T5_40_1 } & 0.111 & 0.739 & -0.039 \\ \text { T5_41_1 } & 0.042 & 0.837 & -0.019 \\ \text { T5_42_1 } & 0.387 & 0.534 & -0.081 \\ \text { T5_2_2 } & 0.049 & 0.825 & -0.038 \\ \text { T5_3_2 } & 0.000 & 0.984 & 0.003 \\ \text { T5_4_2 } & 0.001 & 0.971 & 0.005 \\ \text { T5_6_2 } & 0.001 & 0.976 & -0.009 \\ \text { T5_7_2 } & 0.003 & 0.959 & -0.003 \\ \text { T5_12_2 } & 0.001 & 0.979 & -0.009 \\ \text { T5_13_2 } & 0.001 & 0.971 & 0.005 \\ \text { T5_14_2 } & 0.006 & 0.940 & 0.011 \\ \text { T5_15_2 } & 0.053 & 0.818 & 0.024 \\ \text { T5_17_2 } & 0.000 & 0.984 & 0.003 \\ \text { T5_18_2 } & 0.000 & 0.994 & -0.002 \\ \text { T5_19_2 } & 0.000 & 0.996 & -0.006 \\ \text { T5_20_2 } & 0.001 & 0.982 & 0.003\end{array}$




$\begin{array}{llll}\text { T5_21_2 } & 0.004 & 0.950 & 0.009 \\ \text { T5_22_2 } & 0.029 & 0.866 & 0.025 \\ \text { T5_24_2 } & 0.003 & 0.960 & -0.013 \\ \text { T5_25_2 } & 0.003 & 0.955 & 0.008 \\ \text { T5_28_2 } & 0.020 & 0.887 & 0.015 \\ \text { T5_32_2 } & 0.006 & 0.941 & 0.003 \\ \text { T5_33_2 } & 0.000 & 0.997 & -0.007 \\ \text { T5_34_2 } & 0.048 & 0.827 & 0.026 \\ \text { T5_35_2 } & 0.037 & 0.847 & 0.022 \\ \text { T5_38_2 } & 0.092 & 0.761 & 0.038 \\ \text { T5_40_2 } & 0.025 & 0.874 & 0.017 \\ \text { T5_41_2 } & 0.057 & 0.812 & 0.028 \\ \text { T5_42_2 } & 0.096 & 0.757 & 0.039 \\ \text { T5_43_2 } & 0.002 & 0.963 & -0.001 \\ \text { T5_4_3 } & 0.062 & 0.804 & 0.024 \\ \text { T5_533 } & 0.014 & 0.906 & 0.005 \\ \text { T5_6_3 } & 0.032 & 0.858 & 0.014 \\ \text { T5_733 } & 0.045 & 0.833 & 0.019 \\ \text { T5_8_3 } & 0.019 & 0.891 & -0.021 \\ \text { T5_9_3 } & 0.031 & 0.861 & -0.027 \\ \text { T5_11_3 } & 0.051 & 0.821 & 0.020 \\ \text { T5_16_3 } & 0.026 & 0.871 & 0.011 \\ \text { T5_17_34.1 } & 0.010 & 0.920 & -0.018 \\ \text { T5_18_3 } & 0.033 & 0.857 & 0.014 \\ \text { T5_19_3 } & 0.038 & 0.846 & 0.016 \\ \text { T5_20_3 } & 0.038 & 0.846 & 0.016 \\ \text { T5_21_3 } & 0.091 & 0.763 & -0.047 \\ \text { T5_22_3 } & 0.000 & 0.988 & -0.020 \\ \text { T5_23_3 } & 0.022 & 0.881 & 0.009 \\ \text { T5_24_3 } & 0.015 & 0.904 & 0.005 \\ \text { T5_25_3 } & 0.016 & 0.898 & 0.006 \\ \text { T5_27_3 } & 0.012 & 0.914 & -0.001 \\ \text { T5_28_3 } & 0.205 & 0.651 & -0.051 \\ \text { T5_29_3 } & 0.040 & 0.841 & -0.013 \\ \text { T5_30_3 } & 0.195 & 0.659 & -0.051 \\ \text { T5_32_3 } & 0.002 & 0.968 & 0.010 \\ \text { T5_36_3 } & 0.001 & 0.983 & 0.013 \\ \text { T5_37_3 } & 0.183 & 0.669 & -0.048 \\ \text { T5_39_3 } & 0.088 & 0.767 & -0.028 \\ \text { T5_40_3 } & 0.157 & 0.692 & -0.043 \\ \text { T5_41_3 } & 0.050 & 0.823 & -0.018\end{array}$




$\begin{array}{llll}\text { T5_42_3 } & 0.027 & 0.869 & -0.010 \\ \text { T5_14 } & 0.041 & 0.840 & -0.023 \\ \text { T5_2_4 } & 0.001 & 0.977 & 0.024 \\ \text { T5_44 } & 0.000 & 0.996 & 0.013 \\ \text { T5_7_4 } & 0.000 & 0.990 & 0.040 \\ \text { T5_944 } & 0.206 & 0.650 & -0.029 \\ \text { T5_12_4 } & 0.000 & 0.993 & 0.012 \\ \text { T5_13_4 } & 0.033 & 0.856 & -0.013 \\ \text { T5_14_4 } & 0.001 & 0.975 & 0.010 \\ \text { T5_16_4 } & 0.034 & 0.853 & -0.014 \\ \text { T5_17_4 } & 0.022 & 0.883 & -0.008 \\ \text { T5_19_4 } & 0.042 & 0.837 & -0.017 \\ \text { T5_20_4 } & 0.006 & 0.940 & 0.027 \\ \text { T5_2244 } & 0.000 & 0.998 & 0.019 \\ \text { T5_23_4 } & 0.035 & 0.851 & -0.014 \\ \text { T5_2444 } & 0.006 & 0.941 & 0.003 \\ \text { T5_27_4 } & 0.102 & 0.750 & -0.026 \\ \text { T5_2844 } & 0.000 & 0.991 & 0.021 \\ \text { T5_30_4 } & 0.048 & 0.827 & -0.013 \\ \text { T5_3144 } & 0.333 & 0.564 & -0.064 \\ \text { T5_32_4 } & 0.217 & 0.641 & -0.048 \\ \text { T5_33_4 } & 0.000 & 0.992 & 0.024 \\ \text { T5_34_4 } & 0.114 & 0.736 & -0.028 \\ \text { T5_37_4 } & 0.056 & 0.813 & -0.013 \\ \text { T5_38_4 } & 0.000 & 0.994 & 0.027 \\ \text { T5_39_4 } & 0.041 & 0.839 & -0.010 \\ \text { T5_43_4 } & 0.116 & 0.734 & -0.028 \\ \text { T5_1_5 } & 0.000 & 1.000 & -0.003 \\ \text { T5_4_5 } & 0.000 & 0.993 & -0.009 \\ \text { T5_8_5 } & 0.011 & 0.917 & -0.031 \\ \text { T5_9_5 } & 0.001 & 0.971 & -0.013 \\ \text { T5_10_5 } & 0.001 & 0.974 & 0.002 \\ \text { T5_13_5 } & 0.000 & 0.993 & -0.009 \\ \text { T5_15_5 } & 0.000 & 0.991 & -0.009 \\ \text { T5_16_5 } & 0.000 & 0.993 & -0.004 \\ \text { T5_17_5 } & 0.000 & 0.999 & -0.007 \\ \text { T5_18_5 } & 0.000 & 0.996 & -0.003 \\ \text { T5_19_5 } & 0.028 & 0.867 & 0.022 \\ \text { T5_20_56.1 } & 0.006 & 0.938 & 0.009 \\ \text { T5_21_5 } & 0.024 & 0.876 & 0.021 \\ \text { T5_22_5 } & 0.000 & 0.988 & 0.000\end{array}$




\begin{tabular}{|c|c|c|c|}
\hline T5_23_5 & 0.002 & 0.965 & -0.014 \\
\hline T5_25_5 & 0.000 & 0.991 & -0.006 \\
\hline T5_27_5 & 0.462 & 0.497 & 0.096 \\
\hline T5_28_5 & 0.041 & 0.839 & 0.026 \\
\hline T5_31_5 & 0.007 & 0.933 & 0.008 \\
\hline T5_32_5 & 0.008 & 0.929 & -0.022 \\
\hline T5_34_5 & 0.008 & 0.928 & -0.023 \\
\hline T5_35_5 & 0.024 & 0.876 & 0.017 \\
\hline T5_39_5 & 0.020 & 0.889 & 0.017 \\
\hline T5_40_5 & 0.003 & 0.956 & 0.003 \\
\hline T5_41_5 & 0.058 & 0.810 & 0.031 \\
\hline T5_43_5 & 0.012 & 0.912 & 0.011 \\
\hline T5_1_6 & 0.010 & 0.922 & -0.038 \\
\hline T5_2_6 & 0.073 & 0.787 & 0.021 \\
\hline T5_5_6 & 0.325 & 0.568 & 0.066 \\
\hline T5_6_6 & 0.075 & 0.784 & 0.045 \\
\hline T5_7_6 & 0.004 & 0.948 & -0.011 \\
\hline T5_8_6 & 0.000 & 0.995 & -0.003 \\
\hline T5_9_6 & 0.004 & 0.949 & -0.011 \\
\hline T5_10_6 & 0.000 & 0.994 & -0.003 \\
\hline T5_13_6 & 0.003 & 0.955 & 0.012 \\
\hline T5_15_6 & 0.002 & 0.962 & 0.010 \\
\hline T5_20_6 & 0.021 & 0.884 & 0.025 \\
\hline T5_22_6 & 0.017 & 0.896 & 0.022 \\
\hline T5_23_6 & 0.052 & 0.819 & 0.037 \\
\hline T5_24_6 & 0.000 & 0.997 & 0.003 \\
\hline T5_25_6 & 0.003 & 0.954 & 0.011 \\
\hline T5_26_6 & 0.065 & 0.798 & 0.019 \\
\hline T5_27_6 & 0.001 & 0.981 & -0.017 \\
\hline T5_31_6 & 0.028 & 0.866 & 0.006 \\
\hline T5_32_6 & 0.040 & 0.841 & 0.011 \\
\hline T5_33_6 & 0.023 & 0.878 & 0.003 \\
\hline T5_34_6 & 0.000 & 0.997 & -0.021 \\
\hline T5_36_6 & 0.084 & 0.772 & 0.023 \\
\hline T5_38_6 & 0.029 & 0.865 & 0.005 \\
\hline T5_40_6 & 0.012 & 0.912 & -0.003 \\
\hline T5_41_6 & 0.187 & 0.665 & 0.045 \\
\hline T5_42_6 & 0.000 & 0.996 & -0.019 \\
\hline T5_43_6 & 0.101 & 0.751 & 0.027 \\
\hline
\end{tabular}


Table 46

Task 5 DIF: White vs. Sample

\begin{tabular}{lccc}
\hline ItemID & ChiSq & pvalue & EffectSize \\
\hline T5_10_12 & 0.109 & 0.742 & 0.023 \\
T5_18_12 & 0.000 & 0.984 & -0.030 \\
T5_24_12 & 0.013 & 0.910 & -0.015 \\
T5_30_12 & 0.292 & 0.589 & 0.093 \\
T5_32_12 & 0.062 & 0.804 & -0.033 \\
T5_26_23 & 0.154 & 0.695 & -0.041 \\
T5_39_23 & 0.025 & 0.875 & 0.047 \\
T5_10_23 & 0.360 & 0.549 & 0.050 \\
T5_11_23 & 0.022 & 0.881 & -0.020 \\
T5_23_23 & 0.232 & 0.630 & 0.033 \\
T5_3_34 & 0.042 & 0.838 & -0.032 \\
T5_10_34 & 0.010 & 0.922 & -0.018 \\
T5_14_34 & 0.000 & 0.991 & -0.004 \\
T5_17_34 & 0.011 & 0.919 & 0.019 \\
T5_31_34 & 1.218 & 0.270 & -0.116 \\
T5_34_34 & 1.479 & 0.224 & -0.133 \\
T5_26_45 & 0.070 & 0.792 & 0.044 \\
T5_36_45 & 0.517 & 0.472 & -0.111 \\
T5_26_56 & 0.032 & 0.858 & 0.042 \\
T5_30_56 & 1.386 & 0.239 & 0.188 \\
T5_37_56 & 0.650 & 0.420 & 0.132 \\
T5_3_56 & 0.079 & 0.779 & 0.015 \\
T5_6_56 & 0.129 & 0.720 & 0.027 \\
T5_7_56 & 0.182 & 0.670 & 0.038 \\
T5_12_56 & 0.165 & 0.684 & 0.034 \\
T5_20_56 & 0.112 & 0.739 & 0.023 \\
T5_6_456 & 2.315 & 0.128 & 0.141 \\
T5_8_456 & 0.400 & 0.527 & 0.050 \\
T5_15_456 & 0.158 & 0.691 & 0.012 \\
T5_21_456 & 0.221 & 0.639 & 0.025 \\
T5_25_456 & 0.274 & 0.601 & 0.032 \\
T5_1_1 & 0.072 & 0.789 & -0.015 \\
T5_2_1 & 0.081 & 0.776 & -0.017 \\
T5_3_1 & 0.093 & 0.760 & -0.021 \\
T5_4_1 & 0.143 & 0.705 & -0.032 \\
T5_6_1 & 0.143 & 0.705 & -0.032 \\
T5_7_1 & 0.130 & 0.718 & -0.029 \\
\end{tabular}




$\begin{array}{llll}\text { T5_8_1 } & 0.130 & 0.718 & -0.029 \\ \text { T5_9_1 } & 0.191 & 0.662 & -0.037 \\ \text { T5_11_1 } & 0.788 & 0.375 & -0.108 \\ \text { T5_12_1 } & 0.150 & 0.698 & -0.031 \\ \text { T5_13_1 } & 0.130 & 0.718 & -0.029 \\ \text { T5_14_1 } & 0.129 & 0.719 & -0.029 \\ \text { T5_15_1 } & 0.117 & 0.732 & -0.026 \\ \text { T5_16_1 } & 0.140 & 0.708 & -0.031 \\ \text { T5_17_1 } & 0.093 & 0.761 & -0.020 \\ \text { T5_19_1 } & 0.152 & 0.696 & -0.034 \\ \text { T5_20_1 } & 0.118 & 0.731 & -0.026 \\ \text { T5_21_1 } & 0.088 & 0.767 & -0.019 \\ \text { T5_22_1 } & 0.057 & 0.812 & -0.010 \\ \text { T5_23_1 } & 0.130 & 0.718 & -0.029 \\ \text { T5_25_1 } & 0.127 & 0.721 & -0.028 \\ \text { T5_26_1 } & 0.000 & 0.986 & 0.009 \\ \text { T5_27_1 } & 0.003 & 0.960 & 0.013 \\ \text { T5_28_1 } & 0.042 & 0.838 & -0.032 \\ \text { T5_29_1 } & 0.003 & 0.955 & -0.008 \\ \text { T5_33_1 } & 0.428 & 0.513 & -0.098 \\ \text { T5_34_1 } & 0.003 & 0.956 & -0.008 \\ \text { T5_36_1 } & 0.008 & 0.927 & -0.013 \\ \text { T5_37_1 } & 0.061 & 0.805 & 0.043 \\ \text { T5_38_1 } & 0.265 & 0.606 & -0.079 \\ \text { T5_39_1 } & 0.120 & 0.729 & -0.055 \\ \text { T5_40_1 } & 0.050 & 0.823 & -0.034 \\ \text { T5_41_1 } & 0.036 & 0.849 & -0.029 \\ \text { T5_42_1 } & 0.029 & 0.865 & -0.027 \\ \text { T5_2_2 } & 0.152 & 0.697 & -0.001 \\ \text { T5_3_2 } & 0.659 & 0.417 & 0.064 \\ \text { T5_4_2 } & 0.613 & 0.434 & 0.060 \\ \text { T5_6_2 } & 0.600 & 0.439 & 0.058 \\ \text { T5_7_2 } & 0.169 & 0.681 & 0.005 \\ \text { T5_12_2 } & 0.293 & 0.588 & 0.022 \\ \text { T5_13_2 } & 0.439 & 0.508 & 0.041 \\ \text { T5_14_2 } & 0.614 & 0.433 & 0.059 \\ \text { T5_15_2 } & 0.305 & 0.581 & 0.028 \\ \text { T5_17_2 } & 0.659 & 0.417 & 0.064 \\ \text { T5_18_2 } & 0.239 & 0.625 & 0.014 \\ \text { T5_19_2 } & 0.100 & 0.752 & -0.012 \\ \text { T5_20_ } & 0.286 & 0.593 & 0.021\end{array}$




$\begin{array}{llll}\text { T5_21_2 } & 0.237 & 0.627 & 0.014 \\ \text { T5_22_2 } & 0.570 & 0.450 & 0.055 \\ \text { T5_24_2 } & 0.313 & 0.576 & 0.025 \\ \text { T5_25_2 } & 0.298 & 0.585 & 0.023 \\ \text { T5_28_2 } & 0.021 & 0.885 & 0.029 \\ \text { T5_32_2 } & 0.003 & 0.960 & -0.006 \\ \text { T5_33_2 } & 0.003 & 0.956 & 0.017 \\ \text { T5_34_2 } & 0.001 & 0.975 & -0.003 \\ \text { T5_35_2 } & 0.000 & 0.987 & 0.000 \\ \text { T5_38_2 } & 0.008 & 0.930 & -0.012 \\ \text { T5_40_2 } & 0.155 & 0.694 & 0.067 \\ \text { T5_41_2 } & 0.040 & 0.842 & 0.039 \\ \text { T5_42_2 } & 0.169 & 0.681 & 0.069 \\ \text { T5_43_2 } & 0.000 & 0.998 & 0.008 \\ \text { T5_4_3 } & 0.012 & 0.912 & 0.000 \\ \text { T5_5_3 } & 0.040 & 0.842 & -0.014 \\ \text { T5_6_3 } & 0.066 & 0.797 & -0.023 \\ \text { T5_7_3 } & 0.084 & 0.772 & -0.027 \\ \text { T5_8_3 } & 0.220 & 0.639 & -0.041 \\ \text { T5_9_3 } & 0.021 & 0.885 & 0.010 \\ \text { T5_11_3 } & 0.058 & 0.810 & -0.020 \\ \text { T5_16_3 } & 0.040 & 0.841 & -0.014 \\ \text { T5_17_34.1 } & 0.011 & 0.919 & 0.019 \\ \text { T5_18_3 } & 0.065 & 0.798 & -0.022 \\ \text { T5_19_3 } & 0.059 & 0.808 & -0.020 \\ \text { T5_20_3 } & 0.057 & 0.812 & -0.020 \\ \text { T5_21_3 } & 0.000 & 0.998 & 0.030 \\ \text { T5_22_3 } & 0.044 & 0.834 & -0.015 \\ \text { T5_23_3 } & 0.058 & 0.810 & -0.020 \\ \text { T5_24_3 } & 0.000 & 0.993 & 0.015 \\ \text { T5_25_3 } & 0.050 & 0.823 & -0.018 \\ \text { T5_27_3 } & 0.000 & 0.991 & 0.018 \\ \text { T5_28_3 } & 0.001 & 0.974 & 0.004 \\ \text { T5_29_3 } & 0.022 & 0.882 & 0.038 \\ \text { T5_30_3 } & 0.087 & 0.768 & 0.060 \\ \text { T5_32_3 } & 0.000 & 0.987 & 0.014 \\ \text { T5_36_3 } & 0.001 & 0.973 & 0.006 \\ \text { T5_37_3 } & 0.024 & 0.878 & -0.013 \\ \text { T5_39_3 } & 0.194 & 0.660 & -0.055 \\ \text { T5_41_3 } & 0.006 & 0.938 & -0.001\end{array}$




$\begin{array}{llll}\text { T5_42_3 } & 0.001 & 0.974 & 0.022 \\ \text { T5_14 } & 0.107 & 0.743 & -0.005 \\ \text { T5_2_4 } & 0.006 & 0.939 & -0.005 \\ \text { T5_44 } & 0.003 & 0.954 & -0.008 \\ \text { T5_7_4 } & 0.634 & 0.426 & -0.103 \\ \text { T5_944 } & 0.092 & 0.762 & -0.029 \\ \text { T5_12_4 } & 0.011 & 0.916 & -0.001 \\ \text { T5_13_4 } & 0.002 & 0.967 & -0.010 \\ \text { T5_14_4 } & 0.019 & 0.890 & 0.004 \\ \text { T5_16_4 } & 0.081 & 0.776 & 0.026 \\ \text { T5_17_4 } & 0.040 & 0.842 & 0.014 \\ \text { T5_19_4 } & 0.057 & 0.811 & 0.020 \\ \text { T5_20_4 } & 0.079 & 0.779 & -0.026 \\ \text { T5_2244 } & 0.001 & 0.971 & -0.011 \\ \text { T5_23_4 } & 0.066 & 0.798 & 0.022 \\ \text { T5_2444 } & 0.008 & 0.929 & -0.003 \\ \text { T5_27_4 } & 0.179 & 0.672 & -0.028 \\ \text { T5_2844 } & 0.173 & 0.678 & -0.026 \\ \text { T5_30_4 } & 0.358 & 0.550 & -0.053 \\ \text { T5_3144 } & 0.648 & 0.421 & -0.084 \\ \text { T5_32_4 } & 0.286 & 0.593 & -0.044 \\ \text { T5_33_4 } & 0.171 & 0.679 & -0.026 \\ \text { T5_34_4 } & 0.334 & 0.563 & -0.051 \\ \text { T5_37_4 } & 0.111 & 0.739 & -0.014 \\ \text { T5_38_4 } & 0.081 & 0.777 & -0.006 \\ \text { T5_39_4 } & 0.074 & 0.786 & -0.004 \\ \text { T5_43_4 } & 0.476 & 0.490 & -0.067 \\ \text { T5_1_5 } & 0.014 & 0.907 & 0.028 \\ \text { T5_4_5 } & 0.026 & 0.872 & -0.019 \\ \text { T5_8_5 } & 0.063 & 0.802 & 0.010 \\ \text { T5_9_5 } & 0.005 & 0.943 & -0.005 \\ \text { T5_10_5 } & 0.007 & 0.933 & -0.007 \\ \text { T5_13_5 } & 0.004 & 0.949 & -0.004 \\ \text { T5_15_5 } & 0.013 & 0.910 & -0.012 \\ \text { T5_16_5 } & 0.009 & 0.927 & -0.009 \\ \text { T5_17_5 } & 0.009 & 0.926 & -0.008 \\ \text { T5_18_5 } & 0.003 & 0.958 & -0.003 \\ \text { T5_19_5 } & 0.000 & 0.998 & 0.010 \\ \text { T5_20_56.1 } & 0.112 & 0.739 & 0.023 \\ \text { T5_21_5 } & 0.000 & 0.988 & 0.013 \\ \text { T5_22_5 } & 0.034 & 0.853 & -0.023\end{array}$




\begin{tabular}{llll} 
T5_23_5 & 0.005 & 0.946 & 0.021 \\
T5_25_5 & 0.024 & 0.877 & -0.018 \\
T5_27_5 & 0.187 & 0.666 & 0.028 \\
T5_285 & 0.277 & 0.599 & 0.042 \\
T5_31_5 & 0.348 & 0.555 & 0.053 \\
T5_32_5 & 0.381 & 0.537 & 0.057 \\
T5_34_5 & 0.481 & 0.488 & 0.068 \\
T5_35_5 & 0.180 & 0.671 & 0.028 \\
T5_39_5 & 0.188 & 0.665 & 0.030 \\
T5_405 & 0.229 & 0.633 & 0.036 \\
T5_41_5 & 0.120 & 0.729 & 0.017 \\
T5_43_5 & 0.104 & 0.747 & 0.013 \\
T5_1_6 & 0.007 & 0.934 & 0.021 \\
T5_266 & 0.040 & 0.841 & 0.038 \\
T5_5_6 & 0.020 & 0.888 & -0.018 \\
T5_666 & 0.044 & 0.834 & -0.004 \\
T5_766 & 0.169 & 0.681 & 0.027 \\
T5_8_6 & 0.222 & 0.638 & 0.036 \\
T5_9_6 & 0.222 & 0.638 & 0.036 \\
T5_10_6 & 0.355 & 0.551 & 0.055 \\
T5_13_6 & 0.119 & 0.730 & 0.016 \\
T5_15_6 & 0.377 & 0.539 & 0.057 \\
T5_20_6 & 0.173 & 0.678 & 0.027 \\
T5_22_6 & 0.056 & 0.814 & 0.001 \\
T5_23_6 & 0.031 & 0.862 & -0.009 \\
T5_24_6 & 0.017 & 0.896 & -0.016 \\
T5_5_6 & 0.300 & 0.584 & 0.047 \\
T5_26_6 & 0.210 & 0.647 & -0.030 \\
T5_2766 & 0.005 & 0.947 & 0.056 \\
T5_31_6 & 0.156 & 0.693 & -0.018 \\
T5_32_6 & 0.204 & 0.651 & -0.028 \\
T5_33_6 & 0.116 & 0.734 & -0.010 \\
T5_3466 & 0.033 & 0.855 & 0.077 \\
T5_36_6 & 0.152 & 0.697 & -0.019 \\
T5_38_6 & 0.001 & 0.983 & 0.038 \\
T5_40_6 & 0.050 & 0.824 & 0.008 \\
T5_41_6 & 0.215 & 0.643 & -0.031 \\
T5_42_6 & 0.111 & 0.739 & -0.008 \\
T5_43_6 & 0.037 & 0.848 & 0.011 \\
\hline & & & \\
& &
\end{tabular}


Table 47

Task 5 DIF: Black vs. Sample

\begin{tabular}{lccc}
\hline ItemID & ChiSq & pvalue & EffectSize \\
\hline T5_10_12 & 0.022 & 0.883 & -0.008 \\
T5_18_12 & 0.004 & 0.948 & 0.016 \\
T5_24_12 & 0.020 & 0.888 & 0.004 \\
T5_30_12 & 0.724 & 0.395 & -0.130 \\
T5_32_12 & 0.093 & 0.760 & -0.046 \\
T5_26_23 & 0.006 & 0.941 & 0.023 \\
T5_39_23 & 0.213 & 0.645 & -0.066 \\
T5_10_23 & 0.273 & 0.601 & -0.054 \\
T5_11_23 & 0.027 & 0.869 & 0.003 \\
T5_23_23 & 0.129 & 0.720 & -0.029 \\
T5_3_34 & 0.139 & 0.709 & 0.037 \\
T5_10_34 & 0.133 & 0.715 & 0.036 \\
T5_14_34 & 0.142 & 0.707 & 0.036 \\
T5_17_34 & 0.087 & 0.768 & 0.025 \\
T5_31_34 & 0.249 & 0.618 & 0.062 \\
T5_34_34 & 1.277 & 0.258 & 0.165 \\
T5_26_45 & 0.250 & 0.617 & -0.048 \\
T5_36_45 & 0.487 & 0.485 & 0.142 \\
T5_26_56 & 0.011 & 0.915 & -0.029 \\
T5_30_56 & 1.587 & 0.208 & -0.202 \\
T5_37_56 & 0.340 & 0.560 & -0.098 \\
T5_3_56 & 0.000 & 1.000 & 0.005 \\
T5_6_56 & 0.001 & 0.974 & 0.000 \\
T5_7_56 & 0.008 & 0.931 & -0.009 \\
T5_12_56 & 0.004 & 0.951 & -0.005 \\
T5_20_56 & 0.001 & 0.973 & -0.001 \\
T5_6_456 & 1.167 & 0.280 & -0.101 \\
T5_8_456 & 0.000 & 0.986 & -0.005 \\
T5_15_456 & 0.051 & 0.821 & 0.040 \\
T5_21_456 & 0.093 & 0.761 & 0.050 \\
T5_25_456 & 0.001 & 0.979 & -0.004 \\
T5_1_1 & 0.148 & 0.701 & 0.042 \\
T5_2_1 & 0.038 & 0.846 & 0.012 \\
T5_3_1 & 0.061 & 0.804 & 0.021 \\
T5_4_1 & 0.077 & 0.781 & 0.025 \\
T5_6_1 & 0.082 & 0.775 & 0.027 \\
T5_1 & 0.064 & 0.800 & 0.021 \\
& & &
\end{tabular}




$\begin{array}{llll}\text { T5_8_1 } & 0.064 & 0.800 & 0.021 \\ \text { T5_9_1 } & 0.009 & 0.926 & 0.015 \\ \text { T5_11_1 } & 0.117 & 0.733 & 0.056 \\ \text { T5_12_1 } & 0.000 & 0.988 & 0.003 \\ \text { T5_13_1 } & 0.064 & 0.800 & 0.021 \\ \text { T5_14_1 } & 0.069 & 0.792 & 0.023 \\ \text { T5_15_1 } & 0.058 & 0.811 & 0.019 \\ \text { T5_16_1 } & 0.080 & 0.778 & 0.026 \\ \text { T5_17_1 } & 0.039 & 0.845 & 0.012 \\ \text { T5_19_1 } & 0.070 & 0.791 & 0.023 \\ \text { T5_20_1 } & 0.058 & 0.810 & 0.019 \\ \text { T5_21_1 } & 0.095 & 0.758 & 0.030 \\ \text { T5_22_1 } & 0.072 & 0.789 & 0.024 \\ \text { T5_23_1 } & 0.064 & 0.800 & 0.021 \\ \text { T5_25_1 } & 0.087 & 0.768 & 0.028 \\ \text { T5_26_1 } & 0.163 & 0.686 & -0.049 \\ \text { T5_27_1 } & 0.155 & 0.694 & -0.047 \\ \text { T5_28_1 } & 0.004 & 0.949 & 0.006 \\ \text { T5_29_1 } & 0.043 & 0.837 & -0.018 \\ \text { T5_33_1 } & 0.025 & 0.874 & 0.044 \\ \text { T5_34_1 } & 0.028 & 0.868 & -0.011 \\ \text { T5_36_1 } & 0.026 & 0.873 & -0.010 \\ \text { T5_37_1 } & 0.133 & 0.715 & -0.041 \\ \text { T5_38_1 } & 0.006 & 0.939 & 0.034 \\ \text { T5_39_1 } & 0.001 & 0.970 & 0.028 \\ \text { T5_40_1 } & 0.001 & 0.974 & 0.011 \\ \text { T5_41_1 } & 0.035 & 0.852 & -0.014 \\ \text { T5_42_1 } & 0.090 & 0.764 & -0.031 \\ \text { T5_2_2 } & 0.103 & 0.749 & -0.004 \\ \text { T5_3_2 } & 0.370 & 0.543 & -0.050 \\ \text { T5_4_2 } & 0.354 & 0.552 & -0.048 \\ \text { T5_6_2 } & 0.333 & 0.564 & -0.045 \\ \text { T5_7_2 } & 0.005 & 0.944 & 0.024 \\ \text { T5_12_2 } & 0.176 & 0.675 & -0.019 \\ \text { T5_13_2 } & 0.353 & 0.552 & -0.047 \\ \text { T5_14_2 } & 0.366 & 0.545 & -0.049 \\ \text { T5_15_2 } & 0.219 & 0.640 & -0.037 \\ \text { T5_17_2 } & 0.370 & 0.543 & -0.050 \\ \text { T5_18_2 } & 0.221 & 0.638 & -0.027 \\ \text { T5_19_2 } & 0.041 & 0.840 & 0.014 \\ \text { T5_20_2 } & 0.142 & 0.706 & -0.013\end{array}$




$\begin{array}{llll}\text { T5_21_2 } & 0.193 & 0.660 & -0.023 \\ \text { T5_22_2 } & 0.339 & 0.560 & -0.046 \\ \text { T5_24_2 } & 0.227 & 0.634 & -0.029 \\ \text { T5_25_2 } & 0.192 & 0.661 & -0.023 \\ \text { T5_28_2 } & 0.010 & 0.920 & -0.029 \\ \text { T5_32_2 } & 0.001 & 0.971 & -0.002 \\ \text { T5_33_2 } & 0.001 & 0.981 & -0.018 \\ \text { T5_34_2 } & 0.000 & 0.989 & -0.005 \\ \text { T5_35_2 } & 0.019 & 0.891 & 0.013 \\ \text { T5_38_2 } & 0.023 & 0.878 & 0.016 \\ \text { T5_40_2 } & 0.028 & 0.866 & -0.040 \\ \text { T5_41_2 } & 0.063 & 0.802 & -0.053 \\ \text { T5_42_2 } & 0.096 & 0.756 & -0.060 \\ \text { T5_43_2 } & 0.000 & 0.983 & -0.005 \\ \text { T5_4_3 } & 0.010 & 0.921 & 0.000 \\ \text { T5_5_3 } & 0.030 & 0.863 & 0.012 \\ \text { T5_6_3 } & 0.051 & 0.821 & 0.020 \\ \text { T5_7_3 } & 0.052 & 0.820 & 0.020 \\ \text { T5_8_3 } & 0.013 & 0.911 & -0.016 \\ \text { T5_9_3 } & 0.021 & 0.886 & -0.023 \\ \text { T5_11_3 } & 0.041 & 0.839 & 0.016 \\ \text { T5_16_3 } & 0.037 & 0.847 & 0.015 \\ \text { T5_17_34.1 } & 0.087 & 0.768 & 0.025 \\ \text { T5_18_3 } & 0.054 & 0.816 & 0.021 \\ \text { T5_19_3 } & 0.056 & 0.813 & 0.021 \\ \text { T5_20_3 } & 0.047 & 0.829 & 0.018 \\ \text { T5_21_3 } & 0.226 & 0.634 & -0.072 \\ \text { T5_22_3 } & 0.055 & 0.815 & 0.021 \\ \text { T5_23_3 } & 0.041 & 0.839 & 0.016 \\ \text { T5_24_3 } & 0.013 & 0.909 & 0.002 \\ \text { T5_25_3 } & 0.041 & 0.840 & 0.016 \\ \text { T5_27_3 } & 0.323 & 0.570 & -0.069 \\ \text { T5_28_3 } & 0.164 & 0.685 & -0.043 \\ \text { T5_29_3 } & 0.427 & 0.513 & -0.081 \\ \text { T5_30_3 } & 0.489 & 0.484 & -0.089 \\ \text { T5_32_3 } & 0.105 & 0.746 & -0.031 \\ \text { T5_36_3 } & 0.199 & 0.656 & -0.051 \\ \text { T5_37_3 } & 0.121 & 0.728 & -0.035 \\ \text { T5_49_3 } & 0.008 & 0.931 & 0.038 \\ & 0.098 & 0.755 & -0.029 \\ \text { T0_3 } & 0.182 & 0.670 & -0.047\end{array}$




$\begin{array}{llll}\text { T5_42_3 } & 0.220 & 0.639 & -0.055 \\ \text { T5_1_4 } & 0.197 & 0.657 & -0.013 \\ \text { T5_2_4 } & 0.097 & 0.756 & 0.045 \\ \text { T5_4_4 } & 0.012 & 0.912 & 0.014 \\ \text { T5_7_4 } & 0.821 & 0.365 & 0.101 \\ \text { T5_9_4 } & 0.230 & 0.632 & 0.034 \\ \text { T5_12_4 } & 0.005 & 0.947 & 0.007 \\ \text { T5_13_4 } & 0.032 & 0.859 & 0.024 \\ \text { T5_14_4 } & 0.009 & 0.926 & 0.011 \\ \text { T5_16_4 } & 0.010 & 0.919 & 0.012 \\ \text { T5_17_4 } & 0.010 & 0.922 & 0.012 \\ \text { T5_19_4 } & 0.004 & 0.947 & 0.007 \\ \text { T5_20_4 } & 0.270 & 0.603 & 0.041 \\ \text { T5_22_4 } & 0.006 & 0.937 & 0.009 \\ \text { T5_23_4 } & 0.003 & 0.955 & 0.005 \\ \text { T5_24_4 } & 0.034 & 0.853 & 0.025 \\ \text { T5_27_4 } & 0.150 & 0.698 & 0.026 \\ \text { T5_28_4 } & 0.252 & 0.616 & 0.044 \\ \text { T5_30_4 } & 0.151 & 0.698 & 0.026 \\ \text { T5_31_4 } & 0.519 & 0.471 & 0.077 \\ \text { T5_32_4 } & 0.210 & 0.647 & 0.036 \\ \text { T5_33_4 } & 0.163 & 0.687 & 0.029 \\ \text { T5_34_4 } & 0.514 & 0.474 & 0.077 \\ \text { T5_37_4 } & 0.070 & 0.791 & 0.008 \\ \text { T5_38_4 } & 0.070 & 0.792 & 0.007 \\ \text { T5_39_4 } & 0.142 & 0.707 & 0.024 \\ \text { T5_43_4 } & 0.456 & 0.499 & 0.071 \\ \text { T5_1_5 } & 0.086 & 0.769 & 0.011 \\ \text { T5_4_5 } & 0.172 & 0.679 & 0.029 \\ \text { T5_8_5 } & 0.200 & 0.655 & -0.045 \\ \text { T5_9_5 } & 0.139 & 0.709 & 0.023 \\ \text { T5_10_5 } & 0.119 & 0.730 & 0.018 \\ \text { T5_13_5 } & 0.066 & 0.797 & 0.004 \\ \text { T5_15_5 } & 0.121 & 0.728 & 0.019 \\ \text { T5_16_5 } & 0.173 & 0.678 & 0.029 \\ \text { T5_17_5 } & 0.169 & 0.681 & 0.029 \\ \text { T5_18_5 } & 0.259 & 0.611 & 0.044 \\ \text { T5_19_5 } & 0.095 & 0.758 & 0.013 \\ \text { T5_20_56.1 } & 0.001 & 0.973 & -0.001 \\ \text { T5_21_5 } & 0.049 & 0.825 & -0.001 \\ \text { T5_22_5 } & 0.182 & 0.670 & 0.031\end{array}$




\begin{tabular}{llll} 
T5_23_5 & 0.020 & 0.887 & -0.013 \\
T5_25_5 & 0.111 & 0.739 & 0.017 \\
T5_27_5 & 0.317 & 0.573 & -0.025 \\
T5_28_5 & 0.456 & 0.499 & -0.042 \\
T5_31_5 & 0.769 & 0.381 & -0.074 \\
T5_32_5 & 0.494 & 0.482 & -0.047 \\
T5_34_5 & 0.888 & 0.346 & -0.084 \\
T5_35_5 & 0.407 & 0.524 & -0.038 \\
T5_39_5 & 0.502 & 0.479 & -0.049 \\
T5_40_5 & 0.694 & 0.405 & -0.067 \\
T5_41_5 & 0.335 & 0.563 & -0.029 \\
T5_43_5 & 0.279 & 0.597 & -0.021 \\
T5_1_6 & 0.167 & 0.683 & 0.042 \\
T5_2_6 & 0.001 & 0.972 & -0.014 \\
T5_5_6 & 0.218 & 0.641 & 0.052 \\
T5_6_6 & 0.014 & 0.907 & 0.025 \\
T5_7_6 & 0.238 & 0.625 & -0.034 \\
T5_8_6 & 0.289 & 0.591 & -0.042 \\
T5_9_6 & 0.222 & 0.637 & -0.031 \\
T5_10_6 & 0.390 & 0.532 & -0.055 \\
T5_13_6 & 0.069 & 0.793 & 0.002 \\
T5_15_6 & 0.346 & 0.556 & -0.049 \\
T5_20_6 & 0.160 & 0.689 & -0.019 \\
T5_22_6 & 0.044 & 0.834 & 0.010 \\
T5_23_6 & 0.003 & 0.957 & 0.034 \\
T5_24_6 & 0.014 & 0.907 & 0.024 \\
T5_25_6 & 0.368 & 0.544 & -0.052 \\
T5_26_6 & 0.478 & 0.489 & 0.042 \\
T5_27_6 & 0.089 & 0.765 & -0.019 \\
T5_31_6 & 0.574 & 0.449 & 0.051 \\
T5_32_6 & 0.548 & 0.459 & 0.050 \\
T5_33_6 & 0.605 & 0.437 & 0.055 \\
T5_34_6 & 0.002 & 0.961 & -0.062 \\
T5_36_6 & 0.819 & 0.365 & 0.075 \\
T5_38_6 & 0.133 & 0.715 & -0.009 \\
T5_40_6 & 0.248 & 0.619 & 0.011 \\
T5_41_6 & 0.647 & 0.421 & 0.060 \\
T5_42_6 & 0.505 & 0.477 & 0.042 \\
T5_43_6 & 0.319 & 0.572 & 0.022 \\
\hline & & & \\
\hline
\end{tabular}


Table 48

Task 5 DIF: Latinx vs. Sample

\begin{tabular}{|c|c|c|c|}
\hline ItemID & ChiSq & pvalue & EffectSize \\
\hline T5_10_12 & 0.660 & 0.417 & 0.172 \\
\hline T5_18_12 & 0.141 & 0.707 & 0.071 \\
\hline T5_24_12 & 0.154 & 0.695 & 0.077 \\
\hline T5_30_12 & 0.891 & 0.345 & 0.163 \\
\hline T5_32_12 & 2.674 & 0.102 & 0.405 \\
\hline T5_26_23 & 0.516 & 0.473 & 0.141 \\
\hline T5_39_23 & 0.416 & 0.519 & 0.126 \\
\hline T5_10_23 & 0.006 & 0.938 & 0.020 \\
\hline T5_11_23 & 0.051 & 0.821 & 0.067 \\
\hline T5_23_23 & 0.002 & 0.969 & 0.007 \\
\hline T5_3_34 & 0.262 & 0.609 & -0.080 \\
\hline T5_10_34 & 0.376 & 0.540 & -0.116 \\
\hline T5_14_34 & 0.344 & 0.558 & -0.107 \\
\hline T5_17_34 & 0.578 & 0.447 & -0.167 \\
\hline T5_31_34 & 0.638 & 0.424 & 0.121 \\
\hline T5_34_34 & 0.016 & 0.900 & -0.117 \\
\hline T5_26_45 & 0.039 & 0.843 & -0.043 \\
\hline T5_36_45 & 0.021 & 0.886 & -0.056 \\
\hline T5_26_56 & 0.206 & 0.650 & 0.025 \\
\hline T5_30_56 & 0.327 & 0.567 & -0.025 \\
\hline T5_37_56 & 0.859 & 0.354 & -0.145 \\
\hline T5_3_56 & 0.465 & 0.496 & -0.130 \\
\hline T5_6_56 & 0.494 & 0.482 & -0.140 \\
\hline T5_7_56 & 0.383 & 0.536 & -0.111 \\
\hline T5_12_56 & 0.569 & 0.451 & -0.157 \\
\hline T5_20_56 & 0.475 & 0.491 & -0.135 \\
\hline T5_6_456 & 1.178 & 0.278 & -0.177 \\
\hline T5_8_456 & 1.985 & 0.159 & -0.274 \\
\hline T5_15_456 & 2.153 & 0.142 & -0.292 \\
\hline T5_21_456 & 2.267 & 0.132 & -0.309 \\
\hline T5_25_456 & 1.679 & 0.195 & -0.235 \\
\hline T5_1_1 & 0.015 & 0.903 & -0.004 \\
\hline T5_2_1 & 0.068 & 0.794 & 0.042 \\
\hline T5_3_1 & 0.059 & 0.809 & 0.036 \\
\hline T5_4_1 & 0.092 & 0.762 & 0.057 \\
\hline T5_6_1 & 0.091 & 0.763 & 0.056 \\
\hline T5_7_1 & 0.094 & 0.759 & 0.058 \\
\hline
\end{tabular}




$\begin{array}{llll} & & & \\ \text { T5_8_1 } & 0.094 & 0.759 & 0.058 \\ \text { T5_9_1 } & 0.971 & 0.325 & 0.158 \\ \text { T5_11_1 } & 0.889 & 0.346 & 0.141 \\ \text { T5_12_1 } & 1.417 & 0.234 & 0.229 \\ \text { T5_13_1 } & 0.094 & 0.759 & 0.058 \\ \text { T5_14_1 } & 0.091 & 0.763 & 0.056 \\ \text { T5_15_1 } & 0.092 & 0.761 & 0.057 \\ \text { T5_16_1 } & 0.088 & 0.766 & 0.055 \\ \text { T5_17_1 } & 0.074 & 0.786 & 0.046 \\ \text { T5_19_1 } & 0.078 & 0.780 & 0.049 \\ \text { T5_20_1 } & 0.094 & 0.760 & 0.058 \\ \text { T5_21_1 } & 0.049 & 0.825 & 0.029 \\ \text { T5_22_1 } & 0.043 & 0.835 & 0.024 \\ \text { T5_23_1 } & 0.094 & 0.759 & 0.058 \\ \text { T5_25_1 } & 0.083 & 0.773 & 0.052 \\ \text { T5_26_1 } & 0.711 & 0.399 & 0.173 \\ \text { T5_27_1 } & 0.982 & 0.322 & 0.222 \\ \text { T5_28_1 } & 0.705 & 0.401 & 0.171 \\ \text { T5_29_1 } & 0.493 & 0.483 & 0.123 \\ \text { T5_33_1 } & 0.230 & 0.632 & 0.048 \\ \text { T5_34_1 } & 0.564 & 0.453 & 0.141 \\ \text { T5_36_1 } & 0.711 & 0.399 & 0.171 \\ \text { T5_37_1 } & 0.081 & 0.776 & -0.028 \\ \text { T5_38_1 } & 0.532 & 0.466 & 0.132 \\ \text { T5_39_1 } & 0.396 & 0.529 & 0.100 \\ \text { T5_40_1 } & 0.594 & 0.441 & 0.148 \\ \text { T5_41_1 } & 0.677 & 0.411 & 0.166 \\ \text { T5_42_1 } & 0.834 & 0.361 & 0.191 \\ \text { T5_2_2 } & 0.000 & 0.992 & 0.006 \\ \text { T5_3_2 } & 0.000 & 0.985 & 0.010 \\ \text { T5_4_2 } & 0.005 & 0.946 & 0.025 \\ \text { T5_6_2 } & 0.001 & 0.979 & 0.012 \\ \text { T5_7_2 } & 0.277 & 0.599 & 0.134 \\ \text { T5_12_2 } & 0.001 & 0.981 & -0.013 \\ \text { T5_13_2 } & 0.007 & 0.934 & 0.030 \\ \text { T5_14_2 } & 0.000 & 0.985 & -0.003 \\ \text { T5_15_2 } & 0.263 & 0.608 & 0.129 \\ \text { T5_17_2 } & 0.000 & 0.985 & 0.010 \\ \text { T5_20_2 } & 0.000 & 0.988 & -0.016\end{array}$




\begin{tabular}{|c|c|c|c|}
\hline T5_21_2 & 0.001 & 0.975 & 0.014 \\
\hline T5_22_2 & 0.001 & 0.974 & 0.014 \\
\hline T5_24_2 & 0.000 & 0.985 & 0.010 \\
\hline T5_25_2 & 0.001 & 0.971 & 0.016 \\
\hline T5_28_2 & 0.211 & 0.646 & 0.122 \\
\hline T5_32_2 & 0.123 & 0.726 & 0.082 \\
\hline T5_33_2 & 0.223 & 0.637 & 0.126 \\
\hline T5_34_2 & 0.241 & 0.623 & 0.132 \\
\hline T5_35_2 & 0.207 & 0.649 & 0.121 \\
\hline T5_38_2 & 0.057 & 0.811 & 0.048 \\
\hline T5_40_2 & 0.135 & 0.713 & 0.091 \\
\hline T5_41_2 & 0.056 & 0.813 & 0.044 \\
\hline T5_42_2 & 0.062 & 0.804 & 0.051 \\
\hline T5_43_2 & 0.196 & 0.658 & 0.114 \\
\hline T5_4_3 & 0.000 & 0.985 & -0.003 \\
\hline T5_5_3 & 0.000 & 0.993 & 0.006 \\
\hline T5_6_3 & 0.000 & 0.988 & 0.008 \\
\hline T5_7_3 & 0.000 & 0.989 & 0.007 \\
\hline T5_8_3 & 0.120 & 0.729 & 0.060 \\
\hline T5_9_3 & 0.064 & 0.801 & 0.028 \\
\hline T5_11_3 & 0.000 & 0.985 & 0.009 \\
\hline T5_16_3 & 0.000 & 0.984 & -0.004 \\
\hline T5_17_34.1 & 0.578 & 0.447 & -0.167 \\
\hline T5_18_3 & 0.000 & 0.999 & 0.003 \\
\hline T5_19_3 & 0.000 & 0.992 & 0.006 \\
\hline T5_20_3 & 0.000 & 0.985 & 0.009 \\
\hline T5_21_3 & 0.026 & 0.872 & -0.001 \\
\hline T5_22_3 & 0.001 & 0.979 & 0.012 \\
\hline T5_23_3 & 0.002 & 0.961 & 0.019 \\
\hline T5_24_3 & 0.005 & 0.941 & -0.045 \\
\hline T5_25_3 & 0.004 & 0.953 & 0.022 \\
\hline T5_27_3 & 0.013 & 0.909 & -0.007 \\
\hline T5_28_3 & 0.125 & 0.724 & 0.075 \\
\hline T5_29_3 & 0.120 & 0.729 & 0.069 \\
\hline T5_30_3 & 0.017 & 0.898 & -0.003 \\
\hline T5_32_3 & 0.040 & 0.842 & 0.020 \\
\hline T5_36_3 & 0.129 & 0.720 & 0.076 \\
\hline T5_37_3 & 0.190 & 0.663 & 0.097 \\
\hline T5_39_3 & 0.113 & 0.737 & 0.065 \\
\hline T5_40_3 & 0.129 & 0.720 & 0.072 \\
\hline T5_41_3 & 0.119 & 0.730 & 0.069 \\
\hline
\end{tabular}




$\begin{array}{llll} & & & \\ \text { T5_42_3 } & 0.065 & 0.800 & 0.039 \\ \text { T5_1_4 } & 0.238 & 0.626 & -0.077 \\ \text { T5_2_4 } & 0.362 & 0.548 & -0.101 \\ \text { T5_4_4 } & 0.344 & 0.558 & -0.097 \\ \text { T5_7_4 } & 0.041 & 0.841 & -0.070 \\ \text { T5_9_4 } & 0.000 & 0.990 & 0.005 \\ \text { T5_12_4 } & 0.288 & 0.592 & -0.080 \\ \text { T5_13_4 } & 0.413 & 0.520 & -0.115 \\ \text { T5_14_4 } & 0.245 & 0.620 & -0.065 \\ \text { T5_16_4 } & 0.530 & 0.467 & -0.146 \\ \text { T5_17_4 } & 0.482 & 0.488 & -0.133 \\ \text { T5_19_4 } & 0.427 & 0.514 & -0.120 \\ \text { T5_20_4 } & 0.004 & 0.949 & -0.024 \\ \text { T5_22_4 } & 0.137 & 0.711 & -0.023 \\ \text { T5_23_4 } & 0.432 & 0.511 & -0.122 \\ \text { T5_24_4 } & 0.340 & 0.560 & -0.096 \\ \text { T5_27_4 } & 0.236 & 0.627 & 0.075 \\ \text { T5_28_4 } & 0.079 & 0.779 & 0.003 \\ \text { T5_30_4 } & 0.372 & 0.542 & 0.116 \\ \text { T5_31_4 } & 0.187 & 0.665 & 0.056 \\ \text { T5_32_4 } & 0.250 & 0.617 & 0.080 \\ \text { T5_33_4 } & 0.175 & 0.676 & 0.049 \\ \text { T5_34_4 } & 0.051 & 0.821 & -0.012 \\ \text { T5_37_4 } & 0.153 & 0.696 & 0.040 \\ \text { T5_38_4 } & 0.065 & 0.800 & -0.001 \\ \text { T5_39_4 } & 0.174 & 0.677 & 0.054 \\ \text { T5_43_4 } & 0.106 & 0.745 & 0.018 \\ \text { T5_1_5 } & 0.192 & 0.661 & -0.097 \\ \text { T5_4_5 } & 0.038 & 0.846 & -0.015 \\ \text { T5_8_5 } & 0.000 & 0.997 & 0.036 \\ \text { T5_9_5 } & 0.075 & 0.784 & -0.042 \\ \text { T5_10_5 } & 0.045 & 0.833 & -0.020 \\ \text { T5_13_5 } & 0.014 & 0.908 & 0.012 \\ \text { T5_15_5 } & 0.118 & 0.732 & -0.065 \\ \text { T5_16_5 } & 0.149 & 0.699 & -0.080 \\ \text { T5_17_5 } & 0.180 & 0.672 & -0.093 \\ \text { T5_18_5 } & 0.154 & 0.695 & -0.082 \\ \text { T5_19_5 } & 0.133 & 0.716 & -0.072 \\ \text { T5_20_56.1 } & 0.475 & 0.491 & -0.135 \\ \text { T5_5 } & 0.077 & 0.782 & -0.043 \\ \text { T255 } & 0.048 & 0.826 & -0.023\end{array}$




\begin{tabular}{|c|c|c|c|}
\hline T5_23_5 & 0.065 & 0.799 & -0.035 \\
\hline T5_25_5 & 0.042 & 0.838 & -0.018 \\
\hline T5_27_5 & 0.058 & 0.810 & -0.099 \\
\hline T5_28_5 & 0.000 & 0.986 & -0.003 \\
\hline T5_31_5 & 0.006 & 0.940 & 0.028 \\
\hline T5_32_5 & 0.001 & 0.974 & -0.008 \\
\hline T5_34_5 & 0.000 & 0.988 & -0.026 \\
\hline T5_35_5 & 0.000 & 0.989 & 0.010 \\
\hline T5_39_5 & 0.000 & 0.996 & -0.022 \\
\hline T5_40_5 & 0.017 & 0.896 & 0.046 \\
\hline T5_41_5 & 0.009 & 0.924 & 0.035 \\
\hline T5_43_5 & 0.001 & 0.977 & -0.030 \\
\hline T5_1_6 & 2.247 & 0.134 & -0.297 \\
\hline T5_2_6 & 1.578 & 0.209 & -0.214 \\
\hline T5_5_6 & 1.940 & 0.164 & -0.261 \\
\hline T5_6_6 & 0.098 & 0.754 & -0.069 \\
\hline T5_7_6 & 0.057 & 0.812 & -0.044 \\
\hline T5_8_6 & 0.069 & 0.792 & -0.052 \\
\hline T5_9_6 & 0.056 & 0.813 & -0.044 \\
\hline T5_10_6 & 0.079 & 0.778 & -0.058 \\
\hline T5_13_6 & 0.182 & 0.670 & -0.107 \\
\hline T5_15_6 & 0.109 & 0.741 & -0.075 \\
\hline T5_20_6 & 0.079 & 0.779 & -0.058 \\
\hline T5_22_6 & 0.016 & 0.898 & -0.006 \\
\hline T5_23_6 & 0.207 & 0.649 & -0.117 \\
\hline T5_24_6 & 0.027 & 0.869 & -0.018 \\
\hline T5_25_6 & 0.056 & 0.813 & -0.043 \\
\hline T5_26_6 & 0.798 & 0.372 & -0.135 \\
\hline T5_27_6 & 0.702 & 0.402 & -0.113 \\
\hline T5_31_6 & 0.986 & 0.321 & -0.166 \\
\hline T5_32_6 & 1.052 & 0.305 & -0.181 \\
\hline T5_33_6 & 1.490 & 0.222 & -0.247 \\
\hline T5_34_6 & 0.179 & 0.673 & 0.037 \\
\hline T5_36_6 & 1.432 & 0.232 & -0.239 \\
\hline T5_38_6 & 0.630 & 0.427 & -0.099 \\
\hline T5_40_6 & 0.736 & 0.391 & -0.120 \\
\hline T5_41_6 & 1.120 & 0.290 & -0.193 \\
\hline T5_42_6 & 0.725 & 0.395 & -0.116 \\
\hline T5_43_6 & 0.991 & 0.319 & -0.169 \\
\hline
\end{tabular}


Table 49

Task 6 DIF: Male vs. female

\begin{tabular}{lccc}
\hline ItemID & ChiSq & pvalue & EffectSize \\
\hline T6_16_12 & 0.009 & 0.926 & -0.013 \\
T6_19_12 & 0.005 & 0.944 & -0.018 \\
T6_21_12 & 0.003 & 0.957 & -0.017 \\
T6_26_12 & 0.360 & 0.548 & 0.049 \\
T6_41_12 & 0.001 & 0.973 & -0.049 \\
T6_17_23 & 0.093 & 0.760 & 0.020 \\
T6_20_23 & 0.007 & 0.932 & -0.010 \\
T6_36_23 & 0.039 & 0.844 & -0.024 \\
T6_42_23 & 0.108 & 0.743 & -0.044 \\
T6_1_34 & 0.055 & 0.815 & 0.077 \\
T6_5_34 & 0.022 & 0.882 & -0.012 \\
T6_10_34 & 0.000 & 0.997 & 0.009 \\
T6_21_34 & 0.042 & 0.838 & -0.020 \\
T6_24_34 & 0.000 & 0.995 & 0.010 \\
T6_12_3.1 & 0.111 & 0.740 & -0.030 \\
T6_40_34 & 0.020 & 0.888 & 0.099 \\
T6_43_34 & 0.065 & 0.799 & 0.034 \\
T6_27_45 & 0.883 & 0.348 & -0.096 \\
T6_28_45 & 0.035 & 0.852 & 0.072 \\
T6_1_56 & 0.000 & 0.992 & 0.000 \\
T6_2_56 & 0.000 & 0.994 & 0.004 \\
T6_6_56 & 0.000 & 0.987 & 0.004 \\
T6_18_56 & 0.008 & 0.930 & 0.019 \\
T6_28_56 & 0.070 & 0.791 & -0.056 \\
T6_32_56 & 0.052 & 0.820 & -0.053 \\
T6_45_56 & 0.602 & 0.438 & -0.132 \\
T6_1_456 & 0.233 & 0.630 & -0.041 \\
T6_4_456 & 0.027 & 0.870 & 0.004 \\
T6_8_456 & 0.001 & 0.973 & 0.028 \\
T6_14_456 & Inf & 0.000 & NaN \\
T6_22_456 & 0.007 & 0.933 & 0.020 \\
T6_1_1 & 0.075 & 0.784 & -0.004 \\
T6_2_1 & 0.455 & 0.500 & 0.058 \\
T6_3_1 & 0.454 & 0.500 & 0.060 \\
T6_4_1 & 0.001 & 0.977 & -0.010 \\
T6_5_1 & 0.017 & 0.895 & 0.005 \\
T6_6_1 & 0.024 & 0.876 & 0.008 \\
T6 & & & \\
\hline
\end{tabular}




$\begin{array}{llll} & & & \\ \text { T6_7_1 } & 0.294 & 0.588 & 0.037 \\ \text { T6_8_1 } & 0.000 & 1.000 & -0.019 \\ \text { T6_9_1 } & 0.002 & 0.967 & -0.009 \\ \text { T6_11_1 } & 0.015 & 0.903 & 0.003 \\ \text { T6_12_1 } & 0.000 & 0.990 & -0.013 \\ \text { T6_13_1 } & 0.027 & 0.869 & 0.009 \\ \text { T6_14_1 } & 0.030 & 0.862 & 0.011 \\ \text { T6_17_1 } & 0.001 & 0.982 & -0.011 \\ \text { T6_18_1 } & 0.037 & 0.848 & 0.014 \\ \text { T6_19_12.1 } & 0.005 & 0.944 & -0.018 \\ \text { T6_20_1 } & 0.039 & 0.843 & 0.015 \\ \text { T6_22_1 } & 0.013 & 0.910 & 0.002 \\ \text { T6_23_1 } & 0.010 & 0.921 & -0.001 \\ \text { T6_24_1 } & 0.029 & 0.865 & 0.010 \\ \text { T6_25_1 } & 0.016 & 0.900 & 0.004 \\ \text { T6_27_1 } & 0.088 & 0.767 & 0.022 \\ \text { T6_28_1 } & 0.118 & 0.731 & 0.027 \\ \text { T6_29_1 } & 0.090 & 0.764 & 0.018 \\ \text { T6_30_1 } & 0.148 & 0.701 & 0.034 \\ \text { T6_31_1 } & 0.099 & 0.753 & 0.025 \\ \text { T6_32_1 } & 0.195 & 0.659 & 0.043 \\ \text { T6_34_1 } & 0.011 & 0.918 & -0.009 \\ \text { T6_35_1 } & 0.138 & 0.711 & 0.032 \\ \text { T6_36_1 } & 0.006 & 0.940 & -0.011 \\ \text { T6_37_1 } & 0.068 & 0.795 & 0.017 \\ \text { T6_39_1 } & 0.048 & 0.827 & 0.008 \\ \text { T6_40_1 } & 0.008 & 0.929 & -0.006 \\ \text { T6_41_12.1 } & 0.001 & 0.973 & -0.049 \\ \text { T6_42_1 } & 0.636 & 0.425 & 0.090 \\ \text { T6_43_1 } & 0.014 & 0.907 & -0.003 \\ \text { T6_45_1 } & 0.134 & 0.715 & 0.034 \\ \text { T6_46_1 } & 0.004 & 0.951 & -0.007 \\ \text { T6_47_1 } & 0.001 & 0.975 & -0.029 \\ \text { T6_48_1 } & 0.025 & 0.875 & 0.002 \\ \text { T6_1_2 } & 0.127 & 0.722 & 0.029 \\ \text { T6_3_2 } & 0.197 & 0.657 & 0.040 \\ \text { T6_4_2 } & 0.026 & 0.872 & -0.004 \\ \text { T6_5_2 } & 0.001 & 0.972 & -0.019 \\ \text { T6_10_2 } & 0.044 & 0.834 & 0.022\end{array}$




$\begin{array}{llll}\text { T6_12_2 } & 0.004 & 0.951 & 0.000 \\ \text { T6_14_2 } & 0.040 & 0.841 & -0.043 \\ \text { T6_15_2 } & 0.027 & 0.870 & -0.058 \\ \text { T6_16_2 } & 0.067 & 0.795 & 0.029 \\ \text { T6_18_2 } & 0.007 & 0.934 & 0.003 \\ \text { T6_19_2 } & 0.007 & 0.934 & -0.026 \\ \text { T6_22_2 } & 0.050 & 0.823 & 0.025 \\ \text { T6_23_2 } & 0.008 & 0.931 & 0.004 \\ \text { T6_26_2 } & 0.000 & 0.999 & -0.020 \\ \text { T6_28_2 } & 0.006 & 0.939 & -0.030 \\ \text { T6_29_2 } & 0.071 & 0.791 & 0.027 \\ \text { T6_31_2 } & 0.009 & 0.925 & -0.034 \\ \text { T6_32_2 } & 0.015 & 0.901 & 0.006 \\ \text { T6_35_2 } & 0.000 & 0.991 & -0.017 \\ \text { T6_38_2 } & 0.001 & 0.970 & -0.009 \\ \text { T6_39_2 } & 0.003 & 0.956 & -0.006 \\ \text { T6_40_2 } & 0.009 & 0.923 & 0.000 \\ \text { T6_41_2 } & 0.000 & 0.998 & -0.019 \\ \text { T6_43_2 } & 0.028 & 0.868 & 0.010 \\ \text { T6_44_2 } & 0.001 & 0.971 & -0.009 \\ \text { T6_45_2 } & 0.000 & 0.995 & -0.019 \\ \text { T6_46_2 } & 0.212 & 0.646 & 0.054 \\ \text { T6_47_2 } & 0.073 & 0.787 & 0.027 \\ \text { T6_49_2 } & 0.063 & 0.802 & -0.056 \\ \text { T6_2_3 } & 0.034 & 0.853 & 0.038 \\ \text { T6_3_3 } & 0.012 & 0.914 & 0.003 \\ \text { T6_4_3 } & 0.000 & 0.996 & -0.013 \\ \text { T6_8_3 } & 0.000 & 0.999 & -0.013 \\ \text { T6_9_3 } & 0.169 & 0.681 & 0.070 \\ \text { T6_11_3 } & 0.002 & 0.961 & -0.006 \\ \text { T6_12_3 } & 0.084 & 0.773 & 0.030 \\ \text { T6_13_3 } & 0.000 & 0.999 & -0.013 \\ \text { T6_15_3 } & 0.070 & 0.792 & 0.027 \\ \text { T6_16_3 } & 0.000 & 0.988 & 0.007 \\ \text { T6_17_3 } & 0.010 & 0.919 & 0.002 \\ \text { T6_18_3 } & 0.002 & 0.968 & -0.007 \\ \text { T6_19_3 } & 0.000 & 0.991 & -0.015 \\ \text { T6_22_3 } & 0.000 & 0.992 & -0.014 \\ \text { T6_23_3 } & 0.003 & 0.957 & -0.005 \\ \text { T6_27_3 } & 0.026 & 0.871 & -0.004\end{array}$




$\begin{array}{llll}\text { T6_28_3 } & 0.101 & 0.750 & -0.027 \\ \text { T6_29_3 } & 0.030 & 0.863 & 0.051 \\ \text { T6_30_3 } & 0.003 & 0.958 & 0.013 \\ \text { T6_32_3 } & 0.172 & 0.678 & -0.042 \\ \text { T6_33_3 } & 0.001 & 0.982 & 0.030 \\ \text { T6_34_3 } & 0.120 & 0.729 & -0.032 \\ \text { T6_36_3 } & 0.019 & 0.891 & 0.000 \\ \text { T6_37_3 } & 0.006 & 0.936 & 0.010 \\ \text { T6_38_3 } & 0.011 & 0.918 & 0.038 \\ \text { T6_39_3 } & 0.025 & 0.876 & -0.003 \\ \text { T6_44_3 } & 0.175 & 0.676 & -0.042 \\ \text { T6_45_3 } & 0.000 & 0.996 & 0.027 \\ \text { T6_47_3 } & 0.003 & 0.956 & 0.012 \\ \text { T6_2_4 } & 0.003 & 0.956 & 0.065 \\ \text { T6_3_4 } & 0.000 & 0.998 & 0.025 \\ \text { T6_5_4 } & 0.001 & 0.974 & 0.020 \\ \text { T6_10_4 } & 0.000 & 0.990 & 0.028 \\ \text { T6_11_4 } & 0.009 & 0.926 & 0.012 \\ \text { T6_12_4 } & 0.008 & 0.930 & 0.012 \\ \text { T6_16_4 } & 0.021 & 0.886 & 0.054 \\ \text { T6_17_4 } & 0.009 & 0.924 & 0.011 \\ \text { T6_18_4 } & 0.025 & 0.875 & 0.051 \\ \text { T6_19_4 } & 0.015 & 0.904 & 0.007 \\ \text { T6_20_4 } & 0.007 & 0.933 & 0.062 \\ \text { T6_21_4 } & 0.135 & 0.714 & -0.029 \\ \text { T6_23_4 } & 0.133 & 0.715 & -0.030 \\ \text { T6_24_4 } & 0.080 & 0.778 & -0.018 \\ \text { T6_25_4 } & 0.000 & 0.984 & 0.022 \\ \text { T6_26_4 } & 0.479 & 0.489 & -0.063 \\ \text { T6_29_4 } & 0.080 & 0.777 & -0.002 \\ \text { T6_31_4 } & 0.031 & 0.860 & 0.015 \\ \text { T6_33_4 } & 0.004 & 0.948 & 0.031 \\ \text { T6_34_4 } & 0.023 & 0.881 & 0.019 \\ \text { T6_35_4 } & 0.157 & 0.692 & -0.018 \\ \text { T6_36_4 } & 0.091 & 0.763 & -0.004 \\ \text { T6_38_4 } & 0.031 & 0.860 & 0.013 \\ \text { T6_40_4 } & 0.000 & 0.993 & 0.041 \\ \text { T6_42_4 } & 0.006 & 0.937 & 0.059 \\ \text { T6445_4 } & 0.002 & 0.966 & 0.036 \\ \text { T6_4 } & 0.413 & 0.521 & -0.057 \\ \text { T6 } & 0.028 & 0.868 & 0.017\end{array}$




$\begin{array}{llll}\text { T6_46_4 } & 0.000 & 0.985 & 0.040 \\ \text { T6_48_4 } & 0.167 & 0.683 & -0.020 \\ \text { T6_49_4 } & 0.005 & 0.941 & 0.032 \\ \text { T6_3_5 } & 0.005 & 0.947 & -0.014 \\ \text { T6_7_5 } & 0.007 & 0.932 & -0.016 \\ \text { T6_8_5 } & 0.011 & 0.917 & 0.017 \\ \text { T6_9_5 } & 0.068 & 0.794 & -0.041 \\ \text { T6_10_5 } & 0.035 & 0.852 & -0.031 \\ \text { T6_11_5 } & 0.041 & 0.840 & -0.034 \\ \text { T6_12_5 } & 0.005 & 0.946 & 0.011 \\ \text { T6_13_5 } & 0.000 & 0.993 & -0.003 \\ \text { T6_15_5 } & 0.017 & 0.895 & -0.024 \\ \text { T6_16_5 } & 0.002 & 0.968 & -0.009 \\ \text { T6_17_5 } & 0.000 & 1.000 & 0.001 \\ \text { T6_19_5 } & 0.002 & 0.968 & -0.010 \\ \text { T6_20_5 } & 0.208 & 0.648 & -0.071 \\ \text { T6_22_5 } & 0.031 & 0.861 & -0.030 \\ \text { T6_24_5 } & 0.013 & 0.910 & -0.021 \\ \text { T6_26_5 } & 0.000 & 0.989 & 0.002 \\ \text { T6_27_5 } & 0.017 & 0.895 & -0.021 \\ \text { T6_29_5 } & 0.040 & 0.842 & -0.031 \\ \text { T6_30_5 } & 0.004 & 0.951 & -0.011 \\ \text { T6_31_5 } & 0.007 & 0.933 & -0.014 \\ \text { T6_33_5 } & 0.001 & 0.972 & 0.010 \\ \text { T6_34_5 } & 0.003 & 0.956 & 0.012 \\ \text { T6_35_5 } & 0.012 & 0.914 & -0.017 \\ \text { T6_36_5 } & 0.011 & 0.917 & 0.020 \\ \text { T6_37_5 } & 0.001 & 0.977 & -0.006 \\ \text { T6_38_5 } & 0.000 & 0.994 & 0.000 \\ \text { T6_43_5 } & 0.015 & 0.903 & -0.019 \\ \text { T6_46_5 } & 0.007 & 0.934 & -0.014 \\ \text { T6_47_5 } & 0.016 & 0.900 & 0.023 \\ \text { T6_49_5 } & 0.001 & 0.979 & -0.005 \\ \text { T6_1_6 } & 0.001 & 0.982 & -0.014 \\ \text { T6_5_6 } & 0.038 & 0.845 & -0.040 \\ \text { T6_6_6 } & 0.000 & 0.990 & -0.004 \\ \text { T6_7_6 } & 0.000 & 0.987 & -0.003 \\ \text { T6_8_6 } & 0.007 & 0.935 & 0.020 \\ \text { T6_11_6 } & 0.000 & 0.989 & 0.007\end{array}$




\begin{tabular}{llll} 
T6_12_6 & 0.000 & 0.985 & 0.011 \\
T6_16_6 & 0.004 & 0.953 & 0.017 \\
T6_20_6 & 0.000 & 0.995 & 0.009 \\
T6_22_6 & 0.115 & 0.735 & -0.046 \\
T6_23_6 & 0.000 & 0.993 & 0.005 \\
T6_24_6 & 0.023 & 0.879 & -0.019 \\
T6_25_6 & 0.000 & 0.992 & 0.010 \\
T6_27_6 & 0.006 & 0.940 & -0.027 \\
T6_28_6 & 0.001 & 0.982 & -0.009 \\
T6_30_6 & 0.043 & 0.835 & -0.045 \\
T6_31_6 & 0.001 & 0.981 & -0.019 \\
T6_34_6 & 0.000 & 0.986 & -0.018 \\
T6_35_6 & 0.006 & 0.939 & 0.000 \\
T6_36_6 & 0.023 & 0.880 & 0.010 \\
T6_38_6 & 0.004 & 0.950 & -0.025 \\
T6_40_6 & 0.001 & 0.976 & -0.007 \\
T6_41_6 & 0.000 & 0.991 & -0.013 \\
T6_42_6 & 0.000 & 0.987 & -0.008 \\
T6_43_6 & 0.004 & 0.953 & -0.001 \\
T6_44_6 & 0.010 & 0.920 & 0.006 \\
T6_45_6 & 0.076 & 0.782 & -0.057 \\
T6_46_6 & 0.014 & 0.907 & -0.033 \\
T6_47_6 & 0.029 & 0.865 & 0.015 \\
T6_48_6 & 0.000 & 0.991 & -0.018 \\
\hline & & &
\end{tabular}


Table 50

Task 6 DIF: White vs. Sample

\begin{tabular}{|c|c|c|c|}
\hline ItemID & ChiSq & pvalue & EffectSize \\
\hline T6_16_12 & 0.008 & 0.931 & -0.042 \\
\hline T6_19_12 & 0.002 & 0.963 & -0.018 \\
\hline T6_21_12 & 0.005 & 0.946 & -0.038 \\
\hline T6_26_12 & 0.229 & 0.632 & -0.078 \\
\hline T6_41_12 & 0.007 & 0.931 & 0.011 \\
\hline T6_17_23 & 0.000 & 0.993 & -0.027 \\
\hline T6_20_23 & 0.001 & 0.973 & -0.034 \\
\hline T6_36_23 & 0.096 & 0.756 & 0.102 \\
\hline T6_42_23 & 0.026 & 0.871 & 0.026 \\
\hline T6_1_34 & 0.148 & 0.701 & 0.097 \\
\hline T6_5_34 & 0.030 & 0.863 & -0.035 \\
\hline T6_10_34 & 0.000 & 0.995 & -0.005 \\
\hline T6_21_34 & 0.001 & 0.972 & 0.001 \\
\hline T6_24_34 & 0.002 & 0.962 & -0.016 \\
\hline T6_12_3.1 & 0.081 & 0.776 & -0.003 \\
\hline T6_40_34 & 0.000 & 0.992 & 0.097 \\
\hline T6_43_34 & 0.007 & 0.934 & 0.080 \\
\hline T6_27_45 & 0.123 & 0.726 & -0.051 \\
\hline T6_28_45 & 0.060 & 0.807 & -0.031 \\
\hline T6_1_56 & 0.039 & 0.844 & -0.011 \\
\hline T6_2_56 & 0.008 & 0.927 & -0.100 \\
\hline T6_6_56 & 0.013 & 0.911 & -0.023 \\
\hline T6_18_56 & 0.156 & 0.693 & 0.020 \\
\hline T6_28_56 & 0.064 & 0.800 & -0.048 \\
\hline T6_32_56 & 0.094 & 0.760 & 0.040 \\
\hline T6_45_56 & 0.008 & 0.928 & 0.009 \\
\hline T6_1_456 & 0.092 & 0.762 & -0.024 \\
\hline T6_4_456 & 0.020 & 0.889 & -0.045 \\
\hline T6_8_456 & 0.075 & 0.784 & -0.030 \\
\hline T6_14_456 & Inf & 0.000 & $\mathrm{NaN}$ \\
\hline T6_22_456 & 0.030 & 0.863 & -0.044 \\
\hline T6_1_1 & 0.093 & 0.760 & 0.056 \\
\hline T6_2_1 & 0.047 & 0.829 & -0.013 \\
\hline T6_3_1 & 0.257 & 0.612 & 0.082 \\
\hline T6_4_1 & 0.029 & 0.864 & -0.007 \\
\hline T6_5_1 & 0.000 & 0.993 & 0.017 \\
\hline T6_6_1 & 0.011 & 0.916 & 0.002 \\
\hline
\end{tabular}




$\begin{array}{llll} & & & \\ \text { T6_7_1 } & 0.233 & 0.629 & -0.056 \\ \text { T6_8_1 } & 0.001 & 0.980 & 0.014 \\ \text { T6_9_1 } & 0.011 & 0.916 & 0.002 \\ \text { T6_11_1 } & 0.062 & 0.803 & -0.020 \\ \text { T6_12_1 } & 0.033 & 0.856 & -0.008 \\ \text { T6_13_1 } & 0.100 & 0.752 & -0.028 \\ \text { T6_14_1 } & 0.012 & 0.914 & 0.001 \\ \text { T6_17_1 } & 0.005 & 0.942 & 0.008 \\ \text { T6_18_1 } & 0.041 & 0.839 & -0.011 \\ \text { T6_19_12.1 } & 0.002 & 0.963 & -0.018 \\ \text { T6_20_1 } & 0.014 & 0.907 & 0.000 \\ \text { T6_22_1 } & 0.019 & 0.892 & -0.002 \\ \text { T6_23_1 } & 0.006 & 0.937 & 0.006 \\ \text { T6_24_1 } & 0.016 & 0.899 & 0.000 \\ \text { T6_25_1 } & 0.012 & 0.912 & 0.001 \\ \text { T6_27_1 } & 0.065 & 0.798 & -0.044 \\ \text { T6_28_1 } & 0.034 & 0.853 & 0.025 \\ \text { T6_29_1 } & 0.460 & 0.498 & 0.087 \\ \text { T6_30_1 } & 0.180 & 0.672 & -0.070 \\ \text { T6_31_1 } & 0.208 & 0.648 & 0.055 \\ \text { T6_32_1 } & 0.021 & 0.884 & 0.016 \\ \text { T6_34_1 } & 0.065 & 0.799 & -0.043 \\ \text { T6_35_1 } & 0.000 & 0.999 & -0.005 \\ \text { T6_36_1 } & 0.143 & 0.705 & -0.061 \\ \text { T6_37_1 } & 0.074 & 0.786 & -0.046 \\ \text { T6_39_1 } & 0.020 & 0.886 & -0.029 \\ \text { T6_40_1 } & 0.161 & 0.689 & 0.051 \\ \text { T6_41_12.1 } & 0.007 & 0.931 & 0.011 \\ \text { T6_42_1 } & 0.386 & 0.534 & 0.081 \\ \text { T6_43_1 } & 0.096 & 0.757 & 0.037 \\ \text { T6_45_1 } & 0.018 & 0.894 & 0.017 \\ \text { T6_46_1 } & 0.283 & 0.595 & 0.067 \\ \text { T6_47_1 } & 0.062 & 0.803 & 0.028 \\ \text { T6_48_1 } & 0.013 & 0.909 & 0.009 \\ \text { T6_1_2 } & 0.089 & 0.766 & 0.001 \\ \text { T6_3_2 } & 0.235 & 0.628 & 0.030 \\ \text { T6_4_2 } & 0.002 & 0.969 & -0.057 \\ \text { T6_5_2 } & 0.197 & 0.657 & 0.021 \\ \text { T6_8_2 } & 0.161 & 0.688 & 0.014 \\ \text { T6_2 } & 0.048 & 0.826 & -0.013 \\ \text { T6 } & 0.028 & 0.868 & -0.020\end{array}$




$\begin{array}{lccc}\text { T6_12_2 } & 0.018 & 0.894 & -0.026 \\ \text { T6_14_2 } & 0.293 & 0.589 & 0.036 \\ \text { T6_15_2 } & 0.021 & 0.884 & -0.025 \\ \text { T6_16_2 } & 0.047 & 0.828 & -0.012 \\ \text { T6_18_2 } & 0.201 & 0.654 & 0.021 \\ \text { T6_19_2 } & 0.180 & 0.672 & 0.018 \\ \text { T6_22_2 } & 0.256 & 0.613 & 0.031 \\ \text { T6_23_2 } & 0.136 & 0.713 & 0.011 \\ \text { T6_26_2 } & 0.019 & 0.891 & 0.027 \\ \text { T6_28_2 } & 0.032 & 0.859 & -0.026 \\ \text { T6_29_2 } & 0.026 & 0.871 & -0.022 \\ \text { T6_31_2 } & 0.086 & 0.769 & 0.050 \\ \text { T6_32_2 } & 0.002 & 0.968 & -0.005 \\ \text { T6_35_2 } & 0.061 & 0.804 & -0.036 \\ \text { T6_38_2 } & 0.024 & 0.876 & 0.029 \\ \text { T6_39_2 } & 0.007 & 0.932 & 0.019 \\ \text { T6_40_2 } & 0.000 & 0.988 & 0.009 \\ \text { T6_41_2 } & 0.002 & 0.968 & -0.004 \\ \text { T6_43_2 } & 0.043 & 0.835 & -0.030 \\ \text { T6_44_2 } & 0.210 & 0.647 & -0.068 \\ \text { T6_45_2 } & 0.002 & 0.962 & 0.014 \\ \text { T6_46_2 } & 0.004 & 0.951 & -0.008 \\ \text { T6_47_2 } & 0.196 & 0.658 & 0.073 \\ \text { T6_49_2 } & 0.024 & 0.876 & 0.029 \\ \text { T6_2_3 } & 0.217 & 0.641 & -0.003 \\ \text { T6_3_3 } & 0.007 & 0.933 & 0.004 \\ \text { T6_4_3 } & 0.010 & 0.921 & 0.003 \\ \text { T6_8_3 } & 0.000 & 0.993 & 0.023 \\ \text { T6_9_3 } & 0.103 & 0.748 & 0.015 \\ \text { T6_11_3 } & 0.002 & 0.969 & 0.028 \\ \text { T6_12_3 } & 0.001 & 0.976 & 0.012 \\ \text { T6_26_3 } & 0.090 & 0.765 & -0.004 \\ \text { T6_27_3 } & 0.071 & 0.790 & 0.002\end{array}$




$\begin{array}{llll}\text { T6_28_3 } & 0.484 & 0.487 & -0.064 \\ \text { T6_29_3 } & 0.021 & 0.885 & 0.020 \\ \text { T6_30_3 } & 0.001 & 0.973 & 0.051 \\ \text { T6_32_3 } & 0.071 & 0.790 & 0.002 \\ \text { T6_33_3 } & 0.003 & 0.954 & 0.034 \\ \text { T6_34_3 } & 0.038 & 0.846 & 0.013 \\ \text { T6_36_3 } & 0.047 & 0.828 & 0.079 \\ \text { T6_37_3 } & 0.238 & 0.626 & -0.033 \\ \text { T6_38_3 } & 0.407 & 0.524 & -0.056 \\ \text { T6_39_3 } & 0.067 & 0.796 & 0.002 \\ \text { T6_44_3 } & 0.002 & 0.968 & 0.036 \\ \text { T6_45_3 } & 0.219 & 0.640 & -0.031 \\ \text { T6_47_3 } & 0.055 & 0.815 & 0.005 \\ \text { T6_2_4 } & 0.023 & 0.879 & -0.008 \\ \text { T6_3_4 } & 0.001 & 0.981 & -0.032 \\ \text { T6_5_4 } & 0.026 & 0.873 & 0.001 \\ \text { T6_10_4 } & 0.001 & 0.973 & -0.018 \\ \text { T6_11_4 } & 0.000 & 0.988 & -0.021 \\ \text { T6_12_4 } & 0.033 & 0.855 & 0.004 \\ \text { T6_16_4 } & 0.005 & 0.942 & 0.010 \\ \text { T6_17_4 } & 0.011 & 0.918 & -0.008 \\ \text { T6_18_4 } & 0.037 & 0.848 & -0.012 \\ \text { T6_19_4 } & 0.044 & 0.835 & 0.008 \\ \text { T6_20_4 } & 0.024 & 0.878 & 0.048 \\ \text { T6_21_4 } & 0.000 & 0.992 & -0.021 \\ \text { T6_23_4 } & 0.003 & 0.960 & -0.016 \\ \text { T6_24_4 } & 0.010 & 0.921 & -0.008 \\ \text { T6_25_4 } & 0.007 & 0.933 & -0.011 \\ \text { T6_26_4 } & 0.171 & 0.679 & -0.022 \\ \text { T6_29_4 } & 0.144 & 0.705 & -0.014 \\ \text { T6_44_4 } & 0.811 & 0.368 & -0.093 \\ \text { T6_45_4 } & 0.018 & 0.893 & 0.023\end{array}$




$\begin{array}{llll} & & & \\ \text { T6_46_4 } & 0.019 & 0.890 & 0.024 \\ \text { T6_48_4 } & 0.141 & 0.708 & -0.014 \\ \text { T6_49_4 } & 0.112 & 0.738 & -0.006 \\ \text { T6_3_5 } & 0.000 & 0.996 & -0.003 \\ \text { T6_7_5 } & 0.001 & 0.977 & -0.008 \\ \text { T6_8_5 } & 0.011 & 0.915 & 0.017 \\ \text { T6_9_5 } & 0.003 & 0.954 & -0.029 \\ \text { T6_10_5 } & 0.001 & 0.974 & 0.006 \\ \text { T6_11_5 } & 0.000 & 0.988 & 0.004 \\ \text { T6_12_5 } & 0.000 & 0.987 & -0.001 \\ \text { T6_13_5 } & 0.008 & 0.930 & 0.014 \\ \text { T6_15_5 } & 0.000 & 0.995 & -0.003 \\ \text { T6_16_5 } & 0.016 & 0.898 & -0.020 \\ \text { T6_17_5 } & 0.005 & 0.943 & 0.012 \\ \text { T6_19_5 } & 0.017 & 0.896 & 0.021 \\ \text { T6_20_5 } & 0.000 & 0.991 & -0.040 \\ \text { T6_22_5 } & 0.003 & 0.956 & -0.011 \\ \text { T6_24_5 } & 0.001 & 0.983 & 0.004 \\ \text { T6_26_5 } & 0.008 & 0.930 & -0.054 \\ \text { T6_27_5 } & 0.046 & 0.831 & -0.004 \\ \text { T6_29_5 } & 0.077 & 0.781 & 0.008 \\ \text { T6_30_5 } & 0.011 & 0.915 & -0.020 \\ \text { T6_31_5 } & 0.006 & 0.940 & -0.051 \\ \text { T6_33_5 } & 0.052 & 0.820 & -0.002 \\ \text { T6_34_5 } & 0.089 & 0.765 & 0.010 \\ \text { T6_35_5 } & 0.000 & 0.989 & -0.031 \\ \text { T6_36_5 } & 0.021 & 0.884 & -0.014 \\ \text { T6_37_5 } & 0.000 & 0.993 & -0.035 \\ \text { T6_38_5 } & 0.075 & 0.784 & 0.005 \\ \text { T6_43_5 } & 0.000 & 0.997 & -0.036 \\ \text { T6_46_5 } & 0.091 & 0.763 & 0.009 \\ \text { T6_47_5 } & 0.068 & 0.794 & 0.004 \\ \text { T6_49_5 } & 0.014 & 0.907 & -0.057 \\ \text { T6_1_6 } & 0.018 & 0.894 & -0.033 \\ \text { T6_5_6 } & 0.029 & 0.864 & 0.019 \\ \text { T6_6_6 } & 0.035 & 0.851 & 0.020 \\ \text { T6_7_6 } & 0.010 & 0.922 & 0.005 \\ \text { T6_8_6 } & 0.037 & 0.849 & -0.010 \\ \text { T6_11_6 } & 0.115 & 0.734 & 0.014 \\ \text { T6_6 } & 0.005 & 0.943 & -0.028 \\ \text { T6 } & 0.052 & 0.819 & -0.004\end{array}$




\begin{tabular}{lllc} 
T6_12_6 & 0.007 & 0.936 & -0.026 \\
T6_16_6 & 0.048 & 0.826 & -0.005 \\
T6_20_6 & 0.040 & 0.842 & -0.008 \\
T6_22_6 & 0.119 & 0.730 & 0.014 \\
T6_23_6 & 0.040 & 0.842 & -0.008 \\
T6_24_6 & 0.176 & 0.675 & 0.026 \\
T6_25_6 & 0.005 & 0.945 & -0.028 \\
T6_27_6 & 0.005 & 0.944 & 0.019 \\
T6_28_6 & 0.030 & 0.862 & 0.059 \\
T6_30_6 & 0.036 & 0.849 & 0.001 \\
T6_31_6 & 0.000 & 0.990 & 0.030 \\
T6_34_6 & 0.090 & 0.764 & 0.077 \\
T6_35_6 & 0.003 & 0.959 & 0.041 \\
T6_36_6 & 0.051 & 0.821 & -0.005 \\
T6_38_6 & 0.001 & 0.981 & 0.025 \\
T6_40_6 & 0.000 & 0.993 & 0.035 \\
T6_41_6 & 0.036 & 0.849 & -0.002 \\
T6_42_6 & 0.009 & 0.926 & 0.014 \\
T6_43_6 & 0.014 & 0.904 & 0.051 \\
T6_44_6 & 0.002 & 0.969 & 0.020 \\
T6_45_6 & 0.071 & 0.790 & -0.012 \\
T6_46_6 & 0.010 & 0.921 & 0.048 \\
T6_47_6 & 0.024 & 0.878 & 0.054 \\
T6_48_6 & 0.016 & 0.900 & 0.011 \\
\hline
\end{tabular}


Table 51

Task 6 DIF: Black vs. Sample

\begin{tabular}{lccc}
\hline ItemID & ChiSq & pvalue & EffectSize \\
\hline T6_16_12 & 0.110 & 0.740 & 0.063 \\
T6_19_12 & 0.023 & 0.881 & 0.037 \\
T6_21_12 & 0.000 & 0.997 & 0.012 \\
T6_26_12 & 0.112 & 0.738 & 0.058 \\
T6_41_12 & 0.088 & 0.767 & -0.044 \\
T6_17_23 & 0.008 & 0.928 & -0.007 \\
T6_20_23 & 0.024 & 0.878 & 0.034 \\
T6_36_23 & 0.286 & 0.593 & -0.103 \\
T6_42_23 & 0.115 & 0.735 & 0.036 \\
T6_1_34 & 0.038 & 0.845 & -0.058 \\
T6_5_34 & 0.100 & 0.752 & 0.034 \\
T6_10_34 & 0.000 & 0.991 & -0.018 \\
T6_21_34 & 0.011 & 0.915 & 0.003 \\
T6_24_34 & 0.004 & 0.951 & -0.029 \\
T6_12_3.1 & 0.007 & 0.934 & -0.022 \\
T6_40_34 & 0.138 & 0.710 & -0.114 \\
T6_43_34 & 0.065 & 0.798 & -0.090 \\
T6_27_45 & 0.010 & 0.921 & 0.051 \\
T6_28_45 & 0.001 & 0.979 & 0.022 \\
T6_1_56 & 0.008 & 0.927 & 0.041 \\
T6_2_56 & 0.051 & 0.821 & 0.060 \\
T6_6_56 & 0.003 & 0.960 & 0.013 \\
T6_18_56 & 0.040 & 0.842 & -0.011 \\
T6_28_56 & 0.017 & 0.896 & 0.047 \\
T6_32_56 & 0.120 & 0.729 & -0.030 \\
T6_45_56 & 0.037 & 0.847 & -0.008 \\
T6_1_456 & 0.007 & 0.935 & 0.010 \\
T6_4_456 & 0.041 & 0.839 & 0.057 \\
T6_8_456 & 0.010 & 0.922 & 0.010 \\
T6_14_456 & Inf & 0.000 & NaN \\
T6_22_456 & 0.007 & 0.933 & 0.010 \\
T6_1_1 & 0.056 & 0.813 & -0.038 \\
T6_2_1 & 0.039 & 0.843 & 0.028 \\
T6_3_1 & 0.134 & 0.715 & -0.057 \\
T6_4_1 & 0.024 & 0.878 & 0.008 \\
T6_5_1 & 0.000 & 0.987 & -0.017 \\
T6_6_1 & 0.023 & 0.879 & 0.009 \\
\end{tabular}




$\begin{array}{llll}\text { T6_7_1 } & 0.007 & 0.933 & 0.013 \\ \text { T6_8_1 } & 0.002 & 0.967 & -0.008 \\ \text { T6_9_1 } & 0.011 & 0.915 & 0.003 \\ \text { T6_11_1 } & 0.060 & 0.806 & 0.024 \\ \text { T6_12_1 } & 0.089 & 0.765 & 0.030 \\ \text { T6_13_1 } & 0.116 & 0.733 & 0.037 \\ \text { T6_14_1 } & 0.008 & 0.928 & 0.000 \\ \text { T6_17_1 } & 0.000 & 0.997 & -0.015 \\ \text { T6_18_1 } & 0.062 & 0.804 & 0.022 \\ \text { T6_19_12.1 } & 0.023 & 0.881 & 0.037 \\ \text { T6_20_1 } & 0.013 & 0.909 & 0.004 \\ \text { T6_22_1 } & 0.067 & 0.796 & 0.026 \\ \text { T6_23_1 } & 0.012 & 0.911 & 0.003 \\ \text { T6_24_1 } & 0.028 & 0.867 & 0.011 \\ \text { T6_25_1 } & 0.023 & 0.879 & 0.009 \\ \text { T6_27_1 } & 0.013 & 0.911 & 0.032 \\ \text { T6_28_1 } & 0.075 & 0.784 & -0.030 \\ \text { T6_29_1 } & 0.740 & 0.390 & -0.109 \\ \text { T6_30_1 } & 0.000 & 0.990 & 0.020 \\ \text { T6_31_1 } & 0.366 & 0.545 & -0.068 \\ \text { T6_32_1 } & 0.215 & 0.643 & -0.056 \\ \text { T6_34_1 } & 0.018 & 0.894 & -0.006 \\ \text { T6_35_1 } & 0.131 & 0.718 & -0.035 \\ \text { T6_36_1 } & 0.003 & 0.958 & 0.023 \\ \text { T6_37_1 } & 0.000 & 0.994 & 0.013 \\ \text { T6_39_1 } & 0.002 & 0.962 & 0.008 \\ \text { T6_40_1 } & 0.319 & 0.572 & -0.068 \\ \text { T6_41_12.1 } & 0.088 & 0.767 & -0.044 \\ \text { T6_42_1 } & 0.361 & 0.548 & -0.075 \\ \text { T6_43_1 } & 0.192 & 0.661 & -0.048 \\ \text { T6_45_1 } & 0.178 & 0.674 & -0.053 \\ \text { T6_46_1 } & 0.404 & 0.525 & -0.076 \\ \text { T6_47_1 } & 0.357 & 0.550 & -0.071 \\ \text { T6_48_1 } & 0.045 & 0.832 & -0.014 \\ \text { T6_1_2 } & 0.004 & 0.951 & 0.005 \\ \text { T6_3_2 } & 0.014 & 0.908 & -0.003 \\ \text { T6_4_2 } & 0.024 & 0.878 & 0.046 \\ \text { T6_5_2 } & 0.007 & 0.936 & 0.012 \\ \text { T6_7_2 } & 0.127 & 0.722 & -0.029 \\ \text { T6_10_2 } & 0.030 & 0.863 & -0.002\end{array}$




$\begin{array}{llll}\text { T6_12_2 } & 0.010 & 0.920 & 0.010 \\ \text { T6_14_2 } & 0.108 & 0.743 & -0.026 \\ \text { T6_15_2 } & 0.002 & 0.964 & 0.028 \\ \text { T6_16_2 } & 0.019 & 0.891 & 0.004 \\ \text { T6_18_2 } & 0.137 & 0.711 & -0.030 \\ \text { T6_19_2 } & 0.130 & 0.719 & -0.029 \\ \text { T6_22_2 } & 0.164 & 0.685 & -0.037 \\ \text { T6_23_2 } & 0.008 & 0.929 & 0.011 \\ \text { T6_26_2 } & 0.002 & 0.961 & -0.001 \\ \text { T6_28_2 } & 0.094 & 0.759 & 0.039 \\ \text { T6_29_2 } & 0.109 & 0.742 & 0.042 \\ \text { T6_31_2 } & 0.036 & 0.850 & -0.043 \\ \text { T6_32_2 } & 0.031 & 0.859 & 0.020 \\ \text { T6_35_2 } & 0.276 & 0.599 & 0.072 \\ \text { T6_38_2 } & 0.025 & 0.875 & -0.038 \\ \text { T6_39_2 } & 0.026 & 0.871 & -0.039 \\ \text { T6_40_2 } & 0.026 & 0.872 & 0.017 \\ \text { T6_41_2 } & 0.005 & 0.945 & 0.001 \\ \text { T6_43_2 } & 0.018 & 0.894 & 0.011 \\ \text { T6_44_2 } & 0.114 & 0.735 & 0.044 \\ \text { T6_45_2 } & 0.010 & 0.920 & -0.031 \\ \text { T6_46_2 } & 0.003 & 0.956 & 0.001 \\ \text { T6_47_2 } & 0.337 & 0.561 & -0.104 \\ \text { T6_49_2 } & 0.019 & 0.891 & 0.012 \\ \text { T6_2_3 } & 0.382 & 0.537 & 0.068 \\ \text { T6_3_3 } & 0.019 & 0.890 & 0.006 \\ \text { T6_4_3 } & 0.000 & 0.996 & -0.015 \\ \text { T6_8_3 } & 0.012 & 0.913 & -0.038 \\ \text { T6_9_3 } & 0.000 & 0.988 & -0.026 \\ \text { T6_11_3 } & 0.000 & 0.991 & -0.018 \\ \text { T6_12_3 } & 0.002 & 0.961 & -0.008 \\ \text { T6_13_3 } & 0.000 & 0.991 & -0.014 \\ \text { T6_15_3 } & 0.055 & 0.815 & 0.020 \\ \text { T6_16_3 } & 0.066 & 0.798 & 0.017 \\ \text { T6_17_3 } & 0.029 & 0.866 & 0.010 \\ \text { T6_18_3 } & 0.076 & 0.782 & 0.026 \\ \text { T6_19_3 } & 0.004 & 0.950 & -0.007 \\ \text { T623_3 } & 0.006 & 0.937 & -0.004 \\ \text { T6_3 } & 0.000 & 0.993 & -0.020 \\ \text { T6 } & 0.008 & 0.927 & -0.023 \\ \text { T6 } & 0.007 & 0.935 & 0.009\end{array}$




$\begin{array}{lllc}\text { T6_28_3 } & 0.063 & 0.802 & 0.036 \\ \text { T6_29_3 } & 0.026 & 0.873 & -0.035 \\ \text { T6_30_3 } & 0.126 & 0.723 & -0.063 \\ \text { T6_32_3 } & 0.040 & 0.842 & 0.027 \\ \text { T6_33_3 } & 0.020 & 0.889 & 0.018 \\ \text { T6_34_3 } & 0.000 & 0.999 & -0.003 \\ \text { T6_36_3 } & 0.064 & 0.801 & -0.047 \\ \text { T6_37_3 } & 0.003 & 0.955 & 0.006 \\ \text { T6_38_3 } & 0.073 & 0.788 & 0.039 \\ \text { T6_39_3 } & 0.000 & 0.989 & -0.011 \\ \text { T6_44_3 } & 0.021 & 0.885 & -0.031 \\ \text { T6_45_3 } & 0.000 & 0.988 & -0.006 \\ \text { T6_47_3 } & 0.010 & 0.920 & 0.013 \\ \text { T6_244 } & 0.141 & 0.708 & 0.022 \\ \text { T6_3_4 } & 0.016 & 0.899 & 0.024 \\ \text { T6_544 } & 0.001 & 0.978 & -0.005 \\ \text { T6_10_4 } & 0.000 & 0.996 & 0.000 \\ \text { T6_1144 } & 0.000 & 0.985 & -0.004 \\ \text { T6_12_4 } & 0.000 & 0.999 & -0.001 \\ \text { T6_16_4 } & 0.063 & 0.803 & -0.002 \\ \text { T6_17_4 } & 0.000 & 0.998 & 0.000 \\ \text { T6_18_4 } & 0.149 & 0.699 & 0.022 \\ \text { T6_19_4 } & 0.007 & 0.935 & 0.017 \\ \text { T6_20_4 } & 0.000 & 0.997 & -0.039 \\ \text { T6_21_4 } & 0.001 & 0.971 & 0.009 \\ \text { T6_23_4 } & 0.027 & 0.870 & -0.026 \\ \text { T6_24_4 } & 0.012 & 0.911 & -0.018 \\ \text { T6_25_4 } & 0.006 & 0.936 & -0.013 \\ \text { T6_26_4 } & 0.117 & 0.732 & 0.017 \\ \text { T6_29_4 } & 0.220 & 0.639 & 0.034 \\ \text { T6_31_4 } & 0.311 & 0.577 & 0.048 \\ \text { T6_33_4 } & 0.264 & 0.607 & 0.040 \\ \text { T6_34_4 } & 0.140 & 0.708 & 0.020 \\ \text { T6_35_4 } & 0.228 & 0.633 & 0.036 \\ \text { T6_36_4 } & 0.178 & 0.673 & 0.026 \\ \text { T6_38_4 } & 0.468 & 0.494 & 0.068 \\ \text { T6_40_4 } & 0.387 & 0.534 & 0.057 \\ \text { T6_42_4 } & 0.015 & 0.904 & -0.021 \\ \text { T6_43_4 } & 0.087 & 0.769 & 0.006 \\ \text { T6_44_4 } & 0.585 & 0.444 & 0.080 \\ \text { T6_45_4 } & 0.004 & 0.947 & -0.028\end{array}$




$\begin{array}{llll}\text { T6_46_4 } & 0.040 & 0.843 & -0.009 \\ \text { T6_48_4 } & 0.020 & 0.887 & -0.016 \\ \text { T6_49_4 } & 0.082 & 0.775 & 0.005 \\ \text { T6_3_5 } & 0.001 & 0.973 & -0.020 \\ \text { T6_7_5 } & 0.000 & 0.992 & -0.027 \\ \text { T6_8_5 } & 0.007 & 0.935 & -0.012 \\ \text { T6_9_5 } & 0.000 & 0.990 & 0.044 \\ \text { T6_10_5 } & 0.001 & 0.971 & -0.036 \\ \text { T6_11_5 } & 0.013 & 0.909 & -0.048 \\ \text { T6_12_5 } & 0.012 & 0.914 & -0.009 \\ \text { T6_13_5 } & 0.000 & 0.998 & -0.031 \\ \text { T6_15_5 } & 0.000 & 0.992 & -0.024 \\ \text { T6_16_5 } & 0.008 & 0.929 & 0.028 \\ \text { T6_17_5 } & 0.000 & 0.990 & -0.024 \\ \text { T6_19_5 } & 0.000 & 0.986 & -0.023 \\ \text { T6_20_5 } & 0.062 & 0.804 & 0.080 \\ \text { T6_22_5 } & 0.000 & 0.984 & -0.023 \\ \text { T6_24_5 } & 0.019 & 0.891 & -0.004 \\ \text { T6_26_5 } & 0.000 & 0.990 & 0.071 \\ \text { T6_27_5 } & 0.123 & 0.726 & 0.014 \\ \text { T6_29_5 } & 0.439 & 0.508 & -0.038 \\ \text { T6_30_5 } & 0.070 & 0.792 & 0.028 \\ \text { T6_31_5 } & 0.006 & 0.939 & 0.084 \\ \text { T6_33_5 } & 0.039 & 0.843 & 0.038 \\ \text { T6_34_5 } & 0.027 & 0.870 & 0.041 \\ \text { T6_35_5 } & 0.160 & 0.689 & 0.004 \\ \text { T6_36_5 } & 0.037 & 0.847 & 0.039 \\ \text { T6_37_5 } & 0.023 & 0.881 & 0.041 \\ \text { T6_38_5 } & 0.061 & 0.806 & 0.030 \\ \text { T6_43_5 } & 0.002 & 0.967 & 0.063 \\ \text { T6_46_5 } & 0.166 & 0.684 & 0.006 \\ \text { T6_47_5 } & 0.084 & 0.772 & 0.022 \\ \text { T6_49_5 } & 0.022 & 0.883 & 0.044 \\ \text { T6_1_6 } & 0.087 & 0.768 & 0.053 \\ \text { T6_5_6 } & 0.001 & 0.974 & -0.003 \\ \text { T6_6_6 } & 0.025 & 0.875 & -0.021 \\ \text { T6_7_6 } & 0.054 & 0.817 & -0.031 \\ \text { T6_11_6 } & 0.048 & 0.826 & 0.014 \\ & 0.066 & 0.797 & 0.008 \\ \text { T6_6 } & 0.000 & 0.995 & 0.055 \\ \text { T6 } & 0.821 & 0.014\end{array}$




\begin{tabular}{llll} 
T6_12_6 & 0.000 & 0.994 & 0.047 \\
T6_16_6 & 0.012 & 0.914 & 0.032 \\
T6_20_6 & 0.012 & 0.913 & 0.032 \\
T6_22_6 & 0.110 & 0.740 & -0.004 \\
T6_23_6 & 0.008 & 0.930 & 0.035 \\
T6_24_6 & 0.228 & 0.633 & -0.027 \\
T6_25_6 & 0.001 & 0.972 & 0.043 \\
T6_27_6 & 0.025 & 0.874 & -0.022 \\
T6_28_6 & 0.001 & 0.979 & -0.054 \\
T6_30_6 & 0.054 & 0.817 & -0.011 \\
T6_31_6 & 0.001 & 0.978 & -0.042 \\
T6_34_6 & 0.002 & 0.964 & -0.056 \\
T6_35_6 & 0.008 & 0.929 & -0.032 \\
T6_36_6 & 0.130 & 0.719 & 0.011 \\
T6_38_6 & 0.027 & 0.870 & -0.020 \\
T6_40_6 & 0.000 & 0.991 & -0.049 \\
T6_41_6 & 0.156 & 0.693 & 0.018 \\
T6_42_6 & 0.040 & 0.841 & -0.013 \\
T6_43_6 & 0.004 & 0.948 & -0.035 \\
T6_44_6 & 0.137 & 0.711 & 0.014 \\
T6_45_6 & 0.111 & 0.739 & 0.006 \\
T6_46_6 & 0.001 & 0.980 & -0.041 \\
T6_47_6 & 0.002 & 0.969 & -0.036 \\
T6_48_6 & 0.011 & 0.918 & -0.030 \\
\hline
\end{tabular}


Table 52

Task 6 DIF: Latinx vs. Sample

\begin{tabular}{|c|c|c|c|}
\hline ItemID & ChiSq & pvalue & EffectSize \\
\hline T6_16_12 & 0.037 & 0.849 & 0.023 \\
\hline T6_19_12 & 0.000 & 0.997 & -0.041 \\
\hline T6_21_12 & 0.045 & 0.832 & 0.033 \\
\hline T6_26_12 & 0.134 & 0.715 & 0.033 \\
\hline T6_41_12 & 0.433 & 0.510 & 0.129 \\
\hline T6_17_23 & 0.039 & 0.844 & 0.050 \\
\hline T6_20_23 & 0.060 & 0.807 & 0.064 \\
\hline T6_36_23 & 0.287 & 0.592 & 0.068 \\
\hline T6_42_23 & 0.323 & 0.570 & 0.077 \\
\hline T6_1_34 & 0.301 & 0.583 & -0.176 \\
\hline T6_5_34 & 0.445 & 0.505 & -0.148 \\
\hline T6_10_34 & 0.160 & 0.689 & -0.055 \\
\hline T6_21_34 & 0.204 & 0.652 & -0.077 \\
\hline T6_24_34 & 0.016 & 0.898 & 0.039 \\
\hline T6_12_3.1 & 0.230 & 0.632 & 0.102 \\
\hline T6_40_34 & 0.327 & 0.567 & -0.014 \\
\hline T6_43_34 & 0.243 & 0.622 & -0.037 \\
\hline T6_27_45 & 0.233 & 0.629 & 0.005 \\
\hline T6_28_45 & 0.146 & 0.703 & -0.023 \\
\hline T6_1_56 & 0.252 & 0.616 & -0.071 \\
\hline T6_2_56 & 0.000 & 0.987 & 0.085 \\
\hline T6_6_56 & 0.000 & 0.989 & 0.100 \\
\hline T6_18_56 & 0.579 & 0.447 & -0.160 \\
\hline T6_28_56 & 0.034 & 0.855 & 0.245 \\
\hline T6_32_56 & 0.065 & 0.799 & 0.053 \\
\hline T6_45_56 & 0.186 & 0.666 & -0.008 \\
\hline T6_1_456 & 0.444 & 0.505 & -0.015 \\
\hline T6_4_456 & 1.108 & 0.293 & -0.151 \\
\hline T6_8_456 & 0.106 & 0.745 & 0.112 \\
\hline T6_14_456 & Inf & 0.000 & $\mathrm{NaN}$ \\
\hline T6_22_456 & 0.029 & 0.866 & 0.165 \\
\hline T6_1_1 & 0.038 & 0.845 & -0.034 \\
\hline T6_2_1 & 0.067 & 0.796 & -0.014 \\
\hline T6_3_1 & 0.086 & 0.769 & 0.018 \\
\hline T6_4_1 & 0.027 & 0.871 & 0.027 \\
\hline T6_5_1 & 0.012 & 0.914 & 0.007 \\
\hline T6_6_1 & 0.004 & 0.953 & -0.008 \\
\hline
\end{tabular}




$\begin{array}{llll}\text { T6_7_1 } & 0.612 & 0.434 & 0.168 \\ \text { T6_8_1 } & 0.011 & 0.917 & 0.006 \\ \text { T6_9_1 } & 0.010 & 0.919 & 0.004 \\ \text { T6_11_1 } & 0.037 & 0.847 & 0.035 \\ \text { T6_12_1 } & 0.010 & 0.919 & -0.084 \\ \text { T6_13_1 } & 0.002 & 0.962 & -0.013 \\ \text { T6_14_1 } & 0.019 & 0.892 & 0.017 \\ \text { T6_17_1 } & 0.029 & 0.865 & 0.026 \\ \text { T6_18_1 } & 0.004 & 0.948 & -0.006 \\ \text { T6_19_12.1 } & 0.000 & 0.997 & -0.041 \\ \text { T6_20_1 } & 0.044 & 0.834 & 0.041 \\ \text { T6_22_1 } & 0.002 & 0.961 & -0.012 \\ \text { T6_23_1 } & 0.000 & 0.987 & -0.023 \\ \text { T6_24_1 } & 0.000 & 0.984 & -0.046 \\ \text { T6_25_1 } & 0.036 & 0.849 & 0.033 \\ \text { T6_27_1 } & 0.208 & 0.649 & 0.105 \\ \text { T6_28_1 } & 0.100 & 0.752 & 0.061 \\ \text { T6_29_1 } & 0.004 & 0.949 & -0.023 \\ \text { T6_30_1 } & 0.104 & 0.747 & 0.061 \\ \text { T6_31_1 } & 0.048 & 0.826 & 0.021 \\ \text { T6_32_1 } & 0.228 & 0.633 & 0.107 \\ \text { T6_34_1 } & 0.031 & 0.861 & 0.001 \\ \text { T6_35_1 } & 0.042 & 0.838 & 0.006 \\ \text { T6_36_1 } & 0.002 & 0.966 & -0.035 \\ \text { T6_37_1 } & 0.000 & 0.995 & -0.048 \\ \text { T6_39_1 } & 0.028 & 0.867 & 0.008 \\ \text { T6_40_1 } & 0.071 & 0.790 & 0.040 \\ \text { T6_41_12.1 } & 0.433 & 0.510 & 0.129 \\ \text { T6_42_1 } & 0.001 & 0.983 & -0.034 \\ \text { T6_43_1 } & 0.003 & 0.955 & -0.029 \\ \text { T6_45_1 } & 0.171 & 0.679 & 0.085 \\ \text { T6_46_1 } & 0.000 & 0.988 & -0.043 \\ \text { T6_47_1 } & 0.105 & 0.746 & 0.054 \\ \text { T6_48_1 } & 0.050 & 0.824 & 0.023 \\ \text { T6_1_2 } & 0.010 & 0.922 & -0.009 \\ \text { T6_3_2 } & 0.004 & 0.951 & -0.019 \\ \text { T6_4_2 } & 0.071 & 0.790 & 0.039 \\ \text { T6_5_2 } & 0.042 & 0.838 & -0.087 \\ \text { T6_7_2 } & 0.015 & 0.901 & 0.044 \\ \text { T6_8_2 } & 0.045 & 0.832 & -0.090 \\ \text { T6_10_2 } & 0.000 & 0.986 & -0.003\end{array}$




$\begin{array}{llll}\text { T6_12_2 } & 0.002 & 0.969 & 0.015 \\ \text { T6_14_2 } & 0.001 & 0.981 & -0.005 \\ \text { T6_15_2 } & 0.000 & 0.997 & -0.048 \\ \text { T6_16_2 } & 0.001 & 0.977 & -0.006 \\ \text { T6_18_2 } & 0.002 & 0.966 & 0.016 \\ \text { T6_19_2 } & 0.000 & 0.990 & -0.001 \\ \text { T6_22_2 } & 0.001 & 0.983 & 0.008 \\ \text { T6_23_2 } & 0.009 & 0.924 & -0.052 \\ \text { T6_26_2 } & 0.001 & 0.977 & -0.050 \\ \text { T6_28_2 } & 0.001 & 0.974 & -0.044 \\ \text { T6_29_2 } & 0.111 & 0.739 & 0.078 \\ \text { T6_31_2 } & 0.080 & 0.778 & 0.064 \\ \text { T6_32_2 } & 0.036 & 0.850 & 0.031 \\ \text { T6_35_2 } & 0.171 & 0.680 & 0.105 \\ \text { T6_38_2 } & 0.057 & 0.811 & 0.050 \\ \text { T6_39_2 } & 0.007 & 0.933 & -0.005 \\ \text { T6_40_2 } & 0.051 & 0.822 & 0.041 \\ \text { T6_41_2 } & 0.027 & 0.870 & 0.022 \\ \text { T6_43_2 } & 0.003 & 0.961 & -0.015 \\ \text { T6_44_2 } & 0.134 & 0.715 & 0.090 \\ \text { T6_45_2 } & 0.000 & 0.996 & -0.055 \\ \text { T6_46_2 } & 0.032 & 0.859 & 0.026 \\ \text { T6_47_2 } & 0.112 & 0.738 & 0.080 \\ \text { T6_49_2 } & 0.043 & 0.837 & -0.122 \\ \text { T6_2_3 } & 0.001 & 0.971 & -0.118 \\ \text { T6_3_3 } & 0.000 & 0.993 & 0.000 \\ \text { T6_4_3 } & 0.000 & 0.993 & -0.007 \\ \text { T6_8_3 } & 0.001 & 0.980 & 0.005 \\ \text { T6_9_3 } & 0.001 & 0.977 & -0.073 \\ \text { T6_11_3 } & 0.000 & 0.993 & -0.030 \\ \text { T6_12_3 } & 0.001 & 0.978 & 0.006 \\ \text { T6_13_3 } & 0.001 & 0.980 & -0.012 \\ \text { T6_15_3 } & 0.000 & 0.998 & -0.026 \\ \text { T6_16_3 } & 0.123 & 0.726 & 0.035 \\ \text { T6_17_3 } & 0.000 & 0.984 & -0.021 \\ \text { T6_18_3 } & 0.000 & 0.997 & -0.026 \\ \text { T6_19_3 } & 0.004 & 0.947 & -0.049 \\ \text { T6_22_3 } & 0.000 & 0.984 & 0.004 \\ \text { T6_23_3 } & 0.000 & 0.983 & 0.003 \\ \text { T6_26_3 } & 0.057 & 0.812 & 0.017 \\ \text { T6_27_3 } & 0.001 & 0.973 & -0.049\end{array}$




$\begin{array}{llll}\text { T6_28_3 } & 0.010 & 0.920 & -0.028 \\ \text { T6_29_3 } & 0.053 & 0.817 & 0.018 \\ \text { T6_30_3 } & 0.001 & 0.979 & -0.051 \\ \text { T6_32_3 } & 0.001 & 0.974 & -0.099 \\ \text { T6_33_3 } & 0.006 & 0.939 & -0.112 \\ \text { T6_34_3 } & 0.026 & 0.873 & -0.013 \\ \text { T6_36_3 } & 0.012 & 0.914 & -0.124 \\ \text { T6_37_3 } & 0.001 & 0.974 & -0.096 \\ \text { T6_38_3 } & 0.064 & 0.800 & 0.026 \\ \text { T6_39_3 } & 0.008 & 0.928 & -0.029 \\ \text { T6_44_3 } & 0.000 & 0.999 & -0.064 \\ \text { T6_45_3 } & 0.078 & 0.780 & 0.034 \\ \text { T6_47_3 } & 0.316 & 0.574 & 0.123 \\ \text { T6_244 } & 0.048 & 0.828 & -0.098 \\ \text { T6_3_4 } & 0.181 & 0.670 & -0.050 \\ \text { T6_544 } & 0.059 & 0.808 & 0.014 \\ \text { T6_10_4 } & 0.000 & 0.985 & 0.091 \\ \text { T6_1144 } & 0.016 & 0.900 & 0.053 \\ \text { T6_12_4 } & 0.086 & 0.769 & -0.004 \\ \text { T6_16_4 } & 0.176 & 0.675 & -0.171 \\ \text { T6_17_4 } & 0.108 & 0.742 & -0.017 \\ \text { T6_18_4 } & 0.125 & 0.724 & -0.147 \\ \text { T6_19_4 } & 0.390 & 0.532 & -0.118 \\ \text { T6_20_4 } & 0.060 & 0.807 & -0.106 \\ \text { T6_21_4 } & 0.001 & 0.975 & 0.083 \\ \text { T6_23_4 } & 0.007 & 0.931 & 0.065 \\ \text { T6_24_4 } & 0.084 & 0.773 & -0.005 \\ \text { T6_25_4 } & 0.007 & 0.934 & 0.068 \\ \text { T6_26_4 } & 0.161 & 0.689 & 0.031 \\ \text { T6_29_4 } & 0.016 & 0.899 & -0.058 \\ \text { T6_31_4 } & 0.021 & 0.885 & -0.055 \\ \text { T6_33_4 } & 0.011 & 0.918 & -0.066 \\ \text { T6_34_4 } & 0.025 & 0.875 & -0.052 \\ \text { T6_35_4 } & 0.057 & 0.812 & -0.024 \\ \text { T6_36_4 } & 0.012 & 0.913 & -0.066 \\ \text { T6_38_4 } & 0.035 & 0.852 & -0.038 \\ \text { T6_40_4 } & 0.001 & 0.977 & -0.116 \\ \text { T6_42_4 } & 0.000 & 0.994 & -0.130 \\ \text { T6_43_4 } & 0.028 & 0.868 & -0.052 \\ \text { T6_44_4 } & 0.265 & 0.607 & 0.071 \\ \text { T6_45_4 } & 0.013 & 0.908 & -0.067\end{array}$




$\begin{array}{llll}\text { T6_46_4 } & 0.001 & 0.972 & -0.141 \\ \text { T6_48_4 } & 0.068 & 0.794 & -0.018 \\ \text { T6_49_4 } & 0.030 & 0.862 & -0.048 \\ \text { T6_3_5 } & 0.001 & 0.980 & 0.086 \\ \text { T6_7_5 } & 0.000 & 0.990 & 0.082 \\ \text { T6_8_5 } & 0.026 & 0.873 & -0.001 \\ \text { T6_9_5 } & 0.000 & 0.997 & 0.014 \\ \text { T6_10_5 } & 0.000 & 0.994 & 0.074 \\ \text { T6_11_5 } & 0.009 & 0.926 & 0.108 \\ \text { T6_12_5 } & 0.016 & 0.901 & 0.012 \\ \text { T6_13_5 } & 0.001 & 0.976 & 0.064 \\ \text { T6_15_5 } & 0.001 & 0.971 & 0.042 \\ \text { T6_16_5 } & 0.064 & 0.801 & -0.064 \\ \text { T6_17_5 } & 0.014 & 0.907 & 0.016 \\ \text { T6_19_5 } & 0.004 & 0.947 & 0.030 \\ \text { T6_20_5 } & 0.156 & 0.693 & -0.112 \\ \text { T6_22_5 } & 0.018 & 0.895 & 0.122 \\ \text { T6_24_5 } & 0.047 & 0.829 & -0.019 \\ \text { T6_26_5 } & 0.006 & 0.941 & -0.072 \\ \text { T6_27_5 } & 0.001 & 0.980 & -0.040 \\ \text { T6_29_5 } & 0.001 & 0.970 & -0.033 \\ \text { T6_30_5 } & 0.007 & 0.932 & 0.004 \\ \text { T6_31_5 } & 0.001 & 0.971 & -0.060 \\ \text { T6_33_5 } & 0.002 & 0.965 & -0.064 \\ \text { T6_34_5 } & 0.001 & 0.982 & -0.030 \\ \text { T6_35_5 } & 0.130 & 0.718 & 0.095 \\ \text { T6_36_5 } & 0.001 & 0.980 & -0.033 \\ \text { T6_37_5 } & 0.002 & 0.962 & -0.062 \\ \text { T6_38_5 } & 0.010 & 0.921 & -0.080 \\ \text { T6_43_5 } & 0.000 & 0.990 & -0.021 \\ \text { T6_46_5 } & 0.020 & 0.888 & -0.096 \\ \text { T6_47_5 } & 0.000 & 0.990 & -0.044 \\ \text { T6_49_5 } & 0.004 & 0.951 & -0.069 \\ \text { T6_1_6 } & 0.243 & 0.622 & 0.058 \\ \text { T6_5_6 } & 0.161 & 0.689 & 0.082 \\ \text { T6_6_6 } & 0.079 & 0.779 & 0.125 \\ \text { T6_7_6 } & 0.139 & 0.710 & 0.082 \\ \text { T6_1196 } & 0.001 & 0.983 & 0.037 \\ \text { T6_6 } & 0.066 & 0.798 & -0.058 \\ \text { T6_6 } & 0.002 & 0.966 & 0.016 \\ \text { T6 } & 0.003 & 0.960 & 0.013\end{array}$




\begin{tabular}{llll} 
T6_12_6 & 0.013 & 0.911 & -0.008 \\
T6_16_6 & 0.040 & 0.841 & -0.038 \\
T6_20_6 & 0.042 & 0.838 & -0.039 \\
T6_22_6 & 0.063 & 0.801 & -0.055 \\
T6_23_6 & 0.072 & 0.789 & -0.060 \\
T6_24_6 & 0.057 & 0.812 & -0.051 \\
T6_25_6 & 0.008 & 0.927 & -0.001 \\
T6_27_6 & 0.083 & 0.774 & 0.076 \\
T6_28_6 & 0.012 & 0.914 & 0.132 \\
T6_30_6 & 0.009 & 0.923 & 0.138 \\
T6_31_6 & 0.001 & 0.982 & 0.170 \\
T6_34_6 & 0.325 & 0.569 & -0.028 \\
T6_35_6 & 0.016 & 0.900 & 0.128 \\
T6_36_6 & 0.103 & 0.748 & 0.055 \\
T6_38_6 & 0.039 & 0.844 & 0.104 \\
T6_40_6 & 0.000 & 0.992 & 0.202 \\
T6_41_6 & 0.512 & 0.474 & -0.080 \\
T6_42_6 & 0.107 & 0.744 & 0.058 \\
T6_43_6 & 0.084 & 0.771 & 0.069 \\
T6_44_6 & 1.229 & 0.268 & -0.215 \\
T6_45_6 & 0.078 & 0.780 & 0.078 \\
T6_46_6 & 0.061 & 0.804 & 0.088 \\
T6_47_6 & 0.414 & 0.520 & -0.052 \\
T6_48_6 & 0.000 & 0.995 & 0.202 \\
\hline
\end{tabular}


Table 53

Task 7 DIF: Male vs. female

\begin{tabular}{|c|c|c|c|}
\hline ItemID & ChiSq & pvalue & EffectSize \\
\hline T7_6_12 & 0.000 & 0.987 & -0.011 \\
\hline T7_8_12 & 0.011 & 0.917 & 0.006 \\
\hline T7_22_12 & 0.114 & 0.736 & -0.031 \\
\hline T7_29_12 & 0.039 & 0.843 & -0.011 \\
\hline T7_29_12.1 & 0.021 & 0.886 & 0.020 \\
\hline T7_4_23 & 0.057 & 0.811 & 0.011 \\
\hline T7_12_23 & 0.032 & 0.859 & 0.005 \\
\hline T7_15_23 & 0.062 & 0.803 & 0.016 \\
\hline T7_17_23 & 0.009 & 0.925 & 0.059 \\
\hline T7_26_23 & 0.039 & 0.844 & 0.010 \\
\hline T7_2_34 & 0.001 & 0.981 & 0.003 \\
\hline T7_9_34 & 0.037 & 0.847 & 0.045 \\
\hline T7_15_34 & 0.000 & 0.988 & 0.003 \\
\hline T7_29_34 & 0.000 & 0.989 & 0.072 \\
\hline T7_31_34 & 0.304 & 0.582 & -0.024 \\
\hline T7_16_45 & 0.005 & 0.942 & 0.012 \\
\hline T7_1_56 & 0.377 & 0.539 & 0.084 \\
\hline T7_15_56 & 0.001 & 0.979 & -0.002 \\
\hline T7_17_56 & 0.444 & 0.505 & 0.051 \\
\hline T7_9_456 & 0.056 & 0.813 & 0.047 \\
\hline T7_15_456 & 0.030 & 0.862 & -0.019 \\
\hline T7_1_1 & 0.007 & 0.933 & -0.020 \\
\hline T7_2_1 & 0.000 & 0.995 & -0.003 \\
\hline T7_3_1 & 0.127 & 0.721 & -0.037 \\
\hline T7_4_1 & 0.003 & 0.954 & 0.006 \\
\hline T7_5_1 & 0.019 & 0.890 & -0.028 \\
\hline T7_7_1 & 0.000 & 0.998 & -0.008 \\
\hline T7_8_12.1 & 0.011 & 0.917 & 0.006 \\
\hline T7_9_1 & 0.000 & 0.996 & -0.009 \\
\hline T7_10_1 & 0.012 & 0.913 & 0.013 \\
\hline T7_12_1 & 0.017 & 0.898 & -0.027 \\
\hline T7_13_1 & 0.058 & 0.809 & -0.018 \\
\hline T7_14_1 & 0.345 & 0.557 & -0.072 \\
\hline T7_15_1 & 0.001 & 0.980 & -0.012 \\
\hline T7_16_1 & 0.114 & 0.736 & -0.029 \\
\hline T7_17_1 & 0.165 & 0.685 & -0.039 \\
\hline T7_18_1 & 0.052 & 0.820 & -0.012 \\
\hline
\end{tabular}




$\begin{array}{lllc}\text { T7_19_1 } & 0.212 & 0.645 & -0.047 \\ \text { T7_20_1 } & 0.292 & 0.589 & -0.058 \\ \text { T7_21_1 } & 0.369 & 0.543 & -0.069 \\ \text { T7_22_12.1 } & 0.114 & 0.736 & -0.031 \\ \text { T7_25_1 } & 0.068 & 0.794 & -0.015 \\ \text { T7_26_1 } & 0.196 & 0.658 & -0.044 \\ \text { T7_27_1 } & 0.193 & 0.661 & -0.042 \\ \text { T7_28_1 } & 0.023 & 0.879 & 0.002 \\ \text { T7_29_12.2 } & 0.039 & 0.843 & -0.011 \\ \text { T7_30_1 } & 0.161 & 0.688 & -0.038 \\ \text { T7_31_1 } & 0.035 & 0.852 & -0.003 \\ \text { T7_33_1 } & 0.033 & 0.856 & -0.003 \\ \text { T7_1_2 } & 0.004 & 0.950 & 0.006 \\ \text { T7_2_2 } & 0.096 & 0.756 & -0.058 \\ \text { T7_5_2 } & 0.152 & 0.697 & 0.050 \\ \text { T7_6_2 } & 0.000 & 0.992 & -0.007 \\ \text { T7_7_2 } & 0.012 & 0.912 & 0.014 \\ \text { T7_8_2 } & 0.001 & 0.980 & 0.000 \\ \text { T7_9_2 } & 0.001 & 0.976 & 0.001 \\ \text { T7_11_2 } & 0.000 & 0.996 & -0.002 \\ \text { T7_13_2 } & 0.024 & 0.878 & 0.019 \\ \text { T7_16_2 } & 0.000 & 0.991 & 0.001 \\ \text { T7_19_2 } & 0.001 & 0.980 & -0.011 \\ \text { T7_20_2 } & 0.033 & 0.857 & -0.034 \\ \text { T7_21_2 } & 0.012 & 0.914 & -0.020 \\ \text { T7_22_2 } & 0.007 & 0.936 & -0.018 \\ \text { T7_24_2 } & 0.015 & 0.904 & -0.023 \\ \text { T7_27_2 } & 0.031 & 0.860 & -0.032 \\ \text { T7_28_2 } & 0.012 & 0.912 & 0.018 \\ \text { T7_32_2 } & 0.064 & 0.801 & -0.045 \\ \text { T7_33_2 } & 0.003 & 0.956 & -0.015 \\ \text { T7_1_3 } & 0.075 & 0.784 & 0.025 \\ \text { T7_3_3 } & 0.068 & 0.795 & 0.023 \\ \text { T7_4_3 } & 0.003 & 0.959 & -0.030 \\ \text { T7_5_3 } & 0.097 & 0.756 & 0.029 \\ \text { T7_6_3 } & 0.060 & 0.807 & -0.018 \\ \text { T7_8_3 } & 0.056 & 0.814 & 0.018 \\ \text { T7_10_3 } & 0.002 & 0.970 & -0.027 \\ \text { T7_11_3 } & 0.000 & 0.999 & 0.019 \\ \text { T7_14_3 } & 0.230 & 0.631 & -0.053 \\ \text { T7_16_3 } & 0.000 & 0.984 & 0.033\end{array}$




$\begin{array}{llll}\text { T7_17_3 } & 0.008 & 0.930 & 0.056 \\ \text { T7_19_3 } & 0.228 & 0.633 & -0.039 \\ \text { T7_20_3 } & 0.002 & 0.962 & 0.051 \\ \text { T7_22_3 } & 0.040 & 0.841 & 0.006 \\ \text { T7_24_3 } & 0.024 & 0.877 & 0.010 \\ \text { T7_27_3 } & 0.032 & 0.858 & 0.012 \\ \text { T7_28_3 } & 0.009 & 0.924 & 0.021 \\ \text { T7_30_3 } & 0.000 & 0.986 & 0.027 \\ \text { T7_31_34.1 } & 0.304 & 0.582 & -0.024 \\ \text { T7_32_3 } & 0.248 & 0.618 & -0.041 \\ \text { T7_33_3 } & 0.010 & 0.923 & 0.023 \\ \text { T7_2_4 } & 0.074 & 0.785 & -0.027 \\ \text { T7_4_4 } & 0.030 & 0.862 & -0.012 \\ \text { T7_5_4 } & 0.000 & 0.987 & 0.021 \\ \text { T7_6_4 } & 0.034 & 0.854 & -0.014 \\ \text { T7_7_4 } & 0.007 & 0.933 & 0.021 \\ \text { T7_10_4 } & 0.024 & 0.878 & -0.009 \\ \text { T7_11_4 } & 0.100 & 0.752 & -0.016 \\ \text { T7_13_4 } & 0.001 & 0.973 & 0.009 \\ \text { T7_14_4 } & 0.001 & 0.974 & 0.009 \\ \text { T7_17_4 } & 0.000 & 0.997 & 0.021 \\ \text { T7_18_4 } & 0.004 & 0.951 & 0.028 \\ \text { T7_19_4 } & 0.059 & 0.808 & -0.021 \\ \text { T7_20_4 } & 0.000 & 0.992 & 0.015 \\ \text { T7_21_4 } & 0.003 & 0.959 & 0.008 \\ \text { T7_24_4 } & 0.000 & 0.997 & 0.018 \\ \text { T7_26_4 } & 0.066 & 0.798 & -0.022 \\ \text { T7_27_4 } & 0.001 & 0.980 & 0.025 \\ \text { T7_29_4 } & 0.045 & 0.832 & -0.018 \\ \text { T7_32_4 } & 0.084 & 0.772 & -0.030 \\ \text { T7_33_4 } & 0.096 & 0.757 & -0.032 \\ \text { T7_2_5 } & 0.009 & 0.926 & 0.008 \\ \text { T7_3_5 } & 0.008 & 0.929 & 0.008 \\ \text { T7_4_5 } & 0.757 & 0.384 & 0.104 \\ \text { T7_7_5 } & 0.015 & 0.904 & 0.013 \\ \text { T7_9_5 } & 0.033 & 0.856 & -0.036 \\ \text { T7_10_5 } & 0.004 & 0.951 & 0.004 \\ \text { T7_11_5 } & 0.000 & 0.992 & -0.023 \\ \text { T713_5 } & 0.328 & 0.567 & 0.063 \\ \text { T3_5 } & 0.010 & 0.921 & 0.009 \\ \text { T3 } & & 0.799 & 0.023\end{array}$




\begin{tabular}{llll} 
T7_19_5 & 0.003 & 0.958 & -0.008 \\
T7_20_5 & 0.015 & 0.904 & -0.040 \\
T7_23_5 & 0.051 & 0.821 & 0.017 \\
T7_24_5 & 0.092 & 0.762 & 0.030 \\
T7_25_5 & 0.030 & 0.862 & -0.048 \\
T7_26_5 & 0.148 & 0.700 & 0.042 \\
T7_30_5 & 0.030 & 0.864 & 0.010 \\
T7_31_5 & 0.057 & 0.811 & 0.020 \\
T7_32_5 & 0.013 & 0.909 & 0.002 \\
T7_2_6 & 0.001 & 0.970 & 0.007 \\
T7_3_6 & 0.002 & 0.963 & -0.011 \\
T7_4_6 & 0.065 & 0.799 & 0.039 \\
T7_7_6 & 0.000 & 0.992 & -0.003 \\
T7_8_6 & 0.024 & 0.878 & 0.023 \\
T7_9_6 & 0.007 & 0.932 & 0.014 \\
T7_10_6 & 0.426 & 0.514 & 0.062 \\
T7_14_6 & 0.004 & 0.948 & -0.025 \\
T7_15_6 & 0.298 & 0.585 & 0.047 \\
T7_16_6 & 0.001 & 0.974 & -0.028 \\
T7_17_6 & 0.012 & 0.914 & -0.017 \\
T7_21_6 & 0.000 & 0.988 & -0.036 \\
T7_22_6 & 0.003 & 0.956 & -0.024 \\
T7_24_6 & 0.007 & 0.934 & -0.049 \\
T7_25_6 & 0.020 & 0.887 & -0.011 \\
T7_27_6 & 0.053 & 0.818 & 0.001 \\
T7_28_6 & 0.058 & 0.810 & 0.004 \\
T7_29_6 & 0.017 & 0.898 & -0.057 \\
T7_30_6 & 0.008 & 0.927 & -0.019 \\
T7_31_6 & 0.010 & 0.921 & -0.018 \\
T7_32_6 & 0.001 & 0.976 & -0.043 \\
\hline & & & \\
& &
\end{tabular}


Table 54

Task 7 DIF: White vs. Sample

\begin{tabular}{|c|c|c|c|}
\hline ItemID & ChiSq & pvalue & EffectSize \\
\hline T7_6_12 & 0.075 & 0.784 & -0.020 \\
\hline T7_8_12 & 0.066 & 0.797 & -0.018 \\
\hline T7_22_12 & 0.421 & 0.516 & -0.136 \\
\hline T7_29_12 & 5.959 & 0.015 & 0.185 \\
\hline T7_29_12.1 & 0.842 & 0.359 & 0.054 \\
\hline T7_4_23 & 0.829 & 0.363 & 0.095 \\
\hline T7_12_23 & 0.067 & 0.795 & -0.003 \\
\hline T7_15_23 & 0.001 & 0.979 & 0.046 \\
\hline T7_17_23 & 0.003 & 0.953 & -0.217 \\
\hline T7_26_23 & 0.101 & 0.751 & -0.168 \\
\hline T7_2_34 & 1.247 & 0.264 & -0.085 \\
\hline T7_9_34 & 1.397 & 0.237 & -0.043 \\
\hline T7_15_34 & 1.176 & 0.278 & -0.079 \\
\hline T7_29_34 & 1.061 & 0.303 & 0.114 \\
\hline T7_31_34 & 0.302 & 0.583 & 0.048 \\
\hline T7_16_45 & 0.000 & 0.995 & 0.096 \\
\hline T7_1_56 & 0.070 & 0.792 & 0.017 \\
\hline T7_15_56 & 0.000 & 0.988 & 0.064 \\
\hline T7_17_56 & 0.525 & 0.469 & -0.056 \\
\hline T7_9_456 & 0.002 & 0.963 & 0.086 \\
\hline T7_15_456 & 0.707 & 0.400 & -0.043 \\
\hline T7_1_1 & 0.205 & 0.651 & -0.022 \\
\hline T7_2_1 & 0.012 & 0.915 & 0.031 \\
\hline T7_3_1 & 0.819 & 0.365 & 0.084 \\
\hline T7_4_1 & 0.446 & 0.504 & -0.056 \\
\hline T7_5_1 & 0.004 & 0.949 & 0.063 \\
\hline T7_7_1 & 0.139 & 0.709 & -0.010 \\
\hline T7_8_12.1 & 0.066 & 0.797 & -0.018 \\
\hline T7_9_1 & 0.484 & 0.487 & -0.060 \\
\hline T7_10_1 & 0.295 & 0.587 & -0.037 \\
\hline T7_12_1 & 0.022 & 0.883 & 0.026 \\
\hline T7_13_1 & 0.056 & 0.814 & -0.032 \\
\hline T7_14_1 & 0.024 & 0.878 & -0.043 \\
\hline T7_15_1 & 0.120 & 0.729 & -0.003 \\
\hline T7_16_1 & 0.710 & 0.400 & 0.022 \\
\hline T7_17_1 & 2.219 & 0.136 & 0.124 \\
\hline T7_18_1 & 1.427 & 0.232 & 0.080 \\
\hline
\end{tabular}




$\begin{array}{llll}\text { T7_19_1 } & 0.151 & 0.698 & -0.052 \\ \text { T7_20_1 } & 2.385 & 0.123 & 0.124 \\ \text { T7_21_1 } & 0.551 & 0.458 & 0.004 \\ \text { T7_22_12.1 } & 0.421 & 0.516 & -0.136 \\ \text { T7_25_1 } & 1.272 & 0.259 & 0.059 \\ \text { T7_26_1 } & 1.172 & 0.279 & 0.056 \\ \text { T7_27_1 } & 0.271 & 0.603 & -0.038 \\ \text { T7_28_1 } & 0.042 & 0.837 & -0.087 \\ \text { T7_29_12.2 } & 5.959 & 0.015 & 0.185 \\ \text { T7_30_1 } & 0.067 & 0.796 & -0.072 \\ \text { T7_31_1 } & 0.001 & 0.983 & -0.117 \\ \text { T7_33_1 } & 0.025 & 0.875 & -0.095 \\ \text { T7_1_2 } & 0.101 & 0.750 & 0.026 \\ \text { T7_2_2 } & 1.417 & 0.234 & 0.070 \\ \text { T7_5_2 } & 0.577 & 0.448 & 0.002 \\ \text { T7_6_2 } & 0.003 & 0.953 & -0.015 \\ \text { T7_7_2 } & 0.080 & 0.778 & 0.021 \\ \text { T7_8_2 } & 0.016 & 0.900 & -0.005 \\ \text { T7_9_2 } & 0.006 & 0.938 & -0.012 \\ \text { T7_11_2 } & 0.005 & 0.946 & -0.039 \\ \text { T7_13_2 } & 0.000 & 0.991 & -0.021 \\ \text { T7_16_2 } & 1.368 & 0.242 & 0.097 \\ \text { T7_19_2 } & 0.220 & 0.639 & -0.021 \\ \text { T7_20_2 } & 0.029 & 0.864 & -0.068 \\ \text { T7_21_2 } & 0.701 & 0.402 & 0.043 \\ \text { T7_22_2 } & 0.005 & 0.946 & -0.111 \\ \text { T7_24_2 } & 1.299 & 0.254 & 0.092 \\ \text { T7_27_2 } & 0.203 & 0.652 & -0.021 \\ \text { T7_28_2 } & 0.221 & 0.638 & -0.011 \\ \text { T7_32_2 } & 0.080 & 0.778 & -0.049 \\ \text { T7_33_2 } & 0.199 & 0.656 & -0.023 \\ \text { T7_1_3 } & 0.633 & 0.426 & -0.061 \\ \text { T7_3_3 } & 0.602 & 0.438 & -0.058 \\ \text { T7_4_3 } & 0.042 & 0.837 & 0.032 \\ \text { T7_5_3 } & 0.364 & 0.547 & -0.030 \\ \text { T7_6_3 } & 0.384 & 0.535 & 0.078 \\ \text { T7_8_3 } & 0.048 & 0.827 & 0.029 \\ \text { T7_10_3 } & 0.001 & 0.978 & 0.073 \\ \text { T7_11_3 } & 0.014 & 0.905 & -0.043 \\ \text { T7_14_3 } & 0.060 & 0.807 & -0.064 \\ \text { T7_16_3 } & 0.019 & 0.891 & -0.073 \\ & & & \end{array}$




\begin{tabular}{|c|c|c|c|}
\hline T7_17_3 & 0.013 & 0.909 & -0.074 \\
\hline T7_19_3 & 1.455 & 0.228 & 0.105 \\
\hline T7_20_3 & 0.149 & 0.700 & -0.033 \\
\hline T7_22_3 & 0.005 & 0.943 & -0.071 \\
\hline T7_24_3 & 1.708 & 0.191 & 0.117 \\
\hline T7_27_3 & 0.336 & 0.562 & 0.001 \\
\hline T7_28_3 & 0.360 & 0.549 & 0.001 \\
\hline T7_30_3 & 1.314 & 0.252 & 0.093 \\
\hline T7_31_34.1 & 0.302 & 0.583 & 0.048 \\
\hline T7_32_3 & 1.229 & 0.268 & 0.091 \\
\hline T7_33_3 & 0.001 & 0.975 & -0.085 \\
\hline T7_2_4 & 0.000 & 0.990 & 0.021 \\
\hline T7_4_4 & 0.097 & 0.756 & -0.025 \\
\hline T7_5_4 & 0.002 & 0.968 & 0.035 \\
\hline T7_6_4 & 0.010 & 0.921 & 0.008 \\
\hline T7_7_4 & 0.107 & 0.744 & 0.133 \\
\hline T7_10_4 & 0.017 & 0.896 & 0.003 \\
\hline T7_11_4 & 0.807 & 0.369 & -0.069 \\
\hline T7_13_4 & 0.006 & 0.940 & 0.040 \\
\hline T7_14_4 & 0.000 & 0.988 & 0.031 \\
\hline T7_17_4 & 0.000 & 0.990 & 0.050 \\
\hline T7_18_4 & 0.000 & 0.987 & 0.047 \\
\hline T7_19_4 & 0.024 & 0.876 & 0.022 \\
\hline T7_20_4 & 0.188 & 0.665 & -0.025 \\
\hline T7_21_4 & 0.002 & 0.965 & 0.060 \\
\hline T7_24_4 & 0.139 & 0.709 & -0.014 \\
\hline T7_26_4 & 0.001 & 0.976 & 0.058 \\
\hline T7_27_4 & 0.002 & 0.961 & 0.040 \\
\hline T7_29_4 & 0.358 & 0.550 & -0.050 \\
\hline T7_32_4 & 0.205 & 0.651 & -0.028 \\
\hline T7_33_4 & 0.413 & 0.521 & -0.056 \\
\hline T7_2_5 & 0.350 & 0.554 & -0.037 \\
\hline T7_3_5 & 0.456 & 0.499 & -0.051 \\
\hline T7_4_5 & 0.469 & 0.493 & -0.015 \\
\hline T7_7_5 & 0.511 & 0.475 & -0.057 \\
\hline T7_9_5 & 0.008 & 0.928 & 0.041 \\
\hline T7_10_5 & 0.012 & 0.913 & 0.038 \\
\hline T7_11_5 & 0.011 & 0.917 & 0.074 \\
\hline T7_12_5 & 0.800 & 0.371 & -0.054 \\
\hline T7_13_5 & 0.000 & 0.987 & 0.052 \\
\hline T7_18_5 & 0.000 & 0.985 & 0.037 \\
\hline
\end{tabular}




\begin{tabular}{|c|c|c|c|}
\hline T7_19_5 & 0.020 & 0.889 & 0.055 \\
\hline T7_20_5 & 0.078 & 0.780 & 0.076 \\
\hline T7_23_5 & 0.003 & 0.955 & 0.020 \\
\hline T7_24_5 & 0.001 & 0.983 & 0.036 \\
\hline T7_25_5 & 0.001 & 0.972 & 0.039 \\
\hline T7_26_5 & 0.000 & 0.998 & 0.032 \\
\hline T7_30_5 & 0.022 & 0.883 & 0.005 \\
\hline T7_31_5 & 0.001 & 0.979 & 0.025 \\
\hline T7_32_5 & 0.028 & 0.866 & 0.004 \\
\hline T7_2_6 & 0.042 & 0.838 & 0.037 \\
\hline T7_3_6 & 0.036 & 0.850 & 0.035 \\
\hline T7_4_6 & 0.000 & 0.992 & 0.008 \\
\hline T7_7_6 & 0.000 & 0.990 & -0.001 \\
\hline T7_8_6 & 0.005 & 0.946 & 0.016 \\
\hline T7_9_6 & 0.008 & 0.927 & -0.013 \\
\hline T7_10_6 & 0.005 & 0.946 & 0.053 \\
\hline T7_14_6 & 0.019 & 0.891 & 0.063 \\
\hline T7_15_6 & 0.021 & 0.884 & 0.014 \\
\hline T7_16_6 & 0.019 & 0.890 & 0.057 \\
\hline T7_17_6 & 0.000 & 0.997 & 0.032 \\
\hline T7_21_6 & 0.028 & 0.868 & 0.061 \\
\hline T7_22_6 & 0.000 & 0.991 & 0.032 \\
\hline T7_24_6 & 0.033 & 0.856 & 0.063 \\
\hline T7_25_6 & 0.000 & 0.991 & 0.033 \\
\hline T7_27_6 & 0.277 & 0.599 & 0.116 \\
\hline T7_28_6 & 0.017 & 0.895 & 0.010 \\
\hline T7_29_6 & 0.020 & 0.888 & 0.057 \\
\hline T7_30_6 & 0.090 & 0.765 & 0.082 \\
\hline T7_31_6 & 0.001 & 0.978 & 0.026 \\
\hline T7_32_6 & 0.000 & 0.993 & 0.029 \\
\hline
\end{tabular}


Table 55

Task 7 DIF: Black vs. Sample

\begin{tabular}{|c|c|c|c|}
\hline ItemID & ChiSq & pvalue & EffectSize \\
\hline T7_6_12 & 0.036 & 0.850 & 0.011 \\
\hline T7_8_12 & 0.090 & 0.765 & 0.028 \\
\hline T7_22_12 & 0.206 & 0.650 & 0.146 \\
\hline T7_29_12 & 5.979 & 0.015 & -0.208 \\
\hline T7_29_12.1 & 0.494 & 0.482 & -0.045 \\
\hline T7_4_23 & 0.339 & 0.561 & -0.063 \\
\hline T7_12_23 & 0.000 & 0.989 & -0.035 \\
\hline T7_15_23 & 0.000 & 0.992 & -0.035 \\
\hline T7_17_23 & 0.045 & 0.832 & 0.188 \\
\hline T7_26_23 & 0.106 & 0.745 & 0.164 \\
\hline T7_2_34 & 1.303 & 0.254 & 0.081 \\
\hline T7_9_34 & 1.393 & 0.238 & 0.029 \\
\hline T7_15_34 & 1.231 & 0.267 & 0.075 \\
\hline T7_29_34 & 0.784 & 0.376 & -0.071 \\
\hline T7_31_34 & 0.563 & 0.453 & -0.057 \\
\hline T7_16_45 & 0.000 & 0.990 & -0.064 \\
\hline T7_1_56 & 0.157 & 0.692 & -0.001 \\
\hline T7_15_56 & 0.001 & 0.983 & -0.064 \\
\hline T7_17_56 & 0.372 & 0.542 & 0.045 \\
\hline T7_9_456 & 0.039 & 0.844 & -0.094 \\
\hline T7_15_456 & 1.239 & 0.266 & 0.059 \\
\hline T7_1_1 & 0.136 & 0.713 & 0.023 \\
\hline T7_2_1 & 0.000 & 0.993 & -0.035 \\
\hline T7_3_1 & 1.192 & 0.275 & -0.096 \\
\hline T7_4_1 & 0.253 & 0.615 & 0.045 \\
\hline T7_5_1 & 0.000 & 0.988 & -0.038 \\
\hline T7_7_1 & 0.017 & 0.896 & -0.014 \\
\hline T7_8_12.1 & 0.090 & 0.765 & 0.028 \\
\hline T7_9_1 & 0.177 & 0.674 & 0.032 \\
\hline T7_10_1 & 0.141 & 0.707 & 0.025 \\
\hline T7_12_1 & 0.000 & 0.995 & -0.036 \\
\hline T7_13_1 & 0.069 & 0.793 & 0.052 \\
\hline T7_14_1 & 0.214 & 0.643 & 0.016 \\
\hline T7_15_1 & 0.006 & 0.941 & -0.025 \\
\hline T7_16_1 & 1.201 & 0.273 & -0.057 \\
\hline T7_17_1 & 2.851 & 0.091 & -0.151 \\
\hline T7_18_1 & 1.462 & 0.227 & -0.079 \\
\hline
\end{tabular}




$\begin{array}{llll}\text { T7_19_1 } & 0.117 & 0.732 & 0.066 \\ \text { T7_20_1 } & 3.479 & 0.062 & -0.171 \\ \text { T7_21_1 } & 0.736 & 0.391 & -0.017 \\ \text { T7_22_12.1 } & 0.206 & 0.650 & 0.146 \\ \text { T7_25_1 } & 1.851 & 0.174 & -0.090 \\ \text { T7_26_1 } & 1.738 & 0.187 & -0.089 \\ \text { T7_27_1 } & 0.130 & 0.718 & 0.070 \\ \text { T7_28_1 } & 0.080 & 0.777 & 0.084 \\ \text { T7_29_12.2 } & 5.979 & 0.015 & -0.208 \\ \text { T7_30_1 } & 0.295 & 0.587 & 0.033 \\ \text { T7_31_1 } & 0.000 & 1.000 & 0.130 \\ \text { T7_33_1 } & 0.067 & 0.795 & 0.087 \\ \text { T7_1_2 } & 0.035 & 0.852 & -0.016 \\ \text { T7_2_2 } & 0.376 & 0.540 & -0.015 \\ \text { T7_5_2 } & 0.238 & 0.626 & 0.007 \\ \text { T7_6_2 } & 0.009 & 0.926 & -0.001 \\ \text { T7_7_2 } & 0.114 & 0.736 & -0.039 \\ \text { T7_8_2 } & 0.000 & 0.998 & 0.014 \\ \text { T7_9_2 } & 0.004 & 0.948 & 0.029 \\ \text { T7_11_2 } & 0.020 & 0.889 & 0.041 \\ \text { T7_13_2 } & 0.000 & 0.984 & 0.022 \\ \text { T7_16_2 } & 0.834 & 0.361 & -0.080 \\ \text { T7_19_2 } & 0.112 & 0.738 & 0.018 \\ \text { T7_20_2 } & 0.003 & 0.956 & 0.062 \\ \text { T7_21_2 } & 0.574 & 0.449 & -0.054 \\ \text { T7_22_2 } & 0.000 & 0.990 & 0.074 \\ \text { T7_24_2 } & 0.728 & 0.394 & -0.070 \\ \text { T7_27_2 } & 0.060 & 0.806 & 0.029 \\ \text { T7_28_2 } & 0.226 & 0.634 & -0.011 \\ \text { T7_32_2 } & 0.037 & 0.847 & 0.039 \\ \text { T7_33_2 } & 0.102 & 0.749 & 0.018 \\ \text { T7_1_3 } & 0.393 & 0.531 & 0.049 \\ \text { T7_3_3 } & 0.392 & 0.531 & 0.049 \\ \text { T7_4_3 } & 0.059 & 0.808 & -0.013 \\ \text { T7_5_3 } & 0.251 & 0.617 & 0.029 \\ \text { T7_6_3 } & 1.273 & 0.259 & -0.123 \\ \text { T7_8_3 } & 0.039 & 0.843 & -0.019 \\ \text { T7_10_3 } & 0.000 & 0.989 & -0.053 \\ \text { T7_16_3 } & 0.001 & 0.976 & 0.111\end{array}$




$\begin{array}{lllc}\text { T7_17_3 } & 0.017 & 0.898 & 0.094 \\ \text { T7_19_3 } & 2.080 & 0.149 & -0.125 \\ \text { T7_20_3 } & 0.167 & 0.683 & 0.050 \\ \text { T7_22_3 } & 0.104 & 0.747 & 0.057 \\ \text { T7_24_3 } & 2.337 & 0.126 & -0.131 \\ \text { T7_2_3 } & 0.438 & 0.508 & 0.006 \\ \text { T7_28_3 } & 0.334 & 0.563 & 0.024 \\ \text { T7_30_3 } & 1.005 & 0.316 & -0.055 \\ \text { T7_31_34.1 } & 0.563 & 0.453 & -0.057 \\ \text { T7_32_3 } & 1.852 & 0.174 & -0.113 \\ \text { T7_33_3 } & 0.001 & 0.971 & 0.108 \\ \text { T7__4 } & 0.004 & 0.953 & -0.037 \\ \text { T7_4_4 } & 0.335 & 0.563 & 0.045 \\ \text { T7_5_4 } & 0.001 & 0.977 & -0.043 \\ \text { T7_6_4 } & 0.052 & 0.819 & -0.010 \\ \text { T7_74 } & 0.011 & 0.918 & -0.126 \\ \text { T7_10_4 } & 0.085 & 0.770 & -0.001 \\ \text { T7_1144 } & 1.170 & 0.280 & 0.072 \\ \text { T7_13_4 } & 0.000 & 0.992 & -0.049 \\ \text { T7_1444 } & 0.009 & 0.926 & -0.033 \\ \text { T7_17_4 } & 0.002 & 0.967 & -0.062 \\ \text { T7_18_4 } & 0.008 & 0.928 & -0.035 \\ \text { T7_19_4 } & 0.072 & 0.789 & -0.008 \\ \text { T7_0_4 } & 0.222 & 0.638 & 0.027 \\ \text { T7_21_4 } & 0.007 & 0.933 & -0.037 \\ \text { T7_4_4 } & 0.029 & 0.865 & -0.020 \\ \text { T7_26_4 } & 0.011 & 0.918 & -0.072 \\ \text { T7_7_4 } & 0.006 & 0.938 & -0.039 \\ \text { T7_29_4 } & 0.400 & 0.527 & 0.053 \\ \text { T7_32_4 } & 0.251 & 0.617 & 0.032 \\ \text { T7_33_4 } & 0.416 & 0.519 & 0.054 \\ \text { T7_255 } & 0.516 & 0.473 & 0.039 \\ \text { T7_3_5 } & 0.830 & 0.362 & 0.070 \\ \text { T7_4_5 } & 0.257 & 0.612 & 0.000 \\ \text { T7_7_5 } & 0.877 & 0.349 & 0.074 \\ \text { T7_9_5 } & 0.000 & 0.992 & -0.080 \\ \text { T7_10_5 } & 0.049 & 0.826 & -0.040 \\ \text { T7_11_5 } & 0.207 & 0.649 & -0.151 \\ \text { T7_12_5 } & 0.607 & 0.436 & 0.049 \\ \text { T7_13_5 } & 0.013 & 0.910 & -0.057 \\ \text { T7_18_5 } & 0.037 & 0.847 & -0.032 \\ & & & \end{array}$




\begin{tabular}{llll} 
T7_19_5 & 0.066 & 0.797 & -0.042 \\
T7_20_5 & 0.091 & 0.763 & -0.048 \\
T7_23_5 & 0.006 & 0.939 & -0.014 \\
T7_24_5 & 0.267 & 0.605 & -0.082 \\
T7_25_5 & 0.001 & 0.971 & -0.007 \\
T7_26_5 & 0.108 & 0.743 & -0.052 \\
T7_30_5 & 0.000 & 0.987 & 0.006 \\
T7_31_5 & 0.090 & 0.764 & -0.049 \\
T7_32_5 & 0.005 & 0.943 & -0.015 \\
T7_2_6 & 0.023 & 0.879 & -0.016 \\
T7_3_6 & 0.077 & 0.782 & -0.035 \\
T7_4_6 & 0.002 & 0.969 & 0.002 \\
T7_7_6 & 0.013 & 0.911 & -0.010 \\
T7_8_6 & 0.018 & 0.894 & -0.013 \\
T7_9_6 & 0.000 & 0.991 & 0.006 \\
T7_10_6 & 0.030 & 0.862 & -0.021 \\
T7_14_6 & 0.008 & 0.930 & -0.067 \\
T7_15_6 & 0.099 & 0.753 & 0.002 \\
T7_16_6 & 0.000 & 0.997 & -0.056 \\
T7_17_6 & 0.070 & 0.792 & -0.015 \\
T7_21_6 & 0.010 & 0.920 & -0.041 \\
T7_22_6 & 0.083 & 0.774 & -0.011 \\
T7_24_6 & 0.002 & 0.961 & -0.048 \\
T7_25_6 & 0.108 & 0.742 & -0.004 \\
T7_27_6 & 0.024 & 0.878 & -0.084 \\
T7_28_6 & 0.155 & 0.694 & 0.007 \\
T7_29_6 & 0.000 & 0.988 & -0.059 \\
T7_30_6 & 0.001 & 0.976 & -0.066 \\
T7_31_6 & 0.006 & 0.939 & -0.044 \\
T7_32_6 & 0.016 & 0.899 & -0.037 \\
\hline & & & \\
& &
\end{tabular}


Table 56

Task 7 DIF: Latinx vs. Sample

\begin{tabular}{|c|c|c|c|}
\hline ItemID & ChiSq & pvalue & EffectSize \\
\hline T7_6_12 & 0.295 & 0.587 & 0.088 \\
\hline T7_8_12 & 0.389 & 0.533 & 0.115 \\
\hline T7_22_12 & 0.021 & 0.884 & -0.069 \\
\hline T7_29_12 & 0.027 & 0.869 & 0.051 \\
\hline T7_29_12.1 & 0.060 & 0.806 & -0.063 \\
\hline T7_4_23 & 0.019 & 0.891 & -0.013 \\
\hline T7_12_23 & 0.294 & 0.588 & 0.089 \\
\hline T7_15_23 & 0.101 & 0.750 & 0.013 \\
\hline T7_17_23 & 0.002 & 0.962 & 0.042 \\
\hline T7_26_23 & 0.063 & 0.802 & -0.035 \\
\hline T7_2_34 & 0.000 & 0.992 & 0.027 \\
\hline T7_9_34 & 0.321 & 0.571 & 0.134 \\
\hline T7_15_34 & 0.004 & 0.953 & -0.021 \\
\hline T7_29_34 & 0.040 & 0.841 & -0.126 \\
\hline T7_31_34 & 0.048 & 0.827 & -0.124 \\
\hline T7_16_45 & 0.015 & 0.901 & -0.118 \\
\hline T7_1_56 & 0.061 & 0.806 & -0.081 \\
\hline T7_15_56 & 0.007 & 0.935 & -0.020 \\
\hline T7_17_56 & 0.744 & 0.388 & -0.184 \\
\hline T7_9_456 & 0.001 & 0.982 & 0.083 \\
\hline T7_15_456 & 0.152 & 0.696 & -0.054 \\
\hline T7_1_1 & 0.110 & 0.740 & 0.051 \\
\hline T7_2_1 & 0.064 & 0.801 & 0.023 \\
\hline T7_3_1 & 0.600 & 0.439 & 0.159 \\
\hline T7_4_1 & 0.161 & 0.688 & 0.073 \\
\hline T7_5_1 & 0.014 & 0.906 & -0.023 \\
\hline T7_7_1 & 0.231 & 0.631 & 0.098 \\
\hline T7_8_12.1 & 0.389 & 0.533 & 0.115 \\
\hline T7_9_1 & 0.232 & 0.630 & 0.098 \\
\hline T7_10_1 & 0.083 & 0.773 & 0.036 \\
\hline T7_12_1 & 0.013 & 0.909 & -0.023 \\
\hline T7_13_1 & 0.040 & 0.841 & -0.047 \\
\hline T7_14_1 & 0.238 & 0.625 & 0.056 \\
\hline T7_15_1 & 0.037 & 0.849 & 0.001 \\
\hline T7_16_1 & 0.039 & 0.843 & 0.039 \\
\hline T7_17_1 & 0.031 & 0.860 & 0.034 \\
\hline T7_18_1 & 0.071 & 0.790 & 0.061 \\
\hline
\end{tabular}




$\begin{array}{llll}\text { T7_19_1 } & 0.048 & 0.827 & 0.046 \\ \text { T7_20_1 } & 0.098 & 0.754 & 0.077 \\ \text { T7_21_1 } & 0.014 & 0.906 & 0.015 \\ \text { T7_22_12.1 } & 0.021 & 0.884 & -0.069 \\ \text { T7_25_1 } & 0.004 & 0.951 & -0.008 \\ \text { T7_26_1 } & 0.164 & 0.685 & 0.107 \\ \text { T7_27_1 } & 0.022 & 0.882 & -0.105 \\ \text { T7_28_1 } & 0.000 & 0.986 & -0.025 \\ \text { T7_29_12.2 } & 0.027 & 0.869 & 0.051 \\ \text { T7_30_1 } & 0.016 & 0.901 & 0.016 \\ \text { T7_31_1 } & 0.011 & 0.916 & 0.005 \\ \text { T7_33_1 } & 0.017 & 0.897 & 0.014 \\ \text { T7_1_2 } & 0.027 & 0.869 & 0.035 \\ \text { T7_2_2 } & 0.055 & 0.815 & -0.093 \\ \text { T7_5_2 } & 0.001 & 0.971 & 0.022 \\ \text { T7_6_2 } & 0.001 & 0.979 & -0.014 \\ \text { T7_7_2 } & 0.031 & 0.861 & 0.036 \\ \text { T7_8_2 } & 0.001 & 0.972 & -0.035 \\ \text { T7_9_2 } & 0.000 & 0.987 & -0.052 \\ \text { T7_11_2 } & 0.000 & 0.998 & -0.046 \\ \text { T7_13_2 } & 0.010 & 0.920 & -0.079 \\ \text { T7_16_2 } & 0.009 & 0.925 & -0.016 \\ \text { T7_19_2 } & 0.017 & 0.895 & -0.023 \\ \text { T7_20_2 } & 0.000 & 0.999 & 0.021 \\ \text { T7_21_2 } & 0.001 & 0.980 & 0.025 \\ \text { T7_22_2 } & 0.002 & 0.968 & 0.060 \\ \text { T7_24_2 } & 0.032 & 0.857 & -0.041 \\ \text { T7_27_2 } & 0.026 & 0.871 & -0.037 \\ \text { T7_28_2 } & 0.000 & 0.993 & 0.021 \\ \text { T7_32_2 } & 0.026 & 0.872 & -0.034 \\ \text { T7_33_2 } & 0.036 & 0.850 & -0.045 \\ \text { T7_1_3 } & 0.143 & 0.706 & 0.070 \\ \text { T7_3_3 } & 0.137 & 0.711 & 0.067 \\ \text { T7_4_3 } & 0.006 & 0.938 & -0.109 \\ \text { T7_5_3 } & 0.050 & 0.823 & 0.016 \\ \text { T7_6_3 } & 0.102 & 0.749 & 0.072 \\ \text { T7_8_3 } & 0.010 & 0.922 & -0.025 \\ \text { T7_10_3 } & 0.001 & 0.982 & -0.052 \\ \text { T7_11_3 } & 0.001 & 0.972 & -0.044 \\ \text { T7_14_3 } & 0.018 & 0.892 & -0.105 \\ \text { T7_16_3 } & 0.000 & 0.997 & 0.020\end{array}$




$\begin{array}{llll}\text { T7_17_3 } & 0.040 & 0.841 & -0.051 \\ \text { T7_19_3 } & 0.022 & 0.881 & -0.035 \\ \text { T7_20_3 } & 0.003 & 0.959 & 0.001 \\ \text { T7_22_3 } & 0.001 & 0.973 & 0.021 \\ \text { T7_24_3 } & 0.012 & 0.912 & -0.025 \\ \text { T7_27_3 } & 0.001 & 0.981 & 0.028 \\ \text { T7_28_3 } & 0.012 & 0.915 & -0.022 \\ \text { T7_30_3 } & 0.040 & 0.842 & -0.056 \\ \text { T7_31_34.1 } & 0.048 & 0.827 & -0.124 \\ \text { T7_32_3 } & 0.006 & 0.937 & -0.012 \\ \text { T7_33_3 } & 0.000 & 0.990 & 0.022 \\ \text { T7_2_4 } & 0.030 & 0.862 & 0.008 \\ \text { T7_4_4 } & 0.253 & 0.615 & -0.105 \\ \text { T7_5_4 } & 0.021 & 0.886 & 0.022 \\ \text { T7_6_4 } & 0.153 & 0.696 & -0.066 \\ \text { T7_7_4 } & 0.165 & 0.684 & -0.147 \\ \text { T7_10_4 } & 0.029 & 0.865 & 0.011 \\ \text { T7_11_4 } & 0.000 & 0.999 & 0.023 \\ \text { T7_13_4 } & 0.046 & 0.830 & 0.000 \\ \text { T7_14_4 } & 0.031 & 0.861 & 0.012 \\ \text { T7_17_4 } & 0.000 & 0.998 & -0.061 \\ \text { T7_18_4 } & 0.059 & 0.809 & 0.022 \\ \text { T7_19_4 } & 0.000 & 0.993 & -0.053 \\ \text { T7_20_4 } & 0.129 & 0.720 & 0.067 \\ \text { T7_21_4 } & 0.015 & 0.903 & -0.127 \\ \text { T7_24_4 } & 0.102 & 0.750 & 0.050 \\ \text { T7_26_4 } & 0.002 & 0.963 & -0.095 \\ \text { T7_27_4 } & 0.003 & 0.954 & -0.102 \\ \text { T7_29_4 } & 0.141 & 0.707 & 0.072 \\ \text { T7_32_4 } & 0.098 & 0.755 & 0.051 \\ \text { T7_33_4 } & 0.146 & 0.702 & 0.075 \\ \text { T7_2_5 } & 0.011 & 0.916 & 0.060 \\ \text { T7_3_5 } & 0.003 & 0.956 & -0.019 \\ \text { T7_4_5 } & 0.265 & 0.607 & 0.117 \\ \text { T7_7_5 } & 0.002 & 0.962 & -0.017 \\ \text { T7_9_5 } & 0.001 & 0.978 & 0.009 \\ \text { T7_10_5 } & 0.001 & 0.982 & 0.008 \\ \text { T7_11_5 } & 0.433 & 0.511 & 0.161 \\ \text { T7_12_5 } & 0.227 & 0.634 & 0.107 \\ \text { T7_13_5 } & 0.014 & 0.906 & -0.039 \\ \text { T7_18_5 } & 0.025 & 0.875 & -0.014 \\ \end{array}$




\begin{tabular}{|c|c|c|c|}
\hline T7_19_5 & 0.006 & 0.936 & -0.038 \\
\hline T7_20_5 & 0.000 & 0.984 & -0.086 \\
\hline T7_23_5 & 0.035 & 0.851 & -0.003 \\
\hline T7_24_5 & 0.307 & 0.579 & 0.118 \\
\hline T7_25_5 & 0.038 & 0.846 & -0.004 \\
\hline T7_26_5 & 0.125 & 0.724 & 0.052 \\
\hline T7_30_5 & 0.152 & 0.696 & 0.064 \\
\hline T7_31_5 & 0.089 & 0.766 & 0.033 \\
\hline T7_32_5 & 0.084 & 0.773 & 0.030 \\
\hline T7_2_6 & 0.060 & 0.806 & -0.090 \\
\hline T7_3_6 & 0.003 & 0.958 & -0.026 \\
\hline T7_4_6 & 0.000 & 0.996 & -0.010 \\
\hline T7_7_6 & 0.001 & 0.970 & -0.021 \\
\hline T7_8_6 & 0.022 & 0.883 & -0.058 \\
\hline T7_9_6 & 0.011 & 0.916 & -0.044 \\
\hline T7_10_6 & 0.594 & 0.441 & -0.054 \\
\hline T7_14_6 & 0.086 & 0.770 & 0.123 \\
\hline T7_15_6 & 1.069 & 0.301 & -0.144 \\
\hline T7_16_6 & 0.109 & 0.741 & 0.095 \\
\hline T7_17_6 & 0.293 & 0.589 & 0.018 \\
\hline T7_21_6 & 0.177 & 0.674 & 0.060 \\
\hline T7_22_6 & 0.797 & 0.372 & -0.106 \\
\hline T7_24_6 & 0.298 & 0.585 & 0.014 \\
\hline T7_25_6 & 0.750 & 0.387 & -0.101 \\
\hline T7_27_6 & 0.275 & 0.600 & 0.025 \\
\hline T7_28_6 & 0.525 & 0.469 & -0.052 \\
\hline T7_29_6 & 0.034 & 0.854 & 0.148 \\
\hline T7_30_6 & 0.345 & 0.557 & -0.001 \\
\hline T7_31_6 & 0.145 & 0.704 & 0.072 \\
\hline T7_32_6 & 0.264 & 0.608 & 0.029 \\
\hline
\end{tabular}


Table 57

Task 8 DIF: Male vs. female

\begin{tabular}{|c|c|c|c|}
\hline ItemID & ChiSq & pvalue & EffectSize \\
\hline T8_16_34 & 0.000 & 0.988 & 0.005 \\
\hline T8_18_34 & 0.010 & 0.919 & 0.027 \\
\hline T8_20_34 & 0.053 & 0.817 & 0.047 \\
\hline T8_21_34 & 0.015 & 0.904 & 0.030 \\
\hline T8_37_34 & 0.357 & 0.551 & -0.035 \\
\hline T8_46_34 & 0.325 & 0.569 & -0.025 \\
\hline T8_31_45 & 0.398 & 0.528 & -0.061 \\
\hline T8_43_45 & 0.319 & 0.572 & -0.055 \\
\hline T8_4_56 & 0.004 & 0.951 & 0.006 \\
\hline T8_5_56 & 0.002 & 0.965 & -0.014 \\
\hline T8_8_56 & 0.021 & 0.886 & 0.018 \\
\hline T8_27_56 & 0.111 & 0.739 & 0.028 \\
\hline T8_39_56 & 0.001 & 0.983 & -0.033 \\
\hline T8_43_56 & 0.113 & 0.737 & -0.071 \\
\hline T8_9_456 & 0.032 & 0.858 & -0.011 \\
\hline T8_13_456 & 0.023 & 0.880 & 0.035 \\
\hline T8_20_456 & 0.002 & 0.961 & 0.009 \\
\hline T8_24_456 & 0.057 & 0.812 & -0.017 \\
\hline T8_25_456 & 0.122 & 0.727 & -0.037 \\
\hline T8_1_3 & 0.029 & 0.864 & 0.014 \\
\hline T8_2_3 & 0.032 & 0.858 & 0.016 \\
\hline T8_3_3 & 0.029 & 0.864 & 0.014 \\
\hline T8_4_3 & 0.045 & 0.832 & 0.021 \\
\hline T8_5_3 & 0.010 & 0.922 & 0.003 \\
\hline T8_6_3 & 0.000 & 0.989 & -0.009 \\
\hline T8_8_3 & 0.051 & 0.821 & -0.022 \\
\hline T8_9_3 & 0.273 & 0.602 & -0.068 \\
\hline T8_10_3 & 0.032 & 0.859 & -0.016 \\
\hline T8_11_3 & 0.018 & 0.895 & -0.010 \\
\hline T8_12_3 & 0.017 & 0.896 & 0.008 \\
\hline T8_13_3 & 0.047 & 0.828 & 0.021 \\
\hline T8_14_3 & 0.000 & 0.990 & -0.015 \\
\hline T8_15_3 & 0.005 & 0.946 & -0.001 \\
\hline T8_17_3 & 0.026 & 0.872 & 0.013 \\
\hline T8_19_3 & 0.023 & 0.880 & 0.011 \\
\hline T8_22_3 & 0.051 & 0.822 & 0.022 \\
\hline T8_23_3 & 0.006 & 0.940 & 0.000 \\
\hline
\end{tabular}




$\begin{array}{llll} & & & \\ \text { T8_24_3 } & 0.053 & 0.818 & 0.023 \\ \text { T8_25_3 } & 0.018 & 0.894 & 0.008 \\ \text { T8_26_3 } & 0.222 & 0.638 & -0.045 \\ \text { T8_27_3 } & 0.100 & 0.752 & -0.024 \\ \text { T8_28_3 } & 0.011 & 0.918 & 0.005 \\ \text { T8_29_3 } & 0.175 & 0.676 & -0.039 \\ \text { T8_30_3 } & 0.569 & 0.451 & -0.085 \\ \text { T8_32_3 } & 0.202 & 0.653 & -0.043 \\ \text { T8_33_3 } & 0.106 & 0.744 & -0.025 \\ \text { T8_34_3 } & 0.491 & 0.483 & -0.078 \\ \text { T8_36_3 } & 0.209 & 0.648 & -0.045 \\ \text { T8_40_3 } & 0.107 & 0.743 & -0.025 \\ \text { T8_41_3 } & 0.138 & 0.711 & -0.031 \\ \text { T8_42_3 } & 0.028 & 0.866 & -0.003 \\ \text { T8_43_3 } & 0.401 & 0.527 & -0.069 \\ \text { T8_44_3 } & 0.400 & 0.527 & -0.067 \\ \text { T8_45_3 } & 0.002 & 0.963 & 0.032 \\ \text { T8_47_3 } & 0.190 & 0.663 & -0.041 \\ \text { T8_48_3 } & 0.038 & 0.846 & -0.007 \\ \text { T8_49_3 } & 0.009 & 0.924 & 0.008 \\ \text { T8_1_4 } & 0.330 & 0.566 & -0.031 \\ \text { T8_2_4 } & 0.385 & 0.535 & -0.037 \\ \text { T8_3_4 } & 0.700 & 0.403 & -0.069 \\ \text { T8_4_4 } & 0.073 & 0.787 & -0.021 \\ \text { T8_5_4 } & 0.040 & 0.842 & -0.010 \\ \text { T8_6_4 } & 0.034 & 0.855 & -0.007 \\ \text { T8_7_4 } & 0.046 & 0.830 & -0.012 \\ \text { T8_8_4 } & 0.000 & 0.999 & 0.021 \\ \text { T8_10_4 } & 0.102 & 0.750 & -0.027 \\ \text { T8_12_4 } & 0.078 & 0.780 & -0.022 \\ \text { T8_15_4 } & 0.004 & 0.949 & 0.044 \\ \text { T8_16_4 } & 0.032 & 0.858 & -0.007 \\ \text { T8_17_4 } & 0.002 & 0.968 & 0.015 \\ \text { T8_21_4 } & 0.018 & 0.894 & 0.001 \\ \text { T8_23_4 } & 0.048 & 0.827 & -0.012 \\ \text { T8_26_4 } & 0.050 & 0.824 & -0.001 \\ \text { T8_27_4 } & 0.013 & 0.911 & 0.015 \\ \text { T8_43 } & 0.020 & 0.887 & 0.007 \\ \text { T8_4 } & 0.230 & 0.632 & -0.041 \\ \text { T8 } & 0.136 & 0.713 & -0.026 \\ \text { T8 } & 0.230 & 0.631 & -0.040\end{array}$




$\begin{array}{llll} & & & \\ \text { T8_35_4 } & 0.086 & 0.769 & -0.014 \\ \text { T8_38_4 } & 0.043 & 0.837 & 0.000 \\ \text { T8_41_4 } & 0.036 & 0.850 & 0.002 \\ \text { T8_44_4 } & 0.183 & 0.668 & -0.035 \\ \text { T8_45_4 } & 0.052 & 0.820 & -0.005 \\ \text { T8_46_4 } & 0.000 & 0.998 & 0.031 \\ \text { T8_47_4 } & 0.061 & 0.805 & -0.007 \\ \text { T8_48_4 } & 0.138 & 0.711 & -0.027 \\ \text { T8_49_4 } & 0.192 & 0.661 & -0.037 \\ \text { T8_1_5 } & 0.031 & 0.859 & 0.018 \\ \text { T8_2_5 } & 0.004 & 0.953 & 0.003 \\ \text { T8_3_56 } & 0.051 & 0.822 & 0.014 \\ \text { T8_6_5 } & 0.011 & 0.918 & -0.022 \\ \text { T8_7_5 } & 0.001 & 0.976 & -0.011 \\ \text { T8_8_56.1 } & 0.021 & 0.886 & 0.018 \\ \text { T8_10_5 } & 0.014 & 0.907 & -0.025 \\ \text { T8_11_5 } & 0.018 & 0.893 & -0.031 \\ \text { T8_13_5 } & 0.005 & 0.943 & 0.008 \\ \text { T8_14_5 } & 0.030 & 0.864 & -0.033 \\ \text { T8_15_5 } & 0.014 & 0.906 & 0.015 \\ \text { T8_18_5 } & 0.002 & 0.962 & -0.014 \\ \text { T8_19_5 } & 0.006 & 0.941 & -0.018 \\ \text { T8_20_5 } & 0.000 & 0.992 & -0.001 \\ \text { T8_22_5 } & 0.000 & 0.989 & -0.009 \\ \text { T8_23_5 } & 0.000 & 0.998 & -0.008 \\ \text { T8_25_5 } & 0.014 & 0.907 & -0.025 \\ \text { T8_26_5 } & 0.009 & 0.926 & 0.014 \\ \text { T8_29_5 } & 0.029 & 0.866 & -0.031 \\ \text { T8_31_5 } & 0.002 & 0.963 & -0.014 \\ \text { T8_33_5 } & 0.321 & 0.571 & 0.080 \\ \text { T8_35_5 } & 0.004 & 0.951 & -0.017 \\ \text { T8_36_5 } & 0.000 & 0.987 & 0.001 \\ \text { T8_38_5 } & 0.118 & 0.731 & -0.054 \\ \text { T8_40_5 } & 0.003 & 0.959 & -0.013 \\ \text { T8_41_5 } & 0.001 & 0.977 & -0.011 \\ \text { T8_42_5 } & 0.092 & 0.761 & -0.052 \\ \text { T8_44_5 } & 0.003 & 0.960 & 0.007 \\ \text { T8_45_5 } & 0.000 & 0.987 & -0.009 \\ \text { T8_49_5 } & 0.014 & 0.907 & -0.023 \\ \text { T85 } & 0.031 & 0.861 & -0.032 \\ \text { T8 } & 0.013 & 0.909 & -0.022\end{array}$




\begin{tabular}{|c|c|c|c|}
\hline T8_1_6 & 0.074 & 0.786 & 0.025 \\
\hline T8_2_6 & 0.060 & 0.807 & 0.021 \\
\hline T8_5_6 & 0.002 & 0.969 & 0.008 \\
\hline T8_7_6 & 0.037 & 0.847 & 0.014 \\
\hline T8_9_6 & 0.000 & 0.997 & 0.002 \\
\hline T8_10_6 & 0.026 & 0.871 & 0.027 \\
\hline T8_15_6 & 0.001 & 0.972 & 0.007 \\
\hline T8_17_6 & 0.001 & 0.977 & 0.007 \\
\hline T8_19_6 & 0.006 & 0.939 & 0.013 \\
\hline T8_20_6 & 0.014 & 0.908 & 0.020 \\
\hline T8_21_6 & 0.000 & 0.994 & 0.004 \\
\hline T8_22_6 & 0.000 & 0.992 & -0.005 \\
\hline T8_23_6 & 0.004 & 0.947 & 0.012 \\
\hline T8_24_6 & 0.006 & 0.940 & 0.013 \\
\hline T8_25_6 & 0.023 & 0.879 & 0.025 \\
\hline T8_28_6 & 0.046 & 0.831 & 0.016 \\
\hline T8_29_6 & 0.029 & 0.865 & 0.010 \\
\hline T8_30_6 & 0.062 & 0.804 & 0.020 \\
\hline T8_31_6 & 0.085 & 0.770 & 0.027 \\
\hline T8_32_6 & 0.000 & 0.990 & -0.018 \\
\hline T8_34_6 & 0.045 & 0.833 & 0.017 \\
\hline T8_35_6 & 0.001 & 0.972 & -0.025 \\
\hline T8_36_6 & 0.005 & 0.943 & -0.031 \\
\hline T8_37_6 & 0.039 & 0.843 & 0.014 \\
\hline T8_38_6 & 0.048 & 0.826 & 0.016 \\
\hline T8_39_6 & 0.001 & 0.979 & -0.025 \\
\hline T8_40_6 & 0.006 & 0.941 & -0.005 \\
\hline T8_42_6 & 0.013 & 0.911 & 0.002 \\
\hline T8_43_6 & 0.000 & 0.988 & -0.022 \\
\hline T8_44_6 & 0.002 & 0.964 & -0.010 \\
\hline T8_46_6 & 0.030 & 0.862 & 0.009 \\
\hline T8_49_6 & 0.057 & 0.811 & 0.020 \\
\hline
\end{tabular}


Table 58

Task 8 DIF: White vs. Sample

\begin{tabular}{lccc}
\hline ItemID & ChiSq & pvalue & EffectSize \\
\hline T8_16_34 & 0.126 & 0.722 & -0.051 \\
T8_18_34 & 0.033 & 0.856 & -0.026 \\
T8_20_34 & 0.056 & 0.814 & -0.034 \\
T8_21_34 & 0.006 & 0.940 & -0.010 \\
T8_37_34 & 0.138 & 0.710 & 0.074 \\
T8_46_34 & 0.095 & 0.758 & 0.066 \\
T8_31_45 & 0.233 & 0.630 & -0.088 \\
T8_43_45 & 0.885 & 0.347 & -0.151 \\
T8_4_56 & 0.597 & 0.440 & 0.064 \\
T8_5_56 & 0.301 & 0.583 & 0.030 \\
T8_8_56 & 0.359 & 0.549 & 0.036 \\
T8_27_56 & 0.052 & 0.820 & 0.041 \\
T8_39_56 & 0.264 & 0.608 & 0.082 \\
T8_43_56 & 0.251 & 0.617 & 0.077 \\
T8_9_456 & 0.572 & 0.450 & 0.041 \\
T8_13_456 & 0.617 & 0.432 & -0.023 \\
T8_20_456 & 0.003 & 0.957 & -0.066 \\
T8_24_456 & 0.230 & 0.632 & -0.004 \\
T8_25_456 & 1.195 & 0.274 & 0.090 \\
T8_1_3 & 0.112 & 0.738 & -0.027 \\
T8_2_3 & 0.097 & 0.755 & -0.024 \\
T8_3_3 & 0.112 & 0.738 & -0.027 \\
T8_4_3 & 0.089 & 0.766 & -0.021 \\
T8_5_3 & 0.114 & 0.735 & -0.027 \\
T8_6_3 & 0.058 & 0.810 & -0.013 \\
T8_8_3 & 0.003 & 0.953 & 0.024 \\
T8_9_3 & 0.033 & 0.856 & 0.045 \\
T8_10_3 & 0.026 & 0.872 & -0.015 \\
T8_11_3 & 0.033 & 0.856 & -0.015 \\
T8_12_3 & 0.111 & 0.739 & -0.027 \\
T8_13_3 & 0.066 & 0.797 & -0.015 \\
T8_14_3 & 0.069 & 0.794 & -0.016 \\
T8_15_3 & 0.047 & 0.829 & -0.009 \\
T8_17_3 & 0.056 & 0.812 & -0.011 \\
T8_19_3 & 0.082 & 0.775 & -0.019 \\
T8_23_3 & 0.129 & 0.720 & -0.030
\end{tabular}




$\begin{array}{llll}\text { T8_24_3 } & 0.038 & 0.846 & -0.006 \\ \text { T8_25_3 } & 0.012 & 0.915 & 0.007 \\ \text { T8_26_3 } & 0.021 & 0.886 & 0.012 \\ \text { T8_27_3 } & 0.125 & 0.724 & 0.042 \\ \text { T8_28_3 } & 0.100 & 0.751 & 0.037 \\ \text { T8_29_3 } & 0.139 & 0.709 & 0.045 \\ \text { T8_30_3 } & 0.050 & 0.824 & 0.023 \\ \text { T8_32_3 } & 0.032 & 0.857 & -0.039 \\ \text { T8_33_3 } & 0.237 & 0.627 & 0.061 \\ \text { T8_34_3 } & 0.013 & 0.911 & 0.008 \\ \text { T8_36_3 } & 0.001 & 0.973 & -0.004 \\ \text { T8_40_3 } & 0.238 & 0.626 & 0.064 \\ \text { T8_41_3 } & 0.033 & 0.857 & 0.016 \\ \text { T8_42_3 } & 0.125 & 0.723 & 0.043 \\ \text { T8_43_3 } & 0.030 & 0.864 & 0.017 \\ \text { T8_44_3 } & 0.434 & 0.510 & 0.087 \\ \text { T8_45_3 } & 0.005 & 0.942 & 0.002 \\ \text { T8_47_3 } & 0.063 & 0.802 & 0.029 \\ \text { T8_48_3 } & 0.157 & 0.692 & 0.048 \\ \text { T8_49_3 } & 0.056 & 0.812 & 0.027 \\ \text { T8_1_4 } & 0.037 & 0.847 & 0.037 \\ \text { T8_2_4 } & 0.017 & 0.897 & -0.016 \\ \text { T8_3_4 } & 0.005 & 0.946 & 0.019 \\ \text { T8_4_4 } & 0.081 & 0.776 & 0.025 \\ \text { T8_5_4 } & 0.017 & 0.898 & 0.001 \\ \text { T8_6_4 } & 0.034 & 0.854 & 0.009 \\ \text { T8_7_4 } & 0.025 & 0.874 & 0.005 \\ \text { T8_8_4 } & 0.031 & 0.861 & 0.007 \\ \text { T8_10_4 } & 0.067 & 0.797 & 0.020 \\ \text { T8_12_4 } & 0.032 & 0.858 & 0.009 \\ \text { T8_15_4 } & 0.035 & 0.852 & -0.024 \\ \text { T8_16_4 } & 0.027 & 0.870 & 0.006 \\ \text { T8_17_4 } & 0.005 & 0.941 & -0.008 \\ \text { T8_21_4 } & 0.014 & 0.905 & -0.041 \\ \text { T8_23_4 } & 0.010 & 0.922 & -0.004 \\ \text { T8_26_4 } & 0.234 & 0.629 & -0.044 \\ \text { T8_27_4 } & 0.041 & 0.839 & -0.002 \\ \text { T8_29_4 } & 0.007 & 0.935 & 0.042 \\ \text { T8_32_4 } & 0.003 & 0.956 & 0.038 \\ \text { T8_33_4 } & 0.080 & 0.778 & -0.016 \\ \text { T8_34_4 } & 0.220 & 0.639 & -0.042 \\ & & & \end{array}$




$\begin{array}{llll} & & & \\ \text { T8_35_4 } & 0.141 & 0.707 & -0.030 \\ \text { T8_38_4 } & 0.014 & 0.905 & 0.009 \\ \text { T8_41_4 } & 0.333 & 0.564 & -0.059 \\ \text { T8_44_4 } & 0.151 & 0.697 & -0.032 \\ \text { T8_45_4 } & 0.003 & 0.956 & 0.018 \\ \text { T8_46_4 } & 0.160 & 0.689 & -0.032 \\ \text { T8_47_4 } & 0.028 & 0.867 & 0.001 \\ \text { T8_48_4 } & 0.154 & 0.695 & -0.033 \\ \text { T8_49_4 } & 0.255 & 0.614 & -0.049 \\ \text { T8_1_5 } & 0.228 & 0.633 & 0.026 \\ \text { T8_2_5 } & 0.301 & 0.584 & 0.035 \\ \text { T8_3_56 } & 0.000 & 0.985 & 0.001 \\ \text { T8_6_5 } & 0.006 & 0.939 & 0.012 \\ \text { T8_7_5 } & 0.017 & 0.896 & -0.024 \\ \text { T8_8_56.1 } & 0.359 & 0.549 & 0.036 \\ \text { T8_10_5 } & 0.000 & 0.996 & 0.000 \\ \text { T8_11_5 } & 0.269 & 0.604 & 0.031 \\ \text { T8_13_5 } & 0.003 & 0.955 & 0.008 \\ \text { T8_14_5 } & 0.008 & 0.930 & 0.014 \\ \text { T8_15_5 } & 0.020 & 0.887 & 0.021 \\ \text { T8_18_5 } & 0.005 & 0.943 & -0.016 \\ \text { T8_19_5 } & 0.007 & 0.935 & -0.017 \\ \text { T8_20_5 } & 0.011 & 0.915 & -0.021 \\ \text { T8_22_5 } & 0.031 & 0.860 & -0.031 \\ \text { T8_23_5 } & 0.000 & 0.996 & -0.005 \\ \text { T8_25_5 } & 0.016 & 0.899 & 0.020 \\ \text { T8_26_5 } & 0.383 & 0.536 & 0.048 \\ \text { T8_29_5 } & 0.055 & 0.815 & -0.010 \\ \text { T8_31_5 } & 0.293 & 0.588 & 0.033 \\ \text { T8_33_5 } & 0.642 & 0.423 & 0.073 \\ \text { T8_35_5 } & 0.153 & 0.696 & 0.011 \\ \text { T8_36_5 } & 0.271 & 0.603 & 0.032 \\ \text { T8_38_5 } & 0.944 & 0.331 & 0.099 \\ \text { T8_40_5 } & 0.089 & 0.766 & 0.000 \\ \text { T8_41_5 } & 0.143 & 0.705 & 0.010 \\ \text { T8_42_5 } & 0.057 & 0.812 & -0.010 \\ \text { T8_44_5 } & 0.363 & 0.547 & 0.046 \\ \text { T8_45_5 } & 0.640 & 0.424 & 0.073 \\ \text { T8_5 } & 0.126 & 0.722 & 0.007 \\ \text { T8_5 } & 0.023 & 0.881 & -0.022 \\ \text { T8 } & 0.204 & 0.652 & 0.024\end{array}$




\begin{tabular}{lllc} 
T8_1_6 & 0.000 & 0.985 & 0.003 \\
T8_2_6 & 0.048 & 0.827 & 0.032 \\
T8_5_6 & 0.148 & 0.701 & 0.007 \\
T8_7_6 & 0.061 & 0.805 & 0.036 \\
T8_9_6 & 0.490 & 0.484 & 0.056 \\
T8_10_6 & 0.059 & 0.808 & -0.014 \\
T8_15_6 & 0.083 & 0.773 & -0.007 \\
T8_17_6 & 0.269 & 0.604 & 0.028 \\
T8_19_6 & 0.255 & 0.614 & 0.026 \\
T8_20_6 & 0.038 & 0.846 & -0.022 \\
T8_21_6 & 0.311 & 0.577 & 0.034 \\
T8_22_6 & 0.410 & 0.522 & 0.047 \\
T8_23_6 & 0.046 & 0.830 & -0.018 \\
T8_24_6 & 0.141 & 0.707 & 0.007 \\
T8_25_6 & 0.041 & 0.840 & -0.021 \\
T8_28_6 & 0.001 & 0.972 & 0.040 \\
T8_29_6 & 0.386 & 0.535 & -0.047 \\
T8_30_6 & 0.011 & 0.916 & 0.034 \\
T8_31_6 & 0.108 & 0.742 & -0.003 \\
T8_32_6 & 0.002 & 0.966 & 0.044 \\
T8_34_6 & 0.459 & 0.498 & -0.056 \\
T8_35_6 & 0.269 & 0.604 & -0.030 \\
T8_36_6 & 0.010 & 0.922 & 0.036 \\
T8_37_6 & 0.126 & 0.723 & -0.004 \\
T8_38_6 & 0.270 & 0.603 & -0.029 \\
T8_39_6 & 0.039 & 0.844 & 0.085 \\
T8_40_6 & 0.335 & 0.563 & -0.036 \\
T8_42_6 & 0.113 & 0.737 & -0.001 \\
T8_43_6 & 0.196 & 0.658 & -0.020 \\
T8_44_6 & 0.001 & 0.971 & 0.061 \\
T8_46_6 & 0.065 & 0.799 & 0.011 \\
T8_49_6 & 0.193 & 0.660 & -0.019 \\
\hline
\end{tabular}


Table 59

Task 8 DIF: Black vs. Sample

\begin{tabular}{|c|c|c|c|}
\hline ItemID & ChiSq & pvalue & EffectSize \\
\hline T8_16_34 & 0.395 & 0.530 & 0.069 \\
\hline T8_18_34 & 0.156 & 0.693 & 0.033 \\
\hline T8_20_34 & 0.160 & 0.690 & 0.032 \\
\hline T8_21_34 & 0.004 & 0.951 & -0.020 \\
\hline T8_37_34 & 0.367 & 0.545 & -0.069 \\
\hline T8_46_34 & 0.388 & 0.534 & -0.066 \\
\hline T8_31_45 & 0.000 & 0.991 & 0.051 \\
\hline T8_43_45 & 0.027 & 0.869 & 0.075 \\
\hline T8_4_56 & 0.189 & 0.664 & -0.036 \\
\hline T8_5_56 & 0.285 & 0.594 & -0.051 \\
\hline T8_8_56 & 0.028 & 0.867 & 0.006 \\
\hline T8_27_56 & 0.124 & 0.725 & -0.052 \\
\hline T8_39_56 & 0.435 & 0.510 & -0.098 \\
\hline T8_43_56 & 0.580 & 0.446 & -0.111 \\
\hline T8_9_456 & 0.082 & 0.775 & -0.022 \\
\hline T8_13_456 & 0.624 & 0.430 & -0.005 \\
\hline T8_20_456 & 0.259 & 0.611 & 0.105 \\
\hline T8_24_456 & 0.000 & 0.995 & 0.029 \\
\hline T8_25_456 & 0.102 & 0.749 & -0.027 \\
\hline T8_1_3 & 0.083 & 0.773 & 0.024 \\
\hline T8_2_3 & 0.075 & 0.785 & 0.022 \\
\hline T8_3_3 & 0.083 & 0.773 & 0.024 \\
\hline T8_4_3 & 0.076 & 0.782 & 0.022 \\
\hline T8_5_3 & 0.041 & 0.840 & 0.011 \\
\hline T8_6_3 & 0.022 & 0.883 & 0.001 \\
\hline T8_8_3 & 0.272 & 0.602 & -0.053 \\
\hline T8_9_3 & 0.493 & 0.483 & -0.081 \\
\hline T8_10_3 & 0.174 & 0.677 & -0.037 \\
\hline T8_11_3 & 0.073 & 0.786 & -0.017 \\
\hline T8_12_3 & 0.078 & 0.780 & 0.023 \\
\hline T8_13_3 & 0.045 & 0.833 & 0.012 \\
\hline T8_14_3 & 0.012 & 0.914 & -0.004 \\
\hline T8_15_3 & 0.042 & 0.837 & 0.011 \\
\hline T8_17_3 & 0.032 & 0.857 & 0.006 \\
\hline T8_19_3 & 0.005 & 0.945 & -0.010 \\
\hline T8_22_3 & 0.110 & 0.741 & 0.031 \\
\hline T8_23_3 & 0.079 & 0.779 & 0.022 \\
\hline
\end{tabular}




$\begin{array}{llll}\text { T8_24_3 } & 0.019 & 0.890 & 0.001 \\ \text { T8_25_3 } & 0.000 & 0.989 & -0.018 \\ \text { T8_26_3 } & 0.170 & 0.680 & -0.011 \\ \text { T8_27_3 } & 0.697 & 0.404 & -0.077 \\ \text { T8_28_3 } & 0.831 & 0.362 & -0.089 \\ \text { T8_29_3 } & 0.537 & 0.464 & -0.058 \\ \text { T8_30_3 } & 0.374 & 0.541 & -0.045 \\ \text { T8_32_3 } & 0.046 & 0.830 & 0.014 \\ \text { T8_33_3 } & 0.667 & 0.414 & -0.074 \\ \text { T8_34_3 } & 0.086 & 0.770 & 0.003 \\ \text { T8_36_3 } & 0.367 & 0.544 & -0.041 \\ \text { T8_40_3 } & 0.899 & 0.343 & -0.093 \\ \text { T8_41_3 } & 0.531 & 0.466 & -0.062 \\ \text { T8_42_3 } & 0.573 & 0.449 & -0.065 \\ \text { T8_43_3 } & 0.430 & 0.512 & -0.051 \\ \text { T8_44_3 } & 1.227 & 0.268 & -0.118 \\ \text { T8_45_3 } & 0.216 & 0.642 & -0.022 \\ \text { T8_47_3 } & 0.373 & 0.541 & -0.046 \\ \text { T8_48_3 } & 0.524 & 0.469 & -0.062 \\ \text { T8_49_3 } & 0.537 & 0.464 & -0.062 \\ \text { T8_1_4 } & 0.018 & 0.893 & -0.007 \\ \text { T8_2_4 } & 0.027 & 0.869 & -0.001 \\ \text { T8_3_4 } & 0.083 & 0.773 & 0.016 \\ \text { T8_4_4 } & 0.005 & 0.943 & 0.006 \\ \text { T8_5_4 } & 0.008 & 0.930 & 0.008 \\ \text { T8_6_4 } & 0.002 & 0.967 & -0.016 \\ \text { T8_7_4 } & 0.014 & 0.904 & 0.014 \\ \text { T8_8_4 } & 0.001 & 0.978 & -0.001 \\ \text { T8_10_4 } & 0.007 & 0.935 & 0.008 \\ \text { T8_12_4 } & 0.016 & 0.898 & 0.014 \\ \text { T8_15_4 } & 0.046 & 0.831 & 0.006 \\ \text { T8_16_4 } & 0.023 & 0.881 & 0.019 \\ \text { T8_373343 } & 0.000 & 0.996 & -0.011 \\ \text { T8_21_4 } & 0.052 & 0.819 & 0.030 \\ \text { T8_23_4 } & 0.042 & 0.837 & 0.027 \\ \text { T8_26_4 } & 0.161 & 0.688 & 0.040 \\ \text { T8_43 } & 0.049 & 0.825 & 0.013 \\ \text { T8_4 } & 0.011 & 0.916 & -0.040 \\ \text { T8 } & 0.018 & 0.894 & -0.044 \\ \text { T8 } & 0.011 & 0.918 & -0.004 \\ \text { T8 } & & 0.637 & 0.050\end{array}$




$\begin{array}{llll} & & & \\ \text { T8_35_4 } & 0.040 & 0.841 & 0.010 \\ \text { T8_38_4 } & 0.023 & 0.880 & 0.002 \\ \text { T8_41_4 } & 0.220 & 0.639 & 0.049 \\ \text { T8_44_4 } & 0.087 & 0.768 & 0.025 \\ \text { T8_45_4 } & 0.000 & 0.985 & -0.027 \\ \text { T8_46_4 } & 0.036 & 0.849 & 0.008 \\ \text { T8_47_4 } & 0.039 & 0.844 & 0.010 \\ \text { T8_48_4 } & 0.092 & 0.762 & 0.027 \\ \text { T8_49_4 } & 0.091 & 0.763 & 0.025 \\ \text { T8_1_5 } & 0.292 & 0.589 & -0.041 \\ \text { T8_2_5 } & 0.405 & 0.525 & -0.054 \\ \text { T8_3_56 } & 0.266 & 0.606 & 0.045 \\ \text { T8_6_5 } & 0.000 & 0.987 & -0.032 \\ \text { T8_7_5 } & 0.132 & 0.716 & 0.033 \\ \text { T8_8_56.1 } & 0.028 & 0.867 & 0.006 \\ \text { T8_10_5 } & 0.012 & 0.912 & -0.006 \\ \text { T8_11_5 } & 0.586 & 0.444 & -0.073 \\ \text { T8_13_5 } & 0.031 & 0.861 & 0.004 \\ \text { T8_14_5 } & 0.000 & 0.991 & -0.025 \\ \text { T8_15_5 } & 0.034 & 0.854 & 0.006 \\ \text { T8_18_5 } & 0.085 & 0.771 & 0.022 \\ \text { T8_19_5 } & 0.031 & 0.860 & 0.004 \\ \text { T8_20_5 } & 0.145 & 0.703 & 0.035 \\ \text { T8_22_5 } & 0.049 & 0.825 & 0.011 \\ \text { T8_23_5 } & 0.035 & 0.852 & 0.005 \\ \text { T8_25_5 } & 0.000 & 0.991 & -0.026 \\ \text { T8_26_5 } & 0.764 & 0.382 & -0.066 \\ \text { T8_29_5 } & 0.080 & 0.778 & 0.028 \\ \text { T8_31_5 } & 0.212 & 0.645 & 0.002 \\ \text { T8_33_5 } & 0.466 & 0.495 & -0.033 \\ \text { T8_35_5 } & 0.276 & 0.599 & -0.007 \\ \text { T8_36_5 } & 0.596 & 0.440 & -0.048 \\ \text { T8_38_5 } & 1.358 & 0.244 & -0.107 \\ \text { T8_40_5 } & 0.203 & 0.653 & 0.002 \\ \text { T8_41_5 } & 0.120 & 0.729 & 0.021 \\ \text { T8_42_5 } & 0.146 & 0.703 & 0.014 \\ \text { T8_44_5 } & 0.683 & 0.409 & -0.059 \\ \text { T845_5 } & 0.451 & 0.502 & -0.031 \\ \text { T8_5 } & 0.281 & 0.596 & -0.007 \\ \text { T8_5 } & 0.062 & 0.804 & 0.032 \\ \text { T8 } & 0.536 & 0.464 & -0.043\end{array}$




\begin{tabular}{lccc} 
T8_1_6 & 0.015 & 0.902 & 0.010 \\
T8_2_6 & 0.006 & 0.938 & 0.004 \\
T8_5_6 & 0.053 & 0.818 & 0.021 \\
T8_7_6 & 0.000 & 0.988 & -0.016 \\
T8_9_6 & 0.668 & 0.414 & -0.070 \\
T8_10_6 & 0.031 & 0.859 & 0.030 \\
T8_15_6 & 0.063 & 0.802 & 0.019 \\
T8_17_6 & 0.151 & 0.698 & -0.003 \\
T8_19_6 & 0.175 & 0.676 & -0.008 \\
T8_20_6 & 0.020 & 0.889 & 0.036 \\
T8_21_6 & 0.259 & 0.611 & -0.023 \\
T8_22_6 & 0.403 & 0.526 & -0.043 \\
T8_23_6 & 0.013 & 0.910 & 0.040 \\
T8_24_6 & 0.100 & 0.752 & 0.008 \\
T8_25_6 & 0.008 & 0.928 & 0.044 \\
T8_28_6 & 0.118 & 0.732 & -0.014 \\
T8_29_6 & 0.788 & 0.375 & 0.069 \\
T8_30_6 & 0.091 & 0.763 & -0.026 \\
T8_31_6 & 0.434 & 0.510 & 0.034 \\
T8_32_6 & 0.052 & 0.820 & -0.038 \\
T8_34_6 & 0.900 & 0.343 & 0.079 \\
T8_35_6 & 0.684 & 0.408 & 0.058 \\
T8_36_6 & 0.021 & 0.885 & -0.049 \\
T8_37_6 & 0.354 & 0.552 & 0.020 \\
T8_38_6 & 0.502 & 0.479 & 0.039 \\
T8_39_6 & 0.023 & 0.880 & -0.100 \\
T8_40_6 & 0.670 & 0.413 & 0.054 \\
T8_42_6 & 0.261 & 0.610 & 0.008 \\
T8_43_6 & 0.661 & 0.416 & 0.058 \\
T8_44_6 & 0.001 & 0.979 & -0.068 \\
T8_46_6 & 0.106 & 0.745 & -0.020 \\
T8_49_6 & 0.518 & 0.472 & 0.042 \\
\hline
\end{tabular}


Table 60

Task 8 DIF: Latinx vs. Sample

\begin{tabular}{lccc}
\hline ItemID & ChiSq & pvalue & EffectSize \\
\hline T8_16_34 & 0.182 & 0.670 & -0.087 \\
T8_18_34 & 0.091 & 0.764 & -0.044 \\
T8_20_34 & 0.129 & 0.719 & -0.063 \\
T8_21_34 & 0.014 & 0.905 & 0.020 \\
T8_37_34 & 0.518 & 0.472 & 0.045 \\
T8_46_34 & 0.254 & 0.614 & -0.042 \\
T8_31_45 & 0.280 & 0.597 & 0.012 \\
T8_43_45 & 0.681 & 0.409 & 0.088 \\
T8_4_56 & 0.407 & 0.523 & -0.123 \\
T8_5_56 & 0.034 & 0.855 & 0.029 \\
T8_8_56 & 0.326 & 0.568 & -0.096 \\
T8_27_56 & 0.140 & 0.708 & -0.039 \\
T8_39_56 & 0.083 & 0.773 & 0.003 \\
T8_43_56 & 0.397 & 0.529 & -0.122 \\
T8_9_456 & 1.390 & 0.239 & -0.216 \\
T8_13_456 & 0.146 & 0.702 & 0.025 \\
T8_20_456 & 0.795 & 0.373 & -0.105 \\
T8_24_456 & 0.879 & 0.349 & -0.123 \\
T8_25_456 & 1.713 & 0.191 & -0.259 \\
T8_1_3 & 0.018 & 0.893 & 0.029 \\
T8_2_3 & 0.018 & 0.894 & 0.029 \\
T8_3_3 & 0.018 & 0.893 & 0.029 \\
T8_4_3 & 0.004 & 0.953 & 0.004 \\
T8_5_3 & 0.048 & 0.827 & 0.055 \\
T8_6_3 & 0.001 & 0.981 & -0.007 \\
T8_8_3 & 0.441 & 0.507 & 0.134 \\
T8_9_3 & 0.244 & 0.621 & 0.070 \\
T8_10_3 & 0.384 & 0.536 & 0.119 \\
T8_11_3 & 0.226 & 0.634 & 0.072 \\
T8_12_3 & 0.015 & 0.903 & 0.025 \\
T8_13_3 & 0.006 & 0.939 & 0.009 \\
T8_14_3 & 0.053 & 0.817 & 0.059 \\
T8_15_3 & 0.004 & 0.953 & 0.004 \\
T8_17_3 & 0.007 & 0.933 & 0.012 \\
T8_19_3 & 0.011 & 0.917 & 0.018 \\
T8_23_3 & 0.003 & 0.956 & 0.003 \\
& 0.000 & 0.983 & -0.009 \\
\hline
\end{tabular}




$\begin{array}{llll}\text { T8_24_3 } & 0.000 & 0.988 & -0.011 \\ \text { T8_25_3 } & 0.025 & 0.874 & 0.035 \\ \text { T8_26_3 } & 0.017 & 0.897 & -0.021 \\ \text { T8_27_3 } & 0.148 & 0.700 & 0.073 \\ \text { T8_28_3 } & 0.224 & 0.636 & 0.098 \\ \text { T8_29_3 } & 0.115 & 0.734 & 0.048 \\ \text { T8_30_3 } & 0.182 & 0.669 & 0.084 \\ \text { T8_32_3 } & 0.208 & 0.648 & 0.087 \\ \text { T8_33_3 } & 0.090 & 0.764 & 0.044 \\ \text { T8_34_3 } & 0.078 & 0.780 & 0.032 \\ \text { T8_36_3 } & 0.158 & 0.691 & 0.068 \\ \text { T8_40_3 } & 0.223 & 0.637 & 0.095 \\ \text { T8_41_3 } & 0.177 & 0.674 & 0.081 \\ \text { T8_42_3 } & 0.008 & 0.928 & -0.023 \\ \text { T8_43_3 } & 0.078 & 0.780 & 0.031 \\ \text { T8_44_3 } & 0.105 & 0.745 & 0.052 \\ \text { T8_45_3 } & 0.104 & 0.747 & 0.047 \\ \text { T8_47_3 } & 0.151 & 0.697 & 0.069 \\ \text { T8_48_3 } & 0.071 & 0.790 & 0.033 \\ \text { T8_49_3 } & 0.055 & 0.815 & 0.018 \\ \text { T8_1_4 } & 0.013 & 0.908 & -0.078 \\ \text { T8_2_4 } & 0.048 & 0.826 & 0.050 \\ \text { T8_3_4 } & 0.001 & 0.971 & -0.027 \\ \text { T8_4_4 } & 0.314 & 0.575 & -0.104 \\ \text { T8_5_4 } & 0.285 & 0.593 & -0.095 \\ \text { T8_6_4 } & 0.157 & 0.692 & -0.046 \\ \text { T8_7_4 } & 0.320 & 0.572 & -0.106 \\ \text { T8_32_4 } & 0.261 & 0.609 & 0.055 \\ \text { T8_33_4 } & 0.452 & 0.501 & 0.111 \\ \text { T8_10_4 } & 0.343 & 0.558 & -0.113 \\ \text { T8_12_4 } & 0.329 & 0.566 & -0.109 \\ \text { T8_15_4 } & 0.101 & 0.750 & 0.079 \\ \text { T8_16_4 } & 0.421 & 0.517 & -0.133 \\ \text { T8_17_4 } & 0.023 & 0.879 & 0.036 \\ \text { T8_21_4 } & 0.093 & 0.761 & -0.016 \\ \text { T8_23_4 } & 0.315 & 0.575 & -0.104 \\ \text { T8_26_4 } & 0.019 & 0.892 & -0.077 \\ \text { T8244 } & 0.001 & 0.971 & -0.135 \\ \text { T844 } & 0.415 & 0.520 & 0.099 \\ \text { T8 } & 0.685 & 0.020\end{array}$




$\begin{array}{lllc}\text { T8_35_4 } & 0.362 & 0.547 & 0.086 \\ \text { T8_38_4 } & 0.027 & 0.870 & -0.063 \\ \text { T8_41_4 } & 0.234 & 0.629 & 0.046 \\ \text { T8_44_4 } & 0.550 & 0.458 & 0.134 \\ \text { T8_45_4 } & 0.105 & 0.746 & -0.009 \\ \text { T8_4644 } & 0.103 & 0.749 & -0.014 \\ \text { T8_47_4 } & 0.072 & 0.788 & -0.023 \\ \text { T8_48_4 } & 0.461 & 0.497 & 0.112 \\ \text { T8_49_4 } & 0.420 & 0.517 & 0.103 \\ \text { T8_155 } & 0.014 & 0.906 & 0.064 \\ \text { T8_2_5 } & 0.003 & 0.957 & -0.012 \\ \text { T8_356 } & 2.064 & 0.151 & -0.258 \\ \text { T8_6_5 } & 0.001 & 0.971 & 0.035 \\ \text { T8_755 } & 0.036 & 0.849 & -0.019 \\ \text { T8_8_56.1 } & 0.326 & 0.568 & -0.096 \\ \text { T8_10_5 } & 0.000 & 0.996 & 0.047 \\ \text { T8_11_5 } & 0.003 & 0.958 & 0.043 \\ \text { T8_13_5 } & 0.026 & 0.873 & -0.008 \\ \text { T8_14_5 } & 0.019 & 0.892 & 0.000 \\ \text { T8_15_5 } & 0.132 & 0.716 & -0.077 \\ \text { T8_18_5 } & 0.028 & 0.867 & -0.010 \\ \text { T8_19_5 } & 0.001 & 0.983 & 0.039 \\ \text { T8_20_5 } & 0.068 & 0.795 & -0.041 \\ \text { T8_22_5 } & 0.001 & 0.977 & 0.054 \\ \text { T8_23_5 } & 0.024 & 0.877 & -0.005 \\ \text { T8_25_5 } & 0.002 & 0.964 & 0.032 \\ \text { T8_26_5 } & 0.067 & 0.796 & 0.057 \\ \text { T8_29_5 } & 0.000 & 0.989 & -0.028 \\ \text { T8_31_5 } & 0.001 & 0.977 & -0.040 \\ \text { T8_33_5 } & 0.000 & 0.989 & -0.050 \\ \text { T8_35_5 } & 0.000 & 0.994 & -0.059 \\ \text { T8_36_5 } & 0.015 & 0.902 & 0.011 \\ \text { T8_38_5 } & 0.004 & 0.949 & -0.009 \\ \text { T8_40_5 } & 0.016 & 0.901 & 0.007 \\ \text { T8_41_5 } & 0.052 & 0.819 & -0.131 \\ \text { T8_42_5 } & 0.012 & 0.914 & 0.002 \\ \text { T8_44_5 } & 0.034 & 0.853 & 0.033 \\ \text { T8_45_5 } & 0.001 & 0.981 & -0.040 \\ \text { T8_46_5 } & 0.040 & 0.841 & 0.030 \\ \text { T8_48_5 } & 0.000 & 0.986 & -0.025 \\ \text { T8_49_5 } & 0.083 & 0.773 & 0.063\end{array}$




\begin{tabular}{|c|c|c|c|}
\hline T8_1_6 & 1.319 & 0.251 & -0.212 \\
\hline T8_2_6 & 1.539 & 0.215 & -0.243 \\
\hline T8_5_6 & 0.136 & 0.712 & -0.089 \\
\hline T8_7_6 & 1.259 & 0.262 & -0.202 \\
\hline T8_9_6 & 0.016 & 0.899 & -0.009 \\
\hline T8_10_6 & 0.024 & 0.878 & -0.017 \\
\hline T8_15_6 & 0.014 & 0.907 & -0.006 \\
\hline T8_17_6 & 0.086 & 0.769 & -0.064 \\
\hline T8_19_6 & 0.088 & 0.766 & -0.065 \\
\hline T8_20_6 & 0.008 & 0.928 & 0.005 \\
\hline T8_21_6 & 0.105 & 0.746 & -0.075 \\
\hline T8_22_6 & 0.074 & 0.786 & -0.057 \\
\hline T8_23_6 & 0.024 & 0.878 & -0.017 \\
\hline T8_24_6 & 0.080 & 0.777 & -0.060 \\
\hline T8_25_6 & 0.053 & 0.818 & -0.042 \\
\hline T8_28_6 & 0.727 & 0.394 & -0.152 \\
\hline T8_29_6 & 0.734 & 0.392 & -0.155 \\
\hline T8_30_6 & 0.091 & 0.763 & 0.041 \\
\hline T8_31_6 & 0.913 & 0.339 & -0.187 \\
\hline T8_32_6 & 0.095 & 0.758 & 0.041 \\
\hline T8_34_6 & 0.701 & 0.403 & -0.149 \\
\hline T8_35_6 & 0.559 & 0.455 & -0.112 \\
\hline T8_36_6 & 0.411 & 0.521 & -0.071 \\
\hline T8_37_6 & 0.438 & 0.508 & -0.082 \\
\hline T8_38_6 & 0.408 & 0.523 & -0.078 \\
\hline T8_39_6 & 0.118 & 0.732 & 0.032 \\
\hline T8_40_6 & 0.368 & 0.544 & -0.067 \\
\hline T8_42_6 & 0.305 & 0.581 & -0.046 \\
\hline T8_43_6 & 0.918 & 0.338 & -0.189 \\
\hline T8_44_6 & 0.058 & 0.809 & 0.063 \\
\hline T8_46_6 & 0.167 & 0.683 & 0.003 \\
\hline T8_49_6 & 0.396 & 0.529 & -0.077 \\
\hline
\end{tabular}


Table 61

Year 1, Grade 1 correlations

\begin{tabular}{lccccccccc}
\hline Task & Task 1 & Task 3 & Task 5 & Task 6 & Task 7 & PPVT & SWE & PDE & TWS \\
\hline Task 1 & 1.00 & & & & & & & & \\
Task 3 & 0.07 & 1.00 & & & & & & & \\
Task 5 & 0.05 & 0.46 & 1.00 & & & & & & \\
Task 6 & 0.03 & 0.53 & 0.62 & 1.00 & & & & & \\
Task 7 & 0.15 & 0.25 & 0.33 & 0.23 & 1.00 & & & & \\
PPVT & 0.00 & 0.14 & 0.23 & 0.14 & 0.23 & 1.00 & & & \\
SWE & 0.06 & 0.33 & 0.39 & 0.40 & 0.18 & 0.16 & 1.00 & & \\
PDE & 0.01 & 0.38 & 0.48 & 0.44 & 0.12 & 0.19 & 0.41 & 1.00 & \\
TWS & 0.04 & 0.39 & 0.47 & 0.46 & 0.18 & 0.18 & 0.46 & 0.33 & 1.00 \\
\hline
\end{tabular}

Note. PPVT = Peabody Picture Vocabulary Test, SWE = TOWRE Sight Word Efficiency, PDE = TOWRE Phoneme Deletion, TWS = Test of Written Spelling 
Table 62

Year 1, Grade 2 correlations

\begin{tabular}{lccccccccc}
\hline Task & Task 1 & Task 3 & Task 5 & Task 6 & Task 7 & PPVT & SWE & PDE & TWS \\
\hline Task 1 & 1.00 & & & & & & & & \\
Task 3 & 0.23 & 1.00 & & & & & & & \\
Task 5 & 0.21 & 0.57 & 1.00 & & & & & & \\
Task 6 & 0.23 & 0.58 & 0.72 & 1.00 & & & & & \\
Task 7 & 0.26 & 0.49 & 0.43 & 0.42 & 1.00 & & & & \\
PPVT & 0.17 & 0.22 & 0.28 & 0.22 & 0.31 & 1.00 & & & \\
SWE & 0.05 & 0.32 & 0.48 & 0.32 & 0.19 & 0.25 & 1.00 & & \\
PDE & 0.05 & 0.09 & 0.31 & 0.17 & 0.05 & 0.05 & 0.19 & 1.00 & \\
TWS & 0.03 & 0.33 & 0.53 & 0.41 & 0.16 & 0.24 & 0.33 & 0.28 & 1.00 \\
\hline
\end{tabular}

Note. PPVT = Peabody Picture Vocabulary Test, SWE = TOWRE Sight Word Efficiency, PDE = TOWRE Phoneme Deletion, TWS = Test of Written Spelling 
Table 63

Year 1, Grade 3 correlations

\begin{tabular}{|c|c|c|c|c|c|c|c|c|c|c|c|}
\hline Task & Task 1 & Task 3 & Task 4 & Task 5 & Task 6 & Task 7 & Task 8 & PPVT & SWE & PDE & TWS \\
\hline Task 1 & 1.00 & & & & & & & & & & \\
\hline Task 3 & 0.09 & 1.00 & & & & & & & & & \\
\hline Task 4 & 0.08 & 0.66 & 1.00 & & & & & & & & \\
\hline Task 5 & 0.09 & 0.61 & 0.67 & 1.00 & & & & & & & \\
\hline Task 6 & 0.10 & 0.62 & 0.62 & 0.73 & 1.00 & & & & & & \\
\hline Task 7 & 0.12 & 0.45 & 0.46 & 0.41 & 0.38 & 1.00 & & & & & \\
\hline Task 8 & 0.12 & 0.67 & 0.73 & 0.74 & 0.68 & 0.51 & 1.00 & & & & \\
\hline PPVT & 0.04 & 0.35 & 0.22 & 0.20 & 0.08 & 0.22 & 0.23 & 1.00 & & & \\
\hline SWE & 0.05 & 0.17 & 0.24 & 0.30 & 0.15 & 0.23 & 0.26 & 0.17 & 1.00 & & \\
\hline PDE & 0.12 & 0.20 & 0.23 & 0.32 & 0.27 & 0.04 & 0.25 & 0.15 & 0.45 & 1.00 & \\
\hline TWS & 0.00 & 0.39 & 0.44 & 0.48 & 0.37 & 0.28 & 0.41 & 0.16 & 0.41 & 0.29 & 1.00 \\
\hline
\end{tabular}

Note. PPVT = Peabody Picture Vocabulary Test, SWE = TOWRE Sight Word Efficiency, PDE = TOWRE Phoneme Deletion, TWS = Test of Written Spelling 
Table 64

Year 1, Grade 4 correlations

\begin{tabular}{|c|c|c|c|c|c|c|c|c|c|c|c|}
\hline Task & Task 1 & Task 3 & Task 4 & Task 5 & Task 6 & Task 7 & Task 8 & PPVT & SWE & PDE & TWS \\
\hline Task 1 & 1.00 & & & & & & & & & & \\
\hline Task 3 & 0.08 & 1.00 & & & & & & & & & \\
\hline Task 4 & 0.08 & 0.65 & 1.00 & & & & & & & & \\
\hline Task 5 & 0.09 & 0.54 & 0.69 & 1.00 & & & & & & & \\
\hline Task 6 & 0.06 & 0.58 & 0.71 & 0.67 & 1.00 & & & & & & \\
\hline Task 7 & 0.08 & 0.47 & 0.49 & 0.45 & 0.38 & 1.00 & & & & & \\
\hline Task 8 & 0.10 & 0.66 & 0.77 & 0.76 & 0.71 & 0.55 & 1.00 & & & & \\
\hline PPVT & -0.05 & 0.40 & 0.36 & 0.35 & 0.28 & 0.43 & 0.34 & 1.00 & & & \\
\hline SWE & 0.00 & 0.25 & 0.23 & 0.36 & 0.18 & 0.19 & 0.25 & 0.19 & 1.00 & & \\
\hline PDE & -0.03 & 0.25 & 0.23 & 0.36 & 0.14 & 0.26 & 0.30 & 0.16 & 0.33 & 1.00 & \\
\hline TWS & 0.01 & 0.32 & 0.38 & 0.49 & 0.38 & 0.20 & 0.38 & 0.15 & 0.30 & 0.22 & 1.00 \\
\hline
\end{tabular}

Note. PPVT = Peabody Picture Vocabulary Test, SWE = TOWRE Sight Word Efficiency, PDE = TOWRE Phoneme Deletion, TWS = Test of Written Spelling 
Table 65

Year 1, Grade 5 correlations

\begin{tabular}{|c|c|c|c|c|c|c|c|c|c|c|c|}
\hline Task & Task 1 & Task 3 & Task 4 & Task 5 & Task 6 & Task 7 & Task 8 & PPVT & SWE & PDE & TWS \\
\hline Task 1 & 1.00 & & & & & & & & & & \\
\hline Task 3 & 0.22 & 1.00 & & & & & & & & & \\
\hline Task 4 & 0.15 & 0.67 & 1.00 & & & & & & & & \\
\hline Task 5 & 0.16 & 0.57 & 0.72 & 1.00 & & & & & & & \\
\hline Task 6 & 0.18 & 0.56 & 0.66 & 0.72 & 1.00 & & & & & & \\
\hline Task 7 & 0.11 & 0.50 & 0.56 & 0.58 & 0.39 & 1.00 & & & & & \\
\hline Task 8 & 0.17 & 0.62 & 0.73 & 0.77 & 0.67 & 0.61 & 1.00 & & & & \\
\hline PPVT & 0.07 & 0.34 & 0.35 & 0.31 & 0.12 & 0.36 & 0.28 & 1.00 & & & \\
\hline SWE & 0.02 & 0.08 & 0.21 & 0.22 & 0.08 & 0.25 & 0.30 & 0.20 & 1.00 & & \\
\hline PDE & 0.01 & 0.03 & 0.04 & 0.21 & 0.04 & 0.16 & 0.13 & 0.13 & 0.44 & 1.00 & 0.24 \\
\hline TWS & 0.00 & 0.30 & 0.39 & 0.51 & 0.28 & 0.29 & 0.41 & 0.18 & 0.30 & 0.24 & 1.00 \\
\hline
\end{tabular}

Note. PPVT = Peabody Picture Vocabulary Test, SWE = TOWRE Sight Word Efficiency, PDE = TOWRE Phoneme Deletion, TWS = Test of Written Spelling 
Table 66

Year 1, Grade 6 correlations

\begin{tabular}{|c|c|c|c|c|c|c|c|c|c|c|c|}
\hline Task & Task 1 & Task 3 & Task 4 & Task 5 & Task 6 & Task 7 & Task 8 & PPVT & SWE & PDE & TWS \\
\hline Task 1 & 1.00 & & & & & & & & & & \\
\hline Task 3 & 0.03 & 1.00 & & & & & & & & & \\
\hline Task 4 & -0.03 & 0.64 & 1.00 & & & & & & & & \\
\hline Task 5 & -0.04 & 0.58 & 0.71 & 1.00 & & & & & & & \\
\hline Task 6 & -0.02 & 0.57 & 0.64 & 0.65 & 1.00 & & & & & & \\
\hline Task 7 & 0.11 & 0.59 & 0.57 & 0.59 & 0.52 & 1.00 & & & & & \\
\hline Task 8 & 0.04 & 0.63 & 0.71 & 0.75 & 0.62 & 0.61 & 1.00 & & & & \\
\hline PPVT & 0.00 & 0.30 & 0.26 & 0.37 & 0.18 & 0.39 & 0.37 & 1.00 & & & \\
\hline SWE & -0.07 & 0.26 & 0.31 & 0.23 & 0.17 & 0.22 & 0.28 & 0.30 & 1.00 & & \\
\hline PDE & -0.08 & 0.09 & 0.23 & 0.26 & 0.14 & 0.20 & 0.22 & 0.10 & 0.32 & 1.00 & \\
\hline TWS & -0.01 & 0.13 & 0.35 & 0.48 & 0.19 & 0.22 & 0.45 & 0.12 & 0.16 & 0.17 & 1.00 \\
\hline
\end{tabular}

Note. PPVT = Peabody Picture Vocabulary Test, SWE = TOWRE Sight Word Efficiency, PDE = TOWRE Phoneme Deletion, TWS = Test of Written Spelling 
Table 67

Year 1, summary r-squared for main effect and interaction effect models of MATRS tasks by grade and outcome

\begin{tabular}{lccccc}
\hline Model & Grade & PPVT & SWE & PDE & TWS \\
\hline Main & 1 & 0.08 & 0.20 & 0.26 & 0.27 \\
& 2 & 0.15 & 0.25 & 0.10 & 0.30 \\
& 3 & 0.16 & 0.11 & 0.14 & 0.27 \\
& 4 & 0.24 & 0.15 & 0.18 & 0.26 \\
& 5 & 0.21 & 0.13 & 0.08 & 0.27 \\
& 6 & 0.2 & 0.13 & 0.12 & 0.35 \\
\hline Interaction & Grade & PPVT & SWE & PDE & TWS \\
& 1 & 0.17 & 0.30 & 0.36 & 0.40 \\
& 2 & 0.29 & 0.33 & 0.17 & 0.42 \\
& 3 & 0.82 & 0.81 & 0.81 & 0.81 \\
& 4 & 0.66 & 0.60 & 0.66 & 0.67 \\
& 5 & 0.72 & 0.71 & 0.73 & 0.76 \\
& 6 & 0.86 & 0.79 & 0.81 & 0.83 \\
\hline
\end{tabular}

Note. PPVT $=$ Peabody Picture Vocabulary Test, SWE $=$ TOWRE Sight Word Efficiency, PDE $=$ TOWRE Phoneme Deletion, TWS $=$ Test of Written Spelling 
Table 68

Year 3 Grade 1, Form A correlations

\begin{tabular}{|c|c|c|c|c|c|c|c|c|c|c|c|c|c|c|c|c|c|c|}
\hline & Task & 1 & 2 & 3 & 4 & 5 & 6 & 7 & 8 & 9 & 10 & 11 & 12 & 13 & 14 & 15 & 16 & 17 \\
\hline 1 & Task 1 & 1.00 & & & & & & & & & & & & & & & & \\
\hline 2 & Task 2 & 0.05 & 1.00 & & & & & & & & & & & & & & & \\
\hline 3 & Task 3 & 0.06 & 0.19 & 1.00 & & & & & & & & & & & & & & \\
\hline 4 & Task 5 & 0.14 & 0.08 & 0.27 & 1.00 & & & & & & & & & & & & & \\
\hline 5 & Task 6 & 0.04 & 0.07 & 0.12 & 0.28 & 1.00 & & & & & & & & & & & & \\
\hline 6 & Task 7 & 0.05 & 0.16 & 0.36 & 0.34 & 0.08 & 1.00 & & & & & & & & & & & \\
\hline 7 & LWID1 & 0.10 & 0.06 & 0.23 & 0.48 & 0.00 & 0.24 & 1.00 & & & & & & & & & & \\
\hline 8 & WA1 & 0.07 & 0.11 & 0.17 & 0.49 & 0.04 & 0.26 & 0.84 & 1.00 & & & & & & & & & \\
\hline 9 & PC1 & 0.04 & 0.14 & 0.19 & 0.43 & -0.02 & 0.26 & 0.84 & 0.80 & 1.00 & & & & & & & & \\
\hline 10 & PPVT1 & 0.07 & 0.13 & 0.30 & 0.26 & 0.01 & 0.44 & 0.38 & 0.41 & 0.44 & 1.00 & & & & & & & \\
\hline 11 & TWS1 & 0.06 & 0.10 & 0.13 & 0.51 & 0.09 & 0.17 & 0.82 & 0.80 & 0.74 & 0.30 & 1.00 & & & & & & \\
\hline 12 & ELI1 & 0.06 & 0.16 & 0.12 & 0.48 & 0.11 & 0.23 & 0.74 & 0.78 & 0.74 & 0.51 & 0.69 & 1.00 & & & & & \\
\hline 13 & BLEND1 & 0.08 & 0.09 & 0.18 & 0.45 & 0.07 & 0.33 & 0.63 & 0.63 & 0.65 & 0.54 & 0.59 & 0.66 & 1.00 & & & & \\
\hline 14 & LWID2 & 0.10 & 0.06 & 0.23 & 0.53 & 0.08 & 0.29 & 0.88 & 0.80 & 0.76 & 0.36 & 0.80 & 0.69 & 0.60 & 1.00 & & & \\
\hline 15 & WA2 & 0.08 & 0.01 & 0.27 & 0.53 & 0.17 & 0.34 & 0.79 & 0.83 & 0.71 & 0.31 & 0.76 & 0.64 & 0.57 & 0.88 & 1.00 & & \\
\hline 16 & PC2 & 0.11 & 0.12 & 0.22 & 0.44 & 0.06 & 0.31 & 0.77 & 0.74 & 0.79 & 0.42 & 0.67 & 0.67 & 0.56 & 0.80 & 0.74 & 1.00 & \\
\hline 17 & TWS2 & 0.05 & 0.09 & 0.29 & 0.56 & 0.19 & 0.26 & 0.84 & 0.80 & 0.73 & 0.24 & 0.81 & 0.72 & 0.60 & 0.87 & 0.82 & 0.70 & 1.00 \\
\hline
\end{tabular}

Note. Outcomes with an appended " 1 " denote fall outcomes; outcomes with an appended "2" denote spring outcomes. PPVT =

Peabody Picture Vocabulary Test, SWE = TOWRE Sight Word Efficiency, PDE $=$ TOWRE Phoneme Deletion, TWS $=$ Test of Written Spelling, LWID = WRMT-3 Letter Word Identification, WA = WRMT-3 Word Attack, ELI = Elision, BLEND = Blending, $\mathrm{PC}=$ Passage Comprehension. 
Table 69

Grade 2, Form A correlations

\begin{tabular}{|c|c|c|c|c|c|c|c|c|c|c|c|c|c|c|c|c|c|c|}
\hline & Task & 1 & 2 & 3 & 4 & 5 & 6 & 7 & 8 & 9 & 10 & 11 & 12 & 13 & 14 & 15 & 16 & 17 \\
\hline 1 & Task 1 & 1.00 & & & & & & & & & & & & & & & & \\
\hline 2 & Task 2 & 0.03 & 1.00 & & & & & & & & & & & & & & & \\
\hline 3 & Task 3 & 0.05 & 0.39 & 1.00 & & & & & & & & & & & & & & \\
\hline 4 & Task 5 & 0.10 & 0.34 & 0.52 & 1.00 & & & & & & & & & & & & & \\
\hline 5 & Task 6 & 0.06 & 0.37 & 0.50 & 0.65 & 1.00 & & & & & & & & & & & & \\
\hline 6 & Task 7 & 0.34 & 0.20 & 0.26 & 0.35 & 0.35 & 1.00 & & & & & & & & & & & \\
\hline 7 & LWID1 & 0.20 & 0.37 & 0.47 & 0.73 & 0.63 & 0.45 & 1.00 & & & & & & & & & & \\
\hline 8 & WA1 & 0.13 & 0.23 & 0.47 & 0.75 & 0.59 & 0.40 & 0.83 & 1.00 & & & & & & & & & \\
\hline 9 & PC1 & 0.18 & 0.35 & 0.47 & 0.56 & 0.53 & 0.44 & 0.73 & 0.68 & 1.00 & & & & & & & & \\
\hline 10 & PPVT1 & 0.21 & 0.43 & 0.60 & 0.54 & 0.51 & 0.64 & 0.62 & 0.58 & 0.59 & 1.00 & & & & & & & \\
\hline 11 & TWS1 & 0.11 & 0.23 & 0.45 & 0.74 & 0.58 & 0.35 & 0.82 & 0.78 & 0.75 & 0.51 & 1.00 & & & & & & \\
\hline 12 & ELI1 & 0.05 & 0.21 & 0.49 & 0.50 & 0.45 & 0.34 & 0.68 & 0.73 & 0.59 & 0.51 & 0.64 & 1.00 & & & & & \\
\hline 13 & BLEND1 & 0.07 & 0.14 & 0.28 & 0.44 & 0.26 & 0.29 & 0.44 & 0.52 & 0.43 & 0.39 & 0.44 & 0.55 & 1.00 & & & & \\
\hline 14 & LWID2 & 0.34 & 0.24 & 0.46 & 0.68 & 0.46 & 0.44 & 0.81 & 0.80 & 0.82 & 0.58 & 0.83 & 0.79 & 0.80 & 1.00 & & & \\
\hline 15 & WA2 & 0.26 & 0.24 & 0.57 & 0.72 & 0.57 & 0.38 & 0.71 & 0.84 & 0.74 & 0.63 & 0.73 & 0.84 & 0.73 & 0.80 & 1.00 & & \\
\hline 16 & PC2 & 0.35 & 0.25 & 0.51 & 0.61 & 0.48 & 0.50 & 0.74 & 0.64 & 0.78 & 0.77 & 0.71 & 0.64 & 0.71 & 0.76 & 0.67 & 1.00 & \\
\hline 17 & TWS2 & 0.34 & 0.33 & 0.60 & 0.78 & 0.58 & 0.43 & 0.85 & 0.83 & 0.76 & 0.65 & 0.84 & 0.89 & 0.76 & 0.86 & 0.83 & 0.71 & 1.00 \\
\hline
\end{tabular}

Note. Outcomes with an appended "1" denote fall outcomes; outcomes with an appended "2" denote spring outcomes. PPVT =

Peabody Picture Vocabulary Test, SWE = TOWRE Sight Word Efficiency, PDE = TOWRE Phoneme Deletion, TWS $=$ Test of Written Spelling, LWID = WRMT-3 Letter Word Identification, WA = WRMT-3 Word Attack, ELI = Elision, BLEND = Blending, PC = Passage Comprehension. 
Table 70

Grade 3, Form A correlations

\begin{tabular}{|c|c|c|c|c|c|c|c|c|c|c|c|c|c|c|c|c|c|c|c|c|}
\hline & Task & 1 & 2 & 3 & 4 & 5 & 6 & 7 & 8 & 9 & 10 & 11 & 12 & 13 & 14 & 15 & 16 & 17 & 18 & 19 \\
\hline 1 & Task 1 & 1.00 & & & & & & & & & & & & & & & & & & \\
\hline 2 & Task 2 & 0.20 & 1.00 & & & & & & & & & & & & & & & & & \\
\hline 3 & Task 3 & 0.07 & 0.34 & 1.00 & & & & & & & & & & & & & & & & \\
\hline 4 & Task 5 & 0.00 & 0.41 & 0.43 & 1.00 & & & & & & & & & & & & & & & \\
\hline 5 & Task 6 & -0.04 & 0.48 & 0.41 & 0.67 & 1.00 & & & & & & & & & & & & & & \\
\hline 6 & Task 7 & 0.12 & 0.33 & 0.39 & 0.40 & 0.44 & 1.00 & & & & & & & & & & & & & \\
\hline 7 & Task 4 & -0.14 & 0.34 & 0.56 & 0.65 & 0.66 & 0.44 & 1.00 & & & & & & & & & & & & \\
\hline 8 & Task 8 & 0.00 & 0.45 & 0.60 & 0.73 & 0.73 & 0.65 & 0.75 & 1.00 & & & & & & & & & & & \\
\hline 9 & LWID1 & -0.05 & 0.38 & 0.48 & 0.63 & 0.63 & 0.51 & 0.70 & 0.74 & 1.00 & & & & & & & & & & \\
\hline 10 & WA1 & 0.06 & 0.43 & 0.39 & 0.74 & 0.59 & 0.43 & 0.65 & 0.67 & 0.80 & 1.00 & & & & & & & & & \\
\hline 11 & PC1 & -0.10 & 0.34 & 0.40 & 0.39 & 0.42 & 0.41 & 0.50 & 0.57 & 0.63 & 0.36 & 1.00 & & & & & & & & \\
\hline 12 & PPVT1 & 0.06 & 0.33 & 0.63 & 0.27 & 0.40 & 0.59 & 0.49 & 0.53 & 0.49 & 0.29 & 0.60 & 1.00 & & & & & & & \\
\hline 13 & TWS1 & 0.01 & 0.39 & 0.32 & 0.76 & 0.70 & 0.42 & 0.59 & 0.71 & 0.73 & 0.71 & 0.49 & 0.28 & 1.00 & & & & & & \\
\hline 14 & ELI1 & -0.05 & 0.44 & 0.38 & 0.61 & 0.62 & 0.40 & 0.57 & 0.57 & 0.59 & 0.57 & 0.42 & 0.42 & 0.61 & 1.00 & & & & & \\
\hline 15 & BLEND1 & 0.12 & 0.35 & 0.42 & 0.34 & 0.41 & 0.42 & 0.48 & 0.50 & 0.52 & 0.46 & 0.34 & 0.45 & 0.43 & 0.63 & 1.00 & & & & \\
\hline 16 & LWID2 & -0.04 & 0.42 & 0.54 & 0.70 & 0.68 & 0.59 & 0.69 & 0.81 & 0.86 & 0.72 & 0.62 & 0.48 & 0.78 & 0.59 & 0.55 & 1.00 & & & \\
\hline 17 & WA2 & 0.01 & 0.32 & 0.42 & 0.66 & 0.58 & 0.54 & 0.66 & 0.74 & 0.69 & 0.72 & 0.49 & 0.40 & 0.67 & 0.55 & 0.54 & 0.77 & 1.00 & & \\
\hline 18 & PC2 & 0.05 & 0.57 & 0.46 & 0.46 & 0.57 & 0.63 & 0.55 & 0.68 & 0.72 & 0.58 & 0.63 & 0.60 & 0.49 & 0.43 & 0.36 & 0.74 & 0.58 & 1.00 & \\
\hline 19 & TWS2 & 0.06 & 0.36 & 0.44 & 0.76 & 0.69 & 0.52 & 0.63 & 0.74 & 0.79 & 0.78 & 0.45 & 0.32 & 0.82 & 0.64 & 0.54 & 0.84 & 0.75 & 0.59 & 1.00 \\
\hline
\end{tabular}

Note. Outcomes with an appended "1" denote fall outcomes; outcomes with an appended "2" denote spring outcomes. PPVT =

Peabody Picture Vocabulary Test, SWE = TOWRE Sight Word Efficiency, PDE $=$ TOWRE Phoneme Deletion, TWS $=$ Test of Written Spelling, LWID = WRMT-3 Letter Word Identification, WA = WRMT-3 Word Attack, ELI = Elision, BLEND = Blending, $\mathrm{PC}=$ Passage Comprehension. 
Table 71

Grade 4, Form A correlations

\begin{tabular}{rlcccccccccccccccccccc}
\hline & Task & 1 & 2 & 3 & 4 & 5 & 6 & 7 & 8 & 9 & 10 & 11 & 12 & 13 & 14 & 15 & 16 & 17 & 18 & 19 \\
\hline 1 & Task 1 & 1.00 & & & & & & & & & & & & & & & \\
2 & Task 2 & 0.04 & 1.00 & & & & & & & & & & & & & \\
3 & Task 3 & 0.05 & 0.42 & 1.00 & & & & & & & & & & & & \\
4 & Task 5 & 0.18 & 0.33 & 0.17 & 1.00 & & & & & & & & & & & \\
5 & Task 6 & 0.03 & 0.44 & 0.33 & 0.51 & 1.00 & 0.32 & & & & & & & & & & \\
6 & Task 7 & 0.02 & 0.24 & 0.16 & 0.39 & 0.32 & 1.00 & & & & & & & & & & \\
7 & Task 4 & 0.33 & 0.47 & 0.36 & 0.64 & 0.56 & 0.28 & 1.00 & & & & & & & & & \\
8 & Task 8 & 0.14 & 0.47 & 0.41 & 0.73 & 0.57 & 0.49 & 0.65 & 1.00 & & & & & & & & \\
9 & LWID1 & 0.18 & 0.48 & 0.41 & 0.72 & 0.61 & 0.35 & 0.68 & 0.66 & 1.00 & & & & & & & \\
10 & WA1 & 0.15 & 0.37 & 0.26 & 0.73 & 0.60 & 0.35 & 0.59 & 0.63 & 0.80 & 1.00 & & & & & & \\
11 & PC1 & 0.32 & 0.52 & 0.37 & 0.64 & 0.59 & 0.46 & 0.72 & 0.64 & 0.70 & 0.62 & 1.00 & & & & & \\
12 & PPVT1 & 0.19 & 0.45 & 0.39 & 0.31 & 0.32 & 0.47 & 0.47 & 0.45 & 0.49 & 0.32 & 0.64 & 1.00 & & & & \\
13 & TWS1 & 0.08 & 0.49 & 0.33 & 0.73 & 0.72 & 0.38 & 0.64 & 0.67 & 0.76 & 0.73 & 0.66 & 0.42 & 1.00 & & & \\
14 & ELI1 & 0.14 & 0.36 & 0.28 & 0.62 & 0.40 & 0.32 & 0.50 & 0.55 & 0.65 & 0.50 & 0.43 & 0.32 & 0.60 & 1.00 & & \\
15 & BLEND1 & 0.13 & 0.04 & 0.06 & 0.13 & 0.03 & 0.12 & 0.08 & 0.12 & 0.28 & 0.09 & 0.21 & 0.25 & 0.11 & 0.27 & 1.00 & \\
16 & LWID2 & 0.09 & 0.49 & 0.37 & 0.77 & 0.58 & 0.48 & 0.66 & 0.70 & 0.85 & 0.78 & 0.67 & 0.37 & 0.68 & 0.60 & 0.11 & 1.00 & \\
17 & WA2 & 0.19 & 0.35 & 0.36 & 0.70 & 0.50 & 0.40 & 0.59 & 0.67 & 0.65 & 0.72 & 0.54 & 0.33 & 0.67 & 0.52 & -0.09 & 0.75 & 1.00 \\
18 & PC2 & 0.26 & 0.47 & 0.39 & 0.45 & 0.39 & 0.38 & 0.56 & 0.54 & 0.64 & 0.50 & 0.68 & 0.57 & 0.54 & 0.42 & 0.20 & 0.63 & 0.58 & 1.00 \\
19 & TWS2 & 0.16 & 0.47 & 0.41 & 0.66 & 0.56 & 0.31 & 0.60 & 0.60 & 0.67 & 0.67 & 0.57 & 0.26 & 0.66 & 0.62 & 0.17 & 0.68 & 0.57 & 0.48 & 1.00 \\
\hline
\end{tabular}

Note. Outcomes with an appended " 1 ” denote fall outcomes; outcomes with an appended "2" denote spring outcomes. PPVT =

Peabody Picture Vocabulary Test, SWE = TOWRE Sight Word Efficiency, PDE $=$ TOWRE Phoneme Deletion, TWS $=$ Test of Written Spelling, LWID = WRMT-3 Letter Word Identification, WA = WRMT-3 Word Attack, ELI = Elision, BLEND = Blending, $\mathrm{PC}=$ Passage Comprehension. 
Table 72

Grade 5, Form A correlations

\begin{tabular}{|c|c|c|c|c|c|c|c|c|c|c|c|c|c|c|c|c|c|c|c|c|}
\hline & Task & 1 & 2 & 3 & 4 & 5 & 6 & 7 & 8 & 9 & 10 & 11 & 12 & 13 & 14 & 15 & 16 & 17 & 18 & 19 \\
\hline 1 & Task 1 & 1.00 & & & & & & & & & & & & & & & & & & \\
\hline 2 & Task 2 & 0.03 & 1.00 & & & & & & & & & & & & & & & & & \\
\hline 3 & Task 3 & 0.16 & 0.33 & 1.00 & & & & & & & & & & & & & & & & \\
\hline 4 & Task 5 & 0.11 & 0.48 & 0.53 & 1.00 & & & & & & & & & & & & & & & \\
\hline 5 & Task 6 & 0.27 & 0.23 & 0.44 & 0.63 & 1.00 & & & & & & & & & & & & & & \\
\hline 6 & Task 7 & 0.27 & 0.36 & 0.62 & 0.51 & 0.56 & 1.00 & & & & & & & & & & & & & \\
\hline 7 & Task 4 & 0.21 & 0.60 & 0.46 & 0.58 & 0.35 & 0.46 & 1.00 & & & & & & & & & & & & \\
\hline 8 & Task 8 & 0.29 & 0.65 & 0.59 & 0.62 & 0.45 & 0.62 & 0.76 & 1.00 & & & & & & & & & & & \\
\hline 9 & LWID1 & 0.25 & 0.55 & 0.61 & 0.72 & 0.51 & 0.65 & 0.70 & 0.85 & 1.00 & & & & & & & & & & \\
\hline 10 & WA1 & 0.15 & 0.44 & 0.48 & 0.63 & 0.50 & 0.54 & 0.55 & 0.61 & 0.75 & 1.00 & & & & & & & & & \\
\hline 11 & PC1 & 0.14 & 0.53 & 0.67 & 0.68 & 0.58 & 0.63 & 0.63 & 0.85 & 0.77 & 0.58 & 1.00 & & & & & & & & \\
\hline 12 & PPVT1 & 0.24 & 0.41 & 0.71 & 0.64 & 0.46 & 0.68 & 0.54 & 0.73 & 0.73 & 0.56 & 0.82 & 1.00 & & & & & & & \\
\hline 13 & TWS1 & 0.29 & 0.50 & 0.49 & 0.63 & 0.66 & 0.51 & 0.67 & 0.75 & 0.80 & 0.69 & 0.69 & 0.56 & 1.00 & & & & & & \\
\hline 14 & ELI1 & 0.34 & 0.24 & 0.40 & 0.51 & 0.46 & 0.54 & 0.52 & 0.54 & 0.62 & 0.69 & 0.60 & 0.59 & 0.62 & 1.00 & & & & & \\
\hline 15 & BLEND1 & 0.28 & 0.23 & 0.33 & 0.40 & 0.41 & 0.47 & 0.32 & 0.43 & 0.30 & 0.19 & 0.43 & 0.52 & 0.22 & 0.41 & 1.00 & & & & \\
\hline 16 & LWID2 & 0.31 & 0.65 & 0.47 & 0.54 & 0.58 & 0.46 & 0.79 & 0.83 & 0.86 & 0.74 & 0.77 & 0.38 & 0.87 & 0.76 & 0.20 & 1.00 & & & \\
\hline 17 & WA2 & 0.17 & 0.54 & 0.45 & 0.42 & 0.77 & 0.53 & 0.87 & 0.77 & 0.65 & 0.63 & 0.87 & 0.37 & 0.76 & 0.61 & 0.39 & 0.84 & 1.00 & & \\
\hline 18 & PC2 & 0.04 & 0.62 & 0.48 & 0.59 & 0.61 & 0.50 & 0.86 & 0.77 & 0.80 & 0.61 & 0.90 & 0.81 & 0.74 & 0.56 & 0.49 & 0.78 & 0.75 & 1.00 & \\
\hline 19 & TWS2 & 0.39 & 0.70 & 0.43 & 0.52 & 0.63 & 0.44 & 0.86 & 0.92 & 0.87 & 0.80 & 0.77 & 0.51 & 0.93 & 0.73 & 0.37 & 0.93 & 0.83 & 0.83 & 1.00 \\
\hline
\end{tabular}

Note. Outcomes with an appended "1" denote fall outcomes; outcomes with an appended "2" denote spring outcomes. PPVT =

Peabody Picture Vocabulary Test, SWE = TOWRE Sight Word Efficiency, PDE = TOWRE Phoneme Deletion, TWS Test of Written Spelling, LWID = WRMT-3 Letter Word Identification, WA = WRMT-3 Word Attack, ELI = Elision, BLEND = Blending, PC = Passage Comprehension. 
Table 73

Grade 6, Form A correlations

\begin{tabular}{|c|c|c|c|c|c|c|c|c|c|c|c|c|c|c|c|c|c|c|c|c|}
\hline & Task & 1 & 2 & 3 & 4 & 5 & 6 & 7 & 8 & 9 & 10 & 11 & 12 & 13 & 14 & 15 & 16 & 17 & 18 & 19 \\
\hline 1 & Task 1 & 1.00 & & & & & & & & & & & & & & & & & & \\
\hline 2 & Task 2 & 0.20 & 1.00 & & & & & & & & & & & & & & & & & \\
\hline 3 & Task 3 & 0.06 & 0.42 & 1.00 & & & & & & & & & & & & & & & & \\
\hline 4 & Task 5 & -0.06 & 0.38 & 0.44 & 1.00 & & & & & & & & & & & & & & & \\
\hline 5 & Task 6 & -0.06 & 0.19 & 0.27 & 0.46 & 1.00 & & & & & & & & & & & & & & \\
\hline 6 & Task 7 & -0.15 & 0.44 & 0.44 & 0.60 & 0.47 & 1.00 & & & & & & & & & & & & & \\
\hline 7 & Task 4 & 0.05 & 0.42 & 0.45 & 0.53 & 0.44 & 0.52 & 1.00 & & & & & & & & & & & & \\
\hline 8 & Task 8 & 0.12 & 0.42 & 0.50 & 0.70 & 0.61 & 0.61 & 0.64 & 1.00 & & & & & & & & & & & \\
\hline 9 & LWID1 & -0.03 & 0.39 & 0.38 & 0.70 & 0.48 & 0.68 & 0.62 & 0.71 & 1.00 & & & & & & & & & & \\
\hline 10 & WA1 & -0.02 & 0.33 & 0.42 & 0.69 & 0.46 & 0.56 & 0.63 & 0.62 & 0.73 & 1.00 & & & & & & & & & \\
\hline 11 & PC1 & -0.09 & 0.34 & 0.38 & 0.52 & 0.35 & 0.61 & 0.38 & 0.46 & 0.59 & 0.53 & 1.00 & & & & & & & & \\
\hline 12 & PPVT1 & -0.04 & 0.42 & 0.49 & 0.44 & 0.39 & 0.65 & 0.50 & 0.53 & 0.58 & 0.34 & 0.56 & 1.00 & & & & & & & \\
\hline 13 & TWS1 & 0.02 & 0.41 & 0.48 & 0.81 & 0.60 & 0.66 & 0.57 & 0.84 & 0.80 & 0.69 & 0.55 & 0.53 & 1.00 & & & & & & \\
\hline 14 & ELI1 & -0.01 & 0.34 & 0.35 & 0.60 & 0.32 & 0.55 & 0.43 & 0.47 & 0.66 & 0.72 & 0.49 & 0.36 & 0.65 & & & & & & \\
\hline 15 & BLEND1 & -0.10 & 0.35 & 0.36 & 0.53 & 0.52 & 0.53 & 0.51 & 0.47 & 0.52 & 0.51 & 0.37 & 0.43 & 0.60 & 0.49 & 1.00 & & & & \\
\hline 16 & LWID2 & -0.18 & 0.38 & 0.54 & 0.58 & 0.74 & 0.47 & 0.33 & 0.33 & 0.48 & 0.52 & 0.68 & 0.43 & 0.65 & 0.48 & 0.48 & 1.00 & & & \\
\hline 17 & WA2 & -0.23 & 0.11 & 0.42 & 0.57 & 0.59 & 0.51 & 0.38 & 0.41 & 0.41 & 0.77 & 0.41 & 0.19 & 0.65 & 0.62 & 0.55 & 0.68 & 1.00 & & \\
\hline 18 & PC2 & -0.03 & 0.63 & 0.63 & 0.53 & 0.73 & 0.66 & 0.70 & 0.63 & 0.65 & 0.51 & 0.64 & 0.71 & 0.67 & 0.59 & 0.35 & 0.75 & 0.51 & 1.00 & \\
\hline 19 & TWS2 & -0.13 & 0.40 & 0.47 & 0.82 & 0.64 & 0.62 & 0.66 & 0.63 & 0.60 & 0.78 & 0.53 & 0.28 & 0.86 & 0.70 & 0.48 & 0.71 & 0.75 & 0.73 & 1.00 \\
\hline
\end{tabular}

Note. Outcomes with an appended " 1 ” denote fall outcomes; outcomes with an appended "2" denote spring outcomes. PPVT =

Peabody Picture Vocabulary Test, SWE = TOWRE Sight Word Efficiency, PDE = TOWRE Phoneme Deletion, TWS = Test of Written Spelling, LWID = WRMT-3 Letter Word Identification, WA = WRMT-3 Word Attack, ELI = Elision, BLEND = Blending, $\mathrm{PC}=$ Passage Comprehension. 
Table 74

Grade 1, Form B correlations

\begin{tabular}{|c|c|c|c|c|c|c|c|c|c|c|c|c|c|c|c|c|c|c|}
\hline & Task & 1 & 2 & 3 & 4 & 5 & 6 & 7 & 8 & 9 & 10 & 11 & 12 & 13 & 14 & 15 & 16 & 17 \\
\hline 1 & Task 1 & 1.00 & & & & & & & & & & & & & & & & \\
\hline 2 & Task 2 & 0.08 & 1.00 & & & & & & & & & & & & & & & \\
\hline 3 & Task 3 & 0.23 & 0.20 & 1.00 & & & & & & & & & & & & & & \\
\hline 4 & Task 5 & 0.08 & 0.23 & 0.36 & 1.00 & & & & & & & & & & & & & \\
\hline 5 & Task 6 & 0.15 & 0.24 & 0.25 & 0.63 & 1.00 & & & & & & & & & & & & \\
\hline 6 & Task 7 & 0.17 & 0.31 & 0.15 & 0.35 & 0.10 & 1.00 & & & & & & & & & & & \\
\hline 7 & LWID1 & 0.07 & 0.25 & 0.22 & 0.71 & 0.42 & 0.33 & 1.00 & & & & & & & & & & \\
\hline 8 & WA1 & 0.09 & 0.20 & 0.20 & 0.71 & 0.44 & 0.38 & 0.88 & 1.00 & & & & & & & & & \\
\hline 9 & PC1 & 0.05 & 0.24 & 0.21 & 0.53 & 0.24 & 0.41 & 0.82 & 0.77 & 1.00 & & & & & & & & \\
\hline 10 & PPVT1 & 0.30 & 0.36 & 0.30 & 0.38 & 0.24 & 0.42 & 0.46 & 0.42 & 0.50 & 1.00 & & & & & & & \\
\hline 11 & TWS1 & 0.08 & 0.23 & 0.25 & 0.81 & 0.52 & 0.36 & 0.83 & 0.82 & 0.72 & 0.53 & 1.00 & & & & & & \\
\hline 12 & ELI1 & 0.11 & 0.17 & 0.26 & 0.69 & 0.41 & 0.31 & 0.65 & 0.73 & 0.62 & 0.49 & 0.78 & 1.00 & & & & & \\
\hline 13 & BLEND1 & 0.06 & 0.18 & 0.22 & 0.49 & 0.22 & 0.33 & 0.61 & 0.66 & 0.54 & 0.43 & 0.58 & 0.54 & 1.00 & & & & \\
\hline 14 & LWID2 & 0.22 & 0.11 & 0.31 & 0.71 & 0.32 & 0.39 & 0.93 & 0.86 & 0.86 & 0.30 & 0.81 & 0.51 & 0.65 & 1.00 & & & \\
\hline 15 & WA2 & 0.03 & 0.10 & 0.14 & 0.71 & 0.32 & 0.41 & 0.86 & 0.89 & 0.75 & 0.30 & 0.79 & 0.56 & 0.67 & 0.86 & 1.00 & & \\
\hline 16 & PC2 & 0.25 & 0.08 & 0.25 & 0.63 & 0.20 & 0.35 & 0.70 & 0.65 & 0.81 & 0.37 & 0.71 & 0.64 & 0.62 & 0.76 & 0.63 & 1.00 & \\
\hline 17 & TWS2 & 0.12 & -0.02 & 0.16 & 0.78 & 0.23 & 0.46 & 0.76 & 0.80 & 0.63 & 0.29 & 0.86 & 0.53 & 0.75 & 0.75 & 0.79 & 0.60 & 1.00 \\
\hline
\end{tabular}

Note. Outcomes with an appended "1" denote fall outcomes; outcomes with an appended "2" denote spring outcomes. PPVT =

Peabody Picture Vocabulary Test, SWE $=$ TOWRE Sight Word Efficiency, PDE $=$ TOWRE Phoneme Deletion, TWS $=$ Test of Written Spelling, LWID = WRMT-3 Letter Word Identification, WA = WRMT-3 Word Attack, ELI = Elision, BLEND = Blending, PC = Passage Comprehension. 
Table 75

Grade 2, Form B correlations

\begin{tabular}{|c|c|c|c|c|c|c|c|c|c|c|c|c|c|c|c|c|c|c|}
\hline & Task & 1 & 2 & 3 & 4 & 5 & 6 & 7 & 8 & 9 & 10 & 11 & 12 & 13 & 14 & 15 & 16 & 17 \\
\hline 1 & Task 1 & 1.00 & & & & & & & & & & & & & & & & \\
\hline 2 & Task 2 & 0.15 & 1.00 & & & & & & & & & & & & & & & \\
\hline 3 & Task 3 & 0.20 & 0.22 & 1.00 & & & & & & & & & & & & & & \\
\hline 4 & Task 5 & 0.16 & 0.34 & 0.36 & 1.00 & & & & & & & & & & & & & \\
\hline 5 & Task 6 & 0.11 & 0.33 & 0.36 & 0.58 & 1.00 & & & & & & & & & & & & \\
\hline 6 & Task 7 & 0.11 & 0.40 & 0.24 & 0.31 & 0.20 & 1.00 & & & & & & & & & & & \\
\hline 7 & LWID1 & 0.19 & 0.23 & 0.31 & 0.68 & 0.36 & 0.17 & 1.00 & & & & & & & & & & \\
\hline 8 & WA1 & 0.27 & 0.24 & 0.31 & 0.66 & 0.33 & 0.24 & 0.83 & 1.00 & & & & & & & & & \\
\hline 9 & PC1 & 0.20 & 0.23 & 0.31 & 0.45 & 0.30 & 0.15 & 0.70 & 0.65 & 1.00 & & & & & & & & \\
\hline 10 & PPVT1 & 0.09 & 0.08 & 0.25 & 0.23 & 0.11 & 0.31 & 0.39 & 0.36 & 0.40 & 1.00 & & & & & & & \\
\hline 11 & TWS1 & 0.18 & 0.22 & 0.26 & 0.68 & 0.40 & 0.09 & 0.78 & 0.76 & 0.67 & 0.33 & 1.00 & & & & & & \\
\hline 12 & ELI1 & 0.21 & 0.27 & 0.35 & 0.62 & 0.34 & 0.33 & 0.62 & 0.67 & 0.48 & 0.30 & 0.60 & 1.00 & & & & & \\
\hline 13 & BLEND1 & 0.19 & 0.20 & 0.21 & 0.39 & 0.21 & 0.09 & 0.51 & 0.62 & 0.43 & 0.26 & 0.53 & 0.45 & 1.00 & & & & \\
\hline 14 & LWID2 & 0.34 & 0.20 & 0.34 & 0.61 & 0.28 & 0.20 & 0.82 & 0.71 & 0.53 & 0.14 & 0.60 & 0.66 & 0.26 & 1.00 & & & \\
\hline 15 & WA2 & 0.18 & 0.31 & 0.17 & 0.70 & 0.40 & 0.25 & 0.77 & 0.77 & 0.51 & 0.12 & 0.63 & 0.74 & 0.40 & 0.77 & 1.00 & & \\
\hline 16 & PC2 & 0.23 & 0.15 & 0.34 & 0.50 & 0.19 & 0.00 & 0.74 & 0.69 & 0.66 & 0.03 & 0.70 & 0.59 & 0.37 & 0.74 & 0.60 & 1.00 & \\
\hline 17 & TWS2 & 0.23 & 0.31 & 0.28 & 0.79 & 0.37 & 0.18 & 0.80 & 0.84 & 0.54 & 0.20 & 0.85 & 0.79 & 0.48 & 0.71 & 0.79 & 0.74 & 1.00 \\
\hline
\end{tabular}

Note. Outcomes with an appended " 1 " denote fall outcomes; outcomes with an appended "2" denote spring outcomes. PPVT =

Peabody Picture Vocabulary Test, SWE = TOWRE Sight Word Efficiency, PDE = TOWRE Phoneme Deletion, TWS $=$ Test of Written Spelling, LWID = WRMT-3 Letter Word Identification, WA = WRMT-3 Word Attack, ELI = Elision, BLEND = Blending, $\mathrm{PC}=$ Passage Comprehension. 
Table 76

Grade 3, Form B correlations

\begin{tabular}{|c|c|c|c|c|c|c|c|c|c|c|c|c|c|c|c|c|c|c|c|c|}
\hline & Task & 1 & 2 & 3 & 4 & 5 & 6 & 7 & 8 & 9 & 10 & 11 & 12 & 13 & 14 & 15 & 16 & 17 & 18 & 19 \\
\hline 1 & Task 1 & 1.00 & & & & & & & & & & & & & & & & & & \\
\hline 2 & Task 2 & 0.01 & 1.00 & & & & & & & & & & & & & & & & & \\
\hline 3 & Task 3 & 0.15 & 0.37 & 1.00 & & & & & & & & & & & & & & & & \\
\hline 4 & Task 5 & 0.13 & 0.49 & 0.46 & 1.00 & & & & & & & & & & & & & & & \\
\hline 5 & Task 6 & 0.15 & 0.35 & 0.28 & 0.61 & 1.00 & & & & & & & & & & & & & & \\
\hline 6 & Task 7 & 0.10 & 0.17 & 0.44 & 0.36 & 0.34 & 1.00 & & & & & & & & & & & & & \\
\hline 7 & Task 4 & 0.25 & 0.22 & 0.56 & 0.55 & 0.56 & 0.34 & 1.00 & & & & & & & & & & & & \\
\hline 8 & Task 8 & 0.11 & 0.41 & 0.56 & 0.73 & 0.58 & 0.50 & 0.71 & 1.00 & & & & & & & & & & & \\
\hline 9 & LWID1 & 0.17 & 0.36 & 0.49 & 0.75 & 0.61 & 0.42 & 0.72 & 0.79 & 1.00 & & & & & & & & & & \\
\hline 10 & WA1 & 0.15 & 0.29 & 0.35 & 0.71 & 0.55 & 0.32 & 0.62 & 0.69 & 0.81 & 1.00 & & & & & & & & & \\
\hline 11 & PC1 & 0.13 & 0.35 & 0.52 & 0.51 & 0.39 & 0.38 & 0.61 & 0.62 & 0.68 & 0.61 & 1.00 & & & & & & & & \\
\hline 12 & PPVT1 & 0.23 & 0.27 & 0.56 & 0.36 & 0.26 & 0.60 & 0.48 & 0.54 & 0.56 & 0.39 & 0.57 & 1.00 & & & & & & & \\
\hline 13 & TWS1 & 0.13 & 0.45 & 0.46 & 0.78 & 0.70 & 0.34 & 0.71 & 0.75 & 0.84 & 0.74 & 0.65 & 0.40 & 1.00 & & & & & & \\
\hline 14 & ELI1 & 0.15 & 0.35 & 0.52 & 0.62 & 0.45 & 0.42 & 0.61 & 0.60 & 0.72 & 0.66 & 0.60 & 0.51 & 0.67 & 1.00 & & & & & \\
\hline 15 & BLEND1 & 0.12 & 0.25 & 0.38 & 0.40 & 0.20 & 0.43 & 0.28 & 0.39 & 0.40 & 0.33 & 0.34 & 0.33 & 0.33 & 0.49 & 1.00 & & & & \\
\hline 16 & LWID2 & 0.07 & 0.33 & 0.46 & 0.77 & 0.58 & 0.21 & 0.68 & 0.69 & 0.89 & 0.76 & 0.59 & 0.54 & 0.88 & 0.67 & 0.28 & 1.00 & & & \\
\hline 17 & WA2 & -0.01 & 0.28 & 0.43 & 0.77 & 0.51 & 0.20 & 0.56 & 0.67 & 0.78 & 0.70 & 0.50 & 0.42 & 0.79 & 0.56 & 0.20 & 0.81 & 1.00 & & \\
\hline 18 & PC2 & 0.03 & 0.11 & 0.38 & 0.38 & 0.24 & 0.22 & 0.55 & 0.50 & 0.59 & 0.49 & 0.45 & 0.46 & 0.50 & 0.53 & 0.23 & 0.60 & 0.53 & 1.00 & \\
\hline 19 & TWS2 & -0.02 & 0.39 & 0.28 & 0.78 & 0.58 & 0.06 & 0.61 & 0.67 & 0.85 & 0.76 & 0.47 & 0.29 & 0.91 & 0.63 & 0.16 & 0.83 & 0.75 & 0.46 & 1.00 \\
\hline
\end{tabular}

Note. Outcomes with an appended " 1 " denote fall outcomes; outcomes with an appended " 2 " denote spring outcomes. PPVT $=$

Peabody Picture Vocabulary Test, SWE = TOWRE Sight Word Efficiency, PDE = TOWRE Phoneme Deletion, TWS $=$ Test of Written Spelling, LWID = WRMT-3 Letter Word Identification, WA = WRMT-3 Word Attack, ELI = Elision, BLEND = Blending, $\mathrm{PC}=$ Passage Comprehension. 
Table 77

Grade 4, Form B correlations

\begin{tabular}{|c|c|c|c|c|c|c|c|c|c|c|c|c|c|c|c|c|c|c|c|c|}
\hline & Task & 1 & 2 & 3 & 4 & 5 & 6 & 7 & 8 & 9 & 10 & 11 & 12 & 13 & 14 & 15 & 16 & 17 & 18 & 19 \\
\hline 1 & Task 1 & 1.00 & & & & & & & & & & & & & & & & & & \\
\hline 2 & Task 2 & 0.21 & 1.00 & & & & & & & & & & & & & & & & & \\
\hline 3 & Task 3 & 0.15 & 0.32 & 1.00 & & & & & & & & & & & & & & & & \\
\hline 4 & Task 5 & 0.19 & 0.35 & 0.34 & 1.00 & & & & & & & & & & & & & & & \\
\hline 5 & Task 6 & 0.15 & 0.38 & 0.38 & 0.59 & 1.00 & & & & & & & & & & & & & & \\
\hline 6 & Task 7 & 0.00 & 0.16 & 0.25 & 0.34 & 0.21 & 1.00 & & & & & & & & & & & & & \\
\hline 7 & Task 4 & 0.12 & 0.24 & 0.41 & 0.49 & 0.47 & 0.31 & 1.00 & & & & & & & & & & & & \\
\hline 8 & Task 8 & 0.13 & 0.42 & 0.40 & 0.70 & 0.62 & 0.42 & 0.61 & 1.00 & & & & & & & & & & & \\
\hline 9 & LWID1 & 0.22 & 0.36 & 0.40 & 0.75 & 0.56 & 0.33 & 0.51 & 0.66 & 1.00 & & & & & & & & & & \\
\hline 10 & WA1 & 0.27 & 0.33 & 0.29 & 0.72 & 0.52 & 0.32 & 0.51 & 0.66 & 0.81 & 1.00 & & & & & & & & & \\
\hline 11 & PC1 & 0.10 & 0.37 & 0.50 & 0.43 & 0.41 & 0.34 & 0.38 & 0.44 & 0.54 & 0.42 & 1.00 & & & & & & & & \\
\hline 12 & PPVT1 & -0.01 & 0.24 & 0.50 & 0.35 & 0.34 & 0.37 & 0.38 & 0.38 & 0.39 & 0.24 & 0.52 & 1.00 & & & & & & & \\
\hline 13 & TWS1 & 0.18 & 0.45 & 0.39 & 0.74 & 0.65 & 0.33 & 0.57 & 0.77 & 0.84 & 0.78 & 0.51 & 0.35 & 1.00 & & & & & & \\
\hline 14 & ELI1 & 0.26 & 0.26 & 0.34 & 0.57 & 0.44 & 0.30 & 0.34 & 0.55 & 0.67 & 0.63 & 0.43 & 0.16 & 0.70 & 1.00 & & & & & \\
\hline 15 & BLEND1 & 0.06 & 0.12 & 0.28 & 0.31 & 0.21 & 0.34 & 0.15 & 0.27 & 0.38 & 0.37 & 0.36 & 0.24 & 0.42 & 0.56 & 1.00 & & & & \\
\hline 16 & LWID2 & 0.16 & 0.46 & 0.46 & 0.71 & 0.57 & 0.37 & 0.51 & 0.73 & 0.83 & 0.77 & 0.60 & 0.42 & 0.86 & 0.70 & 0.31 & 1.00 & & & \\
\hline 17 & WA2 & 0.27 & 0.30 & 0.43 & 0.64 & 0.57 & 0.23 & 0.40 & 0.66 & 0.72 & 0.80 & 0.36 & 0.27 & 0.79 & 0.53 & 0.29 & 0.76 & 1.00 & & \\
\hline 18 & PC2 & 0.27 & 0.36 & 0.47 & 0.35 & 0.40 & 0.19 & 0.26 & 0.55 & 0.37 & 0.39 & 0.46 & 0.38 & 0.55 & 0.44 & 0.19 & 0.49 & 0.44 & 1.00 & \\
\hline 19 & TWS2 & 0.17 & 0.46 & 0.47 & 0.70 & 0.59 & 0.37 & 0.50 & 0.78 & 0.84 & 0.81 & 0.54 & 0.41 & 0.92 & 0.65 & 0.36 & 0.86 & 0.80 & 0.49 & 1.00 \\
\hline
\end{tabular}

Note. Outcomes with an appended " 1 ” denote fall outcomes; outcomes with an appended "2" denote spring outcomes. PPVT =

Peabody Picture Vocabulary Test, SWE = TOWRE Sight Word Efficiency, PDE $=$ TOWRE Phoneme Deletion, TWS $=$ Test of Written Spelling, LWID = WRMT-3 Letter Word Identification, WA = WRMT-3 Word Attack, ELI = Elision, BLEND = Blending, $\mathrm{PC}=$ Passage Comprehension. 
Table 78

Grade 5, Form B correlations

\begin{tabular}{|c|c|c|c|c|c|c|c|c|c|c|c|c|c|c|c|c|c|c|c|c|}
\hline & Task & 1 & 2 & 3 & 4 & 5 & 6 & 7 & 8 & 9 & 10 & 11 & 12 & 13 & 14 & 15 & 16 & 17 & 18 & 19 \\
\hline 1 & Task 1 & 1.00 & & & & & & & & & & & & & & & & & & \\
\hline 2 & Task 2 & 0.29 & 1.00 & & & & & & & & & & & & & & & & & \\
\hline 3 & Task 3 & 0.09 & 0.33 & 1.00 & & & & & & & & & & & & & & & & \\
\hline 4 & Task 5 & 0.18 & 0.18 & 0.46 & 1.00 & & & & & & & & & & & & & & & \\
\hline 5 & Task 6 & 0.29 & 0.37 & 0.30 & 0.44 & 1.00 & & & & & & & & & & & & & & \\
\hline 6 & Task 7 & 0.15 & 0.28 & 0.54 & 0.39 & 0.30 & 1.00 & & & & & & & & & & & & & \\
\hline 7 & Task 4 & 0.23 & 0.27 & 0.45 & 0.58 & 0.52 & 0.42 & 1.00 & & & & & & & & & & & & \\
\hline 8 & Task 8 & 0.31 & 0.40 & 0.51 & 0.66 & 0.49 & 0.48 & 0.65 & 1.00 & & & & & & & & & & & \\
\hline 9 & LWID1 & 0.29 & 0.32 & 0.52 & 0.74 & 0.56 & 0.47 & 0.70 & 0.68 & 1.00 & & & & & & & & & & \\
\hline 10 & WA1 & 0.27 & 0.18 & 0.29 & 0.63 & 0.60 & 0.28 & 0.58 & 0.53 & 0.75 & 1.00 & & & & & & & & & \\
\hline 11 & PC1 & 0.28 & 0.35 & 0.46 & 0.51 & 0.39 & 0.45 & 0.69 & 0.59 & 0.67 & 0.51 & 1.00 & & & & & & & & \\
\hline 12 & PPVT1 & 0.12 & 0.29 & 0.46 & 0.36 & 0.22 & 0.46 & 0.53 & 0.42 & 0.50 & 0.29 & 0.71 & 1.00 & & & & & & & \\
\hline 13 & TWS1 & 0.34 & 0.37 & 0.46 & 0.71 & 0.63 & 0.48 & 0.68 & 0.74 & 0.83 & 0.70 & 0.62 & 0.43 & 1.00 & & & & & & \\
\hline 14 & ELI1 & 0.27 & 0.19 & 0.30 & 0.49 & 0.50 & 0.26 & 0.46 & 0.44 & 0.58 & 0.54 & 0.49 & 0.45 & 0.64 & 1.00 & & & & & \\
\hline 15 & BLEND1 & 0.13 & 0.30 & 0.35 & 0.40 & 0.33 & 0.39 & 0.42 & 0.39 & 0.48 & 0.40 & 0.48 & 0.49 & 0.45 & 0.38 & 1.00 & & & & \\
\hline 16 & LWID2 & 0.39 & 0.41 & 0.53 & 0.61 & 0.57 & 0.35 & 0.64 & 0.67 & 0.85 & 0.71 & 0.64 & 0.53 & 0.78 & 0.48 & 0.58 & 1.00 & & & \\
\hline 17 & WA2 & 0.38 & 0.21 & 0.46 & 0.62 & 0.47 & 0.25 & 0.67 & 0.52 & 0.75 & 0.71 & 0.67 & 0.41 & 0.70 & 0.60 & 0.49 & 0.74 & 1.00 & & \\
\hline 18 & PC2 & 0.22 & 0.38 & 0.37 & 0.51 & 0.50 & 0.41 & 0.51 & 0.50 & 0.53 & 0.38 & 0.56 & 0.46 & 0.61 & 0.53 & 0.47 & 0.57 & 0.43 & 1.00 & \\
\hline 19 & TWS2 & 0.28 & 0.48 & 0.44 & 0.59 & 0.61 & 0.21 & 0.75 & 0.63 & 0.80 & 0.65 & 0.59 & 0.38 & 0.84 & 0.52 & 0.54 & 0.79 & 0.69 & 0.55 & 1.00 \\
\hline
\end{tabular}

Note. Outcomes with an appended " 1 " denote fall outcomes; outcomes with an appended " 2 " denote spring outcomes. PPVT $=$

Peabody Picture Vocabulary Test, SWE = TOWRE Sight Word Efficiency, PDE = TOWRE Phoneme Deletion, TWS $=$ Test of Written Spelling, LWID = WRMT-3 Letter Word Identification, WA = WRMT-3 Word Attack, ELI = Elision, BLEND = Blending, $\mathrm{PC}=$ Passage Comprehension. 
Table 79

Grade 6, Form B correlations

\begin{tabular}{|c|c|c|c|c|c|c|c|c|c|c|c|c|c|c|c|c|c|c|c|c|}
\hline & Task & 1 & 2 & 3 & 4 & 5 & 6 & 7 & 8 & 9 & 10 & 11 & 12 & 13 & 14 & 15 & 16 & 17 & 18 & 19 \\
\hline 1 & Task 1 & 1.00 & & & & & & & & & & & & & & & & & & \\
\hline 2 & Task 2 & -0.04 & 1.00 & & & & & & & & & & & & & & & & & \\
\hline 3 & Task 3 & -0.02 & 0.56 & 1.00 & & & & & & & & & & & & & & & & \\
\hline 4 & Task 5 & -0.03 & 0.49 & 0.63 & 1.00 & & & & & & & & & & & & & & & \\
\hline 5 & Task 6 & -0.13 & 0.37 & 0.50 & 0.43 & 1.00 & & & & & & & & & & & & & & \\
\hline 6 & Task 7 & -0.02 & 0.37 & 0.53 & 0.58 & 0.40 & 1.00 & & & & & & & & & & & & & \\
\hline 7 & Task 4 & -0.05 & 0.41 & 0.59 & 0.64 & 0.37 & 0.46 & 1.00 & & & & & & & & & & & & \\
\hline 8 & Task 8 & -0.10 & 0.54 & 0.62 & 0.70 & 0.56 & 0.61 & 0.53 & 1.00 & & & & & & & & & & & \\
\hline 9 & LWID1 & -0.05 & 0.42 & 0.55 & 0.71 & 0.38 & 0.61 & 0.56 & 0.68 & 1.00 & & & & & & & & & & \\
\hline 10 & WA1 & 0.02 & 0.18 & 0.37 & 0.66 & 0.32 & 0.42 & 0.41 & 0.54 & 0.66 & 1.00 & & & & & & & & & \\
\hline 11 & PC1 & 0.03 & 0.32 & 0.54 & 0.51 & 0.36 & 0.53 & 0.40 & 0.57 & 0.67 & 0.42 & 1.00 & & & & & & & & \\
\hline 12 & PPVT1 & -0.09 & 0.34 & 0.46 & 0.43 & 0.32 & 0.57 & 0.39 & 0.50 & 0.60 & 0.28 & 0.61 & 1.00 & & & & & & & \\
\hline 13 & TWS1 & 0.00 & 0.44 & 0.59 & 0.78 & 0.55 & 0.56 & 0.52 & 0.72 & 0.75 & 0.71 & 0.56 & 0.42 & 1.00 & & & & & & \\
\hline 14 & ELI1 & 0.00 & 0.29 & 0.24 & 0.54 & 0.17 & 0.44 & 0.24 & 0.46 & 0.60 & 0.59 & 0.39 & 0.40 & 0.55 & 1.00 & & & & & \\
\hline 15 & BLEND1 & 0.00 & 0.36 & 0.28 & 0.35 & 0.27 & 0.34 & 0.21 & 0.46 & 0.44 & 0.31 & 0.40 & 0.32 & 0.41 & 0.46 & 1.00 & & & & \\
\hline 16 & LWID2 & 0.27 & 0.44 & 0.71 & 0.88 & 0.24 & 0.59 & 0.39 & 0.82 & 0.84 & 0.67 & 0.63 & 0.53 & 0.86 & 0.68 & 0.61 & 1.00 & & & \\
\hline 17 & WA2 & 0.30 & 0.34 & 0.64 & 0.80 & 0.29 & 0.63 & 0.29 & 0.84 & 0.84 & 0.77 & 0.66 & 0.60 & 0.81 & 0.68 & 0.60 & 0.91 & 1.00 & & \\
\hline 18 & PC2 & 0.28 & 0.54 & 0.55 & 0.58 & 0.38 & 0.62 & 0.22 & 0.68 & 0.66 & 0.39 & 0.72 & 0.70 & 0.76 & 0.52 & 0.28 & 0.68 & 0.65 & 1.00 & \\
\hline 19 & TWS2 & 0.29 & 0.52 & 0.66 & 0.70 & 0.50 & 0.53 & 0.29 & 0.78 & 0.70 & 0.47 & 0.68 & 0.59 & 0.80 & 0.56 & 0.65 & 0.81 & 0.70 & 0.67 & 1.00 \\
\hline
\end{tabular}

Note. Outcomes with an appended "1" denote fall outcomes; outcomes with an appended "2" denote spring outcomes. PPVT =

Peabody Picture Vocabulary Test, SWE = TOWRE Sight Word Efficiency, PDE = TOWRE Phoneme Deletion, TWS $=$ Test of Written Spelling, LWID = WRMT-3 Letter Word Identification, WA = WRMT-3 Word Attack, ELI = Elision, BLEND = Blending, $\mathrm{PC}=$ Passage Comprehension. 
Table 80

Year 3 summary predictive validity of MATRS tasks by form, grade, and outcome.

\begin{tabular}{lccccccccc}
\hline & \multicolumn{2}{c}{ LWID } & \multicolumn{2}{c}{ WA } & \multicolumn{2}{c}{ PC } & \multicolumn{2}{c}{ TWS } \\
Form & Grade & $\mathrm{R} 2$ & $\mathrm{~N}$ & $\mathrm{R} 2$ & $\mathrm{~N}$ & $\mathrm{R} 2$ & $\mathrm{~N}$ & $\mathrm{R} 2$ & $\mathrm{~N}$ \\
\hline Form A & 1 & 0.30 & 123 & 0.31 & 123 & 0.23 & 123 & 0.33 & 123 \\
& 2 & 0.48 & 58 & 0.5 & 58 & 0.47 & 58 & 0.65 & 58 \\
& 3 & 0.71 & 76 & 0.61 & 76 & 0.64 & 76 & 0.68 & 73 \\
& 4 & 0.73 & 58 & 0.58 & 58 & 0.46 & 58 & 0.7 & 58 \\
& 5 & 0.76 & 13 & 0.83 & 13 & 0.88 & 13 & 0.92 & 13 \\
& 6 & 0.77 & 21 & 0.59 & 21 & 0.82 & 21 & 0.86 & 22 \\
\hline Form B & 1 & 0.54 & 27 & 0.56 & 27 & 0.44 & 27 & 0.66 & 27 \\
& 2 & 0.48 & 48 & 0.57 & 48 & 0.37 & 48 & 0.65 & 47 \\
& 3 & 0.67 & 61 & 0.63 & 59 & 0.35 & 60 & 0.69 & 61 \\
& 4 & 0.67 & 72 & 0.58 & 72 & 0.42 & 72 & 0.58 & 70 \\
& 5 & 0.61 & 54 & 0.6 & 54 & 0.45 & 55 & 0.7 & 54 \\
& 6 & 0.91 & 27 & 0.89 & 28 & 0.59 & 27 & 0.67 & 27 \\
\hline
\end{tabular}

Note. TWS $=$ Test of Written Spelling, LWID = WRMT-3 Letter Word Identification, WA = WRMT-3 Word Attack, PC = Passage Comprehension. 
Figure 1. Test Characteristic Curves by Task
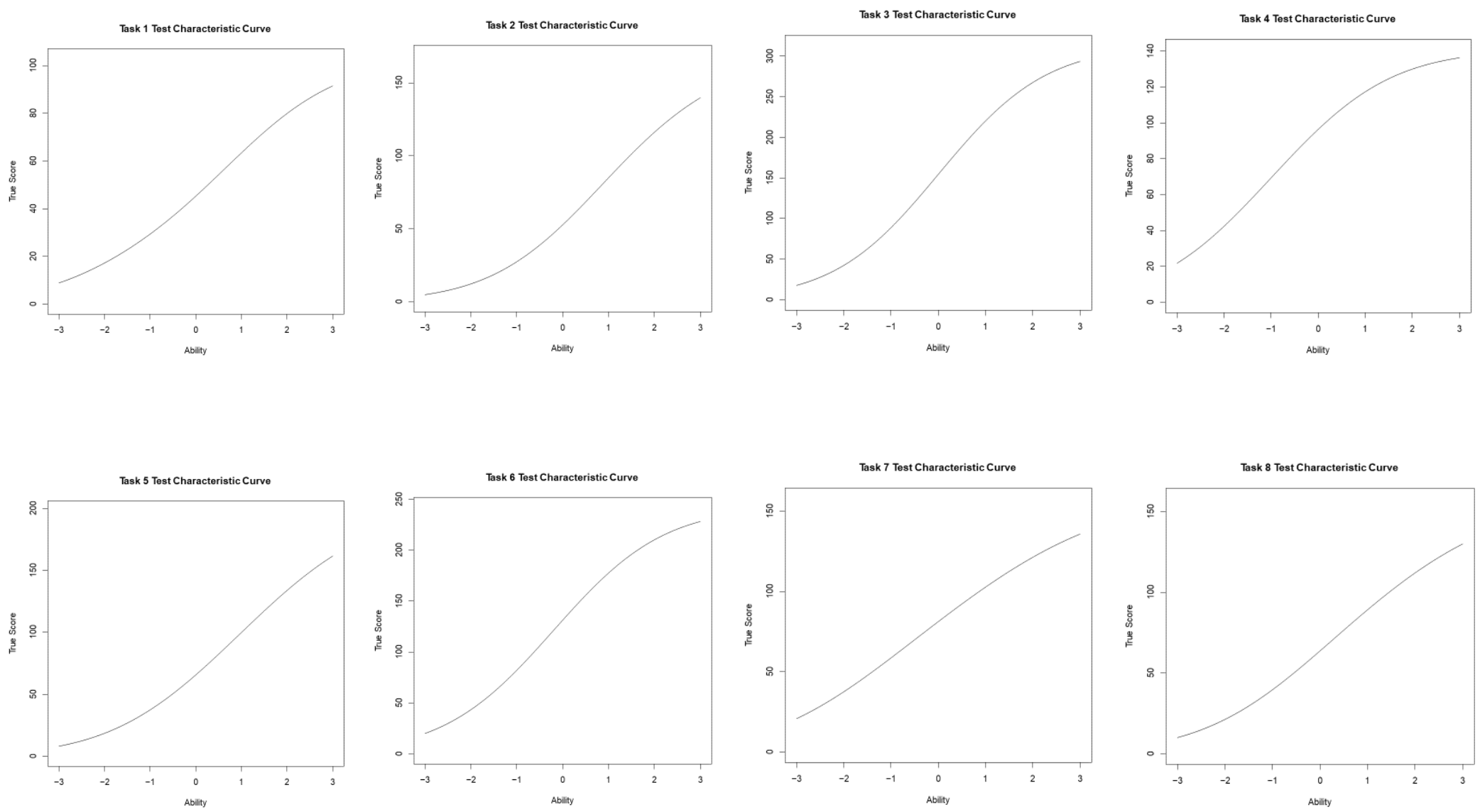
Figure 2. Test Information Functions by Task
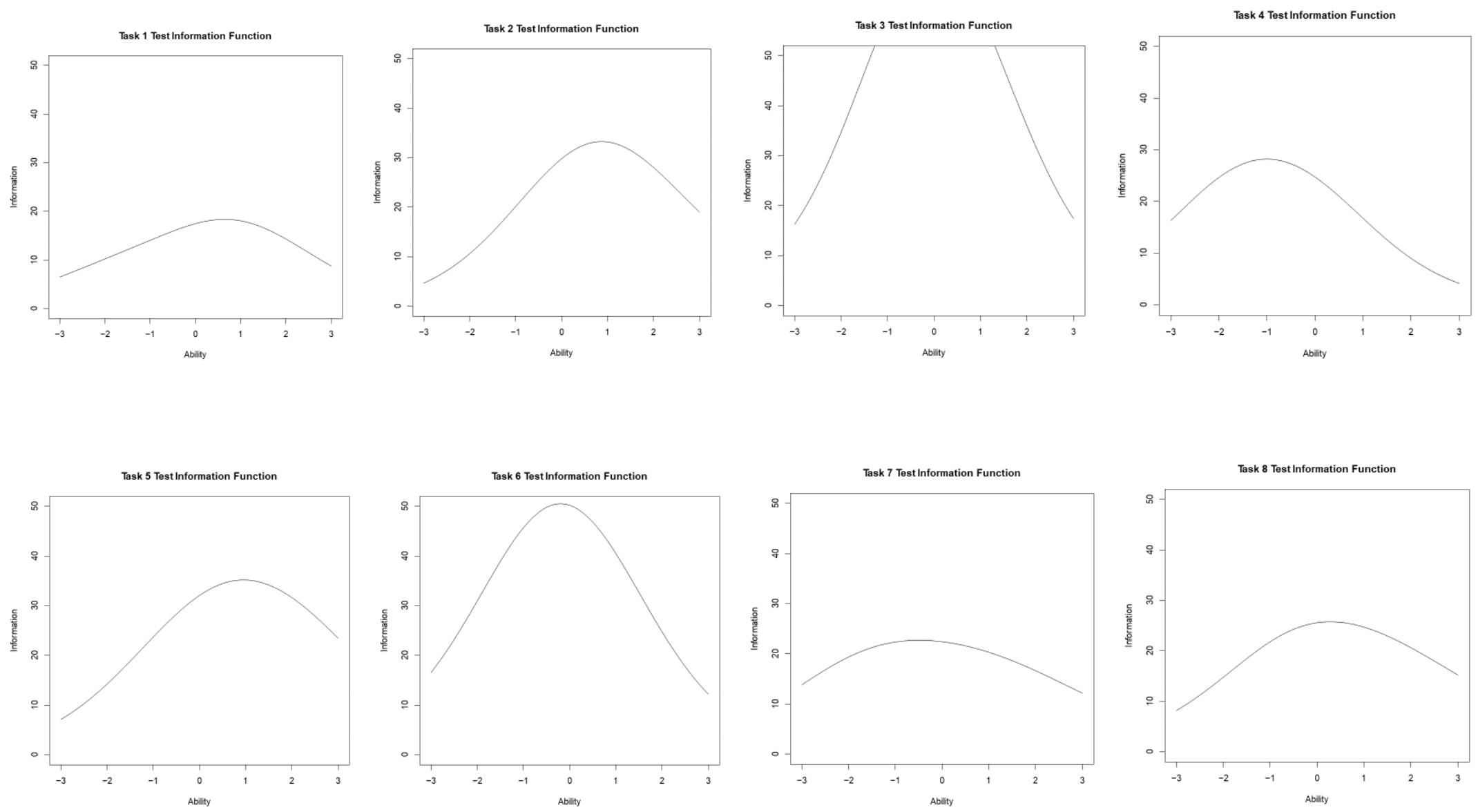